\title{
A TWO WEIGHT LOCAL $T b$ THEOREM FOR THE HILBERT TRANSFORM
}

\author{
ERIC T. SAWYER, CHUN-YEN SHEN, AND IGNACIO URIARTE-TUERO
}

\begin{abstract}
We obtain a two weight local $T b$ theorem for any elliptic and gradient elliptic fractional singular integral operator $T^{\alpha}$ on the real line $\mathbb{R}$, and any pair of locally finite positive Borel measures $(\sigma, \omega)$ on $\mathbb{R}$. The Hilbert transform is included in the case $\alpha=0$, and is bounded from $L^{2}(\sigma)$ to $L^{2}(\omega)$ if and only if the Muckenhoupt and energy conditions hold, as well as $b_{Q}$ and $b_{Q}^{*}$ testing conditions over intervals $Q$, where the families $\left\{b_{Q}\right\}$ and $\left\{b_{Q}^{*}\right\}$ are $p$-weakly accretive for some $p>2$. A number of new ideas are needed to accommodate weak goodness, including a new method for handling the stubborn nearby form, and an additional corona construction to deal with the stopping form. In a sense, this theorem improves the $T 1$ theorem obtained by the authors and M. Lacey in the two part paper LaSaShUr3], Lac].
\end{abstract}

\section{CONTENTS}

1. Introduction

1.1. New ideas

1.2. Standard fractional singular integrals

1.3. Weakly accretive $\mathbf{b}$-testing and $\mathbf{b}^{*}$-testing conditions

1.4. Poisson integrals and the Muckenhoupt condition $\mathfrak{A}_{2}^{\alpha}$

1.5. Energy Conditions

2. The local $T b$ theorem and proof preliminaries

2.1. Reduction to the pointwise lower bound property

2.2. Reduction to real bounded accretive families

2.3. Reverse Hölder control of children

2.4. Three corona decompositions

2.5. Iterated coronas and general stopping data

2.6. Grid parameterizations

2.7. The Monotonicity Lemma

2.8. Organization of the proof

3. Form splittings

3.1. The Hytönen-Martikainen decomposition and a weak variant of NTV goodness

3.2. A characterization of bilinear forms supported on disjoint intervals

4. Disjoint form

4.1. Long range form

4.2. Short range form

5. Nearby form

5.1. Random surgery

5.2. Return of the original testing function

5.3. A finite iteration and final random surgery

6. Main below form

6.1. The canonical splitting and local below forms

6.2. Diagonal and far below forms

6.3. Intertwining Proposition

6.4. Paraproduct, neighbour and broken forms

7. Stopping form

Date: September 25, 2017.

Research supported in part by NSERC.

C.-Y. Shen supported in part by MOST, through grant 104-2628-M-002 -015-MY4.

I. Uriarte-Tuero has been partially supported by grant MTM2015-65792-P (MINECO, Spain). 
7.1. The bound for the second sublinear inequality

7.2. The bound for the first sublinear inequality

7.3. Straddling, Substraddling and Corona-straddling Lemmas 99

7.4. The bottom/up stopping time argument of M. Lacey 106

\begin{tabular}{ll|} 
7.5. The indented corona construction & 109 \\
\hline
\end{tabular}

7.6. Size estimates $\quad 112$

8. Wrapup of the proof $\quad 120$

9. Appendix A: Martingale differences $\quad 121$

9.1. Convergence for weakly and controlled accretive families $\quad 121$

9.2. Frame inequalities 123

9.3. Unbroken projections, broken differences and a Calderón reproducing formula 128

9.4. Weak Riesz inequalities 132

10. Appendix B: Control of functional energy $\quad 136$

10.1. Various energy conditions 138

10.2. The Poisson formulation $\quad 143$

10.3. Poisson testing $\quad 144$

10.4. The forward Poisson testing inequality $\quad 146$

10.5. The backward Poisson testing inequality 156

11. Appendix C: Errata for the Revista paper $\quad 168$

12. Appendix D: Glossary $\quad 172$

References $\quad 175$

\section{INTRODUCTION}

The original $T 1$ theorem of David and Journé [DaJo, which characterized boundedness of a singular integral operator by testing over indicators $\mathbf{1}_{Q}$ of cubes $Q$, was quickly extended to a $T b$ theorem by David, Journé and Semmes [DaJoSe, in which the indicators $\mathbf{1}_{Q}$ were replaced by testing functions $b \mathbf{1}_{Q}$ for an accretive function $b$, i.e. $0<c \leq \operatorname{Re} b \leq|b| \leq C<\infty$. Here the accretive function $b$ could be chosen to adapt well to the operator at hand, resulting in almost immediate verification of the $b$-testing conditions, despite difficulty in verifying the 1-testing conditions. One motivating example of this phenomenon is the boundedness of the Cauchy integral on Lipschitz curves]. See e.g. [Ste, pages 310-316].

Subsequently, M. Christ $\mathrm{Chr}$. obtained a far more robust local $\mathrm{Tb}$ theorem in the setting of homogeneous spaces, in which the testing functions could be further specialized to $b_{Q} \mathbf{1}_{Q}$, where now the accretive functions $b_{Q}$ can be chosen to differ for each cube $Q$. Applications of the local $T b$ theorem included boundedness of layer potentials, see e.g. [AAAHK] and references there; and the Kato problem, see [HoMc], [HoLaMc] and AuHoMcTc: and many authors, including G. David [Dav1]; Nazarov, Treil and Volberg [NTV3, NTV2; Auscher, Hofmann, Muscalu, Tao and Thiele AuHoMuTaTh, Hytönen and Martikainen HyMa, and more recently Lacey and Martikainen LaMa, set about proving extensions of the local $T b$ theorem, for example to include a single upper doubling weight together with weaker upper bounds on the function $b$. But these extensions were modelled on the 'nondoubling' methods that arose in connection with upper doubling measures in the analytic capacity problem, see Mattila, Melnikov and Verdera MaMeVe, G. David Dav1, Dav2, X. Tolsa [Tol], and alsoVolberg [Vol, and were thus constrained to a single weight - a setting in which both the Muckenhoupt and energy conditions follow from the upper doubling condition.

In this paper, we consider only the case of dimension $n=1$, and we adapt methods from the theory of two weight $T 1$ theorems, which arose from [NTV4, Vol, LaSaShUr3, [Lac, [SaShUr7] and [SaShUr9], and were used in $\mathrm{HyMa}$ as well, to prove a two weight local $T b$ theorem. These methods involve the 'testing' perspective toward characterizing two weight norm inequalities for an operator $T$. As suggested by work originating in $[\mathrm{DaJ}]$ and $\underline{\mathrm{Saw}}$, it is plausible to conjecture that a given operator $T$ is bounded from one

\footnotetext{
${ }^{1}$ The problem reduces to boundedness on $L^{2}(\mathbb{R})$ of the singular integral operator $C_{A}$ with kernel $K_{A}(x, y) \equiv$ $\frac{1}{x-y+i(A(x)-A(y))}$, where the curve has graph $\{x+i A(x): x \in \mathbb{R}\}$. Now $b(x) \equiv 1+i A^{\prime}(x)$ is accretive and the $b$-testing condition $\int_{I}\left|C_{A}\left(\mathbf{1}_{I} b\right)(x)\right|^{2} d x \leq \mathfrak{T}_{H}^{b}|I|$ follows from $\left|C_{A}\left(\mathbf{1}_{I} b\right)(x)\right|^{2} \approx \ln \frac{x-\alpha}{\beta-x}$, for $x \in I=[\alpha, \beta]$. In the case of a $C^{1, \delta}$ curve, the kernel $K_{A}$ is $C^{1, \delta}$ and any $T b$ theorem applies with $T=C_{A}$ and $\sigma=\omega=d x$, to show that $C_{A}$ is bounded on $L^{2}(\mathbb{R})$.
} 
weighted space to another if and only if both it and its dual are bounded when tested over a suitable family of functions related geometrically to $T$, e.g. testing over indicators of intervals for fractional integrals $T$ as in $\underline{\mathrm{Saw}}$.

Muckenhoupt conditions: However, for even the simplest singular integral, the Hilbert transform, testing over indicators of intervals no longer suffices, and an additional 'side condition' on the weight pair is required - namely the Muckenhoupt $\mathfrak{A}_{2}$ condition, a simpler form of which was shown by Hunt, Muckenhoupt and Wheeden HuMuWh to characterize the one weight inequality for the Hilbert transform. This side condition is a size condition on the weight pair that is typically shown to be necessary by testing over so-called tails of indicators of intervals, and indeed is known to be necessary for boundedness of a broad class of fractional singular integrals that are 'strongly elliptic'. Using this side condition of Muckenhoupt, the solution of the NTV conjecture, due to the authors and M. Lacey in the two part paper [LaSaShUr3-[Lac, shows that the Hilbert transform $H$ is bounded between weighted $L^{2}$ spaces if and only if the Muckenhoupt condition and the two testing conditions over indicators of intervals all hold. However, the testing conditions for singular integrals, unlike those for positive operators such as fractional integrals, are extremely unstable and in principle difficult to check [LaSaUr2]. On the other hand, given a weight pair, it may be possible to produce a family of testing functions adapted to intervals on which the boundedness of the operator is evident. In such a case, one would like to conclude that finding an appropriately nondegenerate family of such testing functions, for which the corresponding testing conditions hold, is enough to guarantee boundedness of the operator - bringing us back to a local $T b$ theorem. In any event, one would in general like to understand the weakest testing conditions that are sufficient for two weight boundedness of a given operator.

Energy conditions: Our $T b$ theorem lies in this direction, but the method of proof requires in addition a second 'side condition', namely the so-called energy condition, introduced in [LaSaUr2]. The energy condition is necessary for the boundedness of the Hilbert transform, and actually follows there from testing over indicators of intervals and, through the Muckenhoupt condition, testing over tails of indicators of intervals as well. More generally, it is known that the energy condition is necessary for boundedness of gradient elliptic fractional singular integrals on the real line [SaShUr11, but fails to be necessary for certain elliptic singular integrals on the line.

Failure of sufficiency of Muckenhoupt and Energy conditions: However, the weight pair $(\omega, \ddot{\sigma})$ constructed in [LaSaUr2] satisfies the Muckenhoupt and energy conditions, yet fails to satisfy the norm inequality for the Hilbert transform 3 . This shows that, even assuming the necessary conditions of Muckenhoupt and energy, we still need some sort of testing conditions, and our $T b$ theorem essentially leaves the choice of testing conditions at our disposal - subject only to nondegeneracy and size conditions.

For example, in the case of the Hilbert transform, Theorem 7 below roughly says this. As we are dealing with the case of general locally finite positive Borel measures, all intervals appearing in this paper should be assumed to closed on the left and open on the right, except when otherwise noted.

Theorem 1 ( $T b$ for Hilbert transform). Let $H$ denote the Hilbert transform on the real line $\mathbb{R}$, let $\sigma$ and $\omega$ be locally finite positive Borel measures on $\mathbb{R}$. Then $H_{\sigma}$, where $H_{\sigma} f \equiv H(f \sigma)$, is bounded from $L^{2}(\sigma)$ to $L^{2}(\omega)$ if and only if the Muckenhoupt and energy side conditions hold, as well as the $\mathbf{b}$-testing and $\mathbf{b}^{*}$-testing conditions

$$
\int_{I}\left|T_{\sigma}^{\alpha} b_{I}\right|^{2} d \omega \leq\left(\mathfrak{T}_{T^{\alpha}}^{\mathbf{b}}\right)^{2}|I|_{\sigma} \text { and } \int_{J}\left|T_{\omega}^{\alpha, *} b_{J}^{*}\right|^{2} d \sigma \leq\left(\mathfrak{T}_{T^{\alpha}}^{\mathbf{b}^{*}, *}\right)^{2}|J|_{\omega},
$$

taken over two families of test functions $\left\{b_{I}\right\}_{I \in \mathcal{P}}$ and $\left\{b_{J}^{*}\right\}_{J \in \mathcal{P}}$, where $b_{I}$ and $b_{J}^{*}$ are only required to be nondegenerate in an average sense, and to be just slightly better than $L^{2}$ functions themselves, namely $L^{p}$ for some $p>2$.

The families of test functions $\left\{b_{I}\right\}_{I \in \mathcal{P}}$ and $\left\{b_{J}^{*}\right\}_{J \in \mathcal{P}}$ in the $T b$ theorem above are nondegenerate and slightly better than $L^{2}$ functions, but otherwise remain at the disposal of the reader. It is this flexibility in choosing families of test functions that distinguishes this characterization as compared to the corresponding $T 1$ theorem 4 . The $T b$ theorem here generalizes many of the one-weight $T b$ theorems in one dimension, since

\footnotetext{
${ }^{2}$ consider e.g. $d \omega(x)=\delta_{0}(x)$ and $d \sigma(x)=|x| d x$.

${ }^{3}$ The interested reader can easily verify this, or see previous versions of the current paper where details are included.

${ }^{4}$ The energy conditions in (1.12) and (1.13) below are relatively simple, stable and checkable conditions on a weight pair, that are in addition, an almost immediate consequence of the Muckenhoupt side condition and the testing conditions for $H$ over indicators of intervals [LaSaUr2].
} 
in the upper doubling case, the Muckenhoupt $\mathfrak{A}_{2}$ condition and the energy condition easily follow from the upper doubling condition. Recall that in the one-weight case with doubling and upper doubling measures $\mu$, there has been a long and sustained effort to relax the integrability conditions of the testing functions: see e.g. S. Hofmann [Hof] and Alfonseca, Auscher, Axelsson, Hofmann and Kim [AAAHK]. Subsequently, Hytönen- Martikainen HyMa assumed $T b$ in $L^{s}(\mu)$ for some $s>2$, and the one weight theorem with testing functions $b$ in $L^{2}(\mu)$ was attained by Lacey-Martikainen LaMa, but their argument strongly uses methods not immediately available in the two weight setting.

Finally, we point out that the proof of our $T b$ theorem is mostly self-contained, but at the expense of considerable length. This is not just for the convenience of the reader, but mainly because we must repeat much of the proof strategy from [NTV4, LaSaShUr3, Lac, [SaShUr7, SaShUr9] and [SaShUr10], as the new ideas used here force redevelopment of many of the previous arguments in these papers. We now turn to a brief discussion of these new ideas for those readers already acquainted with the theory of $T 1$ and local $T b$ theorems. See the brief schematic (3.4) below for a picture summary of the decompositions involved.

1.1. New ideas. For those readers already familiar with the theory of local $T b$ theorems, we describe here some of the new techniques introduced in this paper to handle the two weight situation. There are many difficulties to be overcome in proving a local $T b$ theorem, even in the one weight setting, as compared to the corresponding $T 1$ theorem, and we indicate four of them now.

(1) First difficulty: In order to control the dual martingale differences for 'breaking' children, i.e. when the testing function corresponding to a child is not the restriction of the testing function of the parent, we need to construct coronas in which the restrictions don't change, and for which the 'breaking' intervals satisfy a Carleson condition. This makes the so-called 'nearby' inner products $\left\langle T_{\sigma}^{\alpha} b_{I}, b_{J}^{*}\right\rangle_{\omega}$, i.e. those in which the intervals $I$ and $J$ are close in both position and scale, extremely difficult to estimate due to the fact that the testing conditions are lost in the corona, except at the tops of coronas, and are replaced with just a weak testing condition. Ironically, these nearby inner products are the easiest to estimate in the proof of a $T 1$ theorem since the testing conditions remain in force in the coronas there. In the one weight setting in [NTV3], HyMa and LaMa, special considerations, such as boundedness of Poisson integrals, are taken into account in handling nearby inner products with random surgery, and are unavailable to us here.

Resolution: We develop a new recursive method for controlling the nearby form by the energy conditions and testing at the tops of the coronas, in which we resurrect the original testing functions discarded during the corona construction. This is presented in Section 5 ,

(2) Second difficulty: Both dual martingale and martingale differences fail to satisfy two-sided framelike and Riesz-like inequalities in the setting of a $T b$ theorem when $p=2$, complicating the treatment of paraproducts.

Resolution: We assume $p>2$ in the upper $L^{p}$ control of testing functions, and then reduce this case to that of bounded testing functions using an absorption and recursion argument. For families of bounded testing functions, we prove two-sided Riesz-like inequalities for dual martingale differences that are more robust than frame inequalities (but only one-sided Riesz-like inequalities for martingale differences), and that enable many of the $T 1$ two weight techniques to carry over here in the $T b$ setting. In particular these are key to controlling paraproducts here.

(3) Third difficulty: Only a weaker form of goodness due to Hytönen and Martikainen HyMa is available for use in two weight $T b$ theorems, complicating Lacey's treatment of the stopping form.

Resolution: We adapt the two weight $T 1$ arguments to accommodate weak goodness in two ways, the first highly nontrivial and second more straightforward:

(1) in bounding the stopping form by Lacey's size functional on admissible collections and bottom/up corona construction in [Lac, as adapted in [SaShUr9] and SaShUr10, but using an additional top/down 'indented' corona construction, along with an enlargement of the skeleton skel $(I)$ of an interval $I$ to the body body $(I)$ of $I$, in order to deal with the lack of goodness in telescoping intervals - see Section 7 .

(2) in controlling functional energy as in [SaShUr9] and [SaShUr10], but with a different decomposition of the stopping intervals into 'Whitney' intervals, and modified pseudoprojections to accommodate two independent families of grids - see Appendix B. 
(4) Fourth difficulty: The dual martingale differences are not in general projections when some of the children 'break', and the Monotonicity Lemma fails to hold in any of the traditional forms in the setting of $T 1$ theorems.

Resolution: We introduce an additional square function bound on the right hand side involving an infimum of averages, $\inf _{z \in \mathbb{R}} \sum_{J^{\prime} \in \mathfrak{C}_{\text {broken }}(J)}\left|J^{\prime}\right|_{\omega}\left(E_{J^{\prime}}^{\omega}|x-z|\right)^{2}$, summed over broken children. We also use the fact that the corresponding 'unbroken' dual martingale differences form projections, but then we also need to modify the testing function at the top of a corona, and also refine the triple corona construction, so that dual martingale differences have controlled averages on children throughout the corona.

1.2. Standard fractional singular integrals. Let $0 \leq \alpha<1$. We define a standard $\alpha$-fractional CZ kernel $K^{\alpha}(x, y)$ to be a real-valued function defined on $\mathbb{R} \times \mathbb{R}$ satisfying the following fractional size and smoothness conditions of order $1+\delta$ for some $\delta>0$ : For $x \neq y$,

$$
\begin{aligned}
\left|K^{\alpha}(x, y)\right| & \leq C_{C Z}|x-y|^{\alpha-1} \text { and }\left|\nabla K^{\alpha}(x, y)\right| \leq C_{C Z}|x-y|^{\alpha-2}, \\
\left|\nabla K^{\alpha}(x, y)-\nabla K^{\alpha}\left(x^{\prime}, y\right)\right| & \leq C_{C Z}\left(\frac{\left|x-x^{\prime}\right|}{|x-y|}\right)^{\delta}|x-y|^{\alpha-2}, \quad \frac{\left|x-x^{\prime}\right|}{|x-y|} \leq \frac{1}{2},
\end{aligned}
$$

and the last inequality also holds for the adjoint kernel in which $x$ and $y$ are interchanged. We note that a more general definition of kernel has only order of smoothness $\delta>0$, rather than $1+\delta$, but the use of the Monotonicity and Energy Lemmas in arguments below involves first order Taylor approximations to the kernel functions $K^{\alpha}(\cdot, y)$.

1.2.1. Defining the norm inequality. We now turn to a precise definition of the weighted norm inequality

$$
\left\|T_{\sigma}^{\alpha} f\right\|_{L^{2}(\omega)} \leq \mathfrak{N}_{T_{\sigma}^{\alpha}}\|f\|_{L^{2}(\sigma)}, \quad f \in L^{2}(\sigma) .
$$

For this we follow the lead of [NTV3] and introduce a family $\left\{\eta_{\delta, R}^{\alpha}\right\}_{0<\delta<R<\infty}$ of nonnegative functions on $[0, \infty)$ so that the truncated kernels $K_{\delta, R}^{\alpha}(x, y)=\eta_{\delta, R}^{\alpha}(|x-y|) K^{\alpha}(x, y)$ are bounded with compact support for fixed $x$ or $y$. Then the truncated operators

$$
T_{\sigma, \delta, R}^{\alpha} f(x) \equiv \int_{\mathbb{R}} K_{\delta, R}^{\alpha}(x, y) f(y) d \sigma(y), \quad x \in \mathbb{R},
$$

are pointwise well-defined, and we will refer to the pair $\left(K^{\alpha},\left\{\eta_{\delta, R}^{\alpha}\right\}_{0<\delta<R<\infty}\right)$ as an $\alpha$-fractional singular integral operator, which we typically denote by $T^{\alpha}$, suppressing the dependence on the truncations.

Definition 2. We say that an $\alpha$-fractional singular integral operator $T^{\alpha}=\left(K^{\alpha},\left\{\eta_{\delta, R}^{\alpha}\right\}_{0<\delta<R<\infty}\right)$ satisfies the norm inequality (1.2) provided

$$
\left\|T_{\sigma, \delta, R}^{\alpha} f\right\|_{L^{2}(\omega)} \leq \mathfrak{N}_{T_{\sigma}^{\alpha}}\|f\|_{L^{2}(\sigma)}, \quad f \in L^{2}(\sigma), 0<\delta<R<\infty
$$

It turns out that, in the presence of the Muckenhoupt conditions (1.11) below, the norm inequality (1.2) is essentially independent of the choice of truncations used, and this is explained in some detail in [NTV3], LaSaShUr3] and SaShUr10. Thus, as in [SaShUr10, we are free to use the tangent line truncations described there throughout the proofs of our results.

1.3. Weakly accretive $\mathbf{b}$-testing and $\mathbf{b}^{*}$-testing conditions. Denote by $\mathcal{P}$ the collection of intervals in $\mathbb{R}$. Note that we include an $L^{p}$ upper bound in our definition of ' $p$-weakly accretive family' of functions.

Definition 3. Let $p \geq 2$ and let $\mu$ be a locally finite positive Borel measure on $\mathbb{R}$. We say that a family $\mathbf{b}=\left\{b_{Q}\right\}_{Q \in \mathcal{P}}$ of functions indexed by $\mathcal{P}$ is a $p$-weakly $\mu$-accretive family of functions on $\mathbb{R}$ if

$$
\begin{aligned}
& \operatorname{support} b_{Q} \subset Q, \quad Q \in \mathcal{P}, \\
& 1 \leq\left|\frac{1}{|Q|_{\mu}} \int_{Q} b_{Q} d \mu\right| \leq\left(\frac{1}{|Q|_{\mu}} \int_{Q}\left|b_{Q}\right|^{p} d \mu\right)^{\frac{1}{p}} \leq C_{\mathbf{b}}<\infty, \quad Q \in \mathcal{P} .
\end{aligned}
$$


Suppose $\sigma$ and $\omega$ are locally finite positive Borel measures on $\mathbb{R}$. The $\mathbf{b}$-testing conditions for $T^{\alpha}$ and $\mathbf{b}^{*}$-testing conditions for the dual $T^{\alpha, *}$ are given by

$$
\begin{aligned}
\int_{Q}\left|T_{\sigma}^{\alpha} b_{Q}\right|^{2} d \omega & \leq\left(\mathfrak{T}_{T^{\alpha}}^{\mathbf{b}}\right)^{2}|Q|_{\sigma}, \quad \text { for all intervals } Q, \\
\int_{Q}\left|T_{\omega}^{\alpha, *} b_{Q}^{*}\right|^{2} d \sigma & \leq\left(\mathfrak{T}_{T^{\alpha, *}}^{\mathbf{b}^{*}}\right)^{2}|Q|_{\omega}, \quad \text { for all intervals } Q,
\end{aligned}
$$

where these inequalities are interpreted as holding uniformly over truncations of $T_{\sigma}^{\alpha}$ and $T^{\alpha, *}$.

1.4. Poisson integrals and the Muckenhoupt condition $\mathfrak{A}_{2}^{\alpha}$. Let $\mu$ be a locally finite positive Borel measure on $\mathbb{R}$, and suppose $Q$ is an interval in $\mathbb{R}$. Recall that $|Q|=\ell(Q)$ for an interval $Q$. The two $\alpha$-fractional Poisson integrals of $\mu$ on an interval $Q$ are given by the following expressions:

$$
\begin{aligned}
\mathrm{P}^{\alpha}(Q, \mu) & \equiv \int_{\mathbb{R}} \frac{|Q|}{\left(|Q|+\left|x-x_{Q}\right|\right)^{2-\alpha}} d \mu(x), \\
\mathcal{P}^{\alpha}(Q, \mu) & \equiv \int_{\mathbb{R}}\left(\frac{|Q|}{\left(|Q|+\left|x-x_{Q}\right|\right)^{2}}\right)^{1-\alpha} d \mu(x),
\end{aligned}
$$

where $\left|x-x_{Q}\right|$ denotes distance between $x$ and $x_{Q}$ and $|Q|$ denotes the Lebesgue measure of the interval $Q$. We refer to $\mathrm{P}^{\alpha}$ as the standard Poisson integral and to $\mathcal{P}^{\alpha}$ as the reproducing Poisson integral. Note that these two kernels satisfy

$$
0 \leq \mathrm{P}^{\alpha}(Q, \mu) \leq C \mathcal{P}^{\alpha}(Q, \mu), \quad \text { for all intervals } Q \text { and positive measures } \mu .
$$

We now define the one-tailed Muckenhoupt constant with holes $\mathcal{A}_{2}^{\alpha}$ using the reproducing Poisson kernel $\mathcal{P}^{\alpha}$. On the other hand, the standard Poisson integral $\mathrm{P}^{\alpha}$ arises naturally throughout the proof of the $T b$ theorem in estimating oscillation of the fractional singular integral $T^{\alpha}$, and in the definition of the energy conditions below.

Definition 4. Suppose $\sigma$ and $\omega$ are locally finite positive Borel measures on $\mathbb{R}$. The one-tailed Muckenhoupt constants $\mathcal{A}_{2}^{\alpha}$ and $\mathcal{A}_{2}^{\alpha, *}$ with holes for the weight pair $(\sigma, \omega)$ are given by

$$
\begin{aligned}
\mathcal{A}_{2}^{\alpha} & \equiv \sup _{Q \in \mathcal{P}} \mathcal{P}^{\alpha}\left(Q, \mathbf{1}_{Q^{c}} \sigma\right) \frac{|Q|_{\omega}}{|Q|^{1-\alpha}}<\infty, \\
\mathcal{A}_{2}^{\alpha, *} & \equiv \sup _{Q \in \mathcal{P}} \mathcal{P}^{\alpha}\left(Q, \mathbf{1}_{Q^{c}} \omega\right) \frac{|Q|_{\sigma}}{|Q|^{1-\alpha}}<\infty .
\end{aligned}
$$

Note that these definitions are the conditions with 'holes' introduced by Hytönen [Hyt - the supports of the measures $\mathbf{1}_{Q^{c}} \sigma$ and $\mathbf{1}_{Q} \omega$ in the definition of $\mathcal{A}_{2}^{\alpha}$ are disjoint, and so any common point masses of $\sigma$ and $\omega$ do not appear simultaneously in the factors of any of the products $\mathcal{P}^{\alpha}\left(Q, \mathbf{1}_{Q^{c} \sigma} \sigma\right) \frac{|Q|_{\omega}}{|Q|^{1-\alpha}}$. We will also use the smaller 'offset' Muckenhoupt condition

$$
A_{2}^{\alpha} \equiv \sup _{Q \text { and } Q^{\prime} \text { are adjacent, } \ell(Q)=\ell\left(Q^{\prime}\right)} \frac{|Q|_{\omega}}{|Q|^{1-\alpha}} \frac{\left|Q^{\prime}\right|_{\sigma}}{\left|Q^{\prime}\right|^{1-\alpha}}<\infty,
$$

but the classical Muckenhoupt condition $A_{2}^{\alpha, \text { class }} \equiv \sup _{Q \in \mathcal{P}} \frac{|Q|_{\omega}|Q|_{\sigma}}{|Q|^{2-2 \alpha}}<\infty$ will find no use in the two weight setting with common point masses permitted.

Remark 5. Initially, these definitions of Muckenhoupt type were given in the following 'one weight' case, $d \omega(x)=w(x) d x$ and $d \sigma(x)=\frac{1}{w(x)} d x$, where $\mathcal{A}_{2}^{\alpha}\left(\lambda w,(\lambda w)^{-1}\right)=\mathcal{A}_{2}^{\alpha}\left(w, w^{-1}\right)$ is homogeneous of degree 0. Of course the two weight version is homogeneous of degree 2 in the weight pair, $\mathcal{A}_{2}^{\alpha}(\lambda \sigma, \lambda \omega)=\lambda^{2} \mathcal{A}_{2}^{\alpha}(\sigma, \omega)$, while all of the other conditions we consider in connection with two weight norm inequalities, including the operator norm $\mathfrak{N}_{T^{\alpha}}(\sigma, \omega)$ itself, are homogeneous of degree 1 in the weight pair. This awkwardness regarding the homogeneity of Muckenhoupt conditions could be rectified by simply taking the square root of $\mathcal{A}_{2}^{\alpha}$ and renaming it, but the current definition is so entrenched in the literature, in particular in connection with the $A_{2}$ conjecture, that we will leave it as is. 
1.4.1. Punctured $A_{2}^{\alpha}$ conditions. The classical $A_{2}^{\alpha}$ characteristic $\sup _{Q \in \mathcal{P}} \frac{|Q|_{\omega}}{|Q|^{1-\alpha}} \frac{|Q|_{\sigma}}{|Q|^{1-\alpha}}$ fails to be finite when the measures $\sigma$ and $\omega$ have a common point mass - simply let $Q$ in the sup above shrink to a common mass point. But there is a substitute that is quite similar in character that is motivated by the fact that for large intervals $Q$, the sup above is problematic only if just one of the measures is mostly a point mass when restricted to $Q$.

Given an at most countable set $\mathfrak{P}=\left\{p_{k}\right\}_{k=1}^{\infty}$ in $\mathbb{R}$, an interval $Q \in \mathcal{P}$, and a positive locally finite Borel measure $\mu$, define

$$
\mu(Q, \mathfrak{P}) \equiv|Q|_{\mu}-\sup \left\{\mu\left(p_{k}\right): p_{k} \in Q \cap \mathfrak{P}\right\}
$$

where the supremum is actually achieved since $\sum_{p_{k} \in Q \cap \mathfrak{P}} \mu\left(p_{k}\right)<\infty$ as $\mu$ is locally finite. The quantity $\mu(Q, \mathfrak{P})$ is simply the $\widetilde{\mu}$ measure of $Q$ where $\widetilde{\mu}$ is the measure $\mu$ with its largest point mass from $\mathfrak{P}$ in $Q$ removed. Given a locally finite positive measure pair $(\sigma, \omega)$, let

$$
\mathfrak{P}_{(\sigma, \omega)}=\left\{p_{k}\right\}_{k=1}^{\infty}
$$

be the at most countable set of common point masses of $\sigma$ and $\omega$. Then the weighted norm inequality (1.2) typically implies finiteness of the following punctured Muckenhoupt conditions:

$$
\begin{aligned}
A_{2}^{\alpha, p u n c t}(\sigma, \omega) & \equiv \sup _{Q \in \mathcal{P}} \frac{\omega\left(Q, \mathfrak{P}_{(\sigma, \omega)}\right)}{|Q|^{1-\alpha}} \frac{|Q|_{\sigma}}{|Q|^{1-\alpha}}, \\
A_{2}^{\alpha, *, p u n c t}(\sigma, \omega) & \equiv \sup _{Q \in \mathcal{P}} \frac{|Q|_{\omega}}{|Q|^{1-\alpha}} \frac{\sigma\left(Q, \mathfrak{P}_{(\sigma, \omega)}\right)}{|Q|^{1-\alpha}} .
\end{aligned}
$$

All of the above Muckenhuopt conditions $\mathcal{A}_{2}^{\alpha}, \mathcal{A}_{2}^{\alpha, *}, A_{2}^{\alpha, \text { punct }}$ and $A_{2}^{\alpha, *, \text { punct }}$ are necessary for boundedness of an elliptic $\alpha$-fractional singular integral $T_{\sigma}^{\alpha}$ on the line from $L^{2}(\sigma)$ to $L^{2}(\omega)$ (see SaShUr10]). It is convenient to define

$$
\mathfrak{A}_{2}^{\alpha} \equiv \mathcal{A}_{2}^{\alpha}+\mathcal{A}_{2}^{\alpha, *}+A_{2}^{\alpha, \text { punct }}+A_{2}^{\alpha, *, \text { punct }}
$$

1.5. Energy Conditions. Here is the definition of the strong energy conditions, which we sometimes refer to simply as the energy conditions. Let $m_{I}^{\mu} \equiv \frac{1}{\mid I} \int x d \mu(x)$ be the average of $x$ over $I$ with respect to the measure $\mu$, which we often abbreviate to $m_{I}$ when the measure $\mu$ is understood.

Definition 6. Let $0 \leq \alpha<1$. Suppose $\sigma$ and $\omega$ are locally finite positive Borel measures on $\mathbb{R}$. Then the strong energy constant $\mathcal{E}_{2}^{\alpha}$ is defined by

$$
\left(\mathcal{E}_{2}^{\alpha}\right)^{2} \equiv \sup _{I=\dot{\cup} I_{r}} \frac{1}{|I|_{\sigma}} \sum_{r=1}^{\infty}\left(\frac{\mathrm{P}^{\alpha}\left(I_{r}, \mathbf{1}_{I} \sigma\right)}{\left|I_{r}\right|}\right)^{2}\left\|x-m_{I_{r}}^{\omega}\right\|_{L^{2}\left(\mathbf{1}_{I_{r}} \omega\right)}^{2},
$$

where the supremum is taken over arbitrary decompositions of an interval I using a pairwise disjoint union

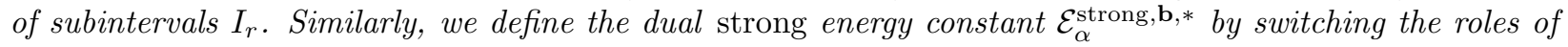
$\sigma$ and $\omega$ :

$$
\left(\mathcal{E}_{2}^{\alpha, *}\right)^{2} \equiv \sup _{I=\dot{\cup} I_{r}} \frac{1}{|I|_{\omega}} \sum_{r=1}^{\infty}\left(\frac{\mathrm{P}^{\alpha}\left(I_{r}, \mathbf{1}_{I} \omega\right)}{\left|I_{r}\right|}\right)^{2}\left\|x-m_{I_{r}}^{\sigma}\right\|_{L^{2}\left(\mathbf{1}_{I_{r}} \sigma\right)}^{2}
$$

These energy conditions are necessary for boundedness of elliptic and gradient elliptic operators, including the Hilbert transform (but not for certain elliptic singular operators that fail to be gradient elliptic) - see SaShUr11, and see also (3.27) below for control of the energy constants $\mathcal{E}_{2}^{\alpha}$ and $\mathcal{E}_{2}^{\alpha, *}$ by the 1-testing and Muckenhoupt constants $\mathfrak{T}_{T^{\alpha}}^{\mathbf{1}}, \mathfrak{T}_{T^{\alpha}}^{\mathbf{1 , *}}$ and $\sqrt{\mathfrak{A}_{2}^{\alpha}}$. It is convenient to define

$$
\mathfrak{E}_{2}^{\alpha} \equiv \mathcal{E}_{2}^{\alpha}+\mathcal{E}_{2}^{\alpha, *}
$$

as well as

$$
\mathcal{N} \mathcal{T} \mathcal{V}_{\alpha} \equiv \mathfrak{T}_{T^{\alpha}}^{\mathbf{b}}+\mathfrak{T}_{T^{\alpha}}^{\mathbf{b}^{*}, *}+\sqrt{\mathfrak{A}_{2}^{\alpha}}+\mathfrak{E}_{2}^{\alpha}
$$




\section{THE LOCAL $T b$ THEOREM AND PROOF PRELIMINARIES}

We derive a local $T b$ theorem based in part on our proof of the $T 1$ theorem in [SaShUr7, SaShUr6], SaShUr9] and SaShUr10, in turn based on prior work in [NTV4, LaSaShUr3 and Lac, and in part on the proof of one weight $T b$ theorems in Nazarov, Treil and Volberg [NTV3] and Hytönen and Martikainen HyMa. Recall from SaShUr11 that an $\alpha$-fractional singular integral $T^{\alpha}$ with kernel $K^{\alpha}$ is said to be elliptic if $\left|K^{\alpha}(x, y)\right| \geq c|x-y|^{\alpha-1}$, and gradient elliptic if the kernel $K^{\alpha}(x, y)$ satisfies

$$
\frac{d}{d x} K^{\alpha}(x, y),-\frac{d}{d y} K^{\alpha}(x, y) \geq c|x-y|^{\alpha-2} .
$$

The Hilbert transform kernel $K(x, y)=\frac{1}{y-x}$ satisfies (2.1) with $\alpha=0$. In dimension $n=1$ the Muckenhoupt conditions are necessary for norm boundedness of elliptic operators by results in LaSaUr2, [Hyt2 and SaShUr9], and the energy conditions are necessary for norm boundedness of gradient elliptic operators by results in [SaShUr11]. Moreover, in dimension $n=1$, Hytönen [Hyt2, Corollary 3.10] proves that full testing is controlled by testing and the Muckenhoupt conditions for the Hilbert transform, and this is easily extended to $0 \leq \alpha<1$ - see (3.25) below. Here is our two weight local $T b$ theorem.

Theorem 7. Suppose that $\sigma$ and $\omega$ are locally finite positive Borel measures on the real line $\mathbb{R}$.

(1) Assume that $T^{\alpha}$ is a standard $\alpha$-fractional elliptic and gradient elliptic singular integral operator on $\mathbb{R}$, and set $T_{\sigma}^{\alpha} f=T^{\alpha}(f \sigma)$ for any smooth truncation of $T_{\sigma}^{\alpha}$, so that $T_{\sigma}^{\alpha}$ is apriori bounded from $L^{2}(\sigma)$ to $L^{2}(\omega)$.

(2) Let $p>2$ and let $\mathbf{b}=\left\{b_{Q}\right\}_{Q \in \mathcal{P}}$ be a p-weakly $\sigma$-accretive family of functions on $\mathbb{R}$, and let $\mathbf{b}^{*}=$ $\left\{b_{Q}^{*}\right\}_{Q \in \mathcal{P}}$ be a p-weakly $\omega$-accretive family of functions on $\mathbb{R}$.

(3) Then for $0 \leq \alpha<1$, the operator $T_{\sigma}^{\alpha}$ is bounded from $L^{2}(\sigma)$ to $L^{2}(\omega)$ with operator norm $\mathfrak{N}_{T_{\sigma}^{\alpha}}$, i.e.

$$
\left\|T_{\sigma}^{\alpha} f\right\|_{L^{2}(\omega)} \leq \mathfrak{N}_{T_{\sigma}^{\alpha}}\|f\|_{L^{2}(\sigma)}, \quad f \in L^{2}(\sigma)
$$

uniformly in smooth truncations of $T^{\alpha}$ if and only if the Muckenhoupt and energy conditions hold, i.e. $\mathcal{A}_{2}^{\alpha}, \mathcal{A}_{2}^{\alpha, *}, A_{2}^{\alpha, \text { punct }}, A_{2}^{\alpha, *, \text { punct }}, \mathcal{E}_{2}^{\alpha}, \mathcal{E}_{2}^{\alpha, *}<\infty$, and the $\mathbf{b}$-testing conditions for $T^{\alpha}$ and the $\mathbf{b}^{*}$ testing conditions for the dual $T^{\alpha, *}$ both hold. Moreover, we have the equivalence,

$$
\mathfrak{N}_{T^{\alpha}} \approx \mathcal{N} \mathcal{T} \mathcal{V}_{\alpha}=\mathfrak{T}_{\mathbf{R}^{\alpha}}^{\mathbf{b}}+\mathfrak{T}_{\mathbf{R}^{\alpha}}^{\mathbf{b}^{*}}+\sqrt{\mathfrak{A}_{2}^{\alpha}}+\mathfrak{E}_{2}^{\alpha}
$$

Remark 8. In the special case that $\sigma=\omega=\mu$, the classical Muckenhoupt $A_{2}^{\alpha}$ condition is

$$
\sup _{Q \in \mathcal{P}} \frac{|Q|_{\mu}}{|Q|^{1-\alpha}} \frac{|Q|_{\mu}}{|Q|^{1-\alpha}}<\infty,
$$

which is the upper doubling measure condition with exponent $1-\alpha$, i.e.

$$
|Q|_{\mu} \leq C \ell(Q)^{1-\alpha}, \quad \text { for all intervals } Q,
$$

which of course prohibits point masses in $\mu$. Both Poisson integrals are then bounded,

$$
\begin{aligned}
\mathrm{P}^{\alpha}(Q, \mu) & \lesssim \sum_{k=0}^{\infty} \frac{|Q|}{\left(2^{k}|Q|\right)^{2-\alpha}}\left|2^{k} Q\right|_{\mu} \lesssim \sum_{k=0}^{\infty} \frac{|Q|}{\left(2^{k}|Q|\right)^{2-\alpha}}\left(2^{k}|Q|\right)^{1-\alpha}=2 \\
\mathcal{P}^{\alpha}(Q, \mu) & \lesssim \sum_{k=0}^{\infty}\left(\frac{|Q|}{\left(2^{k}|Q|\right)^{2}}\right)^{1-\alpha}\left|2^{k} Q\right|_{\mu} \lesssim \sum_{k=0}^{\infty}\left(\frac{|Q|}{\left(2^{k}|Q|\right)^{2}}\right)^{1-\alpha}\left(2^{k}|Q|\right)^{1-\alpha}=C_{\alpha}<\infty
\end{aligned}
$$

and it follows easily that the equal weight pair $(\mu, \mu)$ satisfies not only the Muckenhoupt $\mathfrak{A}_{2}^{\alpha}$ condition, but also the strong energy condition $\mathfrak{E}_{2}^{\alpha}$ :

$$
\begin{aligned}
& \sum_{r=1}^{\infty}\left(\frac{\mathrm{P}^{\alpha}\left(I_{r}, \mathbf{1}_{I} \sigma\right)}{\left|I_{r}\right|}\right)^{2}\left\|x-\mathbb{E}_{I_{r}}^{\omega} x\right\|_{L^{2}(\omega)}^{2} \leq C \sum_{r=1}^{\infty}\left\|\frac{x-\mathbb{E}_{I_{r}}^{\omega} x}{\left|I_{r}\right|}\right\|_{L^{2}(\omega)}^{2} \\
& \leq C \sum_{r=1}^{\infty}\left|I_{r}\right|_{\omega} \leq C|I|_{\omega}=C|I|_{\sigma}
\end{aligned}
$$

since $\omega=\sigma$. Thus Theorem [7, when restricted to a single weight $\sigma=\omega$, recovers a weaker version of the one weight theorem of Lacey and Martikainen [LaMa, Theorem 1.1] for dimension $n=1$ - weaker due to our 
assumption that $p>2$. On the other hand, the possibility of a two weight theorem for a 2-weakly $\mu$-accretive family is highly problematic, as one of the key proof strategies used in [LaMa in the one weight case is a reduction to testing over $f$ and $g$ with controlled $L^{\infty}$ norm via interpolation, a strategy that appears to be unavailable in the two weight setting.

Problem 9. Does Theorem 7remain true in the case $p=2$, i.e. when $\mathbf{b}=\left\{b_{Q}\right\}_{Q \in \mathcal{P}}$ is a 2 -weakly $\sigma$-accretive family of functions, and $\mathbf{b}^{*}=\left\{b_{Q}^{*}\right\}_{Q \in \mathcal{P}}$ is a 2-weakly $\omega$-accretive family of functions?

Problem 10. Are the energy conditions in Theorem 7 already implied by the Muckenhoupt, $\mathbf{b}$-testing and dual $\mathbf{b}^{*}$-testing conditions for a pair of $p$-weakly accretive families when $p>2$ ?

In order to prove Theorem 7 , we first establish some improved properties for a $p$-weakly $\mu$-accretive family, and establish some improved energy conditions related to the families of testing functions $\mathbf{b}$ and $\mathbf{b}^{*}$. We turn to these matters in the next three subsections.

2.1. Reduction to the pointwise lower bound property. Here we show that we may assume without loss of generality that the $p$-weakly accretive families of testing functions $b_{Q}$ and $b_{Q}^{*}$ for $Q \in \mathcal{P}$ satisfy the pointwise lower bound property, written $P L B P$ :

$$
\left|b_{Q}(x)\right| \geq c_{1}>0 \quad \text { for } Q \in \mathcal{P} \text { and } \sigma \text {-a.e. } x \in \mathbb{R},
$$

for some positive constant $c_{1}$. Of course if $b_{Q}=\mathbf{1}_{Q} b$ for some globally defined $b$, then the $P L B P$ is immediate from Lebesgue's dyadic differentiation theorem. We make the following definition of a $p$-strongly $\mu$-accretive family in $\mathbb{R}$.

Definition 11. Let $\mu$ be a positive Borel measure on $\mathbb{R}$. We say that a family $\mathbf{b}=\left\{b_{Q}\right\}_{Q \in \mathcal{P}}$ of functions indexed by $\mathcal{P}$ is a $p$-strongly $\mu$-accretive family of functions on $\mathbb{R}$ if the $b_{Q}$ are real-valued and there are positive constants $C_{\mathbf{b}}$, and $c_{1}$ such that

$$
\begin{aligned}
& \text { support } b_{Q} \subset Q, \quad Q \in \mathcal{P}, \\
& 0<1 \leq\left|\frac{1}{|Q|_{\mu}} \int_{Q} b_{Q} d \mu\right| \leq\left(\frac{1}{|Q|_{\mu}} \int_{Q}\left|b_{Q}\right|^{p} d \mu\right)^{\frac{1}{p}} \leq C_{\mathbf{b}}<\infty, \quad Q \in \mathcal{P}, \\
& \left|b_{Q}(x)\right| \geq c_{1}>0 \quad \text { for } \sigma \text {-a.e. } x \in \mathbb{R} .
\end{aligned}
$$

To obtain that the families $\mathbf{b}=\left\{b_{Q}\right\}_{Q \in \mathcal{P}^{n}}$ and $\mathbf{b}^{*}=\left\{b_{Q}^{*}\right\}_{Q \in \mathcal{P}^{n}}$ can be assumed to satisfy the $P L B P$ requires some effort. But first, let us make a simple observation (essentially in HyMa]) under the additional assumption that the breaking intervals $Q$, those for which there is a dyadic child $Q^{\prime}$ of $Q$ with $b_{Q^{\prime}} \neq \mathbf{1}_{Q^{\prime}} b_{Q}$, satisfy a $\mu$-Carleson condition. If $G_{k} \equiv \cup \mathcal{G}_{k}$ where

$$
\mathcal{G}_{k} \equiv\left\{A \in \mathcal{A}: A \text { is a } k^{t h} \text { generation breaking interval }\right\}
$$

then $\left|\bigcap_{k=1}^{\infty} G_{k}\right|_{\sigma}=0$ since $\left|G_{k}\right|_{\sigma} \lesssim 2^{-\delta k}$ for some $\delta>0$ by the Carleson condition on breaking intervals. Thus for $\sigma$-almost every $x$, the sequence of test functions $\left\{b_{Q}\right\}_{Q: x \in Q}$, when arranged in order of decreasing length of $Q$, has the property that all sufficiently small $Q$ with $x \in Q$ belong to the same corona $\mathcal{C}_{A}$ with $x \in A$, and hence $b_{Q}=\mathbf{1}_{Q} b_{A}$ for sufficiently small intervals $Q$ containing $x$. Suppose that $A \in \mathcal{G}_{k}$. Then by Lebesgue's dyadic differentiation theorem, we have

$$
\left|b_{A}(x)\right|=\left|\lim _{Q \searrow x} \frac{1}{|Q|_{\mu}} \int_{Q} b_{A} d \mu\right|=\lim _{Q \searrow x}\left|\frac{1}{|Q|_{\mu}} \int_{Q} b_{Q} d \mu\right| \geq c>0
$$

for $\sigma$-a.e. $x \in A \backslash\left(\cup \mathcal{G}_{k+1}\right)$. But this misses showing that $\left|b_{A}(x)\right| \geq c>0$ on $A \cap\left(\cup \mathcal{G}_{k+1}\right)$, and for this we must work harder.

Proposition 12. Let $p \geq 2$. Suppose that $\mathbf{b}=\left\{b_{Q}\right\}_{Q \in \mathcal{P}}$ is a p-weakly $\sigma$-accretive family of complex-valued functions on $\mathbb{R}$ that satisfies the $\mathbf{b}$-testing condition (1.5) for a fractional singular integral operator $T^{\alpha}$. Then 
there is a p-weakly $\sigma$-accretive family $\widetilde{\mathbf{b}}=\left\{\widetilde{b}_{Q}\right\}_{Q \in \mathcal{P}}$ that satisfies the PLBP. Moreover, the full $\widetilde{\mathbf{b}}$-testing condition (1.5) for $T^{\alpha}$ holds and we have the estimate,

$$
\begin{aligned}
& \left\{\widetilde{b}_{Q}\right\}_{Q \in \mathcal{P}^{n}} \text { is p-strongly } \sigma \text {-accretive, } \\
& \mathfrak{F} \mathfrak{T}_{T^{\alpha}}^{\widetilde{\mathbf{b}}}(\sigma, \omega) \leq C_{\alpha}\left(\mathfrak{T}_{T^{\alpha}}^{\mathbf{b}}(\sigma, \omega)+\mathfrak{T}_{T^{\alpha}}^{\mathbf{b}^{*} *}(\sigma, \omega)+\sqrt{\mathcal{A}_{2}^{\alpha}(\sigma, \omega)}+\sqrt{\mathcal{A}_{2}^{\alpha, *}(\sigma, \omega)}\right) .
\end{aligned}
$$

Proof. For every interval $Q \in \mathcal{P}$ define

$$
E(Q) \equiv\left\{x \in Q:\left|b_{Q}(x)\right|<\frac{1}{4}\right\} .
$$

Momentarily fix an interval $Q \in \mathcal{P}$ and $\delta>0$, and let $\left\{I_{j}(Q)\right\}_{j=1}^{\infty}$ be a collection of pairwise disjoint intervals such that

$$
\begin{aligned}
& E(Q)=\left\{x \in Q:\left|b_{Q}(x)\right|<\frac{1}{4}\right\} \subset \bigcup_{j=1}^{\infty} I_{j}(Q) ; \\
& \left|\bigcup_{j=1}^{\infty} I_{j}(Q) \backslash E(Q)\right|_{\sigma}<\delta|Q|_{\sigma} .
\end{aligned}
$$

Note that

$$
\begin{aligned}
|Q|_{\sigma} & \leq\left|\int_{Q} b_{Q} d \sigma\right|=\left|\int_{E(Q)} b_{Q} d \sigma\right|+\left|\int_{Q \backslash E(Q)} b_{Q} d \sigma\right| \\
& \leq \frac{1}{4}|E(Q)|_{\sigma}+\eta \int_{Q \backslash E(Q)}\left|b_{Q}\right|^{2} d \sigma+\frac{1}{\eta} \int_{Q \backslash E(Q)} d \sigma \\
& \leq \frac{1}{4}|E(Q)|_{\sigma}+\eta \int_{Q}\left|b_{Q}\right|^{2} d \sigma+\frac{1}{\eta}|Q \backslash E(Q)|_{\sigma} \\
& \leq \frac{1}{4}|E(Q)|_{\sigma}+\eta C|Q|_{\sigma}+\frac{1}{\eta}|Q \backslash E(Q)|_{\sigma} .
\end{aligned}
$$

Thus taking $\eta=\frac{1}{2 C}$, and dividing through by $|Q|_{\sigma}$, we get

$$
\begin{aligned}
1 & \leq \frac{1}{4} \frac{|E(Q)|_{\sigma}}{|Q|_{\sigma}}+\frac{1}{2}+2 C \frac{|Q \backslash E(Q)|_{\sigma}}{|Q|_{\sigma}} \leq \frac{3}{4}+2 C \frac{|Q \backslash E(Q)|_{\sigma}}{|Q|_{\sigma}} ; \\
& \Longrightarrow \frac{1}{4} \leq 2 C \frac{|Q \backslash E(Q)|_{\sigma}}{|Q|_{\sigma}} \Longrightarrow|Q \backslash E(Q)|_{\sigma} \geq \frac{1}{8 C}|Q|_{\sigma} \\
& \Longrightarrow|E(Q)|_{\sigma} \leq\left(1-\frac{1}{8 C}\right)|Q|_{\sigma}=\beta|Q|_{\sigma},
\end{aligned}
$$

where $\beta=1-\frac{1}{8 C}$. Now we note that since $\delta>0$ can be taken arbitrarily small, we may without loss of generality take $\delta=05$. Altogether then, we have shown that for every $Q \in \mathcal{P}$, there is a pairwise disjoint collection of intervals $\left\{I_{j}^{Q}\right\}_{j}$ such that

$$
\begin{aligned}
E(Q) & \equiv\left\{x \in Q:\left|b_{Q}(x)\right|<\frac{1}{4}\right\}=\bigcup_{j=1}^{\infty} I_{j}(Q), \\
\text { and }|E(Q)|_{\sigma} & =\sum_{j=1}^{\infty}\left|I_{j}(Q)\right|_{\sigma} \leq \beta|Q|_{\sigma}, \text { where } 0<\beta=1-\frac{1}{8 C}<1 .
\end{aligned}
$$

Now we begin the first step of the construction of a new family $\left\{\widetilde{b}_{Q}\right\}_{Q \in \mathcal{D}}$ that satisfies both the accretivity conditions and the testing conditions, as well as the pointwise lower bound condition. We start by defining

\footnotetext{
${ }^{5} \mathrm{~A}$ rigorous limiting argument can be modelled after that given here.
} 
for $\epsilon=\left\{\epsilon_{j}\right\}_{j=1}^{\infty}$,

$$
\widetilde{b}_{Q}^{\epsilon}(x) \equiv b_{Q}(x)+\sum_{j=1}^{\infty} \epsilon_{j} b_{I_{j}(Q)}(x),
$$

where $\epsilon_{j} \in\{-1,1\}$ for all $j \geq 1$.

We first note that we can assume that the collection of intervals $\left\{I_{i}(Q)\right\}_{i}$ is subordinate to the collection of children of $Q$, i.e. $I_{i}(Q) \subset Q^{\prime}$ for some $Q^{\prime} \in \mathfrak{C}(Q)$ depending on $i$. Then we have for each $Q^{\prime} \in \mathfrak{C}(Q)$ that

$$
\begin{aligned}
\int_{Q^{\prime}}\left|\widetilde{b}_{Q}^{\epsilon}\right|^{p} d \sigma & =\int_{Q^{\prime}}\left|b_{Q}+\sum_{j=1}^{\infty} \epsilon_{j} b_{I_{j}}\right|^{p} d \sigma \\
& \leq 2^{p}\left\{\int_{Q^{\prime}}\left|b_{Q}\right|^{2 p} d \sigma+\sum_{j: I_{j} \subset Q^{\prime}} \int\left|b_{I_{j}}\right|^{p} d \sigma\right\} \\
& \leq C_{p}\left\{\left|Q^{\prime}\right|_{\sigma}+\sum_{j: I_{j} \subset Q^{\prime}}\left|I_{j}\right|_{\sigma}\right\} \leq C_{p}(1+\beta)\left|Q^{\prime}\right|_{\sigma} .
\end{aligned}
$$

Now with $\mathbb{E}$ denoting expectation with respect to the uniform probability measure on $\Omega_{\infty} \equiv\{-1,1\}^{\mathbb{N}}$, we have

$$
\mathbb{E} \int \widetilde{b}_{Q}^{\epsilon} d \sigma=\int b_{Q} d \sigma \geq 1>0
$$

and

$$
\begin{aligned}
& \mathbb{E} \int\left|T_{\sigma}^{\alpha}\left(\mathbf{1}_{Q} \widetilde{b}_{Q}^{\epsilon}\right)\right|^{2} d \omega=\mathbb{E} \int\left|T_{\sigma}^{\alpha}\left(b_{Q}+\sum_{j=1}^{\infty} \epsilon_{j} b_{I_{j}}\right)\right|^{2} d \omega \\
= & \mathbb{E} \int\left\{\left|T_{\sigma}^{\alpha} b_{Q}\right|^{2}+2 \operatorname{Re} \sum_{j=1}^{\infty} \epsilon_{j}\left(T_{\sigma}^{\alpha} b_{Q}\right) \overline{\left(T_{\sigma}^{\alpha} b_{I_{j}}\right)}+\sum_{j, k=1}^{\infty} \epsilon_{j} \epsilon_{k}\left(T_{\sigma}^{\alpha} b_{I_{j}}\right) \overline{\left(T_{\sigma}^{\alpha} b_{I_{k}}\right)}\right\} d \omega \\
\leq & \int\left|T_{\sigma}^{\alpha} b_{Q}\right|^{2} d \omega+\sum_{j=1}^{\infty} \int\left|T_{\sigma}^{\alpha} b_{I_{j}}\right|^{2} d \omega \\
\leq & \mathfrak{F T}_{T^{\alpha}}^{\mathbf{b}}|Q|_{\sigma}+\sum_{j=1}^{\infty} \mathfrak{F T}_{T^{\alpha}}^{\mathbf{b}}\left|I_{i}(Q)\right|_{\sigma} \leq \mathfrak{F}_{T^{\alpha}}^{\mathbf{b}}[1+\beta]|Q|_{\sigma} .
\end{aligned}
$$

So altogether, at this point in the first step of the construction, we have for each pair $(Q, E(Q))$ consisting of an interval $Q$ and a subset $E(Q)$ having measure at most $\beta|Q|_{\sigma}$ :

$$
\begin{aligned}
|E(Q)|_{\sigma} & \leq \beta|Q|_{\sigma} \\
\mathbb{E} \int \widetilde{b}_{Q}^{\epsilon} d \sigma & =\int b_{Q} d \sigma \geq|Q|_{\sigma}>0, \\
\int_{Q^{\prime}}\left|\widetilde{b}_{Q}^{\epsilon}\right|^{p} d \sigma & \leq C_{p}(1+\beta)\left|Q^{\prime}\right|_{\sigma}, \quad Q^{\prime} \in \mathfrak{C}(Q), \\
\mathbb{E} \int\left|T_{\sigma}^{\alpha}\left(\mathbf{1}_{Q} \widetilde{b}_{Q}^{\epsilon}\right)\right|^{2} d \omega & \leq \mathfrak{F T}_{T^{\alpha}}^{\mathbf{b}}[1+\beta]|Q|_{\sigma} .
\end{aligned}
$$


Now we choose a positive constant $A$ large enough so that with probabilities $\frac{1}{2}$ for $\int \widetilde{b}_{Q}^{\epsilon} d \sigma$ and $\frac{3}{4}$ for $\int_{Q}\left|T_{\sigma}^{\alpha}\left(\mathbf{1}_{Q} \widetilde{b}_{Q}^{\epsilon}\right)\right|^{2} d \omega$ (note that $\left(1-\frac{1}{2}\right)+\left(1-\frac{3}{4}\right)=\frac{3}{4}<1$ ), there exists $\epsilon \in \Omega_{\infty}$ so that $\widetilde{b}_{Q}^{1} \equiv \widetilde{b}_{Q}^{\epsilon}$ satisfies

$$
\begin{aligned}
\int \widetilde{b}_{Q}^{1} d \sigma & \geq|Q|_{\sigma}>0, \\
\int_{Q^{\prime}}\left|\widetilde{b}_{Q}^{1}\right|^{p} d \sigma & \leq A C_{p}(1+\beta)\left|Q^{\prime}\right|_{\sigma}, \quad Q^{\prime} \in \mathfrak{C}(Q), \\
\int\left|T_{\sigma}^{\alpha}\left(\mathbf{1}_{Q} \widetilde{b}_{Q}^{1}\right)\right|^{2} d \omega & \leq A \mathfrak{F T}_{T^{\alpha}}^{\mathbf{b}}[1+\beta] \beta|Q|_{\sigma} .
\end{aligned}
$$

To see how big $A$ must be taken to achieve (2.3), we use Chebyshev's inequality as follows. Take $N$ large, set

$$
\widetilde{b}_{Q}^{\epsilon, N}(x) \equiv b_{Q}(x)+\sum_{j=1}^{N} \epsilon_{j} b_{I_{j}(Q)}(x),
$$

and equip $\Omega_{N}=\{-1,1\}^{N}$ with the uniform probability measure that assigns mass $\frac{1}{2^{N}}$ to each $\epsilon \in \Omega_{N}$. Then for each child $Q^{\prime}$ of $Q$ we have as above that

$$
\frac{1}{\# \Omega_{N}} \sum_{\boldsymbol{\epsilon} \in \Omega_{N}} \int\left|T_{\sigma}^{\alpha}\left(\mathbf{1}_{Q} \widetilde{b}_{Q}^{\epsilon, N}\right)\right|^{2} d \omega=\mathbb{E} \int\left|T_{\sigma}^{\alpha}\left(\mathbf{1}_{Q} \widetilde{b}_{Q}^{\epsilon, N}\right)\right|^{2} d \omega \leq C(1+\beta)|Q|_{\sigma},
$$

which by Chebyshev's inequality implies that

$$
\begin{gathered}
A C(1+\beta)|Q|_{\sigma} \#\left\{\boldsymbol{\epsilon} \in \Omega_{N}: \int\left|T_{\sigma}^{\alpha}\left(\mathbf{1}_{Q} \widetilde{b}_{Q}^{\epsilon, N}\right)\right|^{2} d \omega>A C(1+\beta)|Q|_{\sigma}\right\} \\
\leq \sum_{\boldsymbol{\epsilon} \in \Omega_{N}} \int\left|T_{\sigma}^{\alpha}\left(\mathbf{1}_{Q} \widetilde{b}_{Q}^{\epsilon, N}\right)\right|^{2} d \omega \leq \# \Omega_{N} C(1+\beta)|Q|_{\sigma}
\end{gathered}
$$

which gives in turn that

$$
\frac{1}{\# \Omega_{N}} \#\left\{\epsilon \in \Omega_{N}: \int\left|T_{\sigma}^{\alpha}\left(\mathbf{1}_{Q} \widetilde{b}_{Q}^{\epsilon, N}\right)\right|^{2} d \omega>A C(1+\beta)|Q|_{\sigma}\right\} \leq \frac{1}{A} .
$$

This of course just says that the probability that $\int\left|T_{\sigma}^{\alpha}\left(\mathbf{1}_{Q} \widetilde{b}_{Q}^{\epsilon, N}\right)\right|^{2} d \omega$ exceeds $A$ times $C(1+\beta)|Q|_{\sigma}$ is less than $\frac{1}{A}$. So in order to achieve that this latter probability is at most $\frac{1}{4}$, we take $A \geq 4$. Then we get that

$$
\int\left|T_{\sigma}^{\alpha}\left(\mathbf{1}_{Q} \widetilde{b}_{Q}^{\epsilon, N}\right)\right|^{2} d \omega \leq A C(1+\beta)|Q|_{\sigma}, \quad Q^{\prime} \in \mathfrak{C}(Q)
$$

holds for a set of $\epsilon$ in $\Omega_{N}$ of probability at least $1-\frac{1}{4}=\frac{3}{4}$.

So altogether, provided that we take $A=4$, we obtain that all inequalities,

$$
\begin{aligned}
\int \widetilde{b}_{Q}^{\epsilon, N} d \sigma & \geq|Q|_{\sigma}>0, \\
\int_{Q^{\prime}}\left|\widetilde{b}_{Q}^{\epsilon, N}\right|^{p} d \sigma & \leq C_{p}(1+\beta)\left|Q^{\prime}\right|_{\sigma}, \quad Q^{\prime} \in \mathfrak{C}(Q), \\
\int\left|T_{\sigma}^{\alpha}\left(\mathbf{1}_{Q^{b}} \widetilde{b}_{Q}^{\epsilon, N}\right)\right|^{2} d \omega & \leq A \mathfrak{F}_{T^{\alpha}}^{\mathbf{b}}[1+\beta] \beta|Q|_{\sigma},
\end{aligned}
$$

hold simultaneously for a set of $\epsilon$ in $\Omega_{N}$ of probability at least

$$
1-\left\{\left(1-\frac{1}{2}\right)+\left(1-\frac{3}{4}\right)\right\}=1-\left\{\frac{1}{2}+\frac{1}{4}\right\}=\frac{1}{4}
$$

Since these estimates are independent of $N$, we can let $N \rightarrow \infty$ to obtain that there is at least one $\epsilon \in \Omega_{\infty}$ for which (2.3) holds with $\widetilde{b}_{Q}^{1} \equiv \widetilde{b}_{Q}^{\epsilon, N}$.

We also have $\left|\widetilde{b}_{Q}^{1}\right| \geq \frac{3}{4}$ in $Q$ except on the exceptional set

$$
G \equiv \bigcup_{j=1}^{\infty} E\left(I_{j}^{Q}\right)
$$


whose $\sigma$-measure satisfies

$$
|G|_{\sigma}=\sum_{j=1}^{\infty}\left|E\left(I_{j}^{Q}\right)\right|_{\sigma} \leq \sum_{j=1}^{\infty} \beta\left|I_{j}^{Q}\right|_{\sigma}=\beta|E(Q)|_{\sigma} \leq \beta^{2}|Q|_{\sigma} .
$$

Now we consider the set

$$
F\left(I_{j}(Q)\right) \equiv\left\{x \in E\left(I_{j}(Q)\right):\left|b_{Q}(x)+\epsilon_{j} b_{I_{j}^{Q}}(x)\right|<\frac{1}{4}\right\} .
$$

We may assume that

$$
F\left(I_{j}(Q)\right)=\bigcup_{k=1}^{\infty} I_{k}\left(I_{j}(Q)\right)
$$

where $\left\{I_{k}\left(I_{j}(Q)\right)\right\}_{k=1}^{\infty}$ is a pairwise disjoint collection of intervals for each $j$. Now we apply the above step to each pair $\left(I_{j}(Q), F\left(I_{j}(Q)\right)\right)$ consisting of an interval $I_{j}^{Q}$ and a subset $F\left(I_{j}^{Q}\right)$ having measure at most $\beta\left|I_{j}^{Q}\right|_{\sigma}$. Then arguing as above for each such pair, and adding results, we obtain that there exists $\boldsymbol{\epsilon}_{j}$ so that $\widetilde{b}_{Q}^{2} \equiv \sum_{j=1}^{\infty}{\widetilde{b^{1}}}_{Q}^{\epsilon_{j}}$ satisfies

$$
\begin{aligned}
\int \widetilde{b}_{Q}^{2} d \sigma & \geq|Q|_{\sigma}>0 \\
\int_{Q^{\prime}}\left|\widetilde{b}_{Q}^{2}\right|^{p} d \sigma & \leq A C_{p}\left(1+\beta+\beta^{2}\right)\left|Q^{\prime}\right|_{\sigma}, \quad Q^{\prime} \in \mathfrak{C}(Q), \\
\int\left|T_{\sigma}^{\alpha}\left(\mathbf{1}_{Q} \widetilde{b}_{Q}^{2}\right)\right|^{2} d \omega & \leq A \mathfrak{F}_{T^{\alpha}}^{\mathbf{b}}\left[1+\beta+\beta^{2}\right] \beta|Q|_{\sigma},
\end{aligned}
$$

as well as $\left|\widetilde{b}_{Q}^{2}\right| \geq \frac{3}{4}$ in $Q$ except on the exceptional set

$$
G \equiv \bigcup_{j, k=1}^{\infty} E\left(I_{k}\left(I_{j}(Q)\right)\right)
$$

whose $\sigma$-measure satisfies

$$
\begin{aligned}
|G|_{\sigma} & =\sum_{j, k=1}^{\infty}\left|E\left(I_{k}\left(I_{j}(Q)\right)\right)\right|_{\sigma} \leq \sum_{j=1}^{\infty} \beta\left|E\left(I_{j}(Q)\right)\right|_{\sigma} \\
& \leq \sum_{j=1}^{\infty} \beta^{2}\left|I_{j}^{Q}\right|_{\sigma}=\beta^{2}|E(Q)|_{\sigma} \leq \beta^{3}|Q|_{\sigma} .
\end{aligned}
$$

Continuing in this way, we end up with the desired function $\widetilde{b}_{Q}(x)=\lim _{n \rightarrow \infty} \widetilde{b}_{Q}^{n}(x)$, since $\beta<1$ implies that the collection of nested intervals $\left\{I_{j_{N}}\left(\ldots I_{j_{2}}\left(I_{j_{1}}(Q)\right) \ldots\right)\right\}$ satisfy a $\sigma$-Carleson condition. We emphasize that for each interval $Q$, we then have $\left|\widetilde{b}_{Q}(x)\right| \geq \frac{3}{4}$ for $\sigma$-a.e. $x \in Q$, as well as $\int_{Q^{\prime}}\left|\widetilde{b}_{Q}\right|^{2} d \sigma \leq C^{\prime}\left|Q^{\prime}\right|_{\sigma}$ for all $Q^{\prime} \in \mathfrak{C}(Q)$. The full $\widetilde{b}$-testing condition constant that we have obtained satisfies

$$
\mathfrak{F} \widetilde{T}_{T^{\alpha}}^{\widetilde{\mathbf{b}}}(\sigma, \omega) \leq C_{\alpha}\left(\mathfrak{F}_{T^{\alpha}}^{\mathbf{b}}(\sigma, \omega)+\mathfrak{F T}_{T^{\alpha}}^{\mathbf{b}^{*}, *}(\sigma, \omega)+\sqrt{\mathcal{A}_{2}^{\alpha}(\sigma, \omega)}+\sqrt{\mathcal{A}_{2}^{\alpha, *}(\sigma, \omega)}\right),
$$

and since the full testing constant is controlled by the testing constant and the Muckenhoupt constant in (3.25) below, the proof is complete.

2.2. Reduction to real bounded accretive families. Recall that a vector of 'complex-valued testing functions' $\mathbf{b} \equiv\left\{b_{Q}\right\}_{Q \in \mathcal{D}}$ is a $p$-strongly $\mu$-accretive family if

$$
\begin{gathered}
\text { support } b_{Q} \subset Q, \quad Q \in \mathcal{P}, \\
1 \leq\left|\frac{1}{|Q|_{\mu}} \int_{Q} b_{Q} d \mu\right| \leq\left(\frac{1}{|Q|_{\mu}} \int_{Q}\left|b_{Q}\right|^{p} d \mu\right)^{\frac{1}{p}} \leq C_{\mathbf{b}}(p)<\infty, \quad Q \in \mathcal{P},
\end{gathered}
$$


and if $\mathbf{b}$ satisfies the $P L B P$, i.e.

$$
\left|b_{Q}(x)\right| \geq c_{1}>0 \quad \text { for } Q \in \mathcal{D} \text { and } \sigma-\text { a.e. } x \in \mathbb{R}^{n} .
$$

We begin by noting that if $b_{Q}$ satisfies (2.4) with $\mu=\sigma$, and satisfies a given $\mathbf{b}$-testing condition for a weight pair $(\sigma, \omega)$, then $\operatorname{Re} b_{Q}$ satisfies $\left(\frac{1}{|Q|_{\mu}} \int_{Q}\left|\operatorname{Re} b_{Q}\right|^{p} d \mu\right)^{\frac{1}{p}} \leq C_{\mathbf{b}}(p)$ and the given b-testing condition for $(\sigma, \omega)$ with $\operatorname{Re} b_{Q}$ in place of $b_{Q}$.

Conclusion 13. We may assume throughout the proof of Theorem 7 that our p-weakly $\mu$-accretive families $\mathbf{b} \equiv\left\{b_{Q}\right\}_{Q \in \mathcal{D}}$ and $\mathbf{b}^{*} \equiv\left\{b_{Q}^{*}\right\}_{Q \in \mathcal{G}}$ consist of real-valued functions that in addition satisfy the PLBP and $1 \leq \frac{1}{|Q|_{\sigma}} \int_{Q} b_{Q} d \sigma$ and $1 \leq \frac{1}{|Q|_{\sigma}} \int_{Q} b_{Q}^{*} d \sigma$.

Next we show that the assumption of testing conditions for a Calderón-Zygmund operator $T$ and $p$ strongly $\mu$-accretive testing functions $\mathbf{b}=\left\{b_{Q}\right\}_{Q \in \mathcal{P}}$ and $\mathbf{b}^{*}=\left\{b_{Q}^{*}\right\}_{Q \in \mathcal{P}}$ with $p>2$ can always be replaced with real-valued $\infty$-strongly $\mu$-accretive testing functions, thus reducing the $T b$ theorem for the case $p>2$ to the case when $p=\infty$ and the PLBP (2.2) holds. We now proceed to develop a precise statement. We extend (2.4) to $2 \leq p \leq \infty$ by

$$
\begin{aligned}
& \text { support } b_{Q} \subset Q, \quad Q \in \mathcal{P}, \\
& 1 \leq\left|\frac{1}{|Q|_{\mu}} \int_{Q} b_{Q} d \mu\right| \leq\left\{\begin{array}{cc}
\left(\frac{1}{|Q|_{\mu}} \int_{Q}\left|b_{Q}\right|^{p} d \mu\right)^{\frac{1}{p}} \leq C_{\mathbf{b}}(p)<\infty & \text { for } 2 \leq p<\infty \\
\left\|b_{Q}\right\|_{L^{\infty}(\mu)} \leq C_{\mathbf{b}}(\infty)<\infty & \text { for } p=\infty
\end{array}, \quad Q \in \mathcal{P} .\right.
\end{aligned}
$$

Proposition 14. Let $0 \leq \alpha<1$, and let $\sigma$ and $\omega$ be locally finite positive Borel measures on the real line $\mathbb{R}$, and let $T^{\alpha}$ be a standard $\alpha$-fractional elliptic and gradient elliptic singular integral operator on $\mathbb{R}$. Set $T_{\sigma}^{\alpha} f=T^{\alpha}(f \sigma)$ for any smooth truncation of $T_{\sigma}^{\alpha}$, so that $T_{\sigma}^{\alpha}$ is apriori bounded from $L^{2}(\sigma)$ to $L^{2}(\omega)$. Finally, define the sequence of positive extended real numbers

$$
\left\{p_{n}\right\}_{n=0}^{\infty}=\left\{\frac{2}{1-\left(\frac{2}{3}\right)^{n}}\right\}_{n=0}^{\infty}=\left\{\infty, 6, \frac{18}{5}, \frac{162}{65}, \ldots\right\} .
$$

Suppose that the following statement is true:

$\left(\mathcal{S}_{\infty}\right):$ If $\mathbf{b}=\left\{b_{Q}\right\}_{Q \in \mathcal{P}}$ is an $\infty$-strongly $\sigma$-accretive family of functions on $\mathbb{R}$, and if $\mathbf{b}^{*}=\left\{b_{Q}^{*}\right\}_{Q \in \mathcal{P}}$ is an $\infty$-strongly $\omega$-accretive family of functions on $\mathbb{R}$, then the operator norm $\mathfrak{N}_{T_{\sigma}^{\alpha}}$ of $T_{\sigma}^{\alpha}$ from $L^{2}(\sigma)$ to $L^{2}(\omega)$, i.e. the best constant in

$$
\left\|T_{\sigma}^{\alpha} f\right\|_{L^{2}(\omega)} \leq \mathfrak{N}_{T_{\sigma}^{\alpha}}\|f\|_{L^{2}(\sigma)}, \quad f \in L^{2}(\sigma),
$$

uniformly in smooth truncations of $T^{\alpha}$, satisfies

$$
\mathfrak{T}_{T^{\alpha}}^{\mathbf{b}}+\mathfrak{T}_{T^{\alpha}}^{\mathbf{b}^{*}}+\sqrt{\mathfrak{A}_{2}^{\alpha}}+\mathfrak{E}_{2}^{\alpha} \lesssim \mathfrak{N}_{T^{\alpha}} \lesssim\left(C_{\mathbf{b}}(\infty)+C_{\mathbf{b}^{*}}(\infty)\right)\left(\mathfrak{T}_{T^{\alpha}}^{\mathbf{b}}+\mathfrak{T}_{T^{\alpha}}^{\mathbf{b}^{*}}+\sqrt{\mathfrak{A}_{2}^{\alpha}}+\mathfrak{E}_{2}^{\alpha}\right),
$$

where $C_{\mathbf{b}}(\infty), C_{\mathbf{b}^{*}}(\infty)$ are the accretivity constants in (2.5), and the constants implied by $\lesssim$ depend on $\alpha$ and the Calderón-Zygmund constant $C_{C Z}$ in (1.1).

Then for each $n \geq 0$, the following statements hold:

$\left(\mathcal{S}_{n}\right):$ Let $p \in\left(p_{n+1}, p_{n}\right]$. If $\mathbf{b}=\left\{b_{Q}\right\}_{Q \in \mathcal{P}}$ is a p-strongly $\sigma$-accretive family of functions on $\mathbb{R}$, and if $\mathbf{b}^{*}=\left\{b_{Q}^{*}\right\}_{Q \in \mathcal{P}}$ is a p-strongly $\omega$-accretive family of functions on $\mathbb{R}$, then the operator norm $\mathfrak{N}_{T_{\sigma}^{\alpha}}$ of $T_{\sigma}^{\alpha}$ from $L^{2}(\sigma)$ to $L^{2}(\omega)$, i.e. the best constant in

$$
\left\|T_{\sigma}^{\alpha} f\right\|_{L^{2}(\omega)} \leq \mathfrak{N}_{T_{\sigma}^{\alpha}}\|f\|_{L^{2}(\sigma)}, \quad f \in L^{2}(\sigma),
$$

uniformly in smooth truncations of $T^{\alpha}$, satisfies

$$
\mathfrak{T}_{T^{\alpha}}^{\mathbf{b}}+\mathfrak{T}_{T^{\alpha}}^{\mathbf{b}^{*}}+\sqrt{\mathfrak{A}_{2}^{\alpha}}+\mathfrak{E}_{2}^{\alpha} \lesssim \mathfrak{N}_{T^{\alpha}} \lesssim\left(C_{\mathbf{b}}(p)+C_{\mathbf{b}^{*}}(p)\right)^{3^{n+1}}\left(\mathfrak{T}_{T^{\alpha}}^{\mathbf{b}}+\mathfrak{T}_{T^{\alpha}}^{\mathbf{b}^{*}}+\sqrt{\mathfrak{A}_{2}^{\alpha}}+\mathfrak{E}_{2}^{\alpha}\right),
$$

where $C_{\mathbf{b}}(p), C_{\mathbf{b}^{*}}(p)$ are the accretivity constants in $(2.4)$, and the constants implied by $\lesssim$ depend on $p, \alpha$, and the Calderón-Zygmund constant $C_{C Z}$ in (1.1). 
Proof of Proposition 14. We first prove $\left(\mathcal{S}_{0}\right)$. So fix $p \in\left(p_{1}, p_{0}\right)=(6, \infty)$, and let $\mathbf{b}=\left\{b_{Q}\right\}_{Q \in \mathcal{P}}$ be a $p$ weakly $\sigma$-accretive family of functions on $\mathbb{R}$, and let $\mathbf{b}^{*}=\left\{b_{Q}^{*}\right\}_{Q \in \mathcal{P}}$ be a $p$-weakly $\omega$-accretive family of functions on $\mathbb{R}$. Let $0<\varepsilon<1$ (to be chosen differently at various points in the argument below) and define

$$
\lambda=\lambda(\varepsilon)=\left(\frac{p}{p-2} C_{\mathbf{b}}(p)^{p} \frac{1}{\varepsilon}\right)^{\frac{1}{p-2}},
$$

and a new collection of test functions,

$$
\widehat{b}_{Q} \equiv 2 b_{Q}\left(\mathbf{1}_{\left\{\left|b_{Q}\right| \leq \lambda\right\}}+\frac{\lambda}{\left|b_{Q}\right|} \mathbf{1}_{\left\{\left|b_{Q}\right|>\lambda\right\}}\right), \quad Q \in \mathcal{P},
$$

which continue to satisfy the $P L B P(2.2)$. We compute

$$
\begin{aligned}
\int_{\left\{\left|b_{Q}\right|>\lambda\right\}}\left|b_{Q}\right|^{2} d \sigma & =\int_{\left\{\left|b_{Q}\right|>\lambda\right\}}\left[\int_{0}^{\left|b_{Q}\right|} 2 t d t\right] d \sigma \\
& =\iint_{\left\{(x, t) \in \mathbb{R} \times(0, \infty): \max \{t, \lambda\}<\left|b_{Q}(x)\right|\right\}} 2 t d t d \sigma(x) \\
& =\int_{[0, \lambda]} \int_{\left\{x \in \mathbb{R}: \lambda<\left|b_{Q}(x)\right|\right\}} d \sigma(x) 2 t d t+\int_{(\lambda, \infty)} \int_{\left\{x \in \mathbb{R}: t<\left|b_{Q}(x)\right|\right\}} d \sigma(x) 2 t d t \\
& =\lambda^{2}\left|\left\{\left|b_{Q}\right|>\lambda\right\}\right|_{\sigma}+\int_{\lambda}^{\infty}\left|\left\{\left|b_{Q}\right|>t\right\}\right|_{\sigma} 2 t d t,
\end{aligned}
$$

and hence

$$
\begin{aligned}
\int_{\left\{\left|b_{Q}\right|>\lambda\right\}}\left|b_{Q}\right|^{2} d \sigma & \leq \lambda^{2} \frac{1}{\lambda^{p}}\left(\int\left|b_{Q}\right|^{p} d \sigma\right)+\int_{\lambda}^{\infty} \frac{1}{t^{p}}\left(\int\left|b_{Q}\right|^{p} d \sigma\right) 2 t d t \\
& =\left\{\lambda^{2-p}+\int_{\lambda}^{\infty} 2 t^{1-p} d t\right\} C_{\mathbf{b}}(p)^{p}|Q|_{\sigma} \\
& =\frac{p}{p-2} \lambda^{2-p} C_{\mathbf{b}}(p)^{p}|Q|_{\sigma}=\varepsilon|Q|_{\sigma},
\end{aligned}
$$

by (2.6). Thus we have the lower bound,

$$
\begin{aligned}
\left|\frac{1}{|Q|_{\sigma}} \int_{Q} \widehat{b}_{Q} d \sigma\right| & =2\left|\frac{1}{|Q|_{\sigma}} \int_{Q} b_{Q} d \sigma-\frac{1}{|Q|_{\sigma}} \int_{Q} b_{Q}\left(\frac{\lambda}{\left|b_{Q}\right|}-1\right) \mathbf{1}_{\left\{\left|b_{Q}\right|>\lambda\right\}} d \sigma\right| \\
& \geq 2\left|\frac{1}{|Q|_{\sigma}} \int_{Q} b_{Q} d \mu\right|-2\left(\frac{1}{|Q|_{\sigma}} \int_{Q}\left|b_{Q}\right|^{2} \mathbf{1}_{\left\{\left|b_{Q}\right|>\lambda\right\}} d \sigma\right)^{\frac{1}{2}} \\
& \geq 2-2\left(\frac{1}{|Q|_{\sigma}} \varepsilon|Q|_{\sigma}\right)^{\frac{1}{2}}=2-2 \sqrt{\varepsilon} \geq 1>0, \quad Q \in \mathcal{P},
\end{aligned}
$$

if we choose $0<\varepsilon \leq \frac{1}{4}$. For an upper bound we have

$$
\left\|\widehat{b}_{Q}\right\|_{L^{\infty}(\sigma)} \leq 2 \lambda=2 \lambda(\varepsilon)=2\left(\frac{p}{p-2} C_{\mathbf{b}}(p)^{p} \frac{1}{\varepsilon}\right)^{\frac{1}{p-2}},
$$

which altogether shows that

Similarly we have

$$
\begin{aligned}
C_{\widehat{\mathbf{b}}}(\infty) \leq 2\left(\frac{p}{p-2} C_{\mathbf{b}}(p)^{p} \frac{1}{\varepsilon}\right)^{\frac{1}{p-2}} & =2\left(\frac{p}{p-2}\right)^{\frac{1}{p-2}} C_{\mathbf{b}}(p)^{\frac{p}{p-2}} \varepsilon^{-\frac{1}{p-2}}, \\
\text { for } 0<\varepsilon & \leq \frac{1}{4}
\end{aligned}
$$

$$
\begin{aligned}
& C_{\widehat{\mathbf{b}}^{*}}(\infty) \leq 2\left(\frac{p}{p-2} C_{\mathbf{b}^{*}}(p)^{p} \frac{1}{\varepsilon^{*}}\right)^{\frac{1}{p-2}}=2\left(\frac{p}{p-2}\right)^{\frac{1}{p-2}} C_{\mathbf{b}^{*}}(p)^{\frac{p}{p-2}}\left(\varepsilon^{*}\right)^{-\frac{1}{p-2}} \\
& \text { for } 0<\varepsilon^{*} \leq \frac{1}{4}
\end{aligned}
$$


Moreover, we also have

$$
\begin{aligned}
\sqrt{\int_{Q}\left|T_{\sigma}^{\alpha} \widehat{b}_{Q}\right|^{2} d \omega} & \leq 2 \sqrt{\int_{Q}\left|T_{\sigma}^{\alpha} b_{Q}\right|^{2} d \omega}+2 \sqrt{\int_{Q}\left|T_{\sigma}^{\alpha} \mathbf{1}_{\left\{\left|b_{Q}\right|>\lambda\right\}}\left(\frac{\lambda}{\left|b_{Q}\right|}-1\right) b_{Q}\right|^{2} d \omega} \\
& \leq 2 \mathfrak{T}_{T^{\alpha}}^{\mathbf{b}} \sqrt{|Q|_{\sigma}}+2 \mathfrak{N}_{T^{\alpha}} \sqrt{\int_{\left\{\left|b_{Q}\right|>\lambda\right\}}\left|b_{Q}\right|^{2} d \sigma} \\
& =2\left\{\mathfrak{T}_{T^{\alpha}}^{\mathbf{b}}+\sqrt{\varepsilon} \mathfrak{N}_{T^{\alpha}}\right\} \sqrt{|Q|_{\sigma}}, \quad \text { for all intervals } Q,
\end{aligned}
$$

which shows that

$$
\mathfrak{T}_{T^{\alpha}}^{\widehat{\mathbf{b}}} \leq 2 \mathfrak{T}_{T^{\alpha}}^{\mathbf{b}}+2 \sqrt{\varepsilon} \mathfrak{N}_{T^{\alpha}}
$$

Now we take $\varepsilon=\varepsilon^{*}$ and apply the fact that $\left(\mathcal{S}_{\infty}\right)$ holds to obtain

$$
\begin{aligned}
\mathfrak{N}_{T^{\alpha}} \lesssim\left(C_{\widehat{\mathbf{b}}}(\infty)+C_{\widehat{\mathbf{b}}^{*}}(\infty)\right)\left\{\mathfrak{T}_{T^{\alpha}}^{\widehat{\mathbf{b}}}+\mathfrak{T}_{T^{\alpha, *}}^{\widehat{\mathbf{b}}^{*}}+\sqrt{\mathfrak{A}_{2}^{\alpha}}+\mathfrak{E}_{2}^{\alpha}\right\} \\
\lesssim\left(C_{\mathbf{b}}(p)+C_{\mathbf{b}^{*}}(p)\right)^{\frac{p}{p-2}} \varepsilon^{-\frac{1}{p-2}}\left\{\left[\mathfrak{T}_{T^{\alpha}}^{\mathbf{b}}+\sqrt{\varepsilon} \mathfrak{N}_{T^{\alpha}}\right]+\left[\mathfrak{T}_{T^{\alpha, *}}^{\mathbf{b}^{*}}+\sqrt{\varepsilon} \mathfrak{N}_{T^{\alpha}}\right]+\sqrt{\mathfrak{A}_{2}^{\alpha}}+\mathfrak{E}_{2}^{\alpha}\right\} \\
\lesssim\left(C_{\mathbf{b}}(p)+C_{\mathbf{b}^{*}}(p)\right)^{\frac{p}{p-2}} \varepsilon^{-\frac{1}{p-2}}\left\{\mathfrak{T}_{T^{\alpha}}^{\mathbf{b}}+\mathfrak{T}_{T^{\alpha, *}}^{\mathbf{b}^{*}}+\sqrt{\mathfrak{A}_{2}^{\alpha}}+\mathfrak{E}_{2}^{\alpha}\right\} \\
+\left(C_{\mathbf{b}}(p)+C_{\mathbf{b}^{*}}(p)\right)^{\frac{p}{p-2}} \varepsilon^{\frac{1}{2}-\frac{1}{p-2}} \mathfrak{N}_{T^{\alpha}} .
\end{aligned}
$$

Now we choose

$$
\varepsilon=\frac{1}{\Gamma}\left(C_{\mathbf{b}}(p)+C_{\mathbf{b}^{*}}(p)\right)^{-\frac{p}{\frac{1}{2-2}}}
$$

with $\Gamma$ large enough, depending only on the implied constant $C_{\text {implied }}$ (where $\lesssim$ is written $\leq C_{\text {implied }}$ ), so that the final term on the right satisfies

$$
\begin{aligned}
& C_{\text {implied }}\left(C_{\mathbf{b}}(p)+C_{\mathbf{b}^{*}}(p)\right)^{\frac{p}{p-2}} \varepsilon^{\frac{1}{2}-\frac{1}{p-2}} \mathfrak{N}_{T^{\alpha}} \\
= & C_{\text {implied }}\left(C_{\mathbf{b}}(p)+C_{\mathbf{b}^{*}}(p)\right)^{\frac{p}{p-2}}\left(\frac{1}{\Gamma}\right)^{\frac{1}{2}-\frac{1}{p-2}}\left(C_{\mathbf{b}}(p)+C_{\mathbf{b}^{*}}(p)\right)^{-\frac{p}{p-2}} \mathfrak{N}_{T^{\alpha}} \\
\leq & C_{\text {implied }}\left(\frac{1}{\Gamma}\right)^{\frac{1}{4}} \mathfrak{N}_{T^{\alpha}}=\frac{1}{2} \mathfrak{N}_{T^{\alpha}},
\end{aligned}
$$

i.e, we choose $\Gamma=\left(2 C_{\text {implied }}\right)^{4}$, and where we have used $\frac{1}{2}-\frac{1}{p-2} \geq \frac{1}{4}$ for $p>6$. This term can then be absorbed into the left hand side to obtain

$$
\begin{gathered}
\mathfrak{N}_{T^{\alpha}} \lesssim\left(C_{\mathbf{b}}(p)+C_{\mathbf{b}^{*}}(p)\right)^{\frac{p}{p-2}}\left(\left(C_{\mathbf{b}}(p)+C_{\mathbf{b}^{*}}(p)\right)^{-\frac{p}{\frac{1}{2}-\frac{1}{p-2}}}\right)^{-\frac{1}{p-2}} \\
\times\left\{\mathfrak{T}_{T^{\alpha}}^{\mathbf{b}}+\mathfrak{T}_{T^{\alpha}, *}^{\mathbf{b}^{*}}+\sqrt{\mathfrak{A}_{2}^{\alpha}}+\mathfrak{E}_{2}^{\alpha}\right\} \\
\lesssim\left(C_{\mathbf{b}}(p)+C_{\mathbf{b}^{*}}(p)\right)^{\frac{p}{p-2}\left\{1+\frac{\frac{1}{p-2}}{\frac{1}{2}-\frac{1}{p-2}}\right\}\left\{\mathfrak{T}_{T^{\alpha}}^{\mathbf{b}}+\mathfrak{T}_{T^{\alpha, *}}^{\mathbf{b}^{*}}+\sqrt{\mathfrak{A}_{2}^{\alpha}}+\mathfrak{E}_{2}^{\alpha}\right\}}
\end{gathered}
$$

Since

we get

$$
\frac{p}{p-2}\left\{1+\frac{\frac{1}{p-2}}{\frac{1}{2}-\frac{1}{p-2}}\right\}=\left(1+\frac{2}{p-2}\right)\left(1+\frac{2}{p-4}\right) \leq 3 \text { for } p>6
$$

$$
\mathfrak{N}_{T^{\alpha}} \lesssim\left(C_{\mathbf{b}}(p)+C_{\mathbf{b}^{*}}(p)\right)^{3}\left\{\mathfrak{T}_{T^{\alpha}}^{\mathbf{b}}+\mathfrak{T}_{T^{\alpha, *}}^{\mathbf{b}^{*}}+\sqrt{\mathfrak{A}_{2}^{\alpha}}+\mathfrak{E}_{2}^{\alpha}\right\},
$$

which completes the proof of $\left(\mathcal{S}_{0}\right)$.

Suppose now, in order to derive a contradiction, that $\left(\mathcal{S}_{n}\right)$ fails for some $n \geq 1$. Then there exists an integer $n \geq 1$ and an exponent $r \in\left(p_{n+1}, p_{n}\right]$ such that

$\left(\mathcal{S}_{r}\right)$ fails and $\left(\mathcal{S}_{q}\right)$ holds for $q>p_{n}$. 
We now show that $\left(\mathcal{S}_{p}\right)$ holds for all $p \in\left(p_{n+1}, p_{n}\right]$, contradicting the fact that $\left(\mathcal{S}_{r}\right)$ fails. So fix $n \geq 1$, $p \in\left(p_{n+1}, p_{n}\right]$, and suppose that $\mathbf{b}=\left\{b_{Q}\right\}_{Q \in \mathcal{P}}$ is a $p$-weakly $\sigma$-accretive family of functions on $\mathbb{R}$, and that $\mathbf{b}^{*}=\left\{b_{Q}^{*}\right\}_{Q \in \mathcal{P}}$ is a $p$-weakly $\omega$-accretive family of functions on $\mathbb{R}$. Note that the sequence $\left\{p_{n}\right\}_{n=0}^{\infty}=$ $\left\{\frac{2}{1-\left(\frac{2}{3}\right)^{n}}\right\}_{n=0}^{\infty}$ satisfies the recursion relation

$$
p_{n+1}=\frac{6}{1+\frac{4}{p_{n}}}, \text { equivalently } p_{n}=\frac{4}{\frac{6}{p_{n+1}}-1}, \quad n \geq 0 .
$$

Choose $q \in\left(p_{n}, p_{n-1}\right]$ so that

$$
p>\frac{6}{1+\frac{4}{q}}, \text { i.e. } q<\frac{4}{\frac{6}{p}-1}
$$

which can be done since $p>p_{n+1}=\frac{2}{1-\left(\frac{2}{3}\right)^{n+1}}$ is equivalent to $p_{n}=\frac{2}{1-\left(\frac{2}{3}\right)^{n}}<\frac{4}{\frac{6}{p}-1}$, which leaves room to choose $q$ satisfying $p_{n}<q<\frac{4}{\frac{6}{p}-1}$.

Now let $0<\varepsilon<1$ (to be fixed later), define $\lambda=\lambda(\varepsilon)$ as in (2.6), and define $\widehat{b}_{Q}$ as in (2.7). Recall from (2.8) and (2.9) that we then have

$$
\int_{\left\{\left|b_{Q}\right|>\lambda\right\}}\left|b_{Q}\right|^{2} d \sigma \leq \varepsilon|Q|_{\sigma}
$$

and

$$
\left|\frac{1}{|Q|_{\sigma}} \int_{Q} \widehat{b}_{Q} d \sigma\right| \geq 1, \quad Q \in \mathcal{P},
$$

if we choose $0<\varepsilon \leq \frac{1}{4}$. We of course have the previous upper bound

$$
\left\|\widehat{b}_{Q}\right\|_{L^{\infty}(\sigma)} \leq 2 \lambda=2 \lambda(\varepsilon)=2\left(\frac{p}{p-2} C_{\mathbf{b}}(p)^{p} \frac{1}{\varepsilon}\right)^{\frac{1}{p-2}}
$$

and while this turned out to be sufficient in the case $n=0$, we must do better than $O\left(\frac{1}{\varepsilon}\right)^{\frac{1}{p-2}}$ in the case $n \geq 1$. In fact we compute the $L^{q}$ norm instead, recalling that $q>p$ :

$$
\begin{aligned}
& \left(\frac{1}{|Q|_{\mu}} \int_{Q}\left|\widehat{b}_{Q}\right|^{q} d \mu\right)^{\frac{1}{q}}=2\left(\frac{1}{|Q|_{\mu}} \int_{Q}\left|b_{Q}\left(\mathbf{1}_{\left\{\left|b_{Q}\right| \leq \lambda\right\}}+\frac{\lambda}{\left|b_{Q}\right|} \mathbf{1}_{\left\{\left|b_{Q}\right|>\lambda\right\}}\right)\right|^{q} d \mu\right)^{\frac{1}{q}} \\
= & 2\left(\frac{1}{|Q|_{\mu}} \int_{\left\{\left|b_{Q}\right| \leq \lambda\right\}}\left[\int_{0}^{\left|b_{Q}\right|} q t^{q-1} d t\right] d \sigma+\frac{\lambda^{q}\left|\left\{\left|b_{Q}\right|>\lambda\right\}\right|_{\mu}}{|Q|_{\mu}}\right)^{\frac{1}{q}} \\
\leq & 2\left(\frac{1}{|Q|_{\mu}} \int_{0}^{\lambda}\left[\int_{\left\{t<\left|b_{Q}\right| \leq \lambda\right\}} d \sigma\right] q t^{q-1} d t+C_{\mathbf{b}}(p)^{p} \lambda^{q-p}\right)^{\frac{1}{q}} \\
\leq & 2\left(\frac{1}{|Q|_{\mu}} \int_{0}^{\lambda}\left[\frac{1}{t^{p}} \int\left|b_{Q}\right|^{p} d \sigma\right] q t^{q-1} d t+C_{\mathbf{b}}(p)^{p} \lambda^{q-p}\right)^{\frac{1}{q}} \\
\leq & 2 C_{\mathbf{b}}(p)^{\frac{p}{q}}\left(\int_{0}^{\lambda} q t^{q-p-1} d t+\lambda^{q-p}\right)^{\frac{1}{q}}=2\left(C_{\mathbf{b}}(p)^{p} \frac{2 q-p}{q-p} \lambda^{q-p}\right)^{\frac{1}{q}}
\end{aligned}
$$

which shows that $C_{\widehat{\mathbf{b}}}(q)$ satisfies the estimate

$$
\begin{aligned}
C_{\widehat{\mathbf{b}}}(q) & \leq 2 C_{\mathbf{b}}(p)^{\frac{p}{q}}\left(\frac{2 q-p}{q-p}\right)^{\frac{1}{q}}\left[\left(\frac{p}{p-2} C_{\mathbf{b}}(p)^{p} \frac{1}{\varepsilon}\right)^{\frac{1}{p-2}}\right]^{1-\frac{p}{q}} \\
& \lesssim C_{\mathbf{b}}(p)^{\frac{p}{q}\left(1+\frac{q-p}{p-2}\right)} \varepsilon^{-\frac{1-\frac{p}{q}}{p-2}} \lesssim C_{\mathbf{b}}(p)^{\frac{3}{2}} \varepsilon^{-\frac{1-\frac{p}{q}}{p-2}}
\end{aligned}
$$


a significant improvement over the bound $O\left(\varepsilon^{-\frac{1}{p-2}}\right)$. Here we have used that if $p>\frac{6}{1+\frac{4}{q}}=\frac{6 q}{q+4}$, then

$$
\begin{aligned}
\frac{p}{q}\left(1+\frac{q-p}{p-2}\right) & =\frac{p}{p-2} \frac{q-2}{q}<\frac{\frac{6 q}{q+4}}{\frac{6 q}{q+4}-2} \frac{q-2}{q} \\
& =\frac{6 q}{6 q-(2 q+8)} \frac{q-2}{q}=\frac{6(q-2)}{4 q-8}=\frac{3}{2}
\end{aligned}
$$

Moreover, from (2.10) we also have

$$
\mathfrak{T}_{T^{\alpha}}^{\widehat{\mathbf{b}}} \leq 2 \mathfrak{T}_{T^{\alpha}}^{\mathbf{b}}+2 \sqrt{\varepsilon} \mathfrak{N}_{T^{\alpha}}
$$

We can do the same for the dual testing functions $\mathbf{b}^{*}=\left\{b_{Q}^{*}\right\}_{Q \in \mathcal{P}}$, and then altogether we have both

$$
\begin{gathered}
1 \leq\left|\frac{1}{|Q|_{\sigma}} \int_{Q} \widehat{b}_{Q} d \sigma\right| \leq\left\|\widehat{b}_{Q}\right\|_{L^{q}(\sigma)} \leq C_{\mathbf{b}}(p)^{\frac{3}{2}} \varepsilon^{-\frac{1-\frac{p}{q}}{p^{2}}}, \quad Q \in \mathcal{P}, \\
\mathfrak{T}_{T^{\alpha}}^{\widehat{\mathbf{b}}} \leq 2 \mathfrak{T}_{T^{\alpha}}^{\mathbf{b}}+2 \sqrt{\varepsilon} \mathfrak{N}_{T^{\alpha}},
\end{gathered}
$$

as well as

$$
\begin{gathered}
1 \leq\left|\frac{1}{|Q|_{\omega}} \int_{Q} \widehat{b^{*}} Q_{Q} d \omega\right| \leq\left\|\widehat{b^{*}} Q\right\|_{L^{q}(\omega)} \leq C_{\mathbf{b}^{*}}(p)^{\frac{3}{2}} \varepsilon^{-\frac{1-\frac{p}{q}}{p-2}}, \quad Q \in \mathcal{P}, \\
\mathfrak{T}_{T^{\alpha}}^{\widehat{\mathbf{b}^{*}}} \leq 2 \mathfrak{T}_{T^{\alpha}}^{\mathbf{b}^{*}}+2 \sqrt{\varepsilon^{*}} \mathfrak{N}_{T^{\alpha}},
\end{gathered}
$$

provided

$$
0<\varepsilon=\varepsilon^{*} \leq \frac{1}{4} .
$$

We now use these estimates, together with the fact that $\left(\mathcal{S}_{n-1}\right)$ holds, to obtain

$$
\begin{gathered}
\mathfrak{N}_{T^{\alpha}} \lesssim\left(C_{\widehat{\mathbf{b}}}(q)+C_{\widehat{\mathbf{b}}^{*}}(q)\right)^{3^{n}}\left\{\mathfrak{T}_{T^{\alpha}}^{\widehat{\mathbf{b}}}+\mathfrak{T}_{T^{\alpha, *}}^{\widehat{\mathbf{b}}^{*}}+\sqrt{\mathfrak{A}_{2}^{\alpha}}+\mathfrak{E}_{2}^{\alpha}\right\} \\
\lesssim\left(C_{\mathbf{b}}(p)+C_{\mathbf{b}^{*}}(p)\right)^{\frac{3}{2} 3^{n}} \varepsilon^{-\frac{1-\frac{p}{q}}{p-2}} \\
\quad \times\left\{\left[\mathfrak{T}_{T^{\alpha}}^{\mathbf{b}}+\sqrt{\varepsilon} \mathfrak{N}_{T^{\alpha}}\right]+\left[\mathfrak{T}_{T^{\alpha, *}}^{\mathbf{b}^{*}}+\sqrt{\varepsilon} \mathfrak{N}_{T^{\alpha}}\right]+\sqrt{\mathfrak{A}_{2}^{\alpha}}+\mathfrak{E}_{2}^{\alpha}\right\} \\
\lesssim\left(C_{\mathbf{b}}(p)+C_{\mathbf{b}^{*}}(p)\right)^{\frac{3}{2} 3^{n}} \varepsilon^{-\frac{1-\frac{p}{q}}{p-2}}\left\{\mathfrak{T}_{T^{\alpha}}^{\mathbf{b}}+\mathfrak{T}_{T^{\alpha, *}}^{\mathbf{b}^{*}}+\sqrt{\mathfrak{A}_{2}^{\alpha}}+\mathfrak{E}_{2}^{\alpha}\right\} \\
+\left(C_{\mathbf{b}}(p)+C_{\mathbf{b}^{*}}(p)\right)^{\frac{3}{2} 3^{n}} \sqrt{\varepsilon \varepsilon} \varepsilon^{-\frac{1-\frac{p}{q}}{p-2}} \mathfrak{N}_{T^{\alpha}} .
\end{gathered}
$$

We can absorb the last term on the right hand side above into the left hand side for $\varepsilon>0$ sufficiently small, since (2.11) gives $\frac{\frac{6}{p}-1}{2}<\frac{2}{q}$, and hence

$$
\frac{1}{2}-\frac{1-\frac{p}{q}}{p-2}=\frac{p\left(1+\frac{2}{q}\right)-4}{2 p-4}>\frac{p\left(1+\frac{\frac{6}{p}-1}{2}\right)-4}{2 p-4}=\frac{1}{4} .
$$

In fact, we choose

$$
\varepsilon=\frac{1}{\Gamma}\left(C_{\mathbf{b}}(p)+C_{\mathbf{b}^{*}}(p)\right)^{\left[\frac{3}{2} 3^{n}\right]}\left[\frac{\frac{1}{q-\frac{p}{q}}-\frac{1}{2}}{\frac{1-2}{p-2}}\right.
$$


with $\Gamma$ sufficiently large, depending only on the implied constant, to get

$$
\begin{gathered}
\left.\mathfrak{N}_{T^{\alpha}} \lesssim\left(C_{\mathbf{b}}(p)+C_{\mathbf{b}^{*}}(p)\right)^{\left[\frac{3}{2} 3^{n}\right]}\left(\left(C_{\mathbf{b}}(p)+C_{\mathbf{b}^{*}}(p)\right)^{\left[\frac{3}{2} 3^{n}\right]\left[\frac{1}{\frac{1-\frac{p}{q}}{p-2}-\frac{1}{2}}\right]}\right)^{-\frac{1-\frac{p}{q}}{p-2}}\right) \\
\times\left\{\mathfrak{T}_{T^{\alpha}}^{\mathbf{b}}+\mathfrak{T}_{T^{\alpha, *}}^{\mathbf{b}^{*}}+\sqrt{\mathfrak{A}_{2}^{\alpha}}+\mathfrak{E}_{2}^{\alpha}\right\} \\
\lesssim\left(C_{\mathbf{b}}(p)+C_{\mathbf{b}^{*}}(p)\right)^{\left[\frac{3}{2} 3^{n}\right][1+1]}\left\{\mathfrak{T}_{T^{\alpha}}^{\mathbf{b}}+\mathfrak{T}_{T^{\alpha, *}}^{\mathbf{b}^{*}}+\sqrt{\mathfrak{A}_{2}^{\alpha}}+\mathfrak{E}_{2}^{\alpha}\right\} .
\end{gathered}
$$

Here we have used that (2.13) applied twice implies

$$
\frac{\frac{1-\frac{p}{q}}{p-2}}{\frac{1}{2}-\frac{1-\frac{p}{q}}{p-2}}<4 \frac{1-\frac{p}{q}}{p-2} \leq 1
$$

So we finally have

$$
\mathfrak{N}_{T^{\alpha}} \lesssim\left(C_{\mathbf{b}}(p)+C_{\mathbf{b}^{*}}(p)\right)^{3^{n+1}}\left\{\mathfrak{T}_{T^{\alpha}}^{\mathbf{b}}+\mathfrak{T}_{T^{\alpha, *}}^{\mathbf{b}^{*}}+\sqrt{\mathfrak{A}_{2}^{\alpha}}+\mathfrak{E}_{2}^{\alpha}\right\},
$$

which completes the proof of Proposition 14

Remark 15. Propositions 12 and 14 extend to higher dimensions with analogous proofs.

Conclusion 16. We may assume for the proof of Theorem 7 given below that $p=\infty$ and that the testing functions are real-valued, satisfy the PLBP and satisfy

$$
\begin{aligned}
& \text { support } b_{Q} \subset Q, \quad Q \in \mathcal{P}, \\
& 1 \leq \frac{1}{|Q|_{\mu}} \int_{Q} b_{Q} d \mu \leq\left\|b_{Q}\right\|_{L^{\infty}(\mu)} \leq C_{\mathbf{b}}(\infty)<\infty, \quad Q \in \mathcal{P} .
\end{aligned}
$$

2.3. Reverse Hölder control of children. Here we begin to further reduce the proof of Theorem 7 to the case of bounded real testing functions $\mathbf{b}=\left\{b_{Q}\right\}_{Q \in \mathcal{P}}$ having reverse Hölder control

$$
\left|\frac{1}{\left|Q^{\prime}\right|_{\sigma}} \int_{Q^{\prime}} b_{Q} d \sigma\right| \geq c\left\|\mathbf{1}_{Q^{\prime}} b_{Q}\right\|_{L^{\infty}(\sigma)}>0,
$$

for all children $Q^{\prime} \in \mathfrak{C}(Q)$ with $\left|Q^{\prime}\right|_{\sigma}>0$ and $Q \in \mathcal{P}$.

2.3.1. Control of averages over children. Here we address the case of a single interval $Q$.

Lemma 17. Suppose that $\sigma$ and $\omega$ are locally finite positive Borel measures on the real line $\mathbb{R}$. Assume that $T^{\alpha}$ is a standard $\alpha$-fractional elliptic and gradient elliptic singular integral operator on $\mathbb{R}$, and set $T_{\sigma}^{\alpha} f=T^{\alpha}(f \sigma)$ for any smooth truncation of $T_{\sigma}^{\alpha}$, so that $T_{\sigma}^{\alpha}$ is apriori bounded from $L^{2}(\sigma)$ to $L^{2}(\omega)$. Let $Q \in \mathcal{P}$ and let $\mathfrak{N}_{T^{\alpha}}(Q)$ be the best constant in the local inequality

$$
\sqrt{\int_{Q^{\prime}}\left|T_{\sigma}^{\alpha}\left(\mathbf{1}_{Q} f\right)\right|^{2} d \omega} \leq \mathfrak{N}_{T^{\alpha}}(Q) \sqrt{\int_{Q}|f|^{2} d \sigma}, \quad f \in L^{2}\left(\mathbf{1}_{Q} \sigma\right) .
$$

Suppose that $b_{Q}$ is a real-valued function supported in $Q$ such that

$$
\begin{gathered}
1 \leq \frac{1}{|Q|_{\sigma}} \int_{Q} b_{Q} d \sigma \leq\left\|\mathbf{1}_{Q} b_{Q}\right\|_{L^{\infty}(\sigma)} \leq C_{b_{Q}}, \\
\sqrt{\int_{Q}\left|T_{\sigma}^{\alpha} b_{Q}\right|^{2} d \omega} \leq \mathfrak{T}_{T^{\alpha}}^{b_{Q}}(Q) \sqrt{|Q|_{\sigma}} .
\end{gathered}
$$


Then for every $0<\delta<\frac{1}{4 C_{\mathrm{b}}^{3}}$, there exists a real-valued function $\widetilde{b}_{Q}$ supported in $Q$ such that

$$
\begin{aligned}
& 1 \leq \frac{1}{|Q|_{\sigma}} \int_{Q} \widetilde{b}_{Q} d \sigma \leq\left\|\mathbf{1}_{Q} \widetilde{b}_{Q}\right\|_{L^{\infty}(\sigma)} \leq 2\left(1+\sqrt{C_{b_{Q}}}\right) C_{b_{Q}}, \\
& \sqrt{\int_{Q}\left|T_{\sigma}^{\alpha} \widetilde{b}_{Q}\right|^{2} d \omega} \leq\left[2 \widetilde{T}_{T^{\alpha}}^{b_{Q}}(Q)+4 C_{b_{Q}}^{\frac{3}{2}} \delta^{\frac{1}{4}} \mathfrak{N}_{T^{\alpha}}(Q)\right] \sqrt{|Q|_{\sigma}}, \\
& 0<\left\|\mathbf{1}_{Q_{i}} \widetilde{b}_{Q}\right\|_{L^{\infty}(\sigma)} \leq \frac{16 C_{b_{Q}}}{\delta}\left|\frac{1}{\left|Q_{i}\right|_{\sigma}} \int_{Q_{i}} \widetilde{b}_{Q} d \sigma\right|, \quad Q_{i} \in \mathfrak{C}(Q) .
\end{aligned}
$$

Moreover, if $\left|b_{Q}\right| \geq c_{1}>0$, then we may take $\left|\widetilde{b}_{Q}\right| \geq c_{1}$ as well.

Proof. Let $0<\delta<1$ and fix $Q \in \mathcal{P}$. By assumption we have

$$
1 \leq \frac{1}{|Q|_{\sigma}} \int_{Q} b_{Q} d \sigma \leq\left\|\mathbf{1}_{Q} b_{Q}\right\|_{L^{\infty}(\sigma)} \leq C_{b_{Q}}
$$

Let $Q_{\text {left }}$ and $Q_{\text {right }}$ be the children of $Q$. We now define $\widetilde{b}_{Q}$. First we note that the inequality

$$
\left|\frac{1}{\left|Q^{\prime}\right|_{\sigma}} \int_{Q^{\prime}} b_{Q} d \sigma\right|<\frac{\delta}{C_{b_{Q}}}\left\|\mathbf{1}_{Q^{\prime}} b_{Q}\right\|_{L^{\infty}(\sigma)}
$$

cannot hold for $Q^{\prime}$ equal to both $Q_{\text {left }}$ and $Q_{\text {right }}$, since otherwise we obtain the contradiction

$$
\begin{aligned}
\left|\int_{Q} b_{Q} d \sigma\right| & \leq\left|\int_{Q_{\text {left }}} b_{Q} d \sigma\right|+\left|\int_{Q_{\mathrm{right}}} b_{Q} d \sigma\right| \\
& <\frac{\delta}{C_{\mathbf{b}}}\left(\left|Q_{\text {left }}\right|_{\sigma}\left\|\mathbf{1}_{Q_{\text {left }}} b_{Q}\right\|_{L^{\infty}(\sigma)}+\left|Q_{\text {right }}\right|_{\sigma}\left\|\mathbf{1}_{Q_{\mathrm{right}}} b_{Q}\right\|_{L^{\infty}(\sigma)}\right) \\
& \leq \frac{\delta}{C_{b_{Q}}}|Q|_{\sigma}\left\|\mathbf{1}_{Q} b_{Q}\right\|_{L^{\infty}(\sigma)} \leq \delta\left|\int_{Q} b_{Q} d \sigma\right|<\left|\int_{Q} b_{Q} d \sigma\right| .
\end{aligned}
$$

If (2.16) holds for neither $Q_{\text {left }}$ nor $Q_{\text {right }}$, then we simply define $\widetilde{b}_{Q}=b_{Q}$. If (2.16) holds for just one of the children, say $Q_{\text {left }}$, then we define $\widetilde{b}_{Q}$ differently according to how large the $L^{1}(\sigma)$-average $\frac{1}{\left|Q_{\text {left }}\right|_{\sigma}} \int_{Q_{\text {left }}}\left|b_{Q}\right| d \sigma$ is.

Case (0) $\frac{1}{\left|Q_{\text {left }}\right|_{\sigma}} \int_{Q_{\text {left }}}\left|b_{Q}\right| d \sigma=0$ : In this case we define

$$
\widetilde{b}_{Q} \equiv \delta \mathbf{1}_{Q_{\text {left }}}+b_{Q} \mathbf{1}_{Q_{\text {right }}},
$$

and the reader can easily verify that the conclusions of Lemma 17 hold.

Case (1) $0<\frac{1}{\left.Q_{\text {left }}\right|_{\sigma}} \int_{Q_{\text {left }}}\left|b_{Q}\right| d \sigma \leq \sqrt{C_{b_{Q}} \delta}$ : In this case we define

$$
\widetilde{b}_{Q} \equiv\left(\frac{1}{\left|Q_{\text {left }}\right|_{\sigma}} \int_{Q_{\text {left }}}\left|b_{Q}\right| d \sigma\right) \mathbf{1}_{Q_{\text {left }}}+b_{Q} \mathbf{1}_{Q_{\mathrm{right}}} .
$$

With this definition we then have

and both

$$
\begin{aligned}
1 & \leq \frac{1}{|Q|_{\sigma}} \int_{Q} b_{Q} d \sigma \leq \frac{1}{|Q|_{\sigma}}\left(\int_{Q_{\text {left }}}\left|b_{Q}\right| d \sigma+\int_{Q_{\mathrm{right}}} b_{Q} d \sigma\right) \\
& =\frac{1}{|Q|_{\sigma}} \int_{Q} \widetilde{b}_{Q} d \sigma \leq\left\|\widetilde{b}_{Q}\right\|_{L^{\infty}(\sigma)} \leq\left\|b_{Q}\right\|_{L^{\infty}(\sigma)} \leq C_{b_{Q}},
\end{aligned}
$$

$$
\begin{gathered}
\frac{\left\|\mathbf{1}_{Q_{\text {left }}} \widetilde{b}_{Q}\right\|_{L^{\infty}(\sigma)}}{\left|\frac{1}{\left|Q_{\text {left }}\right|_{\sigma}} \int_{Q_{\text {left }}} \widetilde{b}_{Q} d \sigma\right|}=\frac{\frac{1}{\left|Q_{\text {left }}\right|_{\sigma}} \int_{Q_{\text {left }}}\left|b_{Q}\right| d \sigma}{\left|\frac{1}{\left|Q_{\text {left }}\right|_{\sigma}} \int_{Q_{\text {left }}}\right| b_{Q}|d \sigma|}=1<\frac{1}{\delta} C_{b_{Q}}, \\
\frac{\left\|\mathbf{1}_{Q_{\text {right }}} \widetilde{b}_{Q}\right\|_{L^{\infty}(\sigma)}}{\left|\frac{1}{\left|Q_{\text {right }}\right|_{\sigma}} \int_{Q_{\text {right }}} \widetilde{b}_{Q} d \sigma\right|}=\frac{\left\|\mathbf{1}_{Q_{\text {right }}} b_{Q}\right\|_{L^{\infty}(\sigma)}}{\left|\frac{1}{\left|Q_{\text {right }}\right|_{\sigma}} \int_{Q_{\text {right }}} b_{Q} d \sigma\right|}<\frac{1}{\delta} C_{b_{Q}},
\end{gathered}
$$


where the second line follows since (2.16) fails for $Q^{\prime}=Q_{\text {right }}$.

Finally we check the testing condition in this case. We have from Minkowski's inequality,

$$
\begin{aligned}
\sqrt{\frac{1}{|Q|_{\sigma}} \int_{Q}\left|T_{\sigma}^{\alpha} \widetilde{b}_{Q}\right|^{2} d \omega} & \leq \sqrt{\frac{1}{|Q|_{\sigma}} \int_{Q}\left|T_{\sigma}^{\alpha} b_{Q}\right|^{2} d \omega}+\sqrt{\frac{1}{|Q|_{\sigma}} \int_{Q}\left|T_{\sigma}^{\alpha}\left(\widetilde{b}_{Q}-b_{Q}\right)\right|^{2} d \omega} \\
& \leq \mathfrak{T}_{T^{\alpha}}^{b_{Q}}(Q)+\mathfrak{N}_{T^{\alpha}}(Q) \sqrt{\frac{1}{|Q|_{\sigma}} \int_{Q}\left|\widetilde{b}_{Q}-b_{Q}\right|^{2} d \sigma .}
\end{aligned}
$$

In the case (2.16) holds for neither $Q_{\text {left }}$ nor $Q_{\text {right }}$, then $\widetilde{b}_{Q}-b_{Q}=0$ and so

$$
\sqrt{\frac{1}{|Q|_{\sigma}} \int_{Q}\left|T_{\sigma}^{\alpha} \widetilde{b}_{Q}\right|^{2} d \omega} \leq \mathfrak{T}_{T^{\alpha}}^{b_{Q}}(Q)
$$

In the case (2.16) holds for just one child, say $Q_{\text {left }}$, then

$$
\begin{aligned}
\sqrt{\frac{1}{|Q|_{\sigma}} \int_{Q}\left|\tilde{b}_{Q}-b_{Q}\right|^{2} d \omega} & =\sqrt{\frac{1}{|Q|_{\sigma}} \int_{Q_{\text {left }}}\left|\left(\frac{1}{\left|Q_{\text {left }}\right|_{\sigma}} \int_{Q_{\text {left }}}\left|b_{Q}\right| d \sigma\right)-b_{Q}\right|^{2} d \sigma} \\
& \leq \sqrt{\frac{1}{|Q|_{\sigma}} \int_{Q_{\text {left }}}\left|\frac{1}{\left|Q_{\text {left }}\right|_{\sigma}} \int_{Q_{\text {left }}}\right| b_{Q}|d \sigma|^{2} d \sigma}+\sqrt{\frac{1}{|Q|_{\sigma}}} \int_{Q_{\text {left }}}\left|b_{Q}\right|^{2} d \sigma \\
& \leq \sqrt{\frac{1}{|Q|_{\sigma}} \int_{Q_{\text {left }}} C_{\mathbf{b}} \delta d \sigma}+\sqrt{C_{\mathbf{b}} \frac{1}{|Q|_{\sigma}} \int_{Q_{\text {left }}}\left|b_{Q}\right| d \sigma} \\
& \leq \sqrt{C_{b_{Q}} \delta}+\sqrt{C_{b_{Q}} \sqrt{C_{b_{Q}}}} \leq 2 C_{b_{Q}}^{\frac{3}{4}} \delta^{\frac{1}{4}} .
\end{aligned}
$$

Case (2) $\frac{1}{\left|Q_{\text {left }}\right|_{\sigma}} \int_{Q_{\text {left }}}\left|b_{Q}\right| d \sigma>\sqrt{C_{b_{Q}} \delta}$ : Let

$$
\begin{aligned}
\mathbf{1}_{Q_{\text {left }}}(x) b_{Q}(x) & =p(x)-n(x), \\
\mathbf{1}_{Q_{\text {left }}}(x)\left|b_{Q}(x)\right| & =p(x)+n(x),
\end{aligned}
$$

where $p(x)$ and $n(x)$ are the positive and negative parts respectively of $b_{Q}$ on $Q_{\text {left. Then define }} \widetilde{b}_{Q}$ by

$$
\widetilde{b}_{Q} \equiv\left\{\begin{array}{l}
\left(\frac{1}{\left|Q_{\text {left }}\right|_{\sigma}} \int_{Q_{\text {left }}}\left[p-n\left(1+\sqrt{C_{\mathbf{b}} \delta}\right)\right] d \sigma\right. \\
\left.\frac{1}{\left|Q_{\text {left }}\right|_{\sigma}} \int_{Q_{\text {left }}}\left[\left(1+\sqrt{C_{\mathbf{b}} \delta}\right) p-n\right] d \sigma\right) \mathbf{1}_{Q_{\text {left }}+b_{Q} \mathbf{1}_{Q_{\text {right }}}} \text { if } \quad \mathbf{1}_{Q_{\text {left }}+b_{Q} \mathbf{1}_{Q_{\text {right }}}} \text { if } \quad \int_{Q_{\text {left }}} p d \sigma<\int_{Q_{\text {left }}} n d \sigma \\
Q_{Q_{\text {left }}} n d \sigma
\end{array} .\right.
$$

Subcase (2a) $\int_{Q_{\text {left }}} p d \sigma<\int_{Q_{\text {left }}} n d \sigma:$ In this case we have

$$
\begin{aligned}
1 \leq & \frac{1}{|Q|_{\sigma}} \int_{Q} b_{Q} d \sigma=\frac{1}{|Q|_{\sigma}}\left(\int_{Q_{\text {left }}}(p-n) d \sigma+\int_{Q_{\mathrm{right}}} b_{Q} d \sigma\right) \\
& -\frac{\sqrt{C_{b_{Q}}}}{|Q|_{\sigma}} \int_{Q_{\text {left }}} n d \sigma+\frac{\sqrt{C_{b_{Q}}}}{|Q|_{\sigma}} \int_{Q_{\text {left }}} n d \sigma \\
= & \frac{1}{|Q|_{\sigma}} \int_{Q} \widetilde{b}_{Q} d \sigma+\frac{\sqrt{C_{b_{Q}} \delta}}{|Q|_{\sigma}} \int_{Q_{\text {left }}} n d \sigma \leq \frac{1}{|Q|_{\sigma}} \int_{Q} \widetilde{b}_{Q} d \sigma+\frac{\sqrt{C_{b_{Q}}}}{|Q|_{\sigma}} C_{b_{Q}}\left|Q_{\text {left }}\right|_{\sigma} \\
\leq & \frac{1}{|Q|_{\sigma}} \int_{Q} \widetilde{b}_{Q} d \sigma+\sqrt{C_{b_{Q}} \delta} C_{b_{Q}} \leq \frac{1}{|Q|_{\sigma}} \int_{Q} \widetilde{b}_{Q} d \sigma+\frac{1}{2},
\end{aligned}
$$

if we choose $0<\delta<\frac{1}{4 C_{\mathrm{b}}^{3}}$. This gives the lower bound $\frac{1}{|Q|_{\sigma}} \int_{Q} \widetilde{b}_{Q} d \sigma \geq \frac{1}{2}$, and for an upper bound we have

$$
\left\|\widetilde{b}_{Q}\right\|_{L^{\infty}(\sigma)} \leq\left(1+\sqrt{C_{b_{Q}}}\right) C_{b_{Q}} .
$$


Since we are taking $\delta<\frac{1}{4 C_{\mathrm{b}}^{3}}$, we have $1+\sqrt{C_{b_{Q}} \delta} \leq 2$, and so we also have both

$$
\begin{aligned}
& \frac{\left\|\mathbf{1}_{Q_{\text {left }}} \widetilde{b}_{Q}\right\|_{L^{\infty}(\sigma)}}{\left|\frac{1}{\left|Q_{\text {left }}\right|_{\sigma}} \int_{Q_{\text {left }}} \widetilde{b}_{Q} d \sigma\right|} \leq \frac{\left(1+\sqrt{C_{b_{Q}} \delta}\right) C_{b_{Q}}}{\left|\frac{1}{\left|Q_{\text {left }}\right|_{\sigma}} \int_{Q_{\text {left }}}\left[p-n\left(1+\sqrt{C_{b_{Q}} \delta}\right)\right] d \sigma\right|} \\
& \leq \frac{\left(1+\sqrt{C_{b_{Q}} \delta}\right) C_{b_{Q}}}{\left|\sqrt{C_{b_{Q}} \delta} \frac{1}{\left|Q_{\text {left }}\right|_{\sigma}} \int_{Q_{\text {left }}} n d \sigma\right|} \leq \frac{4 C_{b_{Q}}}{\sqrt{C_{b_{Q}} \delta} \frac{1}{\left|Q_{\text {left }}\right|_{\sigma}} \int_{Q_{\text {left }}}\left|b_{Q}\right| d \sigma} \\
& \leq \frac{4 C_{b_{Q}}}{C_{b_{Q}} \delta}=\frac{4}{\delta} \text {, } \\
& \frac{\left\|\mathbf{1}_{Q_{\mathrm{right}}} \widetilde{b}_{Q}\right\|_{L^{\infty}(\sigma)}}{\left|\frac{1}{\left|Q_{\mathrm{right}}\right|_{\sigma}} \int_{Q_{\mathrm{right}}} \widetilde{b}_{Q} d \sigma\right|}=\frac{\left\|\mathbf{1}_{Q_{\mathrm{right}}} b_{Q}\right\|_{L^{\infty}(\sigma)}}{\left|\frac{1}{\left|Q_{\mathrm{right}}\right|_{\sigma}} \int_{Q_{\mathrm{right}}} b_{Q} d \sigma\right|}<\frac{1}{\delta} C_{b_{Q}},
\end{aligned}
$$

where the second line follows since (2.16) fails for $Q^{\prime}=Q_{\text {right }}$.

Finally we check the testing condition in this case. We have from Minkowski's inequality,

$$
\begin{aligned}
\sqrt{\frac{1}{|Q|_{\sigma}} \int_{Q}\left|T_{\sigma}^{\alpha} \widetilde{b}_{Q}\right|^{2} d \omega} & \leq \sqrt{\frac{1}{|Q|_{\sigma}} \int_{Q}\left|T_{\sigma}^{\alpha} b_{Q}\right|^{2} d \omega}+\sqrt{\frac{1}{|Q|_{\sigma}} \int_{Q}\left|T_{\sigma}^{\alpha}\left(\widetilde{b}_{Q}-b_{Q}\right)\right|^{2} d \omega} \\
& \leq \mathfrak{T}_{T^{\alpha}}^{b_{Q}}(Q)+\mathfrak{N}_{T^{\alpha}}(Q) \sqrt{\frac{1}{|Q|_{\sigma}} \int_{Q}\left|\widetilde{b}_{Q}-b_{Q}\right|^{2} d \sigma .}
\end{aligned}
$$

Now recall we are assuming $\int_{Q_{\text {left }}} n d \sigma>\int_{Q_{\text {left }}} p d \sigma$, so that

$$
\begin{aligned}
\sqrt{\frac{1}{|Q|_{\sigma}} \int_{Q}\left|\widetilde{b}_{Q}-b_{Q}\right|^{2} d \sigma} & =\sqrt{\frac{1}{|Q|_{\sigma}} \int_{Q_{\text {left }}}\left|\sqrt{C_{\mathbf{b}} \delta}\right|^{2} d \sigma} \leq \sqrt{C_{b_{Q}} \delta} \sqrt{\frac{1}{|Q|_{\sigma}} \int_{Q_{\text {left }}}|n|^{2} d \sigma} \\
& \lesssim \sqrt{C_{b_{Q}} \delta} C_{b_{Q}}=C_{b_{Q}}^{\frac{3}{2}} \sqrt{\delta}
\end{aligned}
$$

Thus in any case we have

$$
\sqrt{\frac{1}{|Q|_{\sigma}} \int_{Q}\left|T_{\sigma}^{\alpha} \widetilde{b}_{Q}\right|^{2} d \omega} \leq \mathfrak{T}_{T^{\alpha}}^{b_{Q}}(Q)+2 C_{b_{Q}}^{\frac{3}{2}} \delta^{\frac{1}{2}} \mathfrak{N}_{T^{\alpha}}(Q) .
$$

Subcase (2b) $\int_{Q_{\text {left }}} p d \sigma \geq \int_{Q_{\text {left }}} n d \sigma$ : The same estimates arise in this case, except that we get the better lower bound $\frac{1}{|Q|_{\sigma}} \int_{Q} \widetilde{b}_{Q} d \sigma \geq 1$.

Collecting all of our estimates for $\widetilde{b}_{Q}$ in the various cases above we have

$$
\begin{aligned}
& \frac{1}{2} \leq \frac{1}{|Q|_{\sigma}} \int_{Q} \widetilde{b}_{Q} d \sigma \leq\left\|\mathbf{1}_{Q} \widetilde{b}_{Q}\right\|_{L^{\infty}(\sigma)} \leq\left(1+\sqrt{C_{b_{Q}}}\right) C_{b_{Q}}, \quad Q \in \mathcal{P}, \\
& \frac{\left\|\mathbf{1}_{Q^{\prime}} \widetilde{b}_{Q}\right\|_{L^{\infty}(\sigma)}}{\left|\frac{1}{\left|Q^{\prime}\right|_{\sigma}} \int_{Q^{\prime}} \widetilde{b}_{Q} d \sigma\right|}, \frac{\left\|\mathbf{1}_{Q^{\prime}} \widetilde{b}_{Q}^{*}\right\|_{L^{\infty}(\omega)}}{\left|\frac{1}{\left|Q^{\prime}\right|_{\omega}} \int_{Q^{\prime}} \widetilde{b}_{Q}^{*} d \omega\right|}<\frac{4}{\delta} C_{b_{Q}}, \quad Q \in \mathcal{P} \text { and } Q^{\prime} \in \mathfrak{C}(Q), \\
& \sqrt{\frac{1}{|Q|_{\sigma}} \int_{Q}\left|T_{\sigma}^{\alpha} \widetilde{b}_{Q}\right|^{2} d \omega} \leq \widetilde{T}_{T^{\alpha}}^{b_{Q}}(Q)+2 C_{b_{Q}}^{\frac{3}{2}} \delta^{\frac{1}{4}} \mathfrak{N}_{T^{\alpha}}(Q) \quad Q \in \mathcal{P} .
\end{aligned}
$$

In order to obtain the inequalities for $\widetilde{b}_{Q}$ in the conclusion of Lemma 17, we simply multiply the above function $\widetilde{b}_{Q}$ by a factor of 2 .

Finally, if $\left|b_{Q}\right| \geq c_{1}>0$, we need only consider Case (2) above, in which case we have $\left|b_{Q}\right| \geq\left|\widetilde{b}_{Q}\right|$. This completes the proof of Lemma 17. 
2.3.2. Control of averages in coronas. Let $\mathcal{D}_{Q}$ be the grid of dyadic subintervals of $Q$. In the construction of the triple corona below, we will need to repeat the construction in the previous subsubsection for a subdecomposition $\left\{Q_{i}\right\}_{i=1}^{\infty}$ of dyadic subintervals $Q_{i} \in \mathcal{D}_{Q}$ of an interval $Q$. Define the corona corresponding to the subdecomposition $\left\{Q_{i}\right\}_{i=1}^{\infty}$ by

$$
\mathcal{C}_{Q} \equiv \mathcal{D}_{Q} \backslash \bigcup_{i=1}^{\infty} \mathcal{D}_{Q_{i}}
$$

Lemma 18. Suppose that $\sigma$ and $\omega$ are locally finite positive Borel measures on the real line $\mathbb{R}$. Assume that $T^{\alpha}$ is a standard $\alpha$-fractional elliptic and gradient elliptic singular integral operator on $\mathbb{R}$, and set $T_{\sigma}^{\alpha} f=T^{\alpha}(f \sigma)$ for any smooth truncation of $T_{\sigma}^{\alpha}$, so that $T_{\sigma}^{\alpha}$ is apriori bounded from $L^{2}(\sigma)$ to $L^{2}(\omega)$. Let $Q \in \mathcal{P}$ and let $\mathfrak{N}_{T^{\alpha}}(Q)$ be the best constant in the local inequality

$$
\sqrt{\int_{Q^{\prime}}\left|T_{\sigma}^{\alpha}\left(\mathbf{1}_{Q} f\right)\right|^{2} d \omega} \leq \mathfrak{N}_{T^{\alpha}}(Q) \sqrt{\int_{Q}|f|^{2} d \sigma}, \quad f \in L^{2}\left(\mathbf{1}_{Q} \sigma\right) .
$$

Let $\left\{Q_{i}\right\}_{i=1}^{\infty} \subset \mathcal{D}_{Q}$ be a collection of pairwise disjoint dyadic subintervals of $Q$. Suppose that $b_{Q}$ is a realvalued function supported in $Q$ such that

$$
\begin{aligned}
& 1 \leq \frac{1}{\left|Q^{\prime}\right|_{\sigma}} \int_{Q^{\prime}} b_{Q} d \sigma \leq\left\|\mathbf{1}_{Q^{\prime}} b_{Q}\right\|_{L^{\infty}(\sigma)} \leq C_{\mathbf{b}}, \quad Q^{\prime} \in \mathcal{C}_{Q} \\
& \sqrt{\int_{Q}\left|T_{\sigma}^{\alpha} b_{Q}\right|^{2} d \omega} \leq \mathfrak{T}_{T^{\alpha}}^{b_{Q}}(Q) \sqrt{|Q|_{\sigma}}
\end{aligned}
$$

Then for every $0<\delta<\frac{1}{4 C_{\mathrm{b}}^{3}}$, there exists a real-valued function $\widetilde{b}_{Q}$ supported in $Q$ such that

$$
\begin{aligned}
& 1 \leq \frac{1}{\left|Q^{\prime}\right|_{\sigma}} \int_{Q^{\prime}} \widetilde{b}_{Q} d \sigma \leq\left\|\mathbf{1}_{Q^{\prime}} \widetilde{b}_{Q}\right\|_{L^{\infty}(\sigma)} \leq 2\left(1+\sqrt{C_{\mathbf{b}}}\right) C_{\mathbf{b}}, \quad Q^{\prime} \in \mathcal{C}_{Q}, \\
& \sqrt{\int_{Q}\left|T_{\sigma}^{\alpha} \widetilde{b}_{Q}\right|^{2} d \omega} \leq\left[2 \mathfrak{T}_{T^{\alpha}}^{b_{Q}}(Q)+4 C_{\mathbf{b}}^{\frac{3}{2}} \delta^{\frac{1}{4}} \mathfrak{N}_{T^{\alpha}}(Q)\right] \sqrt{|Q|_{\sigma}}, \\
& 0<\left\|\mathbf{1}_{Q_{i}} \widetilde{b}_{Q}\right\|_{L^{\infty}(\sigma)} \leq \frac{16 C_{\mathbf{b}}}{\delta}\left|\frac{1}{\left|Q_{i}\right|_{\sigma}} \int_{Q_{i}} \widetilde{b}_{Q} d \sigma\right|, \quad 1 \leq i<\infty .
\end{aligned}
$$

Moreover, if $\left|b_{Q}\right| \geq c_{1}>0$, then we may take $\left|\widetilde{b}_{Q}\right| \geq c_{1}$ as well.

The additional gain in the lemma is in the final line that controls the degeneracy of $\widetilde{b}_{Q}$ at the 'bottom' of the corona $\mathcal{C}_{Q}$ by establishing a reverse Hölder control. Note that if we combine this control with the accretivity control in the corona $\mathcal{C}_{Q}$, namely

$$
\left\|\mathbf{1}_{Q^{\prime}} \widetilde{b}_{Q}\right\|_{L^{\infty}(\sigma)} \leq 2\left(1+\sqrt{C_{\mathbf{b}}}\right) C_{\mathbf{b}} \leq 2\left(1+\sqrt{C_{\mathbf{b}}}\right) C_{\mathbf{b}} \frac{1}{\left|Q^{\prime}\right|_{\sigma}} \int_{Q^{\prime}} \widetilde{b}_{Q} d \sigma,
$$

we obtain reverse Hölder control throughout the entire collection $\mathcal{C}_{Q} \cup\left\{Q_{i}\right\}_{i=1}^{\infty}$ :

$$
\left\|\mathbf{1}_{I} \widetilde{b}_{Q^{\prime}}\right\|_{L^{\infty}(\sigma)} \leq C_{\delta, \mathbf{b}}\left|\frac{1}{|I|_{\sigma}} \int_{I} \widetilde{b}_{Q^{\prime}} d \sigma\right|, \quad I \in \mathfrak{C}\left(Q^{\prime}\right), Q^{\prime} \in \mathcal{C}_{Q}
$$

This has the crucial consequence that the martingale and dual martingale differences $\triangle_{Q^{\prime}}^{\sigma, \mathbf{b}}$ and $\square_{Q^{\prime}}^{\sigma, \mathbf{b}}$ associated with these functions as defined in (9.5) of Appendix A, satisfy

$$
\left|\triangle_{Q^{\prime}}^{\sigma, \mathbf{b}} h\right|,\left|\square_{Q^{\prime}}^{\sigma, \mathbf{b}} h\right| \leq C_{\delta, \mathbf{b}} \sum_{I \in \mathfrak{C}\left(Q^{\prime}\right)}\left(\frac{1}{|I|_{\sigma}} \int_{I}|h| d \sigma+\frac{1}{\left|Q^{\prime}\right|_{\sigma}} \int_{Q^{\prime}}|h| d \sigma\right) \mathbf{1}_{I}
$$

See Appendix A for more detail on this. However, the defect in this lemma is that we lose the weak testing condition for $\widetilde{b}_{Q}$ in the corona even if we had assumed it at the outset for $b_{Q}$. 
Proof. The proof of Lemma 18 is similar to that of the special case given by Lemma 17. Indeed, we define

$$
\begin{aligned}
\widetilde{b}_{Q} \equiv & \sum_{i \in G_{0}} \delta \mathbf{1}_{Q_{i}}+\sum_{i \in G_{+}}\left(\frac{1}{\left|Q_{i}\right|_{\sigma}} \int_{Q_{i}}\left|b_{Q}\right| d \sigma\right) \mathbf{1}_{Q_{i}} \\
& +\sum_{i \in B_{-}}\left(\frac{1}{\left|Q_{i}\right|_{\sigma}} \int_{Q_{i}}\left[p_{i}-n_{i}\left(1+\sqrt{C_{\mathbf{b}} \delta}\right)\right] d \sigma\right) \mathbf{1}_{Q_{i}} \\
& +\sum_{i \in B_{+}}\left(\frac{1}{\left|Q_{i}\right|_{\sigma}} \int_{Q_{i}}\left[\left(1+\sqrt{C_{\mathbf{b}} \delta}\right) p_{i}-n_{i}\right] d \sigma\right) \mathbf{1}_{Q_{i}} \\
& +b_{Q} \mathbf{1}_{Q \backslash \cup_{i=1}^{\infty} Q_{i}},
\end{aligned}
$$

where

$$
\begin{aligned}
G_{0} & \equiv\left\{i: \frac{1}{\left|Q_{i}\right|_{\sigma}} \int_{Q_{i}}\left|b_{Q}\right| d \sigma=0\right\} \\
G_{+} & \equiv\left\{i: 0<\frac{1}{\left|Q_{i}\right|_{\sigma}} \int_{Q_{i}}\left|b_{Q}\right| d \sigma \leq \sqrt{C_{\mathbf{b}} \delta}\right\}, \\
B_{-} & \equiv\left\{i: \frac{1}{\left|Q_{i}\right|_{\sigma}} \int_{Q_{i}}\left|b_{Q}\right| d \sigma>\sqrt{C_{\mathbf{b}} \delta} \text { and } \int_{Q_{i}} n d \sigma>\int_{Q_{i}} p d \sigma\right\}, \\
B_{+} & \equiv\left\{i: \frac{1}{\left|Q_{i}\right|_{\sigma}} \int_{Q_{i}}\left|b_{Q}\right| d \sigma>\sqrt{C_{\mathbf{b}} \delta} \text { and } \int_{Q_{i}} p d \sigma \geq \int_{Q_{i}} n d \sigma\right\} .
\end{aligned}
$$

First we note that

$$
\begin{aligned}
1 & \leq \frac{1}{\left|Q^{\prime}\right|_{\sigma}} \int_{Q^{\prime}} b_{Q} d \sigma \leq \frac{1}{\left|Q^{\prime}\right|_{\sigma}} \int_{Q^{\prime}} \widetilde{b}_{Q} d \sigma+\frac{1}{\left|Q^{\prime}\right|_{\sigma}} \int_{Q^{\prime}}\left(b_{Q}-\widetilde{b}_{Q}\right) d \sigma \\
& \leq \frac{1}{\left|Q^{\prime}\right|_{\sigma}} \int_{Q^{\prime}} \widetilde{b}_{Q} d \sigma+\sum_{i: Q_{i} \subset Q^{\prime}} \frac{1}{\left|Q^{\prime}\right|_{\sigma}}\left\{\begin{array}{cc}
\int_{Q_{i}}\left|b_{Q}\right| d \sigma-\delta & \text { in Case (0) } \\
2 \int_{Q_{i}}\left|b_{Q}\right| d \sigma & \text { in Case (1) } \\
\sqrt{C_{\mathbf{b}} \delta} \int_{Q_{i}}\left|b_{Q}\right| d \sigma & \text { in Case (2) }
\end{array}\right. \\
& \leq \frac{1}{\left|Q^{\prime}\right|_{\sigma}} \int_{Q^{\prime}} \widetilde{b}_{Q} d \sigma+2 \sqrt{C_{\mathbf{b}} \delta} C_{\mathbf{b}} \sum_{i: Q_{i} \subset Q^{\prime}} \frac{\left|Q_{i}\right|_{\sigma}}{\left|Q^{\prime}\right|_{\sigma}} \leq \frac{1}{\left|Q^{\prime}\right|_{\sigma}} \int_{Q^{\prime}} \widetilde{b}_{Q} d \sigma+\frac{1}{2},
\end{aligned}
$$

if $0<\delta<\frac{1}{4 C_{\mathrm{b}}^{3}}$.

Then we estimate the testing condition for the interval $Q$ by

$$
\begin{aligned}
\sqrt{\frac{1}{|Q|_{\sigma}} \int_{Q}\left|T_{\sigma}^{\alpha} \widetilde{b}_{Q}\right|^{2} d \omega} & \leq \sqrt{\frac{1}{|Q|_{\sigma}} \int_{Q}\left|T_{\sigma}^{\alpha} b_{Q}\right|^{2} d \omega}+\sqrt{\frac{1}{|Q|_{\sigma}} \int_{Q}\left|T_{\sigma}^{\alpha}\left(\widetilde{b}_{Q}-b_{Q}\right)\right|^{2} d \omega} \\
& \leq \mathfrak{T}_{T^{\alpha}}^{b_{Q}}(Q)+\mathfrak{N}_{T^{\alpha}}(Q) \sqrt{\frac{1}{|Q|_{\sigma}} \int_{Q}\left|\widetilde{b}_{Q}-b_{Q}\right|^{2} d \sigma},
\end{aligned}
$$

and note that the arguments above show that

$$
\begin{aligned}
\widetilde{b}_{Q}-b_{Q}= & \sum_{i \in G}\left(\frac{1}{\left|Q_{i}\right|_{\sigma}} \int_{Q_{i}}\left|b_{Q}\right| d \sigma\right) \mathbf{1}_{Q_{i}}-\sum_{i \in G} b_{Q} \mathbf{1}_{Q_{i}} \\
& -\sum_{i \in B_{-}}\left(\frac{\sqrt{C_{\mathbf{b}} \delta}}{\left|Q_{i}\right|_{\sigma}} \int_{Q_{i}} n_{i} d \sigma\right) \mathbf{1}_{Q_{i}}+\sum_{i \in B_{+}}\left(\frac{\sqrt{C_{\mathbf{b}} \delta}}{\left|Q_{i}\right|_{\sigma}} \int_{Q_{i}} p_{i} d \sigma\right) \mathbf{1}_{Q_{i}},
\end{aligned}
$$

satisfies an inequality of the form

$$
\int_{Q}\left|\widetilde{b}_{Q}-b_{Q}\right|^{2} d \sigma \leq C\left(C_{\mathbf{b}}\right) \delta^{\frac{1}{4}} \sum_{i=1}^{\infty}\left|Q_{i}\right|_{\sigma} \leq C\left(C_{\mathbf{b}}\right) \delta^{\frac{1}{4}}|Q|_{\sigma} .
$$


Remark 19. The estimate $\int_{Q}\left|\widetilde{b}_{Q}-b_{Q}\right|^{2} d \sigma \leq C\left(C_{\mathbf{b}}\right) \delta^{\frac{1}{4}} \sum_{i=1}^{\infty}\left|Q_{i}\right|_{\sigma}$ in the last line of the above proof is of course too large in general to be dominated by a fixed multiple of $\left|Q^{\prime}\right|_{\sigma}$ for $Q^{\prime} \in \mathcal{C}_{Q}$, and this is the reason we have no control of weak testing for $\widetilde{b}_{Q}$ in the rest of the corona even if we assume weak testing for $b_{Q}$ in the corona $\mathcal{C}_{Q}$. This defect is addressed in the next subsection below.

2.4. Three corona decompositions. We will use multiple corona constructions, namely a CalderónZygmund decomposition, an accretive decomposition, a weak testing decomposition, and an energy decomposition, in order to reduce matters to the stopping form, which is treated in Section 7 by adapting the bottom/up stopping time in the argument of M. Lacey in Lac, and using an additional 'indented' top/down corona construction, in order to accommodate weak goodness. We will then iterate these corona decompositions into a single corona decomposition, which we refer to as the triple corona. More precisely, we iterate the first generation of common stopping times with an infusion of the reverse Hölder condition on children, followed by another iteration of the first generation of weak testing stopping times. Recall that we must show the bilinear inequality

$$
\left|\int\left(T_{\sigma}^{\alpha} f\right) g d \omega\right| \leq \mathfrak{N}_{T^{\alpha}}\|f\|_{L^{2}(\sigma)}\|g\|_{L^{2}(\omega)}, \quad f \in L^{2}(\sigma) \text { and } g \in L^{2}(\omega) .
$$

2.4.1. The Calderón-Zygmund corona decomposition. We first introduce the Calderón-Zygmund stopping times $\mathcal{F}$ for a function $\phi \in L^{2}(\mu)$ relative to an interval $S_{0}$ and a positive constant $C_{0} \geq 4$. Let $\mathcal{F}=\{F\}_{F \in \mathcal{F}}$ be the collection of Calderón-Zygmund stopping intervals for $\phi$ defined so that $F \subset S_{0}, S_{0} \in \mathcal{F}$, and for all $F \in \mathcal{F}$ with $F \varsubsetneqq S_{0}$ we have

$$
\begin{aligned}
\frac{1}{|F|_{\mu}} \int_{F}|\phi| d \mu & >C_{0} \frac{1}{\mid \pi_{\left.\mathcal{F} F\right|_{\mu}}} \int_{\pi_{\mathcal{F} F}}|\phi| d \mu ; \\
\frac{1}{\left|F^{\prime}\right|_{\mu}} \int_{F^{\prime}}|\phi| d \mu & \leq C_{0} \frac{1}{\left|\pi_{\mathcal{F}} F\right|_{\mu}} \int_{\pi_{\mathcal{F} F}}|\phi| d \mu \quad \text { for } F \varsubsetneqq F^{\prime} \subset \pi_{\mathcal{F} F} F .
\end{aligned}
$$

To achieve this construction we use the following definition.

Definition 20. Let $C_{0} \geq 4$. Given a dyadic grid $\mathcal{D}$ an interval $S_{0} \in \mathcal{D}$, define $\mathcal{S}\left(S_{0}\right)$ to be the maximal $\mathcal{D}$-subintervals $I \subset S_{0}$ such that

$$
\frac{1}{|I|_{\mu}} \int_{I}|\phi| d \mu>C_{0} \frac{1}{\left|S_{0}\right|_{\mu}} \int_{S_{0}}|\phi| d \mu
$$

and then define the $C Z$ stopping intervals of $S_{0}$ to be the collection

$$
\mathcal{S}=\left\{S_{0}\right\} \cup \bigcup_{n=0}^{\infty} \mathcal{S}_{n}
$$

where $\mathcal{S}_{0}=\mathcal{S}\left(S_{0}\right)$ and $\mathcal{S}_{n+1}=\bigcup_{S \in \mathcal{S}_{n}} \mathcal{S}(S)$ for $n \geq 0$.

Let $\mathcal{D}=\bigcup_{F \in \mathcal{F}} \mathcal{C}_{F}$ be the associated corona decomposition of the dyadic grid $\mathcal{D}$ where

$$
\mathcal{C}_{F} \equiv\left\{F^{\prime} \in \mathcal{D}: F \supset F^{\prime} \supsetneqq H \text { for some } H \in \mathfrak{C}_{\mathcal{F}}(F)\right\} .
$$

We now recall some of the definitions just used above. See [SaShUr7] and/or [SaShUr6] for more detail. For an interval $I \in \mathcal{D}$ let $\pi_{\mathcal{D}} I$ be the $\mathcal{D}$-parent of $I$ in the grid $\mathcal{D}$, and let $\pi_{\mathcal{F}} I$ be the smallest member of $\mathcal{F}$ that strictly contains $I$. For $F, F^{\prime} \in \mathcal{F}$, we say that $F^{\prime}$ is an $\mathcal{F}$-child of $F$ if $\pi_{\mathcal{F}}\left(F^{\prime}\right)=F$ (it could be that $F=\pi_{\mathcal{D}} F^{\prime}$ ), and we denote by $\mathfrak{C}_{\mathcal{F}}(F)$ the set of $\mathcal{F}$-children of $F$. We call $\pi_{\mathcal{F}}\left(F^{\prime}\right)$ the $\mathcal{F}$-parent of $F^{\prime} \in \mathcal{F}$.

The stopping intervals $\mathcal{F}$ above satisfy a Carleson condition:

$$
\sum_{F \in \mathcal{F}: F \subset \Omega}|F|_{\mu} \leq C|\Omega|_{\mu}, \quad \text { for all open sets } \Omega \text {. }
$$

Indeed,

$$
\sum_{F^{\prime} \in \mathfrak{C}_{\mathcal{F}}(F)}\left|F^{\prime}\right|_{\mu} \leq \sum_{F^{\prime} \in \mathfrak{C}_{\mathcal{F}}(F)} \frac{\int_{F^{\prime}}|\phi| d \mu}{C_{0} \frac{1}{|F|_{\mu}} \int_{F}|\phi| d \mu} \leq \frac{1}{C_{0}}
$$


and standard arguments now complete the proof of the Carleson condition.

We emphasize that accretive functions $b$ play no role in the Calderón-Zygmund corona decomposition.

2.4.2. The b-accretive / weak testing corona decomposition. Recall that we are assuming $p=\infty$, and that our testing functions $\mathbf{b}$ and $\mathbf{b}^{*}$ are real-valued, in the proof of Theorem $\mathbf{7}$. We use a corona construction modelled after that of Hytönen and Martikainen HyMa, that delivers a weak corona testing condition that coincides with the testing condition itself only at the tops of the coronas. This corona decomposition is developed to optimize the choice of a new family of testing functions $\left\{\widehat{b}_{Q}\right\}_{Q \in \mathcal{D}}$ taken from the vector $\mathbf{b} \equiv\left\{b_{Q}\right\}_{Q \in \mathcal{D}}$ so that we have

(1) the telescoping property at our disposal in each accretive corona,

(2) a weak corona testing condition remains in force for the new testing functions $\widehat{b}_{Q}$ that coincides with the usual testing condition at the tops of the coronas,

(3) the tops of the coronas, i.e. the stopping intervals, enjoy a Carleson condition.

We will sometimes refer to the old family as the original family, and denote it by $\left\{b_{Q}^{\text {orig }}\right\}_{Q \in \mathcal{D}}$. The original family will reappear later in helping to estimate the nearby form in Section 5 .

Let $\sigma$ and $\omega$ be locally finite Borel measures on $\mathbb{R}$. We assume that the vector of 'testing functions' $\mathbf{b} \equiv\left\{b_{Q}\right\}_{Q \in \mathcal{D}}$ is an $\infty$-strongly $\sigma$-accretive real-valued family, i.e.

$$
\text { support } b_{Q} \subset Q, \quad Q \in \mathcal{D},
$$

and

$$
1 \leq \frac{1}{|Q|_{\mu}} \int_{Q} b_{Q} d \sigma \leq\left\|b_{Q}\right\|_{L^{\infty}(\sigma)} \leq C_{\mathbf{b}}<\infty, \quad Q \in \mathcal{D},
$$

and also that $\mathbf{b}^{*} \equiv\left\{b_{Q}\right\}_{Q \in \mathcal{D}}$ is an $\infty$-strongly $\omega$-accretive real-valued family, and we assume in addition the testing conditions

$$
\begin{gathered}
\int_{Q}\left|T_{\sigma}^{\alpha}\left(\mathbf{1}_{Q} b_{Q}\right)\right|^{2} d \omega \leq \mathfrak{T}_{T^{\alpha}}^{\mathbf{b}}|Q|_{\sigma}, \quad \text { for all intervals } Q, \\
\int_{Q}\left|T_{\omega}^{\alpha, *}\left(\mathbf{1}_{Q} b_{Q}^{*}\right)\right|^{2} d \sigma \leq \mathfrak{T}_{T^{\alpha}}^{\mathbf{b}^{*}, *}|Q|_{\omega}, \quad \text { for all intervals } Q .
\end{gathered}
$$

Definition 21. Given a dyadic grid $\mathcal{D}$ an interval $S_{0} \in \mathcal{D}$, define $\mathcal{S}\left(S_{0}\right)$ to be the maximal $\mathcal{D}$-subintervals $I \subset S_{0}$ such that

$$
\begin{aligned}
& \text { either }\left|\frac{1}{|I|_{\sigma}} \int_{I} b_{S_{0}} d \sigma\right|<\gamma, \\
& \quad \text { or } \int_{I}\left|T_{\sigma}^{\alpha}\left(b_{S_{0}}\right)\right|^{2} d \omega>\Gamma\left(\mathfrak{T}_{T^{\alpha}}^{\mathbf{b}}\right)^{2}|I|_{\sigma},
\end{aligned}
$$

where the positive constants $\gamma, \Gamma$ satisfy $0<\gamma<1<\Gamma<\infty$. Then define the $\mathbf{b}$-accretive stopping intervals of $S_{0}$ to be the collection

$$
\mathcal{S}=\left\{S_{0}\right\} \cup \bigcup_{n=0}^{\infty} \mathcal{S}_{n}
$$

where $\mathcal{S}_{0}=\mathcal{S}\left(S_{0}\right)$ and $\mathcal{S}_{n+1}=\bigcup_{S \in \mathcal{S}_{n}} \mathcal{S}(S)$ for $n \geq 0$.

For $\gamma<1$ chosen small enough and $\Gamma>1$ chosen large enough, the $\mathbf{b}$-accretive stopping intervals satisfy a $\sigma$-Carleson condition relative to the measure $\sigma$, and the corresponding stopping functions $b_{S_{0}}$ satisfy weak testing inequalities in the corona. The following lemma is essentially in $\mathrm{HyMa}$, but we include a proof for completeness.

Lemma 22 ([HyMa $)$. For $\gamma<1$ small enough and $\Gamma>1$ large enough we have the following:

(1) For every open set $\Omega$ we have we have the inequality,

$$
\sum_{S \in \mathcal{S}: S \subset \Omega}|S|_{\sigma} \leq C\left(\mathfrak{T}_{T^{\alpha}}^{\mathbf{b}}\right)^{2}|\Omega|_{\sigma} .
$$


(2) For every interval $S \in \mathcal{C}_{S_{0}}$ we have the weak corona testing inequality,

$$
\int_{S}\left|T_{\sigma}^{\alpha} b_{S_{0}}\right|^{2} d \omega \leq C\left(\mathfrak{T}_{T^{\alpha}}^{\mathbf{b}}\right)^{2}|S|_{\sigma}
$$

Proof. We first address the Carleson condition (2.19). A standard argument reduces matters to the case where $\Omega$ is an interval $Q \in \mathcal{S}$ with $|Q|_{\sigma}>0$. It suffices to consider each of the two stopping criteria separately. We first address the stopping condition $\left|\frac{1}{|I|_{\sigma}} \int_{I} b_{S_{0}} d \sigma\right|<\gamma$. Throughout this proof we will denote the union of these children $\mathcal{S}(Q)$ of $Q$ by $E(Q) \equiv \bigcup_{S \in \mathcal{S}(Q)} S$. Then we have

$$
\left|\int_{E(Q)} b_{Q} d \sigma\right| \leq \sum_{S \in \mathcal{S}(Q)}\left|\int_{S} b_{Q} d \sigma\right|<\gamma \sum_{S \in \mathcal{S}(Q)}|S|_{\sigma} \leq \gamma|Q|_{\sigma}
$$

which together with our hypotheses on $b_{Q}$ gives

$$
\begin{aligned}
|Q|_{\sigma} & <\left|\int_{Q} b_{Q} d \sigma\right|=\left|\int_{E(Q)} b_{Q} d \sigma\right|+\left|\int_{Q \backslash E(Q)} b_{Q} d \sigma\right| \\
& \leq \gamma|Q|_{\sigma}+\sqrt{\int_{Q \backslash E(Q)}\left|b_{Q}\right|^{2} d \sigma \sqrt{|Q \backslash E(Q)|_{\sigma}}} \\
& \leq \gamma|Q|_{\sigma}+\Gamma C_{\mathbf{b}} \sqrt{|Q|_{\sigma}} \sqrt{|Q \backslash E(Q)|_{\sigma}} .
\end{aligned}
$$

Rearranging the inequality yields successively

$$
\begin{aligned}
(1-\gamma)|Q|_{\sigma} & \leq \Gamma C_{\mathbf{b}} \sqrt{|Q|_{\sigma}} \sqrt{|Q \backslash E(Q)|_{\sigma}} ; \\
(1-\gamma)^{2}|Q|_{\sigma}^{2} & \leq \Gamma^{2} C_{\mathbf{b}}^{2}|Q|_{\sigma}|Q \backslash E(Q)|_{\sigma} ; \\
\frac{(1-\gamma)^{2}}{\Gamma^{2} C_{\mathbf{b}}^{2}}|Q|_{\sigma} & \leq|Q \backslash E(Q)|_{\sigma}
\end{aligned}
$$

which in turn gives

$$
\begin{aligned}
\sum_{S \in \mathcal{S}(Q)}|S|_{\sigma} & =|Q|_{\sigma}-|Q \backslash E(Q)|_{\sigma} \\
& \leq|Q|_{\sigma}-\frac{(1-\gamma)^{2}}{\Gamma^{2} C_{\mathbf{b}}^{2}}|Q|_{\sigma}=\left(1-\frac{(1-\gamma)^{2}}{\Gamma^{2} C_{\mathbf{b}}^{2}}\right)|Q|_{\sigma} \equiv \beta|Q|_{\sigma},
\end{aligned}
$$

where $0<\beta<1$ since $1 \leq C_{\mathbf{b}}$. If we now iterate this inequality, we obtain for each $k \geq 1$,

$$
\begin{aligned}
\sum_{\substack{S \in \mathcal{S}: S \subset Q \\
\pi_{\mathcal{S}}^{(k)}(S)=Q}}|S|_{\sigma} & =\sum_{\substack{S \in \mathcal{S}: S \subset Q \\
\pi_{\mathcal{S}}^{(k-1)}(S)=Q}} \sum_{S^{\prime} \in \mathcal{S}(S)}\left|S^{\prime}\right|_{\sigma} \leq \sum_{\substack{S \in \mathcal{S}: S \subset Q \\
\pi_{\mathcal{S}}^{(k-1)}(S)=Q}} \beta|S|_{\sigma} \\
& \vdots \sum_{\substack{S \in \mathcal{S}: S \subset Q \\
\pi_{\mathcal{S}}^{(1)}(S)=Q}}\left(1-\gamma^{2}\right)^{k-1}|S|_{\sigma} \leq \beta^{k}|Q|_{\sigma} \\
\leq &
\end{aligned}
$$

Finally then

$$
\sum_{S \in \mathcal{S}: S \subset Q}|S|_{\sigma} \leq|Q|_{\sigma}+\sum_{\substack { k=1 \\
\begin{subarray}{c}{S \in \mathcal{S}: S \subset Q \\
\pi_{\mathcal{S}}^{(k)}(S)=Q{ k = 1 \\
\begin{subarray} { c } { S \in \mathcal { S } : S \subset Q \\
\pi _ { \mathcal { S } } ^ { ( k ) } ( S ) = Q } }\end{subarray}}^{\infty}|S|_{\sigma} \leq \sum_{k=0}^{\infty} \beta^{k}|Q|_{\sigma}=\frac{1}{1-\beta}|Q|_{\sigma}=\frac{\Gamma^{2} C_{\mathbf{b}}^{2}}{(1-\gamma)^{2}}|Q|_{\sigma} .
$$

Now we turn to the second stopping criterion

$$
\int_{I}\left|T_{\sigma}^{\alpha}\left(b_{S_{0}}\right)\right|^{2} d \omega>\Gamma\left(\mathfrak{T}_{T^{\alpha}}^{\mathbf{b}}\right)^{2}|I|_{\sigma}
$$


We have

$$
\begin{aligned}
\sum_{S \in \mathfrak{C}_{\mathcal{S}}\left(S_{0}\right)}|S|_{\sigma} & \leq \frac{1}{\Gamma\left(\mathfrak{T}_{T^{\alpha}}^{\mathbf{b}}\right)^{2}} \sum_{S \in \mathfrak{C}_{\mathcal{S}}\left(S_{0}\right)} \int_{S}\left|T_{\sigma}^{\alpha}\left(b_{S_{0}}\right)\right|^{2} d \omega \\
& \leq \frac{1}{\Gamma\left(\mathfrak{T}_{T^{\alpha}}^{\mathbf{b}}\right)^{2}} \sum_{S_{0}} \int_{S}\left|T_{\sigma}^{\alpha}\left(b_{S_{0}}\right)\right|^{2} d \omega \leq \frac{1}{\Gamma}\left|S_{0}\right|_{\sigma} .
\end{aligned}
$$

Iterating this inequality gives

$$
\sum_{\substack{S \in \mathcal{S} \\ S \subset S_{0}}}|S|_{\sigma} \leq \sum_{k=0}^{\infty} \frac{1}{\Gamma^{k}}\left|S_{0}\right|_{\sigma}=\frac{\Gamma}{\Gamma-1}\left|S_{0}\right|_{\sigma}
$$

and then

$$
\sum_{\substack{S \in \mathcal{S} \\ S \subset \Omega}}|S|_{\sigma}=\sum_{\substack{\operatorname{maximal} \\ S_{0} \subset \Omega}} \sum_{\substack{S \in \mathcal{S} \\ S}}|S|_{\sigma} \leq \frac{\Gamma}{\Gamma-1} \sum_{\substack{\text { maximal } S_{0} \in \mathcal{S} \\ S_{0} \subset \Omega}}\left|S_{0}\right|_{\sigma}=\frac{\Gamma}{\Gamma-1}|\Omega|_{\sigma} .
$$

Finally, for $I \in \mathcal{C}_{S_{0}}$ we have the weak testing inequality

$$
\int_{I}\left|T_{\sigma}^{\alpha}\left(b_{S_{0}}\right)\right|^{2} d \omega \leq \Gamma\left(\mathfrak{T}_{T^{\alpha}}^{\mathbf{b}}\right)^{2}|I|_{\sigma},
$$

and this completes the proof of Lemma 22

2.4.3. The energy corona decompositions. Given a weight pair $(\sigma, \omega)$, we now construct an energy corona decomposition for $\sigma$, and an energy corona decomposition for $\omega$, that uniformize estimates (c.f. NTV3, LaSaUr2, SaShUr6] and [SaShUr7]). In order to define these constructions, we recall that the energy condition constant $\mathcal{E}_{2}^{\alpha}$ in Definition $[6$ is given by

$$
\left(\mathcal{E}_{2}^{\alpha}\right)^{2} \equiv \sup _{\substack{Q \in \mathcal{P} \\ Q \supset \cup J_{r}}} \frac{1}{|Q|_{\sigma}} \sum_{r=1}^{\infty}\left(\frac{\mathrm{P}^{\alpha}\left(J_{r}, \mathbf{1}_{Q} \sigma\right)}{\left|J_{r}\right|}\right)^{2}\left\|x-m_{J_{r}}\right\|_{L^{2}\left(\mathbf{1}_{J_{r}} \omega\right)}^{2},
$$

where $\dot{\cup} J_{r}$ is an arbitary subdecomposition of $Q$ into intervals $J_{r} \in \mathcal{P}$. In the next definition we restrict the intervals $Q$ to a dyadic grid $\mathcal{D}$, but keep the subintervals $J_{r}$ unrestricted.

Definition 23. Given a dyadic grid $\mathcal{D}$ and an interval $S_{0} \in \mathcal{D}$, define $\mathcal{S}\left(S_{0}\right)$ to be the maximal $\mathcal{D}$ subintervals $I \subset S_{0}$ such that

$$
\sup _{I \supset \cup J_{r}} \sum_{r=1}^{\infty}\left(\frac{\mathrm{P}^{\alpha}\left(J_{r}, \mathbf{1}_{S_{0}} \sigma\right)}{\left|J_{r}\right|}\right)^{2}\left\|x-m_{J_{r}}\right\|_{L^{2}\left(\mathbf{1}_{J_{r}} \omega\right)}^{2} \geq C_{\text {energy }}\left[\left(\mathfrak{E}_{2}^{\alpha}\right)^{2}+\mathfrak{A}_{2}^{\alpha}\right]|I|_{\sigma},
$$

where the intervals $J_{r} \in \mathcal{P}$ are pairwise disjoint in $I, \mathcal{E}_{2}^{\alpha}$ is the energy condition constant, and $C_{\text {energy }}$ is a sufficiently large positive constant depending only on $\alpha$. Then define the $\sigma$-energy stopping intervals of $S_{0}$ to be the collection

$$
\mathcal{S}=\left\{S_{0}\right\} \cup \bigcup_{n=0}^{\infty} \mathcal{S}_{n}
$$

where $\mathcal{S}_{0}=\mathcal{S}\left(S_{0}\right)$ and $\mathcal{S}_{n+1}=\bigcup_{S \in \mathcal{S}_{n}} \mathcal{S}(S)$ for $n \geq 0$.

We now claim that from the energy condition $\mathcal{E}_{2}^{\alpha}<\infty$, we obtain the $\sigma$-Carleson estimate,

$$
\sum_{S \in \mathcal{S}: S \subset I}|S|_{\sigma} \leq 2|I|_{\sigma}, \quad I \in \mathcal{D}^{\sigma} .
$$

Indeed, for any $S_{1} \in \mathcal{S}$ we have

$$
\begin{aligned}
\sum_{S \in \mathfrak{C}_{\mathcal{S}}\left(S_{1}\right)}|S|_{\sigma} & \leq \frac{1}{C_{\text {energy }}\left(\mathcal{E}_{2}^{\alpha}\right)^{2}} \sum_{S \in \mathfrak{C}_{\mathcal{S}}\left(S_{1}\right)} \sup _{S \supset \cup J_{r}} \sum_{r=1}^{\infty}\left(\frac{\mathrm{P}^{\alpha}\left(J_{r}, \mathbf{1}_{S_{1}} \sigma\right)}{\left|J_{r}\right|}\right)^{2}\left\|x-m_{J_{r}}\right\|_{L^{2}\left(\mathbf{1}_{J_{r}} \omega\right)}^{2} \\
& \leq \frac{1}{C_{\text {energy }}\left(\mathcal{E}_{2}^{\alpha}\right)^{2}}\left(\mathcal{E}_{2}^{\alpha}\right)^{2}\left|S_{1}\right|_{\sigma}=\frac{1}{C_{\text {energy }}}\left|S_{1}\right|_{\sigma},
\end{aligned}
$$


upon noting that the union of the subdecompositions $\dot{\cup} J_{r} \subset S$ over $S \in \mathfrak{C}_{\mathcal{S}}\left(S_{1}\right)$ is a subdecomposition of $S_{1}$, and the proof of the Carleson estimate is now finished by iteration in the standard way.

Finally, we record the reason for introducing energy stopping times. If

$$
\mathbf{X}_{\alpha}\left(\mathcal{C}_{S}\right)^{2} \equiv \sup _{I \in \mathcal{C}_{S}} \frac{1}{|I|_{\sigma}} \sup _{I \supset \cup J_{r}} \sum_{r=1}^{\infty}\left(\frac{\mathrm{P}^{\alpha}\left(J_{r}, \mathbf{1}_{S} \sigma\right)}{\left|J_{r}\right|}\right)^{2}\left\|x-m_{J_{r}}\right\|_{L^{2}\left(\mathbf{1}_{J_{r}} \omega\right)}^{2}
$$

is (the square of) the $\alpha$-stopping energy of the weight pair $(\sigma, \omega)$ with respect to the corona $\mathcal{C}_{S}$, then we have the stopping energy bounds

$$
\mathbf{X}_{\alpha}\left(\mathcal{C}_{S}\right) \leq \sqrt{C_{\text {energy }}} \sqrt{\left(\mathfrak{E}_{2}^{\alpha}\right)^{2}+\mathfrak{A}_{2}^{\alpha}}, \quad S \in \mathcal{S},
$$

where $\mathfrak{A}_{2}^{\alpha}$ and the energy constant $\mathfrak{E}_{2}^{\alpha}$ are controlled by the assumptions in Theorem 7.

2.5. Iterated coronas and general stopping data. We will use a construction that permits iteration of the above three corona decompositions by combining Definitions [20, 21] and 23] into a single stopping condition. However, there is one remaining difficulty with the triple corona constructed in this way, namely if a stopping interval $I \in \mathcal{A}$ is a child of an interval $Q$ in the corona $\mathcal{C}_{A}$, then the modulus of the average $\left|\frac{1}{|I|_{\sigma}} \int_{I} b_{Q} d \sigma\right|$ of $b_{Q}$ on $I$ may be far smaller than the sup norm of $\left|b_{Q}\right|$ on the child $I$, indeed it may be that $\frac{1}{|I|_{\sigma}} \int_{I} b_{Q} d \sigma=0$. This of course destroys any reasonable estimation of the martingale and dual martingale differences $\triangle_{Q}^{\sigma, \mathbf{b}} f$ and $\square_{Q}^{\sigma, \mathbf{b}} f$ used in the proof of Theorem 7 and so we will use Lemma 18 on the function $b_{A}$ to obtain a new function $\widetilde{b}_{A}$ for which this problem is circumvented at the 'bottom' of the corona, i.e. for those $A^{\prime} \in C_{\mathcal{A}}(A)$. We then refer to the stopping times $A^{\prime} \in C_{\mathcal{A}}(A)$ as 'shadow' stopping times since we have lost control of the weak testing condition relative to the new function $\widetilde{b}_{A}$. Thus we must redo the weak testing stopping times for the new function $\widetilde{b}_{A}$, but also stopping if we hit one of the shadow stopping times. Here are the details.

Definition 24. Let $C_{0} \geq 4,0<\gamma<1$ and $1<\Gamma<\infty$. Suppose that $\mathbf{b}=\left\{b_{Q}\right\}_{Q \in \mathcal{P}}$ is an $\infty$-strongly $\sigma$-accretive family on $\mathbb{R}$. Given a dyadic grid $\mathcal{D}$ and an interval $Q \in \mathcal{D}$, define the collection of 'shadow' stopping times $\mathcal{S}_{\text {shadow }}(Q)$ to be the maximal $\mathcal{D}$-subintervals $I \subset Q$ such that either

or

$$
\frac{1}{|I|_{\sigma}} \int_{I}|f| d \sigma>C_{0} \frac{1}{|Q|_{\sigma}} \int_{Q}|f| d \sigma
$$

or

$$
\left|\frac{1}{|I|_{\mu}} \int_{I} b_{Q} d \sigma\right|<\gamma \text { or } \int_{I}\left|T_{\sigma}^{\alpha}\left(b_{Q}\right)\right|^{2} d \omega>\Gamma\left(\mathfrak{T}_{T^{\alpha}}^{\mathbf{b}}\right)^{2}|I|_{\sigma}
$$

$$
\sup _{I \supset \dot{\cup} J_{r}} \sum_{r=1}^{\infty}\left(\frac{\mathrm{P}^{\alpha}\left(J_{r}, \sigma\right)}{\left|J_{r}\right|}\right)^{2}\left\|x-m_{J_{r}}\right\|_{L^{2}\left(\mathbf{1}_{J_{r}} \omega\right)}^{2} \geq C_{\text {energy }}\left[\left(\mathcal{E}_{2}^{\alpha, \mathbf{b}, \mathbf{b}^{*}}\right)^{2}+\mathfrak{A}_{2}^{\alpha}\right]|I|_{\sigma} \text {. }
$$

Now we apply Lemma 18 to the function $b_{Q}$ with the subdecomposition $\mathcal{S}_{\text {shadow }}(Q) \equiv\left\{Q_{i}\right\}_{i=1}^{\infty}$ to obtain a new function $\widetilde{b}_{Q}$ satisfying the properties

$$
\begin{aligned}
& \text { support } \widetilde{b}_{Q} \subset Q \\
& 1 \leq \frac{1}{\left|Q^{\prime}\right|_{\sigma}} \int_{Q^{\prime}} \widetilde{b}_{Q} d \sigma \leq\left\|\mathbf{1}_{Q^{\prime}} \widetilde{b}_{Q}\right\|_{L^{\infty}(\sigma)} \leq 2\left(1+\sqrt{C_{\mathbf{b}}}\right) C_{\mathbf{b}}, \quad Q^{\prime} \in \mathcal{C}_{Q}, \\
& \sqrt{\int_{Q}\left|T_{\sigma}^{\alpha} b_{Q}\right|^{2} d \omega} \leq\left[2 \widetilde{T}_{T^{\alpha}}^{\mathbf{b}}(Q)+4 C_{\mathbf{b}}^{\frac{3}{2}} \delta^{\frac{1}{4}} \mathfrak{N}_{T^{\alpha}}(Q)\right] \sqrt{|Q|_{\sigma}}, \\
& \left\|\mathbf{1}_{Q_{i}} \widetilde{b}_{Q}\right\|_{L^{\infty}(\sigma)} \leq \frac{16 C_{\mathbf{b}}}{\delta}\left|\frac{1}{\left|Q_{i}\right|_{\sigma}} \int_{Q_{i}} \widetilde{b}_{Q} d \sigma\right|, \quad 1 \leq i<\infty .
\end{aligned}
$$

Note that each of the functions $\widetilde{b}_{Q^{\prime}} \equiv \mathbf{1}_{Q^{\prime}} \widetilde{b}_{Q}$, for $Q^{\prime} \in \mathcal{C}_{Q}$, now satisfies the crucial reverse Hölder property

$$
\left\|\mathbf{1}_{I} \widetilde{b}_{Q^{\prime}}\right\|_{L^{\infty}(\sigma)} \leq C_{\delta, \mathbf{b}}\left|\frac{1}{|I|_{\sigma}} \int_{I} \widetilde{b}_{Q^{\prime}} d \sigma\right|, \quad \text { for all } I \in \mathfrak{C}\left(Q^{\prime}\right), Q^{\prime} \in \mathcal{C}_{Q} .
$$


Indeed, if $I$ equals one of the $Q_{i}$ then the reverse Hölder condition in the last line of (2.25) applies, while if $I \in \mathcal{C}_{Q}$ then the accretivity in the second line of (2.25) applies.

Since we have lost the weak testing condition in the corona for this new function $\widetilde{b}_{Q}$, the next step is to run again the weak testing construction of stopping times, but this time starting with the new function $\widetilde{b}_{Q}$, and also stopping if we hit one of the 'shadow' stopping times $Q_{i}$. Here is the new stopping criterion.

Definition 25. Let $C_{0} \geq 4$ and $1<\Gamma<\infty$. Let $\mathcal{S}_{\text {shadow }}(Q) \equiv\left\{Q_{i}\right\}_{i=1}^{\infty}$ be as in Definition 24. Define $\mathcal{S}_{\text {iterated }}(Q)$ to be the maximal $\mathcal{D}$-subintervals $I \subset Q$ such that either

$$
\int_{I}\left|T_{\sigma}^{\alpha}\left(\widetilde{b}_{Q}\right)\right|^{2} d \omega>\Gamma\left(\mathfrak{T}_{T^{\alpha}}^{\widetilde{\mathbf{b}}}\right)^{2}|I|_{\sigma}
$$

or

$$
I=Q_{i} \text { for some } 1 \leq i<\infty .
$$

Thus for each interval $Q$ we have now constructed iterated stopping children $\mathcal{S}_{\text {iterated }}(Q)$ by first constructing shadow stopping times $\mathcal{S}_{\text {shadow }}(Q)$ using one step of the triple corona construction, then modifying the testing function to have reverse Hölder controlled children, and finally running again the weak testing stopping time construction to get $\mathcal{S}_{\text {iterated }}(Q)$. These iterated stopping times $\mathcal{S}_{\text {iterated }}(Q)$ have control of CZ averages of $f$ and energy control of $\sigma$ and $\omega$, simply because these controls were achieved in the shadow construction, and were unaffected by either the application of Lemma 18 or the rerunning of the weak testing stopping criterion for $\widetilde{b}_{Q}$. And of course we now have weak testing within the corona determined by $Q$ and $\mathcal{S}_{\text {iterated }}(Q)$, and we also have the crucial reverse Hölder condition on all the children of intervals in the corona. With all of this in hand, here then is the definition of the construction of iterated coronas.

Definition 26. Let $C_{0} \geq 4,0<\gamma<1$ and $1<\Gamma<\infty$. Suppose that $\mathbf{b}=\left\{b_{Q}\right\}_{Q \in \mathcal{P}}$ is an $\infty$-strongly $\sigma$ accretive family on $\mathbb{R}$. Given a dyadic grid $\mathcal{D}$ and an interval $S_{0}$ in $\mathcal{D}$, define the iterated stopping intervals of $S_{0}$ to be the collection

$$
\mathcal{S}=\left\{S_{0}\right\} \cup \bigcup_{n=0}^{\infty} \mathcal{S}_{n}
$$

where $\mathcal{S}_{0}=\mathcal{S}_{\text {iterated }}\left(S_{0}\right)$ and $\mathcal{S}_{n+1}=\bigcup_{S \in \mathcal{S}_{n}} \mathcal{S}_{\text {iterated }}(S)$ for $n \geq 0$, and where $\mathcal{S}_{\text {iterated }}(Q)$ is defined in Definition 25.

It is useful to append to the notion of stopping times $\mathcal{S}$ in the above $\sigma$-iterated corona decomposition a positive constant $A_{0}$ and an additional structure $\alpha_{\mathcal{S}}$ called stopping bounds for a function $f$. We will refer to the resulting triple $\left(A_{0}, \mathcal{F}, \alpha_{\mathcal{F}}\right)$ as constituting stopping data for $f$. If $\mathcal{F}$ is a grid, we define $F^{\prime} \prec F$ if $F^{\prime} \varsubsetneqq F$ and $F^{\prime}, F \in \mathcal{F}$. Recall that $\pi_{\mathcal{F}} F^{\prime}$ is the smallest $F \in \mathcal{F}$ such that $F^{\prime} \prec F$.

Definition 27. Suppose we are given a positive constant $A_{0} \geq 4$, a subset $\mathcal{F}$ of the dyadic grid $\mathcal{D}$ (called the stopping times), and a corresponding sequence $\alpha_{\mathcal{F}} \equiv\left\{\alpha_{\mathcal{F}}(F)\right\}_{F \in \mathcal{F}}$ of nonnegative numbers $\alpha_{\mathcal{F}}(F) \geq 0$ (called the stopping bounds). Let $\left(\mathcal{F}, \prec, \pi_{\mathcal{F}}\right)$ be the tree structure on $\mathcal{F}$ inherited from $\mathcal{D}$, and for each $F \in \mathcal{F}$ denote by $\mathcal{C}_{F}=\left\{I \in \mathcal{D}: \pi_{\mathcal{F}} I=F\right\}$ the corona associated with $F$ :

$$
\mathcal{C}_{F}=\left\{I \in \mathcal{D}: I \subset F \text { and } I \not \subset F^{\prime} \text { for any } F^{\prime} \prec F\right\} .
$$

We say the triple $\left(A_{0}, \mathcal{F}, \alpha_{\mathcal{F}}\right)$ constitutes stopping data for a function $f \in L_{\text {loc }}^{1}(\sigma)$ if

(1) $\mathbb{E}_{I}^{\sigma}|f| \leq \alpha_{\mathcal{F}}(F)$ for all $I \in \mathcal{C}_{F}$ and $F \in \mathcal{F}$,

(2) $\sum_{F^{\prime} \preceq F}\left|F^{\prime}\right|_{\sigma} \leq A_{0}|F|_{\sigma}$ for all $F \in \mathcal{F}$,

(3) $\sum_{F \in \mathcal{F}} \alpha_{\mathcal{F}}(F)^{2}|F|_{\sigma} \leq A_{0}^{2}\|f\|_{L^{2}(\sigma)}^{2}$,

(4) $\alpha_{\mathcal{F}}(F) \leq \alpha_{\mathcal{F}}\left(F^{\prime}\right)$ whenever $F^{\prime}, F \in \mathcal{F}$ with $F^{\prime} \subset F$.

Property (1) says that $\alpha_{\mathcal{F}}(F)$ bounds the averages of $f$ in the corona $\mathcal{C}_{F}$, and property (2) says that the intervals at the tops of the coronas satisfy a Carleson condition relative to the weight $\sigma$. Note that a standard 'maximal interval' argument extends the Carleson condition in property (2) to the inequality

$$
\sum_{F^{\prime} \in \mathcal{F}: F^{\prime} \subset A}\left|F^{\prime}\right|_{\sigma} \leq A_{0}|A|_{\sigma} \text { for all open sets } A \subset \mathbb{R} .
$$


Property (3) is the quasi-orthogonality condition that says the sequence of functions $\left\{\alpha_{\mathcal{F}}(F) \mathbf{1}_{F}\right\}_{F \in \mathcal{F}}$ is in the vector-valued space $L^{2}\left(\ell^{2} ; \sigma\right)$ with control, and is often referred to as a Carleson embedding theorem, and property (4) says that the control on stopping data is nondecreasing on the stopping tree $\mathcal{F}$. We emphasize that we are not assuming in this definition the stronger property that there is $C>1$ such that $\alpha_{\mathcal{F}}\left(F^{\prime}\right)>C \alpha_{\mathcal{F}}(F)$ whenever $F^{\prime}, F \in \mathcal{F}$ with $F^{\prime} \varsubsetneqq F$. Instead, the properties (2) and (3) substitute for this lack. Of course the stronger property does hold for the familiar Calderón-Zygmund stopping data determined by the following requirements for $C>1$,

$$
\mathbb{E}_{F^{\prime}}^{\sigma}|f|>C \mathbb{E}_{F}^{\sigma}|f| \text { whenever } F^{\prime}, F \in \mathcal{F} \text { with } F^{\prime} \varsubsetneqq F, \quad \mathbb{E}_{I}^{\sigma}|f| \leq C \mathbb{E}_{F}^{\sigma}|f| \text { for } I \in \mathcal{C}_{F},
$$

which are themselves sufficiently strong to automatically force properties (2) and (3) with $\alpha_{\mathcal{F}}(F)=\mathbb{E}_{F}^{\sigma}|f|$.

We have the following useful consequence of (2) and (3) that says the sequence $\left\{\alpha_{\mathcal{F}}(F) \mathbf{1}_{F}\right\}_{F \in \mathcal{F}}$ has a quasi-orthogonal property relative to $f$ with a constant $A_{0}^{\prime}$ depending only on $A_{0}$ (see e.g. [SaShUr7]):

$$
\left\|\sum_{F \in \mathcal{F}} \alpha_{\mathcal{F}}(F) \mathbf{1}_{F}\right\|_{L^{2}(\sigma)}^{2} \leq A_{0}^{\prime}\|f\|_{L^{2}(\sigma)}^{2} .
$$

Proposition 28. Let $f \in L^{2}(\sigma)$, let $\mathcal{F}$ be the iterated corona $\mathcal{S}\left(S_{0}\right)$ in Definition 26, and define stopping data $\alpha_{\mathcal{F}}$ by $\alpha_{F}=\frac{1}{\left.F\right|_{\sigma}} \int_{F}|f| d \sigma$. Then there is $A_{0} \geq 4$, depending only on the constant $C_{0}$ in Definition 20, such that the triple $\left(A_{0}, \mathcal{F}, \alpha_{\mathcal{F}}\right)$ constitutes stopping data for the function $f$.

Proof. This is an easy exercise using (2.18), (2.19) and (2.22), and is left for the reader.

2.6. Grid parameterizations. It is important to use two independent random grids, one for each function $f$ and $g$ simultaneously, as this is necessary in order to apply probabilistic methods to the dual martingale averages $\square_{I}^{\mu, \mathbf{b}}$ that depend, not only on $I$, but also on the underlying grid in which $I$ lives. The proof methods for functional energy from [SaShUr7] and [SaShUr6] relied heavily on the use of a single grid, and this must now be modified to accommodate two independent grids.

Now we recall the construction from our paper SaShUr10. We momentarily fix a large positive integer $M \in \mathbb{N}$, and consider the tiling of $\mathbb{R}$ by the family of intervals $\mathbb{D}_{M} \equiv\left\{I_{\alpha}^{M}\right\}_{\alpha \in \mathbb{Z}}$ having side length $2^{-M}$ and given by $I_{\alpha}^{M} \equiv I_{0}^{M}+2^{-M} \alpha$ where $I_{0}^{M}=\left[0,2^{-M}\right)$. A dyadic grid $\mathcal{D}$ built on $\mathbb{D}_{M}$ is defined to be a family of intervals $\mathcal{D}$ satisfying:

(1) Each $I \in \mathcal{D}$ has side length $2^{-\ell}$ for some $\ell \in \mathbb{Z}$ with $\ell \leq M$, and $I$ is a union of $2^{M-\ell}$ intervals from the tiling $\mathbb{D}_{M}$,

(2) For $\ell \leq M$, the collection $\mathcal{D}_{\ell}$ of intervals in $\mathcal{D}$ having side length $2^{-\ell}$ forms a pairwise disjoint decomposition of the space $\mathbb{R}$,

(3) Given $I \in \mathcal{D}_{i}$ and $J \in \mathcal{D}_{j}$ with $j \leq i \leq M$, it is the case that either $I \cap J=\emptyset$ or $I \subset J$.

We now momentarily fix a negative integer $N \in-\mathbb{N}$, and restrict the above grids to intervals of side length at most $2^{-N}$ :

$$
\mathcal{D}^{N} \equiv\left\{I \in \mathcal{D}: \text { side length of } I \text { is at most } 2^{-N}\right\} .
$$

We refer to such grids $\mathcal{D}^{N}$ as a (truncated) dyadic grid $\mathcal{D}$ built on $\mathbb{D}_{M}$ of size $2^{-N}$. There are now two traditional means of constructing probability measures on collections of such dyadic grids, namely parameterization by choice of parent, and parameterization by translation.

Construction \#1: For any

$$
\beta=\left\{\beta_{i}\right\}_{i \in_{M}^{N}} \in \omega_{M}^{N} \equiv\{0,1\}^{\mathbb{Z}_{M}^{N}},
$$

where $\mathbb{Z}_{M}^{N} \equiv\{\ell \in \mathbb{Z}: N \leq \ell \leq M\}$, define the dyadic grid $\mathcal{D}_{\beta}$ built on $\mathbb{D}_{m}$ of size $2^{-N}$ by

$$
\mathcal{D}_{\beta}=\left\{2^{-\ell}\left([0,1)+k+\sum_{i: \ell<i \leq m} 2^{-i+\ell} \beta_{i}\right)\right\}_{N \leq \ell \leq m, k \in \mathbb{Z}} .
$$

Place the uniform probability measure $\rho_{M}^{N}$ on the finite index space $\omega_{M}^{N}=\{0,1\}^{\mathbb{Z}_{M}^{N}}$, namely that which charges each $\beta \in \omega_{M}^{N}$ equally.

Construction \#2: Momentarily fix a (truncated) dyadic grid $\mathcal{D}$ built on $\mathbb{D}_{M}$ of size $2^{-N}$. For any

$$
\gamma \in \Gamma_{M}^{N} \equiv\left\{\gamma \in 2^{-M} \mathbb{Z}_{+}:|\gamma|<2^{-N}\right\}
$$


define the dyadic grid $\mathcal{D}^{\gamma}$ built on $\mathbb{D}_{M}$ of size $2^{-N}$ by

$$
\mathcal{D}^{\gamma} \equiv \mathcal{D}+\gamma
$$

Place the uniform probability measure $\nu_{M}^{N}$ on the finite index set $\Gamma_{M}^{N}$, namely that which charges each multiindex $\gamma$ in $\Gamma_{M}^{N}$ equally.

The two probability spaces $\left(\left\{\mathcal{D}_{\beta}\right\}_{\beta \in \Omega_{M}^{N}}, \mu_{M}^{N}\right)$ and $\left(\left\{\mathcal{D}^{\gamma}\right\}_{\gamma \in \Gamma_{M}^{N}}, \nu_{M}^{N}\right)$ are isomorphic since both collections $\left\{\mathcal{D}_{\beta}\right\}_{\beta \in \Omega_{M}^{N}}$ and $\left\{\mathcal{D}^{\gamma}\right\}_{\gamma \in \Gamma_{M}^{N}}$ describe the set $\boldsymbol{A}_{M}^{N}$ of all (truncated) dyadic grids $\mathcal{D}^{\gamma}$ built on $\mathbb{D}_{m}$ of size $2^{-N}$, and since both measures $\mu_{M}^{N}$ and $\nu_{M}^{N}$ are the uniform measure on this space. The first construction may be thought of as being parameterized by scales - each component $\beta_{i}$ in $\beta=\left\{\beta_{i}\right\}_{i \in{ }_{M}^{N}} \in \omega_{M}^{N}$ amounting to a choice of the two possible tilings at level $i$ that respect the choice of tiling at the level below - and since any grid in $\boldsymbol{A}_{M}^{N}$ is determined by a choice of scales, we see that $\left\{\mathcal{D}_{\beta}\right\}_{\beta \in \Omega_{M}^{N}}=\boldsymbol{A}_{M}^{N}$. The second construction may be thought of as being parameterized by translation - each $\gamma \in \Gamma_{M}^{N}$ amounting to a choice of translation of the grid $\mathcal{D}$ fixed in construction \#2 - and since any grid in $\boldsymbol{A}_{M}^{N}$ is determined by any of the intervals at the top level, i.e. with side length $2^{-N}$, we see that $\left\{\mathcal{D}^{\gamma}\right\}_{\gamma \in \Gamma_{M}^{N}}=\boldsymbol{A}_{M}^{N}$ as well, since every interval at the top level in $\boldsymbol{A}_{M}^{N}$ has the form $Q+\gamma$ for some $\gamma \in \Gamma_{M}^{N}$ and $Q \in \mathcal{D}$ at the top level in $\boldsymbol{A}_{M}^{N}$ (i.e. every interval at the top level in $\boldsymbol{A}_{M}^{N}$ is a union of small intervals in $\mathbb{D}_{m}$, and so must be a translate of some $Q \in \mathcal{D}$ by an amount $2^{-M}$ times an element of $\mathbb{Z}_{+}$). Note also that $\# \Omega_{M}^{N}=\# \Gamma_{M}^{N}=2^{M-N}$. We will use $\boldsymbol{E}_{\Omega_{M}^{N}}$ to denote expectation with respect to this common probability measure on $\boldsymbol{A}_{M}^{N}$.

Notation 29. For purposes of notation and clarity, we now suppress all reference to $M$ and $N$ in our families of grids, and in the notations $\Omega$ and $\Gamma$ for the parameter sets, and we use $\boldsymbol{P}_{\Omega}$ and $\boldsymbol{E}_{\Omega}$ to denote probability and expectation with respect to families of grids, and instead proceed as if all grids considered are unrestricted. The careful reader can supply the modifications necessary to handle the assumptions made above on the grids $\mathcal{D}$ and the functions $f$ and $g$ regarding $M$ and $N$.

2.7. The Monotonicity Lemma. As in virtually all proofs of a two weight $T 1$ theorem (see e.g. LaSaShUr3, [Lac, [SaShUr7] and/or [SaShUr6]), the key to starting an estimate for any of the forms we consider below, is the Monotonicity Lemma and the Energy Lemma, to which we now turn. In dimension $n=1$ (LaSaShUr3], $[\mathrm{Lac}]$ ) the Haar functions have opposite sign on their children, and this was exploited in a simple but powerful monotonicity argument. In higher dimensions, this simple argument no longer holds and that Monotonicity Lemma is replaced with the Lacey-Wick formulation of the Monotonicity Lemma (see [LaWi], and also [SaShUr6]) involving the smaller Poisson operator. As the martingale differences with test functions $b_{Q}$ here are no longer of one sign on children, we will adapt the Lacey-Wick formulation of the Monotonicity Lemma to the operator $T^{\alpha}$ and the dual martingale differences $\left\{\square_{J}^{\omega, \mathbf{b}^{*}}\right\}_{J \in \mathcal{G}}$, bearing in mind that the operators $\square_{J}^{\omega, \mathbf{b}^{*}}$ are no longer projections, which results in only a one-sided estimate with additional terms on the right hand side. It is here that we need the crucial property that Range $\square_{J}^{\omega, \mathbf{b}^{*}}$ is orthogonal to constants, $\int\left(\square_{J}^{\omega, \mathbf{b}^{*}} \Psi\right) d \sigma=\int\left(\triangle_{J}^{\sigma, \mathbf{b}^{*}} 1\right) \Psi d \omega=\int(0) \Psi d \omega=0$ (see Appendix A). See Definition 88 in Appendix A for the terminology ' $p$-weakly $\mu$-controlled accretive family' along with more detail on martingale and dual martingale expansions.

Recall from Appendix A that

$$
\begin{aligned}
\mathbb{E}_{Q}^{\mu, \mathbf{b}} f(x) & \equiv \mathbf{1}_{Q}(x) \frac{1}{\int_{Q} b_{Q} d \mu} \int_{Q} f b_{Q} d \mu, \quad Q \in \mathcal{P}, \\
\mathbb{F}_{Q}^{\mu, \mathbf{b}} f(x) & \equiv \mathbf{1}_{Q}(x) b_{Q}(x) \frac{1}{\int_{Q} b_{Q} d \mu} \int_{Q} f d \mu, \quad Q \in \mathcal{P},
\end{aligned}
$$


and

$$
\begin{aligned}
& \triangle_{Q}^{\mu, \mathbf{b}} f(x) \equiv\left(\sum_{Q^{\prime} \in \mathfrak{C}(Q)} \mathbb{E}_{Q^{\prime}}^{\mu, \mathbf{b}} f(x)\right)-\mathbb{E}_{Q}^{\mu, \mathbf{b}} f(x)=\sum_{Q^{\prime} \in \mathfrak{C}(Q)} \mathbf{1}_{Q^{\prime}}(x)\left(\mathbb{E}_{Q^{\prime}}^{\mu, \mathbf{b}} f(x)-\mathbb{E}_{Q}^{\mu, \mathbf{b}} f(x)\right), \\
& \square_{Q}^{\mu, \mathbf{b}} f(x) \equiv\left(\sum_{Q^{\prime} \in \mathfrak{C}(Q)} \mathbb{F}_{Q^{\prime}}^{\mu, \mathbf{b}} f(x)\right)-\mathbb{F}_{Q}^{\mu, \mathbf{b}} f(x)=\sum_{Q^{\prime} \in \mathfrak{C}(Q)} \mathbf{1}_{Q^{\prime}}(x)\left(\mathbb{F}_{Q^{\prime}}^{\mu, \mathbf{b}} f(x)-\mathbb{F}_{Q}^{\mu, \mathbf{b}} f(x)\right),
\end{aligned}
$$

and from (9.6),

$$
\nabla_{Q}^{\mu} h=\sum_{Q^{\prime} \in \mathfrak{C}_{\text {broken }}(Q)}\left(E_{Q^{\prime}}^{\mu}|h|\right)^{2} \mathbf{1}_{Q^{\prime}} .
$$

We will also need the smaller Poisson integral used in the Lacey-Wick formulation of the Monontonicity Lemma,

$$
\mathrm{P}_{1+\delta}^{\alpha}(J, \mu) \equiv \int \frac{|J|^{\frac{1+\delta}{n}}}{\left(|J|+\left|y-c_{J}\right|\right)^{n+1+\delta-\alpha}} d \mu(y),
$$

which is discussed in more detail below.

Lemma 30 (Monotonicity Lemma). Suppose that $I$ and $J$ are intervals in $\mathbb{R}$ such that $J \subset \gamma J \subset I$ for some $\gamma>1$, and that $\mu$ is a signed measure on $\mathbb{R}$ supported outside $I$. Let $0<\delta<1$ and let $\Psi \in L^{2}(\omega)$. Finally suppose that $T^{\alpha}$ is a standard fractional singular integral on $\mathbb{R}$ as in [SaShUr6], [SaShUr7] and [SaShUr9] with $0 \leq \alpha<1$, and suppose that $\mathbf{b}^{*}$ is an $\infty$-weakly $\mu$-controlled accretive family on $\mathbb{R}$. Then we have the estimate

$$
\left|\left\langle T^{\alpha} \mu, \square_{J}^{\omega, \mathbf{b}^{*}} \Psi\right\rangle_{\omega}\right| \lesssim C_{\mathbf{b}^{*}} C_{C Z} \Phi^{\alpha}(J,|\mu|)\left\|\square_{J}^{\omega, \mathbf{b}^{*}} \Psi\right\|_{L^{2}(\omega)}^{\star}
$$

where

$$
\begin{aligned}
\Phi^{\alpha}(J,|\mu|) & \equiv \frac{\mathrm{P}^{\alpha}(J,|\mu|)}{|J|}\left\|\triangle_{J}^{\omega, \mathbf{b}^{*}} x\right\|_{L^{2}(\omega)}^{\infty}+\frac{\mathrm{P}_{1+\delta}^{\alpha}(J,|\mu|)}{|J|}\left\|x-m_{J}\right\|_{L^{2}\left(\mathbf{1}_{J} \omega\right)}, \\
\left\|\triangle_{J}^{\omega, \mathbf{b}^{*}} x\right\|_{L^{2}(\omega)}^{\mathbf{\omega}^{2}} & \equiv\left\|\triangle_{J}^{\omega, \mathbf{b}^{*}} x\right\|_{L^{2}(\omega)}^{2}+\inf _{z \in \mathbb{R}} \sum_{J^{\prime} \in \mathfrak{C}_{\text {broken }}(J)}\left|J^{\prime}\right|_{\omega}\left(E_{J^{\prime}}^{\omega}|x-z|\right)^{2}, \\
\left\|\square_{J}^{\omega, \mathbf{b}^{*}} \Psi\right\|_{L^{2}(\mu)}^{\star 2} & \equiv\left\|\square_{J}^{\omega, \mathbf{b}^{*}} \Psi\right\|_{L^{2}(\mu)}^{2}+\sum_{J^{\prime} \in \mathfrak{C}_{\text {broken }}(J)}\left|J^{\prime}\right|_{\omega}\left[E_{J^{\prime}}^{\omega}|\Psi|\right]^{2} .
\end{aligned}
$$

All of the implied constants above depend only on $\gamma>1,0<\delta<1$ and $0<\alpha<1$.

Using $\nabla_{J}^{\omega} h=\sum_{J^{\prime} \in \mathfrak{C}_{\text {broken }}(J Q)}\left(E_{J^{\prime}}^{\omega}|h|\right)^{2} \mathbf{1}_{J^{\prime}}$ defined in (9.6) in Appendix A, we can rewrite the expressions $\left\|\triangle_{J}^{\omega, \mathbf{b}^{*}} x\right\|_{L^{2}(\omega)}^{\mathbf{A}^{2}}$ and $\left\|\square_{J}^{\omega, \mathbf{b}^{*}} \Psi\right\|_{L^{2}(\mu)}^{\star 2}$ as

$$
\begin{aligned}
& \left\|\triangle_{J}^{\omega, \mathbf{b}^{*}} x\right\|_{L^{2}(\omega)}^{\mathbf{\omega}^{2}} \equiv\left\|\triangle_{J}^{\omega, \mathbf{b}^{*}} x\right\|_{L^{2}(\omega)}^{2}+\inf _{z \in \mathbb{R}}\left\|\nabla_{J}^{\omega}(x-z)\right\|_{L^{2}(\omega)}^{2}, \\
& \left\|\square_{J}^{\omega, \mathbf{b}^{*}} \Psi\right\|_{L^{2}(\mu)}^{\star 2} \equiv\left\|\square_{J}^{\omega, \mathbf{b}^{*}} \Psi\right\|_{L^{2}(\mu)}^{2}+\left\|\nabla_{J}^{\omega} \Psi\right\|_{L^{2}(\omega)}^{2} .
\end{aligned}
$$

Proof. We also use formulas (9.16), (9.18) and the estimate (9.19) from Appendix A:

$$
\begin{aligned}
\square_{Q}^{\mu, \pi, \mathbf{b}} f & =\left[\sum_{Q^{\prime} \in \mathfrak{C}(Q)} \mathbb{F}_{Q^{\prime}}^{\mu, \pi, \mathbf{b}} f\right]-\mathbb{F}_{Q}^{\mu, \mathbf{b}} f=\sum_{Q^{\prime} \in \mathfrak{C}(Q)} \mathbb{F}_{Q^{\prime}}^{\mu, b_{Q}} f-\mathbb{F}_{Q}^{\mu, b_{Q}} f, \\
\mathbb{F}_{Q}^{\mu, \pi, \mathbf{b}} f & =\mathbf{1}_{Q} \frac{b_{\pi Q}}{\int_{Q} b_{\pi Q} d \mu} \int_{Q} f d \mu, \\
\square_{Q}^{\mu, \mathbf{b}} & =\square_{Q}^{\mu, \pi, \mathbf{b}} \square_{Q}^{\mu, \pi, \mathbf{b}}+\square_{Q, \text { broken }}^{\mu, \mathbf{b}} \text { and } \square_{Q, \text { broken }}^{\mu, \mathbf{b}} f=\sum_{Q^{\prime} \in \mathfrak{C}_{\text {broken }}(Q)} \mathbb{F}_{Q^{\prime}}^{\mu, b_{Q^{\prime}}} f-\mathbb{F}_{Q^{\prime}}^{\mu, b_{Q}} f, \\
\left|\square_{Q, \text { broken }}^{\mu, \mathbf{b}} f\right| & \lesssim\left|\nabla_{Q}^{\mu} f\right|,
\end{aligned}
$$


with similar equalities and inequalities for $\triangle$ and $\mathbb{E}$. Here $\mathfrak{C}_{\text {broken }}(Q)$ denotes the set of broken children, i.e. those $Q^{\prime} \in \mathfrak{C}(Q)$ for which $b_{Q^{\prime}} \neq \mathbf{1}_{Q^{\prime}} b_{Q}$, and more generally and typically, $\mathfrak{C}_{\text {broken }}(Q)=\mathfrak{C}(Q) \cap \mathcal{A}$ where $\mathcal{A}$ is a collection of stopping intervals that includes the broken children and satisfies a $\sigma$-Carleson condition. Using $\square_{J}^{\omega, \mathbf{b}^{*}}=\square_{J}^{\omega, \pi, \mathbf{b}^{*}} \square_{J}^{\omega, \pi, \mathbf{b}^{*}}+\square_{J, \text { broken }}^{\omega, \mathbf{b}^{*}}$, we write

$$
\begin{aligned}
\left|\left\langle T^{\alpha} \mu, \square_{J}^{\omega, \mathbf{b}^{*}} \Psi\right\rangle_{\omega}\right| & =\left|\left\langle T^{\alpha} \mu,\left(\square_{J}^{\omega, \pi, \mathbf{b}^{*}} \square_{J}^{\omega, \pi, \mathbf{b}^{*}}+\square_{J, \text { broken }}^{\omega, \mathbf{b}^{*}}\right) \Psi\right\rangle_{\omega}\right| \\
& \leq\left|\left\langle T^{\alpha} \mu, \square_{J}^{\omega, \pi, \mathbf{b}^{*}} \square_{J}^{\omega, \pi, \mathbf{b}^{*}} \Psi\right\rangle_{\omega}\right|+\left|\left\langle T^{\alpha} \mu, \square_{J, \text { broken }}^{\omega, \mathbf{b}^{*}} \Psi\right\rangle_{\omega}\right| \equiv I+I I .
\end{aligned}
$$

Since $\left\langle 1, \square_{J}^{\omega, \pi, \mathbf{b}^{*}} h\right\rangle_{\omega}=0$, we use $m_{J}=\frac{1}{|J|_{\omega}} \int_{J} x d \omega(x)$ to obtain

$$
T^{\alpha} \mu(x)-T^{\alpha} \mu\left(m_{J}\right)=\int\left[\left(K^{\alpha}\right)(x, y)-\left(K^{\alpha}\right)\left(m_{J}, y\right)\right] d \mu(y)=\int\left[\left(K_{y}^{\alpha}\right)^{\prime}\left(\theta\left(x, m_{J}\right)\right)\left(x-m_{J}\right)\right] d \mu(y)
$$

for some $\theta\left(x, m_{J}\right) \in J$ to obtain

$$
\begin{aligned}
I= & \left|\int\left[T^{\alpha} \mu(x)-T^{\alpha} \mu\left(m_{J}\right)\right] \square_{J}^{\omega, \pi, \mathbf{b}^{*}} \square_{J}^{\omega, \pi, \mathbf{b}^{*}} \Psi(x) d \omega(x)\right| \\
= & \left|\int\left\{\int\left[\left(K_{y}^{\alpha}\right)^{\prime}\left(\theta\left(x, m_{J}\right)\right)\right] d \mu(y)\right\}\left(x-m_{J}\right) \square_{J}^{\omega, \pi, \mathbf{b}^{*}} \square_{J}^{\omega, \pi, \mathbf{b}^{*}} \Psi(x) d \omega(x)\right| \\
\leq & \left|\int\left\{\int\left[\left(K_{y}^{\alpha}\right)^{\prime}\left(m_{J}\right)\right] d \mu(y)\right\}\left(x-m_{J}\right) \square_{J}^{\omega, \pi, \mathbf{b}^{*}} \square_{J}^{\omega, \pi, \mathbf{b}^{*}} \Psi(x) d \omega(x)\right| \\
& +\left|\int\left\{\int\left[\left(K_{y}^{\alpha}\right)^{\prime}\left(\theta\left(x, m_{J}\right)\right)-\left(K_{y}^{\alpha}\right)^{\prime}\left(m_{J}\right)\right] d \mu(y)\right\}\left(x-m_{J}\right) \square_{J}^{\omega, \pi, \mathbf{b}^{*}} \square_{J}^{\omega, \pi, \mathbf{b}^{*}} \Psi(x) d \omega(x)\right| \\
\equiv & I_{1}+I_{2} .
\end{aligned}
$$

Now we estimate

$$
\begin{aligned}
I_{1} & =\left|\int\left[\left(K_{y}^{\alpha}\right)^{\prime}\left(m_{J}\right)\right] d \mu(y)\right|\left|\int\left(x-m_{J}\right) \square_{J}^{\omega, \pi, \mathbf{b}^{*}} \square_{J}^{\omega, \pi, \mathbf{b}^{*}} \Psi(x) d \omega(x)\right| \\
& =\left|\int\left[\left(K_{y}^{\alpha}\right)^{\prime}\left(m_{J}\right)\right] d \mu(y)\right|\left|\int\left(\triangle_{J}^{\omega, \pi, \mathbf{b}^{*}} x\right)\left(\square_{J}^{\omega, \pi, \mathbf{b}^{*}} \Psi(x)\right) d \omega(x)\right| \\
& \lesssim C_{C Z} \frac{\mathrm{P}^{\alpha}(J,|\mu|)}{|J|}\left\|\triangle_{J}^{\omega, \pi, \mathbf{b}^{*}} x\right\|_{L^{2}(\omega)}\left\|\square_{J}^{\omega, \pi, \mathbf{b}^{*}} \Psi\right\|_{L^{2}(\omega)},
\end{aligned}
$$

and

$$
\begin{aligned}
I_{2} & \lesssim C_{C Z} \frac{\mathrm{P}_{1+\delta}^{\alpha}(J,|\mu|)}{|J|} \int\left|x-m_{J}\right|\left|\square_{J}^{\omega, \pi, \mathbf{b}^{*}} \square_{J}^{\omega, \pi, \mathbf{b}^{*}} \Psi(x)\right| d \omega(x) \\
& \lesssim C_{C Z} \frac{\mathrm{P}_{1+\delta}^{\alpha}(J,|\mu|)}{|J|} \sqrt{\int_{J}\left|x-m_{J}\right|^{2} d \omega(x)}\left\|\square_{J}^{\omega, \pi, \mathbf{b}^{*}} \square_{J}^{\omega, \pi, \mathbf{b}^{*}} \Psi\right\|_{L^{2}(\omega)} \\
& \lesssim C_{C Z} \frac{\mathrm{P}_{1+\delta}^{\alpha}(J,|\mu|)}{|J|}\left\|x-m_{J}\right\|_{L^{2}\left(\mathbf{1}_{J} \omega\right)}\left\|\square_{J}^{\omega, \pi, \mathbf{b}^{*}} \Psi\right\|_{L^{2}(\omega)} .
\end{aligned}
$$

For term $I I$ we fix $z \in \bar{J}$ for the moment. Then since $\left\langle 1, \square_{J, \text { broken }}^{\omega, \mathbf{b}^{*}} h\right\rangle_{\omega}=\left\langle 1, \square_{J}^{\omega, \mathbf{b}^{*}} h-\square_{J}^{\omega, \pi, \mathbf{b}^{*}} h\right\rangle_{\omega}=0$, we have

$$
I I=\left|\left\langle T^{\alpha} \mu, \square_{J, \text { broken }}^{\omega, \mathbf{b}^{*}} \Psi\right\rangle_{\omega}\right|=\left|\int\left\{\int\left[\left(K_{y}^{\alpha}\right)^{\prime}(\theta(x, z))\right] d \mu(y)\right\}(x-z) \square_{J, \text { broken }}^{\omega, \mathbf{b}^{*}} \Psi(x) d \omega(x)\right| .
$$

Using reverse Hölder control of children (2.15), we obtain the estimate (9.19) from Appendix A,

$$
\left|\square_{J, \text { broken }}^{\omega, \mathbf{b}^{*}} \Psi\right|=\left|\sum_{J^{\prime} \in \mathfrak{C}_{\text {broken }}(J Q)}\left(\mathbb{F}_{J^{\prime}}^{\omega, b_{J^{\prime}}}-\mathbb{F}_{J^{\prime}}^{\omega, b_{J}}\right) \Psi\right| \lesssim \sum_{J^{\prime} \in \mathfrak{C}_{\text {broken }}(J)} \mathbf{1}_{J^{\prime}} E_{J^{\prime}}^{\omega}|\Psi|,
$$


and so

$$
I I \lesssim C_{C Z} \frac{\mathrm{P}^{\alpha}(J,|\mu|)}{|J|} \sqrt{\sum_{J^{\prime} \in \mathfrak{C}_{\text {broken }}(J)}\left|J^{\prime}\right|_{\omega}\left(E_{J^{\prime}}^{\omega}|x-z|\right)^{2}} \sqrt{\sum_{J^{\prime} \in \mathfrak{C}_{\text {broken }}(J)}\left|J^{\prime}\right|_{\omega}\left[E_{J^{\prime}}^{\omega}|\Psi|\right]^{2}} .
$$

Combining the estimates for terms $I$ and $I I$, we obtain

$$
\begin{aligned}
& \left|\left\langle T^{\alpha} \mu, \square_{J}^{\omega, \mathbf{b}^{*}} \Psi\right\rangle_{\omega}\right| \\
\lesssim & C_{C Z} \frac{\mathrm{P}^{\alpha}(J,|\mu|)}{|J|}\left\|\triangle_{J}^{\omega, \pi, \mathbf{b}^{*}} x\right\|_{L^{2}(\omega)}\left\|\square_{J}^{\omega, \pi, \mathbf{b}^{*}} \Psi\right\|_{L^{2}(\omega)} \\
& +C_{C Z} \frac{\mathrm{P}_{1+\delta}^{\alpha}(J,|\mu|)}{|J|}\left\|x-m_{J}\right\|_{L^{2}\left(\mathbf{1}_{J} \omega\right)}\left\|\square_{J}^{\omega, \pi, \mathbf{b}^{*}} \Psi\right\|_{L^{2}(\omega)} \\
& +C_{C Z} \frac{\mathrm{P}^{\alpha}(J,|\mu|)}{|J|} \inf _{z \in \bar{J}} \sqrt{\sum_{J^{\prime} \in \mathfrak{C}_{\text {broken }}(J)}\left|J^{\prime}\right|_{\omega}\left(E_{J^{\prime}}^{\omega}\left|x-z_{2}\right|\right)^{2}} \sqrt{\sum_{J^{\prime} \in \mathfrak{C}_{\text {broken }}(J)}\left|J^{\prime}\right|_{\omega}\left[E_{J^{\prime}}^{\omega}|\Psi|+E_{J}^{\omega}|\Psi|\right]^{2}},
\end{aligned}
$$

and then noting that the infimum over $z \in \mathbb{R}$ is achieved for $z \in \bar{J}$, and using the triangle inequality on $\square_{J}^{\omega, \pi, \mathbf{b}^{*}}=\square_{J}^{\omega, \mathbf{b}^{*}}-\square_{J, \text { broken }}^{\omega, \mathbf{b}^{*}}$ we get (2.29) .

The right hand side of (2.29) in the Monotonicity Lemma will be typically estimated in what follows using the frame inequalities (see Appendix A) for any interval $K$,

$$
\begin{aligned}
\sum_{J \subset K}\left\|\square_{J}^{\omega, \mathbf{b}^{*}} \Psi\right\|_{L^{2}(\omega)}^{\star 2} & \lesssim\|\Psi\|_{L^{2}(\omega)}^{2}, \\
\sum_{J \subset K}\left\|\triangle_{J}^{\omega, \mathbf{b}^{*}} x\right\|_{L^{2}(\omega)}^{\boldsymbol{\omega}^{2}} & \lesssim \int_{K}\left|x-m_{K}\right|^{2} d \omega(x),
\end{aligned}
$$

together with these inequalities for the square function expressions.

Lemma 31. For any interval $K$ we have

$$
\begin{aligned}
\sum_{J \subset K} \sum_{J^{\prime} \in \mathfrak{C}_{\text {broken }}(J)}\left|J^{\prime}\right|_{\omega}\left[E_{J^{\prime}}^{\omega}|\Psi|(x)\right]^{2} & \lesssim \int_{K}|\Psi(x)|^{2} d \omega(x), \\
\text { and } \sum_{J \subset K} \inf _{z \in \mathbb{R}} \sum_{J^{\prime} \in \mathfrak{C}_{\text {broken }(J)}\left|J^{\prime}\right|_{\omega}\left(E_{J^{\prime}}^{\omega}|x-z|\right)^{2}} & \lesssim \int_{K}\left|x-m_{K}\right|^{2} d \omega(x) .
\end{aligned}
$$

Proof. The first inequality in (2.30) is just the Carleson embedding theorem since the intervals $\left\{J^{\prime} \in \mathfrak{C}_{\text {broken }}(J): J \subset K\right\}$ satisfy an $\omega$-Carleson condition, and the second inequality in (2.30) follows by choosing $z=m_{K}$ to obtain

$$
\inf _{z \in \mathbb{R}} \sum_{J^{\prime} \in \mathfrak{C}_{\text {broken }}(J)}\left|J^{\prime}\right|_{\omega}\left(E_{J^{\prime}}^{\omega}|x-z|\right)^{2} \leq \sum_{J^{\prime} \in \mathfrak{C}_{\text {broken }}(J)}\left|J^{\prime}\right|_{\omega}\left(E_{J^{\prime}}^{\omega}\left|x-m_{K}\right|\right)^{2},
$$

and then applying the Carleson embedding theorem again:

$$
\sum_{J \subset K} \sum_{J^{\prime} \in \mathfrak{C}_{\text {broken }}(J)}\left|J^{\prime}\right|_{\omega}\left(E_{J^{\prime}}^{\omega}\left|x-m_{K}\right|\right)^{2} \lesssim \int_{K}\left|x-m_{K}\right|^{2} d \omega(x)
$$

2.7.1. The smaller Poisson integral. The expressions $\inf _{z \in \mathbb{R}} \frac{\mathrm{P}_{1+\delta}^{\alpha}(J,|\mu|)}{|J|}\|x-z\|_{L^{2}\left(\mathbf{1}_{J} \omega\right)}\left\|\square_{J}^{\omega, \mathbf{b}^{*} \Psi}\right\|_{L^{2}(\omega)}^{\star}$ are typically easier to sum due to the small Poisson operator $\mathrm{P}_{1+\delta}^{\alpha}(J,|\mu|)$. To illlustrate, we show here one way in which we can exploit the additional decay in the Poisson integral $\mathrm{P}_{1+\delta}^{\alpha}$. Suppose that $J$ is good in $I$ with 
$\ell(J)=2^{-s} \ell(I)$ (see Definition 38 below for 'goodness'). We then compute

$$
\begin{aligned}
\frac{\mathrm{P}_{1+\delta}^{\alpha}\left(J, \mathbf{1}_{A \backslash I} \sigma\right)}{|J|^{\frac{1}{n}}} & \approx \int_{A \backslash I} \frac{|J|^{\frac{\delta}{n}}}{\left|y-c_{J}\right|^{n+1+\delta-\alpha}} d \sigma(y) \\
& \leq \int_{A \backslash I}\left(\frac{|J|^{\frac{1}{n}}}{\operatorname{qdist}\left(c_{J}, I^{c}\right)}\right)^{\delta} \frac{1}{\left|y-c_{J}\right|^{n+1-\alpha}} d \sigma(y) \\
& \lesssim\left(\frac{|J|^{\frac{1}{n}}}{q \operatorname{qdist}\left(c_{J}, I^{c}\right)}\right)^{\delta} \frac{\mathrm{P}^{\alpha}\left(J, \mathbf{1}_{A \backslash I} \sigma\right)}{|J|^{\frac{1}{n}}},
\end{aligned}
$$

and use the goodness inequality,

$$
d\left(c_{J}, I^{c}\right) \geq \frac{1}{2} \ell(I)^{1-\varepsilon} \ell(J)^{\varepsilon} \geq \frac{1}{2} 2^{s(1-\varepsilon)} \ell(J),
$$

to conclude that

$$
\left(\frac{\mathrm{P}_{1+\delta}^{\alpha}\left(J, \mathbf{1}_{A \backslash I} \sigma\right)}{|J|^{\frac{1}{n}}}\right) \lesssim 2^{-s \delta(1-\varepsilon)} \frac{\mathrm{P}^{\alpha}\left(J, \mathbf{1}_{A \backslash I} \sigma\right)}{|J|^{\frac{1}{n}}} .
$$

Now we can estimate

$$
\begin{aligned}
& \sum_{J \subset K: J \text { is good in } K} \inf _{z \in \mathbb{R}} \frac{\mathrm{P}_{1+\delta}^{\alpha}\left(J, \mathbf{1}_{K^{c}}|\mu|\right)}{|J|}\|x-z\|_{L^{2}\left(\mathbf{1}_{J} \omega\right)}\left\|\square_{J}^{\omega, \mathbf{b}^{*}} \Psi\right\|_{L^{2}(\omega)}^{\star} \\
\leq & \sqrt{\sum_{J \subset K: J \text { is good in } K}\left(\frac{\mathrm{P}_{1+\delta}^{\alpha}\left(J, \mathbf{1}_{K^{c}}|\mu|\right)}{|J|}\right)^{2} \inf _{z \in \mathbb{R}}\|x-z\|_{L^{2}\left(\mathbf{1}_{J} \omega\right)}^{2}} \sqrt{\sum_{J \subset K: J \text { is good in } K}\left\|\square_{J}^{\omega, \mathbf{b}^{*}} \Psi\right\|_{L^{2}(\omega)}^{\star 2}},
\end{aligned}
$$

where

$$
\begin{aligned}
& \sum_{J \subset K: J \text { is good in } K}\left(\frac{\mathrm{P}_{1+\delta}^{\alpha}\left(J, \mathbf{1}_{K^{c}}|\mu|\right)}{|J|}\right)^{2} \inf _{z \in \mathbb{R}}\|x-z\|_{L^{2}\left(\mathbf{1}_{J} \omega\right)}^{2} \\
& =\sum_{s=0}^{\infty} \sum_{J \subset K: J \text { is good in } K}\left(\frac{\mathrm{P}_{1+\delta}^{\alpha}\left(J, \mathbf{1}_{K^{c}}|\mu|\right)}{|J|}\right)^{2} \inf _{z \in \mathbb{R}}\|x-z\|_{L^{2}\left(\mathbf{1}_{J} \omega\right)}^{2} \\
& \ell(J)=2^{-s} \ell(I) \\
& \leq \sum_{s=0}^{\infty} \sum_{\substack{J \subset K: J \text { is good in } K \\
\ell(J)=2^{-s} \ell(I)}}\left(2^{-s \delta(1-\varepsilon)} \frac{\mathrm{P}^{\alpha}\left(J, \mathbf{1}_{K^{c}} \sigma\right)}{|J|^{\frac{1}{n}}}\right)^{2} \inf _{z \in \mathbb{R}}\|x-z\|_{L^{2}\left(\mathbf{1}_{J} \omega\right)}^{2} \\
& \leq\left(\frac{\mathrm{P}^{\alpha}\left(K, \mathbf{1}_{K^{c}} \sigma\right)}{|K|^{\frac{1}{n}}}\right)^{2} \sum_{s=0}^{\infty} \sum_{\substack{J \subset K: J \text { is good in } K \\
\ell(J)=2^{-s} \ell(I)}} 2^{-2 s \delta(1-\varepsilon)} \inf _{z \in \mathbb{R}}\|x-z\|_{L^{2}\left(\mathbf{1}_{K} \omega\right)}^{2} \\
& \lesssim\left(\frac{\mathrm{P}^{\alpha}\left(K, \mathbf{1}_{K^{c}} \sigma\right)}{|K|^{\frac{1}{n}}}\right)^{2} \inf _{z \in \mathbb{R}}\|x-z\|_{L^{2}\left(\mathbf{1}_{K} \omega\right)}^{2},
\end{aligned}
$$

and where we have used (63), which gives in particular

$$
\mathrm{P}^{\alpha}\left(J, \mu \mathbf{1}_{I^{c}}\right) \lesssim\left(\frac{\ell(J)}{\ell(I)}\right)^{1-\varepsilon(2-\alpha)} \mathrm{P}^{\alpha}\left(I, \mu \mathbf{1}_{I^{c}}\right) .
$$

for $J \subset I$ and $d(J, \partial I)>\frac{1}{2} \ell(J)^{\varepsilon} \ell(I)^{1-\varepsilon}$. We will use such arguments repeatedly in the sequel.

Armed with the Monotonicity Lemma and the lower frame inequality

$$
\sum_{I \in \mathcal{D}}\left\|\square_{I}^{\omega, \mathbf{b}^{*}} g\right\|_{L^{2}(\mu)}^{\star 2} \lesssim\|g\|_{L^{2}(\omega)}^{2},
$$

we can obtain a $\mathbf{b}^{*}$-analogue of the Energy Lemma as in [SaShUr7] and/or [SaShUr6]. 
2.7.2. The Energy Lemma. Suppose now we are given a subset $\mathcal{H}$ of the dyadic grid $\mathcal{G}$.

Notation 32. Due to the failure of both martingale and dual martingale pseudoprojections $\mathbf{Q}_{\mathcal{H}}^{\omega, \mathbf{b}^{*}} x$ and $\mathrm{P}_{\mathcal{H}}^{\omega, \mathbf{b}^{*}} g$, as in Definition 94 in Appendix A, to satisfy inequalities of the form $\left\|\mathrm{P}_{\mathcal{H}}^{\omega, \mathbf{b}^{*}} g\right\|_{L^{2}(\omega)} \lesssim\|g\|_{L^{2}(\omega)}$ when the children 'break', it is convenient to define the 'square function norms' $\left\|\mathbf{Q}_{\mathcal{H}}^{\omega, \mathbf{b}^{*}} x\right\|_{L^{2}(\omega)}^{\boldsymbol{\omega}}$ and $\left\|\mathrm{P}_{\mathcal{H}}^{\omega, \mathbf{b}^{*}} g\right\|_{L^{2}(\omega)}^{\star}$ of the pseudoprojections

$$
\mathrm{Q}_{\mathcal{H}}^{\omega, \mathbf{b}^{*}} x=\sum_{J \in \mathcal{H}} \triangle_{J}^{\omega, \mathbf{b}^{*}} x \text { and } \mathrm{P}_{\mathcal{H}}^{\omega, \mathbf{b}^{*}} g=\sum_{J \in \mathcal{H}} \square_{J}^{\omega, \mathbf{b}^{*}} g
$$

by

$$
\begin{aligned}
\left\|Q_{\mathcal{H}}^{\omega, \mathbf{b}^{*}} x\right\|_{L^{2}(\omega)}^{\boldsymbol{\omega}^{2}} & \equiv \sum_{J \in \mathcal{H}}\left\|\triangle_{J}^{\omega, \mathbf{b}^{*}} x\right\|_{L^{2}(\omega)}^{\boldsymbol{\aleph}^{2}}=\sum_{J \in \mathcal{H}}\left\|\triangle_{J}^{\omega, \mathbf{b}^{*}} x\right\|_{L^{2}(\omega)}^{2}+\sum_{J \in \mathcal{H}} \inf _{z \in \mathbb{R}} \sum_{J^{\prime} \in \mathfrak{C}_{\text {broken }}(J)}\left|J^{\prime}\right|_{\omega}\left(E_{J^{\prime}}^{\omega}|x-z|\right)^{2}, \\
\left\|P_{\mathcal{H}}^{\omega, \mathbf{b}^{*}} g\right\|_{L^{2}(\omega)}^{\star 2} & \equiv \sum_{J \in \mathcal{H}}\left\|\square_{J}^{\omega, \mathbf{b}^{*}} g\right\|_{L^{2}(\omega)}^{\star 2}=\sum_{J \in \mathcal{H}}\left\|\square_{J}^{\omega, \mathbf{b}^{*}} g\right\|_{L^{2}(\omega)}^{\star^{2}}+\sum_{J \in \mathcal{H}} \sum_{J^{\prime} \in \mathfrak{C}_{\text {broken }}(J)}\left|J^{\prime}\right|_{\omega}\left[E_{J^{\prime}}^{\omega}|g|+E_{J}^{\omega}|g|\right]^{2},
\end{aligned}
$$

for any subset $\mathcal{H} \subset \mathcal{G}$. The average $E_{J}^{\omega}|x-z|$ above is taken with respect to the variable $x$, i.e. $E_{J}^{\omega}|x-z|=$ $\frac{1}{\Gamma J_{\omega}} \int|x-z| d \omega(x)$, and it is important that the infimum $\inf _{z \in \mathbb{R}}$ is taken inside the sum $\sum_{J \in \mathcal{H}}$.

Note that we are defining here square function expressions related to pseudoprojections, which depend not only on the functions $\mathbf{Q}_{\mathcal{H}}^{\omega, \mathbf{b}^{*}} x$ and $\mathbf{P}_{\mathcal{H}}^{\omega, \mathbf{b}^{*}} g$, but also on the particular representations $\sum_{J \in \mathcal{H}} \triangle_{J}^{\omega, \mathbf{b}^{*}} x$ and $\sum_{J \in \mathcal{H}} \square_{J}^{\omega, \mathbf{b}^{*}} g$. This slight abuse of notation should not cause confusion, and it provides a useful way of bookkeeping the sums of squares of norms of martingale and dual martingale differences $\left\|\triangle_{J}^{\omega, \mathbf{b}^{*}} x\right\|_{L^{2}(\omega)}^{2}$ and $\left\|\square_{J}^{\omega, \mathbf{b}^{*}} g\right\|_{L^{2}(\omega)}^{2}$, along with the norms of the associated Carleson square function expressions

$$
\begin{aligned}
\sum_{J \in \mathcal{H}} \inf _{z \in \mathbb{R}}\left\|\nabla_{J}^{\omega}(x-z)\right\|_{L^{2}(\omega)}^{2} & =\sum_{J \in \mathcal{H}} \inf _{z \in \mathbb{R}} \sum_{J^{\prime} \in \mathfrak{C}_{\text {broken }}(J)}\left|J^{\prime}\right|_{\omega}\left(E_{J^{\prime}}^{\omega}|x-z|\right)^{2} \\
\sum_{J \in \mathcal{H}}\left\|\nabla_{J}^{\omega} \Psi\right\|_{L^{2}(\omega)}^{2} & =\sum_{J \in \mathcal{H}} \sum_{J^{\prime} \in \mathfrak{C}_{\text {broken }}(J)}\left|J^{\prime}\right|_{\omega}\left[E_{J^{\prime}}^{\omega}|\Psi|\right]^{2} .
\end{aligned}
$$

Note also that the upper weak Riesz inequalities in Appendix A below yield the inequalities

$$
\begin{aligned}
\left\|\mathrm{Q}_{\mathcal{H}}^{\omega, \mathbf{b}^{*}} x\right\|_{L^{2}(\omega)}^{2} & \lesssim \sum_{J \in \mathcal{H}}\left\|\triangle_{J}^{\omega, \mathbf{b}^{*}} x\right\|_{L^{2}(\omega)}^{2} \leq\left\|\mathrm{Q}_{\mathcal{H}}^{\omega, \mathbf{b}^{*}} x\right\|_{L^{2}(\omega)}^{\mathbf{\omega}^{2}} \\
\left\|\mathrm{P}_{\mathcal{H}}^{\omega, \mathbf{b}^{*}} g\right\|_{L^{2}(\omega)}^{2} & \lesssim \sum_{J \in \mathcal{H}}\left\|\square_{J}^{\omega, \mathbf{b}^{*}} g\right\|_{L^{2}(\omega)}^{2} \leq\left\|\mathrm{P}_{\mathcal{H}}^{\omega, \mathbf{b}^{*}} g\right\|_{L^{2}(\omega)}^{\star 2}
\end{aligned}
$$

We will exclusively use $\left\|\mathbf{Q}_{\mathcal{H}}^{\omega, \mathbf{b}^{*}} x\right\|_{L^{2}(\omega)}^{\mathbf{A}^{2}}$ in connection with energy terms, and use $\left\|\mathrm{P}_{\mathcal{H}}^{\sigma, \mathbf{b}^{*} f}\right\|_{L^{2}(\sigma)}^{\star 2}$ and $\left\|\mathrm{P}_{\mathcal{H}}^{\omega, \mathbf{b}^{*} g}\right\|_{L^{2}(\omega)}^{\star 2}$ in connection with functions $f \in L^{2}(\sigma)$ and $g \in L^{2}(\omega)$. Finally, note that $\mathbf{Q}_{\mathcal{H}}^{\omega, \mathbf{b}^{*}} x=\mathbf{Q}_{\mathcal{H}}^{\omega, \mathbf{b}^{*}}(x-m)$ for any constant $m$. We also define the 'large $\mathcal{G}$-pseudoprojections'

$$
Q_{L}^{\omega, \mathbf{b}^{*}} \equiv \sum_{J^{\prime} \in \mathcal{G}: J^{\prime} \subset L} \triangle_{J^{\prime}}^{\omega, \mathbf{b}^{*}}, \quad \text { for any interval } L
$$

Recall that

$$
\Phi^{\alpha}(J, \nu) \equiv \frac{\mathrm{P}^{\alpha}(J, \nu)}{|J|}\left\|\triangle_{J}^{\omega, \mathbf{b}^{*}} x\right\|_{L^{2}(\omega)}^{\boldsymbol{\omega}}+\frac{\mathrm{P}_{1+\delta}^{\alpha}(J, \nu)}{|J|}\left\|x-m_{J}\right\|_{L^{2}\left(\mathbf{1}_{J} \omega\right)} .
$$

Lemma 33 (Energy Lemma). Let $J$ be an interval in $\mathcal{G}$. Let $\Psi_{J}$ be an $L^{2}(\omega)$ function supported in $J$ with vanishing $\omega$-mean, and let $\mathcal{H} \subset \mathcal{G}$ be such that $J^{\prime} \subset J$ for every $J^{\prime} \in \mathcal{H}$. Let $\nu$ be a positive measure supported in $\mathbb{R} \backslash \gamma J$ with $\gamma>1$, and for each $J^{\prime} \in \mathcal{H}$, let $d \nu_{J^{\prime}}=\varphi_{J^{\prime}} d \nu$ with $\left|\varphi_{J^{\prime}}\right| \leq 1$. Suppose that $\mathbf{b}^{*}$ is an 
$\infty$-weakly $\mu$-controlled accretive family on $\mathbb{R}$. Let $T^{\alpha}$ be a standard $\alpha$-fractional singular integral operator with $0 \leq \alpha<1$. Then we have

$$
\begin{aligned}
& \left|\sum_{J^{\prime} \in \mathcal{H}}\left\langle T^{\alpha}\left(\nu_{J^{\prime}}\right), \square_{J^{\prime}}^{\omega, \mathbf{b}^{*}} \Psi_{J}\right\rangle_{\omega}\right| \lesssim C_{\gamma} \sum_{J^{\prime} \in \mathcal{H}} \Phi^{\alpha}\left(J^{\prime}, \nu\right)\left\|\square_{J^{\prime}}^{\omega, \mathbf{b}^{*}} \Psi_{J}\right\|_{L^{2}(\mu)}^{\star} \\
\lesssim & C_{\gamma} \sqrt{\sum_{J^{\prime} \in \mathcal{H}} \Phi^{\alpha}\left(J^{\prime}, \nu\right)^{2}} \sqrt{\sum_{J^{\prime} \in \mathcal{H}}\left\|\square_{J^{\prime}}^{\omega, \mathbf{b}^{*}} \Psi_{J}\right\|_{L^{2}(\mu)}^{\star 2}} \\
\leq & C_{\gamma}\left(\frac{\mathrm{P}^{\alpha}(J, \nu)}{|J|}\left\|\mathrm{Q}_{\mathcal{H}}^{\omega, \mathbf{b}^{*}} x\right\|_{L^{2}(\omega)}^{\mathbf{a}}+\frac{\mathrm{P}_{1+\delta}^{\alpha}(J, \nu)}{|J|}\left\|x-m_{J}\right\|_{L^{2}\left(\mathbf{1}_{J} \omega\right)}\right)\left\|\mathrm{P}_{\mathcal{H}}^{\omega, \mathbf{b}^{*}} \Psi_{J}\right\|_{L^{2}(\mu)}^{\star},
\end{aligned}
$$

and in particular the 'energy' estimate

$$
\left|\left\langle T^{\alpha} \varphi \nu, \Psi_{J}\right\rangle_{\omega}\right| \lesssim C_{\gamma}\left(\frac{\mathrm{P}^{\alpha}(J, \nu)}{|J|}\left\|\mathrm{Q}_{J}^{\omega, \mathbf{b}^{*}} x\right\|_{L^{2}(\omega)}^{\mathbf{\omega}}+\frac{\mathrm{P}_{1+\delta}^{\alpha}(J, \nu)}{|J|}\left\|x-m_{J}\right\|_{L^{2}\left(\mathbf{1}_{J} \omega\right)}\right)\left\|\sum_{J^{\prime} \subset J} \square_{J^{\prime}}^{\omega, \mathbf{b}^{*}} \Psi_{J}\right\|_{L^{2}(\mu)}^{\star}
$$

where $\left\|\sum_{J^{\prime} \subset J} \square_{J^{\prime}}^{\omega, \mathbf{b}^{*}} \Psi_{J}\right\|_{L^{2}(\mu)}^{\star} \lesssim\left\|\Psi_{J}\right\|_{L^{2}(\mu)}$, and the 'pivotal' bound

$$
\left|\left\langle T^{\alpha}(\varphi \nu), \Psi_{J}\right\rangle_{\omega}\right| \lesssim C_{\gamma} \mathrm{P}^{\alpha}(J,|\nu|) \sqrt{|J|_{\omega}}\left\|\Psi_{J}\right\|_{L^{2}(\omega)}
$$

for any function $\varphi$ with $|\varphi| \leq 1$.

Proof. Beginning with the first display in the conclusion of the Energy Lemma, we need only prove the first line since the next two follow from Poisson inequalities and the definitions in Notation 32 Using the Monotonicity Lemma 30, followed by $\left|\nu_{J^{\prime}}\right| \leq \nu$, the Poisson equivalence

$$
\frac{\mathrm{P}^{\alpha}\left(J^{\prime}, \nu\right)}{\left|J^{\prime}\right|} \approx \frac{\mathrm{P}^{\alpha}(J, \nu)}{|J|}, \quad J^{\prime} \subset J \subset \gamma J, \quad \operatorname{supp} \nu \cap \gamma J=\emptyset
$$

and the weak frame inequalities for dual martingale differences in Appendix A, we have

$$
\begin{aligned}
& \left|\sum_{J^{\prime} \in \mathcal{H}}\left\langle T^{\alpha}\left(\nu_{J^{\prime}}\right), \square_{J^{\prime}}^{\omega, \mathbf{b}^{*}} \Psi_{J}\right\rangle_{\omega}\right| \lesssim \sum_{J^{\prime} \in \mathcal{H}} \Phi^{\alpha}\left(J^{\prime},|\mu|\right)\left\|\square_{J^{\prime}}^{\omega, \mathbf{b}^{*}} \Psi_{J}\right\|_{L^{2}(\mu)}^{\star} \\
& \leq \sum_{J^{\prime} \in \mathcal{H}}\left(\frac{\mathrm{P}^{\alpha}\left(J^{\prime}, \nu\right)}{\left|J^{\prime}\right|}\left\|\triangle_{J^{\prime}}^{\omega, \mathbf{b}^{*}} x\right\|_{L^{2}(\omega), z}^{\mathbf{a}}+\frac{\mathrm{P}_{1+\delta}^{\alpha}\left(J^{\prime}, \nu\right)}{\left|J^{\prime}\right|}\left\|x-m_{J^{\prime}}\right\|_{L^{2}\left(\mathbf{1}_{J^{\prime}} \omega\right)}\right)\left\|\square_{J^{\prime}}^{\omega, \mathbf{b}^{*}} \Psi_{J}\right\|_{L^{2}(\omega)}^{\star} \\
& \lesssim\left(\sum_{J^{\prime} \in \mathcal{H}}\left(\frac{\mathrm{P}^{\alpha}\left(J^{\prime}, \nu\right)}{\left|J^{\prime}\right|}\right)^{2}\left\|\triangle_{J^{\prime}}^{\omega, \mathbf{b}^{*}} x\right\|_{L^{2}(\omega), z}^{\mathbf{A} 2}\right)^{\frac{1}{2}}\left(\sum_{J^{\prime} \in \mathcal{H}}\left\|\square_{J^{\prime}}^{\omega, \mathbf{b}^{*}} \Psi_{J}\right\|_{L^{2}(\omega)}^{\star 2}\right)^{\frac{1}{2}} \\
& +\left(\sum_{J^{\prime} \in \mathcal{H}}\left(\frac{\mathrm{P}_{1+\delta}^{\alpha}\left(J^{\prime},|\mu|\right)}{\left|J^{\prime}\right|}\right)^{2}\left\|x-m_{J^{\prime}}\right\|_{L^{2}\left(\mathbf{1}_{J^{\prime}} \omega\right)}^{2}\right)^{\frac{1}{2}}\left(\sum_{J^{\prime} \in \mathcal{H}}\left\|\square_{J^{\prime}}^{\omega, \mathbf{b}^{*}} \Psi_{J}\right\|_{L^{2}(\omega)}^{\star 2}\right)^{\frac{1}{2}} \\
& \lesssim\left(\frac{\mathrm{P}^{\alpha}(J, \nu)}{|J|}\right)\left\|\mathrm{Q}_{\mathcal{H}}^{\omega, \mathbf{b}^{*}} x\right\|_{L^{2}(\omega)}^{\mathbf{\omega}}\left\|\Psi_{J}\right\|_{L^{2}(\omega)}+\frac{1}{\gamma^{\delta^{\prime}}}\left(\frac{\mathrm{P}_{1+\delta^{\prime}}^{\alpha}(J, \nu)}{|J|}\right)\left\|x-m_{J}\right\|_{L^{2}\left(\mathbf{1}_{J} \omega\right)}\left\|\Psi_{J}\right\|_{L^{2}(\omega)} .
\end{aligned}
$$

The last inequality follows from the following calculation using Haar projections $\triangle_{K}^{\omega}$ :

$$
\begin{aligned}
& \sum_{J^{\prime} \in \mathcal{H}}\left(\frac{\mathrm{P}_{1+\delta}^{\alpha}\left(J^{\prime}, \nu\right)}{\left|J^{\prime}\right|}\right)^{2}\left\|x-m_{J^{\prime}}\right\|_{L^{2}\left(\mathbf{1}_{J^{\prime}} \omega\right)}^{2} \\
= & \sum_{J^{\prime} \in \mathcal{H}}\left(\frac{\mathrm{P}_{1+\delta}^{\alpha}\left(J^{\prime}, \nu\right)}{\left|J^{\prime}\right|}\right)^{2} \sum_{J^{\prime \prime} \subset J^{\prime}}\left\|\triangle_{J^{\prime \prime}}^{\omega} x\right\|_{L^{2}(\omega)}^{2}=\sum_{J^{\prime \prime} \subset J}\left\{\sum_{J^{\prime}: J^{\prime \prime} \subset J^{\prime} \subset J}\left(\frac{\mathrm{P}_{1+\delta}^{\alpha}\left(J^{\prime}, \nu\right)}{\left|J^{\prime}\right|}\right)^{2}\left\|\triangle_{J^{\prime \prime}}^{\omega} x\right\|_{L^{2}(\omega)}^{2}\right. \\
\lesssim & \frac{1}{\gamma^{2 \delta^{\prime}}} \sum_{J^{\prime \prime} \subset J}\left(\frac{\mathrm{P}_{1+\delta^{\prime}}^{\alpha}\left(J^{\prime \prime}, \nu\right)}{\left|J^{\prime \prime}\right|}\right)^{2}\left\|\triangle_{J^{\prime \prime}}^{\omega} x\right\|_{L^{2}(\omega)}^{2} \leq \frac{1}{\gamma^{2 \delta^{\prime}}}\left(\frac{\mathrm{P}_{1+\delta^{\prime}}^{\alpha}(J, \nu)}{|J|}\right)^{2} \sum_{J^{\prime \prime} \subset J}\left\|\triangle_{J^{\prime \prime}}^{\omega} x\right\|_{L^{2}(\omega)}^{2}
\end{aligned}
$$


which in turn follows from (recalling $\delta=2 \delta^{\prime}$ and using $\left|J^{\prime}\right|+\left|y-c_{J^{\prime}}\right| \approx|J|+\left|y-c_{J}\right|$ and $\frac{|J|}{|J|+\left|y-c_{J}\right|} \leq \frac{1}{\gamma}$ for $y \in \mathbb{R} \backslash \gamma J)$

$$
\begin{aligned}
& \sum_{J^{\prime}: J^{\prime \prime} \subset J^{\prime} \subset J}\left(\frac{\mathrm{P}_{1+\delta}^{\alpha}\left(J^{\prime}, \nu\right)}{\left|J^{\prime}\right|}\right)^{2}=\sum_{J^{\prime}: J^{\prime \prime} \subset J^{\prime} \subset J}\left|J^{\prime}\right|^{2 \delta}\left(\int_{\mathbb{R} \backslash \gamma J} \frac{1}{\left(\left|J^{\prime}\right|+\left|y-c_{J^{\prime}}\right|\right)^{2+\delta-\alpha}} d \nu(y)\right)^{2} \\
\lesssim & \sum_{J^{\prime}: J^{\prime \prime} \subset J^{\prime} \subset J} \frac{1}{\gamma^{2 \delta^{\prime}}} \frac{\left|J^{\prime}\right|^{2 \delta}}{|J|^{2 \delta}}\left(\int_{\mathbb{R} \backslash \gamma J} \frac{|J|^{\delta^{\prime}}}{\left(|J|+\left|y-c_{J}\right|\right)^{2+\delta^{\prime}-\alpha}} d \nu(y)\right)^{2} \\
= & \frac{1}{\gamma^{2 \delta^{\prime}}}\left(\sum_{J^{\prime}: J^{\prime \prime} \subset J^{\prime} \subset J} \frac{\left|J^{\prime}\right|^{2 \delta}}{|J|^{2 \delta}}\right)\left(\frac{\mathrm{P}_{1+\delta^{\prime}}^{\alpha}(J, \nu)}{|J|}\right)^{2} \lesssim \frac{1}{\gamma^{2 \delta^{\prime}}}\left(\frac{\mathrm{P}_{1+\delta^{\prime}}^{\alpha}(J, \nu)}{|J|}\right)^{2} .
\end{aligned}
$$

Finally we obtain the 'energy' estimate from the equality

$$
\Psi_{J}=\sum_{J^{\prime} \subset J} \square_{J^{\prime}}^{\omega, \mathbf{b}^{*}} \Psi_{J}, \quad \text { (since } \Psi_{J} \text { has vanishing } \omega \text {-mean), }
$$

and we obtain the 'pivotal' bound from the inequality

$$
\sum_{J^{\prime \prime} \subset J}\left\|\triangle_{J^{\prime \prime}}^{\omega, \mathbf{b}^{*}} x\right\|_{L^{2}(\omega)}^{\mathbf{\omega}^{2}} \lesssim\left\|\left(x-m_{J}\right)\right\|_{L^{2}\left(\mathbf{1}_{J} \omega\right)}^{2} \leq|J|^{2}|J|_{\omega}
$$

2.8. Organization of the proof. We adapt the proof of the main theorem in SaShUr7, SaShUr9] and SaShUr10, but beginning instead with the decomposition of Hytönen and Martikainen HyMa, to obtain the norm inequality

$$
\mathfrak{N}_{T^{\alpha}} \lesssim \mathcal{N} \mathcal{T} \mathcal{V}_{\alpha}=\mathfrak{T}_{T^{\alpha}}^{\mathbf{b}}+\mathfrak{T}_{T^{\alpha}}^{\mathbf{b}^{*}}+\sqrt{\mathfrak{A}_{2}^{\alpha}}+\mathfrak{E}_{2}^{\alpha},
$$

under the apriori assumption $\mathfrak{N}_{T^{\alpha}}<\infty$, which is achieved by considering one of the truncations $T_{\sigma, \delta, R}^{\alpha}$ defined in (1.3) above. This will be carried out in the next five sections of this paper. In the next section we consider the various form splittings and reduce matters to the disjoint form, the nearby form and the main below form. Then these latter three forms are taken up in the subsequent three sections, using material from the appendices. Finally, the stopping form is treated in the section following these three.

A major source of difficulty will arise in the infusion of goodness for the intervals $J$ into the main below form where the sum is taken over all pairs $(I, J)$ such that $\ell(J) \leq \ell(I)$. We will infuse goodness in a weak way pioneered by Hytönen and Martikainen in a one weight setting. This weak form of goodness is then exploited in all subsequent constructions by typically replacing $J$ with $J^{\text {in }}$ defining relations, where $J^{\text {s }}$ is the smallest interval $K$ for which $J$ is good in $K$ and beyond (see the next section for terminology, in particular Definition 42).

Another source of difficulty arises in the treatment of the nearby form in the setting of two weights. The one weight proofs in $\mathrm{HyMa}$ and LaMa relied strongly on a property peculiar to the one weight setting - namely the fact already pointed out in Remark 8 above, that both of the Poisson integrals are bounded, namely $\mathrm{P}^{\alpha}(Q, \mu) \lesssim 1$ and $\mathcal{P}^{\alpha}(Q, \mu) \lesssim 1$. We will circumvent this difficulty by combining a recursive energy argument with the full testing conditions assumed for the $\infty$-weakly accretive family of original testing functions $b_{Q}^{\text {orig }}$, before these conditions were suppressed by corona constructions that delivered only weak testing conditions for the new family of testing functions $b_{Q}$.

In Section 7 we bound the stopping form using the arguments from [SaShUr7], [SaShUr9] and [SaShUr10], which were in turn based on the bottom/up stopping time and recursion of M. Lacey in [Lac]. Here we introduce an additional top/down 'indented' corona construction to handle the lack of goodness in size testing intervals, and we use an absorption in place of recursion. Finally, the treatment of various 'straddling lemmas' is complicated by weak goodness, and we use a stronger form of weak goodness defined with the three point 'skeleton' of an interval replaced by an infinite 'body', coupled with two geometric 'Key Facts' to establish these lemmas.

Of particular importance will be two independent results proved in Appendices A and B that follow from known work with some new twists. In Appendix A we establish convergence and frame inequalities for martingale and dual martingale differences, and derive certain weak Riesz inequalities associated with $\infty$-weakly $\mu$-accretive families of testing functions, which will find application in treating the paraproduct 
form below. The boundedness of testing functions, and the reverse Hölder condition on their children, is important here.

In Appendix B we show that the functional energy for an arbitrary pair of grids is controlled by the Muckenhoupt and energy side conditions. The somewhat lengthy proof of this latter assertion is similar to the corresponding proof in the $T 1$ setting - see e.g. [SaShUr9] - but requires a different decomposition of the stopping intervals into 'Whitney intervals' in order to accommodate the weaker notion of goodness used here, as well as the usual decomposition into maximal deeply embedded intervals that is used to control expressions involving the 'small' Poisson integral.

Finally, we include in Appendix C an up-to-date list of errata for our most often referred to paper SaShUr7.

\section{FORM SPLitTings}

Notation 34. Fix grids $\mathcal{D}$ and $\mathcal{G}$. We will use $\mathcal{D}$ to denote the grid associated with $f \in L^{2}(\sigma)$, and we will use $\mathcal{G}$ to denote the grid associated with $g \in L^{2}(\omega)$.

We have defined corona decompositions of $f$ and $g$ in the $\sigma$-iterated triple corona construction above, but in order to start these corona decompositions for $f$ and $g$ respectively within the dyadic grids $\mathcal{D}$ and $\mathcal{G}$, we need to first restrict $f$ and $g$ to be supported in a large common interval $Q_{\infty}$. Then we cover $Q_{\infty}$ with two pairwise disjoint intervals $I_{\infty} \in \mathcal{D}$ with $\ell\left(I_{\infty}\right)=\ell\left(Q_{\infty}\right)$, and similarly cover $Q_{\infty}$ with two pairwise disjoint intervals $J_{\infty} \in \mathcal{G}$ with $\ell\left(J_{\infty}\right)=\ell\left(Q_{\infty}\right)$. We can now use the broken martingale decompositions from Appendix A, together with full b-testing (see (3.24) and (3.25) below), to reduce matters to consideration of the four forms

$$
\sum_{I \in \mathcal{D}: I \subset I_{\infty}} \sum_{J \in \mathcal{G}: J \subset J_{\infty}} \int\left(T_{\sigma}^{\alpha} \square_{I}^{\sigma, \mathbf{b}} f\right) \square_{J}^{\omega, \mathbf{b}^{*}} g d \omega,
$$

with $I_{\infty}$ and $J_{\infty}$ as above, and where we can then use the intervals $I_{\infty}$ and $J_{\infty}$ as the starting intervals in our corona constructions below. Indeed, the identities in Lemma 89 from Appendix A below, give

$$
\begin{aligned}
f & =\sum_{I \in \mathcal{D}_{N}} \mathbb{F}_{I}^{\sigma, \mathbf{b}} f+\sum_{I \in \mathcal{D}: I \subset I_{\infty}, \ell(I) \geq N+1} \square_{I}^{\sigma, \mathbf{b}} f+\mathbb{F}_{I_{\infty}}^{\sigma, \mathbf{b}} f, \\
g & =\sum_{J \in \mathcal{G}_{N}} \mathbb{F}_{J}^{\omega, \mathbf{b}^{*}} g+\sum_{J \in \mathcal{G}: J \subset J_{\infty}, \ell(J) \geq N+1} \square_{J}^{\omega, \mathbf{b}^{*}} g+\mathbb{F}_{J_{\infty}}^{\omega, \mathbf{b}^{*}} g,
\end{aligned}
$$

which can then be used to write the bilinear form $\int\left(T_{\sigma} f\right) g d \omega$ as a sum of the forms

$$
\begin{aligned}
\int\left(T_{\sigma} f\right) g d \omega & =\sum_{\substack{\text { four pairs } \\
\left(I_{\infty}, J_{\infty}\right)}}\left\{\sum_{I \in \mathcal{D}: I \subset I_{\infty}} \sum_{J \in \mathcal{G}: J \subset J_{\infty}} \int\left(T_{\sigma}^{\alpha} \square_{I}^{\sigma, \mathbf{b}} f\right) \square_{J}^{\omega, \mathbf{b}^{*}} g d \omega\right. \\
+ & \left.\sum_{I \in \mathcal{D}: I \subset I_{\infty}} \int\left(T_{\sigma}^{\alpha} \square_{I}^{\sigma, \mathbf{b}} f\right) \mathbb{F}_{J_{\infty}}^{\omega, \mathbf{b}^{*}} g d \omega+\sum_{J \in \mathcal{G}: J \subset J_{\infty}} \int\left(T_{\sigma}^{\alpha} \mathbb{F}_{I_{\infty}}^{\sigma, \mathbf{b}} f\right) \square_{J}^{\omega, \mathbf{b}^{*}} g d \omega+\int\left(T_{\sigma}^{\alpha} \mathbb{F}_{I_{\infty}}^{\sigma, \mathbf{b}} f\right) \mathbb{F}_{J_{\infty}}^{\omega, \mathbf{b}^{*}} g d \omega\right\},
\end{aligned}
$$

taken over the four pairs of intervals $\left(I_{\infty}, J_{\infty}\right)$ above, plus the limit of the sum of terms involving $\sum_{I \in \mathcal{D}_{N}} \mathbb{F}_{I}^{\sigma, \mathbf{b}} f$ and $\sum_{J \in \mathcal{G}_{N}} \mathbb{F}_{J}^{\omega, \mathbf{b}^{*}} g$. This latter limit is easily shown to vanish due to the strong convergence of the dual martingale differences $\square_{I}^{\sigma, \mathbf{b}} f$ and $\square_{J}^{\omega, \mathbf{b}^{*}} g$ in $L^{2}(\sigma)$ and $L^{2}(\omega)$ respectively. More precisely, we have

$$
\begin{aligned}
& \left|\int\left(T_{\sigma}^{\alpha} \sum_{I \in \mathcal{D}_{N}} \mathbb{F}_{I}^{\sigma, \mathbf{b}} f\right) \sum_{J \in \mathcal{G}_{N}} \mathbb{F}_{J}^{\omega, \mathbf{b}^{*}} g d \omega\right| \lesssim \mathfrak{N}_{T^{\alpha}}\left\|\sum_{I \in \mathcal{D}_{N}} \mathbb{F}_{I}^{\sigma, \mathbf{b}} f\right\|_{L^{2}(\sigma)}\left\|\sum_{J \in \mathcal{G}_{N}} \mathbb{F}_{J}^{\omega, \mathbf{b}^{*}} g\right\|_{L^{2}(\omega)} \\
& =\mathfrak{N}_{T^{\alpha}} \sum_{I \in \mathcal{D}: \ell(I) \geq 2^{N}} \square_{I}^{\sigma, \mathbf{b}} f\left\|_{L^{2}(\sigma)}\right\| \sum_{J \in \mathcal{G}: \ell(J) \geq 2^{N}} \square_{J}^{\omega, \mathbf{b}^{*}} g \|_{L^{2}(\omega)} \\
& \lesssim \mathfrak{N}_{T^{\alpha}}\left(\sum_{I \in \mathcal{D}: \ell(I) \geq 2^{N}}\left\|\square_{I}^{\sigma, \mathbf{b}} f\right\|_{L^{2}(\sigma)}^{2}\right)^{\frac{1}{2}}\left(\sum_{J \in \mathcal{G}: \ell(J) \geq 2^{N}}\left\|\square_{J}^{\omega, \mathbf{b}^{*}} g\right\|_{L^{2}(\omega)}^{2}\right)^{\frac{1}{2}}
\end{aligned}
$$


which tends to 0 as $N \rightarrow \infty$ since

$$
\left(\sum_{I \in \mathcal{D}}\left\|\square_{I}^{\sigma, \mathbf{b}} f\right\|_{L^{2}(\sigma)}^{2}\right)^{\frac{1}{2}}\left(\sum_{J \in \mathcal{G}}\left\|\square_{J}^{\omega, \mathbf{b}^{*}} g\right\|_{L^{2}(\omega)}^{2}\right)^{\frac{1}{2}} \lesssim\|f\|_{L^{2}(\sigma)}\|g\|_{L^{2}(\omega)} .
$$

Remark 35. In particular,

$$
\lim _{N \rightarrow \infty} \sup _{I \in \mathcal{D}_{N}}\left\|\mathbb{F}_{I}^{\sigma, \mathbf{b}} f\right\|_{L^{2}(\sigma)}=0=\lim _{N \rightarrow \infty} \sup _{J \in \mathcal{G}_{N}}\left\|\mathbb{F}_{J}^{\omega, \mathbf{b}^{*}} g\right\|_{L^{2}(\sigma)}
$$

and so we can use the pointwise telescoping identities

$$
\mathbb{F}_{I}^{\sigma, \mathbf{b}} f(x)=\sum_{I^{\prime} \in \mathcal{D}: I \subset I^{\prime}} \square_{I^{\prime}}^{\sigma, \mathbf{b}} f(x) \text { and } \mathbb{F}_{J}^{\omega, \mathbf{b}^{*}} g(x)=\sum_{J \in \mathcal{G}: J \subset J^{\prime}} \square_{J^{\prime}}^{\omega, \mathbf{b}^{*}} g(x) .
$$

The second, third and fourth sums in (3.1) can be controlled by the full testing conditions, e.g.

$$
\begin{aligned}
& \left|\sum_{I \in \mathcal{D}: I \subset I_{\infty}} \int\left(T_{\sigma}^{\alpha} \square_{I}^{\sigma, \mathbf{b}} f\right) \mathbb{F}_{J_{\infty}}^{\omega, \mathbf{b}^{*}} g d \omega\right|=\left|\int\left(\sum_{I \in \mathcal{D}: I \subset I_{\infty}} \square_{I}^{\sigma, \mathbf{b}} f\right) T_{\omega}^{\alpha, *}\left(\mathbb{F}_{J_{\infty}}^{\omega, \mathbf{b}^{*}} g\right) d \sigma\right| \\
\leq & \left\|\sum_{I \in \mathcal{D}: I \subset I_{\infty}} \square_{I}^{\sigma, \mathbf{b}} f\right\|_{L^{2}(\sigma)}\left\|T_{\omega}^{\alpha, *}\left(\mathbb{F}_{J_{\infty}}^{\omega, \mathbf{b}^{*}} g\right)\right\|_{L^{2}(\sigma)} \lesssim\|f\|_{L^{2}(\sigma)}\left(\mathfrak{F} \mathfrak{T}_{T_{\omega}^{\alpha, *}}+\sqrt{\mathfrak{A}_{2}^{\alpha}}\right)\|g\|_{L^{2}(\omega)}
\end{aligned}
$$

since $\mathbb{F}_{J_{\infty}}^{\omega, \mathbf{b}^{*}} g=b_{J_{\infty}}^{*} \frac{E_{J_{\infty}}^{\omega} g}{E_{J_{\infty}}^{\omega} b_{J_{\infty}}^{*}}$ is $b_{J_{\infty}}^{*}$ times an 'accretive' average of $g$ on $J_{\infty}$, and similarly for the third and fourth sum. Finally, the full testing conditions $\mathfrak{F} \mathfrak{T}_{T_{\sigma}^{\alpha}}^{\mathbf{b}}$ and $\mathfrak{F} \mathfrak{T}_{T_{\omega}^{\alpha}}^{\mathbf{b}^{*} *}$ are controlled by the usual testing conditions $\mathfrak{T}_{T_{\sigma}^{\alpha}}^{\mathbf{b}}$ and $\mathfrak{T}_{T_{\omega}^{\alpha, *}}^{\mathbf{b}^{*}}$ together with the Muckenhoupt condition $\mathfrak{A}_{2}^{\alpha}$, by (3.25) below.

Important: In the $\sigma$-iterated triple corona construction we redefined the family $\mathbf{b}=\left\{b_{Q}\right\}_{Q \in \mathcal{D}}$ so that the new functions $b_{Q}^{\text {new }}$ are given in terms of the original functions $b_{Q}^{\text {orig }}$ by $b_{Q}^{\text {new }}=\mathbf{1}_{Q} b_{A}^{\text {orig }}$ for $Q \in \mathcal{C}_{A}^{\sigma}$, and of course we then dropped the superscript 'new'. We continue to refer to the triple stopping intervals $A$ as 'breaking' intervals even if $b_{A}$ happens to equal $\mathbf{1}_{A} b_{\pi A}$. The results of Appendix A apply with this more inclusive definition of 'breaking' intervals, and the associated definition of 'broken' children, since only the Carleson condition on stopping intervals is relevant here.

Altogether this and Proposition 28 give us the triple corona decomposition of $f=\sum_{A \in \mathcal{A}} \mathrm{P}_{\mathcal{C}_{A}}^{\sigma, \mathbf{b}} f$, where the pseudoprojection $\mathrm{P}_{\mathcal{C}_{A}}^{\sigma, \mathbf{b}}$ is defined in Appendix A:

$$
\mathrm{P}_{\mathcal{C}_{A}}^{\mu, \mathbf{b}} f=\sum_{I \in \mathcal{C}_{A}} \square_{I}^{\mu, \mathbf{b}} f
$$

We now record the main facts proved above, and in Appendix A below, for the triple corona.

Lemma 36. Let $f \in L^{2}(\sigma)$. We have

$$
\begin{gathered}
f=\sum_{A \in \mathcal{A}} \mathrm{P}_{\mathcal{C}_{A}}^{\sigma} f, \quad \text { both in the sense of norm convergence in } L^{2}(\sigma) \\
\text { and pointwise } \sigma \text {-a.e. }
\end{gathered}
$$

The corona tops $\mathcal{A}$ and stopping bounds $\left\{\alpha_{\mathcal{A}}(A)\right\}_{A \in \mathcal{A}}$ satisfy properties (1), (2), (3) and (4) in Definition 27. hence constitute stopping data for $f$. Moreover, $\mathbf{b}=\left\{b_{I}\right\}_{I \in \mathcal{D}}$ is an $\infty$-strongly $\sigma$-controlled accretive family on $\mathcal{D}$ with corona tops $\mathcal{A} \subset \mathcal{D}$, where $b_{I}=\mathbf{1}_{I} b_{A}$ has reverse Hölder control on children for all $I \in \mathcal{C}_{A}$, and the weak corona forward testing condition holds uniformly in coronas, i.e.

$$
\frac{1}{|I|_{\sigma}} \int_{I}\left|T_{\sigma}^{\alpha} b_{A}\right|^{2} d \sigma \leq C, \quad I \in \mathcal{C}_{A}^{\sigma}
$$

Similar statements hold for $g \in L^{2}(\omega)$.

Now we turn to the various splittings of forms, beginning with the two weight analogue of the decomposition of Hytönen and Martikainen HyMa. Fix the stopping data $\mathcal{A}$ and $\left\{\alpha_{\mathcal{A}}(A)\right\}_{A \in \mathcal{A}}$ and dual martingale differences $\square_{I}^{\sigma, \mathbf{b}}$ constructed above with the triple iterated coronas, as well as the corresponding data for $g$. Here is a brief schematic diagram of the splittings and decompositions we will describe below, with associated 
bounds given in $\square$. We split the form $\left\langle T_{\sigma}^{\alpha} f, g\right\rangle_{\omega}$ into the sum of two essentially symmetric forms by interval size,

$$
\int\left(T_{\sigma} f\right) g d \omega=\left\{\sum_{\substack{I \in \mathcal{D}: J \in \mathcal{G} \\ \ell(J) \leq \ell(I)}}+\sum_{\substack{I \in \mathcal{D}: J \in \mathcal{G} \\ \ell(J)>\ell(I)}}\right\} \int\left(T_{\sigma} \square_{I}^{\sigma, \mathbf{b}} f\right) \square_{J}^{\omega, \mathbf{b}^{*}} g d \omega,
$$

and focus on the first sum,

$$
\Theta(f, g)=\sum_{I \in \mathcal{D} \text { and } J \in \mathcal{G}: \ell(J) \leq \ell(I)}\left\langle T_{\sigma}^{\alpha} \square_{I}^{\sigma, \mathbf{b}} f, \square_{J}^{\omega, \mathbf{b}^{*}}\right\rangle_{\omega},
$$

since the second sum is handled dually, but is easier due to the missing diagonal.

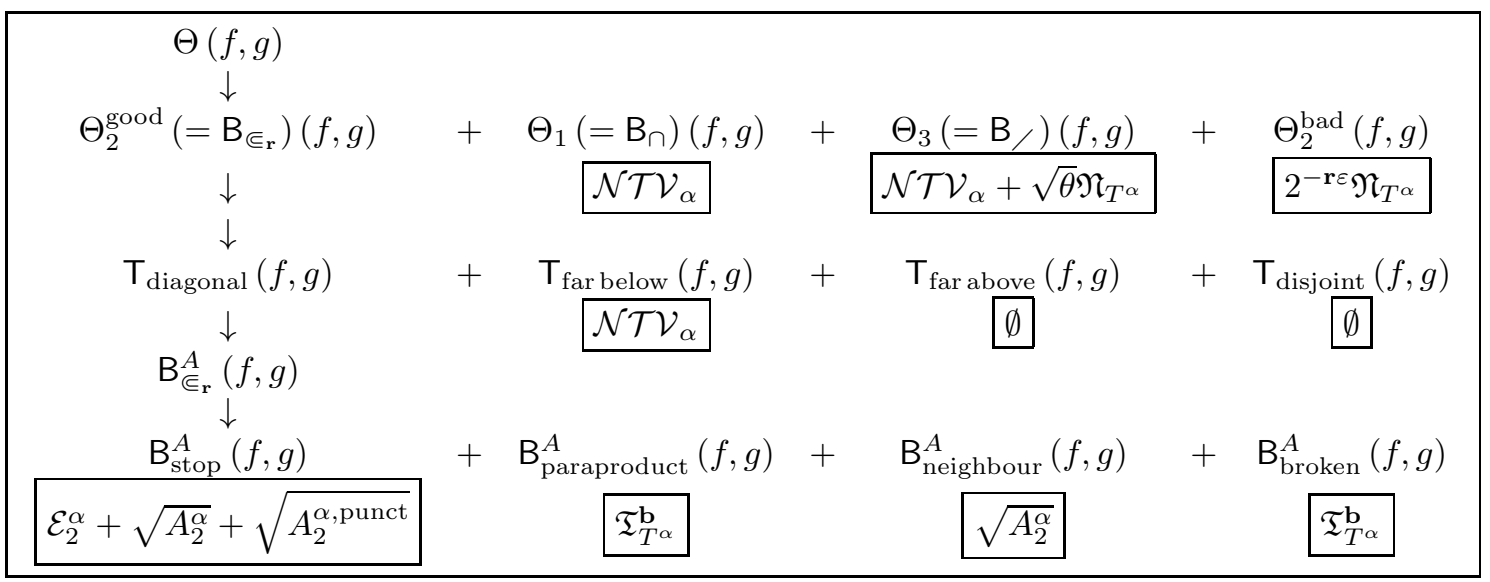

For the reader's convenience we now collect the various martingale and probability estimates that will be used in the proof that follows. First we summarize the martingale identities and estimates from Appendix A that we will use in our proof, noting in particular that lower weak Riesz inequalities are not used in the proof of our $T b$ theorem. Suppose $\mu$ is a positive locally finite Borel measure, and that $\mathbf{b}$ is an $\infty$-strongly $\mu$-controlled accretive family. Then the following martingale identities and estimates hold:

Martingale identities: Both of the following identities hold pointwise $\mu$-almost everywhere, as well as in the sense of strong convergence in $L^{2}(\mu)$ :

$$
\begin{aligned}
f & =\sum_{I \in \mathcal{D}_{N}} \mathbb{F}_{I}^{\mu, \mathbf{b}} f+\sum_{I \in \mathcal{D}: \ell(I) \geq N+1} \square_{I}^{\mu, \mathbf{b}} f \\
f & =\sum_{I \in \mathcal{D}_{N}} \mathbb{E}_{I}^{\mu, \mathbf{b}} f+\sum_{I \in \mathcal{D}: \ell(I) \geq N+1} \triangle_{I}^{\mu, \mathbf{b}} f .
\end{aligned}
$$

Frame estimates: Both of the following frame estimates hold:

$$
\begin{aligned}
\|f\|_{L^{2}(\mu)}^{2} & \approx \sum_{Q \in \mathcal{D}}\left\{\left\|\square_{Q}^{\mu, \mathbf{b}} f\right\|_{L^{2}(\mu)}^{2}+\left\|\nabla_{Q}^{\mu} f\right\|_{L^{2}(\mu)}^{2}\right\} \\
& \approx \sum_{Q \in \mathcal{D}}\left\{\left\|\triangle_{Q}^{\mu, \mathbf{b}} f\right\|_{L^{2}(\mu)}^{2}+\left\|\nabla_{Q}^{\mu} f\right\|_{L^{2}(\mu)}^{2}\right\} .
\end{aligned}
$$

Weak upper Riesz estimates: Define the pseudoprojections,

$$
\begin{aligned}
\Psi_{\mathcal{B}}^{\mu, \mathbf{b}} f & \equiv \sum_{I \in \mathcal{B}} \square_{I}^{\mu, \mathbf{b}} f \\
\left(\Psi_{\mathcal{B}}^{\mu, \mathbf{b}}\right)^{*} f & \equiv \sum_{I \in \mathcal{B}}\left(\square_{I}^{\mu, \mathbf{b}}\right)^{*} f=\sum_{I \in \mathcal{B}} \triangle_{I}^{\mu, \mathbf{b}} f .
\end{aligned}
$$


We have the 'upper Riesz' inequalities for pseudoprojections $\Psi_{\mathcal{B}}^{\mu, \mathbf{b}}$ and $\left(\Psi_{\mathcal{B}}^{\mu, \mathbf{b}}\right)^{*}$ :

$$
\begin{aligned}
\left\|\Psi_{\mathcal{B}}^{\mu, \mathbf{b}} f\right\|_{L^{2}(\mu)}^{2} & \leq C \sum_{I \in \mathcal{B}}\left\|\square_{I}^{\mu, \mathbf{b}} f\right\|_{L^{2}(\mu)}^{2}+\sum_{I \in \mathcal{B}}\left\|\nabla_{I}^{\mu} f\right\|_{L^{2}(\mu)}^{2}, \\
\left\|\left(\Psi_{\mathcal{B}}^{\mu, \mathbf{b}}\right)^{*} f\right\|_{L^{2}(\mu)}^{2} & \leq C \sum_{I \in \mathcal{B}}\left\|\triangle_{I}^{\mu, \mathbf{b}} f\right\|_{L^{2}(\mu)}^{2}+\sum_{I \in \mathcal{B}}\left\|\nabla_{I}^{\mu} f\right\|_{L^{2}(\mu)}^{2},
\end{aligned}
$$

for all $f \in L^{2}(\mu)$ and all subsets $\mathcal{B}$ of the grid $\mathcal{D}$, and where the positive constant $C$ is independent of the subset $\mathcal{B}$. Here $\nabla_{I}^{\mu}$ is the Carleson averaging operator defined in (9.6) in Appendix A.

Now we turn to the probability estimates for martingale differences and halos that we will use. Recall that given $0<\lambda<\frac{1}{2}$, the $\lambda$-halo of $J$ is defined to be

$$
\partial_{\lambda} J \equiv(1+\lambda) J \backslash(1-\lambda) J
$$

Suppose $\mu$ is a positive locally finite Borel measure, and that $\mathbf{b}$ is an $\infty$-weakly $\mu$-controlled accretive family. Then the following probability estimates hold. See Definition 44 below for the notation $\mathcal{G}_{k \text {-bad }}^{\mathcal{D}}$.

Bad cube probability estimates: Suppose that $\mathcal{D}$ and $\mathcal{G}$ are independent random dyadic grids. With $\Psi_{\mathcal{G}_{k-\text { bad }}^{D}}^{\mu, \mathbf{b}^{*}} g \equiv \sum_{J \in \mathcal{G}_{k-\text { bad }}^{\mathcal{D}}} \square_{J}^{\mu, \mathbf{b}^{*}} g$ equal to the pseudoprojection of $g$ onto $k$-bad $\mathcal{G}$-intervals, we have

$$
\boldsymbol{E}_{\Omega}^{\mathcal{D}}\left(\left\|\Psi_{\mathcal{G}_{k-\mathrm{bad}}^{\mathcal{D}}}^{\mu, \mathbf{b}^{*}} g\right\|_{L^{2}(\mu)}^{2}\right) \lesssim \boldsymbol{E}_{\Omega}^{\mathcal{D}}\left(\sum_{J \in \mathcal{G}_{k-\text { bad }}^{\mathcal{D}}}\left[\left\|\square_{J, \mathcal{G}}^{\mu, \mathbf{b}^{*} g}\right\|_{L^{2}(\mu)}^{2}+\left\|\nabla_{J, \mathcal{G}}^{\mu} g\right\|_{L^{2}(\mu)}^{2}\right]\right) \leq C e^{-k \varepsilon}\|g\|_{L^{2}(\mu)}^{2}
$$

where the first inequality is the 'weak upper half Riesz' inequality for the pseudoprojection $\Psi_{\mathcal{G}_{k-\text { bad }}^{D}}^{\mu, \mathbf{b}^{*}}$ ' and the second inequality is proved using the frame inequality in (3.13) below.

Halo probability estimates: Suppose that $\mathcal{D}$ and $\mathcal{G}$ are independent random grids. Using the $p a-$ rameterization by translations of grids and taking the average over certain translates $\tau+\mathcal{D}$ of the grid $\mathcal{D}$ we have

$$
\begin{aligned}
& \boldsymbol{E}_{\Omega}^{\mathcal{D}} \sum_{I^{\prime} \in \mathcal{D}: \ell\left(I^{\prime}\right) \approx \ell\left(J^{\prime}\right)} \int_{J^{\prime} \cap \partial_{\delta} I^{\prime}} d \omega \lesssim \delta \int_{J^{\prime}} d \omega, \quad J^{\prime} \in \mathfrak{C}(J), J \in \mathcal{G}, \\
& \boldsymbol{E}_{\Omega}^{\mathcal{G}} \sum_{J^{\prime} \in \mathcal{G}: \ell\left(J^{\prime}\right) \approx \ell\left(I^{\prime}\right)} \int_{I^{\prime} \cap \partial_{\delta} J^{\prime}} d \sigma \lesssim \delta \int_{I^{\prime}} d \sigma, \quad I^{\prime} \in \mathfrak{C}(I), I \in \mathcal{D},
\end{aligned}
$$

and where the expectations $\boldsymbol{E}_{\Omega}^{\mathcal{D}}$ and $\boldsymbol{E}_{\Omega}^{\mathcal{G}}$ are taken over grids $\mathcal{D}$ and $\mathcal{G}$ respectively. Indeed, it is geometrically evident that for any fixed pair of side lengths $\ell_{1} \approx \ell_{2}$, the average of the measure $\left|J^{\prime} \cap \partial_{\delta} I^{\prime}\right|_{\omega}$ of the set $J^{\prime} \cap \partial_{\delta} I^{\prime}$, as an interval $I^{\prime} \in \mathcal{D}$ with side length $\ell\left(I^{\prime}\right)=\ell_{1}$ is translated across an interval $J^{\prime} \in \mathcal{G}$ of side length $\ell\left(J^{\prime}\right)=\ell_{2}$, is at most $C\left|J^{\prime}\right|_{\omega}$. Using this observation it is now easy to see that (3.7) holds.

3.1. The Hytönen-Martikainen decomposition and a weak variant of NTV goodness. Let $b$ (respectively $\mathbf{b}^{*}$ ) be $\infty$-weakly $\sigma$-controlled (respectively $\omega$-controlled) accretive families. At the beginning of this section, we reduced the estimation of the bilinear form $\int_{\mathbb{R}}\left(T_{\sigma} f\right) g d \omega$ to that of the sum

$$
\sum_{I \in \mathcal{D}} \sum_{J \in \mathcal{G}} \int\left(T_{\sigma}^{\alpha} \square_{I}^{\sigma, \mathbf{b}} f\right) \square_{J}^{\omega, \mathbf{b}^{*}} g d \omega
$$

and then we decomposed this sum by interval side length,

$$
\begin{aligned}
\sum_{I \in \mathcal{D}} \sum_{J \in \mathcal{G}} \int\left(T_{\sigma}^{\alpha} \square_{I}^{\sigma, \mathbf{b}} f\right) \square_{J}^{\omega, \mathbf{b}^{*}} g d \omega & =\left\{\sum_{\substack{I \in \mathcal{D}: J \in \mathcal{G} \\
\ell(J) \leq \ell(I)}}+\sum_{\substack{I \in \mathcal{D}: J \in \mathcal{G} \\
\ell(J)>\ell(I)}}\right\} \int\left(T_{\sigma}^{\alpha} \square_{I}^{\sigma, \mathbf{b}} f\right) \square_{J}^{\omega, \mathbf{b}^{*}} g d \omega \\
& \equiv \Theta(f, g)+\Theta^{*}(f, g),
\end{aligned}
$$


and noted that by symmetry, it suffices to estimate the first form $\Theta(f, g)$. Before introducing goodness into the sum, we follow HyMa and split the form $\Theta(f, g)$ into 3 pieces:

$$
\begin{aligned}
& \Theta(f, g) \equiv \sum_{\substack{I \in \mathcal{D}: J \in \mathcal{G} \\
\ell(J) \leq \ell(I)}} \int\left(T_{\sigma}^{\alpha} \square_{I}^{\sigma, \mathbf{b}} f\right) \square_{J}^{\omega, \mathbf{b}^{*}} g d \omega \\
& =\sum_{I \in \mathcal{D}}\left\{\sum_{\substack{J \in \mathcal{G}: \ell(J) \leq \ell(I) \\
d(J, I)>2 \ell(J)^{\varepsilon} \ell(I)^{1-\varepsilon}}}+\sum_{\substack{J \in \mathcal{G}: \ell(J) \leq 2^{-\mathbf{r}} \ell(I) \\
d(J, I) \leq 2 \ell(J)^{\varepsilon} \ell(I)^{1-\varepsilon}}}+\sum_{\substack{J \in \mathcal{G}: 2^{-\mathbf{r}} \ell(I)<\ell(J) \leq \ell(I) \\
d(J, I) \leq 2 \ell(J)^{\varepsilon} \ell(I)^{1-\varepsilon}}}\right\} \int\left(T_{\sigma}^{\alpha} \square_{I}^{\sigma, \mathbf{b}} f\right) \square_{J}^{\omega, \mathbf{b}^{*}} g d \omega \\
& \equiv \Theta_{1}(f, g)+\Theta_{2}(f, g)+\Theta_{3}(f, g) \text {, }
\end{aligned}
$$

where $\varepsilon>0$ will be chosen to satisfy $0<\varepsilon<\frac{1}{2} \leq \frac{1}{2-\alpha}$ later, and the goodness parameter $\mathbf{r}$ is then determined in (3.19) below. Now the disjoint form $\Theta_{1}(f, g)$ can be handled by 'long-range' and 'short-range' arguments which we give in the next section below, and the nearby form $\Theta_{3}(f, g)$ will be handled in the subsequent section using probabilistic surgery methods and a new deterministic surgery involving energy conditions and the 'original' testing functions discarded in the corona construction. The remaining form $\Theta_{2}(f, g)$ will be treated further in this section after introducing weak goodness.

3.1.1. Good intervals with body'. We begin with the weaker extension of goodness introduced in HyMa, except that we will make it a bit stronger by replacing the skeleton 'skel $K$ ' of an interval $K$, as used in HyMa, by a larger collection of points 'body $K$ ', which we call the dyadic body of $K$. This modification will prove useful in establishing the Straddling Lemma in the treatment of the stopping form in Section 7 below. Let $\mathcal{P}$ denote the collection of all intervals in $\mathbb{R}$. The content of the next four definitions is inspired by, or sometimes identical with, that already appearing in the work of Nazarov, Treil and Volberg in [NTV1] and NTV3].

Definition 37. Let $K \in \mathcal{P}$.

(1) Define the skeleton 'skel $K$ ' of $K$ to consist of its two endpoints and its midpoint.

(2) For a point $x$ in $\mathbb{R}$, define the dyadic spray $\mathbb{S}_{x}^{\mathrm{dy}} \equiv\{x\} \cup\left\{x \pm \frac{1}{2^{j}}\right\}_{k \in \mathbb{Z}}$ of $x$ to consist of $x$ and all points $y$ in $\mathbb{R}$ that have distance $\frac{1}{2^{j}}$ from $x$ for some $j \in \mathbb{Z}$.

(3) Then define the dyadic body body $K$ ' of an interval $K \in \mathcal{P}$ to be the intersection of $\bar{K}$ with the union of the dyadic sprays of its two endpoints, i.e. if $K=[a, b)$, then

$$
\text { body } K=\bar{K} \cap\left(\mathbb{S}_{a}^{\mathrm{dy}} \cup \mathbb{S}_{b}^{\mathrm{dy}}\right)
$$

Thus the body of the unit interval $[0,1)$ consists of the points

$$
\{0\} \dot{\cup}\left\{\frac{1}{2^{j}}\right\}_{j=1}^{\infty} \dot{\cup}\left\{1-\frac{1}{2^{j}}\right\}_{j=2}^{\infty} \dot{\cup}\{1\},
$$

which have the endpoints of $[0,1)$ as cluster points.

Definition 38. Let $0<\varepsilon<1$ (to be chosen later). For intervals $J, K \in \mathcal{P}$ with $\ell(J) \leq \ell(K)$, we define $J$ to be $\varepsilon-$ good with respect to an interval $K$ if

$$
d(J, \operatorname{body} K)>2|J|^{\varepsilon}|K|^{1-\varepsilon},
$$

and we say $J$ is $\varepsilon$ - bad with respect to $K$ if (3.8) fails. We also say that $J$ is $\varepsilon$ - good inside an interval $K$ if $J$ is $\varepsilon-$ good with respect to $K$ and $J \subset K$.

A key consequence of an interval $J$ being $\varepsilon-$ good inside an interval $S$, is that $J$ must then be contained in some dyadic subinterval $K$ of $S$ with $3 K \subset S$ :

$$
\text { If } J \text { is } \varepsilon-\text { good inside } S \text {, then } J \subset K \text { for some } K \in \mathcal{W}(S),
$$

where $\mathcal{W}(S)$ is the collection of maximal dyadic subintervals of $S$ whose triples are contained in $S$. Indeed, the endpoints of the intervals in $\mathcal{W}(S)$ are precisely the body of $S$. Note that this property can fail if we use the smaller set skel $S$ in place of body $S$ in Definition 38 since then an $\varepsilon-$ good interval $J$ could intersect one of the sprays. Of course we will also need to know that body $S$ is not so much larger than skel $S$ that the 
crucial probability estimate for good intervals fails - namely we need to know that given $k \gg 1$, an interval $J \subset S$ of side length $\ell(J)=2^{-k} \ell(S)$ is $\varepsilon$ - good inside $S$ with 'large probability'. This will be made precise below using random dyadic grids.

Definition 39. Let $\mathcal{D}$ and $\mathcal{G}$ be dyadic grids. Define $\mathcal{G}_{(k, \varepsilon)-\operatorname{Dood}}^{\mathcal{D}}$ to consist of those $J \in \mathcal{G}$ such that $J$ is $\varepsilon-\operatorname{good}$ inside every interval $K \in \mathcal{D}$ with $K \cap J \neq \emptyset$ that lies at least $k$ levels 'above' J, i.e. $\ell(K) \geq 2^{k} \ell(J)$ (note that the use of inside forces such $K$ with $K \cap J \neq \emptyset$ to actually contain $J$ ). We also define $J$ to be ' $\varepsilon-\operatorname{good}$ inside an interval $K$ and beyond' if $J \in \mathcal{G}_{(k, \varepsilon)-\text { good }}^{\mathcal{D}}$ where $k=\log _{2} \frac{\ell(K)}{\ell(J)}$ and where $K \cap J \neq \emptyset$, equivalently in this situation $K \supset J$. As the goodness parameter $\varepsilon$ will eventually be fixed throughout the proof, we sometimes suppress it, and simply say ' $J$ is good inside an interval $K$ and beyond' instead of ' $J$ is $\varepsilon-\operatorname{good}$ inside an interval $K$ and beyond'. When $\varepsilon>0$ is understood, we will often write $\mathcal{G}_{k-\operatorname{Dood}}^{\mathcal{D}}=\mathcal{G}_{(k, \varepsilon)-\operatorname{good}}^{\mathcal{D}}$.

Remark 40. Note that

$$
\mathcal{G}_{(k, \varepsilon)-\operatorname{good}}^{\mathcal{D}} \equiv\left\{J \in \mathcal{G}: J \text { is } \varepsilon-\operatorname{good} \text { with respect to every } K \in \mathcal{D} \text { with } \ell(K) \geq 2^{k} \ell(J) .\right\}
$$

Indeed, if $J$ is $\varepsilon$ - bad with respect to some $K \in \mathcal{D}$ with $K \cap J=\emptyset$, then $J$ is also $\varepsilon$ - bad with respect to one of the two neighbours (of the same side length) of $K$ in $\mathcal{D}$.

3.1.2. Grid probability. As pointed out on page 14 of $\mathrm{HyMa}$ by Hytönen and Martikainen, there are subtle difficulties associated in using dual martingale decompositions of functions which depend on the entire dyadic grid, rather than on just the local interval in the grid. We will proceed at first in the spirit of $\mathrm{HyMa}$, and the goodness that we will infuse below into the main 'below' form $\mathrm{B}_{\Subset_{\mathbf{r}}}(f, g)$ will be the Hytönen-Martikainen 'weak' version of NTV goodness, but using the body 'body $I$ ' of an interval rather than its skeleton 'skel $I$ ': every pair $(I, J) \in \mathcal{D} \times \mathcal{G}$ that arises in the form $\mathrm{B}_{\Subset_{\mathbf{r}}}(f, g)$ will satisfy $J \in \mathcal{G}_{(k, \varepsilon) \text {-good }}^{\mathcal{D}}$ where $\ell(I)=2^{k} \ell(J)$.

Now we return to the martingale differences $\square_{I}^{\sigma, \mathbf{b}}$ and $\square_{J}^{\omega, \mathbf{b}^{*}}$ with controlled families $\mathbf{b}$ and $\mathbf{b}^{*}$ in the real line $\mathbb{R}$. When we want to emphasize that the grid in use is $\mathcal{D}$ or $\mathcal{G}$, we will denote the martingale difference by $\square_{I, \mathcal{D}}^{\sigma, \mathbf{b}}$, and similarly for $\square_{J, \mathcal{G}}^{\omega, \mathbf{b}^{*}}$. Recall Definition 38 for the meaning of when an interval $J$ is $\varepsilon$-bad with respect to another interval $K$.

Definition 41. We say that $J \in \mathcal{P}$ is $k$-bad in a grid $\mathcal{D}$ if there is an interval $K \in \mathcal{D}$ with $\ell(K)=2^{k} \ell(J)$ such that $J$ is $\varepsilon$-bad with respect to $K$ (context should eliminate any ambiguity between the different use of $k$-bad when $k \in \mathbb{N}$ and $\varepsilon$-bad when $0<\varepsilon<\frac{1}{2}$ ).

A key observation here (see [NTV1, [NTV2], NTV3] or [NTV4] for the case when goodness is defined using the skeleton instead of the body) is that for any $J \in \mathcal{G}$ where $\mathcal{D}$ and $\mathcal{G}$ are independent random grids,

$$
\boldsymbol{P}_{\Omega}^{\mathcal{D}}(\mathcal{D}: J \text { is } k \text {-bad in } \mathcal{D}) \equiv \int_{\Omega} \mathbf{1}_{\{\mathcal{D}: J \text { is } k \text {-bad in } \mathcal{D}\}} d \mu_{\Omega}(\mathcal{D}) \leq C \varepsilon k 2^{-\varepsilon k} .
$$

Indeed, it suffices to consider the case when $J \in \mathcal{G}$ with $J \subset[0,1)$ and $\ell(J)=2^{-k}$. So fix such an interval $J$. For each $m \in \mathbb{Z}_{2^{k}} \equiv\left\{\ell \in \mathbb{Z}: 0 \leq \ell \leq 2^{k}-1\right\}$, consider the collection $\mathfrak{D}_{m}$ of all grids $\mathcal{D}$ that contain the interval $I_{m} \equiv[0,1)+\frac{m}{2^{k}}=\left[\frac{m}{2^{k}}, 1+\frac{m}{2^{k}}\right)$. Then for every $m$, it is the case that

(1) either $J$ is $\varepsilon$-bad in $\mathcal{D}$ for all $\mathcal{D} \in \mathfrak{D}_{m}$,

(2) or $J$ is $\varepsilon$-good in $\mathcal{D}$ for all $\mathcal{D} \in \mathfrak{D}_{m}$.

We will say that the collection $\mathfrak{D}_{m}$ is $k$-bad if the first case holds. We have the same dichotomy for $\mathfrak{D}_{m}+s$ if we replace $[0,1)$ with the translate $[0,1)+s=[s, 1+s)$ where $0 \leq s<2^{-k}$. We now claim that for any fixed $0 \leq s<2^{-k}$, the number of $k$-bad collections $\mathfrak{D}_{m}+s$ is at most $C \varepsilon k 2^{(1-\varepsilon) k}$, hence the proportion of $k$-bad collections is $\frac{C \varepsilon k 2^{(1-\varepsilon) k}}{2^{k}}=C \varepsilon k 2^{-\varepsilon k}$, from which we obtain the estimate (3.10) as follows. Every grid $\mathcal{D} \in \Omega$ is contained in exactly one of the collections $\left\{\mathfrak{D}_{m}+s\right\}_{m \in \mathbb{Z}_{2^{k}}}$ and $s \in\left[0,2^{-k}\right)$, and so

$$
\begin{aligned}
\boldsymbol{P}_{\Omega}^{\mathcal{D}}(\mathcal{D}: J \text { is } k \text {-bad in } \mathcal{D}) & =\frac{1}{2^{-k}} \int_{\left[0,2^{-k}\right)}\left\{\frac{\#\left\{m \in \mathbb{Z}_{2^{k}}: \mathfrak{D}_{m}+s \text { is } k-\mathrm{bad}\right\}}{\# \mathbb{Z}_{2^{k}}}\right\} d s \\
& \leq \frac{1}{2^{-k}} \int_{\left[0,2^{-k}\right)}\left\{\frac{C \varepsilon k 2^{(1-\varepsilon) k}}{2^{k}}\right\} d s=C \varepsilon k 2^{-\varepsilon k} .
\end{aligned}
$$

To see our claim, it suffices to consider the case $s=0$, to keep the interval $I_{0}=[0,1)$ fixed, and consider instead the translates $J_{m} \equiv J+\frac{m}{2^{k}}$ of the interval $J$ for $0 \leq m \leq 2^{k}-1$. Moreover we can assume without 
loss of generality that $J$ intersects the point $\frac{1}{2^{k}}$ so that all of the intervals $J_{m}$ in the collection $\left\{J_{m}\right\}_{m=0}^{2^{k}-1}$ lie in $I_{0}$ except for the last one $J_{2^{k}-1}=J+1-\frac{1}{2^{k}}$, which intersects the point 1 . In this situation our claim becomes

$$
\#\left\{m \in \mathbb{Z}_{2^{k}}: J_{m} \text { is } \varepsilon-\text { bad in }[0,1)\right\} \leq C \varepsilon k 2^{(1-\varepsilon) k} .
$$

To prove (3.11), we begin by defining

$$
d \equiv \varepsilon k-1 \text { and } L \equiv 2 \ell(J)^{\varepsilon} \ell\left(I_{0}\right)^{1-\varepsilon}=2^{1-\varepsilon k}=2^{-d},
$$

where we may assume $k>\frac{1}{\varepsilon}$ so that $d>0$. Then if $J_{m}$ is $\varepsilon-$ bad in $[0,1)$, at least one of the following two inequalities must hold:

$$
\operatorname{dist}\left(J_{m}, \mathbb{S}_{0}^{\mathrm{dy}}\right) \leq L, \operatorname{dist}\left(J_{m}, \mathbb{S}_{1}^{\mathrm{dy}}\right) \leq L,
$$

where we recall that $\mathbb{S}_{a}^{\mathrm{dy}}$ is the dyadic spray of $a$. Now if $\operatorname{dist}\left(J_{m}, \mathbb{S}_{0}^{\mathrm{dy}}\right) \leq L$, then

$$
\begin{aligned}
\text { either } \operatorname{dist}\left(J_{m},\{0\} \cup\left\{\frac{1}{2^{j}}\right\}_{j>d}\right) & \leq L, \\
\text { or } \operatorname{dist}\left(J_{m}, \frac{1}{2^{j}}\right) & \leq L, \quad \text { for some } 0 \leq j \leq d .
\end{aligned}
$$

However, if

$$
\operatorname{dist}\left(J_{m},\{0\} \cup\left\{\frac{1}{2^{j}}\right\}_{j>d}\right) \leq L
$$

then we must have

$$
m \ell(J) \leq \frac{1}{2^{d}}+L=2 L,
$$

and if $\operatorname{dist}\left(J_{m}, \frac{1}{2^{j}}\right) \leq L$ for some $0 \leq j \leq d$, then we must have

$$
\left|\frac{m}{2^{k}}-\frac{1}{2^{j}}\right| \leq 2 L \text {. }
$$

So altogether the number of indices $m \in \mathbb{Z}_{2^{k}}$ for which dist $\left(J_{m}, \mathbb{S}_{0}^{\mathrm{dy}}\right) \leq L$ holds is at most

$$
\begin{aligned}
2 \frac{L}{\ell(J)}+1+(d+1)\left(2^{k+2} L+1\right) & =(2 d+3) 2^{k+1} L+d+2 \\
& =(2 \varepsilon k+1) \cdot 2^{k+1} \cdot 2^{1-\varepsilon k}+\varepsilon k+1 \leq 20 \varepsilon k 2^{(1-\varepsilon) k} .
\end{aligned}
$$

Similarly the number of indices $m \in \mathbb{Z}_{2^{k}}$ for which dist $\left(J_{m}, \mathbb{S}_{1}^{\text {dy }}\right) \leq L$ holds is at most $20 \varepsilon k 2^{(1-\varepsilon) k}$. Thus we conclude that (3.11) holds with $C=40$.

Then we obtain from (3.10), using the lower frame inequality, the expectation estimate

$$
\begin{aligned}
& \int_{\Omega} \sum_{J \in \mathcal{G}_{k-\text { bad }}^{\mathcal{D}}}\left[\left\|\square_{J, \mathcal{G}}^{\omega, \mathbf{b}^{*}} g\right\|_{L^{2}(\omega)}^{2}+\left\|\nabla_{J, \mathcal{G}}^{\omega} g\right\|_{L^{2}(\omega)}^{2}\right] d \mu_{\Omega}(\mathcal{D}) \\
= & \sum_{J \in \mathcal{G}}\left[\left\|\square_{J, \mathcal{G}}^{\omega, \mathbf{b}^{*}} g\right\|_{L^{2}(\omega)}^{2}+\left\|\nabla_{J, \mathcal{G}}^{\omega} g\right\|_{L^{2}(\omega)}^{2}\right] \int_{\Omega} \mathbf{1}_{\{\mathcal{D}: J \text { is } k \text {-bad in } \mathcal{D}\}} d \mu_{\Omega}(\mathcal{D}) \\
\leq & C k \varepsilon 2^{-k \varepsilon} \sum_{J \in \mathcal{G}}\left[\left\|\square_{J, \mathcal{G}}^{\omega, \mathbf{b}^{*}} g\right\|_{L^{2}(\omega)}^{2}+\left\|\nabla_{J, \mathcal{G}}^{\omega} g\right\|_{L^{2}(\omega)}^{2}\right] \leq C k \varepsilon 2^{-k \varepsilon}\|g\|_{L^{2}(\omega)}^{2},
\end{aligned}
$$

where $\nabla_{J, \mathcal{G}}^{\omega}$ denotes the 'broken' Carleson averaging operator in (9.6) that depends on the broken children in the grid $\mathcal{G}$. Altogether then it follows easily that

$$
\boldsymbol{E}_{\Omega}^{\mathcal{D}}\left(\sum_{J \in \bigcup_{\ell=k}^{\infty} \mathcal{G}_{\ell-\text { bad }}^{\mathcal{D}}}\left[\left\|\square_{J, \mathcal{G}}^{\omega, \mathbf{b}^{*}} g\right\|_{L^{2}(\omega)}^{2}+\left\|\nabla_{J, \mathcal{G}}^{\omega} g\right\|_{L^{2}(\omega)}^{2}\right]\right) \leq C k \varepsilon 2^{-k \varepsilon}\|g\|_{L^{2}(\omega)}^{2},
$$

for some large positive constant $C$. 
From such inequalities summed for $k \geq \mathbf{r}$, it can be concluded as in NTV3 that there is an absolute choice of $\mathbf{r}$ depending on $0<\varepsilon<\frac{1}{2}$ so that the following holds. Let $T: L^{2}(\sigma) \rightarrow L^{2}(\omega)$ be a bounded linear operator. We then have the following traditional inequality for two random grids in the case that $\mathbf{b}$ is an $\infty$-strongly $\mu$-controlled accretive family:

$$
\|T\|_{L^{2}(\sigma) \rightarrow L^{2}(\omega)} \leq 2 \sup _{\|f\|_{L^{2}(\sigma)}=1\|g\|_{L^{2}(\omega)}=1} \sup _{\Omega} \boldsymbol{E}_{\Omega^{\prime}}\left|\left\langle\sum_{I, J \in \mathcal{D}_{\mathbf{r}-\operatorname{good}}^{\mathcal{G}}} T\left(\square_{I, \mathcal{D}}^{\sigma, \mathbf{b}} f\right) f, \square_{J, \mathcal{D}}^{\omega, \mathbf{b}^{*}} g\right\rangle\right| .
$$

However, this traditional method of introducing goodness is flawed here in the general setting of dual martingale differences, since these differences are no longer orthogonal projections, and as emphasized in HyMa, we cannot simply add back in bad intervals whenever we want telescoping identities to hold - but these are needed in order to control the right hand side of (3.14). In fact, in the analysis of the form $\Theta(f, g)$ above, it is necessary to have goodness for the intervals $J$ and telescoping for the intervals $I$. On the other hand, in the analysis of the form $\Theta^{*}(f, g)$ above, it is necessary to have just the opposite - namely goodness for the intervals $I$ and telescoping for the intervals $J$.

Thus, because in this unfortunate set of circumstances we can no longer 'add back in' bad cubes to achieve telescoping, we are prevented from introducing goodness in the full sum (3.3) over all $I$ and $J$, prior to splitting according to side lengths of $I$ and $J$. Thus the infusion of goodness must come after the splitting by side length, but one must work much harder to introduce goodness directly into the form $\Theta(f, g)$ after we have restricted the sum to intervals $J$ that have smaller side length than $I$. This is accomplished in the next subsubsection using the weaker form of $N T V$ goodness introduced by Hytönen and Martikainen in HyMa (that permits certain additional pairs $(I, J)$ in the good forms where $\ell(J) \leq 2^{-\mathbf{r}} \ell(I)$ and yet $J$ is bad in the traditional sense), and that will prevail later in the treatment of the far below forms $\mathrm{T}_{\text {far below }}^{1}(f, g)$, and of the local forms $\mathrm{B}_{\subseteq_{\mathbf{r}}}^{A}(f, g)$ (see Subsection 8 ) where the need for using the 'body' of an interval will become apparent in dealing with the stopping form, and also in the treatment of the functional energy in Appendix B.

3.1.3. Weak goodness. Let $\mathcal{D}$ and $\mathcal{G}$ be dyadic grids. It remains to estimate the form $\Theta_{2}(f, g)$ which, following HyMa, we will split into a 'bad' part and a 'good' part. For this we introduce our main definition associated with the above modification of the weak goodness of Hytönen and Martikainen, namely the definition of the interval $R^{\mathbb{*}}$ in a grid $\mathcal{D}$, given an arbitrary interval $R \in \mathcal{P}$.

Definition 42. Let $\mathcal{D}$ be a dyadic grid. Given $R \in \mathcal{P}$, let $R^{\mathbb{*}}$ be the smallest (if any such exist) $\mathcal{D}$-dyadic superinterval $Q$ of $R$ such that $R$ is good inside all $\mathcal{D}$-dyadic superintervals $K$ of $Q$. Of course $R^{*}$ will not exist if there is no $\mathcal{D}$-dyadic interval $Q$ containing $R$ in which $R$ is good. For intervals $R, Q \in \mathcal{P}$ let $\kappa(Q, R)=\log _{2} \frac{\ell(Q)}{\ell(R)}$. For $R \in \mathcal{P}$ for which $R^{*}$ exists, let $\kappa(R) \equiv \kappa\left(R^{*}, R\right)$.

Note that we typically suppress the dependence of $R^{*}$ on the grid $\mathcal{D}$, since the grid is usually understood

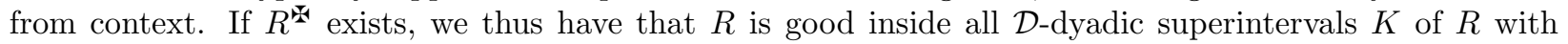
$\ell(K) \geq \ell\left(R^{*}\right)$. Note in particular the monotonicity property for $J^{\prime}, J \in \mathcal{P}$ :

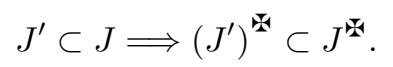

Here now is the decomposition:

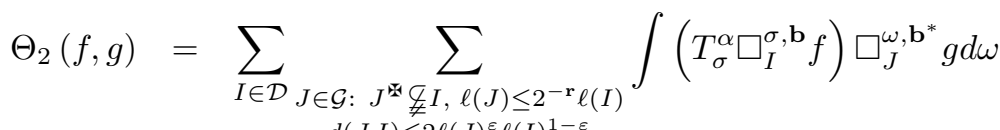

$$
\begin{aligned}
& d(J, I) \leq 2 \ell(J)^{\varepsilon} \ell(I)^{1-\varepsilon} \\
& +\sum_{I \in \mathcal{D}} \sum_{J \in \mathcal{G}: J^{\mathbf{*}} \subsetneq I, \ell(J) \leq 2^{-\mathbf{r}} \ell(I)} \int\left(T_{\sigma}^{\alpha} \square_{I}^{\sigma, \mathbf{b}} f\right) \square_{J}^{\omega, \mathbf{b}^{*}} g d \omega \\
& d(J, I) \leq 2 \ell(J)^{\varepsilon} \ell(I)^{1-\varepsilon} \\
& \equiv \Theta_{2}^{\text {bad }}(f, g)+\Theta_{2}^{\text {good }}(f, g) \text {, }
\end{aligned}
$$

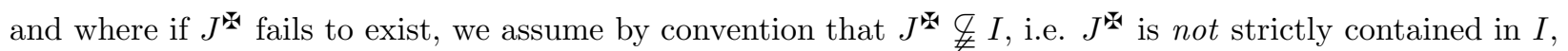
so that the pair $(I, J)$ is then included in the bad form $\Theta_{2}^{\text {bad }}(f, g)$. We will in fact estimate a larger quantity 
corresponding to the bad form, namely

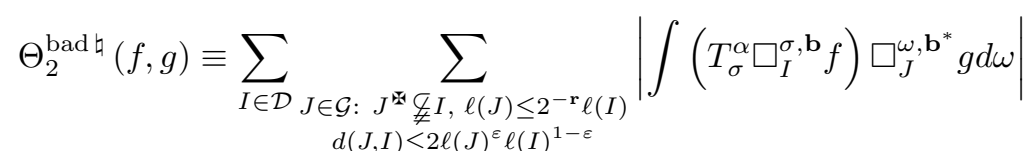

with absolute value signs inside the sum.

Remark 43. We now make some general comments on where we now stand and where we are going.

(1) In the first sum $\Theta_{2}^{\text {bad }}(f, g)$ above, we are roughly keeping the pairs of intervals $(I, J)$ such that $J$ is bad with respect to some 'nearby' interval having side length larger than that of $I$.

(2) We have defined energy and dual energy conditions that are independent of the testing families (because the definition of $\mathrm{E}(J, \omega)=\mathbb{E}_{J}^{\omega, x} \mathbb{E}_{J}^{\omega, x^{\prime}}\left(\left|\frac{x-x^{\prime}}{\ell(J)}\right|^{2}\right)$ does not involve pseudoprojections $\left.\square_{J, \mathcal{D}}^{\omega, \mathbf{b}^{*}}\right)$, but the functional energy condition defined below does involve the dual martingale pseudoprojections $\square_{J, \mathcal{D}}^{\omega, \mathbf{b}^{*}}$.

(3) Using the notion of weak goodness above, we will be able to eliminate all pairs of intervals with $J$ bad in I, which then permits control of the short range form in Section 4 and the neighbour form in Section 6 provided $0<\varepsilon<\frac{1}{2-\alpha}$. Defining shifted coronas in terms of $J^{-}$will then allow existing arguments to prove the Intertwining Proposition and obtain control of the functional energy in Appendix B, as well as permitting control of the stopping form in Section 7 , but all of this with some new twists, for example the introduction of a top/down 'indented corona' in the analysis of the stopping form.

(4) The nearby form $\Theta_{3}(f, g)$ is handled in Section 5 using the energy condition assumption along with the original testing functions $b_{Q}^{\text {orig }}$ discarded during the construction of the testing/accretive corona.

These remarks will become clear in this and the following sections. Recall that we earlier defined in Definition 39, the set $\mathcal{G}_{k \text {-good }}^{\mathcal{D}}=\mathcal{G}_{(k, \varepsilon) \text {-good }}^{\mathcal{D}}$ to consist of those $J \in \mathcal{G}$ such that $J$ is $\varepsilon$-good inside every interval $K \in \mathcal{D}$ with $K \cap J \neq \emptyset$ that lies at least $k$ levels 'above' $J$, i.e. $\ell(K) \geq 2^{k} \ell(J)$. We now define an analogous notion of $\mathcal{G}_{k-\text { bad }}^{\mathcal{D}}$.

Definition 44. Let $\varepsilon>0$. Define the set $\mathcal{G}_{k-\text { bad }}^{\mathcal{D}}=\mathcal{G}_{(k, \varepsilon) \text {-bad }}^{\mathcal{D}}$ to consist of all $J \in \mathcal{G}$ such that there is a $\mathcal{D}$-interval $K$ with sidelength $\ell(K)=2^{k} \ell(J)$ for which $J$ is $\varepsilon$ - bad with respect to $K$.

Note that for grids $\mathcal{D}$ and $\mathcal{G}$, the complement of $\mathcal{G}_{k-\text { good }}^{\mathcal{D}}$ is the union of $\mathcal{G}_{\ell-\text { bad }}^{\mathcal{D}}$ for $\ell \geq k$, i.e.

$$
\mathcal{G} \backslash \mathcal{G}_{k-\text { good }}^{\mathcal{D}}=\bigcup_{\ell \geq k} \mathcal{G}_{\ell-\text { bad }}^{\mathcal{D}} .
$$

Now assume $\varepsilon>0$. We then have the following important property, namely for all intervals $R$, and all $k \geq \mathbf{r}$ (where the goodness parameter $\mathbf{r}$ will be fixed given $\varepsilon>0$ in (3.19) below):

$$
\#\left\{Q: \kappa(Q, R)=k \text { and } d(R, Q) \leq 2 \ell(R)^{\varepsilon} \ell(Q)^{1-\varepsilon}\right\} \lesssim 1 .
$$

As in $\mathrm{HyMa}$, set

$$
\mathcal{G}_{\text {bad }, n}^{\mathcal{D}} \equiv\{J \in \mathcal{G}: J \text { is } \varepsilon-\text { bad with respect to some } K \in \mathcal{D} \text { with } \ell(K) \geq n\} .
$$

We will now use the set equality

$$
\begin{aligned}
& \left\{J \in \mathcal{G}: J^{\mathscr{W}} \not \subset I, \ell(J) \leq 2^{-\mathbf{r}} \ell(I), d(J, I) \leq 2 \ell(J)^{\varepsilon} \ell(I)^{1-\varepsilon}\right\} \\
= & \left\{R \in \mathcal{G}_{\text {bad }, \ell(Q)}^{\mathcal{D}}: \mathbf{r} \leq \kappa(Q, R)<\kappa(R), d(R, Q) \leq 2 \ell(R)^{\varepsilon} \ell(Q)^{1-\varepsilon}\right\},
\end{aligned}
$$

which the careful reader can prove by painstakingly verifying both containments.

Assuming only that $\mathbf{b}$ is 2 -weakly $\mu$-controlled accretive (recall we are assuming the stronger condition that $\mathbf{b}$ is $\infty$-strongly $\mu$-controlled accretive in our proof here), and following the proof in HyMa, we use (3.17) to show that for any fixed grids $\mathcal{D}$ and $\mathcal{G}$, and any bounded linear operator $T_{\sigma}^{\alpha}$ we have the following 
inequality for the form $\Theta_{2}^{\text {bad } \natural, \text { strict }}(f, g)$, defined to be $\Theta_{2}^{\text {bad } \natural}(f, g)$ as in (3.15) with the pairs $(I, J)$ removed when $J^{*}=I$. We use $\varepsilon_{Q, R}= \pm 1$ to obtain

$$
\begin{aligned}
& \Theta_{2}^{\text {bad } \text {, strict }}(f, g)=\sum_{Q \in \mathcal{D}} \sum_{\substack{R \in \mathcal{G}_{\text {bad, } \ell(Q)}^{\mathcal{D}}: \mathbf{r} \leq \kappa(Q, R)<\kappa(R) \\
d(R, Q) \leq 2 \ell(R)^{\varepsilon} \ell(Q)^{1-\varepsilon}}}\left|\left\langle T_{\sigma}^{\alpha}\left(\square_{Q, \mathcal{D}}^{\sigma, \mathbf{b}} f\right), \square_{R, \mathcal{G}}^{\omega, \mathbf{b}^{*} g}\right\rangle\right| \\
& =\sum_{\substack{Q \in \mathcal{D} \\
\begin{array}{r}
R \in \mathcal{G}_{\text {bad }, \ell(Q)}^{\mathcal{D}} \\
d(R, Q) \leq 2 \ell(R)^{\varepsilon} \ell(Q)^{1-\varepsilon}
\end{array}}} \sum_{Q, R}\left\langle T_{\sigma}^{\alpha}\left(\square_{Q, \mathcal{D}}^{\sigma, \mathbf{b}} f\right), \square_{R, \mathcal{G}}^{\omega, \mathbf{b}^{*}} g\right\rangle \\
& \leq \sum_{Q \in \mathcal{D}}\left|\left\langle T_{\sigma}^{\alpha}\left(\square_{Q, \mathcal{D}}^{\sigma, \mathbf{b}} f\right), \sum_{\substack{R \in \mathcal{G}_{\text {bad, } \ell(Q)}^{\mathcal{D}}: \mathbf{r} \leq \kappa(Q, R)<\kappa(R) \\
d(R, Q) \leq 2 \ell(R)^{\ell} \ell(Q)^{1-\varepsilon}}} \varepsilon_{Q, R} \square_{R, \mathcal{G}}^{\omega, \mathbf{b}^{*}} g\right\rangle\right| \\
& \leq \mathfrak{N}_{T^{\alpha}} \sum_{Q \in \mathcal{D}}\left\|\square_{Q, \mathcal{D}}^{\sigma, \mathbf{b}} f\right\|_{L^{2}(\sigma)}\left\|\sum_{\substack{R \in \mathcal{G}_{\text {bad }, \ell(Q)}^{\mathcal{D}}: \mathbf{r} \leq \kappa(Q, R)<\kappa(R) \\
d(R, Q) \leq 2 \ell(R)^{\varepsilon} \ell(Q)^{1-\varepsilon}}} \varepsilon_{Q, R} \square_{R, \mathcal{G}}^{\omega, \mathbf{b}^{*}} g\right\| \|_{L^{2}(\omega)} \\
& \leq \mathfrak{N}_{T^{\alpha}} \sum_{Q \in \mathcal{D}}\left\|\square_{Q, \mathcal{D}}^{\sigma, \mathbf{b}} f\right\|_{L^{2}(\sigma)} \sum_{k=\mathbf{r}}^{\infty}\left\|\sum_{\substack{R \in \mathcal{G}_{\text {bad, } \ell(Q)}^{\mathcal{D}}: k=\kappa(Q, R)<\kappa(R) \\
d(R, Q) \leq 2 \ell(R)^{\varepsilon} \ell(Q)^{1-\varepsilon}}} \varepsilon_{Q, R} \square_{R, \mathcal{G}}^{\omega, \mathbf{b}^{*}} g\right\|,
\end{aligned}
$$

by Minkowski's inequality, and we continue with

$$
\begin{aligned}
& \leq 2 \mathfrak{N}_{T^{\alpha}} \sum_{k=\mathbf{r}}^{\infty}\left(\sum_{Q \in \mathcal{D}}\left\|\square_{Q, \mathcal{D}}^{\sigma, \mathbf{b}} f\right\|_{L^{2}(\sigma)}^{2}\right)^{\frac{1}{2}}\left(\sum_{\substack{Q \in \mathcal{D} \\
R \in \mathcal{G}_{\text {bad }, \ell(Q)}^{\mathcal{D}}: k=\kappa(Q, R)<\kappa(R) \\
d(R, Q) \leq 2 \ell(R)^{\varepsilon} \ell(Q)^{1-\varepsilon}}}\left(\left\|\square_{R, \mathcal{G}}^{\omega, \mathbf{b}^{*} g}\right\|_{L^{2}(\omega)}^{2}+\left\|\nabla_{R, \mathcal{G}}^{\omega} g\right\|_{L^{2}(\omega)}^{2}\right)\right)^{\frac{1}{2}} \\
& \lesssim \mathfrak{N}_{T^{\alpha}}\|f\|_{L^{2}(\sigma)} \sum_{k=\mathbf{r}}^{\infty}\left(\sum_{R \in \mathcal{G}_{\text {bad, } 2^{\mathcal{D}} \ell(R)}}\left(\left\|\square_{R, \mathcal{G}}^{\omega, \mathbf{b}^{*}} g\right\|_{L^{2}(\omega)}^{2}+\left\|\nabla_{R, \mathcal{G}}^{\omega} g\right\|_{L^{2}(\omega)}^{2}\right)\right)^{\frac{1}{2}}
\end{aligned}
$$

where $\nabla_{R, \mathcal{G}}^{\omega}$ denotes the 'broken' Carleson averaging operator in (9.6) that depends on the grid $\mathcal{G}$, and

(1) the penultimate inequality uses Cauchy-Schwarz in $Q$ and the weak upper Riesz inequalities (3.6) for $\sum_{R \in \mathcal{G}_{\text {bad } \ell(Q)}^{\mathcal{D}}: k=\kappa(Q, R)<\kappa(R)} \varepsilon_{Q, R} \square_{R, \mathcal{G}}^{\omega, \mathbf{b}^{*}}$, once for the sum when $\varepsilon_{Q, R}=1$, and again for the sum $d(R, Q) \leq 2 \ell(R)^{\varepsilon} \ell(Q)^{1-\varepsilon}$

when $\varepsilon_{Q, R}=-1$. However, we note that since the sum in $R$ is pigeonholed by $k=\kappa(Q, R)$, the $R$ 's are pairwise disjoint intervals and the pseudoprojections $\square_{R, \mathcal{G}}^{\omega, \mathbf{b}^{*}} g$ are pairwise orthogonal. Thus we could instead apply Cauchy-Schwarz first in $R$, and then in $Q$ as was done in HyMa, but we must still apply weak upper Riesz inequalities as above.

(2) and the final inequality uses the frame inequality (3.5) together with (3.16), namely the fact that there are at most $C$ intervals $Q$ such that $\kappa(Q, R) \geq \mathbf{r}$ is fixed and $d(R, Q) \leq 2 \ell(R)^{\varepsilon} \ell(Q)^{1-\varepsilon}$. 
Now it is easy to verify that we have the same inequality for the pairs $\left(J^{*}, J\right)$ that were removed, and then we take grid expectations and use the probability estimate (3.13) to obtain for $\varepsilon^{\prime}=\frac{1}{2} \varepsilon$ that

$$
\begin{aligned}
& \boldsymbol{E}_{\Omega}^{\mathcal{D}}\left(\Theta_{2}^{\text {bad } \boxminus}(f, g)\right) \\
& \leq \boldsymbol{E}_{\Omega}^{\mathcal{D}} \mathfrak{N}_{T^{\alpha}}\|f\|_{L^{2}(\sigma)} \sum_{k=\mathbf{r}}^{\infty}\left(\sum_{R \in \mathcal{G}_{{\text {bad }, 2^{k} \ell(R)}^{\mathcal{D}}}}\left(\left\|\square_{R, \mathcal{G}}^{\omega, \mathbf{b}^{*}} g\right\|_{L^{2}(\omega)}^{2}+\left\|\nabla_{R, \mathcal{G}}^{\omega} g\right\|_{L^{2}(\omega)}^{2}\right)\right)^{\frac{1}{2}} \\
& \leq \mathfrak{N}_{T^{\alpha}}\|f\|_{L^{2}(\sigma)} \sum_{k=\mathbf{r}}^{\infty}\left(\boldsymbol{E}_{\Omega}^{\mathcal{D}} \sum_{R \in \mathcal{G}_{\mathrm{bad}, 2^{\mathcal{D}} \ell(R)}}\left(\left\|\square_{R, \mathcal{G}}^{\omega, \mathbf{b}^{*}} g\right\|_{L^{2}(\omega)}^{2}+\left\|\nabla_{R, \mathcal{G}}^{\omega} g\right\|_{L^{2}(\omega)}^{2}\right)\right)^{\frac{1}{2}} \\
& \lesssim 2^{-\frac{1}{2} \varepsilon^{\prime} \mathbf{r}} \mathfrak{N}_{T^{\alpha}}\|f\|_{L^{2}(\sigma)} \sum_{k=\mathbf{r}}^{\infty}\left(C_{1} 2^{-\varepsilon k}\|g\|_{L^{2}(\omega)}^{2}\right)^{\frac{1}{2}} \leq C_{\text {good }} 2^{-\frac{1}{2} \varepsilon \mathbf{r}} \mathfrak{N}_{T^{\alpha}}\|f\|_{L^{2}(\sigma)}\|g\|_{L^{2}(\omega)} .
\end{aligned}
$$

Clearly we can now fix $\mathbf{r}$ sufficiently large depending on $\varepsilon>0$ so that

$$
C_{\text {good }} 2^{-\frac{1}{2} \varepsilon \mathbf{r}}<\frac{1}{100}
$$

and then the final term above, namely $C_{\text {good }} 2^{-\frac{1}{2} \varepsilon \mathbf{r}} \mathfrak{N}_{T^{\alpha}}\|f\|_{L^{2}(\sigma)}\|g\|_{L^{2}(\omega)}$, can be absorbed at the end of the proof in Subsection 8. Note that (3.19) fixes our choice of the parameter $\mathbf{r}$ for any given $\varepsilon>0$. Later we will choose $0<\varepsilon<\frac{1}{2} \leq \frac{1}{2-\alpha}$. It is this type of weak goodness that we will exploit in the local forms $\mathrm{B}_{\Subset_{\mathbf{r}}}^{A}(f, g)$ treated below in Section 6 .

We are now left with the following 'good' form to control:

$$
\Theta_{2}^{\text {good }}(f, g)=\sum_{I \in \mathcal{D}} \sum_{\substack{J^{*} \varsubsetneqq I: \ell(J) \leq 2^{-\mathbf{r}} \ell(I) \\ d(J, I) \leq 2 \ell(J)^{\varepsilon} \ell(I)^{1-\varepsilon}}} \int\left(T_{\sigma}^{\alpha} \square_{I}^{\sigma, \mathbf{b}} f\right) \square_{J}^{\omega, \mathbf{b}^{*}} g d \omega .
$$

The first thing we observe regarding this form is that the intervals $J$ which arise in the sum for $\Theta_{2}^{\text {good }}(f, g)$ must lie entirely inside $I$ since $J \subset J^{w} \varsubsetneqq I$. Then in the remainder of the paper, we proceed to analyze

$$
\Theta_{2}^{\text {good }}(f, g)=\sum_{I \in \mathcal{D}} \sum_{J \neq I: \ell(J) \leq 2^{-\mathbf{r}} \ell(I)} \int\left(T_{\sigma}^{\alpha} \square_{I}^{\sigma, \mathbf{b}} f\right) \square_{J}^{\omega, \mathbf{b}^{*}} g d \omega,
$$

in the same way we analyzed the below term $\mathrm{B}_{\Subset_{\mathrm{r}}}(f, g)$ in SaShUr6; namely, by implementing the canonical corona splitting and the decomposition into paraproduct, neighbour and stopping forms, but now with an additional broken form. We have $(\kappa, \varepsilon)$-goodness available for all the intervals $J \in \mathcal{G}$ arising in the form $\Theta_{2}^{\text {good }}(f, g)$, and moreover, the intervals $I \in \mathcal{D}$ arising in the form $\Theta_{2}^{\text {good }}(f, g)$ for a fixed $J$ are tree-connected, so that telescoping identities hold for these intervals $I$. This will prove decisive in the following three sections of the paper.

The forms $\Theta_{1}(f, g)$ and $\Theta_{3}(f, g)$ are analogous to the disjoint and nearby forms $\mathrm{B}_{\cap}(f, g)$ and $\mathrm{B} /(f, g)$ in SaShUr6] respectively. In the next two sections, we control the disjoint form $\Theta_{1}(f, g)$ in essentially the same way that the disjoint form $\mathrm{B}_{\cap}(f, g)$ was treated in [SaShUr6] and in earlier papers of many authors beginning with Nazarov, Treil and Volberg (see e.g. [Vol]), and we control the nearby form $\Theta_{3}(f, g)$ using the probabilistic surgery of Hytönen and Martikainen building on that of NTV, together with a new deterministic surgery involving the energy condition and the original testing functions. But first we recall, in the following subsection, the characterization of boundedness of one-dimensional forms supported on disjoint intervals Hyt2.

3.2. A characterization of bilinear forms supported on disjoint intervals . Matters here in the one-dimensional setting are greatly simplified by a generalization to fractional integrals of Hytönen's characterization of the restricted bilinear inequality,

$$
\left|\int_{\mathbb{R} \backslash I}\left(\int_{I} \frac{f(y)}{|x-y|} d \sigma(y)\right) g(x) d \omega(x)\right| \lesssim \mathfrak{D}\|f\|_{L^{2}(\sigma)}\|g\|_{L^{2}(\omega)},
$$


for all intervals $I$, in terms of the Muckenhoupt conditions, namely

$$
\mathfrak{D} \approx \sqrt{\mathcal{A}_{2}}+\sqrt{\mathcal{A}_{2}^{*}}
$$

where $\mathfrak{D}$ is the best constant in (3.21). In $\mathrm{HyMa}$ this inequality was proved for complementary half-lines, where it was pointed out that the passage to an interval and its complement is then routine.

We claim that Hytönen's characterization extends immediately to fractional integrals on the line with the same proof. Namely, we have,

$$
\begin{aligned}
\left|\int_{(-\infty, a)}\left(\int_{(a, \infty)} \frac{f(y)}{|x-y|^{1-\alpha}} d \sigma(y)\right) g(x) d \omega(x)\right| & \lesssim\left(\sqrt{\mathcal{A}_{2}^{\alpha}}+\sqrt{\mathcal{A}_{2}^{\alpha, *}}\right)\|f\|_{L^{2}(\sigma)}\|g\|_{L^{2}(\omega)}, \\
\left|\int_{\mathbb{R} \backslash I}\left(\int_{I} \frac{f(y)}{|x-y|^{1-\alpha}} d \sigma(y)\right) g(x) d \omega(x)\right| & \lesssim\left(\sqrt{\mathcal{A}_{2}^{\alpha}}+\sqrt{\mathcal{A}_{2}^{\alpha, *}}\right)\|f\|_{L^{2}(\sigma)}\|g\|_{L^{2}(\omega)},
\end{aligned}
$$

and that $\sqrt{\mathcal{A}_{2}^{\alpha}}+\sqrt{\mathcal{A}_{2}^{\alpha, *}} \approx \mathfrak{D}^{\alpha}$ where $\mathfrak{D}^{\alpha}$ is the best constant in the inequality above (a limiting argument shows that we may take one of the half-lines to be closed in (3.22) ). First, the proof that $\sqrt{\mathcal{A}_{2}^{\alpha}}+\sqrt{\mathcal{A}_{2}^{\alpha, *}} \lesssim \mathfrak{D}^{\alpha}$ is the standard proof of necessity of the one-tailed Muckenhoupt conditions. In the other direction, we use the general two weight Hardy inequality of Muckenhoupt as presented in [Hyt2, Theorem 3.3], see also LaSaUr2]: if $\sigma$ and $\omega$ are locally finite positive Borel measures on the interval $(0, \infty)$, then

$$
\int_{0}^{\infty}\left(\int_{(0, x]} f d \sigma\right)^{2} d \omega(x) \leq C \int_{0}^{\infty} f(y)^{2} d \sigma(y)
$$

holds for all $f \in L^{2}(\sigma)$ if and only if

$$
A \equiv \sup _{t>0}\left(\int_{(0, t]} d \sigma\right)\left(\int_{[t, \infty)} d \omega\right)<\infty .
$$

Moreover, if $C$ is the best constant above, then

$$
A \leq C \leq 4 A
$$

We easily obtain the following characterization of an intermediate inequality:

$$
\int_{0}^{\infty} \int_{0}^{\infty} \frac{f(y) g(x)}{(x+y)^{1-\alpha}} d \sigma(y) d \omega(x) \leq C\|f\|_{L^{2}(\sigma)}\|g\|_{L^{2}(\omega)}
$$

if and only if

$$
A \equiv \sup _{t>0} \sqrt{\left(\int_{(0, t]} d \sigma\right)\left(\int_{[t, \infty)} \frac{d \omega(x)}{x^{2-2 \alpha}}\right)}+\sup _{t>0} \sqrt{\left(\int_{(0, t]} d \omega\right)\left(\int_{[t, \infty)} \frac{d \sigma(y)}{y^{2-2 \alpha}}\right)}<\infty,
$$

and moreover the best constant $C$ satisfies $\frac{1}{4} A \leq C \leq 2 A$. To see this we simply use the estimates

$$
\frac{1}{2} \max \left\{\frac{1}{x^{1-\alpha}} \mathbf{1}_{(0, x]}(y), \frac{1}{y^{1-\alpha}} \mathbf{1}_{(0, y]}(x)\right\} \leq \frac{1}{(x+y)^{1-\alpha}} \leq \frac{1}{x^{1-\alpha}} \mathbf{1}_{(0, x]}(y)+\frac{1}{y^{1-\alpha}} \mathbf{1}_{(0, y]}(x),
$$

together with Hardy's inequality. From this and duality, we immediately obtain (3.22).

3.2.1. Control of triple testing and triple energy. We also define the triple $\mathbf{b}$-testing conditions for $T^{\alpha}$ and triple $\mathbf{b}^{*}$-testing conditions for the dual $T^{\alpha, *}$ given by

$$
\begin{aligned}
\int_{3 Q}\left|T_{\sigma}^{\alpha} b_{Q}\right|^{2} d \omega & \leq\left(3 \mathfrak{T}_{T^{\alpha}}^{\mathbf{b}}\right)^{2}|Q|_{\sigma}, \quad \text { for all intervals } Q \\
\int_{3 Q}\left|T_{\omega}^{\alpha, *} b_{Q}^{*}\right|^{2} d \sigma & \leq\left(3 \mathfrak{T}_{T^{\alpha}}^{\mathbf{b}^{*}, *}\right)^{2}|Q|_{\omega}, \quad \text { for all intervals } Q
\end{aligned}
$$


as well as the full $\mathbf{b}$-testing conditions for $T^{\alpha}$ and full $\mathbf{b}^{*}$-testing conditions for the dual $T^{\alpha, *}$ given by

$$
\begin{aligned}
\int_{\mathbb{R}}\left|T_{\sigma}^{\alpha} b_{Q}\right|^{2} d \omega & \leq\left(\mathfrak{F} \mathfrak{T}_{T^{\alpha}}^{\mathbf{b}}\right)^{2}|Q|_{\sigma}, \quad \text { for all intervals } Q, \\
\int_{\mathbb{R}}\left|T_{\omega}^{\alpha, *} b_{Q}^{*}\right|^{2} d \sigma & \leq\left(\mathfrak{F T}_{T^{\alpha}}^{\mathbf{b}^{*}, *}\right)^{2}|Q|_{\omega}, \quad \text { for all intervals } Q .
\end{aligned}
$$

Note that the full testing conditions are implied by the triple testing conditions and the Muckenhoupt conditions (e.g. use the above characterization on complementary half-lines),

$$
\mathfrak{F} \mathfrak{T}_{T^{\alpha}}^{\mathbf{b}} \lesssim 3 \mathfrak{T}_{T^{\alpha}}^{\mathbf{b}}+\sqrt{\mathcal{A}_{2}^{\alpha}} \text { and } \mathfrak{F} \mathfrak{T}_{T^{\alpha, *}}^{\mathbf{b}^{*}} \lesssim 3 \mathfrak{T}_{T^{\alpha, *}}^{\mathbf{b}^{*}}+\sqrt{\mathcal{A}_{2}^{\alpha, *}} .
$$

Since dimension $n=1$, the full testing conditions are controlled by testing and Muckenhoupt, as we now show. Indeed, if we now set $f=b_{I}$ in the second line of (3.22), and take the supremum over all $g \in L^{2}(\omega)$ with $\|g\|_{L^{2}(\omega)}=1$, we obtain

$$
\begin{aligned}
\sqrt{\int_{\mathbb{R} \backslash I}\left(\int_{I} \frac{f(y)}{|x-y|^{1-\alpha}} d \sigma(y)\right)^{2} d \omega(x)} & \lesssim\left(\sqrt{\mathcal{A}_{2}^{\alpha}}+\sqrt{\mathcal{A}_{2}^{\alpha, *}}\right)\left\|b_{I}\right\|_{L^{2}(\sigma)} \\
& \lesssim\left(\sqrt{\mathcal{A}_{2}^{\alpha}}+\sqrt{\mathcal{A}_{2}^{\alpha, *}}\right) \sqrt{|I|_{\sigma}}
\end{aligned}
$$

which gives

$$
\begin{aligned}
\int_{\mathbb{R}}\left|T_{\sigma}^{\alpha} b_{I}\right|^{2} d \omega(x) & =\int_{I}\left|T_{\sigma}^{\alpha} b_{I}\right|^{2} d \omega(x)+\int_{\mathbb{R} \backslash I}\left|T_{\sigma}^{\alpha} b_{I}\right|^{2} d \omega(x) \\
& \lesssim\left(\mathfrak{T}_{T^{\alpha}}^{\mathbf{b}}\right)^{2}|I|_{\sigma}+\int_{\mathbb{R} \backslash I}\left(\int_{I} \frac{f(y)}{|x-y|^{1-\alpha}} d \sigma(y)\right)^{2} d \omega(x) \\
& \lesssim\left\{\left(\mathfrak{T}_{T^{\alpha}}^{\mathbf{b}}\right)^{2}+\mathcal{A}_{2}^{\alpha}+\mathcal{A}_{2}^{\alpha, *}\right\}|I|_{\sigma} .
\end{aligned}
$$

Thus we have obtained control of full $\mathbf{b}$-testing by just $\mathbf{b}$-testing and the Muckenhoupt conditions in dimension $n=1$ :

$$
\mathfrak{F}_{T^{\alpha}}^{\mathbf{b}} \lesssim \mathfrak{T}_{T^{\alpha}}^{\mathbf{b}}+\sqrt{\mathcal{A}_{2}^{\alpha}}+\sqrt{\mathcal{A}_{2}^{\alpha, *}} \text { and } \mathfrak{F T}_{T^{\alpha, *}}^{\mathbf{b}^{*}} \lesssim \mathfrak{T}_{T^{\alpha, *}}^{\mathbf{b}^{*}}+\sqrt{\mathcal{A}_{2}^{\alpha}}+\sqrt{\mathcal{A}_{2}^{\alpha, *}}
$$

Now we turn to the analogous notion of triple energy conditions defined in analogy with the triple testing condtions. Namely, the sum over the intervals $I_{r}$ in the energy condition in Definition 6 is permitted to extend to the triple $3 I$ of the interval $I$, but with the additional proviso that the distance of $I_{r}$ from the boundary of $I$ is at least a positive multiple $\delta$ (the exact value of which is immaterial) of the side length of $I_{r}$.

Definition 45. Let $0 \leq \alpha<1$ and $0<\delta \leq \frac{1}{2}$. Suppose $\sigma$ and $\omega$ are locally finite positive Borel measures on $\mathbb{R}$. Then the triple energy constant $\mathcal{E}_{2}^{\alpha, \text { triple }}$ is defined by

$$
\left(\mathcal{E}_{2}^{\alpha, \text { triple }}\right)^{2} \equiv \sup _{\substack{3 I=\dot{3} I_{r} \\ d\left(I_{r}, \partial I\right) \geq \delta \ell\left(I_{r}\right) \text { when } I_{r} \cap I^{c}=\emptyset}} \frac{1}{|I|_{\sigma}} \sum_{r=1}^{\infty}\left(\frac{\mathrm{P}^{\alpha}\left(I_{r}, \mathbf{1}_{I} \sigma\right)}{\left|I_{r}\right|}\right)^{2}\left\|x-m_{I_{r}}\right\|_{L^{2}\left(\mathbf{1}_{I_{r}} \omega\right)}^{2},
$$

where the supremum is taken over arbitrary decompositions of the triple $3 I$ of an interval I using a pairwise disjoint union of subintervals $I_{r}$ whose distance to the boundary of $I$ is at least a positive multiple of $\ell\left(I_{r}\right)$

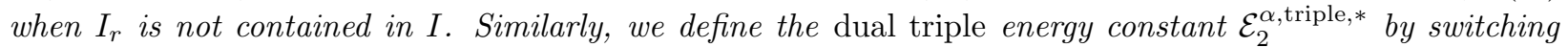
the roles of $\sigma$ and $\omega$ :

$$
\left(\mathcal{E}_{2}^{\alpha, \text { triple, } *}\right)^{2} \equiv \sup _{\substack{3 I=\dot{\cup} I_{r} \\ d\left(I_{r}, \partial I\right) \geq \delta \ell\left(I_{r}\right) \text { when } I_{r} \cap I^{c}=\emptyset}} \sum_{r=1}^{\infty}\left(\frac{\mathrm{P}^{\alpha}\left(I_{r}, \mathbf{1}_{I} \omega\right)}{\left|I_{r}\right|}\right)^{2}\left\|x-m_{I_{r}}\right\|_{L^{2}\left(\mathbf{1}_{I_{r}} \sigma\right)}^{2} .
$$

We now show that in dimension $n=1$, the triple energy conditions are controlled by the energy and Muckenhoupt conditions, namely

$$
\mathcal{E}_{2}^{\alpha, \text { triple }}+\mathcal{E}_{2}^{\alpha, \text { triple, } *} \lesssim \mathcal{E}_{2}^{\alpha}+\mathcal{E}_{2}^{\alpha, *}+\sqrt{\mathfrak{A}_{2}^{\alpha}}
$$


Indeed, assuming for convenience that $\delta=1$, we need only control by $C|I|_{\sigma}$ the sum,

$$
\sum_{r=1}^{\infty}\left(\frac{\mathrm{P}^{\alpha}\left(I_{r}, \mathbf{1}_{I} \sigma\right)}{\left|I_{r}\right|}\right)^{2}\left\|x-m_{I_{r}}\right\|_{L^{2}\left(\mathbf{1}_{I_{r}} \omega\right)}^{2}
$$

over adjacent intervals $J$ and $I$ of equal length where $\left\{I_{r}\right\}_{r=1}^{\infty}$ is a disjoint decomposition of $J=\dot{U} I_{r}$ with $d\left(I_{r}, \partial I\right) \geq \ell\left(I_{r}\right)$ for all $r \geq 1$. However, using reversal of energy for the standard gradient elliptic operator $T^{\alpha}$ with convolution kernel $K^{\alpha}(x)=\frac{x}{|x|^{2-\alpha}}$ (see e.g. [SaShUr10]), we have since $2 I_{r} \cap I=\emptyset$,

$$
\begin{aligned}
& \sum_{r=1}^{\infty}\left(\frac{\mathrm{P}^{\alpha}\left(I_{r}, \mathbf{1}_{I} \sigma\right)}{\left|I_{r}\right|}\right)^{2}\left\|x-m_{I_{r}}\right\|_{L^{2}\left(\mathbf{1}_{I_{r}} \omega\right)}^{2} \\
= & \frac{1}{2} \sum_{r=1}^{\infty}\left(\frac{\mathrm{P}^{\alpha}\left(I_{r}, \mathbf{1}_{I} \sigma\right)}{\left|I_{r}\right|}\right)^{2} \frac{1}{\left|I_{r}\right|_{\omega}} \int_{I_{r}} \int_{I_{r}}|x-z|^{2} d \omega(x) d \omega(z) \\
\lesssim & \sum_{r=1}^{\infty} \frac{1}{\left|I_{r}\right|_{\omega}} \int_{I_{r}} \int_{I_{r}}\left|T_{\sigma}^{\alpha} \mathbf{1}_{I}(x)-T_{\sigma}^{\alpha} \mathbf{1}_{I}(z)\right|^{2} d \omega(x) d \omega(z) \\
\lesssim & \sum_{r=1}^{\infty} \frac{1}{\left|I_{r}\right|_{\omega}} \int_{I_{r}} \int_{I_{r}}\left|T_{\sigma}^{\alpha} \mathbf{1}_{I}(x)\right|^{2} d \omega(x) d \omega(z)+\sum_{r=1}^{\infty} \frac{1}{\left|I_{r}\right|_{\omega}} \int_{I_{r}} \int_{I_{r}}\left|T_{\sigma}^{\alpha} \mathbf{1}_{I}(z)\right|^{2} d \omega(x) d \omega(z) \\
\lesssim & \sum_{r=1}^{\infty} \int_{I_{r}}\left|T_{\sigma}^{\alpha} \mathbf{1}_{I}\right|^{2} d \omega \lesssim \int_{J}\left|T_{\sigma}^{\alpha} \mathbf{1}_{I}\right|^{2} d \omega \lesssim \mathfrak{A}_{2}^{\alpha}|I|_{\sigma},
\end{aligned}
$$

which completes the proof of (3.26).

Finally, we note that a modification of this last argument also shows that the energy condition itself is controlled by the 1-testing condition and the Muckenhoupt condition. Indeed, as shown in [SaShUr11],

$$
\begin{aligned}
& \sum_{r=1}^{\infty}\left(\frac{\mathrm{P}^{\alpha}\left(I_{r}, \mathbf{1}_{I} \sigma\right)}{\left|I_{r}\right|}\right)^{2}\left\|x-m_{I_{r}}\right\|_{L^{2}\left(\mathbf{1}_{I_{r}} \omega\right)}^{2} \\
= & \frac{1}{2} \sum_{r=1}^{\infty}\left(\frac{\mathrm{P}^{\alpha}\left(I_{r},\left[\mathbf{1}_{I \backslash I_{r}}+\mathbf{1}_{I_{r}}\right] \sigma\right)}{\left|I_{r}\right|}\right)^{2} \frac{1}{\left|I_{r}\right|_{\omega}} \int_{I_{r}} \int_{I_{r}}|x-z|^{2} d \omega(x) d \omega(z) \\
\lesssim & \sum_{r=1}^{\infty}\left(\frac{\mathrm{P}^{\alpha}\left(I_{r}, \mathbf{1}_{I \backslash I_{r}} \sigma\right)}{\left|I_{r}\right|}\right)^{2} \frac{1}{\left|I_{r}\right|_{\omega}} \int_{I_{r}} \int_{I_{r}}|x-z|^{2} d \omega(x) d \omega(z)+\sum_{r=1}^{\infty} A_{2}^{\alpha, \text { energy }}\left|I_{r}\right|_{\sigma} \\
\lesssim & \sum_{r=1}^{\infty} \frac{1}{\left|I_{r}\right|_{\omega}} \int_{I_{r}} \int_{I_{r}}\left|T_{\sigma}^{\alpha} \mathbf{1}_{I \backslash I_{r}}(x)-T_{\sigma}^{\alpha} \mathbf{1}_{I \backslash I_{r}}(z)\right|^{2} d \omega(x) d \omega(z)+\mathfrak{A}_{2}^{\alpha}|I|_{\sigma} \\
\lesssim & \sum_{r=1}^{\infty} \frac{1}{\left|I_{r}\right|_{\omega}} \int_{I_{r}} \int_{I_{r}}\left(\left|T_{\sigma}^{\alpha} \mathbf{1}_{I \backslash I_{r}}(x)\right|^{2}+\left|T_{\sigma}^{\alpha} \mathbf{1}_{I \backslash I_{r}}(z)\right|^{2}\right) d \omega(x) d \omega(z)+\mathfrak{A}_{2}^{\alpha}|I|_{\sigma} \\
\lesssim & \sum_{r=1}^{\infty} \int_{I_{r}}\left|T_{\sigma}^{\alpha} \mathbf{1}_{I \backslash I_{r}}(x)\right|^{2} d \omega(x)+\mathfrak{A}_{2}^{\alpha}|I|_{\sigma},
\end{aligned}
$$

and now we 'plug the hole' to continue with

$$
\begin{aligned}
\sum_{r=1}^{\infty} \int_{I_{r}}\left|T_{\sigma}^{\alpha} \mathbf{1}_{I \backslash I_{r}}(x)\right|^{2} d \omega(x) & \lesssim \sum_{r=1}^{\infty} \int_{I_{r}}\left|T_{\sigma}^{\alpha} \mathbf{1}_{I}(x)\right|^{2} d \omega(x)+\sum_{r=1}^{\infty} \int_{I_{r}}\left|T_{\sigma}^{\alpha} \mathbf{1}_{I_{r}}(x)\right|^{2} d \omega(x) \\
& \lesssim \int_{I}\left|T_{\sigma}^{\alpha} \mathbf{1}_{I}\right|^{2} d \omega+\left(\mathfrak{T}_{T^{\alpha}}\right)^{2} \sum_{r=1}^{\infty}\left|I_{r}\right|_{\sigma} \lesssim\left(\mathfrak{T}_{T^{\alpha}}\right)^{2}|I|_{\sigma} .
\end{aligned}
$$

Altogether this gives

$$
\mathcal{E}_{2}^{\alpha}+\mathcal{E}_{2}^{\alpha, *} \lesssim \mathfrak{T}_{T^{\alpha}}+\mathfrak{T}_{T^{\alpha}}^{*}+\mathfrak{A}_{2}^{\alpha}
$$




\section{DisJOINT FORM}

Here we control the disjoint form $\Theta_{1}(f, g)$ by further decomposing it as follows:

$$
\begin{aligned}
& (4.1) \Theta_{1}(f, g)=\sum_{I \in \mathcal{D}} \sum_{\substack{J \in \mathcal{G}: \ell(J) \leq \ell(I) \\
d(J, I)>2 \ell(J)^{\varepsilon} \ell(I)^{1-\varepsilon}}} \int\left(T_{\sigma} \square_{I}^{\sigma, \mathbf{b}} f\right) \square_{J}^{\omega, \mathbf{b}^{*}} g d \omega \\
& =\sum_{I \in \mathcal{D}}\left\{\sum_{\substack{J \in \mathcal{G}: \ell(J) \leq \ell(I) \\
d(J, I)>\max \left(\ell(I), 2 \ell(J)^{\varepsilon} \ell(I)^{1-\varepsilon}\right)}}+\sum_{\substack{J \in \mathcal{G}: \ell(J) \leq \ell(I) \\
\ell(I) \geq d(J, I)>2 \ell(J)^{\varepsilon} \ell(I)^{1-\varepsilon}}}\right\} \int\left(T_{\sigma} \square_{I}^{\sigma, \mathbf{b}} f\right) \square_{J}^{\omega, \mathbf{b}^{*}} g d \omega \\
& \equiv \Theta_{1}^{\text {long }}(f, g)+\Theta_{1}^{\text {short }}(f, g),
\end{aligned}
$$

where $\Theta_{1}^{\text {long }}(f, g)$ is a 'long range' form in which $J$ is far from $I$, and where $\Theta_{1}^{\text {short }}(f, g)$ is a short range form. It should be noted that weak goodness plays no role in treating the disjoint form.

\subsection{Long range form.}

Lemma 46. We have

$$
\sum_{I \in \mathcal{D}} \sum_{\substack{J \in \mathcal{G}: \ell(J) \leq \ell(I) \\ d(J, I)>\ell(I)}}\left|\int\left(T_{\sigma} \square_{I}^{\sigma, \mathbf{b}} f\right) \square_{J}^{\omega, \mathbf{b}^{*}} g d \omega\right| \lesssim \sqrt{A_{2}^{\alpha}}\|f\|_{L^{2}(\sigma)}\|g\|_{L^{2}(\omega)}
$$

Proof. Since $J$ and $I$ are separated by at least $\max \{\ell(J), \ell(I)\}$, we have the inequality

$$
\mathrm{P}^{\alpha}\left(J,\left|\square_{I}^{\sigma, \mathbf{b}} f\right| \sigma\right) \approx \int_{I} \frac{\ell(J)}{\left|y-c_{J}\right|^{2-\alpha}}\left|\square_{I}^{\sigma, \mathbf{b}} f(y)\right| d \sigma(y) \lesssim\left\|\square_{I}^{\sigma, \mathbf{b}} f\right\|_{L^{2}(\sigma)} \frac{\ell(J) \sqrt{|I|_{\sigma}}}{d(I, J)^{2-\alpha}},
$$

since $\int_{I}\left|\square_{I}^{\sigma, \mathbf{b}} f(y)\right| d \sigma(y) \leq\left\|\square_{I}^{\sigma, \mathbf{b}} f\right\|_{L^{2}(\sigma)} \sqrt{|I|_{\sigma}}$. Thus if $A(f, g)$ denotes the left hand side of the conclusion of Lemma 46, we have

$$
\begin{aligned}
& A(f, g) \lesssim \sum_{I \in \mathcal{D} J: \ell(J) \leq \ell(I): d(I, J) \geq \ell(I)}\left\|\square_{I}^{\sigma, \mathbf{b}} f\right\|_{L^{2}(\sigma)}\left\|\square_{J}^{\omega, \mathbf{b}^{*}} g\right\|_{L^{2}(\omega)} \\
& \times \frac{\ell(J)}{d(I, J)^{2-\alpha}} \sqrt{|I|_{\sigma}} \sqrt{|J|_{\omega}} \\
& \equiv \sum_{(I, J) \in \mathcal{P}}\left\|\square_{I}^{\sigma, \mathbf{b}} f\right\|_{L^{2}(\sigma)}\left\|\square_{J}^{\omega, \mathbf{b}^{*}} g\right\|_{L^{2}(\omega)} A(I, J) ; \\
& \text { with } A(I, J) \equiv \frac{\ell(J)}{d(I, J)^{2-\alpha}} \sqrt{|I|_{\sigma}} \sqrt{|J|_{\omega}} ; \\
& \text { and } \mathcal{P} \equiv\{(I, J) \in \mathcal{D} \times \mathcal{G}: \ell(J) \leq \ell(I) \text { and } d(I, J) \geq \ell(I)\} .
\end{aligned}
$$

Now let $\mathcal{D}_{N} \equiv\left\{K \in \mathcal{D}: \ell(K)=2^{N}\right\}$ for each $N \in \mathbb{Z}$. For $N \in \mathbb{Z}$ and $s \in \mathbb{Z}_{+}$, we further decompose $A(f, g)$ by pigeonholing the sidelengths of $I$ and $J$ by $2^{N}$ and $2^{N-s}$ respectively:

$$
\begin{aligned}
A(f, g) & =\sum_{s=0}^{\infty} \sum_{N \in \mathbb{Z}} A_{N}^{s}(f, g) ; \\
A_{N}^{s}(f, g) & \equiv \sum_{(I, J) \in \mathcal{P}_{N}^{s}}\left\|\square_{I}^{\sigma, \mathbf{b}} f\right\|_{L^{2}(\sigma)}\left\|\square_{J}^{\omega, \mathbf{b}^{*}} g\right\|_{L^{2}(\omega)} A(I, J) \\
\text { where } \mathcal{P}_{N}^{s} & \equiv\left\{(I, J) \in \mathcal{D}_{N} \times \mathcal{G}_{N-s}: d(I, J) \geq \ell(I)\right\} .
\end{aligned}
$$

Now let $\mathrm{P}_{M}^{\sigma}=\sum_{K \in \mathcal{D}_{M}} \square_{K}^{\sigma, \mathbf{b}}$ denote the dual martingale pseudoprojection onto Span $\left\{\square_{K}^{\sigma, \mathbf{b}}\right\}_{K \in \mathcal{D}_{M}}$. Since the intervals $K$ in $\mathcal{D}_{M}$ are pairwise disjoint, the pseudoprojections $\square_{K}^{\sigma, \mathbf{b}}$ are mutually orthogonal, which 
means that $\left\|\mathrm{P}_{M}^{\sigma} f\right\|_{L^{2}(\sigma)}^{2}=\sum_{K \in \mathcal{D}_{M}}\left\|\square_{K}^{\sigma, \mathbf{b}} f\right\|_{L^{2}(\sigma)}^{2}$. We claim that

$$
\left|A_{N}^{s}(f, g)\right| \leq C 2^{-s} \sqrt{A_{2}^{\alpha}}\left\|\mathrm{P}_{N}^{\sigma} f\right\|_{L^{2}(\sigma)}^{\star}\left\|\mathrm{P}_{N-s}^{\omega} g\right\|_{L^{2}(\omega)}^{\star}, \quad \text { for } s \geq 0 \text { and } N \in \mathbb{Z} .
$$

With this proved, we can then obtain

$$
\begin{aligned}
A(f, g) & =\sum_{s=0}^{\infty} \sum_{N \in \mathbb{Z}} A_{N}^{s}(f, g) \leq C \sqrt{A_{2}^{\alpha}} \sum_{s=0}^{\infty} 2^{-s} \sum_{N \in \mathbb{Z}}\left\|\mathrm{P}_{N}^{\sigma} f\right\|_{L^{2}(\sigma)}^{\star}\left\|\mathrm{P}_{N-s}^{\omega} g\right\|_{L^{2}(\omega)}^{\star} \\
& \leq C \sqrt{A_{2}^{\alpha}} \sum_{s=0}^{\infty} 2^{-s}\left(\sum_{N \in \mathbb{Z}}\left\|\mathrm{P}_{N}^{\sigma} f\right\|_{L^{2}(\sigma)}^{\star 2}\right)^{\frac{1}{2}}\left(\sum_{N \in \mathbb{Z}}\left\|\mathrm{P}_{N-s}^{\omega} g\right\|_{L^{2}(\omega)}^{\star 2}\right)^{\frac{1}{2}} \\
& \leq C \sqrt{A_{2}^{\alpha}} \sum_{s=0}^{\infty} 2^{-s}\|f\|_{L^{2}(\sigma)}\|g\|_{L^{2}(\omega)}=C \sqrt{A_{2}^{\alpha}}\|f\|_{L^{2}(\sigma)}\|g\|_{L^{2}(\omega)}
\end{aligned}
$$

where in the last line we have used the lower frame inequality for $\square_{I}^{\sigma, \mathbf{b}}$ in Appendix A.

To prove (4.2), we pigeonhole the distance between $I$ and $J$ :

$$
\begin{aligned}
A_{N}^{s}(f, g) & =\sum_{\ell=0}^{\infty} A_{N, \ell}^{s}(f, g) ; \\
A_{N, \ell}^{s}(f, g) & \equiv \sum_{(I, J) \in \mathcal{P}_{N, \ell}^{s}}\left\|\square_{I}^{\sigma, \mathbf{b}} f\right\|_{L^{2}(\sigma)}\left\|\square_{J}^{\omega, \mathbf{b}^{*}} g\right\|_{L^{2}(\omega)} A(I, J) \\
\text { where } \mathcal{P}_{N, \ell}^{s} & \equiv\left\{(I, J) \in \mathcal{D}_{N} \times \mathcal{G}_{N-s}: d(I, J) \approx 2^{N+\ell}\right\} .
\end{aligned}
$$

If we define $\mathcal{H}\left(A_{N, \ell}^{s}\right)$ to be the bilinear form on $\ell^{2} \times \ell^{2}$ with matrix $[A(I, J)]_{(I, J) \in \mathcal{P}_{N, \ell}^{s}}$, then it remains to show that the norm $\left\|\mathcal{H}\left(A_{N, \ell}^{s}\right)\right\|_{\ell^{2} \rightarrow \ell^{2}}$ of $\mathcal{H}\left(A_{N, \ell}^{s}\right)$ on the sequence space $\ell^{2}$ is bounded by $C 2^{-s-\ell} \sqrt{A_{2}^{\alpha}}$. In turn, this is equivalent to showing that the norm $\left\|\mathcal{H}\left(B_{N, \ell}^{s}\right)\right\|_{\ell^{2} \rightarrow \ell^{2}}$ of the bilinear form $\mathcal{H}\left(B_{N, \ell}^{s}\right) \equiv$ $\mathcal{H}\left(A_{N, \ell}^{s}\right)^{\operatorname{tr}} \mathcal{H}\left(A_{N, \ell}^{s}\right)$ on the sequence space $\ell^{2}$ is bounded by $C^{2} 2^{-2 s-2 \ell} \mathfrak{A}_{2}^{\alpha}$. Here $\mathcal{H}\left(B_{N, \ell}^{s}\right)$ is the quadratic form with matrix kernel $\left[B_{N, \ell}^{s}\left(J, J^{\prime}\right)\right]_{J, J^{\prime} \in \mathcal{D}_{N-s}}$ having entries:

$$
B_{N, \ell}^{s}\left(J, J^{\prime}\right) \equiv \sum_{I \in \mathcal{D}_{N}: d(I, J) \approx d\left(I, J^{\prime}\right) \approx 2^{N+\ell}} A(I, J) A\left(I, J^{\prime}\right), \quad \text { for } J, J^{\prime} \in \mathcal{G}_{N-s} .
$$

We are reduced to showing the bilinear form inequality,

$$
\left\|\mathcal{H}\left(B_{N, \ell}^{s}\right)\right\|_{\ell^{2} \rightarrow \ell^{2}} \leq C 2^{-2 s-2 \ell} A_{2}^{\alpha} \quad \text { for } s \geq 0, \ell \geq 0 \text { and } N \in \mathbb{Z} .
$$

We begin by computing $B_{N, \ell}^{s}\left(J, J^{\prime}\right)$ :

$$
\begin{aligned}
& B_{N, \ell}^{s}\left(J, J^{\prime}\right)=\sum_{\substack{I \in \mathcal{D}_{N} \\
d(I, J) \approx d\left(I, J^{\prime}\right) \approx 2^{N+\ell}}} \frac{\ell(J)}{d(I, J)^{2-\alpha}} \sqrt{|I|_{\sigma}} \sqrt{|J|_{\omega}} \frac{\ell\left(J^{\prime}\right)}{d\left(I, J^{\prime}\right)^{2-\alpha}} \sqrt{|I|_{\sigma}} \sqrt{\left|J^{\prime}\right|_{\omega}} \\
& =\left\{\sum_{\substack{I \in \mathcal{D}_{N} \\
d(I, J) \approx d\left(I, J^{\prime}\right) \approx 2^{N+\ell}}}|I|_{\sigma} \frac{1}{d(I, J)^{2-\alpha} d\left(I, J^{\prime}\right)^{2-\alpha}}\right\} \ell(J) \ell\left(J^{\prime}\right) \sqrt{|J|_{\omega}} \sqrt{\left|J^{\prime}\right|_{\omega}} .
\end{aligned}
$$

Now we show that

$$
\left\|\mathcal{H}\left(B_{N, \ell}^{s}\right)\right\|_{\ell^{2} \rightarrow \ell^{2}}=\left\|B_{N, \ell}^{s}\right\|_{\ell^{2} \rightarrow \ell^{2}} \lesssim 2^{-2 s-2 \ell} A_{2}^{\alpha},
$$

by applying the proof of Schur's lemma. Fix $\ell \geq 0$ and $s \geq 0$. Choose the Schur function $\beta(K)=\frac{1}{\sqrt{|K|_{\omega}}}$. Fix $J \in \mathcal{D}_{N-s}$. We now group those $I \in \mathcal{D}_{N}$ with $d(I, J) \approx 2^{N+\ell}$ into finitely many groups $G_{1}, \ldots G_{C}$ for 
which the union of the $I$ in each group is contained in an interval of side length roughly $\frac{1}{100} 2^{N+\ell}$, and we set $I_{k}^{*} \equiv \bigcup_{I \in G_{k}} I$ for $1 \leq k \leq C$. We then have

$$
\begin{aligned}
& \sum_{J^{\prime} \in \mathcal{G}_{N-s}} \frac{\beta(J)}{\beta\left(J^{\prime}\right)} B_{N, \ell}^{s}\left(J, J^{\prime}\right) \\
= & \sum_{\substack{J^{\prime} \in \mathcal{G}_{N-s} \\
d\left(J^{\prime}, J\right) \leq \frac{1}{100} 2^{N+\ell+2}}} \frac{\beta(J)}{\beta\left(J^{\prime}\right)} B_{N, \ell}^{s}\left(J, J^{\prime}\right)+\sum_{\substack{J^{\prime} \in \mathcal{G}_{N-s}-\\
d\left(J^{\prime}, J\right)>\frac{1}{100} 2^{N+\ell+2}}} \frac{\beta(J)}{\beta\left(J^{\prime}\right)} B_{N, \ell}^{s}\left(J, J^{\prime}\right) \\
= & A+B,
\end{aligned}
$$

where

$$
\begin{aligned}
A \lesssim & \sum_{\substack{J^{\prime} \in \mathcal{G}_{N-s} \\
d\left(J, J^{\prime}\right) \leq \frac{1}{100} 2^{N+\ell+2}}}\left\{\sum_{\substack{I \in \mathcal{D}_{N} \\
d(I, J) \approx 2^{N+\ell}}}|I|_{\sigma}\right\} \frac{2^{2(N-s)}}{2^{2(\ell+N)(2-\alpha)}}\left|J^{\prime}\right|_{\omega} \\
= & \sum_{\substack{J^{\prime} \in \mathcal{G}_{N-s} \\
d\left(J, J^{\prime}\right) \leq \frac{1}{100} 2^{N+\ell+2}}}\left\{\sum_{k=1}^{C}\left|I_{k}^{*}\right|_{\sigma}\right\} \frac{2^{2(N-s)}}{2^{2(\ell+N)(2-\alpha)}}\left|J^{\prime}\right|_{\omega}=\frac{2^{2(N-s)}}{2^{2(\ell+N)(2-\alpha)}} \sum_{k=1}^{C} \sum_{\substack{\left.J^{\prime} \in \mathcal{G}_{N-s}-J^{\prime}\right) \leq \frac{1}{100} 2^{N+\ell+2} \\
d\left(J, J^{\prime}\right.}}\left|I_{k}^{*}\right|_{\sigma}\left|J^{\prime}\right|_{\omega} \\
\lesssim & 2^{-2 s-2 \ell} \sum_{k=1}^{C} \frac{\left|I_{k}^{*}\right|_{\sigma}}{2^{(\ell+N)(1-\alpha)}} \frac{\left|\frac{1}{100} 2^{s+\ell+4} J\right|_{\omega}}{2^{(\ell+N)(1-\alpha)}} \lesssim 2^{-2 s-2 \ell} A_{2}^{\alpha},
\end{aligned}
$$

since the intervals $I_{k}^{*}$ and $\frac{1}{100} 2^{s+\ell+4} J$ are well separated.

Define

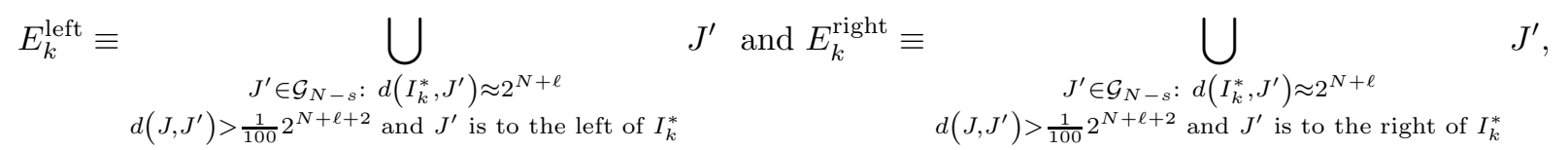

and let $Q_{k}^{\text {left }}$ (respectively $Q_{k}^{\text {right }}$ ) be the smallest interval containing $E_{k}^{\text {left }}$ (respectively $E_{k}^{\text {right }}$ ). Then we have

$$
\begin{aligned}
& B \lesssim \sum_{\substack{J^{\prime} \in \mathcal{G}_{N-s} \\
d\left(J, J^{\prime}\right)>\frac{1}{100} 2^{N+\ell+2}}}\left\{\sum_{\substack{I \in \mathcal{D}_{N} \\
\left(I, J^{\prime}\right) \approx d(I, J) \approx 2^{N+\ell}}}|I|_{\sigma}\right\} \frac{2^{2(N-s)}}{2^{2(\ell+N)(2-\alpha)}}\left|J^{\prime}\right|_{\omega} \\
& \lesssim \sum_{\substack{J^{\prime} \in \mathcal{G}_{N-s}-\ell \\
d\left(J, J^{\prime}\right)>\frac{1}{100} 2^{N+\ell+2}}}\left\{\sum_{k: d\left(I_{k}^{*}, J^{\prime}\right) \approx 2^{N+\ell}}\left|I_{k}^{*}\right|_{\sigma}\right\} \frac{2^{2(N-s)}}{2^{2(\ell+N)(2-\alpha)}}\left|J^{\prime}\right|_{\omega} \\
& \lesssim \frac{2^{2(N-s)}}{2^{2(\ell+N)(2-\alpha)}} \sum_{k=1}^{C}\left|I_{k}^{*}\right|_{\sigma}\left|E_{k}^{\text {left }} \cup E_{k}^{\text {right }}\right|_{\omega} \\
& \lesssim 2^{-2 s-2 \ell} \sum_{k=1}^{C} \frac{\left|I_{k}^{*}\right|_{\sigma}}{2^{(\ell+N)(1-\alpha)}} \frac{\left|Q_{k}^{\text {left }}\right|_{\omega}+\left|Q_{k}^{\text {right }}\right|_{\omega}}{2^{(\ell+N)(1-\alpha)}} \lesssim 2^{-2 s-2 \ell^{2}} \mathfrak{A}_{2}^{\alpha},
\end{aligned}
$$

since the interval $I_{k}^{*}$ is well separated from each of the intervals $Q_{k}^{\text {left }}$ and $Q_{k}^{\text {right }}$. 
Thus we can now apply Schur's argument with $\sum_{J}\left(a_{J}\right)^{2}=\sum_{J^{\prime}}\left(b_{J^{\prime}}\right)^{2}=1$ to obtain

$$
\begin{aligned}
& \sum_{J, J^{\prime} \in \mathcal{G}_{N-s}} a_{J} b_{J^{\prime}} B_{N, \ell}^{s}\left(J, J^{\prime}\right) \\
= & \sum_{J, J^{\prime} \in \mathcal{G}_{N-s}} a_{J} \beta(J) b_{J^{\prime}} \beta\left(J^{\prime}\right) \frac{B_{N, \ell}^{s}\left(J, J^{\prime}\right)}{\beta(J) \beta\left(J^{\prime}\right)} \\
\leq & \sum_{J}\left(a_{J} \beta(J)\right)^{2} \sum_{J^{\prime}} \frac{B_{N, \ell}^{s}\left(J, J^{\prime}\right)}{\beta(J) \beta\left(J^{\prime}\right)}+\sum_{J^{\prime}}\left(b_{J^{\prime}} \beta\left(J^{\prime}\right)\right)^{2} \sum_{J} \frac{B_{N, \ell}^{s}\left(J, J^{\prime}\right)}{\beta(J) \beta\left(J^{\prime}\right)} \\
= & \sum_{J}\left(a_{J}\right)^{2}\left\{\sum_{J^{\prime}} \frac{\beta(J)}{\beta\left(J^{\prime}\right)} B_{N, \ell}^{s}\left(J, J^{\prime}\right)\right\}+\sum_{J^{\prime}}\left(b_{J^{\prime}}\right)^{2}\left\{\sum_{J} \frac{\beta\left(J^{\prime}\right)}{\beta(J)} B_{N, \ell}^{s}\left(J, J^{\prime}\right)\right\} \\
\lesssim & 2^{-2 s-2 \ell} A_{2}^{\alpha}\left(\sum_{J}\left(a_{J}\right)^{2}+\sum_{J^{\prime}}\left(b_{J^{\prime}}\right)^{2}\right)=2^{1-2 s-2 \ell} A_{2}^{\alpha} .
\end{aligned}
$$

This completes the proof of (4.3). We can now sum in $\ell$ to get (4.2) and we are done. This completes our proof of the long range estimate

$$
\mathcal{A}(f, g) \lesssim \sqrt{A_{2}^{\alpha}}\|f\|_{L^{2}(\sigma)}\|g\|_{L^{2}(\omega)}
$$

4.2. Short range form. The form $\Theta_{1}^{\text {short }}(f, g)$ is handled by the following lemma.

Lemma 47. We have

$$
\sum_{I \in \mathcal{D}} \sum_{\substack{J \in \mathcal{G}: \ell(J) \leq \ell(I) \\ \ell(I) \geq d(J, I)>2 \ell(J)^{\varepsilon} \ell(I)^{1-\varepsilon}}}\left|\int\left(T_{\sigma} \square_{I}^{\sigma, \mathbf{b}} f\right) \square_{J}^{\omega, \mathbf{b}^{*}} g d \omega\right| \lesssim \sqrt{A_{2}^{\alpha}}\|f\|_{L^{2}(\sigma)}\|g\|_{L^{2}(\omega)}
$$

Proof. The pairs $(I, J)$ that occur in the sum above satisfy $J \subset 4 I \backslash I$ and so we consider

$$
\mathcal{P} \equiv\left\{(I, J) \in \mathcal{D} \times \mathcal{G}: \ell(J) \leq \ell(I), \ell(I) \geq d(J, I)>2 \ell(J)^{\varepsilon} \ell(I)^{1-\varepsilon}, J \subset 4 I \backslash I\right\} .
$$

For $(I, J) \in \mathcal{P}$, the 'pivotal' estimate from the Energy Lemma 33 gives

$$
\left|\left\langle T_{\sigma}^{\alpha}\left(\square_{I}^{\sigma, \mathbf{b}} f\right), \square_{J}^{\omega, \mathbf{b}^{*}} g\right\rangle_{\omega}\right| \lesssim\left\|\square_{J}^{\omega, \mathbf{b}^{*}} g\right\|_{L^{2}(\omega)} \mathrm{P}^{\alpha}\left(J,\left|\square_{I}^{\sigma, \mathbf{b}} f\right| \sigma\right) \sqrt{|J|_{\omega}} .
$$

Now we pigeonhole the lengths of $I$ and $J$ and the distance between them by defining

$$
\mathcal{P}_{N, d}^{s} \equiv\left\{(I, J) \in \mathcal{P}: \ell(I)=2^{N}, \ell(J)=2^{N-s}, 2^{d-1} \leq d(I, J) \leq 2^{d}, J \subset 4 I \backslash I\right\} .
$$

Note that the closest an interval $J$ can come to $I$ is determined by:

$$
2^{d} \geq 2 \ell(I)^{1-\varepsilon} \ell(J)^{\varepsilon}=2^{1+N(1-\varepsilon)+(N-s) \varepsilon}=2^{1+N-\varepsilon s} ;
$$

which implies $N-\varepsilon s+1 \leq d \leq N$.

Thus we have

$$
\begin{gathered}
\sum_{(I, J) \in \mathcal{P}}\left|\left\langle T_{\sigma}^{\alpha}\left(\square_{I}^{\sigma, \mathbf{b}} f\right), \square_{J}^{\omega, \mathbf{b}^{*}} g\right\rangle_{\omega}\right| \lesssim \sum_{(I, J) \in \mathcal{P}}\left\|\square_{J}^{\omega, \mathbf{b}^{*}} g\right\|_{L^{2}(\omega)} \mathrm{P}^{\alpha}\left(J,\left|\square_{I}^{\sigma, \mathbf{b}} f\right| \sigma\right) \sqrt{|J|_{\omega}} \\
=\sum_{s=0}^{\infty} \sum_{N \in \mathbb{Z}} \sum_{d=N-\varepsilon s+1}^{N} \sum_{(I, J) \in \mathcal{P}_{N, d}^{s}}\left\|\square_{J}^{\omega, \mathbf{b}^{*}} g\right\|_{L^{2}(\omega)} \mathrm{P}^{\alpha}\left(J,\left|\square_{I}^{\sigma, \mathbf{b}} f\right| \sigma\right) \sqrt{|J|_{\omega}} .
\end{gathered}
$$

Now we use

$$
\begin{aligned}
\mathrm{P}^{\alpha}\left(J,\left|\square_{I}^{\sigma, \mathbf{b}} f\right| \sigma\right) & =\int_{I} \frac{\ell(J)}{\left(\ell(J)+\left|y-c_{J}\right|\right)^{2-\alpha}}\left|\square_{I}^{\sigma, \mathbf{b}} f(y)\right| d \sigma(y) \\
& \lesssim \frac{2^{N-s}}{2^{d(2-\alpha)}}\left\|\square_{I}^{\sigma, \mathbf{b}} f\right\|_{L^{2}(\sigma)} \sqrt{|I|_{\sigma}}
\end{aligned}
$$


and apply Cauchy-Schwarz in $J$ and use $J \subset 4 I \backslash I$ to get

$$
\begin{aligned}
& \sum_{(I, J) \in \mathcal{P}}\left|\left\langle T_{\sigma}^{\alpha}\left(\square_{I}^{\sigma, \mathbf{b}} f\right), \square_{J}^{\omega, \mathbf{b}^{*}} g\right\rangle_{\omega}\right| \\
& \lesssim \sum_{s=0}^{\infty} \sum_{N \in \mathbb{Z}} \sum_{d=N-\varepsilon s+1}^{N} \sum_{I \in \mathcal{D}_{N}} \frac{2^{N-s} 2^{N(1-\alpha)}}{2^{d(2-\alpha)}}\left\|\square_{I}^{\sigma, \mathbf{b}} f\right\|_{L^{2}(\sigma)} \frac{\sqrt{|I|_{\sigma}} \sqrt{|4 I \backslash I|_{\omega}}}{2^{N(1-\alpha)}} \\
& \times \sqrt{\sum_{\substack{J \in \mathcal{G}_{N-s} \\
J \subset 4 I I \text { and } d(I, J) \approx 2^{d}}}\left\|\square_{J}^{\omega, \mathbf{b}^{*}} g\right\|_{L^{2}(\omega)}^{2}} \\
& \lesssim \sum_{s=0}^{\infty} \sum_{N \in \mathbb{Z}} \frac{2^{N-s} 2^{N(1-\alpha)}}{2^{(N-\varepsilon s)(2-\alpha)}} \sqrt{A_{2}^{\alpha}} \sum_{I \in \mathcal{D}_{N}}\left\|\square_{I}^{\sigma, \mathbf{b}} f\right\|_{L^{2}(\sigma)} \sqrt[\substack{J \in \mathcal{G}_{N-s} \\
J \subset 4 I \backslash I}]{\left\|\square_{J}^{\omega, \mathbf{b}^{*}} g\right\|_{L^{2}(\omega)}^{2}} \\
& \lesssim \sum_{s=0}^{\infty} 2^{-s[1-\varepsilon(2-\alpha)]} \sqrt{A_{2}^{\alpha}}\|f\|_{L^{2}(\sigma)}\|g\|_{L^{2}(\omega)} \lesssim \sqrt{A_{2}^{\alpha}}\|f\|_{L^{2}(\sigma)}\|g\|_{L^{2}(\omega)},
\end{aligned}
$$

where in the third line above we have summed the geometric series in $d$, and in the last line $\frac{2^{N-s} 2^{N(1-\alpha)}}{2^{(N-\varepsilon s)(2-\alpha)}}=$ $2^{-s[1-\varepsilon(2-\alpha)]}$ followed by Cauchy-Schwarz in $I$ and $N$, using that we have bounded overlap in the quadruples of $I$ for $I \in \mathcal{D}_{N}$, and finally using the lower frame inequality for $\square_{I}^{\sigma, \mathbf{b}}$ from Appendix A. More precisely, if we define $f_{k} \equiv \Psi_{\mathcal{D}_{k}}^{\sigma, \mathbf{b}} f=\sum_{I \in \mathcal{D}_{k}} \square_{I}^{\sigma, \mathbf{b}} f$ and $g_{k} \equiv \Psi_{\mathcal{G}_{k}}^{\sigma, \mathbf{b}^{*}} g=\sum_{J \in \mathcal{G}_{k}} \square_{J}^{\omega, \mathbf{b}^{*}} g$, then we have the quasi-orthogonality inequality

$$
\begin{aligned}
\sum_{N \in \mathbb{Z}}\left\|f_{N}\right\|_{L^{2}(\sigma)}\left\|g_{N-s}\right\|_{L^{2}(\omega)} & \leq\left(\sum_{N \in \mathbb{Z}}\left\|f_{N}\right\|_{L^{2}(\sigma)}^{2}\right)^{\frac{1}{2}}\left(\sum_{N \in \mathbb{Z}}\left\|g_{N-s}\right\|_{L^{2}(\omega)}^{2}\right)^{\frac{1}{2}} \\
& \lesssim\|f\|_{L^{2}(\sigma)}\|g\|_{L^{2}(\omega)} .
\end{aligned}
$$

We have assumed that

$$
0<\varepsilon<\frac{1}{2-\alpha}
$$

in the calculations above in order that the sum in $s$ converges, and this completes the proof of Lemma 47

\section{NEARBY FORM}

We dominate the nearby form $\Theta_{3}(f, g)$ by

$$
\left|\Theta_{3}(f, g)\right| \leq \sum_{I \in \mathcal{D}} \sum_{\substack{J \in \mathcal{G}: 2^{-\mathbf{r}} \ell(I)<\ell(J) \leq \ell(I) \\ d(J, I) \leq 2 \ell(J)^{\varepsilon} \ell(I)^{1-\varepsilon}}}\left|\int\left(T_{\sigma}^{\alpha} \square_{I}^{\sigma, \mathbf{b}} f\right) \square_{J}^{\omega, \mathbf{b}^{*}} g d \omega\right|,
$$

and prove the following lemma that controls the expectation, over two independent grids, of the nearby form $\Theta_{3}(f, g)$. It should be noted that weak goodness plays no role in treating the nearby form.

Lemma 48. Suppose $T^{\alpha}$ is a standard fractional singular integral with $0 \leq \alpha<1$. Let $\theta \in(0,1)$ be sufficiently small depending only on $0 \leq \alpha<1$. Then there is a constant $C_{\theta}$ such that for $f \in L^{2}(\sigma)$ and $g \in L^{2}(\omega)$, and dual martingale differences $\square_{I}^{\sigma, \mathbf{b}}$ and $\square_{J}^{\omega, \mathbf{b}^{*}}$ with $\infty$-strongly accretive families of test functions $\mathbf{b}$ and $\mathbf{b}^{*}$, we have

$$
\begin{aligned}
& \boldsymbol{E}_{\Omega}^{\mathcal{D}} \boldsymbol{E}_{\Omega}^{\mathcal{G}} \sum_{I \in \mathcal{D}} \sum_{\substack{J \in \mathcal{G}: 2^{-\mathbf{r}} \ell(I)<\ell(J) \leq \ell(I) \\
d(J, I) \leq 2 \ell(J)^{\varepsilon} \ell(I)^{1}-\varepsilon}}\left|\left\langle T_{\sigma}^{\alpha}\left(\square_{I}^{\sigma, \mathbf{b}} f\right), \square_{J}^{\omega, \mathbf{b}^{*}} g\right\rangle_{\omega}\right| \\
& \lesssim\left(C_{\theta} \mathcal{N} \mathcal{T} \mathcal{V}_{\alpha}+\sqrt{\theta} \mathfrak{N}_{T^{\alpha}}\right)\|f\|_{L^{2}(\sigma)}\|g\|_{L^{2}(\omega)} .
\end{aligned}
$$


Proof: As usual, we continue to write the independent grids for $f$ and $g$ as $\mathcal{D}$ and $\mathcal{G}$ respectively. Write the dual martingale averages $\square_{I}^{\sigma, \mathbf{b}} f$ and $\square_{J}^{\omega, \mathbf{b}^{*}} g$ as linear combinations

$$
\begin{aligned}
& \square_{I}^{\sigma, \mathbf{b}} f=b_{I} \sum_{I^{\prime} \in \mathfrak{C}_{\text {natural }}(I)} \mathbf{1}_{I^{\prime}} E_{I^{\prime}}^{\sigma}\left(\widehat{\square}_{I}^{\sigma, \mathbf{b}} f\right)+\sum_{I^{\prime} \in \mathfrak{C}_{\text {broken }}(I)} b_{I^{\prime}} \mathbf{1}_{I^{\prime}} \widehat{\mathbb{F}}_{I^{\prime}}^{\sigma, b_{I^{\prime}}} f-b_{I} \sum_{I^{\prime} \in \mathfrak{C}_{\text {broken }}(I)} \mathbf{1}_{I^{\prime}} \widehat{\mathbb{F}}_{I}^{\sigma, b_{I}} f, \\
& \square_{J}^{\omega, \mathbf{b}^{*}} g=b_{J}^{*} \sum_{J^{\prime} \in \mathfrak{C}_{\text {natural }}(J)} \mathbf{1}_{J^{\prime}} E_{J^{\prime}}^{\omega}\left(\widehat{\square}_{J}^{\omega, \mathbf{b}^{*}} g\right)+\sum_{J^{\prime} \in \mathfrak{C}_{\text {broken }}(J)} b_{J^{\prime}}^{*} \mathbf{1}_{J^{\prime}} \widehat{\mathbb{F}}_{J^{\prime}}^{\omega, b_{J^{\prime}}^{*}} g-b_{J}^{*} \sum_{J^{\prime} \in \mathfrak{C}_{\text {broken }}(J)} \mathbf{1}_{J^{\prime}} \widehat{\mathbb{F}}_{J}^{\omega, b_{J}^{*}} g,
\end{aligned}
$$

of the appropriate function $b$ times the indicators of their children, denoted $I^{\prime}$ and $J^{\prime}$ respectively. We will regroup the terms as needed below.

Notation 49. On the natural child $I^{\prime}$, the expression $\widehat{\square}_{I}^{\sigma, \mathbf{b}} f=\frac{1}{b_{I}} \square_{I}^{\sigma, \mathbf{b}} f$ simply denotes the dual martingale average with $b_{I}$ removed, so that we need not assume $\left|b_{I}\right|$ is bounded below in order to make sense of $\frac{1}{b_{I}} \square_{I}^{\sigma, \mathbf{b}} f$. Similar comments apply to the expressions $\widehat{\mathbb{F}}_{I^{\prime}}^{\sigma, b_{I^{\prime}}} f=\frac{1}{b_{I^{\prime}}} \mathbb{F}_{I^{\prime}}^{\sigma, b_{I^{\prime}}} f$ and $\widehat{\mathbb{F}}_{I}^{\sigma, b_{I}} f=\frac{1}{b_{I}} \mathbb{F}_{I}^{\sigma, b_{I}} f$. On the other hand, we are assuming from Proposition 12 that the PLBP (2.2) holds, which shows that $\frac{1}{b_{I^{\prime}}}$ is actually a bounded function. This latter fact will be used shortly in (5.5) below.

Recall that the length of $J$ is at most the length of $I$, i.e. $\ell(J) \leq \ell(I)$. If $J$ and $I$ are separated, by which we mean here that $J \cap I=\emptyset$, then by (3.22) we have the satisfactory estimate

$$
\left|\left\langle T_{\sigma}^{\alpha}\left(\square_{I}^{\sigma, \mathbf{b}} f\right), \square_{J}^{\omega, \mathbf{b}^{*}} g\right\rangle_{\omega}\right| \lesssim \sqrt{\mathcal{A}_{2}^{\alpha, *}+\mathcal{A}_{2}^{\alpha}}\left\|\square_{I}^{\sigma, \mathbf{b}} f\right\|_{L^{2}(\sigma)}\left\|\square_{J}^{\omega, \mathbf{b}^{*}} g\right\|_{L^{2}(\omega)} .
$$

Suppose now that $J \cap I \neq \emptyset$. Using (9.26) we have

$$
\begin{aligned}
\left\langle T_{\sigma}^{\alpha}\left(\square_{I}^{\sigma, \mathbf{b}} f\right), \square_{J}^{\omega, \mathbf{b}^{*}} g\right\rangle_{\omega}= & \left\langle T_{\sigma}^{\alpha}\left(\square_{I}^{\sigma, b, \mathbf{b}} f\right), \square_{J}^{\omega, b, \mathbf{b}^{*}} g\right\rangle_{\omega}+\left\langle T_{\sigma}^{\alpha}\left(\square_{I, \text { broken }}^{\sigma, b)} f\right), \square_{J, \mathrm{~b}}^{\omega, b, \mathbf{b}^{*}} g\right\rangle_{\omega} \\
& +\left\langle T_{\sigma}^{\alpha}\left(\square_{I}^{\sigma, b, \mathbf{b}} f\right), \square_{J, \mathrm{broken}}^{\omega, b, \mathbf{b}^{*}} g\right\rangle_{\omega}+\left\langle T_{\sigma}^{\alpha}\left(\square_{I, \text { broken }}^{\sigma, b, \mathbf{b}} f\right), \square_{J}^{\omega, b, \mathbf{b}^{*}} g\right\rangle_{\omega} .
\end{aligned}
$$

The estimation of the latter three inner products, i.e. those in which a broken operator $\square_{I, \text { broken }}^{\sigma, b, \mathbf{b}}$ or $\square_{J, \text { broken }}^{\omega, b, \mathbf{b}^{*}}$ arises, is easy. Indeed, recall that

$$
\begin{aligned}
& \square_{I, \text { broken }}^{\sigma, b, \mathbf{b}} f=\sum_{I^{\prime} \in \mathfrak{C}_{\text {broken }}(I)} \mathbb{F}_{I^{\prime}}^{\sigma, \mathbf{b}} f=\sum_{I^{\prime} \in \mathfrak{C}_{\text {broken }}(I)}\left(E_{I^{\prime}}^{\sigma} \widehat{\mathbb{F}}_{I^{\prime}}^{\sigma, \mathbf{b}} f\right) b_{I^{\prime}}, \\
& \square_{J, \text { broken }}^{\omega, b, \mathbf{b}}=\sum_{J^{\prime} \in \mathfrak{C}_{\text {broken }}(J)} \mathbb{F}_{J^{\prime}}^{\omega, \mathbf{b}^{*}} g=\sum_{J^{\prime} \in \mathfrak{C}_{\text {broken }}(J)}\left(E_{J^{\prime}}^{\omega} \widehat{\mathbb{F}}_{J^{\prime}}^{\omega, \mathbf{b}^{*}} g\right) b_{J^{\prime}}^{*},
\end{aligned}
$$

so that if at least one broken difference appears in the inner product, as is the case for the latter three inner products in (5.2), then testing and Cauchy-Schwarz are all that is needed. For example, the fourth term satisfies

$$
\begin{aligned}
\left|\left\langle T_{\sigma}^{\alpha}\left(\square_{I, \text { broken }}^{\sigma, b, \mathbf{b}} f\right), \square_{J}^{\omega, b, \mathbf{b}^{*}} g\right\rangle_{\omega}\right| & =\left|\sum_{I^{\prime} \in \mathfrak{C}_{\text {broken }}(I)}\left(E_{I^{\prime}}^{\sigma} \widehat{\mathbb{F}}_{I^{\prime}}^{\sigma, \mathbf{b}} f\right)\left\langle T_{\sigma}^{\alpha} b_{I^{\prime}}, \square_{J}^{\omega, b, \mathbf{b}^{*}} g\right\rangle_{\omega}\right| \\
& \lesssim \sum_{I^{\prime} \in \mathfrak{C}_{\text {broken }}(I)}\left|E_{I^{\prime}}^{\sigma} \widehat{\mathbb{F}}_{I^{\prime}}^{\sigma, \mathbf{b}} f\right| \mathfrak{T}_{T^{\alpha}}^{\mathbf{b}} \sqrt{\left|I^{\prime}\right|_{\sigma}}\left\|\square_{J}^{\omega, b, \mathbf{b}^{*}} g\right\|_{L^{2}(\omega)} \\
& \lesssim \mathfrak{T}_{T^{\alpha}}^{\mathbf{b}}\left\|\nabla_{I}^{\sigma} f\right\|_{L^{2}(\sigma)} \sum_{I^{\prime} \in \mathfrak{C}_{\text {broken }}(I)}\left(\left\|\square_{J}^{\omega, \mathbf{b}^{*}} g\right\|_{L^{2}(\omega)}+\left\|\square_{J, \text { broken }}^{\omega, b \mathbf{b}^{*}} g\right\|_{L^{2}(\omega)}\right) \\
& \lesssim \mathcal{N} \mathcal{T} \mathcal{V}_{\alpha}\left\|\square_{I}^{\sigma, \mathbf{b}} f\right\|_{L^{2}(\sigma)}^{\star}\left\|\square_{J}^{\omega, \mathbf{b}^{*}} g\right\|_{L^{2}(\omega)}^{\star},
\end{aligned}
$$

and the third term can be written as $\left\langle\square_{I}^{\sigma, b, \mathbf{b}} f, T_{\omega}^{\alpha, *}\left(\square_{J, \text { broken }}^{\omega, b, \mathbf{b}^{*}} g\right)\right\rangle_{\sigma}$ and handled similarly. 
Thus it remains to consider the first inner product $\left\langle T_{\sigma}^{\alpha}\left(\square_{I}^{\sigma, b, \mathbf{b}} f\right), \square_{J}^{\omega, b, \mathbf{b}^{*}} g\right\rangle_{\omega}$ on the right hand side of (5.2), which we call the problematic term, and write it as

$$
\begin{aligned}
P(I, J) & \equiv\left\langle T_{\sigma}^{\alpha}\left(\square_{I}^{\sigma, b, \mathbf{b}} f\right), \square_{J}^{\omega, b, \mathbf{b}^{*}} g\right\rangle_{\omega} \\
& =\sum_{I^{\prime} \in \mathfrak{C}(I) \text { and } J^{\prime} \in \mathfrak{C}(J)}\left\langle T_{\sigma}^{\alpha}\left(\mathbf{1}_{I^{\prime}} \square_{I}^{\sigma, b, \mathbf{b}} f\right), \mathbf{1}_{J^{\prime}} \square_{J}^{\omega, b, \mathbf{b}^{*}} g\right\rangle_{\omega} \\
& =\sum_{I^{\prime} \in \mathfrak{C}(I) \text { and } J^{\prime} \in \mathfrak{C}(J)} E_{I^{\prime}}^{\sigma}\left(\widehat{\square}_{I}^{\sigma, b, \mathbf{b}} f\right)\left\langle T_{\sigma}^{\alpha}\left(\mathbf{1}_{I^{\prime}} b_{I}\right), \mathbf{1}_{J^{\prime}} b_{J}^{*}\right\rangle_{\omega} E_{J^{\prime}}^{\omega}\left(\widehat{\square}_{J}^{\omega, b, \mathbf{b}^{*}} g\right) .
\end{aligned}
$$

It now remains to show that

$$
\boldsymbol{E}_{\Omega}^{\mathcal{D}} \boldsymbol{E}_{\Omega}^{\mathcal{G}} \sum_{I \in \mathcal{D}} \sum_{\substack{J \in \mathcal{G}: 2^{-\mathbf{r}} \ell(I)<\ell(J) \leq \ell(I) \\ d(J, I) \leq 2 \ell(J)^{\varepsilon} \ell(I)^{1-\varepsilon}}}|P(I, J)| \lesssim\left(C_{\theta} \mathcal{N} \mathcal{T} \mathcal{V}_{\alpha}+\sqrt{\theta} \mathfrak{N}_{T^{\alpha}}\right)\|f\|_{L^{2}(\sigma)}\|g\|_{L^{2}(\omega)} .
$$

We will repeatedly use the inequality $\left\|\hat{\square}_{I}^{\sigma, b, \mathbf{b}} f\right\|_{L^{2}(\sigma)} \lesssim\left\|\square_{I}^{\sigma, \mathbf{b}} f\right\|_{L^{2}(\sigma)}^{\star}$ which, upon noting (9.26), follows from the PLBP (2.2),

$$
\begin{aligned}
\left\|\widehat{\square}_{I}^{\sigma, b, \mathbf{b}} f\right\|_{L^{2}(\sigma)} & \lesssim\left\|b_{I} \widehat{\square}_{I}^{\sigma, b, \mathbf{b}} f\right\|_{L^{2}(\sigma)}=\left\|\square_{I}^{\sigma, b, \mathbf{b}} f\right\|_{L^{2}(\sigma)} \\
& \leq\left\|\square_{I}^{\sigma, \mathbf{b}} f\right\|_{L^{2}(\sigma)}+\left\|\square_{I, \text { broken }}^{\sigma, b, \mathbf{b}} f\right\|_{L^{2}(\sigma)} \lesssim\left\|\square_{I}^{\sigma, \mathbf{b}} f\right\|_{L^{2}(\sigma)}^{\star} .
\end{aligned}
$$

Suppose now that $I \in \mathcal{C}_{A}$ for $A \in \mathcal{A}$, and that $J \in \mathcal{C}_{B}$ for $B \in \mathcal{B}$. Then the inner product in the third line of (5.3) becomes

$$
\left\langle T_{\sigma}^{\alpha}\left(b_{I} \mathbf{1}_{I^{\prime}}\right), b_{J}^{*} \mathbf{1}_{J^{\prime}}\right\rangle_{\omega}=\left\langle T_{\sigma}^{\alpha}\left(b_{A} \mathbf{1}_{I^{\prime}}\right), b_{B}^{*} \mathbf{1}_{J^{\prime}}\right\rangle_{\omega},
$$

and we will write this inner product in either form, depending on context. We also introduce the following notation:

$$
P_{(I, J)}(E, F) \equiv\left\langle T_{\sigma}^{\alpha}\left(b_{I} \mathbf{1}_{E}\right), b_{J}^{*} \mathbf{1}_{F}\right\rangle_{\omega}, \quad \text { for any sets } E \text { and } F
$$

so that

$$
P(I, J)=\sum_{I^{\prime} \in \mathfrak{C}(I) \text { and } J^{\prime} \in \mathfrak{C}(J)} E_{I^{\prime}}^{\sigma}\left(\widehat{\square}_{I}^{\sigma, b, \mathbf{b}} f\right) P_{(I, J)}\left(I^{\prime}, J^{\prime}\right) E_{J^{\prime}}^{\omega}\left(\widehat{\square}_{J}^{\omega, b, \mathbf{b}^{*}} g\right) .
$$

The first thing we do is reduce matters to showing inequality (5.4) in the case of equal intervals, by which we mean that $P_{(I, J)}\left(I^{\prime}, J^{\prime}\right)$ is replaced by $P_{(I, J)}\left(I^{\prime} \cap J^{\prime}, I^{\prime} \cap J^{\prime}\right)$ in the terms $P(I, J)$ appearing in (5.4). To see this let $K \equiv I^{\prime} \cap J^{\prime}$, write

$$
\begin{aligned}
\left|\left\langle T_{\sigma}^{\alpha}\left(b_{I} \mathbf{1}_{I^{\prime}}\right), b_{J}^{*} \mathbf{1}_{J^{\prime}}\right\rangle_{\omega}\right| & =\left|\left\langle T_{\sigma}^{\alpha}\left(b_{I} \mathbf{1}_{I^{\prime} \backslash J^{\prime}}\right), b_{J}^{*} \mathbf{1}_{J^{\prime}}\right\rangle_{\omega}+\left\langle T_{\sigma}^{\alpha}\left(b_{I} \mathbf{1}_{I^{\prime} \cap J^{\prime}}\right), b_{J}^{*} \mathbf{1}_{J^{\prime} \backslash I^{\prime}}\right\rangle_{\omega}+\left\langle T_{\sigma}^{\alpha}\left(b_{I} \mathbf{1}_{I^{\prime} \cap J^{\prime}}\right), b_{J}^{*} \mathbf{1}_{J^{\prime} \cap I^{\prime}}\right\rangle_{\omega}\right| \\
& \leq\left|\left\langle T_{\sigma}^{\alpha}\left(b_{I} \mathbf{1}_{I^{\prime} \backslash J^{\prime}}\right), b_{J}^{*} \mathbf{1}_{J^{\prime}}\right\rangle_{\omega}\right|+\left|\left\langle T_{\sigma}^{\alpha}\left(b_{I} \mathbf{1}_{K}\right), b_{J}^{*} \mathbf{1}_{J^{\prime} \backslash I^{\prime}}\right\rangle_{\omega}\right|+\left|\left\langle T_{\sigma}^{\alpha}\left(b_{I} \mathbf{1}_{K}\right), b_{J}^{*} \mathbf{1}_{K}\right\rangle\right|,
\end{aligned}
$$

and use (3.22) to obtain

$$
\left|\left\langle T_{\sigma}^{\alpha}\left(b_{I} \mathbf{1}_{I^{\prime} \backslash J^{\prime}}\right), b_{J}^{*} \mathbf{1}_{J^{\prime}}\right\rangle_{\omega}\right|+\left|\left\langle T_{\sigma}^{\alpha}\left(b_{I} \mathbf{1}_{K}\right), b_{J}^{*} \mathbf{1}_{J^{\prime} \backslash I^{\prime}}\right\rangle_{\omega}\right| \lesssim \sqrt{\mathfrak{A}_{2}^{\alpha}} \sqrt{\left|I^{\prime}\right|_{\sigma}\left|J^{\prime}\right|_{\omega}} .
$$

It thus remains to consider only the term $P_{(I, J)}(K, K)=\left\langle T_{\sigma}^{\alpha}\left(b_{I} \mathbf{1}_{K}\right), b_{J}^{*} \mathbf{1}_{K}\right\rangle_{\omega}$ where $K=I^{\prime} \cap J^{\prime} \neq \emptyset$, and to show that

$$
\begin{aligned}
& \boldsymbol{E}_{\Omega}^{\mathcal{D}} \boldsymbol{E}_{\Omega}^{\mathcal{G}} \sum_{I \in \mathcal{D}} \sum_{\substack{J \in \mathcal{G}: 2^{-\mathbf{r}} \ell(I)<\ell(J) \leq \ell(I) \\
d(J, I) \leq 2 \ell(J)^{\varepsilon} \ell(I)^{1}-\varepsilon}}\left|\sum_{\substack{I^{\prime} \in \mathfrak{C}(I) \text { and } \\
K=I^{\prime} \cap J^{\prime} \neq \emptyset}} E_{J^{\prime} \in \mathfrak{C}(J)}^{\sigma}\left(\widehat{\square}_{I}^{\sigma, b, \mathbf{b}} f\right) P_{(I, J)}(K, K) E_{J^{\prime}}^{\omega}\left(\widehat{\square}_{J}^{\omega, b, \mathbf{b}^{*}} g\right)\right| \\
& \lesssim\left(C_{\theta} \mathcal{N} \mathcal{T} \mathcal{V}_{\alpha}+\sqrt{\theta} \mathfrak{N}_{T^{\alpha}}\right)\|f\|_{L^{2}(\sigma)}\|g\|_{L^{2}(\omega)},
\end{aligned}
$$


5.1. Random surgery. However, we wish to further reduce matters to the case where $K \in \mathcal{G}$ and contained in $I^{\prime} \cap J^{\prime}$, and for this we use random surgery and (3.22). First, we note that $I^{\prime}$ cannot be strictly contained in $J^{\prime}$ since $\ell(J) \leq \ell(I)$, and in the case that $J^{\prime} \subset I^{\prime}$, then $K=I^{\prime} \cap J^{\prime}=J^{\prime} \in \mathcal{G}$ and there is nothing more to do in this case. So we may assume that $J^{\prime}$ intersects both $I^{\prime}$ and its complement $\left(I^{\prime}\right)^{c}$.

Our first step is to reduce matters to showing inequality (5.6) in the case where $\ell(K) \geq \lambda \ell\left(I^{\prime}\right)$ for a small positive number $\lambda \ll 2^{-\mathbf{r}}$. This small constant $\lambda$, as well as the constant $\eta_{0}$ introduced in probability estimates below, will be chosen sufficiently small at the end of the proof to result in the term $\sqrt{\theta} \mathfrak{N}_{T^{\alpha}}$ appearing on the right hand side of (5.6). This is accomplished by writing (recall that $K=I^{\prime} \cap J^{\prime}$ for the moment)

$$
\begin{aligned}
& \sum_{I \in \mathcal{D}} \sum_{\substack{J \in \mathcal{G}: 2^{-\mathbf{r}} \ell(I)<\ell(J) \leq \ell(I) \\
d(J, I) \leq 2 \ell(J)^{\varepsilon} \ell(I)^{1-\varepsilon}}}\left|\sum_{I^{\prime} \in \mathfrak{C}(I)} \sum_{\substack{\text { and } \\
K \neq \emptyset}} E_{J^{\prime} \in \mathfrak{C}(J)}^{\sigma}\left(\widehat{\square}_{I}^{\sigma, b, \mathbf{b}} f\right) P_{(I, J)}(K, K) E_{J^{\prime}}^{\omega}\left(\widehat{\square}_{J}^{\omega, b, \mathbf{b}^{*}} g\right)\right| \\
& \leq \sum_{I \in \mathcal{D}} \sum_{\substack{J \in \mathcal{G}: 2^{-\mathbf{r}} \ell(I)<\ell(J) \leq \ell(I) \\
d(J, I) \leq 2 \ell(J)^{\varepsilon} \ell(I)^{1-\varepsilon}}}\left|\sum_{\substack{I^{\prime} \in \mathfrak{C}(I) \text { and } J^{\prime} \in \mathfrak{C}(J) \\
\ell(K) \geq \lambda \ell\left(I^{\prime}\right)}} E_{I^{\prime}}^{\sigma}\left(\hat{\square}_{I}^{\sigma, b, \mathbf{b}} f\right) P_{(I, J)}(K, K) E_{J^{\prime}}^{\omega}\left(\hat{\square}_{J}^{\omega, b, \mathbf{b}^{*}} g\right)\right| \\
& +\sum_{I \in \mathcal{D}} \sum_{\substack{J \in \mathcal{G}: 2^{-\mathbf{r}} \ell(I)<\ell(J) \leq \ell(I) \\
(J, I) \leq 2 \ell(J)^{\ell} \ell(I)^{1-\varepsilon}}} \sum_{\substack{I^{\prime} \in \mathfrak{C}(I) \text { and } \\
0<\ell(K)<\lambda \ell\left(I^{\prime}\right)}} E_{I^{\prime}}^{\sigma}\left(\widehat{\square}_{I}^{\sigma, b, \mathbf{b}} f\right) P_{(I, J)}(K, K) E_{J^{\prime}}^{\omega}\left(\widehat{\square}_{J}^{\omega, b, \mathbf{b}^{*}} g\right) \mid \\
& \equiv A+B \text {. }
\end{aligned}
$$

Term $B$ is handled using the norm constant $\mathfrak{N}_{T^{\alpha}}$ and probability, together with the estimate

$$
\begin{aligned}
& \sum_{I \in \mathcal{D}} \sum_{\begin{array}{c}
J \in \mathcal{G}: 2^{-\mathbf{r}} \ell(I)<\ell(J) \leq \ell(I) \\
(J, I) \leq 2 \ell(J)^{\varepsilon} \ell(I)^{1-\varepsilon}
\end{array}} \sum_{\substack{I^{\prime} \in \mathfrak{C}(I) \\
0<\ell(K)<\lambda \ell\left(I^{\prime}\right)}}\left|\sqrt{|K|_{\omega}} E_{J^{\prime}}^{\omega}\left(\widehat{\square}_{J}^{\omega, b, \mathbf{b}^{*}} g\right)\right|^{2}
\end{aligned}
$$

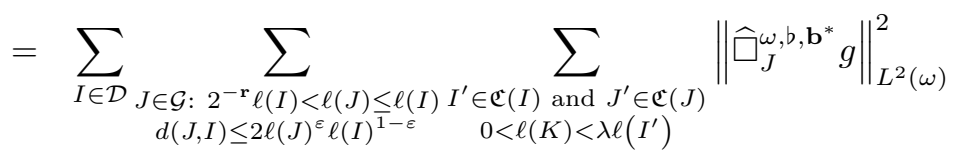

$$
\begin{aligned}
& \lesssim \sum_{J \in \mathcal{G}}\left\|\widehat{\square}_{J}^{\omega, b, \mathbf{b}^{*}} g\right\|_{L^{2}(\omega)}^{2} \lesssim \sum_{J \in \mathcal{G}}\left(\left\|\square_{J}^{\omega, \mathbf{b}^{*}} g\right\|_{L^{2}(\omega)}^{2}+\left\|\widehat{\square}_{J, b r o k e n}^{\omega, b, \mathbf{b}^{*}} g\right\|_{L^{2}(\omega)}^{2}\right) \lesssim\|g\|_{L^{2}(\omega)}^{2},
\end{aligned}
$$

to obtain

$$
\begin{aligned}
& \boldsymbol{E}_{\Omega}^{\mathcal{G}} B \lesssim \boldsymbol{E}_{\Omega}^{\mathcal{G}} \sum_{I \in \mathcal{D}} \sum_{\substack{J \in \mathcal{G}: 2^{-\mathbf{r}} \ell(I)<\ell(J) \leq \ell(I) \\
d(J, I) \leq 2 \ell(J)^{\varepsilon} \ell(I)^{1}-\varepsilon}} \sum_{\substack{I^{\prime} \in \mathfrak{C}(I) \text { and } J^{\prime} \in \mathfrak{C}(J) \\
0<\ell(K)<\lambda \ell\left(I^{\prime}\right)}}\left|E_{I^{\prime}}^{\sigma}\left(\widehat{\square}_{I}^{\sigma, b, \mathbf{b}} f\right) \mathfrak{N}_{T^{\alpha}} \sqrt{|K|_{\sigma}|K|_{\omega}} E_{J^{\prime}}^{\omega}\left(\widehat{\square}_{J}^{\omega, b, \mathbf{b}^{*}} g\right)\right| \\
& \lesssim \boldsymbol{E}_{\Omega}^{\mathcal{G}} \mathfrak{N}_{T^{\alpha}}\left(\sum_{I \in \mathcal{D}} \sum_{I^{\prime} \in \mathfrak{C}(I)}\left(\sum_{\substack{J \in \mathcal{G}: 2^{-\mathbf{r}} \ell(I)<\ell(J) \leq \ell(I) \\
d(J, I) \leq 2 \ell(J)^{\varepsilon} \ell(I)^{1-\varepsilon}}} \sum_{\substack{J^{\prime} \in \mathfrak{C}(J) \\
0<\ell\left(I^{\prime} \cap J^{\prime}\right)<\lambda \ell\left(I^{\prime}\right)}}\left|I^{\prime} \cap J^{\prime}\right|_{\sigma}\right)\left|E_{I^{\prime}}^{\sigma}\left(\widehat{\square}_{I}^{\sigma, b, \mathbf{b}} f\right)\right|^{2}\right)^{\frac{1}{2}}\|g\|_{L^{2}(\omega)} \\
& \leq \mathfrak{N}_{T^{\alpha}}\left(\sum_{I \in \mathcal{D}} \sum_{I^{\prime} \in \mathfrak{C}(I)} \lambda\left|I^{\prime}\right|_{\sigma}\left|E_{I^{\prime}}^{\sigma}\left(\widehat{\square}_{I}^{\sigma, b, \mathbf{b}} f\right)\right|^{2}\right)^{\frac{1}{2}}\|g\|_{L^{2}(\omega)} \lesssim \mathfrak{N}_{T^{\alpha}} \sqrt{\lambda}\|f\|_{L^{2}(\sigma)}\|g\|_{L^{2}(\omega)}
\end{aligned}
$$

since the probability that, given an interval $I^{\prime}$, a grid $\mathcal{G}$ contains an interval $J^{\prime}$ with $2^{-\mathbf{r}} \ell\left(I^{\prime}\right)<\ell\left(J^{\prime}\right) \leq \ell\left(I^{\prime}\right)$ and $0<\ell\left(I^{\prime} \cap J^{\prime}\right)<\lambda \ell\left(I^{\prime}\right)$, is at most $C_{\mathbf{r}} \lambda$ for some large constant $C_{\mathbf{r}}$ depending on the goodness parameter $\mathbf{r}$ (we could also have appealed to the more general halo estimate (3.7)). This term of course contributes to 
the conclusion of the lemma provided $\lambda>0$ is chosen sufficiently small. Thus we are left to control term $A$ in which $\ell(K) \geq \lambda \ell\left(I^{\prime}\right)$ as required.

Now choose $\eta_{0} \in(0,1) \cap\left\{2^{-m}\right\}_{m \in \mathbb{N}}$, say $\eta_{0}=2^{-m}$ for $m$ sufficiently large. We will assume from now on that $\eta_{0}<\frac{1}{2} \lambda$, where $\lambda$ is the small constant above. For any interval $L$ and $0<\eta \leq \frac{1}{2}$, define

$$
\partial_{\eta} L \equiv(1+\eta) L \backslash(1-\eta) L
$$

to be the ' $\eta$-halo' around the boundary, i.e. endpoints, of $L$. One should also recall that all intervals are assumed to be closed on the left and open on the right. For $\eta_{1} \in\left(0, \eta_{0}\right]$ we write

$$
\begin{aligned}
I^{\prime} \cap J^{\prime} & =\left\{\left(I^{\prime} \backslash \partial_{\eta_{1}} I^{\prime}\right) \cap J^{\prime}\right\} \cup\left\{\left[I^{\prime} \cap J^{\prime}\right] \backslash\left[\left(I^{\prime} \backslash \partial_{\eta_{1}} I^{\prime}\right) \cap J^{\prime}\right]\right\} \\
& \equiv M \dot{\cup} L,
\end{aligned}
$$

where $\dot{\cup}$ denotes a disjoint union. At this point we note that there is precisely one endpoint of $I^{\prime \prime} \equiv I^{\prime} \backslash \partial_{\eta_{1}} I^{\prime}$ that lies in $J^{\prime}$ since $\ell\left(I^{\prime} \cap J^{\prime}\right) \geq \lambda \ell\left(I^{\prime}\right)$ and $\eta_{1} \leq \eta_{0}<\frac{1}{2} \lambda$.

Moreover, and this is the key part of the argument, we can choose $\frac{1}{2} \eta_{0} \leq \eta_{1} \leq \eta_{0}$ so that the interval $M=I^{\prime \prime} \cap J^{\prime}$ is a union of a number $B=B\left(I^{\prime}, J^{\prime}\right)=B\left(I^{\prime}, J^{\prime}, \eta_{1}\left(I^{\prime}, J^{\prime}\right)\right)$ of intervals $K_{i} \in \mathcal{G}$ each having side length $\ell\left(K_{i}\right)=2^{-m-1} \ell\left(J^{\prime}\right) \equiv \frac{1}{2} \eta_{0} \ell\left(J^{\prime}\right)$ and where $B \leq C \frac{1}{\eta_{0}}$. Indeed, we take $\frac{1}{2} \eta_{0} \leq \eta_{1} \leq \eta_{0}=2^{-m}$, so that the endpoint of the interval $I^{\prime \prime} \equiv I^{\prime} \backslash \partial_{\eta_{1}} I^{\prime}$ that lies in $J^{\prime}$ coincides with an endpoint of some $K \in \mathcal{G}$ with $\ell(K)=2^{-m-1} \ell\left(J^{\prime}\right)$. This can be arranged by varying $\eta_{1}$ between $\frac{1}{2} \eta_{0}$ and $\eta_{0}$ until the endpoint in question lies among the dyadic numbers that form the endpoints of intervals in the grid $\mathcal{G}$ with side length $2^{-m-1} \ell\left(J^{\prime}\right)$. The choice of intervals $\left\{K_{i}\right\}_{i=1}^{B}$ having common side length $2^{-m-1} \ell\left(J^{\prime}\right)$ is then uniquely determined, and it is easy to see that

$$
B \leq C \frac{1}{\eta_{0}}
$$

Thus the choice of $\eta_{1}=\eta_{1}\left(I^{\prime}, J^{\prime}\right)>0$ is always at most $\eta_{0}$, and at least $\frac{1}{2} \eta_{0}$, but changes according to the relative position of $I^{\prime}$ with respect to $J^{\prime}$ in order that $M$ is a union of intervals $K_{i} \in \mathcal{G}$ of side length at least $\frac{1}{2} \eta_{0} \ell\left(J^{\prime}\right)$, and specified in the manner described above. Define

$$
\mathcal{K}\left(I^{\prime}, J^{\prime}\right) \equiv\left\{K_{i}\right\}_{i=1}^{B\left(I^{\prime}, J^{\prime}\right)}=\left\{K_{i}\right\}_{i=1}^{B}
$$

to be this collection of consecutive adjacent intervals $K_{i}$ uniquely defined here in terms of $I^{\prime}, J^{\prime}$ and $\eta_{1}=$ $\eta_{1}\left(I^{\prime}, J^{\prime}\right)$.

Having chosen the parameter $\eta_{1}$ as above, we now momentarily ignore the decomposition of $M=\bigcup_{i=1}^{B} K_{i}$ into subintervals, and return to the representation $K=I^{\prime} \cap J^{\prime}=M \dot{\cup} L$ determined by our choice of $\eta_{1}$. We have

$$
\begin{aligned}
\left\langle T_{\sigma}^{\alpha}\left(b_{I} \mathbf{1}_{K}\right), b_{J}^{*} \mathbf{1}_{K}\right\rangle_{\omega}= & \left\langle T_{\sigma}^{\alpha}\left(b_{I} \mathbf{1}_{M}\right), b_{J}^{*} \mathbf{1}_{L}\right\rangle_{\omega}+\left\langle T_{\sigma}^{\alpha}\left(b_{I} \mathbf{1}_{L}\right), b_{J}^{*} \mathbf{1}_{M}\right\rangle_{\omega} \\
& +\left\langle T_{\sigma}^{\alpha}\left(b_{I} \mathbf{1}_{L}\right), b_{J}^{*} \mathbf{1}_{L}\right\rangle_{\omega}+\left\langle T_{\sigma}^{\alpha}\left(b_{I} \mathbf{1}_{M}\right), b_{J}^{*} \mathbf{1}_{M}\right\rangle_{\omega}
\end{aligned}
$$

Now we apply (3.22) to the first two terms in (5.9) to obtain that

$$
\begin{gathered}
\left|\left\langle T_{\sigma}^{\alpha}\left(b_{I} \mathbf{1}_{M}\right), b_{J}^{*} \mathbf{1}_{L}\right\rangle_{\omega}\right|+\left|\left\langle T_{\sigma}^{\alpha}\left(b_{I} \mathbf{1}_{L}\right), b_{J}^{*} \mathbf{1}_{M}\right\rangle_{\omega}\right| \\
\lesssim \sqrt{\mathfrak{A}_{2}^{\alpha}}\left\{\sqrt{\int_{M}\left|b_{I}\right|^{2} d \sigma} \sqrt{\int_{L}\left|b_{J}^{*}\right|^{2} d \omega}+\sqrt{\int_{L}\left|b_{I}\right|^{2} d \sigma} \sqrt{\int_{M}\left|b_{J}^{*}\right|^{2} d \omega}\right\} \\
\lesssim \sqrt{\mathfrak{A}_{2}^{\alpha}} \sqrt{\left|I^{\prime}\right|_{\sigma}\left|J^{\prime}\right|_{\omega}},
\end{gathered}
$$


which when plugged appropriately into the left hand side of (5.4) is dominated by the right hand side of (5.4):

$$
\begin{aligned}
& \boldsymbol{E}_{\Omega}^{\mathcal{D}} \boldsymbol{E}_{\Omega}^{\mathcal{G}} \sum_{I \in \mathcal{D}} \sum_{\substack{J \in \mathcal{G}: 2^{-\mathbf{r}} \ell(I)<\ell(J) \leq \ell(I) \\
d(J, I) \leq 2 \ell(J)^{\varepsilon} \ell(I)^{1}-\varepsilon}} \sum_{\substack{I^{\prime} \in \mathfrak{C}(I) \\
J^{\prime} \in \mathfrak{C}(J)}} \\
& \times\left|E_{I^{\prime}}^{\sigma}\left(\widehat{\square}_{I}^{\sigma, b, \mathbf{b}} f\right)\left(\left|\left\langle T_{\sigma}^{\alpha}\left(b_{I} \mathbf{1}_{M}\right), b_{J}^{*} \mathbf{1}_{L}\right\rangle_{\omega}\right|+\left|\left\langle T_{\sigma}^{\alpha}\left(b_{I} \mathbf{1}_{L}\right), b_{J}^{*} \mathbf{1}_{M}\right\rangle_{\omega}\right|\right) E_{J^{\prime}}^{\omega}\left(\widehat{\square}_{J}^{\omega, b, \mathbf{b}^{*}} g\right)\right| \\
\lesssim & \boldsymbol{E}_{\Omega}^{\mathcal{D}} \boldsymbol{E}_{\Omega}^{\mathcal{G}} \sum_{I \in \mathcal{D}} \sum_{\substack{J \in \mathcal{G}: 2^{-\mathbf{r}} \ell(I)<\ell(J) \leq \ell(I) \\
d(J, I) \leq 2 \ell(J)^{\varepsilon} \ell(I)^{1-\varepsilon}}} \sum_{\substack{I^{\prime} \in \mathfrak{C}(I) \\
J^{\prime} \in \mathfrak{C}(J)}}\left|E_{I^{\prime}}^{\sigma}\left(\widehat{\square}_{I}^{\sigma, b, \mathbf{b}} f\right)\right| \sqrt{\mathfrak{A}_{2}^{\alpha}} \sqrt{\left|I^{\prime}\right|_{\sigma}\left|J^{\prime}\right|_{\omega}}\left|E_{J^{\prime}}^{\omega}\left(\widehat{\square}_{J}^{\omega, b, \mathbf{b}^{*}} g\right)\right| \\
\lesssim & \sqrt{\mathfrak{A}_{2}^{\alpha}}\|f\|_{L^{2}(\sigma)}\|g\|_{L^{2}(\omega)},
\end{aligned}
$$

where in the last line we have used $\sum_{\substack{I^{\prime} \in \mathfrak{C}(I) \\ J^{\prime} \in \mathfrak{C}(J)}}\left|E_{I^{\prime}}^{\sigma}\left(\widehat{\square}_{I}^{\sigma, b, \mathbf{b}} f\right)\right|^{2}\left|I^{\prime}\right|_{\sigma}=\left\|\widehat{\square}_{I}^{\sigma, b, \mathbf{b}} f\right\|_{L^{2}(\sigma)}^{2}$ and (5.5) and the frame inequalities in Appendix A. Then we apply Cauchy-Schwarz to the sums in the third term in (5.9) using $L=\partial_{\eta_{1}} I^{\prime} \cap J^{\prime}$ to get

$$
\begin{aligned}
& \boldsymbol{E}_{\Omega}^{\mathcal{D}} \boldsymbol{E}_{\Omega}^{\mathcal{G}} \sum_{I \in \mathcal{D}} \sum_{\substack{J \in \mathcal{G}: 2^{-\mathbf{r}} \ell(I)<\ell(J) \leq \ell(I) \\
d(J, I) \leq 2 \ell(J)^{\varepsilon} \ell(I)^{1-\varepsilon}}} \sum_{\substack{I^{\prime} \in \mathfrak{C}(I) \\
J^{\prime} \in \mathfrak{C}(J)}}\left|E_{I^{\prime}}^{\sigma}\left(\widehat{\square}_{I}^{\sigma, b, \mathbf{b}} f\right)\left\langle T_{\sigma}^{\alpha}\left(b_{I} \mathbf{1}_{L}\right), b_{J}^{*} \mathbf{1}_{L}\right\rangle_{\omega} E_{J^{\prime}}^{\omega}\left(\widehat{\square}_{J}^{\omega, b, \mathbf{b}^{*}} g\right)\right|
\end{aligned}
$$

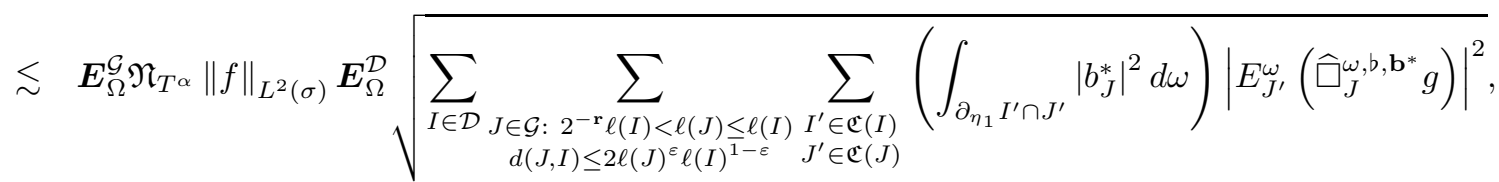

using (5.5) and the frame inequalities in Appendix A again. Then using Cauchy-Schwarz on the expectation $\boldsymbol{E}_{\Omega}^{\mathcal{D}}$, this is dominated by

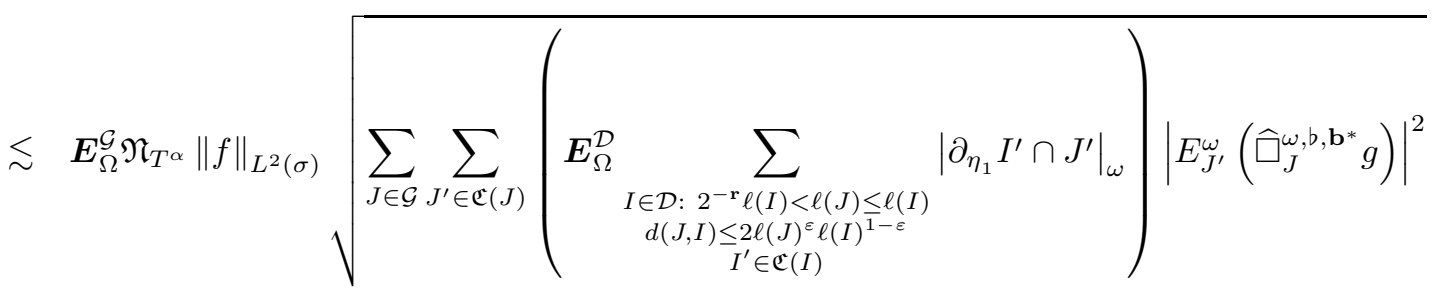

$$
\begin{aligned}
& \lesssim \boldsymbol{E}_{\Omega^{\mathcal{G}}}^{\mathcal{G}} \mathfrak{N}_{T^{\alpha}}\|f\|_{L^{2}(\sigma)} \sqrt{\sum_{J \in \mathcal{G}} \sum_{J^{\prime} \in \mathfrak{C}(J)} C 2^{\mathbf{r}}\left(\boldsymbol{E}_{\Omega}^{\mathcal{D}} \sum_{I^{\prime} \in \mathcal{D}: \ell\left(J^{\prime}\right) \leq \ell\left(I^{\prime}\right) \leq 2^{\mathbf{r}} \ell\left(J^{\prime}\right)}\left|\partial_{\eta_{0}} I^{\prime} \cap J^{\prime}\right|_{\omega}\right)\left|E_{J^{\prime}}^{\omega}\left(\widehat{\square}_{J}^{\omega, b, \mathbf{b}^{*}} g\right)\right|^{2}} \\
& \lesssim \sqrt{\eta_{0}} \mathfrak{N}_{T^{\alpha}}\|f\|_{L^{2}(\sigma)}\|g\|_{L^{2}(\omega)},
\end{aligned}
$$

where in the last line we have used $\eta_{1} \leq \eta_{0}$, and then

$$
\boldsymbol{E}_{\Omega}^{\mathcal{D}} \sum_{I^{\prime} \in \mathcal{D}: \ell\left(J^{\prime}\right) \leq \ell\left(I^{\prime}\right) \leq 2^{\mathrm{r}} \ell\left(J^{\prime}\right)}\left|\partial_{\eta_{0}} I^{\prime} \cap J^{\prime}\right|_{\omega} \lesssim \eta_{0}\left|J^{\prime}\right|_{\omega}
$$

from (3.7) since $\eta_{0} \leq \frac{1}{2} \lambda \ll 2^{-\mathbf{r}}$, and finally quasiorthogonality and (5.5) yet again, to obtain

$$
\begin{aligned}
\sum_{J \in \mathcal{G}} \sum_{J^{\prime} \in \mathfrak{C}(J)}\left|J^{\prime}\right|_{\omega}\left|E_{J^{\prime}}^{\omega}\left(\widehat{\square}_{J}^{\omega, b, \mathbf{b}^{*}} g\right)\right|^{2} & \lesssim \sum_{J \in \mathcal{G}}\left\|\widehat{\square}_{J}^{\omega, b, \mathbf{b}^{*}} g\right\|_{L^{2}(J)}^{2} \lesssim \sum_{J \in \mathcal{G}}\left\|\square_{J}^{\omega, b, \mathbf{b}^{*}} g\right\|_{L^{2}(J)}^{2} \\
& \lesssim \sum_{J \in \mathcal{G}}\left(\left\|\square_{J}^{\omega, \mathbf{b}^{*}} g\right\|_{L^{2}(J)}^{2}+\left\|\nabla_{J}^{\omega} g\right\|_{L^{2}(J)}^{2}\right) \lesssim\|g\|_{L^{2}(\omega)}^{2} .
\end{aligned}
$$


This leaves us to estimate the fourth term in (5.9), i.e. the inner product $\left\langle T_{\sigma}^{\alpha}\left(b_{I} \mathbf{1}_{M}\right), b_{J}^{*} \mathbf{1}_{M}\right\rangle$. It is at this point that we will use the decomposition $M=\bigcup_{1 \leq i \leq B} K_{i}$ constructed above. We have $\left\langle T_{\sigma}^{\alpha}\left(b_{I} \mathbf{1}_{M}\right), b_{J}^{*} \mathbf{1}_{M}\right\rangle_{\omega}=\sum_{i, i^{\prime}=1}^{B}\left\langle T_{\sigma}^{\alpha}\left(b_{I} \mathbf{1}_{K_{i}}\right), b_{J}^{*} \mathbf{1}_{K_{i^{\prime}}}\right\rangle_{\omega}=\sum_{i=1}^{B}\left\langle T_{\sigma}^{\alpha}\left(b_{I} \mathbf{1}_{K_{i}}\right), b_{J}^{*} \mathbf{1}_{K_{i}}\right\rangle_{\omega}+\sum_{i \neq i^{\prime}}\left\langle T_{\sigma}^{\alpha}\left(b_{I} \mathbf{1}_{K_{i}}\right), b_{J}^{*} \sum_{i^{\prime}: i^{\prime} \neq i} \mathbf{1}_{K_{i^{\prime}}}\right\rangle_{\omega}$

and finally, we can use (3.22) once more on the second sum above to reduce matters, modulo a constant multiple of $\frac{1}{\eta_{0}}$, to the case of estimating the inner products $\left\langle T_{\sigma}^{\alpha}\left(b_{I} \mathbf{1}_{K}\right), b_{J}^{*} \mathbf{1}_{K}\right\rangle$ for the intervals $K=K_{i} \in \mathcal{G}$, $1 \leq i \leq B \leq C \frac{1}{\eta_{0}}$, which are contained in $I^{\prime} \cap J^{\prime}$. Thus it remains to show

$$
\begin{aligned}
& \boldsymbol{E}_{\Omega}^{\mathcal{D}} \boldsymbol{E}_{\Omega}^{\mathcal{G}} \sum_{\substack { I \in \mathcal{D} \\
\begin{subarray}{c}{J \in \mathcal{G}: 2^{-\mathbf{r}} \ell(I)<\ell(J) \leq \ell(I) \\
d(J, I) \leq 2 \ell(J)^{\varepsilon} \ell(I)^{1-\varepsilon}{ I \in \mathcal { D } \\
\begin{subarray} { c } { J \in \mathcal { G } : 2 ^ { - \mathbf { r } } \ell ( I ) < \ell ( J ) \leq \ell ( I ) \\
d ( J , I ) \leq 2 \ell ( J ) ^ { \varepsilon } \ell ( I ) ^ { 1 - \varepsilon } } }\end{subarray}}\left|\sum_{\substack{I^{\prime} \in \mathfrak{C}(I) \text { and } \\
K \in \mathcal{K}\left(I^{\prime}, J^{\prime}\right)}} E_{I^{\prime}\left(\mathfrak{C}^{\prime}(J)\right.}^{\sigma}\left(\widehat{\square}_{I}^{\sigma, b, \mathbf{b}} f\right) P_{(I, J)}(K, K) E_{J^{\prime}}^{\omega}\left(\widehat{\square}_{J}^{\omega, b, \mathbf{b}^{*}} g\right)\right| \\
& \lesssim\left(C_{\theta} \mathcal{N} \mathcal{T} \mathcal{V}_{\alpha}+\sqrt{\theta} \mathfrak{N}_{T^{\alpha}}\right)\|f\|_{L^{2}(\sigma)}\|g\|_{L^{2}(\omega)},
\end{aligned}
$$

where we recall that $\mathcal{K}\left(I^{\prime}, J^{\prime}\right)=\left\{K_{i}\right\}_{i=1}^{B}$, and where $B=B\left(I^{\prime}, J^{\prime}\right)$ depends on the pair $\left(I^{\prime}, J^{\prime}\right)$ but is bounded by $C \frac{1}{\eta_{0}}$ independent of $\left(I^{\prime}, J^{\prime}\right)$ and the choice of $\eta_{1}$, and

$$
K_{i} \in \mathcal{G}, K_{i} \subset I^{\prime} \cap J^{\prime}, \ell\left(K_{i}\right)=2^{-m-1} \ell\left(J^{\prime}\right), \quad 1 \leq i \leq B
$$

There will be just one more use of random probability in dealing with the nearby form, and that will occur at the end of the finite iteration in Subsection 5.3 below.

5.2. Return of the original testing function. We now consider the inner product $\left\langle T_{\sigma}^{\alpha}\left(b_{A} \mathbf{1}_{K}\right), b_{B}^{*} \mathbf{1}_{K}\right\rangle_{\omega}$ and estimate the case when

$$
K \in \mathcal{G}, K \subset I^{\prime} \cap J^{\prime}, I^{\prime} \in \mathfrak{C}^{\mathfrak{r}}(I), J^{\prime} \in \mathfrak{C}(J), I \in \mathcal{C}_{A}^{\mathcal{A}}, J \in \mathcal{C}_{B}^{\mathcal{B}}, \ell(K)=2^{-m-1} \ell\left(J^{\prime}\right) .
$$

Recall that for $\eta \in\left(0, \frac{1}{2}\right]$ and any interval $K$, we defined $\partial_{\eta} K \equiv(1+\eta) K \backslash(1-\eta) K$ to be the ' $\eta$-halo' around the boundary, i.e. endpoints, of $K$. In what follows we will now take $\eta=\frac{1}{2}$ and invoke deterministic surgery with $\eta$-halos (which are $\frac{1}{2}$-halos), together with the energy condition and one last application of random surgery, as follows. For subsets $E, F \subset A \cap B$ and intervals $K \subset A \cap B$ we define

$$
\{E, F\} \equiv\left\langle T_{\sigma}^{\alpha}\left(b_{A} \mathbf{1}_{E}\right), b_{B}^{*} \mathbf{1}_{F}\right\rangle_{\omega},
$$

and

$$
K_{\text {in }} \equiv K \backslash \partial_{\eta} K \text { and } K_{\text {out }} \equiv K \cap \partial_{\eta} K
$$

and we write

$$
\{K, K\}=\left\{A, K_{\text {in }}\right\}-\left\{A \backslash K, K_{\text {in }}\right\}+\left\{K_{\text {out }}, K_{\text {out }}\right\}+\left\{K_{\text {in }}, K_{\text {out }}\right\} .
$$

Note that the first two terms on the right hand side of (5.12) decompose the inner product $\left\{K, K_{\text {in }}\right\}$, which 'includes' the difficult symmetric inner product $\left\{K_{\mathrm{in}}, K_{\mathrm{in}}\right\}$. Thus the difficult symmetric inner product is ultimately controlled by testing on the interval $A$ to handle $\left\{A, K_{\text {in }}\right\}$, and by using a trick that resurrects the original testing functions $\left\{b_{J}^{*, \text { orig }}\right\}_{J \in \mathcal{G}}$, discarded in the corona constructions above, to handle $\left\{A \backslash K, K_{\text {in }}\right\}$. More precisely, these original testing functions $b_{J}^{* \text {,orig }}$ are the testing functions obtained after reducing matters to the case of bounded testing functions with the pointwise lower bound property PLBP as in Conclusion [16] above.

The first term on the right side of (5.12) satisfies

$$
\left|\left\{A, K_{\mathrm{in}}\right\}\right|=\left|\int_{K_{\mathrm{in}}}\left(T_{\sigma}^{\alpha} b_{A}\right) b_{B}^{*} d \omega\right| \leq\left\|\mathbf{1}_{K_{\mathrm{in}}} T_{\sigma}^{\alpha} b_{A}\right\|_{L^{2}(\omega)}\left\|\mathbf{1}_{K_{\mathrm{in}}} b_{B}^{*}\right\|_{L^{2}(\omega)} \leq\left\|b_{B}^{*}\right\|_{\infty}\left\|\mathbf{1}_{K_{\mathrm{in}}} T_{\sigma}^{\alpha} b_{A}\right\|_{L^{2}(\omega)} \sqrt{\left|K_{\mathrm{in}}\right|_{\omega}} .
$$


Before proceeding further it will prove convenient to introduce some additional notation, namely we will write the energy estimate in the second display of the Energy Lemma as

$$
\left|\left\langle T^{\alpha} \nu, \Psi_{J}\right\rangle_{\omega}\right| \lesssim C_{\gamma} \mathrm{P}_{\delta}^{\alpha} \mathrm{Q}^{\omega}(J, v)\left\|\Psi_{J}\right\|_{L^{2}(\mu)}^{\star}, \quad \text { if } \int \Psi_{J} d \omega=0 \text { and } \gamma J \cap \operatorname{Supp} \nu=\emptyset, \gamma>1,
$$

where

$$
\mathrm{P}_{\delta}^{\alpha} \mathrm{Q}^{\omega}(J, v) \equiv \frac{\mathrm{P}^{\alpha}(J, \nu)}{|J|}\left\|\mathrm{Q}_{J}^{\omega, \mathbf{b}^{*}} x\right\|_{L^{2}(\omega)}^{\boldsymbol{\omega}}+\frac{\mathrm{P}_{1+\delta}^{\alpha}(J, \nu)}{|J|}\left\|x-m_{J}\right\|_{L^{2}\left(\mathbf{1}_{J} \omega\right)} .
$$

The use of the compact notation $\mathrm{P}_{\delta}^{\alpha} \mathrm{Q}^{\omega}(J, v)$ to denote the complicated expression on the right hand side will considerably reduce the size of many subsequent displays.

Let $K_{\mathrm{in}}^{\text {left }}$ and $K_{\mathrm{in}}^{\text {right }}$ denote the left and right children of $K_{\mathrm{in}}$, which until now have been written as $\left\{K_{\ell}^{\prime \prime}\right\}_{\ell=1}^{2}$ in no particular order, and we will continue to use both of these notations. We now claim that the second term on the right side of (5.12) satisfies

$$
\begin{aligned}
\left|\left\{A \backslash K, K_{\text {in }}\right\}\right| \lesssim & \left\{\mathrm{P}_{\delta}^{\alpha} \mathrm{Q}^{\omega}\left(K_{\mathrm{in}}^{\text {left }}, \mathbf{1}_{A \backslash K} \sigma\right)+\mathrm{P}_{\delta}^{\alpha} \mathrm{Q}^{\omega}\left(K_{\text {in }}^{\text {right }}, \mathbf{1}_{A \backslash K} \sigma\right)\right\} \sqrt{\left|K_{\text {in }}\right|_{\omega}} \\
& +\left(\sqrt{\int_{K_{\text {in }}}\left|T_{\sigma}^{\alpha} b_{A}\right|^{2} d \omega}+\left(\mathfrak{T}_{T^{\alpha}}+\sqrt{\mathfrak{A}_{2}^{\alpha}}\right) \sqrt{\left|K_{\text {in }}\right|_{\sigma}}\right) \sqrt{\left|K_{\text {in }}\right|_{\omega}} \\
& +\sum_{\ell=1}^{2}\left|\left\langle T_{\sigma}^{\alpha} b_{A} \mathbf{1}_{K_{\text {out }}}, b_{K_{\ell}^{\prime \prime}}^{*, \text { orig }}\right\rangle_{\omega}\right|,
\end{aligned}
$$

upon using a trick with the original testing functions $b_{K_{\ell}^{\prime \prime}}^{* \text {,orig }}$ for the two grandchildren $\left\{K_{\ell}^{\prime \prime}\right\}_{\ell=1}^{2}$ of the interval $K$ that lie strictly inside $K$, and whose union is $\frac{1}{2} K$. Indeed, to prove (5.16), we use the following identity whose proof is immediate (and whose origin will be made clear in the discussion below):

$$
\begin{aligned}
\text { 17) } & \left\langle T_{\sigma}^{\alpha}\left(b_{A} \mathbf{1}_{A \backslash K}\right), \mathbf{1}_{K_{\mathrm{in}}} b_{B}^{*}\right\rangle_{\omega}-\sum_{\ell=1}^{2}\left(\frac{\frac{1}{\left|K_{\ell}^{\prime \prime}\right|_{\omega}} \int_{K_{\ell}^{\prime \prime}} b_{B}^{*} d \omega}{\frac{1}{\left.K_{\ell}^{\prime \prime}\right|_{\omega}} \int_{K_{\ell}^{\prime \prime}} b_{K_{\ell}^{\prime \prime}}^{* \text { orig }} d \omega}\right)\left\langle T_{\sigma}^{\alpha}\left(b_{A} \mathbf{1}_{A \backslash K}\right), b_{K_{\ell}^{\prime \prime}}^{* \text { orig }}\right\rangle_{\omega} \\
= & \left\langle T_{\sigma}^{\alpha}\left(b_{A} \mathbf{1}_{A \backslash K}\right), \mathbf{1}_{K_{\mathrm{in}}} b_{B}^{*}\right\rangle_{\omega} \\
& -\sum_{\ell=1}^{2}\left(\frac{\frac{1}{\left|K_{\ell}^{\prime \prime}\right|_{\omega}} \int_{K_{\ell}^{\prime \prime}} b_{B}^{*} d \omega}{\frac{1}{\left|K_{\ell}^{\prime \prime}\right|_{\omega}} \int_{K_{\ell}^{\prime \prime}} b_{K_{\ell}^{\prime \prime}}^{*, \text { orig }} d \omega}\right)\left\{\left\langle T_{\sigma}^{\alpha} b_{A}, b_{K_{\ell}^{\prime \prime}}^{* \text { orig }}\right\rangle_{\omega}-\left\langle b_{A} \mathbf{1}_{K_{\mathrm{in}}}, T_{\omega}^{\alpha, *} b_{K_{\ell}^{\prime \prime}}^{* \text { orig }}\right\rangle_{\sigma}-\left\langle T_{\sigma}^{\alpha} b_{A} \mathbf{1}_{K_{\mathrm{out}}}, b_{K_{\ell}^{\prime \prime}}^{*, \text { orig }}\right\rangle_{\omega}\right\} \\
= & \left\langle T_{\sigma}^{\alpha}\left(b_{A} \mathbf{1}_{A \backslash K}\right), \mathbf{1}_{K_{\mathrm{in}}} b_{B}^{*}\right\rangle_{\omega}+\sum_{\ell=1}^{2}\left(\frac{\frac{1}{\left|K_{\ell}^{\prime \prime}\right|_{\omega}} \int_{K_{\ell}^{\prime \prime}} b_{B}^{*} d \omega}{\frac{1}{\left|K_{\ell}^{\prime \prime}\right|_{\omega}} \int_{K_{\ell}^{\prime \prime}} b_{K_{\ell}^{\prime \prime}}^{* \text { orig }} d \omega}\right)\left\langle T_{\sigma}^{\alpha} b_{A} \mathbf{1}_{K_{\mathrm{out}}}, b_{K_{\ell}^{\prime \prime}}^{* \text { orig }}\right\rangle_{\omega} \\
& -\sum_{\ell=1}^{2}\left(\frac{1}{\frac{1}{\left|K_{\ell}^{\prime \prime}\right|_{\omega}} \int_{K_{\ell}^{\prime \prime}} b_{B}^{*} d \omega}\right)\left\{\left\langle T_{\sigma}^{\alpha} b_{A}, b_{K_{\ell}^{\prime \prime}}^{* \text { orig }}\right\rangle_{\omega}-\left\langle b_{A} \mathbf{1}_{K_{\mathrm{in}}}, T_{\omega}^{\alpha, *} b_{K_{\ell}^{\prime \prime}}^{* \text { orig }}\right\rangle_{\sigma}\right\} .
\end{aligned}
$$

In fact we have the following estimate, more precise than (5.16).

Lemma 50. We have

$$
\begin{aligned}
& \left|\left\{A \backslash K, K_{\mathrm{in}}\right\}+\sum_{\ell=1}^{2}\left(\frac{\frac{1}{\left|K_{\ell}^{\prime \prime}\right|_{\omega}} \int_{K_{\ell}^{\prime \prime}} b_{B}^{*} d \omega}{\frac{1}{\left|K_{\ell}^{\prime \prime}\right|_{\omega}} \int_{K_{\ell}^{\prime \prime}} b_{K_{\ell}^{\prime \prime}}^{*, \text { orig }} d \omega}\right)\left\{K_{\text {out }}, K_{\mathrm{in}}^{\ell}\right\}^{\text {orig }}\right| \\
\lesssim & \left(\left\{\mathrm{P}_{\delta}^{\alpha} \mathrm{Q}^{\omega}\left(K_{\mathrm{in}}^{\mathrm{left}}, \mathbf{1}_{A \backslash K} \sigma\right)+\mathrm{P}_{\delta}^{\alpha} \mathrm{Q}^{\omega}\left(K_{\mathrm{in}}^{\text {right }}, \mathbf{1}_{A \backslash K} \sigma\right)\right\}+\sqrt{\int_{K_{\mathrm{in}}}\left|T_{\sigma}^{\alpha} b_{A}\right|^{2} d \omega}+\left(\mathfrak{T}_{T^{\alpha, *}}+\sqrt{\mathfrak{A}_{2}^{\alpha}}\right) \sqrt{\left|K_{\mathrm{in}}\right|_{\sigma}}\right) \sqrt{\left|K_{\mathrm{in}}\right|_{\omega}},
\end{aligned}
$$

where $b_{K_{\ell}^{\prime \prime}}^{* \text { orig }}$ is the original testing function for the grandchild $K_{\ell}^{\prime \prime}$ of $K$ in Conclusion 16 above, and where

$$
\left\{K_{\text {out }}, K_{\text {in }}^{\ell}\right\}^{\text {orig }} \equiv\left\langle T_{\sigma}^{\alpha} b_{A} \mathbf{1}_{K_{\text {out }}}, b_{K_{\ell}^{\prime \prime}}^{*, \text { orig }}\right\rangle_{\omega} \text { for } \ell \in\{1,2\} .
$$


Before starting the proof of the lemma, we motivate the identity (5.17) with the following discussion. A simpler way to start the analysis for for $\left\{A \backslash K, K_{\text {in }}\right\}=\left\langle T_{\sigma}^{\alpha}\left(b_{A} \mathbf{1}_{A \backslash K}\right), \mathbf{1}_{K_{\text {in }}} b_{B}^{*}\right\rangle_{\omega}$ would be to use instead of (5.17), the more obvious decomposition

$$
\begin{gathered}
\left\langle T_{\sigma}^{\alpha}\left(b_{A} \mathbf{1}_{A \backslash K}\right), \mathbf{1}_{K_{\mathrm{in}}} b_{B}^{*}\right\rangle_{\omega}=\left\langle T_{\sigma}^{\alpha}\left(b_{A} \mathbf{1}_{A \backslash K}\right), \mathbf{1}_{K_{\mathrm{in}}}\left(b_{B}^{*}-\frac{1}{\left|K_{\mathrm{in}}\right|_{\omega}} \int_{K_{\mathrm{in}}} b_{B}^{*} d \omega\right)\right\rangle_{\omega} \\
+\left\langle T_{\sigma}^{\alpha}\left(b_{A} \mathbf{1}_{A \backslash K}\right), \mathbf{1}_{K_{\mathrm{in}}}\left(\frac{1}{\left|K_{\mathrm{in}}\right|_{\omega}} \int_{K_{\mathrm{in}}} b_{B}^{*} d \omega\right)\right\rangle_{\omega} .
\end{gathered}
$$

For the first term in (5.18), we would like to apply the Energy Lemma to obtain

$$
\begin{aligned}
& \left|\left\langle T_{\sigma}^{\alpha}\left(b_{A} \mathbf{1}_{A \backslash K}\right), \mathbf{1}_{K_{\mathrm{in}}}\left(b_{B}^{*}-\frac{1}{\left|K_{\mathrm{in}}\right|_{\omega}} \int_{K_{\mathrm{in}}} b_{B}^{*} d \omega\right)\right\rangle_{\omega}\right| \\
\lesssim & \mathrm{P}_{\delta}^{\alpha} \mathrm{Q}^{\omega}\left(K_{\mathrm{in}}, \mathbf{1}_{A \backslash K} \sigma\right)\left\|\mathbf{1}_{K_{\mathrm{in}}}\left(b_{B}^{*}-\frac{1}{\left|K_{\mathrm{in}}\right|_{\omega}} \int_{K_{\mathrm{in}}} b_{B}^{*} d \omega\right)\right\|_{L^{2}(\omega)},
\end{aligned}
$$

using that the function $h_{K_{\text {in }}}^{*} \equiv \mathbf{1}_{K_{\text {in }}}\left(b_{B}^{*}-\frac{1}{\left|K_{\text {in }}\right|_{\omega}} \int_{K_{\text {in }}} b_{B}^{*} d \omega\right)$ has $\omega$-mean value zero and has support $K_{\text {in }}$ that is strictly separated from the support of $\left|b_{A}\right| \mathbf{1}_{A \backslash K}$. But a problem arises here since $K_{\text {in }}$ is not in the dyadic grid $\mathcal{G}$, despite the fact that $K$ itself is. Indeed, the Energy Lemma requires the dual martingale support of $h_{K_{\text {in }}}^{*}$ to be contained in $K_{\text {in }}$, so that we can take $\mathcal{H}$ in the Energy Lemma to be pseudoprojection onto $K_{\text {in }}$. However, if $K^{\prime}$ is a child of $K$, then $\square_{K^{\prime}}^{\omega, \mathbf{b}^{*}} h_{K_{\text {in }}}^{*}$ could be nonzero, yet $K^{\prime} \not \subset K_{\text {in }}$. This is easily fixed in two steps as follows.

First, recall $\eta=\frac{1}{2}$ so that

$$
K_{\text {in }}=(1-\eta) K=\frac{1}{2} K=\bigcup\left\{K^{\prime \prime} \in \mathfrak{C}^{(2)}(K): \partial K^{\prime \prime} \cap \partial K=\emptyset\right\}
$$

is the union of the 2 grandchildren $K^{\prime \prime}$ of $K$ whose boundaries are disjoint from the boundary of $K$. Then

$$
K_{\text {out }}=\bigcup\left\{K^{\prime \prime} \in \mathfrak{C}^{(2)}(K): \partial K^{\prime \prime} \cap \partial K \neq \emptyset\right\}
$$

is the union of the 2 grandchildren $K^{\prime \prime}$ of $K$ whose boundaries intersect the boundary of $K$. The only possible dyadic subintervals $K^{\prime}$ of $K$ for which both $\square_{K^{\prime}}^{\omega, \mathbf{b}^{*}} h_{K_{\text {in }}}^{*} \neq 0$ and $K^{\prime} \not \subset K_{\text {in }}$ are the children of $K$. Enumerate by $\left\{K_{\ell}^{\prime \prime}\right\}_{\ell=1}^{2}$ the grandchildren of $K$ whose boundaries are disjoint from the boundary of $K$.

Then second, instead of decomposing $\mathbf{1}_{K_{\text {in }}} b_{B}^{*}$ as $h_{K_{\text {in }}}^{*}$ plus $\mathbf{1}_{K_{\text {in }}} \frac{1}{\left|K_{\text {in }}\right|_{\omega}} \int_{K_{\text {in }}} b_{B}^{*} d \omega$, we decompose $\mathbf{1}_{K_{\text {in }}} b_{B}^{*}$ as

$$
\mathbf{1}_{K_{\mathrm{in}}} b_{B}^{*}=\sum_{\ell=1}^{2} \mathbf{1}_{K_{\ell}^{\prime \prime}}\left(b_{B}^{*}-\frac{1}{\left|K_{\ell}^{\prime \prime}\right|_{\omega}} \int_{K_{\ell}^{\prime \prime}} b_{B}^{*} d \omega\right)+\sum_{\ell=1}^{2} \mathbf{1}_{K_{\ell}^{\prime \prime}} \frac{1}{\left|K_{\ell}^{\prime \prime}\right|_{\omega}} \int_{K_{\ell}^{\prime \prime}} b_{B}^{*} d \omega,
$$

and then apply the Energy Lemma to the function

$$
k_{K_{\mathrm{in}}}^{*} \equiv \sum_{\ell=1}^{2} \mathbf{1}_{K_{\ell}^{\prime \prime}}\left(b_{B}^{*}-\frac{1}{\left|K_{\ell}^{\prime \prime}\right|_{\omega}} \int_{K_{\ell}^{\prime \prime}} b_{B}^{*} d \omega\right) \equiv k_{K_{\mathrm{in}}}^{* 1}+k_{K_{\mathrm{in}}}^{*, 2},
$$

which does indeed satisfy $\square_{K^{\prime}}^{\omega, \mathbf{b}^{*}} k_{K_{\text {in }}}^{*}=0$ unless $K^{\prime}$ is a dyadic subinterval of $K$ that is contained in $K_{\text {in }}$. (Furthermore, we could even replace grandchildren by $m$-grandchildren in this argument in order that $\square_{K^{\prime}}^{\omega, \mathbf{b}^{*}} k_{K_{\text {in }}}^{*}=0$ unless $K^{\prime}$ is a dyadic $m$-grandchild of $K$ that is contained in $K_{\text {in }}$, but we will not need this.) If we now use $k_{K_{\text {in }}}^{*}$ instead of $h_{K_{\text {in }}}^{*}$ in (5.18) and (5.19), we obtain

$$
\begin{aligned}
\left\langle T_{\sigma}^{\alpha}\left(b_{A} \mathbf{1}_{A \backslash K}\right), \mathbf{1}_{K_{\text {in }}} b_{B}^{*}\right\rangle_{\omega}= & \left\langle T_{\sigma}^{\alpha}\left(b_{A} \mathbf{1}_{A \backslash K}\right), k_{K_{\text {in }}}^{*}\right\rangle_{\omega} \\
& +\left\langle T_{\sigma}^{\alpha}\left(b_{A} \mathbf{1}_{A \backslash K}\right), \sum_{\ell=1}^{2} \mathbf{1}_{K_{\ell}^{\prime \prime}}\left(\frac{1}{\left|K_{\ell}^{\prime \prime}\right|_{\omega}} \int_{K_{\ell}^{\prime \prime}} b_{B}^{*} d \omega\right)\right\rangle_{\omega},
\end{aligned}
$$

and

$$
\begin{aligned}
(5.21)\left\langle T_{\sigma}^{\alpha}\left(b_{A} \mathbf{1}_{A \backslash K}\right), k_{K_{\text {in }}}^{*}\right\rangle_{\omega} \mid & \leq\left|\left\langle T_{\sigma}^{\alpha}\left(b_{A} \mathbf{1}_{A \backslash K}\right), k_{K_{\text {in }}}^{*, 1}\right\rangle_{\omega}\right|+\left|\left\langle T_{\sigma}^{\alpha}\left(b_{A} \mathbf{1}_{A \backslash K}\right), k_{K_{\text {in }}}^{*, 2}\right\rangle_{\omega}\right| \\
& \leq C_{\eta}\left\{\mathrm{P}_{\delta}^{\alpha} \mathrm{Q}^{\omega}\left(K_{\mathrm{in}}^{\mathrm{left}}, \mathbf{1}_{A \backslash K} \sigma\right)+\mathrm{P}_{\delta}^{\alpha} \mathrm{Q}^{\omega}\left(K_{\mathrm{in}}^{\mathrm{right}}, \mathbf{1}_{A \backslash K} \sigma\right)\right\}\left\|k_{K_{\mathrm{in}}}^{*}\right\|_{L^{2}(\omega)},
\end{aligned}
$$


where the constant $C_{\eta}$ depends on the constant $C_{\gamma}$ in the statement of the Monotonicity Lemma with $\gamma=\frac{1}{1-\eta}$ since $\frac{1}{1-\eta} K_{\text {in }} \cap(A \backslash K)=\emptyset$, and where we have written $\left\{K_{\ell}^{\prime \prime}\right\}_{\ell=1}^{2}=\left\{K_{\text {in }}^{\text {left }}, K_{\text {in }}^{\text {right }}\right\}$ with $K_{\text {in }}^{\text {left }}$ and $K_{\text {in }}^{\text {right }}$ denoting the left hand child and right hand child of $K_{\text {in }}$ respectively.

Conclusion 51. Thus we see that $\mathrm{P}_{\mathcal{H}}^{\omega, \mathbf{b}^{*}}$ and $\mathbf{Q}_{\mathcal{H}}^{\omega, \mathbf{b}^{*}}$ in the Energy Lemma can be taken to be pseudoprojection onto $K_{\mathrm{in}}$, i.e. $\mathrm{P}_{K_{\mathrm{in}}}^{\omega, \mathbf{b}^{*}}=\sum_{J \in \mathcal{G}: J \subset K_{\mathrm{in}}} \square_{J}^{\omega, \mathbf{b}^{*}}$ and $\mathbf{Q}_{K_{\mathrm{in}}}^{\omega, \mathbf{b}^{*}}=\sum_{J \in \mathcal{G}: J \subset K_{\mathrm{in}}} \triangle_{J}^{\omega, \mathbf{b}^{*}}$, and we will see below that the intervals $K_{\mathrm{in}}$ that arise in subsequent arguments will be pairwise disjoint. Furthermore, the energy condition will be used to control these full pseudoprojections $\mathrm{P}_{K_{\mathrm{in}}}^{\omega, \mathbf{b}^{*}}$ when taken over pairwise disjoint decompositions of intervals by subintervals of the form $K_{\text {in }}$.

However, the second line of (5.20) remains problematic, and this is our point of departure for beginning the proof of Lemma 50, in which we exploit the original testing functions $b_{K_{\ell}^{\prime \prime}}^{* \text {,orig }}$ in identity (5.17) in order to handle the second line of (5.20).

Proof of Lemma 50. We begin by rewriting identity (5.17) in the form

$$
\begin{aligned}
\boldsymbol{A} & =\boldsymbol{B}+\boldsymbol{C} \text { where } \\
\boldsymbol{A} & \equiv\left\langle T_{\sigma}^{\alpha}\left(b_{A} \mathbf{1}_{A \backslash K}\right), \mathbf{1}_{K_{\mathrm{in}}} b_{B}^{*}\right\rangle_{\omega}+\sum_{\ell=1}^{2}\left(\frac{\frac{1}{\left|K_{\ell}^{\prime \prime}\right|_{\omega}} \int_{K_{\ell}^{\prime \prime}} b_{B}^{*} d \omega}{\frac{1}{\left|K_{\ell}^{\prime \prime}\right|_{\omega}} \int_{K_{\ell}^{\prime \prime}} b_{K_{\ell}^{\prime \prime}}^{* \text { orig }} d \omega}\right)\left\langle T_{\sigma}^{\alpha} b_{A} \mathbf{1}_{K_{\text {out }}}, b_{K_{\ell}^{\prime \prime}}^{*, \text { orig }}\right\rangle_{\omega}, \\
\boldsymbol{B} & \equiv\left\langle T_{\sigma}^{\alpha}\left(b_{A} \mathbf{1}_{A \backslash K}\right), \mathbf{1}_{K_{\mathrm{in}}} b_{B}^{*}\right\rangle_{\omega}-\sum_{\ell=1}^{2}\left(\frac{\frac{1}{\left.K_{\ell}^{\prime \prime}\right|_{\omega}} \int_{K_{\ell}^{\prime \prime}} b_{B}^{*} d \omega}{\frac{1}{\left.K_{\ell}^{\prime \prime}\right|_{\omega}} \int_{K_{\ell}^{\prime \prime}} b_{K_{\ell}^{\prime \prime}}^{* \text { orig }} d \omega}\right)\left\langle T_{\sigma}^{\alpha}\left(b_{A} \mathbf{1}_{A \backslash K}\right), b_{K_{\ell}^{\prime \prime}}^{* \text { orig }}\right\rangle_{\omega}, \\
\boldsymbol{C} & \equiv \sum_{\ell=1}^{2}\left(\frac{\frac{1}{\left|K_{\ell}^{\prime \prime}\right|_{\omega}} \int_{K_{\ell}^{\prime \prime}} b_{B}^{*} d \omega}{\frac{1}{\left.K_{\ell}^{\prime \prime}\right|_{\omega}} \int_{K_{\ell}^{\prime \prime}} b_{K_{\ell}^{\prime \prime}}^{* \text { orig }} d \omega}\right)\left\{\left\langle T_{\sigma}^{\alpha} b_{A}, b_{K_{\ell}^{\prime \prime}}^{* \text { orig }}\right\rangle_{\omega}-\left\langle b_{A} \mathbf{1}_{K_{\mathrm{in}}}, T_{\omega}^{\alpha, *} b_{K_{\ell}^{\prime \prime}}^{*, \text { orig }}\right\rangle_{\sigma}\right\} .
\end{aligned}
$$

From the discussion above, we recall the identity (5.20) and the estimate (5.21). We also have the analogous identity and estimate with $b_{K_{\ell}^{\prime \prime}}^{* \text {,orig }}$ in place of $\mathbf{1}_{K_{\text {in }}} b_{B}^{*}$ :

$$
\begin{aligned}
\left\langle T_{\sigma}^{\alpha}\left(b_{A} \mathbf{1}_{A \backslash K}\right), b_{K_{\ell}^{\prime \prime}}^{* \text { orig }}\right\rangle_{\omega}= & \left\langle T_{\sigma}^{\alpha}\left(b_{A} \mathbf{1}_{A \backslash K}\right), \mathbf{1}_{K_{\ell}^{\prime \prime}}\left(b_{K_{\ell}^{\prime \prime}}^{* \text { orig }}-\frac{1}{\left|K_{\ell}^{\prime \prime}\right|_{\omega}} \int_{K_{\ell}^{\prime \prime}} b_{K_{\ell}^{\prime \prime}}^{* \text { orig }} d \omega\right)\right\rangle_{\omega} \\
& +\left\langle T_{\sigma}^{\alpha}\left(b_{A} \mathbf{1}_{A \backslash K}\right), \mathbf{1}_{K_{\ell}^{\prime \prime}}\left(\frac{1}{\left|K_{\ell}^{\prime \prime}\right|_{\omega}} \int_{K_{\ell}^{\prime \prime}} b_{K_{\ell}^{\prime \prime}}^{* \text { orig }} d \omega\right)\right\rangle_{\omega},
\end{aligned}
$$

and

$$
\begin{aligned}
& \left|\left\langle T_{\sigma}^{\alpha}\left(b_{A} \mathbf{1}_{A \backslash K}\right), \mathbf{1}_{K_{\ell}^{\prime \prime}}\left(b_{K_{\ell}^{\prime \prime}}^{* \text { orig }}-\frac{1}{\left|K_{\ell}^{\prime \prime}\right|_{\omega}} \int_{K_{\ell}^{\prime \prime}} b_{K_{\ell}^{\prime \prime}}^{* \text { orig }} d \omega\right)\right\rangle_{\omega}\right| \\
\lesssim & \mathrm{P}_{\delta}^{\alpha} \mathrm{Q}^{\omega}\left(K_{\ell}^{\prime \prime}, \mathbf{1}_{A \backslash K} \sigma\right)\left\|\mathbf{1}_{K_{\ell}^{\prime \prime}}\left(b_{K_{\ell}^{\prime \prime}}^{*, \text { orig }}-\frac{1}{\left|K_{\ell}^{\prime \prime}\right|_{\omega}} \int_{K_{\ell}^{\prime \prime}} b_{K_{\ell}^{\prime \prime}}^{* \text { orig }} d \omega\right)\right\|_{L^{2}(\omega)},
\end{aligned}
$$

for $1 \leq \ell \leq 2$, where the implied constants depend on $L^{\infty}$ norms of testing functions and the constant in the Energy Lemma. We will typically suppress dependence on $\eta=\frac{1}{2}$ from now on since there are no other values of $\eta$ used below. Thus we have, using that $K_{\ell}^{\prime \prime}$ is close to $K_{\text {in }}$ in both scale and position,

$$
\begin{aligned}
& \left|\left\langle T_{\sigma}^{\alpha}\left(b_{A} \mathbf{1}_{A \backslash K}\right), \mathbf{1}_{K_{\mathrm{in}}} b_{B}^{*}\right\rangle_{\omega}-\sum_{\ell=1}^{2}\left(\frac{1}{\left|K_{\ell}^{\prime \prime}\right|_{\omega}} \int_{K_{\ell}^{\prime \prime}} b_{B}^{*} d \omega\right)\left\langle T_{\sigma}^{\alpha}\left(b_{A} \mathbf{1}_{A \backslash K}\right), \mathbf{1}_{K_{\ell}^{\prime \prime}}\right\rangle_{\omega}\right| \\
\lesssim & \left\{\mathrm{P}_{\delta}^{\alpha} \mathrm{Q}^{\omega}\left(K_{\mathrm{in}}^{\mathrm{left}}, \mathbf{1}_{A \backslash K} \sigma\right)+\mathrm{P}_{\delta}^{\alpha} \mathrm{Q}^{\omega}\left(K_{\mathrm{in}}^{\mathrm{right}}, \mathbf{1}_{A \backslash K} \sigma\right)\right\}\left\|\mathbf{1}_{K_{\mathrm{in}}}\left(b_{B}^{*}-\frac{1}{\left|K_{\mathrm{in}}\right|_{\omega}} \int_{K_{\mathrm{in}}} b_{B}^{*} d \omega\right)\right\|_{L^{2}(\omega)} \\
\lesssim & \left\{\mathrm{P}_{\delta}^{\alpha} \mathrm{Q}^{\omega}\left(K_{\mathrm{in}}^{\mathrm{left}}, \mathbf{1}_{A \backslash K} \sigma\right)+\mathrm{P}_{\delta}^{\alpha} \mathrm{Q}^{\omega}\left(K_{\mathrm{in}}^{\mathrm{right}}, \mathbf{1}_{A \backslash K} \sigma\right)\right\} \sqrt{\left|K_{\mathrm{in}}\right|_{\omega}} .
\end{aligned}
$$


Then we obtain, upon applying (5.14) with the function $\Psi_{J}^{\ell}$ equal to

$$
\left(\frac{\frac{1}{\left|K_{\ell}^{\prime \prime}\right|_{\omega}} \int_{K_{\ell}^{\prime \prime}} b_{B}^{*} d \omega}{\frac{1}{\left|K_{\ell}^{\prime \prime}\right|_{\omega}} \int_{K_{\ell}^{\prime \prime}} b_{K_{\ell}^{\prime \prime}}^{* \text { orig }} d \omega}\right) b_{K_{\ell}^{\prime \prime}}^{*, \text { orig }}-\left(\frac{1}{\left|K_{\ell}^{\prime \prime}\right|_{\omega}} \int_{K_{\ell}^{\prime \prime}} b_{B}^{*} d \omega\right) \mathbf{1}_{K_{\ell}^{\prime \prime}}
$$

for $\ell=1,2$, and also using (5.24), that

$$
\begin{aligned}
& |\boldsymbol{B}|=\left|\left\langle T_{\sigma}^{\alpha}\left(b_{A} \mathbf{1}_{A \backslash K}\right), \mathbf{1}_{K_{\mathrm{in}}} b_{B}^{*}\right\rangle_{\omega}-\sum_{\ell=1}^{2}\left(\frac{\frac{1}{\left|K_{\ell}^{\prime \prime}\right|_{\omega}} \int_{K_{\ell}^{\prime \prime}} b_{B}^{*} d \omega}{\frac{1}{\left.K_{\ell}^{\prime \prime}\right|_{\omega}} \int_{K_{\ell}^{\prime \prime}} b_{K_{\ell}^{\prime \prime}}^{* \text { orig }} d \omega}\right)\left\langle T_{\sigma}^{\alpha}\left(b_{A} \mathbf{1}_{A \backslash K}\right), b_{K_{\ell}^{\prime \prime}}^{*, \text { orig }}\right\rangle_{\omega}\right| \\
= & \left|\left\langle T_{\sigma}^{\alpha}\left(b_{A} \mathbf{1}_{A \backslash K}\right), \mathbf{1}_{K_{\mathrm{in}}} b_{B}^{*}\right\rangle_{\omega}-\sum_{\ell=1}^{2}\left(\frac{1}{\left|K_{\ell}^{\prime \prime}\right|_{\omega}} \int_{K_{\ell}^{\prime \prime}} b_{B}^{*} d \omega\right)\left\langle T_{\sigma}^{\alpha}\left(b_{A} \mathbf{1}_{A \backslash K}\right), \mathbf{1}_{K_{\ell}^{\prime \prime}}\right\rangle_{\omega}\right| \\
& +O\left\{\sum_{\ell=1}^{2}\left(\frac{\mathrm{P}^{\alpha}\left(K_{\ell}^{\prime \prime}, \mathbf{1}_{A \backslash K} \sigma\right)}{\left|K_{\ell}^{\prime \prime}\right|}\left\|\mathrm{Q}_{K_{\ell}^{\prime \prime}}^{\omega, \mathbf{b}^{*}} x\right\|_{L^{2}(\omega)}^{\omega}+\frac{\mathrm{P}_{1+\delta}^{\alpha}\left(K_{\ell}^{\prime \prime}, \mathbf{1}_{A \backslash K} \sigma\right)}{\left|K_{\ell}^{\prime \prime}\right|}\left\|x-m_{K_{\ell}^{\prime \prime}}\right\|_{L^{2}\left(\mathbf{1}_{K_{\ell}^{\prime \prime}} \omega\right)}\right) \sqrt{\left|K_{\mathrm{in}}\right|_{\omega}}\right\} \\
\lesssim & \left\{\mathrm{P}_{\delta}^{\alpha} \mathrm{Q}^{\omega}\left(K_{\mathrm{in}}^{\text {left }}, \mathbf{1}_{A \backslash K} \sigma\right)+\mathrm{P}_{\delta}^{\alpha} \mathrm{Q}^{\omega}\left(K_{\mathrm{in}}^{\text {right }}, \mathbf{1}_{A \backslash K} \sigma\right)\right\} \sqrt{\left|K_{\mathrm{in}}\right|_{\omega}},
\end{aligned}
$$

since by the triangle inequality

$$
\begin{gathered}
\left\|\Psi_{J}^{\ell}\right\|_{L^{2}(\omega)}=\left\|\left(\frac{\frac{1}{\left|K_{\ell}^{\prime \prime}\right|_{\omega}} \int_{K_{\ell}^{\prime \prime}} b_{B}^{*} d \omega}{\frac{1}{\left|K_{\ell}^{\prime \prime}\right|_{\omega}} \int_{K_{\ell}^{\prime \prime}} b_{K_{\ell}^{\prime \prime}}^{*, \text { orig }} d \omega}\right) b_{K_{\ell}^{\prime \prime}}^{* \text { orig }}-\left(\frac{1}{\left|K_{\ell}^{\prime \prime}\right|_{\omega}} \int_{K_{\ell}^{\prime \prime}} b_{B}^{*} d \omega\right) \mathbf{1}_{K_{\ell}^{\prime \prime}}\right\|_{L^{2}(\omega)} \\
\lesssim\left|\frac{\int_{K_{\ell}^{\prime \prime}} b_{B}^{*} d \omega}{\int_{K_{\ell}^{\prime \prime}} b_{K_{\ell}^{\prime \prime}}^{* \text { orig }} d \omega}\right| \sqrt{\left|K_{\ell}^{\prime \prime}\right|_{\omega}}+\sqrt{\left|K_{\ell}^{\prime \prime}\right|_{\omega}} \lesssim \sqrt{\left|K_{\ell}^{\prime \prime}\right|_{\omega}} \leq \sqrt{\left|K_{\text {in }}\right|_{\omega}}, \quad 1 \leq \ell \leq 2 .
\end{gathered}
$$

Finally, turning our attention to term $C$, the reason for using the identity (5.17) now becomes clear namely the terms $\left\langle T_{\sigma}^{\alpha}\left(b_{A} \mathbf{1}_{A \backslash K}\right), b_{K_{\ell}^{\prime \prime}}^{*, \text { orig }}\right\rangle_{\omega}$, for which multiples are subtracted on the left side above, involves the original testing functions $b_{K_{\ell}^{\prime \prime}}^{*, \text { orig }}$ for which we have the full testing condition. Thus we have

$$
\begin{aligned}
& \left|\sum_{\ell=1}^{2}\left(\frac{\frac{1}{\left|K_{\ell}^{\prime \prime}\right|_{\omega}} \int_{K_{\ell}^{\prime \prime}} b_{B}^{*} d \omega}{\frac{1}{\left.K_{\ell}^{\prime \prime}\right|_{\omega}} \int_{K_{\ell}^{\prime \prime}} b_{K_{\ell}^{\prime \prime}}^{*, \text { orig }} d \omega}\right)\left\langle T_{\sigma}^{\alpha} b_{A}, b_{K_{\ell}^{\prime \prime}}^{* \text { orig }}\right\rangle_{\omega}\right| \\
\lesssim & \sum_{\ell=1}^{2} \sqrt{\int_{K_{\ell}^{\prime \prime}}\left|T_{\sigma}^{\alpha} b_{A}\right|^{2} d \omega} \sqrt{\int_{K_{\ell}^{\prime \prime}}\left|b_{K_{\ell}^{\prime \prime}}^{*, \text { orig }}\right|^{2} d \omega} \lesssim \sqrt{\int_{K_{\text {in }}}\left|T_{\sigma}^{\alpha} b_{A}\right|^{2} d \omega \sqrt{\left|K_{\text {in }}\right|_{\omega}}}
\end{aligned}
$$

and similarly

$$
\begin{aligned}
& \left|\sum_{\ell=1}^{2}\left(\frac{\frac{1}{\left|K_{\ell}^{\prime \prime}\right|_{\omega}} \int_{K_{\ell}^{\prime \prime}} b_{B}^{*} d \omega}{\frac{1}{\left.K_{\ell}^{\prime \prime}\right|_{\omega}} \int_{K_{\ell}^{\prime \prime}} b_{K_{\ell}^{\prime \prime}}^{*, \text { orig }} d \omega}\right)\left\langle b_{A} \mathbf{1}_{K_{\mathrm{in}}}, T_{\omega}^{\alpha, *} b_{K_{\ell}^{\prime \prime}}^{*, \text { orig }}\right\rangle_{\sigma}\right| \lesssim \sqrt{\int_{K_{\mathrm{in}}}\left|b_{A}\right|^{2} d \sigma} \sqrt{\int_{K_{\mathrm{in}}}\left|T_{\omega}^{\alpha, *} \sum_{\ell=1}^{2} b_{K_{\ell}^{\prime \prime}}^{*, \text { orig }}\right|^{2} d \sigma} \\
\lesssim & \left(\mathfrak{T}_{T^{\alpha, *}}+\sqrt{\mathfrak{A}_{2}^{\alpha}}\right) \sqrt{\int_{K_{\mathrm{in}}}\left|b_{A}\right|^{2} d \sigma} \sum_{\ell=1}^{2} \sqrt{\int\left|b_{K_{\ell}^{\prime \prime}}^{* \text { orig }}\right|^{2} d \omega} \lesssim\left(\mathfrak{T}_{T^{\alpha, *}}+\sqrt{\mathfrak{A}_{2}^{\alpha}}\right) \sqrt{\int_{K_{\mathrm{in}}}\left|b_{A}\right|^{2} d \sigma} \sqrt{\left|K_{\mathrm{in}}\right|_{\omega}},
\end{aligned}
$$

which together prove

$$
\begin{aligned}
|\boldsymbol{C}| & =\left|\sum_{\ell=1}^{2}\left(\frac{\frac{1}{\left|K_{\ell}^{\prime \prime}\right|_{\omega}} \int_{K_{\ell}^{\prime \prime}} b_{B}^{*} d \omega}{\frac{1}{\left|K_{\ell}^{\prime \prime}\right|_{\omega}} \int_{K_{\ell}^{\prime \prime}} b_{K_{\ell}^{\prime \prime}}^{* \text {,orig }} d \omega}\right)\left\{\left\langle T_{\sigma}^{\alpha} b_{A}, b_{K_{\ell}^{\prime \prime}}^{*, \text { orig }}\right\rangle_{\omega}-\left\langle b_{A} \mathbf{1}_{K_{\mathrm{in}}}, T_{\omega}^{\alpha, *} b_{K_{\ell}^{\prime \prime}}^{*, \text { orig }}\right\rangle_{\sigma}\right\}\right| \\
& \lesssim\left(\sqrt{\int_{K_{\mathrm{in}}}\left|T_{\sigma}^{\alpha} b_{A}\right|^{2} d \omega}+\left(\mathfrak{T}_{T^{\alpha, *}}+\sqrt{\mathfrak{A}_{2}^{\alpha}}\right) \sqrt{\left|K_{\mathrm{in}}\right|_{\sigma}}\right) \sqrt{\left|K_{\mathrm{in}}\right|_{\omega}} .
\end{aligned}
$$


This completes the proof of Lemma [50, and hence also that of (5.16) since $\left|\frac{\left.\frac{1}{\mid K_{\ell}^{\prime \prime \prime}}\right|_{\omega} \int_{K_{\ell}^{\prime \prime}} b_{B}^{*} d \omega}{\left|\frac{1}{K_{\ell}^{\prime \prime}}\right|_{\omega} \int_{K_{\ell}^{\prime \prime}} b_{K_{\ell}^{\prime \prime}}^{*, \text { orig }} d \omega}\right| \lesssim 1$.

The term $\left\{K_{\text {out }}, K_{\text {in }}^{\ell}\right\}^{\text {orig }}$ will be handled below by relatively crude estimates. If we write

$$
\begin{aligned}
& \{K, K\}=\left\{A, K_{\text {in }}\right\}-\left\{A \backslash K, K_{\text {in }}\right\}+\left\{K_{\text {out }}, K_{\text {out }}\right\}+\left\{K_{\text {in }}, K_{\text {out }}\right\} \\
& =\left\{A, K_{\mathrm{in}}\right\}-\left(\left\{A \backslash K, K_{\mathrm{in}}\right\}+\sum_{\ell=1}^{2}\left(\frac{\frac{1}{\left|K_{\ell}^{\prime \prime}\right|_{\omega}} \int_{K_{\ell}^{\prime \prime}} b_{B}^{*} d \omega}{\frac{1}{\left|K_{\ell}^{\prime \prime \prime}\right|_{\omega}} \int_{K_{\ell}^{\prime \prime}} b_{K_{\ell}^{\prime \prime}}^{* \text { orig }} d \omega}\right)\left\{K_{\text {out }}, K_{\text {in }}^{\ell}\right\}^{\text {orig }}\right) \\
& +\sum_{\ell=1}^{2}\left(\frac{\frac{1}{\left|K_{\ell}^{\prime \prime}\right|_{\omega}} \int_{K_{\ell}^{\prime \prime}} b_{B}^{*} d \omega}{\frac{1}{\left|K_{\ell}^{\prime \prime}\right|_{\omega}} \int_{K_{\ell}^{\prime \prime}} b_{K_{\ell}^{\prime \prime}}^{* \text { orig }} d \omega}\right)\left\{K_{\text {out }}, K_{\text {in }}^{\ell}\right\}^{\text {orig }}+\left\{K_{\text {out }}, K_{\text {out }}\right\}+\left\{K_{\text {in }}, K_{\text {out }}\right\},
\end{aligned}
$$

then, using (5.13) and Lemma 50, we see that we have reduced control of $\{K, K\}$ to control of

$$
\sum_{\ell=1}^{2}\left(\frac{\frac{1}{\left|K_{\ell}^{\prime \prime}\right|_{\omega}} \int_{K_{\ell}^{\prime \prime}} b_{B}^{*} d \omega}{\frac{1}{\left|K_{\ell}^{\prime \prime}\right|_{\omega}} \int_{K_{\ell}^{\prime \prime}} b_{K_{\ell}^{\prime \prime}}^{* \text { orig }} d \omega}\right)\left\{K_{\text {out }}, K_{\text {in }}^{\ell}\right\}^{\text {orig }}+\left\{K_{\text {out }}, K_{\text {out }}\right\}+\left\{K_{\text {in }}, K_{\text {out }}\right\} .
$$

Altogether then, using the above estimates, we have proved

$$
\begin{aligned}
& \left|\{K, K\}-\left\{K_{\text {out }}, K_{\text {out }}\right\}-\left\{K_{\text {in }}, K_{\text {out }}\right\}-\sum_{\ell=1}^{2}\left(\frac{\frac{1}{\left|K_{\ell}^{\prime \prime}\right|_{\omega}} \int_{K_{\ell}^{\prime \prime}} b_{B}^{*} d \omega}{\frac{1}{\left|K_{\ell}^{\prime \prime}\right|_{\omega}} \int_{K_{\ell}^{\prime \prime}} b_{K_{\ell}^{\prime \prime}}^{*, \text { orig }} d \omega}\right)\left\{K_{\text {out }}, K_{\text {in }}^{\ell}\right\}^{\text {orig }}\right| \\
\lesssim & \left\|\mathbf{1}_{K_{\text {in }}} T_{\sigma}^{\alpha} b_{A}\right\|_{L^{2}(\omega)} \sqrt{\left|K_{\text {in }}\right|_{\omega}}+C\left\{\mathrm{P}_{\delta}^{\alpha} \mathrm{Q}^{\omega}\left(K_{\text {in }}^{\text {left }}, \mathbf{1}_{A \backslash K} \sigma\right)+\mathrm{P}_{\delta}^{\alpha} \mathrm{Q}^{\omega}\left(K_{\text {in }}^{\text {right }}, \mathbf{1}_{A \backslash K} \sigma\right)\right\} \sqrt{\left|K_{\text {in }}\right|_{\omega}} \\
\lesssim & C\left(\mathfrak{T}_{T^{\alpha, *}}+\mathfrak{A}_{2}^{\alpha}\right) \sqrt{\left|K_{\text {in }}\right|_{\sigma}} \sqrt{\left|K_{\text {in }}\right|_{\omega}} .
\end{aligned}
$$

We emphasize that this bound did not use any special information regarding $K$ being in the coronas $\mathcal{C}_{A}, \mathcal{C}_{B}$ or not, and thus holds for any interval $K$ contained in $A \cap B$. For clarity of notation we define

$$
\begin{aligned}
\Phi^{A, B}\left(K_{\mathrm{in}}\right) \equiv & \left\|\mathbf{1}_{K_{\mathrm{in}}} T_{\sigma}^{\alpha} b_{A}\right\|_{L^{2}(\omega)} \sqrt{\left|K_{\mathrm{in}}\right|_{\omega}} \\
& +C\left\{\mathrm{P}_{\delta}^{\alpha} \mathrm{Q}^{\omega}\left(K_{\mathrm{in}}^{\text {left }}, \mathbf{1}_{A \backslash K} \sigma\right)+\mathrm{P}_{\delta}^{\alpha} \mathrm{Q}^{\omega}\left(K_{\mathrm{in}}^{\mathrm{right}}, \mathbf{1}_{A \backslash K} \sigma\right)\right\} \sqrt{\left|K_{\mathrm{in}}\right|_{\omega}} \\
& +C\left(\mathfrak{T}_{T^{\alpha, *}}+\mathfrak{A}_{2}^{\alpha}\right) \sqrt{\left|K_{\mathrm{in}}\right|_{\sigma}} \sqrt{\left|K_{\mathrm{in}}\right|_{\omega}},
\end{aligned}
$$

where $\Phi^{A, B}$ should not be confused with the notation $\Phi^{\alpha}$ introduced for the Monotonicity Lemma, and we also define the constants

$$
A_{K_{\mathrm{in}}^{\ell}} \equiv \frac{\frac{1}{\left.K_{\ell}^{\prime \prime}\right|_{\omega}} \int_{K_{\ell}^{\prime \prime}} b_{B}^{*} d \omega}{\frac{1}{\left|K_{\ell}^{\prime \prime}\right|_{\omega}} \int_{K_{\ell}^{\prime \prime}} b_{K_{\ell}^{\prime \prime}}^{*, \text { orig }} d \omega}, \quad \ell \in\{1,2\}
$$

so that we can rewrite (5.25) as

$$
\left|\{K, K\}-\left\{K_{\text {out }}, K_{\text {out }}\right\}-\left\{K_{\text {in }}, K_{\text {out }}\right\}-\sum_{\ell=1}^{2} A_{K_{\text {in }}^{\ell}}\left\{K_{\text {out }}, K_{\text {in }}^{\ell}\right\}^{\text {orig }}\right| \lesssim \Phi^{A, B}\left(K_{\text {in }}\right), \quad K \in \mathcal{G}, K \subset A \cap B .
$$

We can further simplify notation by defining

$$
\left\{K_{\text {out }}, K_{\text {in }}\right\}^{\text {orig }} \equiv \sum_{\ell=1}^{2} A_{K_{\text {in }}^{\ell}}\left\{K_{\text {out }}, K_{\text {in }}^{\ell}\right\}^{\text {orig }},
$$

which we will often use below. At this point, as we will see below, the only problematic inner product subtracted from $\{K, K\}$ on the left hand side of (5.27) is $\left\{K_{\text {out }}, K_{\text {out }}\right\}$, and we will handle this by iterating (5.27) finitely many times and then appealing to a final probability argument starting in (5.32) below. 
5.3. A finite iteration and final random surgery. For $K$ an interval, we write $K_{\text {out }}=K_{\text {left }} \cup K_{\text {right }}$ where $K_{\text {left }}$ and $K_{\text {right }}$ are the two small subintervals on the left and right hand sides of $K$ respectively, and then we have

$$
\left\{K_{\text {out }}, K_{\text {out }}\right\}=\left\{K_{\text {left }}, K_{\text {left }}\right\}+\left\{K_{\text {right }}, K_{\text {right }}\right\}+\left\{K_{\text {left }}, K_{\text {right }}\right\}+\left\{K_{\text {right }}, K_{\text {left }}\right\},
$$

so that (5.27) can be written using (5.28) as

$$
\begin{aligned}
\{K, K\}= & \left\{K_{\text {out }}, K_{\text {out }}\right\}+\left\{K_{\text {in }}, K_{\text {out }}\right\}+\left\{K_{\text {out }}, K_{\text {in }}\right\}^{\text {orig }}+O\left[\Phi^{A, B}\left(K_{\text {in }}\right)\right] \\
= & \left\{K_{\text {left }}, K_{\text {left }}\right\}+\left\{K_{\text {right }}, K_{\text {right }}\right\}+\left\{K_{\text {in }}, K_{\text {out }}\right\}+\left\{K_{\text {out }}, K_{\text {in }}\right\}^{\text {orig }} \\
& +\left\{K_{\text {left }}, K_{\text {right }}\right\}+\left\{K_{\text {right }}, K_{\text {left }}\right\}+O\left[\Phi^{A, B}\left(K_{\text {in }}\right)\right] .
\end{aligned}
$$

At this point we observe that the terms $\left\{K_{\text {in }}, K_{\text {out }}\right\},\left\{K_{\text {out }}, K_{\text {in }}\right\}^{\text {orig }},\left\{K_{\text {left }}, K_{\text {right }}\right\}$, and $\left\{K_{\text {right }}, K_{\text {left }}\right\}$ can all be handled somewhat crudely by separation (3.22):

$$
\begin{aligned}
& \left|\left\{K_{\mathrm{in}}, K_{\mathrm{out}}\right\}\right|=\left|\int_{K_{\mathrm{out}}}\left[T_{\sigma}^{\alpha}\left(\mathbf{1}_{K_{\mathrm{in}}} b_{A}\right)\right] b_{B}^{*} d \omega\right| \\
\lesssim & \sqrt{\mathcal{A}_{2}^{\alpha}}\left(\int_{K_{\mathrm{in}}}\left|b_{A}\right|^{2} d \sigma\right)^{\frac{1}{2}}\left(\int_{K_{\mathrm{out}}}\left|b_{B}^{*}\right|^{2} d \omega\right)^{\frac{1}{2}} \lesssim \sqrt{\mathcal{A}_{2}^{\alpha}} \sqrt{\left|K_{\mathrm{in}}\right|_{\sigma}} \sqrt{\left|K_{\mathrm{out}}\right|_{\omega}},
\end{aligned}
$$

and similarly

$$
\begin{aligned}
& \left|\left\{K_{\text {out }}, K_{\text {in }}\right\}^{\text {orig }}\right| \lesssim \sqrt{\mathcal{A}_{2}^{\alpha}} \sqrt{\left|K_{\text {out }}\right|_{\sigma}} \sqrt{\left|K_{\text {in }}\right|_{\omega}}, \\
& \left|\left\{K_{\text {left }}, K_{\text {right }}\right\}\right|+\left|\left\{K_{\text {right }}, K_{\text {left }}\right\}\right| \lesssim \sqrt{\mathcal{A}_{2}^{\alpha}} \sqrt{\left|K_{\text {out }}\right|_{\sigma}} \sqrt{\left|K_{\text {out }}\right|_{\omega}} .
\end{aligned}
$$

Thus we have

$$
\{K, K\}=\left\{K_{\text {left }}, K_{\text {left }}\right\}+\left\{K_{\text {right }}, K_{\text {right }}\right\}+O\left[\Phi^{A, B}\left(K_{\text {in }}\right)\right]+\mathcal{A}_{2}^{\alpha} \sqrt{|K|_{\sigma}} \sqrt{|K|_{\omega}} .
$$

Upon application of a single iteration we obtain

$$
\begin{aligned}
\{K, K\}= & \left\{K_{\text {left left }}, K_{\text {left left }}\right\}+\left\{K_{\text {left right }}, K_{\text {left right }}\right\}+\left\{K_{\text {right left }}, K_{\text {right left }}\right\}+\left\{K_{\text {right right }}, K_{\text {right right }}\right\} \\
& +O\left[\Phi^{A, B}\left(K_{\text {in }}\right)+\Phi^{A, B}\left(K_{\text {left in }}\right)+\Phi^{A, B}\left(K_{\text {right in }}\right)\right] \\
& +\mathcal{A}_{2}^{\alpha}\left(\sqrt{|K|_{\sigma}} \sqrt{|K|_{\omega}}+\sqrt{\left|K_{\text {left }}\right|_{\sigma}} \sqrt{\left|K_{\text {left }}\right|_{\omega}}+\sqrt{\left|K_{\text {right }}\right|_{\sigma}} \sqrt{\left|K_{\text {right }}\right|_{\omega}}\right)
\end{aligned}
$$

and then iterating finitely many more times gives for $n \in \mathbb{N}$,

$$
\begin{aligned}
\{K, K\} & =\sum_{M \in \mathcal{M}_{n}}\{M, M\}+O\left(\sum_{M \in \mathcal{M}_{n}^{*}}\left[\Phi^{A, B}\left(M_{\mathrm{in}}\right)\right]\right)+\sqrt{\mathcal{A}_{2}^{\alpha}} \sum_{M \in \mathcal{M}_{n}^{*}} \sqrt{|M|_{\sigma}} \sqrt{|M|_{\omega}} \\
& \equiv A(K)+B(K)+C(K)=A_{\left(I^{\prime}, J^{\prime}\right)}(K)+B_{\left(I^{\prime}, J^{\prime}\right)}(K)+C_{\left(I^{\prime}, J^{\prime}\right)}(K),
\end{aligned}
$$

where the collections of intervals $\mathcal{M}_{n}=\mathcal{M}_{n}(K)$ and $\mathcal{M}_{n}^{*}=\mathcal{M}_{n}^{*}(K)$ are defined recursively by

$$
\begin{aligned}
\mathcal{M}_{0} & \equiv\{K\}, \\
\mathcal{M}_{k+1} & \equiv \bigcup_{n}^{n}\left\{M_{\text {left }}, M_{\text {right }}: M \in \mathcal{M}_{k}\right\}, \quad k \geq 0, \\
\mathcal{M}_{n}^{*} & \equiv \bigcup_{k=0}^{n} \mathcal{M}_{k} .
\end{aligned}
$$

We will include the subscript $\left(I^{\prime}, J^{\prime}\right)$ in the notation when we want to indicate the pair $\left(I^{\prime}, J^{\prime}\right)$ for which $K \in \mathcal{K}\left(I^{\prime}, J^{\prime}\right)$ as defined in (5.8) above. Now the term $C(K)$ can be estimated by the crude estimate

$$
C(K)=\sqrt{\mathcal{A}_{2}^{\alpha}} \sum_{M \in \mathcal{M}_{n}^{*}} \sqrt{|M|_{\sigma}} \sqrt{|M|_{\omega}} \leq C_{n} \sqrt{\mathcal{A}_{2}^{\alpha}} \sqrt{|K|_{\sigma}} \sqrt{|K|_{\omega}},
$$


where $n$ is chosen below depending on $\eta_{0}$. For the first term $A(K)$, we will apply the norm inequality and use probability, namely

$$
\begin{aligned}
|A(K)| & \leq \sqrt{C_{\mathbf{b}} C_{\mathbf{b}^{*}}} \mathfrak{N}_{T^{\alpha}} \sum_{M \in \mathcal{M}_{n}} \sqrt{|M|_{\sigma}} \sqrt{|M|_{\omega}} \\
& \leq \sqrt{C_{\mathbf{b} C_{\mathbf{b}^{*}}}} \mathfrak{N}_{T^{\alpha}} \sqrt{\sum_{M \in \mathcal{M}_{n}}|M|_{\sigma}} \sqrt{\sum_{M \in \mathcal{M}_{n}}|M|_{\omega}} \leq \sqrt{C_{\mathbf{b}} C_{\mathbf{b}^{*}}} \mathfrak{N}_{T^{\alpha}} \sqrt{\sum_{M \in \mathcal{M}_{n}}|M|_{\sigma}} \sqrt{|K|_{\omega}}
\end{aligned}
$$

where $\sqrt{C_{\mathbf{b}} C_{\mathbf{b}^{*}}}$ is an upper bound for the testing functions involved, followed by

$$
\boldsymbol{E}_{\Omega}^{\mathcal{G}}\left(\sum_{M \in \mathcal{M}_{n}}|M|_{\sigma}\right) \leq \varepsilon\left|I^{\prime}\right|_{\sigma}
$$

for a sufficiently small $\varepsilon>0$, where roughly speaking, we use the fact that the intervals $M \in \mathcal{M}_{n}$ depend on the grid $\mathcal{G}$ and form a relatively small proportion of $I^{\prime}$, which captures only a small amount of the total mass $\left|I^{\prime}\right|_{\sigma}$ as the grid is translated relative to the grid $\mathcal{D}$ that contains $I^{\prime}$. To be a bit more precise, recall that the intervals $K$ are uniquely constructed as consecutive adjacent intervals of equal length in the grid $\mathcal{G}$ that start out at the endpoint of $J^{\prime}$ that lies inside $I^{\prime}$, and then progress toward the constructed endpoint of $I^{\prime \prime}=I^{\prime} \backslash \partial_{\eta_{1}} I^{\prime}$ lying in the interior of $J^{\prime}$. Thus translates of the grid $\mathcal{G}$ result in translating the intervals $K$ across the interval $I^{\prime}$ a distance comparable to at least the length of $K$, and where $I^{\prime}$ is fixed in the grid $\mathcal{D}$. As a consequence the intervals $M \in \mathcal{M}_{n}(K)$ are also translated across the fixed interval $I^{\prime}$ a distance comparable to at least $\ell(K)$, and the standard halo estimate (3.7) applies since the sum of the lengths of the intervals $M \in \mathcal{M}_{n}$ is a small proportion of the length of $J^{\prime}$, whose length is at most that of $I^{\prime}$.

Here are the specific details. Recall that the intervals $K$ are taken from the set of consecutive intervals $\left\{K_{i}\right\}_{i=1}^{B}$ that lie in $I^{\prime} \cap J^{\prime}$, that the intervals $M \in \mathcal{M}_{n}\left(K_{i}\right)$ have length $\frac{1}{4^{n}} \ell\left(K_{i}\right)$, and that there are $2^{n}$ such intervals in $\mathcal{M}_{n}\left(K_{i}\right)$ for each $i$. Thus we have

$$
\begin{aligned}
\sum_{M \in \mathcal{M}_{n}(K)}|M| & =\sum_{M \in \mathcal{M}_{n}(K)} \frac{1}{4^{n}}|K|=2^{n} \frac{1}{4^{n}}|K|=\frac{1}{2^{n}}|K| \\
& \Longrightarrow \boldsymbol{E}_{\Omega}^{\mathcal{G}}\left(\sum_{i=1}^{B} \sum_{M \in \mathcal{M}_{n}\left(K_{i}\right)}|M|_{\sigma}\right) \leq C \frac{1}{\eta_{0}} \frac{1}{2^{n}}\left|I^{\prime}\right|_{\sigma} \leq \eta_{0}\left|I^{\prime}\right|_{\sigma}
\end{aligned}
$$

where we have used that the variable $B$ is at most $\frac{1}{\eta_{0}}$, and where the final inequality holds if $n$ is chosen large enough that $\frac{1}{2^{n}} \leq \eta_{0}^{2}$. Then we have by Cauchy-Schwarz applied first to $\sum_{i=1}^{B} \sum_{M \in \mathcal{M}_{n}\left(K_{i}\right)}$ and then to $\boldsymbol{E}_{\Omega}^{\mathcal{G}}$

$$
\begin{aligned}
& \boldsymbol{E}_{\Omega}^{\mathcal{G}}\left(\sum_{i=1}^{B}\left|A\left(K_{i}\right)\right|\right) \leq \boldsymbol{E}_{\Omega}^{\mathcal{G}} \sqrt{C_{\mathbf{b}} C_{\mathbf{b}^{*}}} \mathfrak{N}_{T^{\alpha}} \sqrt{\sum_{i=1}^{B} \sum_{M \in \mathcal{M}_{n}\left(K_{i}\right)}|M|_{\sigma} \sqrt{\left|J^{\prime}\right|_{\omega}}} \\
\leq & \sqrt{C_{\mathbf{b}} C_{\mathbf{b}^{*}}} \mathfrak{N}_{T^{\alpha}} \sqrt{\boldsymbol{E}_{\Omega}^{\mathcal{G}} \sum_{i=1}^{B} \sum_{M \in \mathcal{M}_{n}\left(K_{i}\right)}|M|_{\sigma}} \sqrt{\left|J^{\prime}\right|_{\omega}} \\
\leq & \sqrt{C_{\mathbf{b}} C_{\mathbf{b}^{*}}} \mathfrak{N}_{T^{\alpha}} \sqrt{\eta_{0}\left|I^{\prime}\right|_{\sigma}} \sqrt{\left|J^{\prime}\right|_{\omega}}=\sqrt{C_{\mathbf{b}} C_{\mathbf{b}^{*}}} \sqrt{\eta_{0}} \mathfrak{N}_{T^{\alpha}} \sqrt{\left|I^{\prime}\right|_{\sigma}} \sqrt{\left|J^{\prime}\right|_{\omega}},
\end{aligned}
$$

as required.

Now we turn to summing up the remaining terms $B(K)=C \sum_{M \in \mathcal{M}_{n}^{*}} \Phi^{A, B}\left(M_{\text {in }}\right)$ above. We begin by claiming that in the case when the interval $I^{\prime}$ is a natural child of $I$, i.e. $I^{\prime} \in \mathfrak{C}_{\text {natural }}(I)$ so that $I^{\prime} \in \mathcal{C}_{A}^{\mathcal{A}}$, we 
have

$$
\begin{aligned}
& \quad\left(\sum_{M \in \mathcal{M}_{n}^{*}(K)}\left\|\mathbf{1}_{M_{\mathrm{in}}} T_{\sigma}^{\alpha} b_{A}\right\|_{L^{2}(\omega)}^{2}\right)^{\frac{1}{2}}\left(\sum_{M \in \mathcal{M}_{n}^{*}(K)}\left|M_{\mathrm{in}}\right|_{\omega}\right)^{\frac{1}{2}} \\
& +\left(\sum_{M \in \mathcal{M}_{n}^{*}(K)}\left\{\mathrm{P}_{\delta}^{\alpha} \mathrm{Q}^{\omega}\left(M_{\mathrm{in}}^{\text {left }}, \mathbf{1}_{A} \sigma\right)^{2}+\mathrm{P}_{\delta}^{\alpha} \mathrm{Q}^{\omega}\left(M_{\mathrm{in}}^{\mathrm{right}}, \mathbf{1}_{A} \sigma\right)^{2}\right\}\right)^{\frac{1}{2}}\left(\sum_{M \in \mathcal{M}_{n}^{*}(K)}\left|M_{\mathrm{in}}\right|_{\omega}\right)^{\frac{1}{2}} \\
& \lesssim\left(\mathfrak{T}_{T^{\alpha}}^{\mathrm{b}}+\mathcal{E}_{2}^{\alpha}+\sqrt{\mathcal{A}_{2}^{\alpha, *}+\mathcal{A}_{2}^{\alpha}}\right) \sqrt{\left|I^{\prime}\right|_{\sigma}\left|J^{\prime}\right|_{\omega}} \lesssim \mathcal{N} \mathcal{T} \mathcal{V}_{\alpha} \sqrt{\left|I^{\prime}\right|_{\sigma}\left|J^{\prime}\right|_{\omega}},
\end{aligned}
$$

where the last line is a consequence of the crucial fact that the intervals $\left\{M_{\mathrm{in}}\right\}_{M \in \mathcal{M}_{n}^{*}(K)}$ form a pairwise disjoint subdecomposition of $K \subset I^{\prime} \cap J^{\prime}$ (for any $n \geq 1$ ). Indeed, we then have the following inequalities

(1) the first sum on the left hand side satisfies

$$
\sum_{M \in \mathcal{M}_{n}^{*}(K)}\left\|\mathbf{1}_{M_{\mathrm{in}}} T_{\sigma}^{\alpha} b_{A}\right\|_{L^{2}(\omega)}^{2}=\sum_{M \in \mathcal{M}_{n}^{*}(K)} \int_{M_{\mathrm{in}}}\left|T_{\sigma}^{\alpha} b_{A}\right|^{2} d \omega \leq \int_{I^{\prime}}\left|T_{\sigma}^{\alpha} b_{A}\right|^{2} d \omega \lesssim\left(\mathfrak{T}_{T^{\alpha}}^{\mathbf{b}}\right)^{2}\left|I^{\prime}\right|_{\sigma},
$$

by the weak testing condition for $I^{\prime}$ in the corona $\mathcal{C}_{A}$,

(2) and

$$
\sum_{M \in \mathcal{M}_{n}^{*}(K)}\left|M_{\mathrm{in}}\right|_{\omega} \leq|K|_{\omega} \leq\left|J^{\prime}\right|_{\omega}
$$

(3) and, using the definition of $\mathrm{P}_{\delta}^{\alpha} \mathrm{Q}^{\omega}(J, v)$ in (5.15),

$$
\begin{aligned}
& \sum_{M \in \mathcal{M}_{n}^{*}(K)}\left\{\mathrm{P}_{\delta}^{\alpha} \mathrm{Q}^{\omega}\left(M_{\mathrm{in}}^{\text {left }}, \mathbf{1}_{A} \sigma\right)^{2}+\mathrm{P}_{\delta}^{\alpha} \mathrm{Q}^{\omega}\left(M_{\mathrm{in}}^{\text {right }}, \mathbf{1}_{A} \sigma\right)^{2}\right\} \\
\lesssim & \left.\sum_{M_{\mathcal{M} \mathcal{M}_{n}^{*}(K)}\left\{\left(\frac{\mathrm{P}^{\alpha}\left(M_{\mathrm{in}}^{\text {left }}, \mathbf{1}_{A} \sigma\right)}{\left|M_{\mathrm{in}}^{\text {left }}\right|}\right)^{2}\left\|x-m_{M_{\mathrm{in}}^{\text {left }}}\right\|_{L^{2}}^{2}\left(\mathbf{1}_{M_{\mathrm{in}}^{\text {left }}} \omega\right)\right.}+\left(\frac{\mathrm{P}^{\alpha}\left(M_{\mathrm{in}}^{\mathrm{right}}, \mathbf{1}_{A} \sigma\right)}{\left|M_{\mathrm{in}}^{\text {right }}\right|}\right)^{2}\left\|x-m_{M_{\mathrm{in}}^{\text {right }}}\right\|_{L^{2}}^{2}\left(\mathbf{1}_{M_{\mathrm{in}}^{\text {right }}}{ }^{\text {righ }}\right)\right\} \\
\lesssim & \mathcal{N} \mathcal{T} \mathcal{V}_{\alpha}\left|I^{\prime}\right|_{\sigma},
\end{aligned}
$$

upon using the stopping energy condition for $I^{\prime}$ in the corona $\mathcal{C}_{A}$, i.e. the failure of (2.21), in the corona $\mathcal{C}_{A}$ with the subdecomposition $I^{\prime} \supset \bigcup_{M \in \mathcal{M}_{n}^{*}(K)}\left(M_{\mathrm{in}}^{\text {left }} \bigcup M_{\mathrm{in}}^{\text {right }}\right)$.

This completes the proof of (5.34). Our next claim is the inequality

$$
\begin{aligned}
& \left(\sum_{M \in \mathcal{M}_{n}^{*}(K)} \int_{M_{\mathrm{in}}}\left|T_{\sigma}^{\alpha} b_{A}\right|^{2} d \omega+\left(\mathfrak{T}_{T^{\alpha, *}}+\mathfrak{A}_{2}^{\alpha}\right)^{2}\left|M_{\mathrm{in}}\right|_{\sigma}\right)^{\frac{1}{2}}\left(\sum_{M \in \mathcal{M}_{n}^{*}(K)}\left|M_{\mathrm{in}}\right|_{\omega}\right)^{\frac{1}{2}} \\
\lesssim & \left(\int_{I^{\prime}}\left|T_{\sigma}^{\alpha} b_{A}\right|^{2} d \omega+\left(\mathfrak{T}_{T^{\alpha, *}}+\mathfrak{A}_{2}^{\alpha}\right)^{2}\left|I^{\prime}\right|_{\sigma}\right)^{\frac{1}{2}}\left(\left|J^{\prime}\right|_{\omega}\right)^{\frac{1}{2}} \lesssim \mathcal{N} \mathcal{T} \mathcal{V}_{\alpha} \sqrt{\left|I^{\prime}\right|_{\sigma}\left|J^{\prime}\right|_{\omega}},
\end{aligned}
$$

and combining this with (5.34), together with the definition of $\Phi^{A, B}$ in (5.26), gives

$$
\sum_{M \in \mathcal{M}_{n}^{*}(K)} \Phi^{A, B}\left(M_{\text {in }}\right) \lesssim \text { LeftHandSide (5.34) }+ \text { LeftHandSide (5.35) } \lesssim \mathcal{N} \mathcal{T} \mathcal{V}_{\alpha} \sqrt{\left|I^{\prime}\right|_{\sigma}\left|J^{\prime}\right|_{\omega}}
$$

In order to deal with this sum in the case when the child $I^{\prime}$ is broken, we must take the estimate one step further and sum over those broken intervals $I^{\prime}$ whose parents belong to the corona $\mathcal{C}_{A}$, i.e. $\left\{I^{\prime} \in \mathcal{D}: I^{\prime} \in \mathfrak{C}_{\text {broken }}(I)\right.$ for some $\left.I \in \mathcal{C}_{A}\right\}$. Of course this collection is precisely the set of $\mathcal{A}$-children of $A$, i.e.

$$
\left\{I^{\prime} \in \mathcal{D}: I^{\prime} \in \mathfrak{C}_{\text {broken }}(I) \text { for some } I \in \mathcal{C}_{A}\right\}=\mathfrak{C}_{\mathcal{A}}(A) .
$$

To help motivate this, we first recall that we denote the term $B(K)$ by $B_{\left(I^{\prime}, J^{\prime}\right)}(K)$ when we wish to indicate the pair $\left(I^{\prime}, J^{\prime}\right)$ to which $K$ is associated, i.e. $K \in \mathcal{K}\left(I^{\prime}, J^{\prime}\right)$ as in (5.8). Of course the intervals 
$M \in \mathcal{M}(K)$ also depend on the pair of intervals $I^{\prime}$ and $J^{\prime}$, but we will suppress notation to this effect, and the reader should keep this in mind. In particular then, if we now sum over natural children $I^{\prime}$ of $I \in \mathcal{C}_{A}$ and the associated children $J^{\prime}$ of $J \in \mathcal{C}_{A}^{\mathcal{G} \text {, nearby }}(I)$, where

$$
\mathcal{C}_{A}^{\mathcal{G}, \text { nearby }}(I) \equiv\left\{J \in \mathcal{G}: 2^{-\mathbf{r}} \ell(I)<\ell(J) \leq \ell(I) \text { and } d(J, I) \leq 2 \ell(J)^{\varepsilon} \ell(I)^{1-\varepsilon}\right\}, \quad \text { for } I \in \mathcal{C}_{A}
$$

we obtain the following corona estimate, using the collection $\mathcal{K}\left(I^{\prime}, J^{\prime}\right)$ that is defined in (5.8) above with $B \leq C \frac{1}{\eta_{0}}$

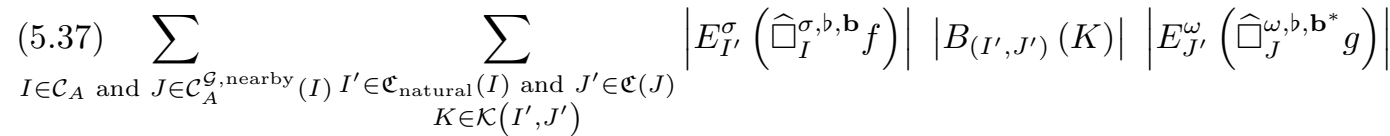

$$
\begin{aligned}
& \lesssim \frac{1}{\eta_{0}} \mathcal{N} \mathcal{T} \mathcal{V}_{\alpha} \sum_{I \in \mathcal{C}_{A} \text { and }} \sum_{J \in \mathcal{C}_{A}^{\mathcal{G}, \text { nearby }}(I)} \sum_{I^{\prime} \in \mathfrak{C}_{\text {natural }}^{I^{\prime}(I) \text { and } J^{\prime} \in \mathfrak{C}(J)}}\left|E_{I^{\prime}}^{\sigma}\left(\widehat{\square}_{I}^{\sigma, b, \mathbf{b}} f\right)\right| \sqrt{\left|I^{\prime}\right|_{\sigma}\left|J^{\prime}\right|_{\omega}}\left|E_{J^{\prime}}^{\omega}\left(\widehat{\square}_{J}^{\omega, b, \mathbf{b}^{*}} g\right)\right| \\
& \lesssim \frac{1}{\eta_{0}} \mathcal{N} \mathcal{T} \mathcal{V}_{\alpha}\left(\sum_{I \in \mathcal{C}_{A}} \sum_{I^{\prime} \in \mathfrak{C}_{\text {natural }}(I)}\left|I^{\prime}\right|_{\sigma}\left|E_{I^{\prime}}^{\sigma}\left(\widehat{\square}_{I}^{\sigma, b, \mathbf{b}} f\right)\right|^{2}\right)^{\frac{1}{2}}\left(\sum_{I \in \mathcal{C}_{A}} \sum_{J \in \mathcal{C}_{A}^{\mathcal{G}, \text { nearby }}(I)} \sum_{J^{\prime} \in \mathfrak{C}(J)}\left|J^{\prime}\right|_{\omega}\left|E_{J^{\prime}}^{\omega}\left(\widehat{\square}_{J}^{\omega, b, \mathbf{b}^{*}} g\right)\right|^{2}\right)^{\frac{1}{2}} \\
& \lesssim \frac{1}{\eta_{0}} \mathcal{N} \mathcal{T} \mathcal{V}_{\alpha}\left\|\mathrm{P}_{\mathcal{C}_{A}}^{\sigma} f\right\|_{L^{2}(\sigma)}^{\star}\left\|\mathrm{P}_{\mathcal{C}_{A}^{\mathcal{G}, \text { nearby }}}^{\omega} g\right\|_{L^{2}(\sigma)}^{\star},
\end{aligned}
$$

where $\mathcal{C}_{A}^{\mathcal{G}, \text { nearby }}=\bigcup_{I \in \mathcal{C}_{A}} \mathcal{C}_{A}^{\mathcal{G}, \text { nearby }}(I)$, and the final line uses (5.5) to obtain

$$
\sum_{I \in \mathcal{C}_{A}} \sum_{I^{\prime} \in \mathfrak{C}_{\text {natural }}(I)}\left|I^{\prime}\right|_{\sigma}\left|E_{I^{\prime}}^{\sigma}\left(\widehat{\square}_{I}^{\sigma, b, \mathbf{b}} f\right)\right|^{2}=\sum_{I \in \mathcal{C}_{A}}\left\|\widehat{\square}_{I}^{\sigma, b, \mathbf{b}} f\right\|_{L^{2}(\sigma)}^{2} \lesssim \sum_{I \in \mathcal{C}_{A}}\left\|\square_{I}^{\sigma, \mathbf{b}} f\right\|_{L^{2}(\sigma)}^{2} \leq\left\|\mathrm{P}_{\mathcal{C}_{A}}^{\sigma} f\right\|_{L^{2}(\sigma)}^{\star 2}
$$

and similarly for the sum in $J$ and $J^{\prime}$, once we note that given $J \in \mathcal{C}_{A}^{\mathcal{G} \text {, nearby }}$, there are only boundedly many $I \in \mathcal{C}_{A}$ for which $J \in \mathcal{C}_{A}^{\mathcal{G} \text {, nearby }}(I)$.

To obtain the same corona estimate when summing over broken $I^{\prime}$, we will exploit the fact that the intervals $A^{\prime} \in \mathfrak{C}_{\mathcal{A}}(A)$ are pairwise disjoint. But first we note that when $I^{\prime}$ is a broken child, neither weak testing nor stopping energy is available. But if we sum over such broken $I^{\prime}$, and use (5.36) to see that the broken children are pairwise disjoint, we obtain the following estimate where for convenience we write

$$
\mathrm{P}_{\delta}^{\alpha} \mathrm{Q}^{\omega}\left(M_{\mathrm{in}}^{\text {left } / \text { right }}, \mathbf{1}_{A} \sigma\right)^{2} \equiv \mathrm{P}_{\delta}^{\alpha} \mathrm{Q}^{\omega}\left(M_{\mathrm{in}}^{\text {left }}, \mathbf{1}_{A} \sigma\right)^{2}+\mathrm{P}_{\delta}^{\alpha} \mathrm{Q}^{\omega}\left(M_{\mathrm{in}}^{\text {right }}, \mathbf{1}_{A} \sigma\right)^{2},
$$

and we use the notation $\mathcal{M}_{n}^{*}\left(I^{\prime}, J^{\prime}\right) \equiv \bigcup_{K \in \mathcal{K}\left(I^{\prime}, J^{\prime}\right)} \mathcal{M}_{n}^{*}(K)$ :

$$
\begin{aligned}
& \sum_{I \in \mathcal{C}_{A} \text { and }} \sum_{J \in \mathcal{C}_{A}^{\mathcal{G}, \text { nearby }}(I)} \sum_{\substack{I^{\prime} \in \mathfrak{C}_{\text {broken }}(I) \text { and } \\
K \in \mathcal{K}\left(I^{\prime}, J^{\prime}\right)}}\left|E_{I^{\prime} \in \mathfrak{C}(J)}^{\sigma}\left(\widehat{\square}_{I}^{\sigma, b, \mathbf{b}} f\right)\right|\left|B_{\left(I^{\prime}, J^{\prime}\right)}(K)\right|\left|E_{J^{\prime}}^{\omega}\left(\widehat{\square}_{J}^{\omega, b, \mathbf{b}^{*}} g\right)\right| \\
& \lesssim \mathcal{N} \mathcal{T} \mathcal{V}_{\alpha} \sum_{I \in \mathcal{C}_{A} \text { and }} \sum_{J \in \mathcal{C}_{A}^{\mathcal{G}, \text { nearby }}(I)} \sum_{I^{\prime} \in \mathfrak{C}_{\text {broken }}(I) \text { and } J^{\prime} \in \mathfrak{C}(J)}\left|E_{I^{\prime}}^{\sigma}\left(\widehat{\square}_{I}^{\sigma, b, \mathbf{b}} f\right)\right| \\
& \times \sqrt{\sum_{M \in \mathcal{M}_{n}^{*}\left(I^{\prime}, J^{\prime}\right)}\left\|\mathbf{1}_{M_{\mathrm{in}}} T_{\sigma}^{\alpha} b_{A}\right\|_{L^{2}(\omega)}^{2}+\sum_{M \in \mathcal{M}_{n}^{*}\left(I^{\prime}, J^{\prime}\right)} \mathrm{P}_{\delta}^{\alpha} \mathrm{Q}^{\omega}\left(M_{\mathrm{in}}^{\text {left } / \text { right }}, \mathbf{1}_{A} \sigma\right)^{2}+\sum_{M \in \mathcal{M}_{n}^{*}\left(I^{\prime}, J^{\prime}\right)}\left|M_{\mathrm{in}}\right|_{\sigma}} \\
& \times \sqrt{\left|J^{\prime}\right|_{\omega}}\left|E_{J^{\prime}}^{\omega}\left(\widehat{\square}_{J}^{\omega, b, \mathbf{b}^{*}} g\right)\right|,
\end{aligned}
$$


which gives

$$
\begin{aligned}
& \sum_{I \in \mathcal{C}_{A} \text { and }} \sum_{J \in \mathcal{C}_{A}^{\mathcal{G}, \text { nearby }}(I)} \sum_{\substack{I^{\prime} \in \mathfrak{C}_{\text {broken }}(I) \text { and } J^{\prime} \in \mathfrak{C}(J) \\
K \in \mathcal{K}\left(I^{\prime}, J^{\prime}\right)}}\left|E_{I^{\prime}}^{\sigma}\left(\widehat{\square}_{I}^{\sigma, b, \mathbf{b}} f\right)\right|\left|B_{\left(I^{\prime}, J^{\prime}\right)}(K)\right|\left|E_{J^{\prime}}^{\omega}\left(\widehat{\square}_{J}^{\omega, b, \mathbf{b}^{*}} g\right)\right| \\
& \lesssim \mathcal{N} \mathcal{T} \mathcal{V}_{\alpha}\left(\sum_{\substack{I \in \mathcal{C}_{A} \\
I^{\prime} \in \mathfrak{C}_{\text {broken }}(I)}} \sum_{\substack{J \in \mathcal{C}^{\mathcal{G}, \text { nearby }} \\
J^{\prime} \in \mathfrak{C}(I)}} \sum_{M \in \mathcal{M}_{n}^{*}\left(I^{\prime}, J^{\prime}\right)}\left\{\left\|\mathbf{1}_{M_{\mathrm{in}}} T_{\sigma}^{\alpha} b_{A}\right\|_{L^{2}(\omega)}^{2}+\mathrm{P}_{\delta}^{\alpha} \mathrm{Q}^{\omega}\left(M_{\mathrm{in}}^{\text {left } / \text { right }}, \mathbf{1}_{A} \sigma\right)^{2}+\left|M_{\mathrm{in}}\right|_{\sigma}\right\}\right)^{\frac{1}{2}}
\end{aligned}
$$

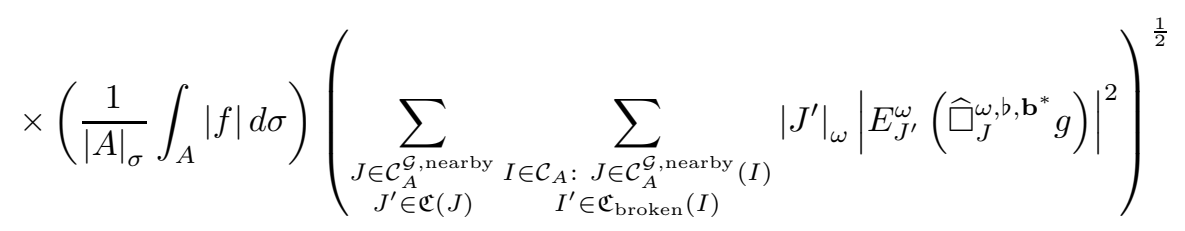

$$
\begin{aligned}
& \lesssim \mathcal{N} \mathcal{T} \mathcal{V}_{\alpha} \sqrt{|A|_{\sigma}\left(\frac{1}{|A|_{\sigma}} \int_{A}|f| d \sigma\right)^{2}}\left\|\mathrm{P}_{\mathcal{C}_{A}^{\mathcal{G}, \text { nearby }}}^{\omega} g\right\|_{L^{2}(\sigma)}^{\star},
\end{aligned}
$$

because

$$
\left|E_{I^{\prime}}^{\sigma}\left(\widehat{\square}_{I}^{\sigma, b, \mathbf{b}} f\right)\right|=\left|\frac{1}{\int_{I} b_{I} d \sigma} \int_{I} f d \sigma\right| \lesssim \frac{1}{|I|_{\sigma}} \int_{I}|f| d \sigma \lesssim \frac{1}{|A|_{\sigma}} \int_{A}|f| d \sigma
$$

if $I^{\prime} \in \mathfrak{C}_{\text {broken }}(I)$ and $I \in \mathcal{C}_{A}$, and because

$$
\begin{aligned}
& \sum_{\substack{I \in \mathcal{C}_{A} \\
I^{\prime} \in \mathfrak{C}_{\text {broken }(I)}}} \sum_{\substack{J \in \mathcal{C}_{\mathcal{G}}^{\mathcal{G}, \text { nearby }} \\
J^{\prime} \in \mathfrak{C}(J)}} \sum_{M \in \mathcal{M}_{n}^{*}\left(I^{\prime}, J^{\prime}\right)}\left\{\left\|\mathbf{1}_{M_{\mathrm{in}}} T_{\sigma}^{\alpha} b_{A}\right\|_{L^{2}(\omega)}^{2}+\mathrm{P}_{\delta}^{\alpha} \mathrm{Q}^{\omega}\left(M_{\mathrm{in}}^{\text {left }}, \mathbf{1}_{A} \sigma\right)^{2}+\mathrm{P}_{\delta}^{\alpha} \mathrm{Q}^{\omega}\left(M_{\mathrm{in}}^{\text {right }}, \mathbf{1}_{A} \sigma\right)^{2}+\left|M_{\mathrm{in}}\right|_{\sigma}\right\} \\
\leq & \int_{A}\left|T_{\sigma}^{\alpha} b_{A}\right|^{2} d \omega+\mathfrak{E}_{2}^{\alpha}|A|_{\sigma}+|A|_{\sigma} \leq\left(\mathfrak{T}_{T^{\alpha}}^{\mathbf{b}}+\mathfrak{E}_{2}^{\alpha}+1\right)^{2}|A|_{\sigma} .
\end{aligned}
$$

Indeed, in this last inequality (5.39), we have used first the testing condition, which applies since the collection of $\mathcal{G}$-dyadic intervals

$$
\mathcal{R} \equiv \bigcup_{I \in \mathcal{C}_{A}} \bigcup_{I^{\prime} \in \mathfrak{C}_{\text {broken }}} \bigcup_{J \in \mathcal{C}_{A}^{\mathcal{G}, \text { nearby }}(I)} \bigcup_{J^{\prime} \in \mathfrak{C}(J)} \bigcup_{M \in \mathcal{M}_{n}^{*}\left(I^{\prime}, J^{\prime}\right)}\left\{M_{\mathrm{in}}^{\text {left }}, M_{\mathrm{in}}^{\text {right }}\right\}
$$

has bounded overlap counting repetitions of intervals. Indeed, given an interval $L \in \mathcal{G}$, there are only a bounded, say $B$, number of pairs $\left(I^{\prime}, J^{\prime}\right)$, comparable in both scale and position, for which $\mathcal{M}_{n}^{*}\left(I^{\prime}, J^{\prime}\right)$ contains an interval $M$ with $M_{\mathrm{in}}^{\text {left }}$ or $M_{\mathrm{in}}^{\text {right }}$ equal to $L$. Thus any tower of such intervals $M_{\mathrm{in}}^{\text {left }}$ or $M_{\mathrm{in}}^{\text {right }}$, that contains a fixed point $x \in \mathbb{R}$, has at most $B$ intervals counting repetitions.

Next we used the energy condition in (5.39), which applies since if $\mathcal{R}$, considered now without repetitions, has bounded overlap $B$, then $\mathcal{R}$ can be decomposed as $B$ pairwise disjoint families $\left\{\mathcal{R}_{i}\right\}_{i=1}^{B}$. Indeed, since all of the intervals lie in the dyadic grid $\mathcal{G}$ and are contained in a fixed interval $A$, the family $\mathcal{R}_{1}$ of maximal intervals in $\mathcal{R}$ are pairwise disjoint, and after removing them, the remaining collection of intervals $\mathcal{R} \backslash \mathcal{R}_{1}$ has bounded overlap $B-1$. Let $\mathcal{R}_{2}$ be the family of maximal dyadic intervals in $\mathcal{R} \backslash \mathcal{R}_{1}$ and continue until all the intervals are exhausted after removing $\mathcal{R}_{R}$.

The inequality (5.38) is a suitable estimate since

$$
\sum_{A \in \mathcal{A}} \sqrt{|A|_{\sigma}\left(\frac{1}{|A|_{\sigma}} \int_{A}|f| d \sigma\right)^{2}}\left\|\mathrm{P}_{\mathcal{C}_{A}^{\mathcal{G}, \text { nearby }}}^{\omega} g\right\|_{L^{2}(\sigma)}^{\star} \lesssim\|f\|_{L^{2}(\sigma)}\|g\|_{L^{2}(\sigma)}
$$

by quasiorthogonality and the frame inequalities in Appendix A, (9.7) and (9.13), together with the bounded overlap of the 'nearby' coronas $\left\{\mathcal{C}_{A}^{\mathcal{G} \text {,nearby }}\right\}_{A \in \mathcal{A}}$. 
Recall that after an initial application of random surgery, we reduced the proof of Lemma 48 to establishing inequality (5.10), in which $P_{(I, J)}(K, K)=\{K, K\}$ in the notation used in (5.30). Now putting all of the above estimates (5.31), (5.33), (5.37) and (5.38) together with (5.30) establishes probabilistic control of the sum of all the inner products $\{K, K\}$ taken over appropriate intervals $K$, yielding (5.10) as required if we choose $\lambda$ and $\eta_{0}$ sufficiently small:

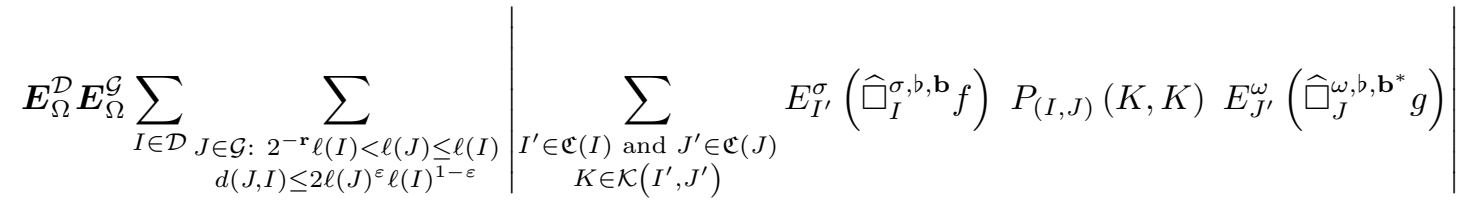

$$
\begin{aligned}
& \leq \boldsymbol{E}_{\Omega}^{\mathcal{D}} \boldsymbol{E}_{\Omega}^{\mathcal{G}} \sum_{A \in \mathcal{A}} \sum_{I \in \mathcal{C}_{A} \text { and }} \sum_{J \in \mathcal{C}_{A}^{\mathcal{G}, \text { nearby }}(I)} \sum_{\substack{I^{\prime} \in \mathfrak{C}(I) \text { and } J^{\prime} \in \mathfrak{C}(J) \\
K \in \mathcal{K}\left(I^{\prime}, J^{\prime}\right)}}\left|E_{I^{\prime}}^{\sigma}\left(\widehat{\square}_{I}^{\sigma, b, \mathbf{b}} f\right)\right|\left|A_{\left(I^{\prime}, J^{\prime}\right)}(K)\right| E_{J^{\prime}}^{\omega}\left(\widehat{\square}_{J}^{\omega, b, \mathbf{b}^{*}} g\right) \\
& +\boldsymbol{E}_{\Omega}^{\mathcal{D}} \boldsymbol{E}_{\Omega}^{\mathcal{G}} \sum_{A \in \mathcal{A}} \sum_{I \in \mathcal{C}_{A} \text { and }} \sum_{J \in \mathcal{C}_{A}^{\mathcal{G}, \text { nearby }}(I)} \sum_{\substack{I^{\prime} \in \mathfrak{C}(I) \\
K \in \mathcal{K}\left(I^{\prime}, J^{\prime}\right)}}\left|E_{I^{\prime}}^{\sigma}\left(\widehat{\square}_{I}^{\sigma, b, \mathbf{b}} f\right)\right|\left(\left|B_{\left(I^{\prime}, J^{\prime}\right)}(K)\right|+\left|C_{\left(I^{\prime}, J^{\prime}\right)}(K)\right|\right)\left|E_{J^{\prime}}^{\omega}\left(\widehat{\square}_{J}^{\omega, b, \mathbf{b}^{*}} g\right)\right| \\
& \lesssim\left(C_{\theta} \mathcal{N} \mathcal{T} \mathcal{V}_{\alpha}+\sqrt{\theta} \mathfrak{N}_{T^{\alpha}}\right)\|f\|_{L^{2}(\sigma)}\|g\|_{L^{2}(\omega)}
\end{aligned}
$$

This completes the proof of Lemma 48 ,

\section{MAIN BELOW FORM}

Now we turn to controlling the main below form (3.20),

$$
\Theta_{2}^{\text {good }}(f, g)=\sum_{I \in \mathcal{D}} \sum_{J \neq I: \ell(J) \leq 2^{-\mathbf{r}} \ell(I)} \int\left(T_{\sigma}^{\alpha} \square_{I}^{\sigma, \mathbf{b}} f\right) \square_{J}^{\omega, \mathbf{b}^{*}} g d \omega .
$$

where we recall that $\mathbf{r}$ is the goodness parameter fixed in (3.19) given $0<\varepsilon<\frac{1}{2}$. The corresponding main below form in SaShUr6] was denoted $\mathrm{B}_{\Subset_{\mathbf{r}}}(f, g)=\mathrm{B}_{\Subset_{\mathbf{r}, \varepsilon}}(f, g)$. However, there are significant differences between the forms $\Theta_{2}^{\text {good }}(f, g)$ and $\mathrm{B}_{\in_{\mathbf{r}}}(f, g)$. In SaShUr6], the Haar martingale averages $\triangle_{I}^{\sigma}$ and $\triangle_{J}^{\omega}$ in $\mathrm{B}_{\Subset_{\mathbf{r}}}(f, g)$ are orthogonal projections, and the intervals $I$ and $J$ that appear in $\mathrm{B}_{\Subset_{\mathbf{r}}}(f, g)$ are all good in the traditional sense. Here, on the other hand, the dual martingale averages $\square_{I}^{\sigma, \mathbf{b}}$ and $\square_{J}^{\omega, \mathbf{b}^{*}}$ in $\Theta_{2}^{\text {good }}(f, g)$ are no longer orthogonal projections, and while the intervals $J$ paired with $I$ remain $\varepsilon$-good inside $I$ and beyond, the lack of orthogonal projections is compensated by the fact that the collections of intervals $I$ associated with any fixed $J$ in $\Theta_{2}^{\text {good }}(f, g)$ are tree-connected. Nevertheless, in order to efficiently import the methods for controlling $\mathrm{B}_{\Subset_{\mathbf{r}}}(f, g)$ from [SaShUr6], we will relabel the main below form $\Theta_{2}^{\text {good }}(f, g)$ as $\mathrm{B}_{\Subset_{\mathbf{r}}}(f, g)=\mathrm{B}_{\leftarrow_{\mathbf{r}, \varepsilon}}(f, g)$ from now on, keeping in mind the aforementioned differences.

To control $\Theta_{2}^{\text {good }}(f, g)=\mathrm{B}_{\Subset_{\mathbf{r}}}(f, g)$ we first perform the canonical corona splitting of $\mathrm{B}_{\Subset_{\mathbf{r}}}(f, g)$ into a diagonal form $\mathrm{T}_{\text {diagonal }}(f, g)$ and a far below form $\mathrm{T}_{\text {far below }}(f, g)$ as in SaShUr6. This canonical splitting of the form $\mathrm{B}_{\Subset_{\mathbf{r}}}(f, g)$ involves the corona pseudoprojections $\mathrm{P}_{\mathcal{C}_{A}^{D}}^{\sigma, \mathbf{b}}$ acting on $f$ and the shifted corona pseudoprojections $\mathrm{P}_{\mathcal{C}_{B}^{\mathcal{G}}}^{\omega, \mathbf{b}^{*} \text {,sift }}$ acting on $g$, where $B$ is a stopping interval in $\mathcal{A}$. The stopping intervals $\mathcal{B}$ constructed relative to $g \in L^{2}(\omega)$ play no role in the analysis here, except to guarantee that the frame and weak Riesz inequalities hold for $g$ and the pseudprojections $\left\{\square_{J}^{\omega, \mathbf{b}^{*}} g\right\}_{J \in \mathcal{G}}$ and Carleson averaging operators $\left\{\nabla_{J, \mathcal{G}}^{\omega} g\right\}_{J \in \mathcal{G}}$. Here the shifted corona $\mathcal{C}_{B}^{\mathcal{G} \text {,shift }}$ is defined for $B \in \mathcal{A}$ - and not for $B \in \mathcal{B}$ - to include those intervals $J \in \mathcal{G}$ such that $J^{*} \in \mathcal{C}_{B}^{\mathcal{D}}$.

Definition 52. For $B \in \mathcal{A}$ we define the shifted $\mathcal{G}$-corona by

$$
\mathcal{C}_{B}^{\mathcal{G} \text {,shift }}=\left\{J \in \mathcal{G}: J^{\mathscr{\text { W }}} \in \mathcal{C}_{B}^{\mathcal{D}}\right\} .
$$

\footnotetext{
${ }^{6}$ While it remains the case with $T b$ arguments that this form is the most challenging, the nearby form also poses great difficulties with $T b$ arguments, especially in contrast with $T 1$ arguments, where the nearby form was handled almost trivially.
} 
The Carleson averaging operator $\nabla_{J, \mathcal{G}}^{\omega}$ is taken over the 'broken' children of $J$ which depend on the grid $\mathcal{G}$ (see (9.6) in Appendix A). We will use repeatedly the fact that the shifted coronas $\mathcal{C}_{B}^{\mathcal{G} \text {,shift }}$ are pairwise disjoint in $B$ :

$$
\sum_{B \in \mathcal{A}} \mathbf{1}_{\mathcal{C}_{B}^{\mathcal{G}, \text { shift }}}(J) \leq \mathbf{1}, \quad J \in \mathcal{G}
$$

It is convenient at this point to introduce the following shorthand notation:

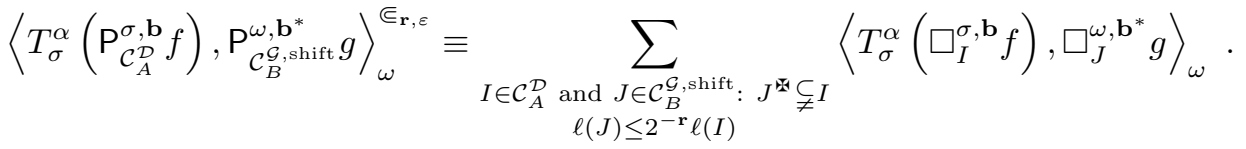

Caution: One musn't assume, from the notation on the left hand side above, that the function $T_{\sigma}^{\alpha}\left(\mathrm{P}_{\mathcal{C}_{A}^{\mathcal{D}}}^{\sigma, \mathbf{b}} f\right)$ is simply integrated against the function $\mathrm{P}_{\mathcal{C}_{B}^{\mathcal{G}, \text { shift }}}^{\omega, \mathbf{b}^{*}} g$. Indeed, the sum on the right hand side is taken over pairs $(I, J)$ such that $J^{*} \in \mathcal{C}_{B}^{\mathcal{D}}$ and $J^{*} \varsubsetneqq I$ and $\ell(J) \leq 2^{-\mathbf{r}} \ell(I)$.

Here is the relevant portion of the brief schematic diagram (3.4) of the decompositions, with bounds in used in the next subsections:

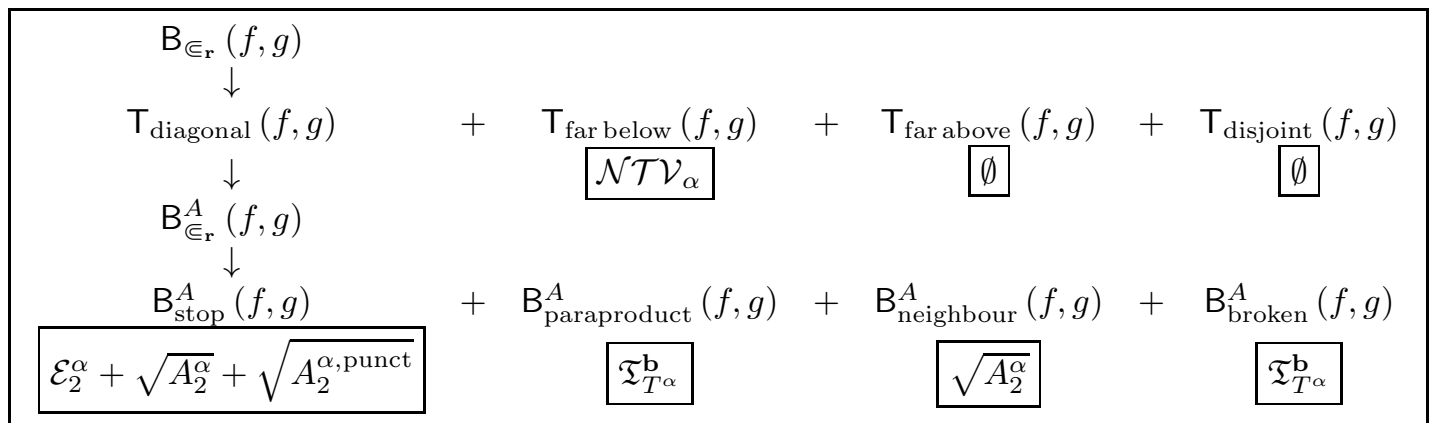

6.1. The canonical splitting and local below forms. We begin with an informal description of decompositions and estimates. The canonical splitting is determined by the coronas $\mathcal{C}_{A}^{\mathcal{D}}$ for $A \in \mathcal{A}$ - note that the stopping times $\mathcal{B}$ play no explicit role in the canonical splitting of the below form, other than to guarantee weak Riesz inequalities for $\square_{J}^{\omega, \mathbf{b}^{*}}$ and $\nabla_{J, \mathcal{G}}^{\omega}$ :

$$
\begin{aligned}
& \mathrm{B}_{\Subset_{\mathbf{r}, \varepsilon}}(f, g) \\
& =\sum_{A, B \in \mathcal{A}}\left\langle T_{\sigma}^{\alpha}\left(\mathrm{P}_{\mathcal{C}_{A}}^{\sigma, \mathbf{b}} f\right), \mathrm{P}_{\mathcal{C}_{B}^{\mathcal{G}, \text { shift }}}^{\omega, \mathbf{b}^{*}} g\right\rangle_{\omega}^{\Subset_{\mathbf{r}, \varepsilon}} \\
& =\sum_{A \in \mathcal{A}}\left\langle T_{\sigma}^{\alpha}\left(\mathrm{P}_{\mathcal{C}_{A}}^{\sigma, \mathbf{b}} f\right), \mathrm{P}_{\mathcal{C}_{A}^{\mathcal{G}, \text { shift }}}^{\omega, \mathbf{b}^{*}} g\right\rangle_{\omega}^{\Subset_{\mathbf{r}, \varepsilon}}+\sum_{\substack{A, B \in \mathcal{A} \\
B \varsubsetneqq A}}\left\langle T_{\sigma}^{\alpha}\left(\mathrm{P}_{\mathcal{C}_{A}}^{\sigma, \mathbf{b}} f\right), \mathrm{P}_{\mathcal{C}_{B}^{\mathcal{G}, \text { shift }}}^{\omega, \mathbf{b}^{*}} g\right\rangle_{\omega}^{\Subset_{\mathbf{r}, \varepsilon}} \\
& +\sum_{\substack{A, B \in \mathcal{A} \\
B \supsetneqq A}}\left\langle T_{\sigma}^{\alpha}\left(\mathrm{P}_{\mathcal{C}_{A}}^{\sigma, \mathbf{b}} f\right), \mathrm{P}_{\mathcal{C}_{B}^{\mathcal{G}, \text { shift }}}^{\omega, \mathbf{b}^{*}} g\right\rangle_{\omega}^{\complement_{\mathbf{r}, \varepsilon}}+\sum_{\substack{A, B \in \mathcal{A} \\
A \cap B=\emptyset}}\left\langle T_{\sigma}^{\alpha}\left(\mathrm{P}_{\mathcal{C}_{A}}^{\sigma, \mathbf{b}} f\right), \mathrm{P}_{\mathcal{C}_{B}^{\mathcal{G}, \text { shift }}}^{\omega, \mathbf{b}^{*}} g\right\rangle_{\omega}^{\Subset_{\mathbf{r}, \varepsilon}} \\
& \equiv \mathrm{T}_{\text {diagonal }}(f, g)+\mathrm{T}_{\text {far below }}(f, g)+\mathrm{T}_{\text {far above }}(f, g)+\mathrm{T}_{\text {disjoint }}(f, g) \text {. }
\end{aligned}
$$

Now the final two terms $\mathrm{T}_{\text {far above }}(f, g)$ and $\mathrm{T}_{\text {disjoint }}(f, g)$ each vanish since there are no pairs $(I, J) \in$ $\mathcal{C}_{A}^{\mathcal{D}} \times \mathcal{C}_{B}^{\mathcal{G} \text {,shift }}$ with both (i) $J^{\mathbf{w}} \varsubsetneqq I$ and (ii) either $B \supsetneqq A$ or $B \cap A=\emptyset$. The far below form $\mathrm{T}_{\text {far below }}(f, g)$ requires functional energy, which we discuss in a moment.

Next we follow this splitting by a further decomposition of the diagonal form into local below forms $\mathrm{B}_{\complement_{\mathbf{r}}}^{A}(f, g)$ given by the individual corona pieces

$$
\mathrm{B}_{\Subset_{\mathbf{r}, \varepsilon}}^{A}(f, g) \equiv\left\langle T_{\sigma}^{\alpha}\left(\mathrm{P}_{\mathcal{C}_{A}}^{\sigma, \mathbf{b}} f\right), \mathrm{P}_{\mathcal{C}_{A}^{\mathcal{G}, \text { shift }}}^{\omega, \mathbf{b}^{*}} g\right\rangle_{\omega}^{\Subset_{\mathbf{r}, \varepsilon}},
$$


and prove the following estimate where $\mathcal{N} \mathcal{T} \mathcal{V}_{\alpha}$ is defined in (1.15):

$$
\left|\mathrm{B}_{\Subset_{\mathbf{r}, \varepsilon}}^{A}(f, g)\right| \lesssim \mathcal{N} \mathcal{T} \mathcal{V}_{\alpha}\left(\alpha_{\mathcal{A}}(A) \sqrt{|A|_{\sigma}}+\left\|\mathrm{P}_{\mathcal{C}_{A}^{\sigma, \mathbf{b}} f}\right\|_{L^{2}(\sigma)}^{\star}\right)\left\|\mathrm{P}_{\mathcal{C}_{A}^{\mathcal{G}, \text { shift }}}^{\omega, \mathbf{b}^{*}} g\right\|_{L^{2}(\omega)}^{\star}
$$

This reduces matters to the local forms since we then have from Cauchy-Schwarz that

$$
\begin{array}{rl}
\sum_{A \in \mathcal{A}}\left|\mathrm{B}_{\Subset_{\mathbf{r}, \varepsilon}}^{A}(f, g)\right| \lesssim \mathcal{N} & \mathcal{T} \mathcal{V}_{\alpha}\left(\sum_{A \in \mathcal{A}} \alpha_{\mathcal{A}}(A)^{2}|A|_{\sigma}+\left\|\mathrm{P}_{\mathcal{C}_{A}^{\mathcal{D}}}^{\sigma, \mathbf{b}} f\right\|_{L^{2}(\sigma)}^{\star 2}\right)^{\frac{1}{2}}\left(\sum_{A \in \mathcal{A}}\left\|\mathrm{P}_{\mathcal{C}_{A}^{\mathcal{G}, \text { shift }}}^{\omega, \mathbf{b}^{*}}\right\|_{L^{2}(\omega)}^{\star 2}\right)^{\frac{1}{2}} \\
& \lesssim \mathcal{N} \mathcal{T} \mathcal{V}_{\alpha}\|f\|_{L^{2}(\sigma)}\|g\|_{L^{2}(\omega)}
\end{array}
$$

by the lower frame inequalities $\sum_{A \in \mathcal{A}}\left\|\mathrm{P}_{\mathcal{C}_{A}^{\sigma}}^{\sigma, \mathbf{b}} f\right\|_{L^{2}(\sigma)}^{\star 2} \lesssim\|f\|_{L^{2}(\sigma)}^{2}$ and $\sum_{A \in \mathcal{A}}\left\|\mathrm{P}_{\mathcal{C}_{A}^{\mathcal{G}, \text { shift }}}^{\omega, \mathbf{b}^{*}}\right\|_{L^{2}(\omega)}^{\star 2} \lesssim\|g\|_{L^{2}(\omega)}^{2}$, using quasi-orthogonality $\sum_{A \in \mathcal{A}} \alpha_{\mathcal{A}}(f)^{2}|A|_{\sigma} \lesssim\|f\|_{L^{2}(\sigma)}^{2}$ in the stopping intervals $\mathcal{A}$, and the pairwise disjointedness of the shifted coronas $\mathcal{C}_{A}^{\mathcal{G} \text {, shift }}$

$$
\sum_{A \in \mathcal{A}} \mathbf{1}_{\mathcal{C}_{A}^{\mathcal{G}, \text { shift }}} \leq \mathbf{1}_{\mathcal{D}}
$$

From now on we will often write $\mathcal{C}_{A}$ in place of $\mathcal{C}_{A}^{\mathcal{D}}$ when no confusion is possible.

Finally, the local forms $\mathrm{B}_{\Subset_{\mathbf{r}, \varepsilon}}^{A}(f, g)$ are decomposed into stopping forms $\mathrm{B}_{\text {stop }}^{A}(f, g)$, paraproduct forms $\mathrm{B}_{\text {paraproduct }}^{A}(f, g)$, neighbour forms $\mathrm{B}_{\text {neighbour }}^{A}(f, g)$ forms and broken forms $\mathrm{B}_{\text {broken }}^{A}(f, g)$. The paraproduct and neighbour terms are handled as in [SaShUr6], which in turn follows the treatment originating in [NTV3], and the broken form is handled with Carleson measure methods, leaving only the stopping form $\mathrm{B}_{\text {stop }}^{A}(f, g)$ to be bounded, which we treat in the next section below, Section 7, by refining the bottom/up stopping time in the argument of M. Lacey in [Lac] with an additional top/down 'indented' corona construction to handle weak goodness.

However, in order to complete the required bounds of the above forms into which the below form $\mathrm{B}_{\Subset_{\mathbf{r}}}(f, g)$ was decomposed, we need functional energy for the far below form. Recall that the vector-valued function b in the accretive coronas 'breaks' only at a collection of intervals satisfying a Carleson condition.

Definition 53. Define the Whitney subintervals $\mathcal{W}(F)$ of an interval $F \in \mathcal{D}$ to consist of the maximal dyadic $\mathcal{D}$-subintervals of a $\mathcal{D}$-interval $F$ that have their triples contained in $F$.

See (10.2) in Appendix B below for more detail on this and the remaining terms in (6.6) below.

Definition 54. Let $\mathfrak{F}_{\alpha}=\mathfrak{F}_{\alpha}(\mathcal{D}, \mathcal{G})=\mathfrak{F}_{\alpha}^{\mathbf{b}^{*}}(\mathcal{D}, \mathcal{G})$ be the smallest constant in the 'functional energy' inequality below, holding for all $h \in L^{2}(\sigma)$ and all $\sigma$-Carleson collections $\mathcal{F} \subset \mathcal{D}$ with Carleson norm $C_{\mathcal{F}}$ bounded by a fixed constant $C$ :

$$
\sum_{F \in \mathcal{F}} \sum_{M \in \mathcal{W}(F)}\left(\frac{\mathrm{P}^{\alpha}(M, h \sigma)}{|M|}\right)^{2}\left\|\mathrm{Q}_{\mathcal{C}_{F}^{\mathcal{G}, \text { shift }} ; M}^{\omega, \mathbf{b}^{*}} x\right\|_{L^{2}(\omega)}^{\mathbf{a}^{2}} \leq\left(\mathfrak{F}_{\alpha}^{\mathbf{b}^{*}}(\mathcal{D}, \mathcal{G})\right)^{2}\|h\|_{L^{2}(\sigma)}^{2}
$$

The main ingredient used in reducing control of the below form $\mathrm{B}_{\Subset_{\mathbf{r}}}(f, g)$ to control of the functional energy $\mathfrak{F}_{\alpha}=\mathfrak{F}_{\alpha}^{\mathbf{b}^{*}}(\mathcal{D}, \mathcal{G})$ constant and the stopping form $\mathrm{B}_{\text {stop }}^{A}(f, g)$, is the Intertwining Proposition from [SaShUr7] and/or [SaShUr6]. The control of the functional energy condition by the energy and Muckenhoupt conditions must also be adapted in light of the $\infty$-strongly accretive function b that only 'breaks' at a collection of intervals satisfying a Carleson condition, but this poses no real difficulties. The fact that the usual Haar bases are orthonormal is here replaced by the weaker condition that the corresponding broken dual martingale 'bases' are merely weak frames satisfying certain weak lower and weak upper Riesz inequalities, but again this poses no real difference in the arguments. Finally, the fact that goodness for $J$ has been replaced with weak goodness, namely $J^{\ddagger} I$ whenever the pair $(I, J)$ occurs in a sum, forces the use of a Whitney decomposition $\mathcal{W}$ of intervals instead of the deeply embedded decomposition $\mathcal{M}_{(\boldsymbol{\rho}, \varepsilon)-\text { deep }}$ used in SaShUr7]. 
We then use the paraproduct / neighbour / broken / stopping splitting mentioned above to reduce boundedness of $\mathrm{B}_{\Subset_{\mathbf{r}, \varepsilon}}^{A}(f, g)$ to boundedness of the associated stopping form

$$
\mathrm{B}_{\text {stop }}^{A}(f, g) \equiv \sum_{\substack{I \in \mathcal{C}_{A} \text { and } J \in \mathcal{C}_{A}^{\mathcal{G} \text {,shift }} \\ J^{\mathbf{*}} \subset I, J^{*} \neq I \text { and } \ell(J) \leq 2^{-\mathbf{r}} \ell(I)}} E_{I_{J}}^{\sigma}\left(\widehat{\square}_{I}^{\sigma, b, \mathbf{b}} f\right)\left\langle T_{\sigma}^{\alpha} b_{A} \mathbf{1}_{A \backslash I_{J}}, \square_{J}^{\omega, \mathbf{b}^{*}} g\right\rangle_{\omega},
$$

where $f$ is supported in the interval $A$ and its expectations $\mathbb{E}_{I}^{\sigma}|f|$ are bounded by $\alpha_{\mathcal{A}}(A)$ for $I \in \mathcal{C}_{A}$, the dual martingale support of $f$ is contained in the corona $\mathcal{C}_{A}^{\sigma}$, and the dual martingale support of $g$ is contained in $\mathcal{C}_{A}^{\mathcal{G} \text {,shift }}$, and where $I_{J}$ is the $\mathcal{D}$-child of $I$ that contains $J$.

6.2. Diagonal and far below forms. Now we turn to estimating the diagonal term $\mathrm{T}_{\text {diagonal }}(f, g)$ and the far below term $\mathrm{T}_{\text {far below }}(f, g)$, where in [SaShUr7 and/or [SaShUr6], the far below terms were bounded using the Intertwining Proposition and the control of functional energy condition by the energy and Muckenhoupt conditions, but of course under the restriction there that the intervals $J$ were good. Here we write

$$
\begin{aligned}
& \text { (6.8) } \\
& \text { 8) } \mathrm{T}_{\text {far below }}(f, g)=\sum_{\substack{A, B \in \mathcal{A} \\
B \varsubsetneqq A}} \sum_{\substack{I \in \mathcal{C}_{A} \text { and } J \in \mathcal{C}_{B}^{\mathcal{G}, \text { shift }} \\
J^{\mathbf{*}} \subsetneq I \text { and } \ell(J) \leq 2^{-\mathbf{r}} \ell(I)}}\left\langle T_{\sigma}^{\alpha}\left(\square_{I}^{\sigma, \mathbf{b}} f\right), \square_{J}^{\omega, \mathbf{b}^{*}} g\right\rangle_{\omega} \\
& =\sum_{B \in \mathcal{A}} \sum_{I \in \mathcal{D}: B \varsubsetneqq I}\left\langle T_{\sigma}^{\alpha}\left(\square_{I}^{\sigma, \mathbf{b}} f\right), \sum_{J \in \mathcal{C}_{B}^{\mathcal{G}, \text { shift }}} \square_{J}^{\omega, \mathbf{b}^{*}} g\right\rangle-\sum_{B \in \mathcal{A}} \sum_{I \in \mathcal{D}: B \varsubsetneqq I}\left\langle T_{\sigma}^{\alpha}\left(\square_{I}^{\sigma, \mathbf{b}} f\right), \sum_{\substack{J \in \mathcal{C}_{B}^{\mathcal{G}, \text { shift }} \\
\ell(J)>2^{-\mathbf{r}} \ell(I)}} \square_{J}^{\omega, \mathbf{b}^{*}} g\right\rangle \\
& =\sum_{B \in \mathcal{A}} \sum_{I \in \mathcal{D}: B \varsubsetneqq I}\left\langle T_{\sigma}^{\alpha}\left(\square_{I}^{\sigma, \mathbf{b}} f\right), g_{B}\right\rangle_{\omega}-\sum_{B \in \mathcal{A}} \sum_{I \in \mathcal{D}: B \varsubsetneqq I}\left\langle T_{\sigma}^{\alpha}\left(\square_{I}^{\sigma, \mathbf{b}} f\right), \sum_{\substack{J \in \mathcal{C}_{B}^{\mathcal{G}, \text { shift }} \\
\ell(J)>2^{-\mathbf{r}} \ell(I)}} \square_{J}^{\omega, \mathbf{b}^{*}} g\right\rangle ; \\
& \text { where } g_{B}=\sum_{J \in \mathcal{C}_{B}^{\mathcal{G}, \text { shift }}} \square_{J}^{\omega, \mathbf{b}^{*}} g=\mathrm{P}_{\mathcal{C}_{F}^{\mathcal{G}}}^{\omega, \text { shift }} \text {, }^{*} g
\end{aligned}
$$

since if $I \in \mathcal{C}_{A}$ and $J \in \mathcal{C}_{B}^{\mathcal{G} \text {,shift }}$, with $J^{\mathbf{w}} \varsubsetneqq I$ and $B \varsubsetneqq A$, then we must have $B \varsubsetneqq I$. First, we note that expectation of the second sum on the right hand side of (6.8) is controlled by (5.1) in Lemma 48, i.e.

$$
\begin{aligned}
& \boldsymbol{E}_{\Omega}^{\mathcal{D}} \boldsymbol{E}_{\Omega}^{\mathcal{G}}\left|\sum_{B \in \mathcal{A}} \sum_{I \in \mathcal{D}: B \varsubsetneqq I}\left\langle T_{\sigma}^{\alpha}\left(\square_{I}^{\sigma, \mathbf{b}} f\right), \sum_{\substack{J \in \mathcal{C}_{B}^{\mathcal{G}, \text { shift }} \\
\ell(J)>2^{-\mathbf{r}} \ell(I)}} \square_{J}^{\omega, \mathbf{b}^{*}} g\right\rangle\right| \\
& \lesssim \boldsymbol{E}_{\Omega}^{\mathcal{D}} \boldsymbol{E}_{\Omega}^{\mathcal{G}} \sum_{I \in \mathcal{D}} \sum_{\substack{J \in \mathcal{G}: 2^{-\mathbf{r}} \ell(I)<\ell(J) \leq \ell(I) \\
d(J, I) \leq 2 \ell(J)^{\varepsilon} \ell(I)^{1-\varepsilon}}}\left|\left\langle T_{\sigma}^{\alpha}\left(\square_{I}^{\sigma, \mathbf{b}} f\right), \square_{J}^{\omega, \mathbf{b}^{*}} g\right\rangle_{\omega}\right|^{\omega} \\
& \lesssim\left(C_{\theta} \mathcal{N} \mathcal{T} \mathcal{V}_{\alpha}+\sqrt{\theta} \mathfrak{N}_{T^{\alpha}}\right)\|f\|_{L^{2}(\sigma)}\|g\|_{L^{2}(\omega)} .
\end{aligned}
$$

Second, we note that the Intertwining Proposition 60, which controls sums of the form

$$
\sum_{F \in \mathcal{F}} \sum_{I: I \supsetneqq F}\left\langle T_{\sigma}^{\alpha} \square_{I}^{\sigma, \mathbf{b}} f, \mathrm{P}_{\mathcal{C}_{F}^{\mathcal{G}, \text { shift }}}^{\omega, \mathbf{b}^{*}} g\right\rangle_{\omega}
$$

can be applied to the first sum on the right hand side of (6.8) to show that it is bounded by $\left(\mathcal{N} \mathcal{T} \mathcal{V}_{\alpha}+\mathfrak{F}_{\alpha}^{\mathbf{b}^{*}}(\mathcal{D}, \mathcal{G})\right)\|f\|_{L^{2}(\sigma)}\|g\|_{L}$ where the goodness parameter $\varepsilon>0$ is chosen sufficiently small. Then Proposition 100 can be applied to show that $\mathfrak{F}_{\alpha}^{\mathbf{b}^{*}}(\mathcal{D}, \mathcal{G}) \lesssim \mathfrak{A}_{2}^{\alpha}+\mathfrak{E}_{2}^{\alpha}$, which completes the proof that

$$
\left|\mathrm{T}_{\text {far below }}(f, g)\right| \lesssim \mathcal{N} \mathcal{T} \mathcal{V}_{\alpha}\|f\|_{L^{2}(\sigma)}\|g\|_{L^{2}(\omega)} .
$$


6.3. Intertwining Proposition. First we adapt the relevant definitions from [SaShUr6].

Definition 55. A collection $\mathcal{F}$ of dyadic intervals is $\sigma$-Carleson if

$$
\sum_{F \in \mathcal{F}: F \subset S}|F|_{\sigma} \leq C_{\mathcal{F}}|S|_{\sigma}, \quad S \in \mathcal{F} .
$$

The constant $C_{\mathcal{F}}$ is referred to as the Carleson norm of $\mathcal{F}$.

Definition 56. Let $\mathcal{F}$ be a collection of dyadic intervals in a grid $\mathcal{D}$. Then for $F \in \mathcal{F}$, we define the shifted corona $\mathcal{C}_{F}^{\mathcal{G} \text {,shift }}$ in analogy with Definition 5 20 by

$$
\mathcal{C}_{F}^{\mathcal{G}, \text { shift }} \equiv\left\{J \in \mathcal{G}: J^{\mathscr{w}} \in \mathcal{C}_{F}\right\}
$$

where $J^{\text {is }}$ is defined in Definition 42 .

Note that the collections $\mathcal{C}_{F}^{\mathcal{G} \text {, shift }}$ are pairwise disjoint in $F$. Let $\mathfrak{C}_{\mathcal{F}}(F)$ denote the set of $\mathcal{F}$-children of $F$. Given any collection $\mathcal{H} \subset \mathcal{G}$ of intervals, a family $\mathbf{b}^{*}$ of dual testing functions, and an arbitrary interval $K \in \mathcal{P}$, we define the corresponding dual pseudoprojection $\mathrm{P}_{\mathcal{H}}^{\omega, \mathbf{b}^{*}}$ and its localization $\mathrm{P}_{\mathcal{H} ; K}^{\omega, \mathbf{b}^{*}}$ to $K$ by

$$
\mathbf{Q}_{\mathcal{H}}^{\omega, \mathbf{b}^{*}}=\sum_{H \in \mathcal{H}} \triangle_{H}^{\omega, \mathbf{b}^{*}} \text { and } \mathbf{Q}_{\mathcal{H} ; K}^{\omega, \mathbf{b}^{*}}=\sum_{H \in \mathcal{H}: H \subset K} \triangle_{H}^{\omega, \mathbf{b}^{*}}
$$

Recall from Definition 54 that $\mathfrak{F}_{\alpha}=\mathfrak{F}_{\alpha}(\mathcal{D}, \mathcal{G})=\mathfrak{F}_{\alpha}^{\mathbf{b}^{*}}(\mathcal{D}, \mathcal{G})$ is the best constant in (6.6), i.e.

$$
\sum_{F \in \mathcal{F}} \sum_{M \in \mathcal{W}(F)}\left(\frac{\mathrm{P}^{\alpha}(M, h \sigma)}{|M|}\right)^{2}\left\|\mathcal{Q}_{\mathcal{C}_{F}^{\mathcal{G}, \text { shift } ; M}}^{\omega,,^{*}} x\right\|_{L^{2}(\omega)}^{\boldsymbol{\omega}^{2}} \leq \mathfrak{F}_{\alpha}\|h\|_{L^{2}(\sigma)} .
$$

Remark 57. If in (6.6), we take $h=\mathbf{1}_{I}$ and $\mathcal{F}$ to be the trivial Carleson collection $\left\{I_{r}\right\}_{r=1}^{\infty}$ where the intervals $I_{r}$ are pairwise disjoint in $I$, then we essentially obtain the Whitney energy condition in Definition 103, but with $Q_{\mathcal{C}_{F}^{\text {g,shift }} ; M}^{\omega, \mathbf{b}^{*}}$ in place of $\mathrm{Q}_{M}^{\text {weak good, } \omega}$. However, the pseudoprojection $\mathrm{Q}_{M}^{\text {weak good, } \omega}$ is larger than $\mathbf{Q}_{\mathcal{C}_{F}^{\mathcal{G}, \text { shift }}}^{\omega, \mathbf{b}^{*}}$, and so we just miss obtaining the Whitney energy condition as a consequence of the functional energy condition. Nevertheless, this near miss with $h=\mathbf{1}_{I}$ explains the terminology 'functional' energy.

We will need an 'indicator' version of the estimate proved above for the disjoint form

$$
\Theta_{1}(f, g)=\sum_{I \in \mathcal{D}} \sum_{\substack{J \in \mathcal{G}: \ell(J) \leq \ell(I) \\ d(J, I)>2 \ell(J)^{\varepsilon} \ell(I)^{1-\varepsilon}}} \int\left(T_{\sigma} \square_{I}^{\sigma, \mathbf{b}} f\right) \square_{J}^{\omega, \mathbf{b}^{*}} g d \omega .
$$

Lemma 58. Fix dyadic grids $\mathcal{D}$ and $\mathcal{G}$. Suppose $T^{\alpha}$ is a standard fractional singular integral with $0 \leq \alpha<1$, that $f \in L^{2}(\sigma)$ and $g \in L^{2}(\omega)$, that $\mathcal{F} \subset \mathcal{D}$ and $\mathcal{H} \subset \mathcal{G}$ are $\sigma$-Carleson and $\omega$-Carleson collections respectively, i.e.,

$$
\sum_{F^{\prime} \in \mathcal{F}: F^{\prime} \subset F}\left|F^{\prime}\right|_{\sigma} \lesssim|F|_{\sigma}, \quad F \in \mathcal{F}, \text { and } \sum_{H^{\prime} \in \mathcal{H}: H^{\prime} \subset H}\left|H^{\prime}\right|_{\omega} \lesssim|H|_{\omega}, \quad H \in \mathcal{H},
$$

and that there are numerical sequences $\left\{\alpha_{\mathcal{F}}(F)\right\}_{F \in \mathcal{F}}$ and $\left\{\beta_{\mathcal{H}}(H)\right\}_{H \in \mathcal{H}}$ such that

$$
\sum_{F \in \mathcal{F}} \alpha_{\mathcal{F}}(F)^{2}|F|_{\sigma} \leq\|f\|_{L^{2}(\sigma)}^{2} \text { and } \sum_{H \in \mathcal{H}} \beta_{\mathcal{H}}(H)^{2}|H|_{\sigma} \leq\|g\|_{L^{2}(\sigma)}^{2} \text {. }
$$

Then

$$
\begin{aligned}
& \sum_{F \in \mathcal{F}} \sum_{\substack{J \in \mathcal{G}: \ell(J) \leq \ell(F) \\
d(J, F)>2 \ell(J)^{\varepsilon} \ell(F)^{1-\varepsilon}}}\left|\int\left(T_{\sigma}^{\alpha} \mathbf{1}_{F} \alpha_{\mathcal{F}}(F)\right) \square_{J}^{\omega, \mathbf{b}^{*}} g d \omega\right| \\
& +\sum_{\substack { G \in \mathcal{G} \\
\begin{subarray}{c}{I \in \mathcal{D}: \ell(I) \leq \ell(G) \\
d(I, G)>2 \ell(I)^{\varepsilon} \ell(G)^{1-\varepsilon}{ G \in \mathcal { G } \\
\begin{subarray} { c } { I \in \mathcal { D } : \ell ( I ) \leq \ell ( G ) \\
d ( I , G ) > 2 \ell ( I ) ^ { \varepsilon } \ell ( G ) ^ { 1 - \varepsilon } } }\end{subarray}}\left|\int\left(T_{\sigma}^{\alpha} \square_{I}^{\sigma, \mathbf{b}} f\right) \mathbf{1}_{G} \beta_{\mathcal{G}}(G) d \omega\right| \\
& \lesssim \sqrt{A_{2}^{\alpha}}\|f\|_{L^{2}(\sigma)}\|g\|_{L^{2}(\omega)} .
\end{aligned}
$$


The proof of this lemma is similar to those of Lemmas 46 and 47 in Section 4 above, using the square function inequalities for $\square_{I}^{\sigma, \mathbf{b}}, \nabla_{I, \mathcal{F}}^{\sigma}$ and $\square_{J}^{\omega, \mathbf{b}^{*}}, \nabla_{J, \mathcal{G}}^{\omega}$ in Appendix A, as well as the quasiorthogonal inequalities assumed in (6.11), which substitute for the square function inequalities when dealing with indicators $\mathbf{1}_{F} \alpha_{\mathcal{F}}(F)$ instead instead of dual martingale differences $\square_{I}^{\sigma, \mathbf{b}} f$. We note that there is no explicit restriction of the type $\ell(J) \leq 2^{-\boldsymbol{\rho}} \ell(I)$ in any of Lemmas 46, 47. or 58.

There is one more simple lemma that we will use in the proof of the Intertwining Proposition, namely that for small $\varepsilon>0$, an interval $J$ is $\varepsilon$-good inside an interval $I$ only if $J$ is many scales smaller in size than $I$. Recall from Definition 38 that if an interval $J$ is $\varepsilon$-good inside an interval $I$, then

$$
J \subset I \text { and } d(J, \text { skel } I) \geq d(J, \text { body } I)>2 \ell(J)^{\varepsilon} \ell(I)^{1-\varepsilon} .
$$

Lemma 59. If $J$ is $\varepsilon$-good inside $I$, then $\ell(J)<2^{-\frac{3}{\varepsilon}} \ell(I)$.

Proof. We have

$$
\frac{1}{4} \ell(I) \geq d(J, \text { skel } I)>2 \ell(J)^{\varepsilon} \ell(I)^{1-\varepsilon}=2\left(\frac{\ell(J)}{\ell(I)}\right)^{\varepsilon} \ell(I),
$$

which gives $\frac{1}{8}>\left(\frac{\ell(J)}{\ell(I)}\right)^{\varepsilon}$, i.e. $\frac{\ell(J)}{\ell(I)}<\left(\frac{1}{8}\right)^{\frac{1}{\varepsilon}}=2^{-\frac{3}{\varepsilon}}$.

Proposition 60 (The Intertwining Proposition). Let $\mathcal{D}$ and $\mathcal{G}$ be grids, and suppose that $\mathbf{b}$ and $\mathbf{b}^{*}$ are $\infty$-strongly $\sigma$-accretive families of intervals in $\mathcal{D}$ and $\mathcal{G}$ respectively. Suppose that $\mathcal{F} \subset \mathcal{D}$ is $\sigma$-Carleson and that the $\mathcal{F}$-coronas

$$
\mathcal{C}_{F} \equiv\left\{I \in \mathcal{D}: I \subset F \text { but } I \not \subset F^{\prime} \text { for } F^{\prime} \in \mathfrak{C}_{\mathcal{F}}(F)\right\}
$$

satisfy

$$
E_{I}^{\sigma}|f| \lesssim E_{F}^{\sigma}|f| \text { and } b_{I}=\mathbf{1}_{I} b_{F}, \quad \text { for all } I \in \mathcal{C}_{F}, F \in \mathcal{F}
$$

Then with the shifted corona in Definition 56 , i.e. $\mathcal{C}_{F}^{\mathcal{G} \text {,shift }}=\left\{J \in \mathcal{G}: J^{*} \in \mathcal{C}_{F}\right\}$ with $J^{*}$ as in Definition 42 that depends on $\varepsilon>0$, we have

$$
\left|\sum_{F \in \mathcal{F}} \sum_{I: I \supsetneqq F}\left\langle T_{\sigma}^{\alpha} \square_{I}^{\sigma, \mathbf{b}} f, \mathrm{P}_{\mathcal{C}_{F}^{\mathcal{G}, \text { shift }}}^{\omega, \mathbf{b}^{*}} g\right\rangle_{\omega}\right| \lesssim\left(\mathfrak{F}_{\alpha}^{\mathbf{b}^{*}}(\mathcal{D}, \mathcal{G})+\mathcal{N} \mathcal{T} \mathcal{V}_{\alpha}\right)\|f\|_{L^{2}(\sigma)}\|g\|_{L^{2}(\omega)},
$$

where the implied constant depends on the $\sigma$-Carleson norm $C_{\mathcal{F}}$ of the family $\mathcal{F}$.

Proof of Proposition [60. We write the sum on the left hand side of the display above as

$$
\begin{aligned}
& \sum_{F \in \mathcal{F}} \sum_{I: I_{\infty} \supset I \supsetneqq F}\left\langle T_{\sigma}^{\alpha} \square_{I}^{\sigma, \mathbf{b}} f, \mathrm{P}_{\mathcal{C}_{F}^{\mathcal{G}, \text { shift }}}^{\omega} g\right\rangle_{\omega}=\sum_{F \in \mathcal{F}}\left\langle T_{\sigma}^{\alpha}\left(\sum_{I: I_{\infty} \supset I \supsetneqq F} \square_{I}^{\sigma, \mathbf{b}} f\right), \mathrm{P}_{\mathcal{C}_{F}^{\mathcal{G}, \text { shift }}}^{\omega} g\right\rangle=\sum_{F \in \mathcal{F}}\left\langle T_{\sigma}^{\alpha}\left(f_{F}^{*}\right), g_{F}\right\rangle_{\omega} ; \\
& \text { where } f_{F}^{*} \equiv \sum_{I: I_{\infty} \supset I \supsetneqq F} \square_{I}^{\sigma, \mathbf{b}} f \text { and } g_{F} \equiv \mathrm{P}_{\mathcal{C}_{F}^{\mathcal{G}, \text { shift }}}^{\omega, \mathbf{b}^{*}},
\end{aligned}
$$

where $I_{\infty}$ is the starting interval for corona constructions in $\mathcal{D}$ as in (3.2) above. Note that $g_{F}$ is supported in $F$. By the telescoping identity for $\square_{I}^{\sigma, \mathbf{b}}$, the function $f_{F}^{*}$ satisfies

$$
\mathbf{1}_{F} f_{F}^{*}=\sum_{I: I_{\infty} \supset I \supsetneqq F} \square_{I}^{\sigma, \mathbf{b}} f=\mathbb{F}_{F}^{\sigma, \mathbf{b}} f-\mathbf{1}_{F} \mathbb{F}_{I_{\infty}}^{\sigma, \mathbf{b}} f=b_{F} \frac{E_{F}^{\sigma} f}{E_{F}^{\sigma} b_{F}}-\mathbf{1}_{F} b_{I_{\infty}} \frac{E_{I_{\infty}}^{\sigma} f}{E_{I_{\infty}}^{\sigma} b_{I_{\infty}}}
$$

However, we cannot apply the testing condition to the function $\mathbf{1}_{F} b_{I_{\infty}}$, and since $E_{I_{\infty}}^{\sigma} f$ does not vanish in

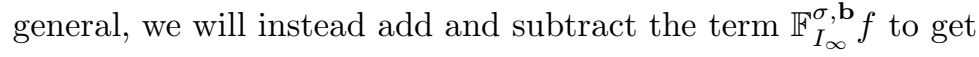

$$
\begin{aligned}
\sum_{F \in \mathcal{F}}\left\langle T_{\sigma}^{\alpha}\left(f_{F}^{*}\right), g_{F}\right\rangle_{\omega} & =\sum_{F \in \mathcal{F}}\left\langle T_{\sigma}^{\alpha}\left(\sum_{I: I_{\infty} \supset I \supsetneqq F} \square_{I}^{\sigma, \mathbf{b}} f\right), \mathrm{P}_{\mathcal{C}_{F}^{\mathcal{G}, \text { shift }}}^{\omega} g\right\rangle \\
& =\sum_{F \in \mathcal{F}}\left\langle T_{\sigma}^{\alpha}\left(\mathbb{F}_{I_{\infty}}^{\sigma, \mathbf{b}} f+\sum_{I: I_{\infty} \supset I \supsetneqq F} \square_{I}^{\sigma, \mathbf{b}} f\right), \mathrm{P}_{\mathcal{C}_{F}^{\mathcal{G}, \text { shift }}} g\right\rangle_{\omega}-\sum_{F \in \mathcal{F}}\left\langle T_{\sigma}^{\alpha}\left(\mathbb{F}_{I_{\infty}}^{\sigma, \mathbf{b}} f\right), \mathrm{P}_{\mathcal{C}_{F}^{\mathcal{G}, \text { shift }}}^{\omega} g\right\rangle_{\omega},
\end{aligned}
$$


where the second sum on the right hand side of the identity satisfies

$$
\begin{aligned}
\left|\sum_{F \in \mathcal{F}}\left\langle T_{\sigma}^{\alpha}\left(\mathbb{F}_{I_{\infty}}^{\sigma, \mathbf{b}} f\right), \mathrm{P}_{\mathcal{C}_{F}^{\mathcal{G}, \text { shift }}}^{\omega} g\right\rangle_{\omega}\right| & =\left|\left\langle T_{\sigma}^{\alpha}\left(\mathbb{F}_{I_{\infty}}^{\sigma, \mathbf{b}} f\right), \sum_{F \in \mathcal{F}} \mathrm{P}_{\mathcal{C}_{F}^{\mathcal{G}, \text { shift }}}^{\omega} g\right\rangle_{\omega}\right| \\
& \leq\left\|T_{\sigma}^{\alpha}\left(\mathbb{F}_{I_{\infty}}^{\sigma, \mathbf{b}} f\right)\right\|_{L^{2}(\omega)}\left\|\sum_{F \in \mathcal{F}} \mathrm{P}_{\mathcal{C}_{F ; \text {; r }}^{\mathcal{G}, \text { shift }}}^{\omega} g\right\|_{L^{2}(\omega)} \\
& \lesssim \mathfrak{F} \mathfrak{T}_{T_{\alpha}}^{\mathbf{b}}\left|E_{I_{\infty}}^{\sigma} f\right|\|g\|_{L^{2}(\omega)} \lesssim\left(\mathfrak{T}_{T_{\alpha}}^{\mathbf{b}}+\mathfrak{A}_{2}^{\alpha}\right)\|f\|_{L^{2}(\sigma)}\|g\|_{L^{2}(\omega)}
\end{aligned}
$$

by (3.25) above, and the Riesz inequalities in Appendix A. The advantage now is that with

$$
f_{F} \equiv \mathbb{F}_{I_{\infty}}^{\sigma, \mathbf{b}} f+f_{F}^{*}=\mathbb{F}_{I_{\infty}}^{\sigma, \mathbf{b}} f+\sum_{I: I_{\infty} \supset I \supsetneqq F} \square_{I}^{\sigma, \mathbf{b}} f
$$

then in the first term on the right hand side of the identity, the telescoping identity gives

$$
\mathbf{1}_{F} f_{F}=\mathbf{1}_{F}\left(\mathbb{F}_{I_{\infty}}^{\sigma, \mathbf{b}} f+\sum_{I: I_{\infty} \supset I \supsetneqq F} \square_{I}^{\sigma, \mathbf{b}} f\right)=\mathbb{F}_{F}^{\sigma, \mathbf{b}} f=b_{F} \frac{E_{F}^{\sigma} f}{E_{F}^{\sigma} b_{F}},
$$

which shows that $f_{F}$ is a controlled constant times $b_{F}$ on $F$.

The intervals $I$ occurring in this sum are linearly and consecutively ordered by inclusion, along with the intervals $F^{\prime} \in \mathcal{F}$ that contain $F$. More precisely we can write

$$
F \equiv F_{0} \varsubsetneqq F_{1} \varsubsetneqq F_{2} \varsubsetneqq \cdots \varsubsetneqq F_{n} \varsubsetneqq F_{n+1} \varsubsetneqq \cdots F_{N}=I_{\infty}
$$

where $F_{m}=\pi_{\mathcal{F}}^{m} F$ for all $m \geq 1$. We can also write

$$
F=F_{0} \equiv I_{0} \varsubsetneqq I_{1} \varsubsetneqq I_{2} \varsubsetneqq \cdots \varsubsetneqq I_{k} \varsubsetneqq I_{k+1} \varsubsetneqq \cdots \varsubsetneqq I_{K}=F_{N}=I_{\infty}
$$

where $I_{k}=\pi_{\mathcal{D}}^{k} F$ for all $k \geq 1$. There is a (unique) subsequence $\left\{k_{m}\right\}_{m=1}^{N}$ such that

$$
F_{m}=I_{k_{m}}, \quad 1 \leq m \leq N .
$$

Then we have

$$
\begin{aligned}
f_{F}(x) & \equiv \mathbb{F}_{I_{\infty}}^{\sigma, \mathbf{b}} f(x)+\sum_{\ell=1}^{K} \square_{I_{\ell}}^{\sigma, \mathbf{b}} f(x), \\
g_{F} & \equiv \sum_{J \in \mathcal{C}_{F}^{\mathcal{G}, \text { shift }}} \square_{J}^{\omega, \mathbf{b}^{*}} g .
\end{aligned}
$$

Assume now that $k_{m} \leq k<k_{m+1}$. We denote the sibling of $I$ by $\theta(I)$, i.e. $\{\theta(I)\}=\mathfrak{C}_{\mathcal{D}}\left(\pi_{\mathcal{D}} I\right) \backslash\{I\}$. There are two cases to consider here:

$$
\theta\left(I_{k}\right) \notin \mathcal{F} \text { and } \theta\left(I_{k}\right) \in \mathcal{F} .
$$

We first note that in either case, using a telescoping sum, we compute that for

$$
x \in \theta\left(I_{k}\right)=I_{k+1} \backslash I_{k} \subset F_{m+1} \backslash F_{m},
$$

we have the formula

$$
\begin{aligned}
f_{F}(x) & =\mathbb{F}_{I_{\infty}}^{\sigma, \mathbf{b}} f(x)+\sum_{\ell \geq k+1} \square_{I_{\ell}}^{\sigma, \mathbf{b}} f(x) \\
& =\mathbb{F}_{\theta\left(I_{k}\right)}^{\sigma, \mathbf{b}} f(x)-\mathbb{F}_{I_{k+1}}^{\sigma, \mathbf{b}} f(x)+\sum_{\ell=k+1}^{K-1}\left(\mathbb{F}_{I_{\ell}}^{\sigma, \mathbf{b}} f(x)-\mathbb{F}_{I_{\ell+1}}^{\sigma, \mathbf{b}} f(x)\right)+\mathbb{F}_{I_{\infty}}^{\sigma, \mathbf{b}} f(x)=\mathbb{F}_{\theta\left(I_{k}\right)}^{\sigma, \mathbf{b}} f(x) .
\end{aligned}
$$

Now fix $x \in \theta\left(I_{k}\right)$. If $\theta\left(I_{k}\right) \notin \mathcal{F}$, then $\theta\left(I_{k}\right) \in \mathcal{C}_{F_{m+1}}^{\sigma}$, and we have

$$
\left|f_{F}(x)\right|=\left|\mathbb{F}_{\theta\left(I_{k}\right)}^{\sigma, \mathbf{b}} f(x)\right| \lesssim\left|b_{\theta\left(I_{k}\right)}(x)\right| \frac{E_{\theta\left(I_{k}\right)}^{\sigma}|f|}{\left|E_{\theta\left(I_{k}\right)}^{\sigma} b_{\theta\left(I_{k}\right)}\right|} \lesssim E_{F_{m+1}}^{\sigma}|f|,
$$


since the testing functions $b_{\theta\left(I_{k}\right)}$ are bounded and accretive, and $E_{\theta\left(I_{k}\right)}^{\sigma}|f| \lesssim E_{F_{m+1}}^{\sigma}|f|$ by hypothesis. On the other hand, if $\theta\left(I_{k}\right) \in \mathcal{F}$, then $I_{k+1} \in \mathcal{C}_{F_{m+1}}^{\sigma}$ and we have

$$
\left|f_{F}(x)\right|=\left|\mathbb{F}_{\theta\left(I_{k}\right)}^{\sigma, \mathbf{b}} f(x)\right| \lesssim E_{\theta\left(I_{k}\right)}^{\sigma}|f| .
$$

Note that $F^{c}=\bigcup_{k \geq 0} \theta\left(I_{k}\right)$. Now we write

$$
\begin{aligned}
f_{F} & =\varphi_{F}+\psi_{F}, \\
\varphi_{F} & \equiv \sum_{k: \theta\left(I_{k}\right) \in \mathcal{F}} \mathbb{F}_{\theta\left(I_{k}\right)}^{\sigma, \mathbf{b}} f \text { and } \psi_{F}=f_{F}-\varphi_{F} \\
\sum_{F \in \mathcal{F}}\left\langle T_{\sigma}^{\alpha} f_{F}, g_{F}\right\rangle_{\omega} & =\sum_{F \in \mathcal{F}}\left\langle T_{\sigma}^{\alpha} \varphi_{F}, g_{F}\right\rangle_{\omega}+\sum_{F \in \mathcal{F}}\left\langle T_{\sigma}^{\alpha} \psi_{F}, g_{F}\right\rangle_{\omega}
\end{aligned}
$$

and note that $\varphi_{F}=0$ on $F$, and $\psi_{F}=b_{F} \frac{E_{F}^{\sigma} f}{E_{F}^{\sigma} b_{F}}$ on $F$. We can apply the first line in (6.12) using $\theta\left(I_{k}\right) \in \mathcal{F}$ to the first sum above since $J \in \mathcal{C}_{F}^{\mathcal{G} \text {,shift }}$ implies $J \subset J^{-} \subset F \subset \theta\left(I_{k}\right)^{c}$, which implies that $d\left(J, \theta\left(I_{k}\right)\right)>$ $2 \ell(J)^{\varepsilon} \ell\left(\theta\left(I_{k}\right)\right)^{1-\varepsilon}$. Thus we obtain after substituting $F^{\prime}$ for $\theta\left(I_{k}\right)$ below,

$$
\begin{aligned}
\left|\sum_{F \in \mathcal{F}}\left\langle T_{\sigma}^{\alpha} \varphi_{F}, g_{F}\right\rangle_{\omega}\right| & =\left|\sum_{F \in \mathcal{F}} \sum_{J \in \mathcal{C}_{F}^{\mathcal{G}, \text { shift }}}\left\langle T_{\sigma}^{\alpha}\left(\sum_{\left.k: I_{k}\right) \in \mathcal{F}} \mathbb{F}_{\theta\left(I_{k}\right)}^{\sigma, \mathbf{b}} f\right), \square_{J}^{\omega, \mathbf{b}^{*}} g\right\rangle\right| \\
& \leq \sum_{F \in \mathcal{F}} \sum_{J \in \mathcal{C}_{F}^{\mathcal{G}, \text { shift }}} \sum_{k:}\left|\left\langle T_{\sigma}^{\alpha}\left(\mathbb{F}_{\theta\left(I_{k}\right) \in \mathcal{F}}^{\sigma, \mathbf{b}} f\right), \square_{J}^{\omega, \mathbf{b}^{*}} g\right\rangle_{\omega}\right| \\
& \leq \sum_{F^{\prime} \in \mathcal{F}} \sum_{\substack{J \in \mathcal{G}: \ell(J) \leq \ell\left(F^{\prime}\right) \\
d\left(J, F^{\prime}\right)>2 \ell(J)^{\varepsilon} \ell\left(F^{\prime}\right)^{1-\varepsilon}}}\left|\left\langle T_{\sigma}^{\alpha}\left(\mathbb{F}_{F^{\prime}}^{\sigma, \mathbf{b}} f\right), \square_{J}^{\omega, \mathbf{b}^{*}} g\right\rangle_{\omega}\right| \\
& \lesssim \sqrt{A_{2}^{\alpha}\|f\|_{L^{2}(\sigma)}\|g\|_{L^{2}(\omega)} .}
\end{aligned}
$$

Turning to the second sum, we note that for $k_{m} \leq k<k_{m+1}$ and $x \in \theta\left(I_{k}\right)$ with $\theta\left(I_{k}\right) \notin \mathcal{F}$, we have

$$
\left|\psi_{F}(x)\right| \lesssim\left|b_{\theta\left(I_{k}\right)}\right| E_{\theta\left(I_{k}\right)}^{\sigma}|f| \mathbf{1}_{F_{m+1} \backslash F_{m}}(x) \lesssim \alpha_{\mathcal{F}}\left(F_{m+1}\right) \mathbf{1}_{F_{m+1} \backslash F_{m}}(x),
$$

and hence the following inequality for $x \notin F$,

$$
\left|\psi_{F}(x)\right| \lesssim \sum_{F^{\prime} \in \mathcal{F}: F \subset F^{\prime}} \alpha_{\mathcal{F}}\left(\pi_{\mathcal{F}} F^{\prime}\right) \mathbf{1}_{\pi_{\mathcal{F}} F^{\prime} \backslash F^{\prime}}(x)=\Phi(x) \mathbf{1}_{F^{c}}(x)
$$

where

$$
\Phi \equiv \sum_{F^{\prime \prime} \in \mathcal{F}} \alpha_{\mathcal{F}}\left(F^{\prime \prime}\right) \mathbf{1}_{F^{\prime \prime} \backslash \cup \mathfrak{C}_{\mathcal{F}}\left(F^{\prime \prime}\right)}=\sum_{F \in \mathcal{F}} \alpha_{\mathcal{F}}(F) \mathbf{1}_{F \backslash \cup \mathfrak{C}_{\mathcal{F}}(F)}
$$

Now we write

$$
\sum_{F \in \mathcal{F}}\left\langle T_{\sigma}^{\alpha} \psi_{F}, g_{F}\right\rangle_{\omega}=\sum_{F \in \mathcal{F}}\left\langle T_{\sigma}^{\alpha}\left(\mathbf{1}_{F} \psi_{F}\right), g_{F}\right\rangle_{\omega}+\sum_{F \in \mathcal{F}}\left\langle T_{\sigma}^{\alpha}\left(\mathbf{1}_{F^{c}} \psi_{F}\right), g_{F}\right\rangle_{\omega} \equiv I+I I
$$

where $I$ and $I I$ are defined at the end of the display. Then by interval testing,

$$
\left|\left\langle T_{\sigma}^{\alpha}\left(b_{F} \mathbf{1}_{F}\right), g_{F}\right\rangle_{\omega}\right|=\left|\left\langle\mathbf{1}_{F} T_{\sigma}^{\alpha}\left(b_{F} \mathbf{1}_{F}\right), g_{F}\right\rangle_{\omega}\right| \lesssim \mathfrak{T}_{T^{\alpha}} \sqrt{|F|_{\sigma}}\left\|g_{F}\right\|_{L^{2}(\omega)}^{\star},
$$

and so quasi-orthogonality, together with the fact that on $F, \psi_{F}=b_{F} \frac{E_{F}^{\sigma} f}{E_{F}^{\sigma} b_{F}}$ is a constant $c=\frac{E_{F}^{\sigma} f}{E_{F}^{\sigma} b_{F}}$ times $b_{F}$, where $|c|$ is bounded by $\alpha_{\mathcal{F}}(F)$, give

$$
\begin{gathered}
|I|=\left|\sum_{F \in \mathcal{F}}\left\langle T_{\sigma}^{\alpha}\left(\mathbf{1}_{F} c b_{F}\right), g_{F}\right\rangle_{\omega}\right| \lesssim \sum_{F \in \mathcal{F}} \alpha_{\mathcal{F}}(F)\left|\left\langle T_{\sigma}^{\alpha} b_{F}, g_{F}\right\rangle_{\omega}\right| \\
\lesssim \sum_{F \in \mathcal{F}} \alpha_{\mathcal{F}}(F) \mathcal{N} \mathcal{T} \mathcal{V}_{\alpha} \sqrt{|F|_{\sigma}}\left\|g_{F}\right\|_{L^{2}(\omega)}^{\star} \lesssim \mathcal{N} \mathcal{T} \mathcal{V}_{\alpha}\|f\|_{L^{2}(\sigma)}\left[\sum_{F \in \mathcal{F}}\left\|g_{F}\right\|_{L^{2}(\omega)}^{\star 2}\right]^{\frac{1}{2}}
\end{gathered}
$$


Now $\mathbf{1}_{F c} \psi_{F}$ is supported outside $F$, and each $J$ in the dual martingale support $\mathcal{C}_{F}^{\mathcal{G} \text {,shift }}$ of $g_{F}=\mathrm{P}_{\mathcal{C}_{F}^{\mathcal{G}, \text { shift }}}^{\omega} g$ is in particular good in the interval $F$, and as a consequence, each such interval $J$ as above is contained in some interval $M$ for $M \in \mathcal{W}(F)$. This containment will be used in the analysis of the term $I I_{G}$ below.

In addition, each $J$ in the dual martingale support $\mathcal{C}_{F}^{\mathcal{G} \text {,shift }}$ of $g_{F}=\mathrm{P}_{\mathcal{C}_{F}^{\mathcal{G}, s h i f t}}^{\omega} g$ is $\left(\left[\frac{3}{\varepsilon}\right], \varepsilon\right)$-deeply embedded in $F$, i.e. $J \Subset_{\left[\frac{3}{\varepsilon}\right], \varepsilon} F$, by Lemma $\left[59\right.$ and the definition of $\mathcal{C}_{F}^{\mathcal{G} \text {,shift }}$ in Definition 56 . As a consequence, each such interval $J$ as above is contained in some interval $M$ for $M \in \mathcal{M}_{\left(\left[\frac{3}{\varepsilon}\right], \varepsilon\right)-\operatorname{deep}, \mathcal{D}}(F)$. This containment will be used in the analysis of the term $I I_{B}$ below.

Notation 61. Define $\boldsymbol{\rho} \equiv\left[\frac{3}{\varepsilon}\right]$, so that for every $J \in \mathcal{C}_{F}^{\mathcal{G} \text {, shift }}$, there is $M \in \mathcal{M}_{(\boldsymbol{\rho}, \varepsilon) \text {-deep, } \mathcal{G}}(F)$ such that $J \subset M$.

The collections $\mathcal{W}(F)$ and $\mathcal{M}_{(\boldsymbol{\rho}, \varepsilon) \text {-deep, } \mathcal{G}}(F)$ used here, and in the display below, are defined in (10.6) in Appendix B. Finally, since the intervals $M \in \mathcal{W}(F)$, as well as the intervals $M \in \mathcal{M}_{\left(\left[\frac{3}{\varepsilon}\right], \varepsilon\right)-\operatorname{deep}, \mathcal{G}}(F)$, satisfy $3 M \subset F$, we can apply (2.29) in the Monotonicity Lemma 30 using (6.14) with $\mu=\mathbf{1}_{F^{c}} \psi_{F}$ and $J^{\prime}$ in place of $J$ there, to obtain

$$
\begin{aligned}
& |I I|=\left|\sum_{F \in \mathcal{F}}\left\langle T_{\sigma}^{\alpha}\left(\mathbf{1}_{F^{c}} \psi_{F}\right), g_{F}\right\rangle_{\omega}\right|=\left|\sum_{F \in \mathcal{F}} \sum_{J^{\prime} \in \mathcal{C}_{F}^{\mathcal{G}, \text { shift }}}\left\langle T_{\sigma}^{\alpha}\left(\mathbf{1}_{F^{c}} \psi_{F}\right), \square_{J^{\prime}}^{\omega, \mathbf{b}^{*}} g\right\rangle_{\omega}\right| \\
& \lesssim \sum_{F \in \mathcal{F}} \sum_{J^{\prime} \in \mathcal{C}_{F}^{\mathcal{G}, \text { shift }}} \frac{\mathrm{P}^{\alpha}\left(J^{\prime}, \mathbf{1}_{F^{c}} \Phi \sigma\right)}{\left|J^{\prime}\right|}\left\|\triangle_{J^{\prime}}^{\omega, \mathbf{b}^{*}} x\right\|_{L^{2}(\omega)}^{\boldsymbol{\omega}}\left\|\square_{J^{\prime}}^{\omega, \mathbf{b}^{*}} g\right\|_{L^{2}(\omega)}^{\star} \\
& +\sum_{F \in \mathcal{F}} \sum_{J^{\prime} \in \mathcal{C}_{F}^{\mathcal{G}, \text { shift }}} \frac{\mathrm{P}_{1+\delta}^{\alpha}\left(J^{\prime}, \mathbf{1}_{F^{c}} \Phi \sigma\right)}{\left|J^{\prime}\right|}\left\|x-m_{J^{\prime}}\right\|_{L^{2}(\omega)}\left\|\square_{J^{\prime}}^{\omega, \mathbf{b}^{*}} g\right\|_{L^{2}(\omega)}^{\star} \\
& \lesssim \sum_{F \in \mathcal{F}} \sum_{M \in \mathcal{W}(F)} \frac{\mathrm{P}^{\alpha}\left(M, \mathbf{1}_{F^{c}} \Phi \sigma\right)}{|M|}\left\|Q_{\mathcal{C}_{F ; M}^{\mathcal{G}, \text { shift }}}^{\omega, \mathbf{b}^{*}} x\right\|_{L^{2}(\omega)}^{\boldsymbol{\omega}}\left\|g_{F ; M}\right\|_{L^{2}(\omega)}^{\star} \\
& +\sum_{F \in \mathcal{F}} \sum_{J \in \mathcal{M}_{(\rho, \varepsilon)-\text { deep }, \mathcal{G}}(F)} \sum_{J^{\prime} \in \mathcal{C}_{F ; J}^{\mathcal{G}, \text { shift }}} \frac{\mathrm{P}_{1+\delta}^{\alpha}\left(J^{\prime}, \mathbf{1}_{F c} \Phi \sigma\right)}{\left|J^{\prime}\right|}\left\|x-m_{J^{\prime}}\right\|_{L^{2}\left(\mathbf{1}_{J^{\prime}} \omega\right)}\left\|\square_{J^{\prime}}^{\omega, \mathbf{b}^{*}} g\right\|_{L^{2}(\omega)}^{\star} \\
& \equiv I I_{G}+I I_{B} \text {. }
\end{aligned}
$$

where $g_{F ; M}$ denotes the pseudoprojection $g_{F ; M}=\sum_{J^{\prime} \in \mathcal{C}_{F}^{\mathcal{G}, \text { shift }}: J^{\prime} \subset M} \square_{J^{\prime}}^{\omega, \mathbf{b}^{*}} g$.

Note: We could also bound $I I_{G}$ by using the decomposition $\mathcal{M}_{(\boldsymbol{\rho}, \varepsilon)-\operatorname{deep}, \mathcal{G}}(F)$ of $F$ into certain maximal $\mathcal{G}$-intervals, but the 'smaller' choice $\mathcal{W}(F)$ of $\mathcal{D}$-intervals is needed for $I I_{G}$ in order to bound it by the corresponding functional energy constant $\mathfrak{F}_{\alpha}^{\mathbf{b}^{*}}$, which can then be controlled by the energy and Muckenhoupt constants in Appendix B.

Then from Cauchy-Schwarz, the functional energy condition, and

$$
\|\Phi\|_{L^{2}(\sigma)}^{2} \leq \sum_{F \in \mathcal{F}} \alpha_{\mathcal{F}}(F)^{2}|F|_{\sigma} \lesssim\|f\|_{L^{2}(\sigma)}^{2},
$$

we obtain

$$
\begin{aligned}
\left|I I_{G}\right| \leq & \left(\sum_{F \in \mathcal{F}} \sum_{M \in \mathcal{W}(F)}\left(\frac{\mathrm{P}^{\alpha}\left(M, \mathbf{1}_{F^{c}} \Phi \sigma\right)}{|M|}\right)^{2}\left\|Q_{\mathcal{C}_{F ; M}^{\mathcal{G}, s h i f t}}^{\omega, \mathbf{b}^{*}} x\right\|_{L^{2}(\omega)}\right)^{\frac{1}{2}} \\
& \times\left(\sum_{F \in \mathcal{F}} \sum_{M \in \mathcal{W}(F)}\left\|g_{F ; M}\right\|_{L^{2}(\omega)}^{\star 2}\right)^{\frac{1}{2}} \\
\lesssim & \mathfrak{F}_{\alpha}^{\mathbf{b}^{*}}\|\Phi\|_{L^{2}(\sigma)}\left[\sum_{F \in \mathcal{F}}\left\|g_{F}\right\|_{L^{2}(\omega)}^{\star 2}\right]^{\frac{1}{2}} \lesssim \mathfrak{F}_{\alpha}^{\mathbf{b}^{*}}\|f\|_{L^{2}(\sigma)}\|g\|_{L^{2}(\omega)}
\end{aligned}
$$


by the pairwise disjointedness of the coronas $\mathcal{C}_{F ; M}^{\mathcal{G} \text {,shift }}$ jointly in $F$ and $M$, which in turn follows from the pairwise disjointedness (6.1) of the shifted coronas $\mathcal{C}_{F}^{\mathcal{G} \text {, shift }}$ in $F$, together with the pairwise disjointedness

of the cubes $M$. Thus we obtain the pairwise disjointedness of both of the pseudoprojections $\mathrm{P}_{\mathcal{C}_{F ; M}^{\mathcal{G}, \text { shift }}}$ and $\mathrm{Q}_{\mathcal{C}_{F ; M}^{\mathcal{G}, \text { shift }}}^{\omega, \mathbf{b}^{*}}$ jointly in $F$ and $M$.

In term $I I_{B}$ the quantities $\left\|x-m_{J^{\prime}}\right\|_{L^{2}\left(\mathbf{1}_{J^{\prime}} \omega\right)}^{2}$ are no longer additive except when the intervals $J^{\prime}$ are pairwise disjoint. As a result we will use (2.33) in the form,

$$
\begin{aligned}
\sum_{J^{\prime} \subset J}\left(\frac{\mathrm{P}_{1+\delta}^{\alpha}\left(J^{\prime}, \nu\right)}{\left|J^{\prime}\right|}\right)^{2}\left\|x-m_{J^{\prime}}\right\|_{L^{2}\left(\mathbf{1}_{J^{\prime}} \omega\right)}^{2} & \lesssim \frac{1}{\gamma^{2 \delta^{\prime}}}\left(\frac{\mathrm{P}_{1+\delta^{\prime}}^{\alpha}(J, \nu)}{|J|}\right)^{2} \sum_{J^{\prime \prime} \subset J}\left\|\triangle_{J^{\prime \prime}}^{\omega} x\right\|_{L^{2}(\omega)}^{2} \\
& \lesssim\left(\frac{\mathrm{P}_{1+\delta^{\prime}}^{\alpha}(J, \nu)}{|J|}\right)^{2}\left\|x-m_{J}\right\|_{L^{2}\left(\mathbf{1}_{J} \omega\right)}^{2},
\end{aligned}
$$

and exploit the decay in the Poisson integral $\mathrm{P}_{1+\delta^{\prime}}^{\alpha}$ along with weak goodness of the intervals $J$. As a consequence we will be able to bound $I I_{B}$ directly by the strong energy condition (1.12), without having to invoke the more difficult functional energy condition. For the decay we compute that for $J \in \mathcal{M}_{(\boldsymbol{\rho}, \varepsilon)-\operatorname{deep}, \mathcal{G}}(F)$

$$
\begin{aligned}
\frac{\mathrm{P}_{1+\delta^{\prime}}^{\alpha}\left(J, \mathbf{1}_{F^{c}} \Phi \sigma\right)}{|J|} & \approx \int_{F^{c}} \frac{|J|^{\delta^{\prime}}}{\left|y-c_{J}\right|^{2+\delta^{\prime}-\alpha}} \Phi(y) d \sigma(y) \\
& \leq \sum_{t=0}^{\infty} \int_{\pi_{\mathcal{F}}^{t+1} F \backslash \pi_{\mathcal{F}}^{t} F}\left(\frac{|J|}{\operatorname{dist}\left(c_{J},\left(\pi_{\mathcal{F}}^{t} F\right)^{c}\right)}\right)^{\delta^{\prime}} \frac{1}{\left|y-c_{J}\right|^{2-\alpha}} \Phi(y) d \sigma(y) \\
& \lesssim \sum_{t=0}^{\infty}\left(\frac{|J|}{\operatorname{dist}\left(c_{J},\left(\pi_{\mathcal{F}}^{t} F\right)^{c}\right)}\right)^{\delta^{\prime}} \frac{\mathrm{P}^{\alpha}\left(J, \mathbf{1}_{\pi_{\mathcal{F}}^{t+1} F \backslash \pi_{\mathcal{F}}^{t} F} \Phi \sigma\right)}{|J|}
\end{aligned}
$$

and then use the weak goodness inequality

$$
\operatorname{dist}\left(c_{J},\left(\pi_{\mathcal{F}}^{t} F\right)^{c}\right) \geq 2 \ell\left(\pi_{\mathcal{F}}^{t} F\right)^{1-\varepsilon} \ell(J)^{\varepsilon} \geq 2 \cdot 2^{t(1-\varepsilon)} \ell(F)^{1-\varepsilon} \ell(J)^{\varepsilon} \geq 2^{t(1-\varepsilon)+1} \ell(J),
$$

to conclude that

$$
\begin{aligned}
\left(\frac{\mathrm{P}_{1+\delta^{\prime}}^{\alpha}\left(J, \mathbf{1}_{F^{c}} \Phi \sigma\right)}{|J|}\right)^{2} & \lesssim\left(\sum_{t=0}^{\infty} 2^{-t \delta^{\prime}(1-\varepsilon)} \frac{\mathrm{P}^{\alpha}\left(J, \mathbf{1}_{\pi_{\mathcal{F}}^{t+1} F \backslash \pi_{\mathcal{F}}^{t} F} \Phi \sigma\right)}{|J|}\right)^{2} \\
& \lesssim \sum_{t=0}^{\infty} 2^{-t \delta^{\prime}(1-\varepsilon)}\left(\frac{\mathrm{P}^{\alpha}\left(J, \mathbf{1}_{\pi_{\mathcal{F}}^{t+1} F \backslash \pi_{\mathcal{F}}^{t} F} \Phi \sigma\right)}{|J|}\right)^{2}
\end{aligned}
$$

Now we first apply Cauchy-Schwarz and (6.15) to obtain

$$
\begin{aligned}
& I I_{B}=\sum_{F \in \mathcal{F}} \sum_{J \in \mathcal{M}} \sum_{(\boldsymbol{\rho}, \varepsilon)-\operatorname{deep}, \mathcal{G}}(F) \frac{\mathrm{P}_{1+\delta}^{\alpha}\left(J^{\prime}, \mathbf{1}_{F^{c}} \Phi \sigma\right)}{\left|J^{\prime}\right|}\left\|x-m_{J^{\prime} \in \mathcal{C}_{F ; J}^{\mathcal{G}, \text { shift }}}\right\|_{L^{2}\left(\mathbf{1}_{J^{\prime}} \omega\right)}\left\|\square_{J^{\prime}}^{\omega, \mathbf{b}^{*}} g\right\|_{L^{2}(\omega)}^{\star}
\end{aligned}
$$

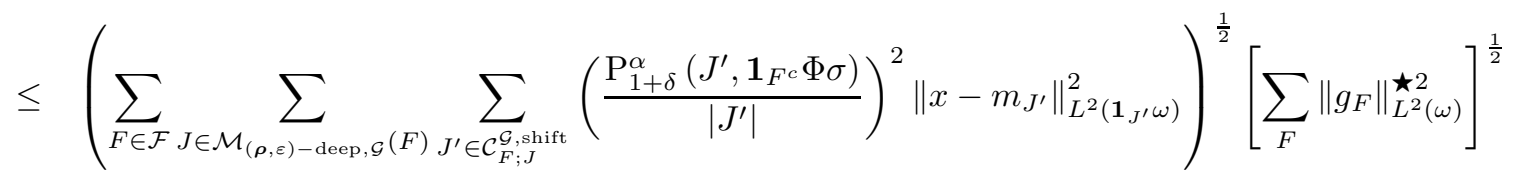

$$
\begin{aligned}
& \leq\left(\sum_{F \in \mathcal{F}} \sum_{J \in \mathcal{M}_{(\boldsymbol{\rho}, \varepsilon)-\text { deep }, \mathcal{G}}(F)}\left(\frac{\mathrm{P}_{1+\delta^{\prime}}^{\alpha}\left(J, \mathbf{1}_{F^{c}} \Phi \sigma\right)}{|J|}\right)^{2}\left\|x-m_{J}\right\|_{L^{2}\left(\mathbf{1}_{J} \omega\right)}^{2}\right)^{\frac{1}{2}}\|g\|_{L^{2}(\omega)} \\
& \equiv \sqrt{I I_{\text {energy }}}\|g\|_{L^{2}(\omega)} \text {, }
\end{aligned}
$$


and it remains to estimate $I I_{\text {energy }}$. From (6.16) and the strong energy condition (1.12), we have

$$
\begin{aligned}
& I I_{\text {energy }}=\sum_{F \in \mathcal{F}} \sum_{J \in \mathcal{M}_{(\boldsymbol{\rho}, \varepsilon)-\text { deep }, \mathcal{G}}(F)}\left(\frac{\mathrm{P}_{1+\delta^{\prime}}^{\alpha}\left(J, \mathbf{1}_{F^{c}} \Phi \sigma\right)}{|J|}\right)^{2}\left\|x-m_{J}\right\|_{L^{2}\left(\mathbf{1}_{J} \omega\right)}^{2} \\
\leq & \sum_{F \in \mathcal{F}} \sum_{J \in \mathcal{M}_{(\boldsymbol{\rho}, \varepsilon) \text {-deep }, \mathcal{G}}(F)} \sum_{t=0}^{\infty} 2^{-t \delta^{\prime}(1-\varepsilon)}\left(\frac{\mathrm{P}^{\alpha}\left(J, \mathbf{1}_{\pi_{\mathcal{F}}^{t+1} F \backslash \pi_{\mathcal{F}}^{t} F} \Phi \sigma\right)}{|J|}\right)^{2}\left\|x-m_{J}\right\|_{L^{2}\left(\mathbf{1}_{J} \omega\right)}^{2} \\
= & \sum_{t=0}^{\infty} 2^{-t \delta^{\prime}(1-\varepsilon)} \sum_{G \in \mathcal{F}} \sum_{F \in \mathfrak{C}_{\mathcal{F}}^{(t+1)}(G)} \sum_{J \in \mathcal{M}_{(\boldsymbol{\rho}, \varepsilon)-\text { deep }, \mathcal{G}}(F)}\left(\frac{\mathrm{P}^{\alpha}\left(J, \mathbf{1}_{G \backslash \pi_{\mathcal{F}}^{t} F} \Phi \sigma\right)}{|J|}\right)^{2}\left\|x-m_{J}\right\|_{L^{2}\left(\mathbf{1}_{J} \omega\right)}^{2} \\
\lesssim & \sum_{t=0}^{\infty} 2^{-t \delta^{\prime}(1-\varepsilon)} \sum_{G \in \mathcal{F}} \alpha_{\mathcal{F}}(G)^{2} \sum_{F \in \mathfrak{C}_{\mathcal{F}}^{(t+1)}(G)} \sum_{J \in \mathcal{M}_{(\boldsymbol{\rho}, \varepsilon) \text {-deep }}(F)}\left(\frac{\mathrm{P}^{\alpha}\left(J, \mathbf{1}_{G \backslash \pi_{\mathcal{F}}^{t} F} \sigma\right)}{|J|}\right)^{2}\left\|x-m_{J}\right\|_{L^{2}\left(\mathbf{1}_{J} \omega\right)}^{2} \\
\lesssim & \sum_{t=0}^{\infty} 2^{-t \delta^{\prime}(1-\varepsilon)} \sum_{G \in \mathcal{F}} \alpha_{\mathcal{F}}(G)^{2}\left(\mathcal{E}_{2}^{\alpha}\right)^{2}|G|_{\sigma} \lesssim\left(\mathcal{E}_{2}^{\alpha}\right)^{2}\|f\|_{L^{2}(\sigma)}^{2} .
\end{aligned}
$$

This completes the proof of the Intertwining Proposition 60,

The task of controlling functional energy is taken up in Appendix B below.

6.4. Paraproduct, neighbour and broken forms. In this subsection we reduce boundedness of the local below form $\mathrm{B}_{\Subset_{\mathbf{r}, \varepsilon}}^{A}(f, g)$ defined in (6.4) to boundedness of the associated stopping form

$$
\mathrm{B}_{\text {stop }}^{A}(f, g) \equiv \sum_{\substack{I \in \mathcal{C}_{A}^{\mathcal{D}} \\ J^{\mathbf{w}} \varsubsetneqq I \text { and } J \in \mathcal{C}_{A}^{\mathcal{G} \text {,shift }} \ell(J) \leq 2^{-\mathbf{r}} \ell(I)}}\left(E_{I_{J}}^{\sigma} \widehat{\square}_{I}^{\sigma, b, \mathbf{b}} f\right)\left\langle T_{\sigma}^{\alpha}\left(\mathbf{1}_{A \backslash I_{J}} b_{A}\right), \square_{J}^{\omega, \mathbf{b}^{*}} g\right\rangle_{\omega},
$$

where the modified difference $\widehat{\square}_{I}^{\sigma, b, \mathbf{b}}$ must be carefully chosen (see (9.21) and (9.23) in Appendix A) in order to control the corresponding paraproduct form below. Indeed, below we will decompose

$$
\mathrm{B}_{\Subset_{\mathbf{r}, \varepsilon}}^{A}(f, g)=\mathrm{B}_{\text {paraproduct }}^{A}(f, g)-\mathrm{B}_{\text {stop }}^{A}(f, g)+\mathrm{B}_{\text {neighbour }}^{A}(f, g)+\mathrm{B}_{\text {broken }}^{A}(f, g),
$$

and then prove in (6.19), (6.25) and (6.18) the estimate

$$
\begin{aligned}
\left|\mathrm{B}_{\mathfrak{\subseteq}_{\mathbf{r}, \varepsilon}}^{A}(f, g)+\mathrm{B}_{\text {stop }}^{A}(f, g)\right| \leq & \left|\mathrm{B}_{\text {paraproduct }}^{A}(f, g)\right|+\left|\mathrm{B}_{\text {neighbour }}^{A}(f, g)\right|+\left|\mathrm{B}_{\text {broken }}^{A}(f, g)\right| \\
\lesssim & \mathfrak{T}_{T^{\alpha}}^{\mathbf{b}} \alpha_{\mathcal{A}}(A) \sqrt{|A|_{\sigma}}\left\|\mathrm{P}_{\mathcal{C}_{A}^{\mathcal{G}, \text { shift }}}^{\omega, \mathbf{b}^{*}} g\right\|_{L^{2}(\omega)}^{\star} \\
& +\sqrt{\mathfrak{A}_{2}^{\alpha}}\left(\left\|\mathrm{P}_{\mathcal{C}_{A}}^{\sigma} f\right\|_{L^{2}(\sigma)}^{\star}+\sqrt{\sum_{A^{\prime} \in \mathfrak{C}_{A}(A)}\left|A^{\prime}\right|_{\sigma} \alpha_{\mathcal{A}}\left(A^{\prime}\right)^{2}}\right)\left\|\mathrm{P}_{\mathcal{C}_{A}^{\mathcal{G}, \text { shift }}}^{\omega, \mathbf{b}^{*}} g\right\|_{L^{2}(\omega)}^{\star},
\end{aligned}
$$

which can of course then be summed in $A \in \mathcal{A}$ to conclude that

$$
\begin{aligned}
& \sum_{A \in \mathcal{A}}\left|\mathrm{B}_{\Subset_{\mathbf{r}, \varepsilon}}^{A}(f, g)+\mathrm{B}_{\text {stop }}^{A}(f, g)\right| \\
\lesssim & \left(\mathfrak{T}_{T^{\alpha}}^{\mathbf{b}}+\sqrt{\mathfrak{A}_{2}^{\alpha}}\right) \sqrt{\sum_{A \in \mathcal{A}}\left\{\alpha_{\mathcal{A}}(A)^{2}|A|_{\sigma}+\left\|\mathrm{P}_{\mathcal{C}_{A}}^{\sigma} f\right\|_{L^{2}(\sigma)}^{\star 2}+\sum_{A^{\prime} \in \mathfrak{C}_{\mathcal{A}}(A)} \alpha_{\mathcal{A}}\left(A^{\prime}\right)^{2}\left|A^{\prime}\right|_{\sigma}\right\}} \sqrt{\sum_{A \in \mathcal{A}}\left\|\mathrm{P}_{\mathcal{C}_{A}^{\mathcal{G}, \text { shift }}}^{\omega, \mathbf{b}^{*}} g\right\|_{L^{2}(\omega)}^{\star 2}} \\
\lesssim & \left(\mathfrak{T}_{T^{\alpha}}^{\mathbf{b}}+\sqrt{\mathfrak{A}_{2}^{\alpha}}\right)\|f\|_{L^{2}(\sigma)}\|g\|_{L^{2}(\omega)} .
\end{aligned}
$$

The stopping form is the subject of the section following this one. 
Note from (9.23) and (9.24) in Appendix A, that the modified dual martingale differences $\square_{I}^{\sigma, b, \mathbf{b}}$ and $\widehat{\square}_{I}^{\sigma, b, \mathbf{b}}$,

$$
\square_{I}^{\sigma, b, \mathbf{b}} f \equiv \square_{I}^{\sigma, \mathbf{b}} f-\sum_{I^{\prime} \in \mathfrak{C}_{\text {broken }}(I)} \mathbb{F}_{I^{\prime}}^{\sigma, \mathbf{b}} f=b_{A} \sum_{I^{\prime} \in \mathfrak{C}(I)} \mathbf{1}_{I^{\prime}} E_{I^{\prime}}^{\sigma}\left(\widehat{\square}_{I}^{\sigma, b, \mathbf{b}} f\right)=b_{A} \widehat{\square}_{I}^{\sigma, b, \mathbf{b}} f,
$$

satisfy the following telescoping property for all $K \in\left(\mathcal{C}_{A} \backslash\{A\}\right) \cup\left(\bigcup_{A^{\prime} \in \mathfrak{C}_{\mathcal{A}}(A)} A^{\prime}\right)$ and $L \in \mathcal{C}_{A}$ with $K \subset L$ :

$$
\sum_{I: \pi K \subset I \subset L} E_{I_{K}}^{\sigma}\left(\widehat{\square}_{I}^{\sigma, b, \mathbf{b}} f\right)=\left\{\begin{array}{ccc}
-E_{L}^{\sigma} \widehat{\mathbb{F}}_{L}^{\sigma, \mathbf{b}} f & \text { if } & K \in \mathfrak{C}_{\mathcal{A}}(A) \\
E_{K}^{\sigma} \widehat{\mathbb{F}}_{K}^{\sigma, \mathbf{b}} f-E_{L}^{\sigma} \widehat{\mathbb{F}}_{L}^{\sigma, \mathbf{b}} f & \text { if } & K \in \mathcal{C}_{A}
\end{array} .\right.
$$

Fix $I \in \mathcal{C}_{A}$ for the moment. We will use

$$
\begin{aligned}
\mathbf{1}_{I} & =\mathbf{1}_{I_{J}}+\mathbf{1}_{\theta\left(I_{J}\right)}, \\
\mathbf{1}_{I_{J}} & =\mathbf{1}_{A}-\mathbf{1}_{A \backslash I_{J}},
\end{aligned}
$$

where $\theta\left(I_{J}\right) \in \mathfrak{C}_{\mathcal{D}}(I) \backslash\left\{I_{J}\right\}$ is the $\mathcal{D}$-child of $I$ other than the child $I_{J}$ that contains $J$. We begin with the splitting

$$
\begin{aligned}
& \left\langle T_{\sigma}^{\alpha} \square_{I}^{\sigma, \mathbf{b}} f, \square_{J}^{\omega, \mathbf{b}^{*}} g\right\rangle_{\omega}=\left\langle T_{\sigma}^{\alpha}\left(\mathbf{1}_{I_{J}} \square_{I}^{\sigma, \mathbf{b}} f\right), \square_{J}^{\omega, \mathbf{b}^{*}} g\right\rangle_{\omega}+\left\langle T_{\sigma}^{\alpha}\left(\mathbf{1}_{\theta\left(I_{J}\right)} \square_{I}^{\sigma, \mathbf{b}} f\right), \square_{J}^{\omega, \mathbf{b}^{*}} g\right\rangle_{\omega} \\
= & \left\langle T_{\sigma}^{\alpha}\left(\mathbf{1}_{I_{J}} \square_{I}^{\sigma, b, \mathbf{b}} f\right), \square_{J}^{\omega, \mathbf{b}^{*}} g\right\rangle_{\omega}+\left\langle T_{\sigma}^{\alpha}\left(\mathbf{1}_{I_{J}} \sum_{I^{\prime} \in \mathfrak{C}_{\text {broken }}(I)} \mathbb{F}_{I^{\prime}}^{\sigma, \mathbf{b}} f\right), \square_{J}^{\omega, \mathbf{b}^{*}} g\right\rangle_{\omega}+\left\langle T_{\sigma}^{\alpha}\left(\mathbf{1}_{\theta\left(I_{J}\right)} \square_{I}^{\sigma, \mathbf{b}} f\right), \square_{J}^{\omega, \mathbf{b}^{*}} g\right\rangle_{\omega} \\
\equiv & I+I I+I I I .
\end{aligned}
$$

From (9.23) we have

$$
\begin{aligned}
I & =\left\langle T_{\sigma}^{\alpha}\left(\mathbf{1}_{I_{J}} \square_{I}^{\sigma, b, \mathbf{b}} f\right), \square_{J}^{\omega, \mathbf{b}^{*}} g\right\rangle_{\omega}=\left\langle T_{\sigma}^{\alpha}\left[b_{A}\left(\mathbf{1}_{I_{J}} \widehat{\square}_{I}^{\sigma, b, \mathbf{b}} f\right)\right], \square_{J}^{\omega, \mathbf{b}^{*}} g\right\rangle_{\omega} \\
& =E_{I_{J}}^{\sigma}\left(\widehat{\square}_{I}^{\sigma, b, \mathbf{b}} f\right)\left\langle T_{\sigma}^{\alpha}\left(\mathbf{1}_{I_{J}} b_{A}\right), \square_{J}^{\omega, \mathbf{b}^{*}} g\right\rangle_{\omega} \\
& =E_{I_{J}}^{\sigma}\left(\widehat{\square}_{I}^{\sigma, b, \mathbf{b}} f\right)\left\langle T_{\sigma}^{\alpha} b_{A}, \square_{J}^{\omega, \mathbf{b}^{*}} g\right\rangle_{\omega}-E_{I_{J}}^{\sigma}\left(\widehat{\square}_{I}^{\sigma, b, \mathbf{b}} f\right)\left\langle T_{\sigma}^{\alpha}\left(\mathbf{1}_{A \backslash I_{J}} b_{A}\right), \square_{J}^{\omega, \mathbf{b}^{*}} g\right\rangle_{\omega} .
\end{aligned}
$$

Since the function $\mathbb{F}_{I_{J}}^{\sigma, \mathbf{b}} f$ is a constant multiple of $b_{I_{J}}$ on $I_{J}$, we can define $\widehat{\mathbb{F}}_{I_{J}}^{\sigma, \mathbf{b}} f \equiv \frac{1}{b_{I_{J}}} \mathbb{F}_{I_{J}}^{\sigma, \mathbf{b}} f$ (or simply use the $P L B P$ we are assuming) and then

$$
I I=\left\langle T_{\sigma}^{\alpha}\left(\mathbf{1}_{I_{J}} \sum_{I^{\prime} \in \mathfrak{C}_{\mathrm{broken}}(I)} \mathbb{F}_{I^{\prime}}^{\sigma, \mathbf{b}} f\right), \square_{J}^{\omega, \mathbf{b}^{*}} g\right\rangle_{\omega}=\mathbf{1}_{\mathfrak{C}_{\mathcal{A}}(A)}\left(I_{J}\right) E_{I_{J}}^{\sigma}\left(\widehat{\mathbb{F}}_{I_{J}}^{\sigma, \mathbf{b}} f\right)\left\langle T_{\sigma}^{\alpha} b_{I_{J}}, \square_{J}^{\omega, \mathbf{b}^{*}} g\right\rangle_{\omega},
$$

where the presence of the indicator function $\mathbf{1}_{\mathfrak{C}_{\mathcal{A}}(A)}\left(I_{J}\right)$ simply means that term $I I$ vanishes unless $I_{J}$ is an $\mathcal{A}$-child of $A$. We now write these terms as

$$
\begin{aligned}
\left\langle T_{\sigma}^{\alpha} \square_{I}^{\sigma, \mathbf{b}} f, \square_{J}^{\omega, \mathbf{b}^{*}} g\right\rangle_{\omega}= & E_{I_{J}}^{\sigma}\left(\widehat{\square}_{I}^{\sigma, b, \mathbf{b}} f\right)\left\langle T_{\sigma}^{\alpha} b_{A}, \square_{J}^{\omega, \mathbf{b}^{*}} g\right\rangle_{\omega} \\
& -E_{I_{J}}^{\sigma}\left(\widehat{\square}_{I}^{\sigma, b, \mathbf{b}} f\right)\left\langle T_{\sigma}^{\alpha}\left(\mathbf{1}_{A \backslash I_{J}} b_{A}\right), \square_{J}^{\omega, \mathbf{b}^{*}} g\right\rangle_{\omega} \\
& +\left\langle T_{\sigma}^{\alpha}\left(\mathbf{1}_{\theta\left(I_{J}\right)} \square_{I}^{\sigma, \mathbf{b}} f\right), \square_{J}^{\omega, \mathbf{b}^{*}} g\right\rangle_{\omega} \\
& +\mathbf{1}_{\left\{I_{J} \in \mathfrak{C}_{\mathcal{A}}(A)\right\}} E_{I_{J}}^{\sigma}\left(\widehat{\mathbb{F}}_{I_{J}}^{\sigma, \mathbf{b}} f\right)\left\langle T_{\sigma}^{\alpha} b_{I_{J}}, \square_{J}^{\omega, \mathbf{b}^{*}} g\right\rangle_{\omega},
\end{aligned}
$$

where the four lines are respectively a paraproduct, stopping, neighbour and broken term.

The corresponding NTV splitting of $\mathrm{B}_{\leftarrow_{\mathbf{r}, \varepsilon}}^{A}(f, g)$ using (6.4) and (6.2) becomes

$$
\begin{aligned}
\mathrm{B}_{\Subset_{\mathbf{r}, \varepsilon}}^{A}(f, g) & =\left\langle T_{\sigma}^{\alpha}\left(\mathrm{P}_{\mathcal{C}_{A}}^{\sigma} f\right), \mathrm{P}_{\mathcal{C}_{A}^{\mathcal{G}, \text { shift }}}^{\omega} g\right\rangle_{\omega}^{\epsilon_{\mathbf{r}, \varepsilon}}=\sum_{\substack{I \in \mathcal{C}_{A} \text { and } J \in \mathcal{C}_{A}^{\mathcal{G}, \text { shift }} \\
J^{\boldsymbol{*}} \varsubsetneqq I \text { and } \ell(J) \leq 2^{-\mathbf{r}} \ell(I)}}\left\langle T_{\sigma}^{\alpha}\left(\square_{I}^{\sigma, \mathbf{b}} f\right), \square_{J}^{\omega, \mathbf{b}^{*}} g\right\rangle_{\omega} \\
& =\mathrm{B}_{\text {paraproduct }}^{A}(f, g)-\mathrm{B}_{\text {stop }}^{A}(f, g)+\mathrm{B}_{\text {neighbour }}^{A}(f, g)+\mathrm{B}_{\text {broken }}^{A}(f, g),
\end{aligned}
$$


where

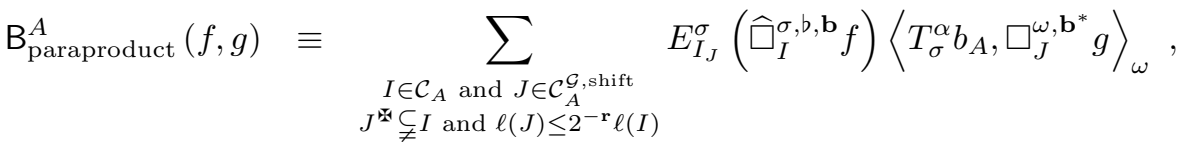

$$
\begin{aligned}
& \mathrm{B}_{\text {stop }}^{A}(f, g) \equiv \sum_{\substack{I \in \mathcal{C}_{A} \text { and } J \in \mathcal{C}_{A}^{\mathcal{G}} \text {, shift } \\
J^{\mathbf{*}} \varsubsetneqq I \text { and } \ell(J) \leq 2^{-\mathbf{r}} \ell(I)}} E_{I_{J}}^{\sigma}\left(\hat{\square}_{I}^{\sigma, b, \mathbf{b}} f\right)\left\langle T_{\sigma}^{\alpha}\left(\mathbf{1}_{A \backslash I_{J}} b_{A}\right), \square_{J}^{\omega, \mathbf{b}^{*}} g\right\rangle_{\omega}, \\
& \mathrm{B}_{\text {neighbour }}^{A}(f, g) \equiv \sum_{\substack{I \in \mathcal{C}_{A} \text { and } J \in \mathcal{C}_{A}^{\mathcal{G}, \text { shift }} \\
J^{\mathbf{w}} \varsubsetneqq I \text { and } \ell(J) \leq 2^{-\mathbf{r}} \ell(I)}}\left\langle T_{\sigma}^{\alpha}\left(\mathbf{1}_{\theta\left(I_{J}\right)} \square_{I}^{\sigma, \mathbf{b}} f\right), \square_{J}^{\omega, \mathbf{b}^{*}} g\right\rangle_{\omega},
\end{aligned}
$$

correspond to the three original NTV forms associated with 1-testing, and where

$$
\mathrm{B}_{\text {broken }}^{A}(f, g) \equiv \sum_{\substack{I \in \mathcal{C}_{A} \text { and } J \in \mathcal{C}_{A}^{\mathcal{G} \text {, shift }} \\ J^{\mathbf{w}} \varsubsetneqq I \text { and } \ell(J) \leq 2^{-\mathbf{r}} \ell(I)}} \mathbf{1}_{\left\{I_{J} \in \mathfrak{C}_{\mathcal{A}}(A)\right\}} E_{I_{J}}^{\sigma}\left(\widehat{\mathbb{F}}_{I_{J}}^{\sigma, \mathbf{b}} f\right)\left\langle T_{\sigma}^{\alpha} b_{I_{J}}, \square_{J}^{\omega, \mathbf{b}^{*}} g\right\rangle_{\omega}=0,
$$

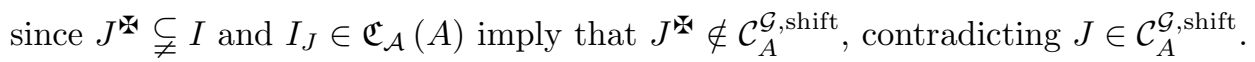

Remark 62. The inquisitive reader will note that the pairs $(I, J)$ arising in the above sum with $J^{*} \subsetneq I$ replaced by $J^{*}=I$ are handled in the probabilistic estimate (3.18) for the bad form $\Theta_{2}^{\text {bad }}$. defined in (3.15).

6.4.1. The paraproduct form. The paraproduct form $\mathrm{B}_{\text {paraproduct }}^{A}(f, g)$ is easily controlled by the testing condition for $T^{\alpha}$ together with weak Riesz inequalities for dual martingale differences. Indeed, recalling the telescoping identity (9.24), and that the collection $\left\{I \in \mathcal{C}_{A}: \ell(J) \leq 2^{-\mathbf{r}} \ell(I)\right\}$ is tree connected for all $J \in \mathcal{C}_{A}^{\mathcal{G} \text {, shift }}$, we have

$$
\begin{aligned}
& \mathrm{B}_{\text {paraproduct }}^{A}(f, g)=\sum_{\substack{I \in \mathcal{C}_{A} \\
J^{\mathbf{w}} \subsetneq I \text { and } J \in \mathcal{C}_{A}^{\mathcal{G}, \text { shift }} \\
\text { and } \ell(J) \leq 2^{-\mathbf{r}} \ell(I)}} E_{I_{J}}^{\sigma}\left(\widehat{\square}_{I}^{\sigma, b, \mathbf{b}} f\right)\left\langle T_{\sigma}^{\alpha} b_{A}, \square_{J}^{\omega, \mathbf{b}^{*}} g\right\rangle_{\omega} \\
& =\sum_{J \in \mathcal{C}_{A}^{\mathcal{G}, \text { shift }}}\left\langle T_{\sigma}^{\alpha} b_{A}, \square_{J}^{\omega, \mathbf{b}^{*}} g\right\rangle_{\omega}\left\{\sum_{I \in \mathcal{C}_{A}: J^{\mathbf{*}} \varsubsetneqq I \text { and } \ell(J) \leq 2^{-\mathbf{r}} \ell(I)} E_{I_{J}}^{\sigma}\left(\widehat{\square}_{I}^{\sigma, b, \mathbf{b}} f\right)\right\} \\
& =\sum_{J \in \mathcal{C}_{A}^{\mathcal{G}, \text { shift }}}\left\langle T_{\sigma}^{\alpha} b_{A}, \square_{J}^{\omega, \mathbf{b}^{*}} g\right\rangle_{\omega}\left\{\mathbf{1}_{\left\{J: I^{\natural}(J)_{J} \in \mathcal{C}_{A}\right\}} E_{I^{\natural}(J)_{J}}^{\sigma \widehat{\mathbb{F}}_{I^{\natural}(J)_{J}}^{\sigma, \mathbf{b}}} f-E_{A}^{\sigma} \widehat{\mathbb{F}}_{A}^{\sigma, \mathbf{b}} f\right\} \\
& =\left\langle T_{\sigma}^{\alpha} b_{A}, \sum_{J \in \mathcal{C}_{A}^{\mathcal{G}, \text { shift }}}\left\{\mathbf{1}_{\left\{J: I^{\natural}(J)_{J} \in \mathcal{C}_{A}\right\}} E_{I^{\natural}(J)_{J}}^{\sigma} \widehat{\mathbb{F}}_{I^{\natural}(J)_{J}}^{\sigma, \mathbf{b}} f-E_{A}^{\sigma} \widehat{\mathbb{F}}_{A}^{\sigma, \mathbf{b}} f\right\} \square_{J}^{\omega, \mathbf{b}^{*}} g\right\rangle_{\omega},
\end{aligned}
$$

where $I^{\natural}(J)$ denotes the smallest interval $I \in \mathcal{C}_{A}$ such that $J^{\natural} \subsetneq I$ and $\ell(J) \leq 2^{-\mathbf{r}} \ell(I)$, and of course $I^{\natural}(J){ }_{J}$ denotes its child containing $J$. Note that by construction of the modified difference operator $\square_{I}^{\sigma, b, \mathbf{b}}$, the only time the average $\widehat{\mathbb{F}}_{I^{\natural}(J)_{J}}^{\sigma} f$ appears in the above sum is when $I^{\natural}(J)_{J} \in \mathcal{C}_{A}$, since the case $I^{\natural}(J)_{J} \in \mathcal{A}$ has been removed to the broken term. This is reflected above with the inclusion of the indicator $\mathbf{1}_{\left\{J: I^{\natural}(J)_{J} \in \mathcal{C}_{A}\right\}}$. It follows that we have the bound $\left|\mathbf{1}_{\left\{J: I^{\natural}(J)_{J} \in \mathcal{C}_{A}\right\}} E_{I^{\natural}(J)_{J}}^{\sigma} \widehat{\mathbb{F}}_{I^{\natural}(J) J}^{\sigma, \mathbf{b}} f\right|+\left|E_{A}^{\sigma} \widehat{\mathbb{F}}_{A}^{\sigma, \mathbf{b}} f\right| \lesssim E_{A}^{\sigma}|f| \leq \alpha_{\mathcal{A}}(A)$.

Thus from Cauchy-Schwarz, the upper weak Riesz inequalities Proposition 95 for the pseudoprojections $\square_{J}^{\omega, \mathbf{b}^{*}} g$ and the bound on the coefficients $\lambda_{J} \equiv\left(\mathbf{1}_{\left\{J: I^{\natural}(J)_{J} \in \mathcal{C}_{A}\right\}} E_{I^{\natural}(J)_{J}}^{\sigma} \widehat{\mathbb{F}}_{I^{\natural}(J)_{J}}^{\sigma, \mathbf{b}} f-E_{A}^{\sigma} \widehat{\mathbb{F}}_{A}^{\sigma, \mathbf{b}} f\right)$ given by $\left|\lambda_{J}\right| \lesssim$ 
$\alpha_{\mathcal{A}}(A)$, we have

$$
\begin{aligned}
\left|\mathrm{B}_{\text {paraproduct }}^{A}(f, g)\right| & =\left|\left\langle T_{\sigma}^{\alpha} b_{A}, \sum_{J \in \mathcal{C}_{A}^{\mathcal{G}, \text { shift }}}\left\{\left(\mathbf{1}_{\left\{J: I^{\natural}(J)_{J} \in \mathcal{C}_{A}\right\}} E_{I^{\natural}(J)_{J}}^{\sigma} \widehat{\mathbb{F}}_{I^{\natural}(J)}^{\sigma, \mathbf{b}} f-E_{A}^{\sigma} \widehat{\mathbb{F}}_{A}^{\sigma, \mathbf{b}} f\right)\right\} \square_{J}^{\omega, \mathbf{b}^{*}} g\right\rangle\right| \\
& \leq\left\|\mathbf{1}_{A} T_{\sigma}^{\alpha} b_{A}\right\|_{L^{2}(\omega)} \| \sum_{J \in \mathcal{C}_{A}^{\mathcal{G}, \text { shift }}} \lambda_{J} \square_{J}^{\omega, \mathbf{b}^{*}} g \mid \\
& \lesssim \alpha_{\mathcal{A}}(A)\left\|\mathbf{1}_{A} T_{\sigma}^{\alpha} b_{A}\right\|_{L^{2}(\omega)}\left\|\sum_{J \in \mathcal{C}_{A}^{\mathcal{G}, \text { shift }}} \square_{J}^{\omega, \mathbf{b}^{*}} g\right\|_{L^{2}(\omega)}^{\star} \\
& \leq \mathfrak{T}_{T^{\alpha}}^{\mathbf{b}} \alpha_{\mathcal{A}}(A) \sqrt{|A|_{\sigma}}\left\|\mathrm{P}_{\mathcal{C}_{A}^{\mathcal{G}, \text { shift }}}^{\omega, \mathbf{b}^{*}} g\right\|_{L^{2}(\omega)}^{\star} .
\end{aligned}
$$

6.4.2. The neighbour form. Next, the neighbour form $\mathrm{B}_{\text {neighbour }}^{A}(f, g)$ is easily controlled by the $\mathfrak{A}_{2}^{\alpha}$ condition using the pivotal estimate in Energy Lemma 33 and the fact that the intervals $J \in \mathcal{C}_{A}^{\mathcal{G} \text {,shift }}$ are good in $I$ and beyond when the pair $(I, J)$ occurs in the sum. In particular, the information encoded in the stopping tree $\mathcal{A}$ plays no role here, apart from appearing in the corona projections on the right hand side of (6.25) below. We have

$$
\mathrm{B}_{\text {neighbour }}^{A}(f, g)=\sum_{\substack{I \in \mathcal{C}_{A} \text { and } J \in \mathcal{C}_{A}^{\mathcal{G}, \text { shift }} \\ J^{\mathbf{*}} \varsubsetneqq I \text { and } \ell(J) \leq 2^{-\mathbf{r}} \ell(I)}}\left\langle T_{\sigma}^{\alpha}\left(\mathbf{1}_{\theta\left(I_{J}\right)} \square_{I}^{\sigma, \mathbf{b}} f\right), \square_{J}^{\omega, \mathbf{b}^{*}} g\right\rangle_{\omega},
$$

where we keep in mind that the pairs $(I, J) \in \mathcal{D} \times \mathcal{G}$ that arise in the sum for $\mathrm{B}_{\text {neighbour }}^{A}(f, g)$ satisfy the property that $J^{\text {F }} \varsubsetneqq I$, so that $J$ is good with respect to all intervals $K$ of size at least that of $J^{\text {, which }}$ includes $I$. Recall that $I_{J}$ is the child of $I$ that contains $J$, and that $\theta\left(I_{J}\right)$ denotes its sibling in $I$, i.e. $\theta\left(I_{J}\right) \in \mathfrak{C}_{\mathcal{D}}(I) \backslash\left\{I_{J}\right\}$. Fix $(I, J)$ momentarily, and an integer $s \geq \mathbf{r}$. Using $\square_{I}^{\sigma, \mathbf{b}}=\square_{I}^{\sigma, b, \mathbf{b}}+\square_{I, \text { broken }}^{\sigma, b, \mathbf{b}}$ and the fact that $\square_{I}^{\sigma, b, \mathbf{b}} f$ is a constant multiple of $b_{\theta\left(I_{J}\right)}$ on the interval $\theta\left(I_{J}\right)$, we have the estimates

$$
\begin{aligned}
\left|\mathbf{1}_{\theta\left(I_{J}\right)} \square_{I}^{\sigma, b, \mathbf{b}} f\right| & =\left|\left(E_{\theta\left(I_{J}\right)}^{\sigma} \widehat{\square}_{I}^{\sigma, b, \mathbf{b}} f\right) b_{\theta\left(I_{J}\right)}\right| \leq C_{\mathbf{b}}\left|E_{\theta\left(I_{J}\right)}^{\sigma} \widehat{\square}_{I}^{\sigma, b, \mathbf{b}} f\right|, \\
\left|\mathbf{1}_{\theta\left(I_{J}\right)} \square_{I, \text { broken }}^{\sigma, b, \mathbf{b}} f\right| & \leq \mathbf{1}_{\mathfrak{C}_{A}(A)}\left(\theta\left(I_{J}\right)\right) E_{\theta\left(I_{J}\right)}^{\sigma}|f|,
\end{aligned}
$$

and hence

$$
\mathbf{1}_{\theta\left(I_{J}\right)}\left|\square_{I}^{\sigma, \mathbf{b}} f\right| \leq C \mathbf{1}_{\theta\left(I_{J}\right)}\left(\left|E_{\theta\left(I_{J}\right)}^{\sigma} \hat{\square}_{I}^{\sigma, b, \mathbf{b}} f\right|+\mathbf{1}_{\mathfrak{C}_{A}(A)}\left(\theta\left(I_{J}\right)\right) E_{\theta\left(I_{J}\right)}^{\sigma}|f|\right),
$$

which will be used below after an application of the Energy Lemma. We can write

$$
\mathrm{B}_{\text {neighbour }}^{A}(f, g)=\sum_{\substack{I \in \mathcal{C}_{A} \text { and } J \in \mathcal{G}_{\left(\kappa\left(I_{J}, J\right), \varepsilon\right)-\text { good }}^{\mathcal{D}} \cap \mathcal{C}_{A}^{\mathcal{G}, \text { shift }} \\ d\left(J, \theta\left(I_{J}\right)\right)>2 \ell(J)^{\varepsilon} \ell\left(\theta\left(I_{J}\right)\right)^{1-\varepsilon} \text { and } \ell(J) \leq 2^{-\mathbf{r}} \ell(I)}}\left\langle T_{\sigma}^{\alpha}\left(\mathbf{1}_{\theta\left(I_{J}\right)} \square_{I}^{\sigma, \mathbf{b}} f\right), \square_{J}^{\omega, \mathbf{b}^{*}} g\right\rangle_{\omega}
$$

where we have included the conditions $J \in \mathcal{G}_{\left(\kappa\left(I_{J}, J\right), \varepsilon\right)-\operatorname{good}}^{\mathcal{D}}$ and $d\left(J, \theta\left(I_{J}\right)\right)>2 \ell(J)^{\varepsilon} \ell\left(\theta\left(I_{J}\right)\right)^{1-\varepsilon}$ in the summation since they are already implied the remaining four conditions, and will be used in estimates below.

We will also use the following fractional analogue of the Poisson inequality in [Vol].

Lemma 63. Suppose $0 \leq \alpha<1$ and $J \subset I \subset K$ and that $d(J, \partial I)>2 \ell(J)^{\varepsilon} \ell(I)^{1-\varepsilon}$ for some $0<\varepsilon<\frac{1}{2-\alpha}$. Then for a positive Borel measure $\mu$ we have

$$
\mathrm{P}^{\alpha}\left(J, \mu \mathbf{1}_{K \backslash I}\right) \lesssim\left(\frac{\ell(J)}{\ell(I)}\right)^{1-\varepsilon(2-\alpha)} \mathrm{P}^{\alpha}\left(I, \mu \mathbf{1}_{K \backslash I}\right)
$$


Proof. We have

$$
\mathrm{P}^{\alpha}\left(J, \mu \mathbf{1}_{K \backslash I}\right) \approx \sum_{k=0}^{\infty} 2^{-k} \frac{1}{\left|2^{k} J\right|^{1-\alpha}} \int_{\left(2^{k} J\right) \cap(K \backslash I)} d \mu,
$$

and $\left(2^{k} J\right) \cap(K \backslash I) \neq \emptyset$ requires

$$
d(J, K \backslash I) \leq c 2^{k} \ell(J),
$$

for some dimensional constant $c>0$. Let $k_{0}$ be the smallest such $k$. By our distance assumption we must then have

$$
2 \ell(J)^{\varepsilon} \ell(I)^{1-\varepsilon} \leq d(J, \partial I) \leq c 2^{k_{0}} \ell(J)
$$

or

$$
2^{-k_{0}+1} \leq c\left(\frac{\ell(J)}{\ell(I)}\right)^{1-\varepsilon}
$$

Now let $k_{1}$ be defined by $2^{k_{1}} \equiv \frac{\ell(I)}{\ell(J)}$. Then assuming $k_{1}>k_{0}$ (the case $k_{1} \leq k_{0}$ is similar) we have

$$
\begin{aligned}
\mathrm{P}^{\alpha}\left(J, \mu \mathbf{1}_{K \backslash I}\right) & \approx\left\{\sum_{k=k_{0}}^{k_{1}}+\sum_{k=k_{1}}^{\infty}\right\} 2^{-k} \frac{1}{\left|2^{k} J\right|^{1-\alpha}} \int_{\left(2^{k} J\right) \cap(K \backslash I)} d \mu \\
& \lesssim 2^{-k_{0}} \frac{|I|^{1-\alpha}}{\left|2^{k_{0}} J\right|^{1-\alpha}}\left(\frac{1}{|I|^{1-\alpha}} \int_{\left(2^{k_{1} J}\right) \cap(K \backslash I)} d \mu\right)+2^{-k_{1}} \mathrm{P}^{\alpha}\left(I, \mu \mathbf{1}_{K \backslash I}\right) \\
& \lesssim\left(\frac{\ell(J)}{\ell(I)}\right)^{(1-\varepsilon)(2-\alpha)}\left(\frac{\ell(I)}{\ell(J)}\right)^{1-\alpha} \mathrm{P}^{\alpha}\left(I, \mu \mathbf{1}_{K \backslash I}\right)+\frac{\ell(J)}{\ell(I)} \mathrm{P}^{\alpha}\left(I, \mu \mathbf{1}_{K \backslash I}\right),
\end{aligned}
$$

which is the inequality (6.22).

Now fix $I_{0}=I_{J}, I_{\theta}=\theta\left(I_{J}\right) \in \mathfrak{C}_{\mathcal{D}}(I)$ and assume that $J \Subset_{\mathbf{r}, \varepsilon} I_{0}$. Let $\frac{\ell(J)}{\ell\left(I_{0}\right)}=2^{-s}$ in the pivotal estimate from Energy Lemma 33 with $J \subset I_{0} \subset I$ to obtain

$$
\begin{aligned}
& \left|\left\langle T_{\sigma}^{\alpha}\left(\mathbf{1}_{\theta\left(I_{J}\right)} \square_{I}^{\sigma, \mathbf{b}} f\right), \square_{J}^{\omega, \mathbf{b}^{*}} g\right\rangle_{\omega}\right| \\
& \lesssim\left\|\square_{J}^{\omega, \mathbf{b}^{*}} g\right\|_{L^{2}(\omega)} \sqrt{|J|_{\omega}} \mathrm{P}^{\alpha}\left(J, \mathbf{1}_{\theta\left(I_{J}\right)}\left|\square_{I}^{\sigma, \mathbf{b}} f\right| \sigma\right) \\
& \lesssim\left\|\square_{J}^{\omega, \mathbf{b}^{*}} g\right\|_{L^{2}(\omega)} \sqrt{|J|_{\omega}} \cdot 2^{-(1-\varepsilon(2-\alpha)) s} \mathrm{P}^{\alpha}\left(I_{0}, \mathbf{1}_{\theta\left(I_{J}\right)}\left|\square_{I}^{\sigma, \mathbf{b}} f\right| \sigma\right) \\
& \lesssim\left\|\square_{J}^{\omega, \mathbf{b}^{*}} g\right\|_{L^{2}(\omega)} \sqrt{|J|_{\omega}} \cdot 2^{-(1-\varepsilon(2-\alpha)) s} \mathrm{P}^{\alpha}\left(I_{0}, \mathbf{1}_{\theta\left(I_{J}\right)}\left(\left|E_{\theta\left(I_{J}\right)}^{\sigma} \hat{\square}_{I}^{\sigma, b, \mathbf{b}} f\right|+\mathbf{1}_{\mathfrak{C}_{A}(A)}\left(\theta\left(I_{J}\right)\right) E_{\theta\left(I_{J}\right)}^{\sigma}|f|\right) \sigma\right) .
\end{aligned}
$$

Here we are using (6.22) in the third line, which applies since $J \subset I_{0}$, and we have used (6.21) in the fourth line. It will be convenient to use the shorthand notation

$$
\mathbf{E}_{\theta\left(I_{J}\right)}^{\sigma} f \equiv\left|E_{\theta\left(I_{J}\right)}^{\sigma} \hat{\square}_{I}^{\sigma, b, \mathbf{b}} f\right|+\mathbf{1}_{\mathfrak{C}_{A}(A)}\left(\theta\left(I_{J}\right)\right) E_{\theta\left(I_{J}\right)}^{\sigma}|f|
$$

where the intervals $I$ and $I_{J}$ on the right hand side are determined uniquely by the interval $\theta\left(I_{J}\right)$.

In the sum below, we keep the side lengths of the intervals $J$ fixed at $2^{-s}$ times that of $I_{0}$, and of course take $J \subset I_{0}$. We also keep the underlying assumptions that $J \in \mathcal{C}_{A}^{\mathcal{G} \text {,shift }}$ and that $J \in \mathcal{G}_{\left(\kappa\left(I_{J}, J\right), \varepsilon\right) \text {-good }}^{\mathcal{D}}$ in mind without necessarily pointing to them in the notation. Matters will shortly be reduced to estimating the following term:

$$
\begin{aligned}
A\left(I, I_{0}, I_{\theta}, s\right) & \equiv \sum_{J: 2^{s+1} \ell(J)=\ell(I): J \subset I_{0}}\left|\left\langle T_{\sigma}^{\alpha}\left(\mathbf{1}_{I_{\theta}} \square_{I}^{\sigma, \mathbf{b}} f\right), \square_{J}^{\omega, \mathbf{b}^{*}} g\right\rangle_{\omega}\right| \\
& \leq 2^{-(1-\varepsilon(2-\alpha)) s}\left(\mathbf{E}_{\theta\left(I_{J}\right)}^{\sigma} f\right) \mathrm{P}^{\alpha}\left(I_{0}, \mathbf{1}_{\theta\left(I_{J}\right)} \sigma\right) \sum_{J: 2^{s+1} \ell(J)=\ell(I): J \subset I_{0}}\left\|\square_{J}^{\omega, \mathbf{b}^{*}} g\right\|_{L^{2}(\omega)} \sqrt{|J|_{\omega}} \\
& \leq 2^{-(1-\varepsilon(2-\alpha)) s}\left(\mathbf{E}_{\theta\left(I_{J}\right)}^{\sigma} f\right) \mathrm{P}^{\alpha}\left(I_{0}, \mathbf{1}_{\theta\left(I_{J}\right)} \sigma\right) \sqrt{\left|I_{0}\right|_{\omega}} \Lambda\left(I, I_{0}, I_{\theta}, s\right), \\
& \text { where } \Lambda\left(I, I_{0}, I_{\theta}, s\right)^{2} \equiv \sum_{J \in \mathcal{C}_{A}^{\mathcal{G}, \text { shift }}: 2^{s+1} \ell(J)=\ell(I): J \subset I_{0}}\left\|\square_{J}^{\omega, \mathbf{b}^{*}} g\right\|_{L^{2}(\omega)}^{2} .
\end{aligned}
$$


The last line follows upon using the Cauchy-Schwarz inequality and the fact that $J \in \mathcal{C}_{A}^{\mathcal{G} \text {,shift }}$. We also note that since $2^{s+1} \ell(J)=\ell(I)$,

$$
\begin{aligned}
\sum_{I_{0} \in \mathfrak{C}_{\mathcal{D}}(I)} \Lambda\left(I, I_{0}, I_{\theta}, s\right)^{2} & \equiv \sum_{J \in \mathcal{C}_{A}^{\mathcal{G}, \text { shift }}: 2^{s+1} \ell(J)=\ell(I): J \subset I}\left\|\square_{J}^{\omega, \mathbf{b}^{*} g}\right\|_{L^{2}(\omega)}^{2} ; \\
\sum_{I \in \mathcal{C}_{A}} \sum_{I_{0} \in \mathfrak{C}_{\mathcal{D}}(I)} \Lambda\left(I, I_{0}, I_{\theta}, s\right)^{2} & \leq\left\|\mathrm{P}_{\mathcal{C}_{A}^{\mathcal{G}, \text { shift }}}^{\omega, \mathbf{b}^{*}} g\right\|_{L^{2}(\omega)}^{\star 2} .
\end{aligned}
$$

Using (5.5) we obtain

$$
\left|E_{I_{\theta}}^{\sigma}\left(\widehat{\square}_{I}^{\sigma, b, \mathbf{b}} f\right)\right| \leq \sqrt{E_{I_{\theta}}^{\sigma}\left|\widehat{\square}_{I}^{\sigma, b, \mathbf{b}} f\right|^{2}} \lesssim\left\|\square_{I}^{\sigma, \mathbf{b}} f\right\|_{L^{2}(\sigma)}^{\star}\left|I_{\theta}\right|_{\sigma}^{-\frac{1}{2}}
$$

and hence

$\mathbf{E}_{\theta\left(I_{J}\right)}^{\sigma} f \equiv\left|E_{\theta\left(I_{J}\right)}^{\sigma} \widehat{\square}_{I}^{\sigma, b, \mathbf{b}} f\right|+\mathbf{1}_{\mathfrak{C}_{A}(A)}\left(\theta\left(I_{J}\right)\right) E_{\theta\left(I_{J}\right)}^{\sigma}|f| \lesssim\left(\left\|\square_{I}^{\sigma, \mathbf{b}} f\right\|_{L^{2}(\sigma)}^{\star}+\mathbf{1}_{\mathfrak{C}_{A}(A)}\left(\theta\left(I_{J}\right)\right)\left|I_{\theta}\right|_{\sigma}^{\frac{1}{2}} E_{\theta\left(I_{J}\right)}^{\sigma}|f|\right)\left|I_{\theta}\right|_{\sigma}^{-\frac{1}{2}}$,

and we can thus estimate $A\left(I, I_{0}, I_{\theta}, s\right)$ as follows:

$$
\begin{aligned}
& A\left(I, I_{0}, I_{\theta}, s\right) \\
\lesssim & 2^{-(1-\varepsilon(2-\alpha)) s}\left(\left\|\square_{I}^{\sigma, \mathbf{b}} f\right\|_{L^{2}(\sigma)}^{\star}+\mathbf{1}_{\mathfrak{C}_{A}(A)}\left(I_{\theta}\right)\left|I_{\theta}\right|_{\sigma}^{\frac{1}{2}} E_{I_{\theta}}^{\sigma}|f|\right) \Lambda\left(I, I_{0}, I_{\theta}, s\right) \cdot\left|I_{\theta}\right|_{\sigma}^{-\frac{1}{2}} \mathrm{P}^{\alpha}\left(I_{0}, \mathbf{1}_{\theta\left(I_{J}\right)} \sigma\right) \sqrt{\left|I_{0}\right|_{\omega}} \\
\lesssim & \sqrt{\mathfrak{A}_{2}^{\alpha}} 2^{-(1-\varepsilon(2-\alpha)) s}\left(\left\|\square_{I}^{\sigma, \mathbf{b}} f\right\|_{L^{2}(\sigma)}^{\star}+\mathbf{1}_{\mathfrak{C}_{A}(A)}\left(I_{\theta}\right)\left|I_{\theta}\right|_{\sigma}^{\frac{1}{2}} E_{I_{\theta}}^{\sigma}|f|\right) \Lambda\left(I, I_{0}, I_{\theta}, s\right),
\end{aligned}
$$

since $\mathrm{P}^{\alpha}\left(I_{0}, \mathbf{1}_{\theta\left(I_{J}\right)} \sigma\right) \lesssim \frac{\left|I_{\theta}\right|_{\sigma}}{\left|I_{\theta}\right|^{1-\alpha}}$ shows that

$$
\left|I_{\theta}\right|_{\sigma}^{-\frac{1}{2}} \mathrm{P}^{\alpha}\left(I_{0}, \mathbf{1}_{\theta\left(I_{J}\right)} \sigma\right) \sqrt{\left|I_{0}\right|_{\omega}} \lesssim \frac{\sqrt{\left|I_{\theta}\right|_{\sigma}} \sqrt{\left|I_{0}\right|_{\omega}}}{|I|^{1-\alpha}} \lesssim \sqrt{\mathfrak{A}_{2}^{\alpha}}
$$

An application of Cauchy-Schwarz to the sum over $I$ using (6.23) then shows that

$$
\begin{aligned}
& \sum_{\substack{I \in \mathcal{C}_{A} \\
I_{0}, I_{\theta} \in \mathfrak{C}_{\mathcal{D}}(I) \\
I_{0} \neq I_{\theta}}} A\left(I, I_{0}, I_{\theta}, s\right) \\
& \lesssim \sqrt{\mathfrak{A}_{2}^{\alpha}} 2^{-(1-\varepsilon(2-\alpha)) s} \sqrt{\sum_{I \in \mathcal{C}_{A}}\left\|\square_{I}^{\sigma, \mathbf{b}} f\right\|_{L^{2}(\sigma)}^{\star 2}+\sum_{I_{\theta} \in \mathfrak{C}_{A}(A)}\left|I_{\theta}\right|_{\sigma}\left(E_{I_{\theta}}^{\sigma}|f|\right)^{2}} \sqrt{\sum_{I \in \mathcal{C}_{A}}\left(\sum_{\substack{I_{0}, I_{\theta} \in \mathfrak{C}_{\mathcal{D}}(I) \\
I_{0} \neq I_{\theta}}} \Lambda\left(I, I_{0}, I_{\theta}, s\right)\right)^{2}} \\
& \lesssim \sqrt{\mathfrak{A}_{2}^{\alpha}} 2^{-(1-\varepsilon(2-\alpha)) s} \sqrt{\left\|\mathrm{P}_{\mathcal{C}_{A}}^{\sigma} f\right\|_{L^{2}(\sigma)}^{\star 2}+\sum_{A^{\prime} \in \mathfrak{C}_{A}(A)}\left|A^{\prime}\right|_{\sigma}\left(E_{A^{\prime}}^{\sigma}|f|\right)^{2}} \sqrt{\sum_{I \in \mathcal{C}_{A}}\left(\sum_{\substack{I_{0} \in \mathfrak{C}_{\mathcal{D}}(I) \\
I_{0} \neq I_{\theta}}} \Lambda\left(I, I_{0}, I_{\theta}, s\right)\right)^{2}} \\
& \lesssim \sqrt{\mathfrak{A}_{2}^{\alpha}} 2^{-(1-\varepsilon(2-\alpha)) s}\left(\left\|\mathrm{P}_{\mathcal{C}_{A}}^{\sigma} f\right\|_{L^{2}(\sigma)}^{\star}+\sqrt{\sum_{A^{\prime} \in \mathfrak{C}_{A}(A)}\left|A^{\prime}\right|_{\sigma}\left(E_{A^{\prime}}^{\sigma}|f|\right)^{2}}\right)\left\|\mathrm{P}_{\mathcal{C}_{A}^{\mathcal{G}, \text {,shift }}}^{\omega, \mathbf{b}^{*}} g\right\|_{L^{2}(\omega)}^{\star} .
\end{aligned}
$$


This estimate is summable in $s \geq \mathbf{r}$ since $\varepsilon<\frac{1}{2-\alpha}$, and so the proof of

$$
\begin{aligned}
& \left|\mathrm{B}_{\text {neighbour }}^{A}(f, g)\right|=\left|\sum_{\substack{I \in \mathcal{C}_{A} \text { and } J \in \mathcal{C}_{A}^{\mathcal{G}, \text { shift }} \\
J^{I * 1} \subsetneq I \text { and } \ell(J) \leq 2^{-r} \ell(I)}}\left\langle T_{\sigma}^{\alpha}\left(\mathbf{1}_{\theta\left(I_{J}\right)} \square_{I}^{\sigma, \mathbf{b}} f\right), \square_{J}^{\omega, \mathbf{b}^{*}} g\right\rangle_{\omega}\right| \\
& \leq \sum_{I \in \mathcal{C}_{A}} \sum_{\substack{I_{0}, I_{\theta} \in \mathfrak{C}_{\mathcal{D}}(I) \\
I_{0} \neq I_{\theta}}} \sum_{s=\mathbf{r}}^{\infty} A\left(I, I_{0}, I_{\theta}, s\right) \\
& \lesssim \sqrt{\mathfrak{A}_{2}^{\alpha}}\left(\left\|\mathrm{P}_{\mathcal{C}_{A}}^{\sigma} f\right\|_{L^{2}(\sigma)}^{\star}+\sqrt{\sum_{A^{\prime} \in \mathfrak{C}_{A}(A)}\left|A^{\prime}\right|_{\sigma} \alpha_{\mathcal{A}}\left(A^{\prime}\right)^{2}}\right)\left\|\mathrm{P}_{\mathcal{C}_{A}^{\mathcal{G}, \text { shift }}}^{\omega, \mathbf{b}^{*}} g\right\|_{L^{2}(\omega)}^{\star}
\end{aligned}
$$

is complete since $E_{A^{\prime}}^{\sigma}|f| \lesssim \alpha_{\mathcal{A}}\left(A^{\prime}\right)$.

\section{STOPPING FORM}

In this section, we modify our adaptation in [SaShUr7, [SaShUr9] and [SaShUr10] of the argument of M. Lacey in $\mathrm{Lac}$ to apply in the setting of a local $T b$ theorem for an $\alpha$-fractional Calderón-Zygmund operator $T^{\alpha}$ in $\mathbb{R}$ using the Monotonicity Lemma 30, the energy condition, and the weak goodness of Hytönen and Martikainen HyMa. Following Lacey in [Lac, we construct $\mathcal{L}$-coronas from the 'bottom up' with stopping times involving the energies $\left\|\triangle_{J}^{\omega, \mathbf{b}^{*}} x\right\|_{L^{2}(\omega)}^{2}$, but then overlay this with an additional top/down 'indented' corona construction in order to accommodate the weaker goodness of Hytönen and Martikainen. We directly control the pairs $(I, J)$ in the stopping form 'essentially' according to the $\mathcal{L}$-coronas to which $I$ and $J^{\text {s }}$

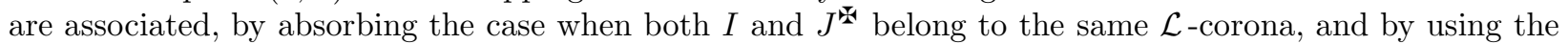
Straddling and Subtraddling Lemmas to control the case when $I$ and $J^{\text {lie }}$ in different coronas, with a geometric gain coming from the separation of the coronas in the 'indented' construction overlaying Lacey's bottom/up construction (we actually use the grandchild $J^{b}$ of $J^{\text {t }}$ that contains $J$ to distinguish aborption cases from straddling cases). We also use a Corona-straddling Lemma to control certain extremal pairs $(I, J)$ that straddle two $\mathcal{A}$-coronas. As in $[\mathrm{Lac}$, an Orthogonality Lemma proves useful in all cases. Finally, since we are using two independent dyadic grids, we must enlarge the skeleton of an interval to include an infinite sequence of points we call the body of the interval.

Apart from these changes, the remaining modifications are more obvious, such as

- the use of the weak goodness of Hytönen and Martikainen HyMa for pairs $(I, J)$ arising in the stopping form, rather than goodness for all intervals $I$ and $J$ that was available in Lac, SaShUr7, [SaShUr9] and [SaShUr10]. For the most part definitions such as admissible collections are modified to require $J^{w} \varsubsetneqq I$. In paricular, Lacey's size functional is enlarged to include more intervals $K \in \mathcal{D}$ that are not good;

- the pseudoprojections $\square_{I}^{\sigma, \mathbf{b}}, \square_{J}^{\omega, \mathbf{b}^{*}}$ and Carleson averaging operators $\nabla_{I}^{\sigma}, \nabla_{J}^{\omega}$ are used in place of the orthogonal Haar projections, and the frame and weak Riesz inequalities compensate for the lack of orthogonality.

Fix grids $\mathcal{D}$ and $\mathcal{G}$. In Section 6 we reduced matters to proving (6.5), i.e.

$$
\left|\mathrm{B}_{\text {stop }}^{A}(f, g)\right| \lesssim \mathcal{N} \mathcal{T} \mathcal{V}_{\alpha}\left(\left\|\mathrm{P}_{\mathcal{C}_{A}^{D}}^{\sigma, \mathbf{b}} f\right\|_{L^{2}(\sigma)}^{\star}+\alpha_{\mathcal{A}}(A) \sqrt{|A|_{\sigma}}\right)\left\|\mathrm{P}_{\mathcal{C}_{A}^{\mathcal{G}, \text { shift }}}^{\omega, \mathbf{b}^{*}} g\right\|_{L^{2}(\omega)}^{\star}
$$

where we recall that $\mathcal{N} \mathcal{T} \mathcal{V}_{\alpha}$ is defined in (1.15), and the nonstandard 'norms' are given in Notation 32 by,

$$
\begin{aligned}
\left\|\mathrm{P}_{\mathcal{C}_{A}^{\mathcal{D}}}^{\sigma, \mathbf{b}} f\right\|_{L^{2}(\sigma)}^{\star 2} & \equiv \sum_{I \in \mathcal{C}_{A}^{\mathcal{D}}}\left(\left\|\square_{I}^{\sigma, \mathbf{b}} f\right\|_{L^{2}(\sigma)}^{2}+\left\|\nabla_{I}^{\sigma} f\right\|_{L^{2}(\sigma)}^{2}\right) \\
\left\|\mathrm{P}_{\mathcal{C}_{A}^{\mathcal{G}, \text { shift }}}^{\omega, \mathbf{b}^{*}} g\right\|_{L^{2}(\omega)}^{\star 2} & \equiv \sum_{J \in \mathcal{C}_{A}^{\mathcal{G}, \text { shift }}}\left(\left\|\square_{J}^{\omega, \mathbf{b}^{*}} g\right\|_{L^{2}(\omega)}^{2}+\left\|\nabla_{J}^{\omega} g\right\|_{L^{2}(\omega)}^{2}\right)
\end{aligned}
$$

\footnotetext{
${ }^{7}$ And correct an error in [SaShUr7] related to the restricted norms of stopping forms for admissible collections.
} 
and that the stopping form is given in (6.17) by

$$
\mathrm{B}_{\text {stop }}^{A}(f, g) \equiv \sum_{\substack{I \in \mathcal{C}_{A}^{\mathcal{D}} \\ J^{\mathbf{w}} \subsetneq I \text { and } J \in \mathcal{C}_{A}^{\mathcal{G}} \text {,shift } \\ \neq(J) \leq 2^{-\mathbf{r}} \ell(I)}}\left(E_{I_{J}}^{\sigma} \widehat{\square}_{I}^{\sigma, b, \mathbf{b}} f\right)\left\langle T_{\sigma}^{\alpha}\left(\mathbf{1}_{A \backslash I_{J}} b_{A}\right), \square_{J}^{\omega, \mathbf{b}^{*}} g\right\rangle_{\omega} .
$$

It is important to note that $J^{f} \varsubsetneqq I$ implies $J^{-} \subset I_{J}$, and it follows that we cannot have $I_{J} \in \mathfrak{C}_{\mathcal{A}}(A)$, i.e. we cannot have that the child of $I$ containing $J$ is a stopping interval in $\mathcal{A}$, since this would then contradict the assumption that $J \in \mathcal{C}_{A}^{\mathcal{G} \text {,shift }}$. Furthermore, the pair $(I, J)=\left(J^{\mathbb{4}}, J\right)$ does not arise in the sum simply because of the requirement $J^{\text {f }} \varsubsetneqq I$. For convenience in notation, and without loss of generality, we now reindex the stopping form with this in mind by replacing the pairs $(I, J)$ in the sum above with new pairs $\left(I^{\prime}, J^{\prime}\right) \equiv\left(I_{J}, J\right)$ (recall that the child of $I$ that contains $J$ is denoted $\left.I_{J}\right)$. The result is that

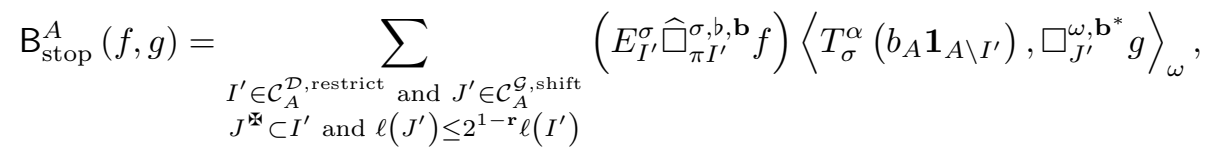

where

$$
\mathcal{C}_{A}^{\mathcal{D}, \text { restrict }} \equiv \mathcal{C}_{A} \backslash\{A\}
$$

is the $A$-corona with its top interval $A$ removed. Now we simply drop the primes from the dummy variables $I^{\prime}$ and $J^{\prime}$ and relabel $1-\mathbf{r}$ as $-\mathbf{r}$ to obtain

$$
\mathrm{B}_{\text {stop }}^{A}(f, g)=\sum_{\substack{I \in \mathcal{C}_{A}^{\mathcal{D}, \text {,restrict }} \\ J^{* \mathbf{A}} \subset I \text { and } \ell(J) \leq 2^{-\mathbf{r}} \ell(I)}}\left(E_{I}^{\sigma} \widehat{\square}_{\pi I}^{\sigma, b, \mathbf{b}} f\right)\left\langle T_{\sigma}^{\alpha}\left(b_{A} \mathbf{1}_{A \backslash I}\right), \square_{J}^{\omega, \mathbf{b}^{*}} g\right\rangle_{\omega} .
$$

Definition 64. Suppose that $A \in \mathcal{A}$ and that $\mathcal{P} \subset \mathcal{C}_{A}^{\mathcal{D} \text {,restrict }} \times \mathcal{C}_{A}^{\mathcal{G} \text {,shift }}$. We say that the collection of pairs $\mathcal{P}$ is $A$-admissible if

- (good and $(\mathbf{r}, \varepsilon)$-deeply embedded) $\ell(J) \leq 2^{-\mathbf{r}} \ell(I)$ and $J^{\mathbf{w}} \subset I \varsubsetneqq A$ for every $(I, J) \in \mathcal{P}$,

- (tree-connected in the first component) if $I_{1} \subset I_{2}$ and both $\left(I_{1}, J\right) \in \mathcal{P}$ and $\left(I_{2}, J\right) \in \mathcal{P}$, then $(I, J) \in \mathcal{P}$ for every $I$ in the geodesic $\left[I_{1}, I_{2}\right]=\left\{I \in \mathcal{D}: I_{1} \subset I \subset I_{2}\right\}$.

From now on we often write $\mathcal{C}_{A}$ and $\mathcal{C}_{A}^{\text {restrict }}$ in place of $\mathcal{C}_{A}^{\mathcal{D}}$ and $\mathcal{C}_{A}^{\mathcal{D} \text {,restrict }}$ respectively, i.e. we drop the superscript $\mathcal{D}$, when there is no possiblility of confusion. The basic example of an admissible collection of pairs is obtained from the pairs of intervals summed in the stopping form $\mathrm{B}_{\text {stop }}^{A}(f, g)$ in (7.3) which occurs in the inequality (7.1) above;

$$
\mathcal{P}^{A} \equiv\left\{(I, J): I \in \mathcal{C}_{A}^{\text {restrict }}, J \in \mathcal{C}_{A}^{\mathcal{G} \text {,shift }}, J^{\mathscr{W}} \subset I \text { and } \ell(J) \leq 2^{-\mathbf{r}} \ell(I)\right\} .
$$

Definition 65. Suppose that $A \in \mathcal{A}$ and that $\mathcal{P}$ is an $A$-admissible collection of pairs. Define the associated stopping form $\mathrm{B}_{\text {stop }}^{A, \mathcal{P}}$ by

$$
\mathrm{B}_{\text {stop }}^{A, \mathcal{P}}(f, g) \equiv \sum_{(I, J) \in \mathcal{P}}\left(E_{I}^{\sigma} \widehat{\square}_{\pi I}^{\sigma, b, \mathbf{b}} f\right)\left\langle T_{\sigma}^{\alpha}\left(b_{A} \mathbf{1}_{A \backslash I}\right), \square_{J}^{\omega, \mathbf{b}^{*}} g\right\rangle_{\omega} .
$$

where $\widehat{\square}_{\pi I}^{\sigma, b, \mathbf{b}}$ is the modified dual martingale difference defined in (9.21) and (9.22).

Recall the strong energy condition constant $\mathcal{E}_{2}^{\alpha}$ defined by

$$
\left(\mathcal{E}_{2}^{\alpha}\right)^{2} \equiv \sup _{I=\dot{\cup} I_{r}} \frac{1}{|I|_{\sigma}} \sum_{r=1}^{\infty}\left(\frac{\mathrm{P}^{\alpha}\left(I_{r}, \mathbf{1}_{I} \sigma\right)}{\left|I_{r}\right|}\right)^{2}\left\|x-m_{I_{r}}^{\omega}\right\|_{L^{2}\left(\mathbf{1}_{I_{r}} \omega\right)}^{2} .
$$

Proposition 66. Suppose that $A \in \mathcal{A}$ and that $\mathcal{P}$ is an $A$-admissible collection of pairs. Then the stopping form $\mathrm{B}_{\text {stop }}^{A, \mathcal{P}}$ satisfies the bound

$$
\left|\mathrm{B}_{\text {stop }}^{A, \mathcal{P}}(f, g)\right| \lesssim\left(\mathcal{E}_{2}^{\alpha}+\sqrt{\mathfrak{A}_{2}^{\alpha}}\right)\left\|\mathrm{P}_{\mathcal{C}_{A}}^{\sigma, \mathbf{b}} f\right\|_{L^{2}(\sigma)}^{\star}\left\|\mathrm{P}_{\mathcal{C}_{A}^{\mathcal{G}, \text { shift }}}^{\omega, \mathbf{b}^{*}} g\right\|_{L^{2}(\omega)}^{\star} .
$$


The above proposition proves (7.1) - even without the term $\alpha_{\mathcal{A}}(A) \sqrt{|A|_{\sigma}}$ on the right - with the choice $\mathcal{P}=\mathcal{P}^{A}$. To prove Proposition 66 we begin by letting

$$
\begin{aligned}
\Pi_{1} \mathcal{P} & \equiv\left\{I \in \mathcal{C}_{A}^{\mathcal{D} \text {,restrict }}:(I, J) \in \mathcal{P} \text { for some } J \in \mathcal{C}_{A}^{\mathcal{G} \text {,shift }}\right\}, \\
\Pi_{2} \mathcal{P} & \equiv\left\{J \in \mathcal{C}_{A}^{\mathcal{G} \text {,shift }}:(I, J) \in \mathcal{P} \text { for some } I \in \mathcal{C}_{A}^{\mathcal{D} \text {,restrict }}\right\},
\end{aligned}
$$

consist of the first and second components respectively of the pairs in $\mathcal{P}$, and writing

$$
\begin{aligned}
\mathrm{B}_{\text {stop }}^{A, \mathcal{P}}(f, g) & =\sum_{J \in \Pi_{2} \mathcal{P}}\left\langle T_{\sigma}^{\alpha} \varphi_{J}^{\mathcal{P}}, \square_{J}^{\omega, \mathbf{b}^{*}} g\right\rangle_{\omega} ; \\
\text { where } \varphi_{J}^{\mathcal{P}} & \equiv \sum_{I \in \mathcal{C}_{A}^{\text {restrict }}:(I, J) \in \mathcal{P}} b_{A}\left(E_{I}^{\sigma} \widehat{\square}_{\pi I}^{\sigma, b, \mathbf{b}} f\right) \mathbf{1}_{A \backslash I},
\end{aligned}
$$

where $E_{I}^{\sigma} h \equiv \frac{1}{|I|_{\sigma}} \int_{I} h d \sigma$ denotes the $\sigma$-average of $h$ on $I$, and where we note that the function $\widehat{\square}_{\pi I}^{\sigma, b, \mathbf{b}} f$ is constant on $I$, so that $E_{I}^{\sigma} \widehat{\square}_{\pi I}^{\sigma, b, \mathbf{b}} f$ simply picks out the value of $\widehat{\square}_{\pi I}^{\sigma, b, \mathbf{b}} f$ on $I$. By the tree-connected property of $\mathcal{P}$, and the telescoping property (9.24), together with the bound $\alpha_{\mathcal{A}}(A)$ on the averages of $f$ in the corona $\mathcal{C}_{A}$, we have

$$
\left|\varphi_{J}^{\mathcal{P}}\right| \lesssim \alpha_{\mathcal{A}}(A) \mathbf{1}_{A \backslash I_{\mathcal{P}}(J)}
$$

where $I_{\mathcal{P}}(J) \equiv \bigcap\{I:(I, J) \in \mathcal{P}\}$ is the smallest interval $I$ for which $(I, J) \in \mathcal{P}$. It is important to note that $J$ is good with respect to $I_{\mathcal{P}}(J)$ and beyond by the infusion of weak goodness above.

Another important property of these functions is the sublinearity:

$$
\left|\varphi_{J}^{\mathcal{P}}\right| \leq\left|\varphi_{J}^{\mathcal{P}_{1}}\right|+\left|\varphi_{J}^{\mathcal{P}_{2}}\right|, \quad \mathcal{P}=\mathcal{P}_{1} \dot{\cup} \mathcal{P}_{2}
$$

which is an immediate consequence of

$$
\varphi_{J}^{\mathcal{P}_{1} \dot{\mathcal{P}} \mathcal{P}_{2}}=\sum_{I \in \mathcal{C}_{A}^{\text {restrict }}:(I, J) \in \mathcal{P}_{1} \dot{\cup} \mathcal{P}_{2}}\{\ldots\}=\sum_{I \in \mathcal{C}_{A}^{\text {restrict }}:(I, J) \in \mathcal{P}_{1}}\{\ldots\}+\sum_{I \in \mathcal{C}_{A}^{\text {restrict }}:(I, J) \in \mathcal{P}_{2}}\{\ldots\}=\varphi_{J}^{\mathcal{P}_{1}}+\varphi_{J}^{\mathcal{P}_{2}} .
$$

Now apply the Monotonicity Lemma 30 to the inner product $\left\langle T_{\sigma}^{\alpha} \varphi_{J}, \square_{J}^{\omega, \mathbf{b}^{*}} g\right\rangle_{\omega}$ (which applies since $J$ is $\operatorname{good}$ in $\left.I_{\mathcal{P}}(J)\right)$ to obtain

$$
\begin{aligned}
\left|\left\langle T_{\sigma}^{\alpha} \varphi_{J}, \square_{J}^{\omega, \mathbf{b}^{*}} g\right\rangle_{\omega}\right| \lesssim & \frac{\mathrm{P}^{\alpha}\left(J,\left|\varphi_{J}^{\mathcal{P}}\right| \mathbf{1}_{A \backslash I_{\mathcal{P}}(J)} \sigma\right)}{|J|}\left\|\triangle_{J}^{\omega, \mathbf{b}^{*}} x\right\|_{L^{2}(\omega)}^{\boldsymbol{\omega}}\left\|\square_{J}^{\omega, \mathbf{b}^{*}} g\right\|_{L^{2}(\omega)}^{\star} \\
& +\frac{\mathrm{P}_{1+\delta}^{\alpha}\left(J,\left|\varphi_{J}^{\mathcal{P}}\right| \mathbf{1}_{A \backslash I_{\mathcal{P}}(J)} \sigma\right)}{|J|}\left\|x-m_{J}^{\omega}\right\|_{L^{2}\left(\mathbf{1}_{J} \omega\right)}\left\|\square_{J}^{\omega, \mathbf{b}^{*}} g\right\|_{L^{2}(\omega)}^{\star}
\end{aligned}
$$

Thus we have

$$
\begin{aligned}
\left|\mathrm{B}_{\text {stop }}^{A, \mathcal{P}}(f, g)\right| \leq & \sum_{J \in \Pi_{2} \mathcal{P}} \frac{\mathrm{P}^{\alpha}\left(J,\left|\varphi_{J}^{\mathcal{P}}\right| \mathbf{1}_{A \backslash I_{\mathcal{P}}(J)} \sigma\right)}{|J|}\left\|\triangle_{J}^{\omega, \mathbf{b}^{*}} x\right\|_{L^{2}(\omega)}^{\boldsymbol{\omega}}\left\|\square_{J}^{\omega, \mathbf{b}^{*}} g\right\|_{L^{2}(\omega)}^{\star} \\
& +\sum_{J \in \Pi_{2} \mathcal{P}} \frac{\mathrm{P}_{1+\delta}^{\alpha}\left(J,\left|\varphi_{J}^{\mathcal{P}}\right| \mathbf{1}_{A \backslash I_{\mathcal{P}}(J)} \sigma\right)}{|J|}\left\|x-m_{J}^{\omega}\right\|_{L^{2}\left(\mathbf{1}_{J} \omega\right)}\left\|\square_{J}^{\omega, \mathbf{b}^{*}} g\right\|_{L^{2}(\omega)}^{\star} \\
\equiv & |\mathrm{B}|_{\text {stop }, 1, \triangle^{\omega}}^{A, \mathcal{P}}(f, g)+|\mathrm{B}|_{\text {stop }, 1+\delta, \mathrm{P} \omega}^{A, \mathcal{P}}(f, g),
\end{aligned}
$$

where we have dominated the stopping form by two sublinear stopping forms that involve the Poisson integrals of order 1 and $1+\delta$ respectively, and where the smaller Poisson integral $\mathrm{P}_{1+\delta}^{\alpha}$ is multiplied by the larger quantity $\left\|x-m_{J}^{\omega}\right\|_{L^{2}\left(\mathbf{1}_{J} \omega\right)}$. It remains to show the following two inequalities where we abbreviate $|\mathrm{B}|_{\text {stop }, 1, \triangle \omega}^{A, \mathcal{P}}$ to $|\mathrm{B}|_{\text {stop }, \triangle \omega}^{A, \mathcal{P}}$ and $|\mathrm{B}|_{\text {stop }, 1+\delta, \mathrm{P} \omega}^{A, \mathcal{P}}$ to $|\mathrm{B}|_{\text {stop }, 1+\delta}^{A, \mathcal{P}}$ :

$$
|\mathrm{B}|_{\text {stop }, \triangle \omega}^{A, \mathcal{P}}(f, g) \lesssim\left(\mathcal{E}_{2}^{\alpha}+\sqrt{\mathfrak{A}_{2}^{\alpha}}\right)\left\|\mathrm{P}_{\pi\left(\Pi_{1} \mathcal{P}\right)}^{\sigma, \mathbf{b}} f\right\|_{L^{2}(\sigma)}^{\star}\left\|\mathrm{P}_{\Pi_{2} \mathcal{P}}^{\omega, \mathbf{b}^{*}} g\right\|_{L^{2}(\omega)}^{\star}
$$


for $f \in L^{2}(\sigma)$ satisfying $E_{I}^{\sigma}|f| \lesssim \alpha_{\mathcal{A}}(A)$ for all $I \in \mathcal{C}_{A}$, and where $\pi\left(\Pi_{1} \mathcal{P}\right) \equiv\left\{\pi_{\mathcal{D}} I: I \in \Pi_{1} \mathcal{P}\right\}$; and

$$
|\mathrm{B}|_{\text {stop }, 1+\delta}^{A, \mathcal{P}}(f, g) \lesssim\left(\mathcal{E}_{2}^{\alpha}+\sqrt{A_{2}^{\alpha}}\right)\left\|\mathrm{P}_{\mathcal{C}_{A}^{D}}^{\sigma, \mathbf{b}} f\right\|_{L^{2}(\sigma)}\left\|\mathrm{P}_{\mathcal{C}_{A}^{\mathcal{G}, \text { shift }}}^{\omega, \mathbf{b}^{*}} g\right\|_{L^{2}(\omega)},
$$

where we only need the case $\mathcal{P}=\mathcal{P}^{A}$ in this latter inequality as there is no absorption involved in treating this second sublinear form. We consider first the easier inequality (7.11) that does not require absorption.

7.1. The bound for the second sublinear inequality. Here we turn to proving (7.11), i.e.

$$
\begin{aligned}
|\mathrm{B}|_{\text {stop }, 1+\delta}^{A, \mathcal{P}}(f, g) & =\sum_{J \in \Pi_{2} \mathcal{P}} \frac{\mathrm{P}_{1+\delta}^{\alpha}\left(J,\left|\varphi_{J}\right| \mathbf{1}_{A \backslash I_{\mathcal{P}}(J)} \sigma\right)}{|J|}\left\|x-m_{J}\right\|_{L^{2}\left(\mathbf{1}_{J} \omega\right)}\left\|\square_{J}^{\omega, \mathbf{b}^{*}} g\right\|_{L^{2}(\omega)}^{\star} \\
& \lesssim\left(\mathcal{E}_{2}^{\alpha}+\sqrt{A_{2}^{\alpha}}\right)\left\|\mathrm{P}_{\mathcal{C}_{A}^{D}}^{\sigma, \mathbf{b}} f\right\|_{L^{2}(\sigma)}\left\|\mathrm{P}_{\mathcal{C}_{A}^{\mathcal{G}, \text { shift }}}^{\omega,,^{*}} g\right\|_{L^{2}(\omega)},
\end{aligned}
$$

where since

$$
\left|\varphi_{J}\right|=\left|\sum_{I \in \mathcal{C}_{A}^{\prime}:(I, J) \in \mathcal{P}}\left(E_{I}^{\sigma} \square_{\pi I}^{\sigma, b, \mathbf{b}} f\right) \mathbf{1}_{A \backslash I}\right| \leq \sum_{I \in \mathcal{C}_{A}^{\prime}:(I, J) \in \mathcal{P}}\left|E_{I}^{\sigma} \square_{\pi I}^{\sigma, b, \mathbf{b}}(f) \mathbf{1}_{A \backslash I}\right|,
$$

the sublinear form $|\mathrm{B}|_{\text {stop }, 1+\delta}^{A, \mathcal{P}}$ can be decomposed by pigeonholing the ratio of side lengths of $J$ and $I$ :

$$
\begin{aligned}
& |\mathrm{B}|_{\text {stop }, 1+\delta}^{A, \mathcal{P}}(f, g)=\sum_{J \in \Pi_{2} \mathcal{P}} \frac{\mathrm{P}_{1+\delta}^{\alpha}\left(J,\left|\varphi_{J}\right| \mathbf{1}_{A \backslash I_{\mathcal{P}}(J)} \sigma\right)}{|J|^{\frac{1}{n}}}\left\|x-m_{J}\right\|_{L^{2}\left(\mathbf{1}_{J} \omega\right)}\left\|\square_{J}^{\omega, \mathbf{b}^{*}} g\right\|_{L^{2}(\omega)}^{\star} \\
& \leq \sum_{(I, J) \in \mathcal{P}} \frac{\mathrm{P}_{1+\delta}^{\alpha}\left(J,\left|E_{I}^{\sigma}\left(\square_{\pi I}^{\sigma, b, \mathbf{b}} f\right)\right| \mathbf{1}_{A \backslash I} \sigma\right)}{|J|^{\frac{1}{n}}}\left\|x-m_{J}\right\|_{L^{2}\left(\mathbf{1}_{J} \omega\right)}\left\|\square_{J}^{\omega, \mathbf{b}^{*}} g\right\|_{L^{2}(\omega)}^{\star} \\
& \equiv \sum_{s=0}^{\infty}|\mathrm{B}|_{\text {stop }, 1+\delta}^{A, \mathcal{P} ; s}(f, g) \text {; } \\
& |\mathrm{B}|_{\text {stop }, 1+\delta}^{A, \mathcal{P} ; s}(f, g) \equiv \sum_{\substack{(I, J) \in \mathcal{P} \\
\ell(J)=2^{-s} \ell(I)}} \frac{\mathrm{P}_{1+\delta}^{\alpha}\left(J,\left|\left(E_{I}^{\sigma} \square_{\pi I}^{\sigma, b, \mathbf{b}} f\right)\right| \mathbf{1}_{A \backslash I} \sigma\right)}{|J|^{\frac{1}{n}}}\left\|x-m_{J}\right\|_{L^{2}\left(\mathbf{1}_{J} \omega\right)}\left\|\square_{J}^{\omega, \mathbf{b}^{*}} g\right\|_{L^{2}(\omega)}^{\star} .
\end{aligned}
$$

We will now adapt the argument for the stopping term starting on page 42 of [LaSaUr2], where the geometric gain from the assumed Energy Hypothesis there will be replaced by a geometric gain from the smaller Poisson integral $\mathrm{P}_{1+\delta}^{\alpha}$ used here.

We exploit the additional decay in the Poisson integral $\mathrm{P}_{1+\delta}^{\alpha}$ as follows. Suppose that $J$ is good in $I$ with $\ell(J)=2^{-s} \ell(I)$. We then obtain from (2.31) above that

$$
\left(\frac{\mathrm{P}_{1+\delta}^{\alpha}\left(J, \mathbf{1}_{A \backslash I} \sigma\right)}{|J|^{\frac{1}{n}}}\right) \lesssim 2^{-s \delta(1-\varepsilon)} \frac{\mathrm{P}^{\alpha}\left(J, \mathbf{1}_{A \backslash I} \sigma\right)}{|J|^{\frac{1}{n}}} .
$$

We next claim that for $s \geq 0$ an integer,

$$
\begin{aligned}
|\mathrm{B}|_{\text {stop }, 1+\delta}^{A, \mathcal{P} ; s}(f, g) & =\sum_{\substack{(I, J) \in \mathcal{P} \\
\ell(J)=2^{-s} \ell(I)}} \frac{\mathrm{P}_{1+\delta}^{\alpha}\left(J,\left|\left(E_{I}^{\sigma} \square_{\pi I}^{\sigma, b, \mathbf{b}} f\right)\right| \mathbf{1}_{A \backslash I} \sigma\right)}{|J|}\left\|x-m_{J}\right\|_{L^{2}\left(\mathbf{1}_{J} \omega\right)}\left\|\square_{J}^{\omega, \mathbf{b}^{*}} g\right\|_{L^{2}(\omega)}^{\star} \\
& \lesssim 2^{-s \delta(1-\varepsilon)}\left(\mathcal{E}_{2}^{\alpha}+\sqrt{A_{2}^{\alpha}}\right)\|f\|_{L^{2}(\sigma)}\|g\|_{L^{2}(\omega)},
\end{aligned}
$$

from which (7.11) follows upon summing in $s \geq 0$. Now using both

$$
\begin{aligned}
\left|E_{I}^{\sigma} \square_{\pi I}^{\sigma, b, \mathbf{b}} f\right| & =\frac{1}{|I|_{\sigma}} \int_{I}\left|\square_{\pi I}^{\sigma, b, \mathbf{b}} f\right| d \sigma \leq\left\|\square_{\pi I}^{\sigma, b, \mathbf{b}} f\right\|_{L^{2}(\sigma)} \frac{1}{\sqrt{|I|_{\sigma}}}, \\
\sum_{I \in \mathcal{D}}\left\|\square_{\pi I}^{\sigma, b, \mathbf{b}} f\right\|_{L^{2}(\sigma)}^{2} & \lesssim \sum_{I \in \mathcal{D}}\left(\left\|\square_{\pi I}^{\sigma, \mathbf{b}} f\right\|_{L^{2}(\sigma)}^{2}+\left\|\nabla_{\pi I}^{\sigma} f\right\|_{L^{2}(\sigma)}^{2}\right) \approx\|f\|_{L^{2}(\sigma)}^{2},
\end{aligned}
$$


where the second line uses frame inequalities in Proposition 91 and displays (9.13) and (9.14) from Appendix A below, we apply Cauchy-Schwarz in the $I$ variable above to see that

$$
\begin{aligned}
& {\left[|\mathrm{B}|_{\text {stop }, 1+\delta}^{A, \mathcal{P} ; s}(f, g)\right]^{2} \lesssim\|f\|_{L^{2}(\sigma)}^{2}} \\
& \times\left[\sum_{I \in \mathcal{C}_{A}^{\text {restrict }}}\left(\frac{1}{\sqrt{|I|_{\sigma}}} \sum_{\substack{J:(I, J) \in \mathcal{P} \\
\ell(J)=2^{-s} \ell(I)}} \frac{\mathrm{P}_{1+\delta}^{\alpha}\left(J, \mathbf{1}_{A \backslash I} \sigma\right)}{|J|}\left\|x-m_{J}\right\|_{L^{2}\left(\mathbf{1}_{J} \omega\right)}\left\|\square_{J}^{\omega, \mathbf{b}^{*}} g\right\|_{L^{2}(\omega)}^{\star}\right)^{2}\right]^{\frac{1}{2}} .
\end{aligned}
$$

We can then estimate the sum inside the square brackets by

$$
\sum_{I \in \mathcal{C}_{A}^{\prime}}\left\{\sum_{\substack{J:(I, J) \in \mathcal{P} \\ \ell(J)=2^{-s} \ell(I)}}\left\|\square_{J}^{\omega, \mathbf{b}^{*}} g\right\|_{L^{2}(\omega)}^{\star 2}\right\} \sum_{\substack{J:(I, J) \in \mathcal{P} \\ \ell(J)=2^{-s} \ell(I)}} \frac{1}{|I|_{\sigma}}\left(\frac{\mathrm{P}_{1+\delta}^{\alpha}\left(J, \mathbf{1}_{A \backslash I} \sigma\right)}{|J|}\right)^{2}\left\|x-m_{J}\right\|_{L^{2}\left(\mathbf{1}_{J} \omega\right)}^{2} \lesssim\|g\|_{L^{2}(\omega)}^{2} A(s)^{2},
$$

where

$$
A(s)^{2} \equiv \sup _{I \in \mathcal{C}_{A}^{\prime}} \sum_{\substack{J:(I, J) \in \mathcal{P} \\ \ell(J)=2^{-s} \ell(I)}} \frac{1}{|I|_{\sigma}}\left(\frac{\mathrm{P}_{1+\delta}^{\alpha}\left(J, \mathbf{1}_{A \backslash I} \sigma\right)}{|J|}\right)^{2}\left\|x-m_{J}\right\|_{L^{2}\left(\mathbf{1}_{J} \omega\right)}^{2} .
$$

Finally then we turn to the analysis of the supremum in last display. From the Poisson decay (7.12) we have

$$
\begin{aligned}
A(s)^{2} & \lesssim \sup _{I \in \mathcal{C}_{A}^{\prime}} \frac{1}{|I|_{\sigma}} 2^{-2 s \delta(1-\varepsilon)} \sum_{\substack{J:(I, J) \in \mathcal{P} \\
\ell(J)=2^{-s} \ell(I)}}\left(\frac{\mathrm{P}^{\alpha}\left(J, \mathbf{1}_{A \backslash I} \sigma\right)}{|J|}\right)^{2}\left\|x-m_{J}\right\|_{L^{2}\left(\mathbf{1}_{J} \omega\right)}^{2} \\
& \lesssim 2^{-2 s \delta(1-\varepsilon)}\left[\left(\mathcal{E}_{2}^{\alpha}\right)^{2}+A_{2}^{\alpha}\right]
\end{aligned}
$$

where the last inequality is the stopping energy inquality (2.23) in the corona $\mathcal{C}_{A}$. Indeed, from Definition 23 , as $(I, J) \in \mathcal{P}$, we have that $I$ is not a stopping interval in $\mathcal{A}$, and hence that (2.21) fails to hold, delivering the estimate above since the terms $\left\|x-m_{J}\right\|_{L^{2}\left(\mathbf{1}_{J} \omega\right)}^{2}$ are additive, as the $J^{\prime} s$ are pigeonholed by $\ell(J)=2^{-s} \ell(I)$ and hence pairwise disjoint.

7.2. The bound for the first sublinear inequality. Recall the definition of the sublinear form $|\mathrm{B}|_{\mathrm{stop}, \triangle^{\omega}}^{A, \mathcal{P}}(f, g)$ in display (7.9), and the inequality (7.10) that we wish to prove.

Definition 67. Denote by $\widehat{\mathfrak{N}}_{\text {stop }, \triangle \omega}^{A, \mathcal{P}}$ the best constant in

$$
|\mathrm{B}|_{\text {stop }, \triangle \omega}^{A, \mathcal{P}}(f, g) \leq \widehat{\mathfrak{N}}_{\text {stop }, \triangle \omega}^{A, \mathcal{P}}\left\|\mathrm{P}_{\pi\left(\Pi_{1} \mathcal{P}\right)}^{\sigma, \mathbf{b}} f\right\|_{L^{2}(\sigma)}^{\star}\left\|\mathrm{P}_{\Pi_{2} \mathcal{P}}^{\omega, \mathbf{b}^{*} g}\right\|_{L^{2}(\omega)}^{\star},
$$

where $f \in L^{2}(\sigma)$ satisfies $E_{I}^{\sigma}|f| \leq \alpha_{\mathcal{A}}(A)$ for all $I \in \mathcal{C}_{A}$, and $g \in L^{2}(\omega)$, and $\pi\left(\Pi_{1} \mathcal{P}\right)=\left\{\pi I: I \in \Pi_{1} \mathcal{P}\right\}$. We refer to $\widehat{\mathfrak{N}}_{\text {stop }, \triangle \omega}^{A, \mathcal{P}}$ as the restricted norm relative to the collection $\mathcal{P}$.

Inequality (7.10) will follow once we have shown that $\widehat{\mathfrak{N}}_{\text {stop }, \triangle \omega}^{A, \mathcal{P}} \lesssim \mathcal{E}_{2}^{\alpha}+\sqrt{\mathfrak{\mathfrak { A }}_{2}^{\alpha}}$. To this end, the following general result on mutually orthogonal admissible collections, given as (3.5) in [Lac, will prove very useful. Given a set $\left\{\mathcal{Q}_{m}\right\}_{m=0}^{\infty}$ of admissible collections for $A$, we say that the collections $\mathcal{Q}_{m}$ are mutually orthogonal, if each collection $\mathcal{Q}_{m}$ satisfies

$$
\mathcal{Q}_{m} \subset \bigcup_{j=0}^{\infty}\left\{\mathcal{A}_{m, j} \times \mathcal{B}_{m, j}\right\}
$$

where the sets $\left\{\mathcal{A}_{m, j}\right\}_{m, j}$ and $\left\{\mathcal{B}_{m, j}\right\}_{m, j}$ are each pairwise disjoint in their respective dyadic grids $\mathcal{D}$ and $\mathcal{G}$ :

$$
\sum_{m, j=0}^{\infty} \mathbf{1}_{\mathcal{A}_{m, j}} \leq \mathbf{1}_{\mathcal{D}} \text { and } \sum_{m, j=0}^{\infty} \mathbf{1}_{\mathcal{B}_{m, j}} \leq \mathbf{1}_{\mathcal{G}}
$$


Lemma 68. Suppose that $\left\{\mathcal{Q}_{m}\right\}_{m=0}^{\infty}$ is a set of admissible collections for $A$ that are mutually orthogonal.

Then $\mathcal{Q} \equiv \bigcup_{m=0}^{\infty} \mathcal{Q}_{m}$ is admissible, and the sublinear stopping form $|\mathrm{B}|_{\text {stop, } \triangle \omega}^{A, \mathcal{Q}}(f, g)$ has its localized norm $\widehat{\mathfrak{N}}_{\mathrm{stop}, \triangle \omega}^{A, \mathcal{Q}}$ controlled by the supremum of the localized norms $\widehat{\mathfrak{N}}_{\mathrm{stop}, \triangle \omega}^{A, \mathcal{Q}_{m}}$ :

$$
\widehat{\mathfrak{N}}_{\mathrm{stop}, \triangle \omega}^{A, \mathcal{Q}} \leq \sup _{m \geq 0} \widehat{\mathfrak{N}}_{\mathrm{stop}, \triangle \omega}^{A, \mathcal{Q}_{m}}
$$

Proof. If $J \in \Pi_{2} \mathcal{Q}_{m}$, then $\varphi_{J}^{\mathcal{Q}}=\varphi_{J}^{\mathcal{Q}_{m}}$ and $I_{\mathcal{Q}}(J)=I_{\mathcal{Q}_{m}}(J)$, since the collection $\left\{\mathcal{Q}_{m}\right\}_{m=0}^{\infty}$ is mutually orthogonal. Thus we have

$$
\begin{aligned}
|\mathrm{B}|_{\mathrm{stop}, \triangle \omega}^{A, \mathcal{Q}}(f, g) & =\sum_{J \in \Pi_{2} \mathcal{Q}} \frac{\mathrm{P}^{\alpha}\left(J,\left|\varphi_{J}^{\mathcal{Q}}\right| \mathbf{1}_{A \backslash I_{\mathcal{Q}}(J)} \sigma\right)}{|J|}\left\|\triangle_{J}^{\omega, \mathbf{b}^{*}} x\right\|_{L^{2}(\omega)}^{\mathbf{\omega}}\left\|\square_{J}^{\omega, \mathbf{b}^{*}} g\right\|_{L^{2}(\omega)}^{\star} \\
& =\sum_{m \geq 0} \sum_{J \in \Pi_{2} \mathcal{Q}_{m}} \frac{\mathrm{P}^{\alpha}\left(J,\left|\varphi_{J}^{\mathcal{Q}_{m}}\right| \mathbf{1}_{A \backslash I_{\mathcal{Q}_{m}}(J)} \sigma\right)}{|J|}\left\|\triangle_{J}^{\omega, \mathbf{b}^{*}} x\right\|_{L^{2}(\omega)}^{\mathbf{\omega}}\left\|\square_{J}^{\omega, \mathbf{b}^{*}} g\right\|_{L^{2}(\omega)}^{\star}=\sum_{m \geq 0}|\mathrm{~B}|_{\mathrm{stop}, \triangle \omega}^{A, \mathcal{Q}_{m}}(f, g),
\end{aligned}
$$

and we can continue with the definition of $\widehat{\mathfrak{N}}_{\text {stop, } \triangle \omega}^{A, \mathcal{Q}_{m}}$ and Cauchy-Schwarz to obtain

$$
\begin{aligned}
|\mathrm{B}|_{\mathrm{stop}, \triangle \omega}^{A, \mathcal{Q}}(f, g) & \leq \sum_{m \geq 0} \widehat{\mathfrak{N}}_{\mathrm{stop}, \triangle \omega}^{A, \mathcal{Q}_{m}}\left\|\mathrm{P}_{\pi\left(\Pi_{1} \mathcal{Q}_{m}\right)}^{\sigma, \mathbf{b}} f\right\|_{L^{2}(\sigma)}^{\star}\left\|\mathrm{P}_{\Pi_{2} \mathcal{Q}_{m}}^{\omega, \mathbf{b}^{*}} g\right\|_{L^{2}(\omega)}^{\star} \\
& \leq\left(\sup _{m \geq 0} \widehat{\mathfrak{N}}_{\mathrm{stop}, \triangle \omega}^{A, \mathcal{Q}_{m}}\right) \sqrt{\sum_{m \geq 0}\left\|\mathrm{P}_{\pi\left(\Pi_{1} \mathcal{Q}_{m}\right)}^{\sigma, \mathbf{b}} f\right\|_{L^{2}(\sigma)}^{\star 2}} \sqrt{\sum_{m \geq 0}\left\|\mathrm{P}_{\Pi_{2} \mathcal{Q}_{m}}^{\omega, \mathbf{b}^{*}} g\right\|_{L^{2}(\omega)}^{\star 2}} \\
& \leq\left(\sup _{m \geq 0} \widehat{\mathfrak{N}}_{\mathrm{stop}, \triangle \omega}^{A, \mathcal{Q}_{m}}\right) \sqrt{\left\|\mathrm{P}_{\pi\left(\Pi_{1} \mathcal{Q}\right)}^{\sigma, \mathbf{b}} f\right\|_{L^{2}(\sigma)}^{\star 2}} \sqrt{\| \mathrm{P}_{\Pi_{2} \mathcal{Q}^{2} \mathbf{b}^{*} \|_{L^{2}(\omega)}^{\star 2}}^{\star 2}} .
\end{aligned}
$$

Now we turn to proving inequality (7.10) for the sublinear form $|\mathrm{B}|_{\mathrm{stop}, \triangle \omega}^{A, \mathcal{P}}(f, g)$, i.e.

$$
\begin{aligned}
|\mathrm{B}|_{\text {stop }, \triangle \omega}^{A, \mathcal{P}}(f, g) & \equiv \sum_{J \in \Pi_{2} \mathcal{P}} \frac{\mathrm{P}^{\alpha}\left(J,\left|\varphi_{J}\right| \mathbf{1}_{A \backslash I_{\mathcal{P}}(J)} \sigma\right)}{|J|}\left\|\triangle_{J}^{\omega, \mathbf{b}^{*}} x\right\|_{L^{2}(\omega)}^{\mathbf{\bullet}}\left\|\square_{J}^{\omega, \mathbf{b}^{*}} g\right\|_{L^{2}(\omega)}^{\star} \\
& \lesssim\left(\mathcal{E}_{2}^{\alpha}+\sqrt{\mathfrak{A}_{2}^{\alpha}}\right)\left\|\mathrm{P}_{\pi\left(\Pi_{1} \mathcal{P}\right)}^{\sigma, \mathbf{b}} f\right\|_{L^{2}(\sigma)}^{\star}\left\|\mathrm{P}_{\Pi_{2} \mathcal{P}}^{\omega, \mathbf{b}^{*}} g\right\|_{L^{2}(\omega)}^{\star} ; \\
\text { where } \varphi_{J} & \equiv \sum_{I \in \mathcal{C}_{A}^{\text {restrict }}:(I, J) \in \mathcal{P}}\left(E_{I}^{\sigma} \widehat{\square}_{\pi I}^{\sigma, b, \mathbf{b}} f\right) b_{A} \mathbf{1}_{A \backslash I} \text { is supported in } A \backslash I_{\mathcal{P}}(J),
\end{aligned}
$$

and $I_{\mathcal{P}}(J)$ denotes the smallest interval $I \in \mathcal{D}$ for which $(I, J) \in \mathcal{P}$. We recall the stopping energy from (2.23),

$$
\mathbf{X}_{\alpha}\left(\mathcal{C}_{A}\right)^{2} \equiv \sup _{I \in \mathcal{C}_{A}} \frac{1}{|I|_{\sigma}} \sup _{I \supset \cup J_{r}} \sum_{r=1}^{\infty}\left(\frac{\mathrm{P}^{\alpha}\left(J_{r}, \mathbf{1}_{A} \sigma\right)}{\left|J_{r}\right|}\right)^{2}\left\|x-m_{J_{r}}\right\|_{L^{2}\left(\mathbf{1}_{J_{r}} \omega\right)}^{2}
$$

where the intervals $J_{r} \in \mathcal{G}$ are pairwise disjoint in $I$.

What now follows is an adaptation to our sublinear form $|\mathrm{B}|_{\mathrm{stop}, \square^{\omega}}^{A, \mathcal{P}}$ of the arguments of M. Lacey in Lac], together with an additional 'indented' corona construction. We have the following Poisson inequality for intervals $B \subset A \subset I$ :

$$
\begin{aligned}
\frac{\mathrm{P}^{\alpha}\left(A, \mathbf{1}_{I \backslash A} \sigma\right)}{|A|} & \approx \int_{I \backslash A} \frac{1}{\left(\left|y-c_{A}\right|\right)^{2-\alpha}} d \sigma(y) \\
& \lesssim \int_{I \backslash A} \frac{1}{\left(\left|y-c_{B}\right|\right)^{2-\alpha}} d \sigma(y) \approx \frac{\mathrm{P}^{\alpha}\left(B, \mathbf{1}_{I \backslash A} \sigma\right)}{|B|}
\end{aligned}
$$

Fix $A \in \mathcal{A}$. Following $\left[\mathrm{Lac}\right.$ we will use a 'decoupled' modification of the stopping energy $\mathbf{X}_{\alpha}\left(\mathcal{C}_{A}\right)$ to define a 'size functional' of an $A$-admissible collection $\mathcal{P}$. So suppose that $\mathcal{P}$ is an $A$-admissible collection of 
pairs of intervals, and recall that $\Pi_{1} \mathcal{P}$ and $\Pi_{2} \mathcal{P}$ denote the intervals in the first and second components of the pairs in $\mathcal{P}$ respectively.

Definition 69. For an $A$-admissible collection of pairs of intervals $\mathcal{P}$, and an interval $K \in \Pi_{1} \mathcal{P}$, define the projection of $\mathcal{P}$ 'relative to $K$ ' by

$$
\Pi_{2}^{K} \mathcal{P} \equiv\left\{J \in \Pi_{2} \mathcal{P}: J^{\mathbf{w}} \subset K\right\}
$$

where we have suppressed dependence on $A$.

Definition 70. We will use as the 'size testing collection' of intervals for $\mathcal{P}$ the collection

$$
\Pi_{1}^{\text {below }} \mathcal{P} \equiv\left\{K \in \mathcal{D}: K \subset I \text { for some } I \in \Pi_{1} \mathcal{P}\right\},
$$

which consists of all intervals contained in an interval from $\Pi_{1} \mathcal{P}$.

Continuing to follow Lacey [Lac, we define a 'size functional' of $\mathcal{P}$, actually two of them, as follows. Recall from Notation 32 that for a pseudoprojection $\mathbf{Q}_{\mathcal{H}}^{\omega}$ on $x$ we have

$$
\left\|Q_{\mathcal{H}}^{\omega, \mathbf{b}^{*}} x\right\|_{L^{2}(\omega)}^{\boldsymbol{\omega}^{2}}=\sum_{J \in \mathcal{H}}\left\|\triangle_{J}^{\omega, \mathbf{b}^{*}} x\right\|_{L^{2}(\omega)}^{\boldsymbol{\omega}^{2}}=\sum_{J \in \mathcal{H}}\left(\left\|\triangle_{J}^{\omega, \mathbf{b}^{*}} x\right\|_{L^{2}(\omega)}^{2}+\inf _{z \in \mathbb{R}} \sum_{J^{\prime} \in \mathfrak{C}_{\text {broken }}(J)}\left|J^{\prime}\right|_{\omega}\left(E_{J^{\prime}}^{\omega}|x-z|\right)^{2}\right) .
$$

Definition 71. If $\mathcal{P}$ is $A$-admissible, define an initial size condition $\mathcal{S}_{\text {init size }}^{\alpha, A}(\mathcal{P})$ by

$$
\mathcal{S}_{\text {init size }}^{\alpha, A}(\mathcal{P})^{2} \equiv \sup _{K \in \Pi_{1}^{\text {below }} \mathcal{P}} \frac{1}{|K|_{\sigma}}\left(\frac{\mathrm{P}^{\alpha}\left(K, \mathbf{1}_{A \backslash K} \sigma\right)}{|K|}\right)^{2}\left\|Q_{\Pi_{2}^{K} \mathcal{P}}^{\omega, \mathbf{b}^{*}} x\right\|_{L^{2}(\omega)}^{\mathbf{\omega}^{2}}
$$

The following key fact is essential.

Key Fact \#1:

$$
K \subset A \text { and } K \notin \mathcal{C}_{A} \Longrightarrow \Pi_{2}^{K} \mathcal{P}=\emptyset
$$

To see this, suppose that $K \subset A$ and $K \notin \mathcal{C}_{A}$. Then $K \subset A^{\prime}$ for some $A^{\prime} \in \mathfrak{C}_{\mathcal{A}}(A)$, and so if there is

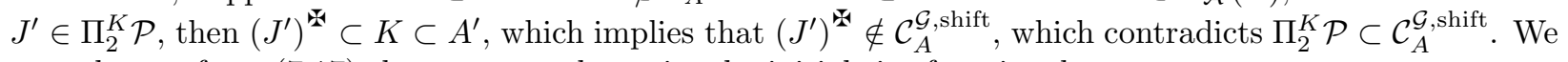
now observe from (17.17) that we may also write the initial size functional as

$$
\mathcal{S}_{\text {init size }}^{\alpha, A}(\mathcal{P})^{2} \equiv \sup _{K \in \Pi_{1}^{\text {below }} \mathcal{P} \cap C_{A}^{\text {restrict }}} \frac{1}{|K|_{\sigma}}\left(\frac{\mathrm{P}^{\alpha}\left(K, \mathbf{1}_{A \backslash K} \sigma\right)}{|K|}\right)^{2}\left\|\mathrm{Q}_{\Pi_{2}^{K} \mathcal{P}}^{\omega, \mathbf{b}^{*}} x\right\|_{L^{2}(\omega)}^{\mathbf{\omega}^{2}}
$$

However, we will also need to control certain pairs $(I, J) \in \mathcal{P}$ using testing intervals $K$ which are strictly smaller than $J^{\mathbb{W}}$, namely those $K \in \mathcal{C}_{A}$ such that $K \subset J^{\mathbb{*}} \subset \pi_{\mathcal{D}}^{(2)} K$. For this, we need a second key fact

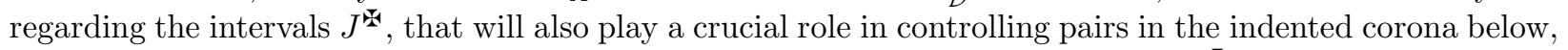
and which is that $J$ is always contained in one of the inner two grandchildren of $J^{*}$.

Key Fact \#2:

$$
\begin{aligned}
& \text { either } 3 J \subset J_{-/+}^{*} \text { or } 3 J \subset J_{+/-}^{*} \text {, }
\end{aligned}
$$

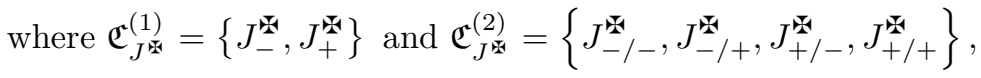

and the children and grandchildren are listed left to right.

To see this, suppose without loss of generality that the child $J_{J}^{*}$ of $J^{*}$ that contains $J$ is the left child $J_{-}^{*}$ (which exists because $J$ is good in $J^{\text {) }}$ ). Then observe that $J$ is by definition $\varepsilon-$ bad in $J_{-}=J_{J}^{\text {U. }}$, i.e.

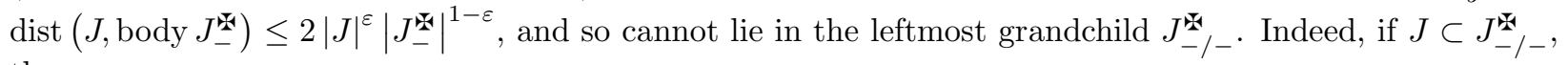
then

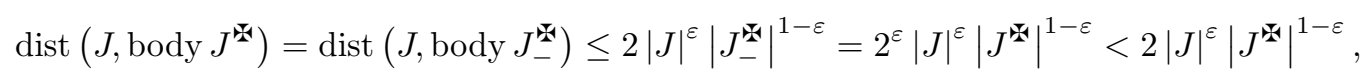

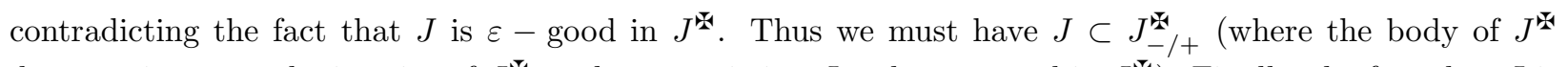
does not intersect the interior of $J_{-/+}$, thus permitting $J$ to be $\varepsilon-\operatorname{good}$ in $J^{-}$). Finally, the fact that $J$ is

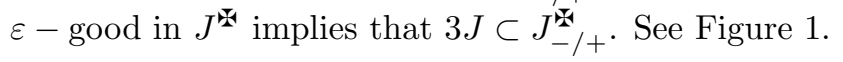


Figure 1. The interval $J$ lies in one of the two inner grandchildren of $J^{\text {t }}$, namely $J_{-/+}^{\text {or }}$

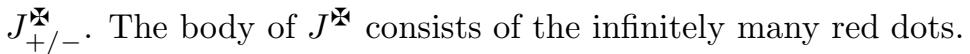

This second key fact is what underlies the construction of the indented corona below, and motivates the next definition of augmented projection, in which we allow intervals $K$ satisfying $J \subset K \varsubsetneqq J^{\mathscr{W}} \subset \pi_{\mathcal{D}}^{(2)} K$, as well as $K \in C_{A}$, to be tested over in the augmented size condition below.

Definition 72. Suppose $\mathcal{P}$ is an A-admissible collection.

(1) For $K \in \Pi_{1} \mathcal{P}$, define the augmented projection of $\mathcal{P}$ relative to $K$ by

$$
\Pi_{2}^{K, \text { aug }} \mathcal{P} \equiv\left\{J \in \Pi_{2} \mathcal{P}: J \subset K \text { and } J^{\text {嵌 }} \subset \pi_{\mathcal{D}}^{(2)} K\right\}=\left\{J \in \Pi_{2} \mathcal{P}: J^{b} \subset K\right\} .
$$

(2) Define the corresponding augmented size functional $\mathcal{S}_{\text {aug size }}^{\alpha, A}(\mathcal{P})$ by

$$
\mathcal{S}_{\text {aug size }}^{\alpha, A}(\mathcal{P})^{2} \equiv \sup _{K \in \Pi_{1}^{\text {below }} \mathcal{P} \cap C_{A}^{\text {restrict }}} \frac{1}{|K|_{\sigma}}\left(\frac{\mathrm{P}^{\alpha}\left(K, \mathbf{1}_{A \backslash K} \sigma\right)}{|K|}\right)^{2}\left\|\mathrm{Q}_{\Pi_{2}^{K, \text { aug }} \mathcal{P}}^{\omega, \mathbf{b}^{*}} x\right\|_{L^{2}(\omega)}^{\boldsymbol{\omega}^{2}} .
$$

We note that the augmented projection $\Pi_{2}^{K \text {,aug }} \mathcal{P}$ includes intervals $J$ for which $J \subset K \varsubsetneqq J^{\mathbb{W}} \subset \pi_{\mathcal{D}}^{(2)} K$, and hence $J$ need not be $\varepsilon$-good inside $K$. For $M \in \mathcal{D}$, denote by $M_{J}$ and $M_{\searrow J}$ the child and grandchild respectively of $M$ that contains $J$, provided they exist. Then by the second key fact (7.19), and using that the endpoints of both $J_{-/+}$and $J_{+/-}^{*}$ lie in the body of $J^{4}$, we have two consequences,

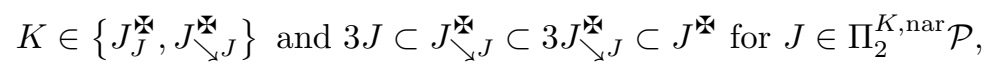

which will play an important role below.

The augmented size functional $\mathcal{S}_{\text {aug size }}^{\alpha, A}(\mathcal{P})$ is a 'decoupled' form of the stopping energy $\mathbf{X}_{\alpha}\left(\mathcal{C}_{A}\right)$ restricted to $\mathcal{P}$, in which the intervals $J$ appearing in $\mathbf{X}_{\alpha}\left(\mathcal{C}_{A}\right)$ no longer appear in the Poisson integral in $\mathcal{S}_{\text {aug size }}^{\alpha, A}(\mathcal{P})$, and it plays a crucial role in Lacey's argument in Lac. We note two essential properties of this definition of size functional:

(1) Monotonicity of size: $\mathcal{S}_{\text {aug size }}^{\alpha, A}(\mathcal{P}) \leq \mathcal{S}_{\text {aug size }}^{\alpha, A}(\mathcal{Q})$ if $\mathcal{P} \subset \mathcal{Q}$,

(2) Control by energy and Muckenhoupt conditions: $\mathcal{S}_{\text {aug size }}^{\alpha, A}(\mathcal{P}) \lesssim \mathcal{E}_{2}^{\alpha}+\sqrt{\mathfrak{A}_{2}^{\alpha}}$.

The monotonicity property follows from $\Pi_{1}^{\text {below }} \mathcal{P} \subset \Pi_{1}^{\text {below }} \mathcal{Q}$ and $\Pi_{2}^{K} \mathcal{P} \subset \Pi_{2}^{K} \mathcal{Q}$. The control property is contained in the next lemma, which uses the stopping energy control for the form $\mathrm{B}_{\text {stop }}^{A}(f, g)$ associated with $A$.

Lemma 73. If $\mathcal{P}^{A}$ is as in 7.4) and $\mathcal{P} \subset \mathcal{P}^{A}$, then

$$
\mathcal{S}_{\text {aug size }}^{\alpha, A}(\mathcal{P}) \leq \mathbf{X}_{\alpha}\left(\mathcal{C}_{A}\right) \lesssim \mathcal{E}_{2}^{\alpha}+\sqrt{A_{2}^{\alpha}}+\sqrt{A_{2}^{\alpha, \text { punct }}} .
$$


Proof. We have

$$
\begin{aligned}
\mathcal{S}_{\text {aug size }}^{\alpha, A}(\mathcal{P})^{2} & =\sup _{K \in \Pi_{1}^{\text {below } \mathcal{P} \cap \mathcal{C}_{A}^{\text {restrict }}}} \frac{1}{|K|_{\sigma}}\left(\frac{\mathrm{P}^{\alpha}\left(K, \mathbf{1}_{A \backslash K} \sigma\right)}{|K|}\right)^{2}\left\|Q_{\Pi_{2}^{K} \mathcal{P} \cup \Pi_{2}^{K, \text { aug }} \mathcal{P}}^{\omega} x\right\|_{L^{2}(\omega)}^{\boldsymbol{中} 2} \\
& \lesssim \sup _{K \in \mathcal{C}_{A}^{\text {restrict }}} \frac{1}{|K|_{\sigma}}\left(\frac{\mathrm{P}^{\alpha}\left(K, \mathbf{1}_{A} \sigma\right)}{|K|}\right)^{2}\left\|x-m_{K}\right\|_{L^{2}\left(\mathbf{1}_{K} \omega\right)}^{2} \leq \mathbf{X}_{\alpha}\left(\mathcal{C}_{A}\right)^{2}
\end{aligned}
$$

which is the first inequality in the statement of the lemma. The second inequality follows from (2.24).

There is an important special circumstance, introduced by M. Lacey in [Lac, in which we can bound our forms by the size functional, namely when the pairs all straddle a subpartition of $A$, and we present this in the next subsection. In order to handle the fact that the intervals in $\Pi_{1}^{\text {below }} \mathcal{P} \cap C_{A}^{\text {restrict }}$ need no longer enjoy any goodness, we will need to formulate a Substraddling Lemma to deal with this situation as well. See Remark on lack of usual goodness after (7.48), where it is explained how this applies to the proof of (7.47). Then in the following subsection, we use the bottom/up stopping time construction of M. Lacey, together with an additional 'indented' top/down corona construction, to reduce control of the sublinear stopping form $|\mathrm{B}|_{\text {stop }, \triangle \omega}^{A, \mathcal{P}}(f, g)$ in inequality (7.10) to the three special cases addressed by the Orthogonality Lemma, the Straddling Lemma and the Substraddling Lemma.

7.3. Straddling, Substraddling and Corona-straddling Lemmas. We begin with the Corona-straddling Lemma in which the straddling collection is the set of $\mathcal{A}$-children of $A$, and applies to the 'corona straddling' subcollection of the initial admissible collection $\mathcal{P}^{A}$ - see (7.4). Define the 'corona straddling' collection $\mathcal{P}_{\text {cor }}^{A}$ by

$$
\mathcal{P}_{\mathrm{cor}}^{A} \equiv \bigcup_{A^{\prime} \in \mathfrak{C}_{\mathcal{A}}(A)}\left\{(I, J) \in \mathcal{P}^{A}: J \subset A^{\prime} \varsubsetneqq J^{\mathcal{A}^{2}} \subset \pi_{\mathcal{D}}^{(2)} A^{\prime}\right\}
$$

Note that $\mathcal{P}_{\text {cor }}^{A}$ is an $A$-admissible collection that consists of just those pairs $(I, J)$ for which $J^{\mathbb{4}}$ is either the $\mathcal{D}$-parent or the $\mathcal{D}$-grandparent of a stopping interval $A^{\prime} \in \mathfrak{C}_{\mathcal{A}}(A)$. The bound for the norm of the corresponding form is controlled by the energy condition.

Lemma 74. We have the sublinear form bound

$$
\mathfrak{N}_{\text {stop }, \triangle \omega}^{A, \mathcal{P}_{\text {cor }}^{A}} \leq C \mathcal{E}_{2}^{\alpha} \text {. }
$$

Proof. The key point here is our assumption that $J \subset A^{\prime} \varsubsetneqq J^{\text {W }} \subset \pi_{\mathcal{D}}^{(2)} A^{\prime}$ for $(I, J) \in \mathcal{P}_{\text {cor }}^{A}$, which implies that in fact $3 J \subset A^{\prime}$ since $J \cap \operatorname{body}\left(\pi_{\mathcal{D}}^{(2)} A^{\prime}\right)=\emptyset$ because $J$ is $\varepsilon-\operatorname{good}$ in $\pi_{\mathcal{D}}^{(2)} A^{\prime}$. We start with

$$
\begin{aligned}
& |\mathrm{B}|_{\text {stop }, \triangle \omega}^{A, \mathcal{P}_{\text {cor }}^{A}}(f, g)=\sum_{J \in \Pi_{2} \mathcal{P}_{\text {cor }}^{A}} \frac{\mathrm{P}^{\alpha}\left(J,\left|\varphi_{J}^{\mathcal{P}_{\text {cor }}^{A}}\right| \mathbf{1}_{A \backslash I_{\mathcal{P}_{\text {cor }}^{A}}(J)} \sigma\right)}{|J|}\left\|\triangle_{J}^{\omega, \mathbf{b}^{*}} x\right\|_{L^{2}(\omega)}^{\infty}\left\|\square_{J}^{\omega, \mathbf{b}^{*} g}\right\|_{L^{2}(\omega)}^{\star} \\
& =\sum_{A^{\prime} \in \mathfrak{C}_{\mathcal{A}}(A)} \sum_{J \in \Pi_{2} \mathcal{P}_{\text {cor }}^{A}: 3 J \subset A^{\prime}} \frac{\mathrm{P}^{\alpha}\left(J,\left|\varphi_{J}^{\mathcal{P}_{\text {cor }}^{A}}\right| \mathbf{1}_{A \backslash I_{\mathcal{P}_{\text {cor }}^{A}}(J)} \sigma\right)}{|J|}\left\|\triangle_{J}^{\omega, \mathbf{b}^{*}} x\right\|_{L^{2}(\omega)}^{\infty}\left\|\square_{J}^{\omega, \mathbf{b}^{*} g}\right\|_{L^{2}(\omega)}^{\star} ; \\
& \text { where } \varphi_{J}^{\mathcal{P}_{\text {cor }}^{A}} \equiv \sum_{I \in \Pi_{1} \mathcal{P}_{\text {cor }}^{A}:(I, J) \in \mathcal{P}_{\text {cor }}^{A}} b_{A} E_{I}^{\sigma}\left(\widehat{\square}_{\pi I}^{\sigma, b, \mathbf{b}} f\right) \mathbf{1}_{A \backslash I} .
\end{aligned}
$$

If $J \in \Pi_{2} \mathcal{P}_{\text {cor }}^{A}$ and $J \subset A^{\prime} \in \mathfrak{C}_{\mathcal{A}}(A)$, then either (1) $A^{\prime}=J_{-/+}$or $J_{+/-}^{*}$ or $(2) A^{\prime}=J_{-}^{+}$or $J_{+}^{*}$, and we have

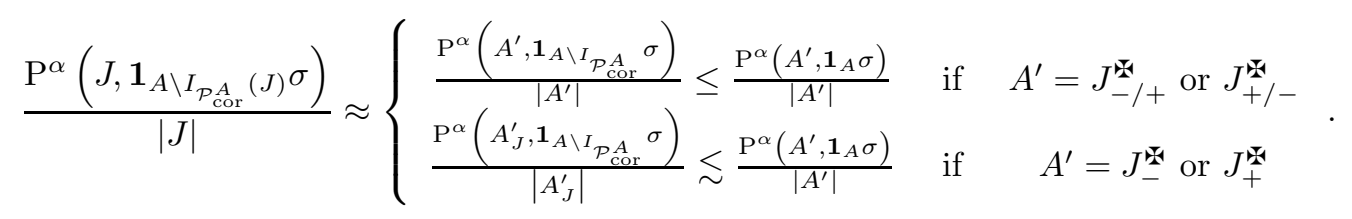


Since $\left|\varphi_{J}^{\mathcal{P}_{\text {cor }}^{A}}\right| \lesssim \alpha_{\mathcal{A}}(A) \mathbf{1}_{A}$ by $(\mathbf{7 . 7})$, we can then bound $|\mathrm{B}|_{\text {stop }, \triangle \omega}^{A, \mathcal{P}_{\text {cor }}^{A}}(f, g)$ by

$$
\begin{aligned}
& \alpha_{\mathcal{A}}(A) \sum_{A^{\prime} \in \mathfrak{C}_{\mathcal{A}}(A)}\left(\frac{\mathrm{P}^{\alpha}\left(A^{\prime}, \mathbf{1}_{A} \sigma\right)}{\left|A^{\prime}\right|}\right)\left\|\mathrm{Q}_{\Pi_{2} \mathcal{P}_{\text {cor }}^{A} ; A^{\prime}}^{\omega, \mathbf{b}^{*}} x\right\|_{L^{2}(\omega)}^{\boldsymbol{\omega}}\left\|\mathrm{P}_{\Pi_{2} \mathcal{P}_{\text {cor }}^{A} ; A^{\prime}}^{\omega, \mathbf{b}^{*}} g\right\|_{L^{2}(\omega)}^{\star} \\
& \leq \alpha_{\mathcal{A}}(A)\left(\sum_{A^{\prime} \in \mathfrak{C}_{\mathcal{A}}(A)}\left(\frac{\mathrm{P}^{\alpha}\left(A^{\prime}, \mathbf{1}_{A} \sigma\right)}{\left|A^{\prime}\right|}\right)^{2}\left\|x-m_{A^{\prime}}^{\sigma}\right\|_{L^{2}\left(\mathbf{1}_{A^{\prime}} \sigma\right)}^{\boldsymbol{\omega}_{2}}\right)^{\frac{1}{2}} \\
& \times\left(\sum_{A^{\prime} \in \mathfrak{C}_{\mathcal{A}}(A)}\left\|\mathrm{P}_{\Pi_{2} \mathcal{P}_{\text {cor }}^{A} ; A^{\prime}}^{\omega, \mathbf{b}^{*}}\right\| \|_{L^{2}(\omega)}^{\star 2}\right)^{\frac{1}{2}} \\
& \leq \mathcal{E}_{2}^{\alpha} \alpha_{\mathcal{A}}(A) \sqrt{|A|_{\sigma}}\left\|\mathrm{P}_{\Pi_{2} \mathcal{P}_{\text {cor }}^{A}}^{\omega, \mathbf{b}^{*}} g\right\|_{L^{2}(\omega)}^{\star} \leq \mathcal{E}_{2}^{\alpha} \alpha_{\mathcal{A}}(A) \sqrt{|A|_{\sigma}}\left\|\mathrm{P}_{\mathcal{C}_{A}^{\text {shift }}}^{\omega, \mathbf{b}^{*}} g\right\|_{L^{2}(\omega)}^{\star},
\end{aligned}
$$

where in the last line we have used the strong energy constant $\mathcal{E}_{2}^{\alpha}$ in (1.12).

Definition 75. We say that an admissible collection of pairs $\mathcal{P}$ is reduced if it contains no pairs from $\mathcal{P}_{\text {cor }}^{A}$, i.e.

$$
\mathcal{P} \cap \mathcal{P}_{\text {cor }}^{A}=\emptyset .
$$

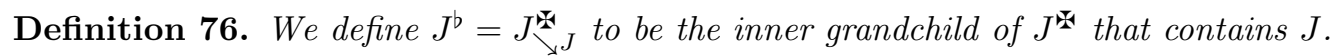

Recall that in terms of $J^{b}$ we rewrite

$$
\Pi_{2}^{K, \text { aug }} \mathcal{P}=\left\{J \in \Pi_{2} \mathcal{P}: J \subset K \text { and } J^{w^{2}} \subset \pi_{\mathcal{D}}^{(2)} K\right\}=\left\{J \in \Pi_{2} \mathcal{P}: J \subset K \text { and } J^{\mathrm{b}} \subset K\right\} .
$$

Definition 77. Given a reduced admissible collection of pairs $\mathcal{Q}$ for $A$, and a subpartition $\mathcal{S} \subset \Pi_{1}^{\text {below }} \mathcal{Q} \cap$ $\mathcal{C}_{A}^{\text {restrict }}$ of pairwise disjoint intervals in $A$, we say that $\mathcal{Q}$ bstraddles $\mathcal{S}$ if for every pair $(I, J) \in \mathcal{Q}$ there is $S \in \mathcal{S} \cap[J, I]$ with $J^{b} \subset S$. To avoid trivialities, we further assume that for every $S \in \mathcal{S}$, there is at least one pair $(I, J) \in \mathcal{Q}$ with $J^{b} \subset S \subset I$. Here $[J, I]$ denotes the geodesic in the dyadic tree $\mathcal{D}$ that connects $J^{\mathcal{D}}$ to $I$, where $J^{\mathcal{D}}$ is the minimal interval in $\mathcal{D}$ that contains $J$.

Definition 78. For any dyadic interval $S \in \mathcal{D}$, define the Whitney collection $\mathcal{W}(S)$ to consist of the maximal subintervals $K$ of $S$ whose triples $3 K$ are contained in $S$. Then set $\mathcal{W}^{*}(S) \equiv \mathcal{W}(S) \cup\{S\}$.

The following geometric proposition will prove useful in proving the bStraddling Lemma 80 below.

Proposition 79. Suppose $\mathcal{Q}$ is reduced admissible and bstraddles a subpartition $\mathcal{S}$ of $A$. Fix $S \in \mathcal{S}$. Define

$$
\varphi_{J}^{\mathcal{Q}^{S}}[h] \equiv \sum_{I \in \Pi_{1} \mathcal{Q}^{S}:(I, J) \in \mathcal{Q}^{S}} b_{A} E_{I}^{\sigma}\left(\widehat{\square}_{\pi I}^{\sigma, b, \mathbf{b}} h\right) \mathbf{1}_{A \backslash I},
$$

assume that $h \in L^{2}(\sigma)$ is supported in the interval $A$, and that there is an interval $H \in \mathcal{C}_{A}$ with $H \supset S$ such that

$$
E_{I}^{\sigma}|h| \leq C E_{H}^{\sigma}|h|, \quad \text { for all } I \in \Pi_{1}^{\text {below }} \mathcal{Q} \cap \mathcal{C}_{A}^{\text {restrict }} \text { with } I \supset S .
$$

Then

$$
\begin{aligned}
& \sum_{J \in \Pi_{2} \mathcal{Q}: J^{b} \subset S} \frac{\mathrm{P}^{\alpha}\left(J,\left|\varphi_{J}^{\mathcal{Q}}[h]\right| \mathbf{1}_{A \backslash I_{\mathcal{Q}}(J)} \sigma\right)}{|J|}\left\|\triangle_{J}^{\omega, \mathbf{b}^{*}} x\right\|_{L^{2}(\omega)}^{\boldsymbol{\omega}}\left\|\square_{J}^{\omega, \mathbf{b}^{*}} g\right\|_{L^{2}(\omega)}^{\star} \\
& \lesssim \alpha_{\mathcal{H}}(H) \frac{\mathrm{P}^{\alpha}\left(S, \mathbf{1}_{A \backslash S} \sigma\right)}{|S|}\left\|\mathrm{Q}_{\Pi_{2}^{S, \text { aug }}}^{\omega, \mathbf{b}^{*}} x\right\|_{L^{2}(\omega)}^{\boldsymbol{\omega}}\left\|\mathrm{P}_{\Pi_{2}^{S, \text { aug }} \mathcal{Q}^{\omega, \mathbf{b}^{*}}} g\right\|_{L^{2}(\omega)}^{\star} \\
& +\alpha_{\mathcal{H}}(H) \sum_{K \in \mathcal{W}(S)} \frac{\mathrm{P}^{\alpha}\left(K, \mathbf{1}_{A \backslash K} \sigma\right)}{|K|}\left\|\mathrm{Q}_{\Pi_{2}^{K, \text { aug }}}^{\omega, \mathbf{b}^{*}} x\right\|_{L^{2}(\omega)}^{\boldsymbol{\omega}} \| \mathrm{P}_{\Pi_{2}^{K, \text { aug }} \mathcal{Q}^{\omega, \mathbf{b}^{*}} g \|_{L^{2}(\omega)}^{\star}}^{\star} .
\end{aligned}
$$

The sum over Whitney intervals $K \in \mathcal{W}(S)$ is only required to bound the sum of those terms on the left for which $J^{\mathrm{b}} \subset S^{\prime \prime}$ for some $S^{\prime \prime} \in \mathfrak{C}_{\mathcal{D}}^{(2)}(S)$. 
Proof. Suppose first that $J^{\mathrm{b}}=S \in \mathcal{C}_{A}^{\text {restrict. }}$. Then $3 S=3 J^{\mathrm{b}} \subset J^{\mathcal{A}} \subset I_{\mathcal{Q}}(J)$ and using (7.7) with $\alpha_{\mathcal{H}}(H)$ in place of $\alpha_{\mathcal{A}}(A)$, we have

$$
\begin{aligned}
\frac{\mathrm{P}^{\alpha}\left(J,\left|\varphi_{J}^{\mathcal{Q}}\right| \mathbf{1}_{A \backslash I_{\mathcal{Q}}(J)} \sigma\right)}{|J|} & \lesssim \alpha_{\mathcal{H}}(H) \frac{\mathrm{P}^{\alpha}\left(J, \mathbf{1}_{\left.A \backslash J^{\boldsymbol{\Psi}} \sigma\right)}\right.}{|J|} \\
& \lesssim \alpha_{\mathcal{H}}(H) \frac{\mathrm{P}^{\alpha}\left(S, \mathbf{1}_{\left.A \backslash J^{\mathcal{H}} \sigma\right)}\right.}{|S|} \leq \alpha_{\mathcal{H}}(H) \frac{\mathrm{P}^{\alpha}\left(S, \mathbf{1}_{A \backslash S} \sigma\right)}{|S|} .
\end{aligned}
$$

Suppose next that $J^{\mathrm{b}}=S^{\prime} \in \mathfrak{C}_{\mathcal{D}}(S)$. Then $3 S^{\prime}=3 J^{\mathrm{b}} \subset J^{\mathbf{w}} \subset I_{\mathcal{Q}}(J)$ and (7.7) give

$$
\begin{aligned}
\frac{\mathrm{P}^{\alpha}\left(J,\left|\varphi_{J}^{\mathcal{Q}}\right| \mathbf{1}_{A \backslash I_{\mathcal{Q}}(J)} \sigma\right)}{|J|} & \lesssim \alpha_{\mathcal{H}}(H) \frac{\mathrm{P}^{\alpha}\left(J, \mathbf{1}_{A \backslash J^{*}} \sigma\right)}{|J|} \\
& \lesssim \alpha_{\mathcal{H}}(H) \frac{\mathrm{P}^{\alpha}\left(S^{\prime}, \mathbf{1}_{A \backslash J^{\mathcal{*}} \sigma} \sigma\right)}{\left|S^{\prime}\right|} \\
& \leq \alpha_{\mathcal{H}}(H) \frac{\mathrm{P}^{\alpha}\left(S^{\prime}, \mathbf{1}_{A \backslash S} \sigma\right)}{\left|S^{\prime}\right|} \approx \alpha_{\mathcal{H}}(H) \frac{\mathrm{P}^{\alpha}\left(S, \mathbf{1}_{A \backslash S} \sigma\right)}{|S|} .
\end{aligned}
$$

Thus in these two cases, by Cauchy-Schwarz, the left hand side of our conclusion is bounded by a multiple of

$$
\begin{aligned}
& \alpha_{\mathcal{H}}(H) \frac{\mathrm{P}^{\alpha}\left(S, \mathbf{1}_{A \backslash S} \sigma\right)}{|S|}\left(\sum_{J \in \Pi_{2} \mathcal{Q}: J^{b} \subset S}\left\|\triangle_{J}^{\omega, \mathbf{b}^{*}} x\right\|_{L^{2}(\omega)}^{\boldsymbol{\omega}^{2}}\right)^{\frac{1}{2}}\left(\sum_{J \in \Pi_{2} \mathcal{Q}: J^{b} \subset S}\left\|\square_{J}^{\omega, \mathbf{b}^{*}} g\right\|_{L^{2}(\omega)}^{\star 2}\right)^{\frac{1}{2}}
\end{aligned}
$$

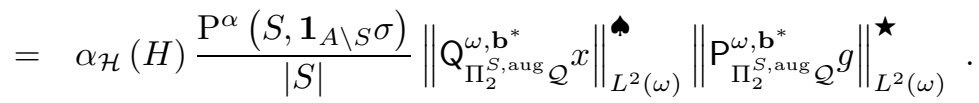

Finally, suppose that $J^{\mathrm{b}} \subset S^{\prime \prime}$ for some $S^{\prime \prime} \in \mathfrak{C}_{\mathcal{D}}^{(2)}(S)$. Then $J^{\text {s }} \subset S$, and Key Fact \#2 in (7.19) shows that $3 J^{b} \subset J^{w}$, so that $3 J^{b} \subset J^{w} \subset S \subset I_{\mathcal{Q}}(J)$. Thus we have $J^{b} \subset K=K[J]$ for some $K \in \mathcal{W}(S)$ and so by (17.7) again,

$$
\begin{aligned}
\frac{\mathrm{P}^{\alpha}\left(J,\left|\varphi_{J}^{\mathcal{Q}}\right| \mathbf{1}_{A \backslash I_{\mathcal{Q}}(J)} \sigma\right)}{|J|} & \lesssim \alpha_{\mathcal{H}}(H) \frac{\mathrm{P}^{\alpha}\left(J, \mathbf{1}_{A \backslash S} \sigma\right)}{|J|} \\
& \lesssim \alpha_{\mathcal{H}}(H) \frac{\mathrm{P}^{\alpha}\left(K, \mathbf{1}_{A \backslash S} \sigma\right)}{|K|} \leq \alpha_{\mathcal{H}}(H) \frac{\mathrm{P}^{\alpha}\left(K, \mathbf{1}_{A \backslash K} \sigma\right)}{|K|} .
\end{aligned}
$$

Now we apply Cauchy-Schwarz again, but noting that $J^{b} \subset K$ this time, to obtain that the left hand side of our conclusion is bounded by a multiple of

$$
\begin{aligned}
& \alpha_{\mathcal{H}}(H) \sum_{K \in \mathcal{W}(S)} \frac{\mathrm{P}^{\alpha}\left(K, \mathbf{1}_{A \backslash K} \sigma\right)}{|K|}\left(\sum_{J \in \Pi_{2} \mathcal{Q}: J^{b} \subset K}\left\|\triangle_{J}^{\omega, \mathbf{b}^{*}} x\right\|_{L^{2}(\omega)}^{\mathbf{\omega}^{2}}\right)^{\frac{1}{2}}\left(\sum_{J \in \Pi_{2} \mathcal{Q}: J^{b} \subset K}\left\|\square_{J}^{\omega, \mathbf{b}^{*} g}\right\|_{L^{2}(\omega)}^{\star 2}\right)^{\frac{1}{2}} \\
& =\alpha_{\mathcal{H}}(H) \sum_{K \in \mathcal{W}(S)} \frac{\mathrm{P}^{\alpha}\left(K, \mathbf{1}_{A \backslash K} \sigma\right)}{|K|}\left\|\mathrm{Q}_{\Pi_{2}^{K, \text { aug }}}^{\omega, \mathbf{b}^{*}} x\right\|_{L^{2}(\omega)}^{\boldsymbol{\omega}}\left\|\mathrm{P}_{\Pi_{2}^{K} \text {,aug }}^{\omega, \mathbf{b}^{*}} g\right\|_{L^{2}(\omega)}^{\star} .
\end{aligned}
$$

This completes the proof of Proposition 79

Recall the family of operators $\left\{\square_{I}^{\sigma, \pi, \mathbf{b}}\right\}_{I \in \mathcal{C}_{A}^{A}}$, where for $I \in \mathcal{C}_{A}^{\mathcal{A}}$, the dual martingale difference $\square_{I}^{\sigma, \pi, \mathbf{b}}$ is defined in (9.16) of Appendix A below, and satisfies

$$
\square_{I}^{\sigma, \pi, \mathbf{b}} f=\left[\sum_{I^{\prime} \in \mathfrak{C}(I)} \mathbb{F}_{I^{\prime}}^{\sigma, \pi, \mathbf{b}} f\right]-\mathbb{F}_{I}^{\sigma, \mathbf{b}} f=\sum_{I^{\prime} \in \mathfrak{C}(I)} \mathbb{F}_{I^{\prime}}^{\sigma, b_{A}} f-\mathbb{F}_{I}^{\sigma, b_{A}} f .
$$

Since $\square_{I}^{\sigma, \pi, \mathbf{b}}$ is the transpose of $\triangle_{I}^{\sigma, \pi, \mathbf{b}}$ for $I \in \mathcal{C}_{A}^{\mathcal{A}}$, the first line of Lemma 93 (where the superscript $\pi$ is suppressed for convenience) shows that $\left\{\square_{I}^{\sigma, \pi, \mathbf{b}}\right\}_{I \in \mathcal{C}_{A}^{A}}$ is a family of projections, and the second line of 
Lemma 93 shows it is an orthogonal family, i.e.

$$
\square_{I}^{\sigma, \pi, \mathbf{b}} \square_{K}^{\sigma, \pi, \mathbf{b}}=\left\{\begin{array}{ccc}
\square_{I}^{\sigma, \pi, \mathbf{b}} & \text { if } \quad I=K \\
0 & \text { if } \quad I \neq K
\end{array}, \quad I, K \in \mathcal{C}_{A}^{\mathcal{A}} .\right.
$$

The orthogonal projections

$$
\begin{aligned}
\mathrm{P}_{\pi\left(\Pi_{1} \mathcal{Q}\right)}^{\sigma, \pi, \mathbf{b}} & \equiv \sum_{I \in \pi_{\left(\Pi_{1} \mathcal{Q}\right)}} \square_{I}^{\sigma, \pi, \mathbf{b}}=\sum_{I \in \Pi_{1} \mathcal{Q}} \square_{\pi I}^{\sigma, \pi, \mathbf{b}}, \\
\text { where } \pi\left(\Pi_{1} \mathcal{Q}\right) & \equiv\left\{\pi_{\mathcal{D}} I: I \in \Pi_{1} \mathcal{Q}\right\} \text { and } \Pi_{1} \mathcal{Q} \subset \mathcal{C}_{A}^{\mathcal{A} \text {,restrict }},
\end{aligned}
$$

thus satisfy the equalities

$$
\square_{\pi I}^{\sigma, \pi, \mathbf{b}} f=\square_{\pi I}^{\sigma, \pi, \mathbf{b}} \mathrm{P}_{\pi\left(\Pi_{1} \mathcal{Q}\right)}^{\sigma, \pi, \mathbf{b}} f \text { and } \widehat{\square}_{\pi I}^{\sigma, \pi, \mathbf{b}} f=\widehat{\square}_{\pi I}^{\sigma, \pi, \mathbf{b}} \mathrm{P}_{\pi\left(\Pi_{1} \mathcal{Q}\right)}^{\sigma, \pi, \mathbf{b}} f, \quad \text { for } I \in \Pi_{1} \mathcal{Q} \subset \mathcal{C}_{A}^{\mathcal{A}, \text { restrict }},
$$

which will permit us to apply certain projection tricks used for Haar projections in the proof of $T 1$ theorems.

However, in our sublinear stopping form $|\mathrm{B}|_{\mathrm{stop}, \triangle^{\omega}}^{A, \mathcal{Q}}$, the dual martingale projections in use in the function

$$
\varphi_{J}^{\mathcal{Q}^{S}} \equiv \sum_{I \in \Pi_{1} \mathcal{Q}^{S}:(I, J) \in \mathcal{Q}^{S}} b_{A} E_{I}^{\sigma}\left(\widehat{\square}_{\pi I}^{\sigma, b, \mathbf{b}} f\right) \mathbf{1}_{A \backslash I}
$$

given in (7.6) above, are the modified pseudoprojections $\left\{\widehat{\square}_{\pi I}^{\sigma, b, \mathbf{b}}\right\}_{I \in \Pi_{1} \mathcal{Q}}$, where $\square_{\pi I}^{\sigma, b, \mathbf{b}}$ differs from the orthogonal projection $\square_{\pi I}^{\sigma, \pi, \mathbf{b}}$ for $I \in \Pi_{1} \mathcal{Q}$ by

$\square_{\pi I}^{\sigma, b, \mathbf{b}} f-\square_{\pi I}^{\sigma, \pi, \mathbf{b}} f=\left\{\left(\sum_{I^{\prime} \in \mathfrak{C}_{\text {natural }}(\pi I)} \mathbb{F}_{I^{\prime}}^{\sigma, b_{A}} f\right)-\mathbb{F}_{\pi I}^{\sigma, b_{A} f}\right\}-\left\{\left(\sum_{I^{\prime} \in \mathfrak{C}^{\prime}(\pi I)} \mathbb{F}_{I^{\prime}}^{\sigma, b_{A}} f\right)-\mathbb{F}_{\pi I}^{\sigma, b_{A}} f\right\}=-\sum_{I^{\prime} \in \mathfrak{C}_{\mathrm{broken}}(\pi I)} \mathbb{F}_{I^{\prime}}^{\sigma, b_{A}} f$.

But the "box support" Supp box of this last expression $\sum_{I^{\prime} \in \mathfrak{C}_{\text {broken }}(\pi I)} \mathbb{F}_{I^{\prime}}^{\sigma, b_{A}} f$ consists of the broken children of $\pi I, \mathfrak{C}_{\text {broken }}(\pi I)$, and is contained in the set $\bigcup_{I \in \mathcal{C}_{A}^{\text {restrict }}} \bigcup_{I^{\prime} \in \mathfrak{C}_{\mathcal{A}}(A) \cap \mathfrak{C}_{\mathcal{D}}(\pi I)}\left\{I^{\prime}\right\}$, i.e.

$$
\begin{aligned}
\operatorname{Supp}_{\text {box }}\left(\sum_{I^{\prime} \in \mathfrak{C}_{\text {broken }}(\pi I)} \mathbb{F}_{I^{\prime}}^{\sigma, b_{A} f}\right) & \subset\left\{I^{\prime} \in \mathfrak{C}_{\mathcal{A}}(A): I^{\prime} \in \mathfrak{C}_{\text {broken }}^{\text {sol }}(\pi I) \text { for some } I \in \mathcal{C}_{A}^{\text {restrict }}\right\} \\
& =\bigcup_{I \in \mathcal{C}_{A}^{\text {restrict }}} \bigcup_{I^{\prime} \in \mathfrak{C}_{\mathcal{A}}(A) \cap \mathfrak{C}_{\mathcal{D}}(\pi I)}\left\{I^{\prime}\right\} .
\end{aligned}
$$

But $I \in \Pi_{1} \mathcal{Q}^{S} \subset \mathcal{C}_{A}^{\text {restrict }}$ is a natural child of $\pi I$, and so

$$
I \cap \operatorname{Supp}_{\text {box }}\left(\sum_{I^{\prime} \in \mathfrak{C}_{\text {broken }}(\pi I)} \mathbb{F}_{I^{\prime}}^{\sigma, b_{A}} f\right)=\emptyset .
$$

It now follows that we have

$$
E_{I}^{\sigma}\left(\widehat{\square}_{\pi I}^{\sigma, b, \mathbf{b}} f\right)=E_{I}^{\sigma}\left(\widehat{\square}_{\pi I}^{\sigma, \pi, \mathbf{b}} f\right), \quad \text { for } I \in \mathcal{C}_{A}^{\text {restrict }}
$$

Returning to (7.22), we have from (7.21) and (7.23) the identity

$$
\begin{aligned}
\varphi_{J}^{\mathcal{Q}^{S}} & \equiv \sum_{I \in \Pi_{1} \mathcal{Q}^{S}:(I, J) \in \mathcal{Q}^{S}} b_{A} E_{I}^{\sigma}\left(\widehat{\square}_{\pi I}^{\sigma, \pi, \mathbf{b}} f\right) \mathbf{1}_{A \backslash I} \\
& =\sum_{I \in \Pi_{1} \mathcal{Q}^{S}:(I, J) \in \mathcal{Q}^{S}} b_{A} E_{I}^{\sigma}\left(\widehat{\square}_{\pi I}^{\sigma, \pi, \mathbf{b}}\left(\mathrm{P}_{\pi\left(\Pi_{1} \mathcal{Q}\right)}^{\sigma, \pi, \mathbf{b}} f\right)\right) \mathbf{1}_{A \backslash I},
\end{aligned}
$$

which will play a critical role in proving the following bStraddling and Substraddling lemmas. The bStraddling Lemma is an adaptation of Lemmas 3.19 and 3.16 in [Lac. 
Lemma 80. Let $\mathcal{Q}$ be a reduced admissible collection of pairs for $A$, and suppose that $\mathcal{S} \subset \Pi_{1}^{\text {below }} \mathcal{Q} \cap \mathcal{C}_{A}^{\text {restrict }}$ is a subpartition of $A$ such that $\mathcal{Q}$ bstraddles $\mathcal{S}$. Then we have the restricted sublinear norm bound

$$
\widehat{\mathfrak{N}}_{\text {stop }, \triangle \omega}^{A, \mathcal{Q}} \leq C_{\mathbf{r}} \sup _{S \in \mathcal{S}} \mathcal{S}_{\text {loc size }}^{\alpha, A ; S}(\mathcal{Q}) \leq C_{\mathbf{r}} \mathcal{S}_{\text {aug size }}^{\alpha, A}(\mathcal{Q}),
$$

where $\mathcal{S}_{\text {loc size }}^{\alpha, A}$ is an $S$-localized size condition with an $S$-hole given by

$$
\mathcal{S}_{\text {loc size }}^{\alpha, A ; S}(\mathcal{Q})^{2} \equiv \sup _{K \in \mathcal{W}^{*}(S) \cap \mathcal{C}_{A}^{\text {restrict }}} \frac{1}{|K|_{\sigma}}\left(\frac{\mathrm{P}^{\alpha}\left(K, \mathbf{1}_{A \backslash S} \sigma\right)}{|K|}\right)^{2} \sum_{J \in \Pi_{2}^{K, \text { aug }} \mathcal{Q}}\left\|\triangle_{J}^{\omega, \mathbf{b}^{*}} x\right\|_{L^{2}(\omega)}^{\mathbf{\omega}^{2}} .
$$

Proof. For $S \in \mathcal{S}$ let $\mathcal{Q}^{S} \equiv\left\{(I, J) \in \mathcal{Q}: J^{\mathrm{b}} \subset S \subset I\right\}$. We begin by using that the reduced collection $\mathcal{Q}$ straddles $\mathcal{S}$, together with the sublinearity property (7.8) of $\varphi_{J}^{\mathcal{Q}}$, and with $|\mathrm{B}|_{\text {stop }, \triangle \omega}^{A, \mathcal{Q}}(f, g)$ as in $(7.9)$, to write

$$
\begin{aligned}
|\mathrm{B}|_{\text {stop }, \triangle \omega}^{A, \mathcal{Q}}(f, g) & =\sum_{J \in \Pi_{2} \mathcal{Q}} \frac{\mathrm{P}^{\alpha}\left(J,\left|\varphi_{J}^{\mathcal{Q}}\right| \mathbf{1}_{A \backslash I_{\mathcal{Q}}(J)} \sigma\right)}{|J|}\left\|\triangle_{J}^{\omega, \mathbf{b}^{*}} x\right\|_{L^{2}(\omega)}^{\boldsymbol{\omega}}\left\|\square_{J}^{\omega, \mathbf{b}^{*}} g\right\|_{L^{2}(\omega)}^{\star} \\
& \leq \sum_{S \in \mathcal{S}} \sum_{J \in \Pi_{2}^{S, \text { aug }} \mathcal{Q}} \frac{\mathrm{P}^{\alpha}\left(J,\left|\varphi_{J}^{\mathcal{Q}^{S}}\right| \mathbf{1}_{A \backslash I_{\mathcal{Q}}(J)} \sigma\right)}{|J|}\left\|\triangle_{J}^{\omega, \mathbf{b}^{*}} x\right\|_{L^{2}(\omega)}^{\boldsymbol{\omega}}\left\|\square_{J}^{\omega, \mathbf{b}^{*}} g\right\|_{L^{2}(\omega)}^{\star} ; \\
\text { where } \varphi_{J}^{\mathcal{Q}^{S}} & \equiv \sum_{I \in \Pi_{1} \mathcal{Q}^{S}:(I, J) \in \mathcal{Q}^{S}} b_{A} E_{I}^{\sigma}\left(\widehat{\square}_{\pi I}^{\sigma, b, \mathbf{b}} f\right) \mathbf{1}_{A \backslash I} .
\end{aligned}
$$

At this point we invoke the identity (17.24),

$$
\varphi_{J}^{\mathcal{Q}^{S}}=\sum_{I \in \Pi_{1} \mathcal{Q}^{S}:(I, J) \in \mathcal{Q}^{S}} b_{A} E_{I}^{\sigma}\left(\widehat{\square}_{\pi I}^{\sigma, \pi, \mathbf{b}}\left(\mathrm{P}_{\pi\left(\Pi_{1} \mathcal{Q}\right)}^{\sigma, \pi, \mathbf{b}} f\right)\right) \mathbf{1}_{A \backslash I}
$$

so that

$$
|\mathrm{B}|_{\text {stop }, \triangle^{\omega}}^{A, \mathcal{Q}}(f, g)=|\mathrm{B}|_{\text {stop }, \triangle^{\omega}}^{A, \mathcal{Q}}(h, g), \quad \text { where } h \equiv \mathrm{P}_{\pi\left(\Pi_{1} \mathcal{Q}\right)}^{\sigma, \pi, \mathbf{b}} f .
$$

We will treat the sublinear form $|\mathrm{B}|_{\mathrm{stop}, \triangle^{\omega}}^{A, \mathcal{Q}}(h, g)$ with $h=\mathrm{P}_{\pi\left(\Pi_{1} \mathcal{Q}\right)}^{\sigma, \pi, \mathbf{b}} f$ using a small variation on the corresponding argument in Lacey Lac 8. Namely, we will apply a Calderón-Zygmund stopping time decomposition to the function $h=\mathrm{P}_{\pi\left(\Pi_{1} \mathcal{Q}\right)}^{\sigma, \pi, \mathbf{b}} f$ on the interval $A$ with 'obstacle' $\mathcal{S} \cup \mathfrak{C}_{A}(A)$, to obtain stopping times $\mathcal{H} \subset \mathcal{C}_{A}$ with the property that for all $H \in \mathcal{H} \backslash\{A\}$ we have

$$
\begin{aligned}
& H \in \mathcal{C}_{A} \text { is not strictly contained in any interval from } \mathcal{S}, \\
& E_{H}^{\sigma}|h|>\Gamma E_{\pi_{\mathcal{H}} H}^{\sigma}|h|, \\
& E_{H^{\prime}}^{\sigma}|h| \leq \Gamma E_{\pi_{\mathcal{H}} H}^{\sigma}|h| \text { for all } H \varsubsetneqq H^{\prime} \subset \pi_{\mathcal{H}} H \text { with } H^{\prime} \in \mathcal{C}_{A} .
\end{aligned}
$$

More precisely, define generation 0 of $\mathcal{H}$ to consist of the single interval $A$. Having defined generation $n$, let generation $n+1$ consist of the union over all intervals $M$ in generation $n$ of the maximal intervals $M^{\prime}$ in $\mathcal{C}_{A}$ that are contained in $M$ with $E_{M^{\prime}}^{\sigma}|h|>\Gamma E_{M}^{\sigma}|h|$, but are not strictly contained in any interval $S$ from $\mathcal{S}$ or contained in any interval $A^{\prime}$ from $\mathfrak{C}_{A}(A)$ - thus the construction stops at the obstacle $\mathcal{S} \cup \mathfrak{C}_{A}(A)$. Then $\mathcal{H}$ is the union of all generations $n \geq 0$.

Denote by

$$
\mathcal{C}_{H}^{\mathcal{H}} \equiv\left\{H^{\prime} \in \mathcal{C}_{A}: H^{\prime} \subset H \text { but } H^{\prime} \not \subset H^{\prime \prime} \text { for any } H^{\prime \prime} \in \mathfrak{C}_{\mathcal{H}}(H)\right\}
$$

the usual $\mathcal{H}$-corona associated with the stopping interval $H$, but restricted to $\mathcal{C}_{A}$, and let $\alpha_{\mathcal{H}}(H)=E_{H}^{\sigma}|f|$ as is customary for a Calderón-Zygmund corona. Since these coronas $\mathcal{C}_{H}^{\mathcal{H}}$ are all contained in $\mathcal{C}_{A}$, we have the stopping energy from the $\mathcal{A}$-corona $\mathcal{C}_{A}$ at our disposal, which as in [Lac], is crucial for the argument. Furthermore, we denote by

$$
\mathcal{Q}_{H} \equiv\left\{(I, J) \in \mathcal{Q}: J \in \mathcal{C}_{H}^{\mathcal{H}, \text { bhift }}\right\}, \quad \text { with } \mathcal{C}_{H}^{\mathcal{H}, \text { shift }} \equiv\left\{J \in \Pi_{2} \mathcal{Q}: J^{b} \in \mathcal{C}_{H}^{\mathcal{H}}\right\}
$$

\footnotetext{
${ }^{8}$ There is a gap in the treatment of the Straddling Lemma 11.10 on page 166 of [SaShUr7]. The wrong restricted norm is used there, but can be fixed by using the corresponding argument of Lacey in [Lac], equivalently adapting the argument here. See Appendix C for a full discussion.
} 
the restriction of the pairs $(I, J)$ in $\mathcal{Q}$ to those for which $J$ lies in the flat shifted $\mathcal{H}$-corona $\mathcal{C}_{H}^{\mathcal{H}, \text { b shift }}$. Since the $\mathcal{H}$-stopping intervals satisfy a $\sigma$-Carleson condition for $\Gamma$ chosen large enough, we have the quasiorthogonal inequality

$$
\sum_{H \in \mathcal{H}} \alpha_{\mathcal{H}}(H)^{2}|H|_{\sigma} \lesssim\|h\|_{L^{2}(\sigma)}^{2}
$$

which below we will see reduces matters to proving inequality (7.25) for the family of reduced admissible collections $\left\{\mathcal{Q}_{H}\right\}_{H \in \mathcal{H}}$ with constants independent of $H$ :

$$
\widehat{\mathfrak{N}}_{\text {stop }, \triangle \omega}^{A, \mathcal{Q}_{H}} \leq C_{\mathbf{r}} \sup _{S \in \mathcal{S}} \mathcal{S}_{\text {loc size }}^{\alpha, A ; S}\left(\mathcal{Q}_{H}\right) \leq C_{\mathbf{r}} \mathcal{S}_{\text {aug size }}^{\alpha, A}\left(\mathcal{Q}_{H}\right), \quad H \in \mathcal{H}
$$

Given $S \in \mathcal{S}$, define $H_{S} \in \mathcal{H}$ to be the minimal interval in $\mathcal{H}$ that contains $S$, and then define

$$
\mathcal{H}_{\mathcal{S}} \equiv\left\{H_{S} \in \mathcal{H}: S \in \mathcal{S}\right\}
$$

Note that a given $H \in \mathcal{H}_{\mathcal{S}}$ may have many intervals $S \in \mathcal{S}$ such that $H=H_{S}$, and we denote the collection of these intervals by $\mathcal{S}_{H} \equiv\left\{S \in \mathcal{S}: H_{S}=H\right\}$. We will organize the straddling intervals $\mathcal{S}$ as

$$
\mathcal{S}=\bigcup_{H \in \mathcal{H}_{\mathcal{S}}} \bigcup_{S \in \mathcal{S}_{H}}
$$

where each $S \in \mathcal{S}$ occurs exactly once in the union on the right hand side, i.e. the collections $\left\{\mathcal{S}_{H}\right\}_{H \in \mathcal{H}}$ are pairwise disjoint.

We now momentarily fix $H \in \mathcal{H}_{\mathcal{S}}$, and consider the reduced admissible collection $\mathcal{Q}_{H}$, so that its projection onto the second component $\Pi_{2} \mathcal{Q}_{H}$ of $\mathcal{Q}_{H}$ is contained in the corona $\mathcal{C}_{H}^{\mathcal{H}, \text { bhift }}$. Then the collection $\mathcal{Q}_{H}$ bstraddles the set $\mathcal{S}_{H}=\left\{S \in \mathcal{S}: H_{S}=H\right\}$. Moreover, $\mathcal{Q}_{H}=\bigcup_{S \in \mathcal{S}: S \subset H} \mathcal{Q}_{H}^{S}$ and $\Pi_{2} \mathcal{Q}_{H}^{S}=\Pi_{2}^{S \text {, aug }} \mathcal{Q}_{H}$.

Recall that a Whitney interval $K$ was required in the right hand side of the conclusion of Proposition 79 only in the case that $J^{b} \subset S^{\prime \prime}$ for some $S^{\prime \prime} \in \mathfrak{C}_{\mathcal{D}}^{(2)}(S)$, which of course implies $3 J^{b} \subset J^{\star} \subset S$. In this case we claim that $K \in \mathcal{C}_{A}$. Indeed, suppose in order to derive a contradiction, that $K \notin \mathcal{C}_{A}$. Then $J^{*} \not \subset K$, and hence $3 J^{*} \not \subset S$. Since $J^{*} \subset S$, it follows that $J^{*}$ shares an endpoint with $S$ (since if not, then $3 J^{*} \subset S$, a contradiction). Now Key Fact \#2 in (7.19) implies that the inner grandchild containing $J$, either $J_{-/+}$ or $J_{+/-}^{*}$, is contained in $K$ where $K \notin \mathcal{C}_{A}$. This then implies that the pair $(I, J)$ belongs to the corona straddling subcollection $\mathcal{P}_{\text {cor }}^{A}$, contradicting the assumption that $\mathcal{Q}$ is reduced.

Thus we have $S \in \Pi_{1}^{\text {below }} \mathcal{Q} \cap \mathcal{C}_{A}^{\text {restrict }}$ and $K \in \mathcal{W}(S) \cap \mathcal{C}_{A}^{\text {restrict }}$ and we can use Proposition 79 with $H=H_{S}$ to bound $|\mathrm{B}|_{\text {stop, } \triangle^{\omega}}^{A, \mathcal{Q}}(f, g)$ by first summing over $H \in \mathcal{H}_{\mathcal{S}}$ and then over $S \in \mathcal{S}_{H}$. Indeed, $\mathcal{Q}_{H}$ bstraddles $\mathcal{S}_{H} \equiv\left\{S \in \mathcal{S}: H_{S}=H\right\}$, so that $\left|\varphi_{J}^{\mathcal{Q}_{H}}\right| \lesssim \alpha_{\mathcal{H}}(H) \mathbf{1}_{A \backslash I_{\mathcal{Q}_{H}}(J)}$ by (17.7), and so the sum over $S \in \mathcal{S}_{H}$ of the first term on the right side of the conclusion of Proposition 79 is bounded by

$$
\begin{aligned}
& \alpha_{\mathcal{H}}(H) \sum_{S \in \mathcal{S}_{H}} \sqrt{|S|_{\sigma}} \frac{1}{\sqrt{|S|_{\sigma}}}\left(\frac{\mathrm{P}^{\alpha}\left(S, \mathbf{1}_{A \backslash S} \sigma\right)}{|S|}\right)\left\|\mathrm{Q}_{\Pi_{2}^{S, \text { aug }} \mathcal{Q}_{H}}^{\omega, \mathbf{b}^{*}} x\right\|_{L^{2}(\omega)}^{\boldsymbol{\omega}}\left\|\mathrm{P}_{\Pi_{2}^{S, \text { aug }} \mathcal{Q}_{H}}^{\omega, \mathbf{b}^{*}} g\right\|_{L^{2}(\omega)}^{\star} \\
\leq & \alpha_{\mathcal{H}}(H)\left\{\sup _{S \in \mathcal{S}_{H}} \frac{1}{\sqrt{|S|_{\sigma}}}\left(\frac{\mathrm{P}^{\alpha}\left(S, \mathbf{1}_{A \backslash S} \sigma\right)}{|S|}\right)\left\|\mathrm{Q}_{\Pi_{2}^{S, \text {,aug }} \mathcal{Q}_{H}}^{\omega, \mathbf{b}^{*}} x\right\|_{L^{2}(\omega)}^{\boldsymbol{\omega}}\right\} \sum_{S \in \mathcal{S}_{H}} \sqrt{|S|_{\sigma}}\left\|\mathrm{P}_{\Pi_{2}^{S, \text { aug }} \mathcal{Q}_{H}}^{\omega, \mathbf{b}^{*}} g\right\|_{L^{2}(\omega)}^{\star} \\
\leq & \alpha_{\mathcal{H}}(H)\left\{\sup _{S \in \mathcal{S}_{H}} \mathcal{S}_{\text {loc size }}^{\alpha, A ; S}\left(\mathcal{Q}_{H}\right)\right\} \sqrt{|H|_{\sigma}}\left\|\mathrm{P}_{\Pi_{2} \mathcal{Q}_{H}}^{\omega, \mathbf{b}^{*}} g\right\|_{L^{2}(\omega)}^{\star},
\end{aligned}
$$


where $\Pi_{2}^{K \text {,aug }} \mathcal{Q}_{H}$ is as in Definition [76, and the corresponding sum over $S \in \mathcal{S}_{H}$ of the second term is bounded by

$$
\begin{aligned}
& \alpha_{\mathcal{H}}(H) \sum_{S \in \mathcal{S}_{H}} \sum_{K \in \mathcal{W}(S) \cap \mathcal{C}_{A}^{\text {restrict }}} \sqrt{|K|_{\sigma}} \frac{1}{\sqrt{|K|_{\sigma}}}\left(\frac{\mathrm{P}^{\alpha}\left(K, \mathbf{1}_{A \backslash S} \sigma\right)}{|K|}\right)\left\|\mathrm{Q}_{\Pi_{2}^{K, \text { aug }} \mathcal{Q}_{H}^{S}}^{\omega, \mathbf{b}^{*}} x\right\|_{L^{2}(\omega)}^{\boldsymbol{\omega}}\left\|\mathrm{P}_{\Pi_{2}^{K, \text { aug }} \mathcal{Q}_{H}^{S}}^{\omega, \mathbf{b}^{*}} g\right\|_{L^{2}(\omega)}^{\star} \\
& \lesssim \alpha_{\mathcal{H}}(H) \sup _{S \in \mathcal{S}_{\mathcal{H}}} \mathcal{S}_{\text {loc size }}^{\alpha, A ; S}\left(\mathcal{Q}_{H}\right)\left(\sum_{S \in \mathcal{S}} \sum_{K \in \mathcal{W}(S)}|K|_{\sigma}\right)^{\frac{1}{2}}\left\|\mathrm{P}_{\Pi_{2} \mathcal{Q}_{H}}^{\omega, \mathbf{b}^{*}} g\right\|_{L^{2}(\omega)}^{\star} \\
& \leq\left\{\sup _{S \in \mathcal{S}_{\mathcal{H}}} \mathcal{S}_{\text {loc size }}^{\alpha, A ; S}\left(\mathcal{Q}_{H}\right)\right\} \alpha_{\mathcal{H}}(H) \sqrt{|H|_{\sigma}}\left\|\mathrm{P}_{\Pi_{2} \mathcal{Q}_{\mathcal{H}}}^{\omega, \mathbf{b}^{*}} g\right\|_{L^{2}(\omega)}^{\star} .
\end{aligned}
$$

Using the definition of $|\mathrm{B}|_{\mathrm{stop}, \triangle^{\omega}}^{A, \mathcal{Q}}(f, g)$ in $(7.9)$, we now sum the previous inequalities over the intervals $H \in \mathcal{H}_{\mathcal{S}}$ to obtain the following string of inequalities (explained in detail after the display)

$$
\begin{aligned}
|\mathrm{B}|_{\text {stop }, \triangle^{\omega}}^{A, \mathcal{Q}}(f, g) & \leq\left\{\sup _{S \in \mathcal{S}} \mathcal{S}_{\text {loc size }}^{\alpha, A ; S}(\mathcal{Q})\right\} \sum_{H \in \mathcal{H}_{\mathcal{S}}} \alpha_{\mathcal{H}}(H) \sqrt{|H|_{\sigma}}\left\|\mathrm{P}_{\Pi_{2} \mathcal{Q}_{H}}^{\omega, \mathbf{b}^{*}} g\right\|_{L^{2}(\omega)}^{\star} \\
& \leq\left\{\sup _{S \in \mathcal{S}} \mathcal{S}_{\text {loc size }}^{\alpha, A ; S}(\mathcal{Q})\right\} \sqrt{\sum_{H \in \mathcal{H}_{\mathcal{S}}} \alpha_{\mathcal{H}}(H)^{2}|H|_{\sigma}} \sqrt{\sum_{H \in \mathcal{H}_{\mathcal{S}}}\left\|\mathrm{P}_{\Pi_{2} \mathcal{Q}_{H}}^{\omega, \mathbf{b}^{*} g}\right\|_{L^{2}(\omega)}^{\star 2}} \\
& \lesssim\left\{\sup _{S \in \mathcal{S}} \mathcal{S}_{\text {loc size }}^{\alpha, A ; S}(\mathcal{Q})\right\}\|h\|_{L^{2}(\sigma)} \sqrt{\sum_{H \in \mathcal{H}_{\mathcal{S}}}\left\|\mathrm{P}_{\Pi_{2} \mathcal{Q}_{H}}^{\omega, \mathbf{b}^{*}} g\right\|_{L^{2}(\omega)}^{\star 2}} \\
& \leq\left\{\sup _{S \in \mathcal{S}} \mathcal{S}_{\text {loc size }}^{\alpha, A ; S}(\mathcal{Q})\right\}\left\|\mathrm{P}_{\pi\left(\Pi_{1} \mathcal{Q}\right)}^{\sigma, \pi, \mathbf{b}} f\right\|_{L^{2}(\sigma)}\left\|\mathrm{P}_{\Pi_{2} \mathcal{Q}}^{\omega, \mathbf{b}^{*} g}\right\|_{L^{2}(\omega)}^{\star} \\
& \lesssim\left\{\sup _{S \in \mathcal{S}} \mathcal{S}_{\text {loc size }}^{\alpha, A ; S}(\mathcal{Q})\right\}\left\|\mathrm{P}_{\pi\left(\Pi_{1} \mathcal{Q}\right)}^{\sigma, \mathbf{b}} f\right\|_{L^{2}(\sigma)}^{\star}\left\|\mathrm{P}_{\Pi_{2} \mathcal{Q}}^{\omega, \mathbf{b}^{*} g}\right\|_{L^{2}(\omega)}^{\star}
\end{aligned}
$$

where in the first line we have used $\mathcal{Q}=\bigcup_{H \in \mathcal{H}_{\mathcal{S}}} \mathcal{Q}_{H}$, which follows from the fact that each $J^{\mathrm{b}}$ is contained in a unique $S \in \mathcal{S}$; in the third line we have used the quasiorthogonal inequality (7.28); in the fourth line we have used that the sets $\Pi_{2} \mathcal{Q}_{H} \subset \mathcal{C}_{H}^{\mathcal{H}, \text { bsift }}$ are pairwise disjoint in $H$ and have union $\Pi_{2} \mathcal{Q}_{=} \bigcup_{H \in \mathcal{H}_{\mathcal{S}}} \Pi_{2} \mathcal{Q}_{H}$. In the final line, we have used first the equality (9.17), second the fact that the functions $\square_{I, \text {, broken }}^{\sigma, \pi} f$ have pairwise disjoint supports, third the upper weak Riesz inequality in Proposition 95, and fourth the estimate (9.19) - which relies on the reverse Hölder property for children in Lemma 18 - to obtain

$$
\begin{aligned}
\left\|\mathrm{P}_{\pi\left(\Pi_{1} \mathcal{Q}\right)}^{\sigma, \pi, \mathbf{b}} f\right\|_{L^{2}(\sigma)}^{2} & =\left\|\sum_{I \in \pi\left(\Pi_{1} \mathcal{Q}\right)} \square_{I}^{\sigma, \mathbf{b}} f-\sum_{I \in \pi\left(\Pi_{1} \mathcal{Q}\right)} \square_{I, \text { broken }}^{\sigma, \pi, \mathbf{b}} f\right\|_{L^{2}(\sigma)}^{2}+\left\|\sum_{I \in \pi\left(\Pi_{1} \mathcal{Q}\right)} \square_{I, \text { broken }}^{\sigma, \pi, \mathbf{b}} f\right\|_{L^{2}(\sigma)}^{2} \\
& \lesssim\left\|\sum_{\left.I \in \Pi_{1} \mathcal{Q}\right)} \square_{I}^{\sigma, \mathbf{b}} f\right\|_{L^{2}(\sigma)}^{2}+\sum_{\pi\left(\Pi_{1} \mathcal{Q}\right)} f\left\|_{L^{2}(\sigma)}^{2}+\sum_{I \in \pi\left(\Pi_{1} \mathcal{Q}\right)}\right\| \square_{I, \text { broken }}^{\sigma, \pi, \mathbf{b}} f \|_{L^{2}(\sigma)}^{2} \\
& \lesssim \sum_{I \in \pi\left(\Pi_{1} \mathcal{Q}\right)}\left\|\square_{I}^{\sigma, \mathbf{b}} f\right\|_{L^{2}(\sigma)}^{2}+\sum_{I \in \pi\left(\Pi_{1} \mathcal{Q}\right)}\left\|\nabla_{I}^{\sigma} f\right\|_{L^{2}(\sigma)}^{2} \lesssim\left\|\mathrm{P}_{\pi\left(\Pi_{1} \mathcal{Q}\right)}^{\sigma, \mathbf{b}} f\right\|_{L^{2}(\sigma)}^{\star 2}
\end{aligned}
$$

We now use the fact that the supremum in the definition of $\mathcal{S}_{\text {loc size }}^{\alpha, A ; S}(\mathcal{Q})$ is taken over $K \in \mathcal{W}^{*}(S) \cap \mathcal{C}_{A}^{\text {restrict }}$ to conclude that

$$
\sup _{S \in \mathcal{S}} \mathcal{S}_{\text {loc size }}^{\alpha, A ; S}(\mathcal{Q}) \leq \mathcal{S}_{\text {aug size }}^{\alpha, A}(\mathcal{Q})
$$

and this completes the proof of Lemma 80 . 
In a similar fashion we can obtain the following Substraddling Lemma.

Definition 81. Given a reduced admissible collection of pairs $\mathcal{Q}$ for $A$, and a $\mathcal{D}$-interval $L$ contained in $A$, we say that $\mathcal{Q}$ substraddles $L$ if for every pair $(I, J) \in \mathcal{Q}$ there is $K \in \mathcal{W}(L) \cap \mathcal{C}_{A}^{\text {restrict }}$ with $J \subset K \subset 3 K \subset I \subset L$.

Lemma 82. Let $L$ be a $\mathcal{D}$-interval contained in $A$, and suppose that $\mathcal{Q}$ is an admissible collection of pairs that substraddles $L$. Then we have the sublinear form bound

$$
\widehat{\mathfrak{N}}_{\text {stop }, \triangle \omega}^{A, \mathcal{Q}} \leq C \mathcal{S}_{\text {aug size }}^{\alpha, A}(\mathcal{Q}) .
$$

Proof. We will show that $\mathcal{Q}$ bstraddles the subset $\mathcal{W}_{L}$ of Whitney intervals for $L$ given by

$$
\mathcal{W}^{\mathcal{Q}}(L) \equiv\left\{K \in \mathcal{W}(L) \cap \mathcal{C}_{A}^{\text {restrict }}: J \subset K \subset 3 K \subset I \subset L \text { for some }(I, J) \in \mathcal{Q}\right\} .
$$

It is clear that $\mathcal{W}^{\mathcal{Q}}(L) \subset \Pi_{1}^{\text {below }} \mathcal{Q} \cap \mathcal{C}_{A}^{\text {restrict }}$ is a subpartition of $A$. It remains to show that for every pair $(I, J) \in \mathcal{Q}$ there is $K \in \mathcal{W}^{\mathcal{Q}}(L) \cap[J, I]$ such that $J^{\mathrm{b}} \subset K$. But our hypothesis implies that there is $K \in \mathcal{W}^{\mathcal{Q}}(L)$ with $J \subset K \subset 3 K \subset I \subset L$. We now consider two cases.

Case 1: If $\pi_{\mathcal{D}}^{(3)} K \subset L$, then by Key Fact \#2 in (7.19), i.e. $3 J$ is contained in an inner grandchild of $J^{\text {W }}$. But $K$ is contained in an outer grandchild of $\pi_{\mathcal{D}}^{(3)} K$ since $\pi_{\mathcal{D}}^{(1)} K$ shares an endpoint with $L$, and so then does $\left.\pi_{\mathcal{D}}^{(3)} K\right)$. We thus have $J^{-} \subset \pi_{\mathcal{D}}^{(2)} K$, which implies that $J^{\mathrm{b}} \subset K$.

Case 2: If $\pi_{\mathcal{D}}^{(3)} K \supseteqq L$, then $K \subset 3 K \subset I \subset L$ implies that $I=L=\pi_{\mathcal{D}}^{(2)} K$. Thus we have $J^{\mathbf{w}} \subset I=\pi_{\mathcal{D}}^{(2)} K$, which again gives $J^{\mathrm{b}} \subset K$.

Now that we know $\mathcal{Q}$ bstraddles the subset $\mathcal{W}^{\mathcal{Q}}(L)$, we can apply Lemma 80 to obtain the required bound $\widehat{\mathfrak{N}}_{\text {stop }, \triangle \omega}^{A, \mathcal{Q}} \leq C \mathcal{S}_{\text {aug size }}^{\alpha, A}(\mathcal{Q})$.

7.4. The bottom/up stopping time argument of M. Lacey. Before introducing Lacey's stopping times, we note that the Corona-straddling Lemma 74 allows us to remove the 'corona straddling' collection $\mathcal{P}_{\text {cor }}^{A}$ of pairs of intervals in (7.20) from the collection $\mathcal{P}^{A}$ in (7.4) used to define the stopping form $\mathrm{B}_{\text {stop }}^{A}(f, g)$. The collection $\mathcal{P}^{A} \backslash \mathcal{P}_{\text {cor }}^{A}$ is of course also $A$-admissible.

Conclusion 83. We assume for the remainder of the proof that all admissible collections $\mathcal{P}$ are reduced, i.e.

$$
\mathcal{P}^{A} \cap \mathcal{P}_{\text {cor }}^{A}=\emptyset \text {, as well as } \mathcal{P} \cap \mathcal{P}_{\text {cor }}^{A}=\emptyset \text { for all } A \text {-admissible } \mathcal{P} \text {. }
$$

We remind the reader again that we will generally use $|J|$ in the Poisson integrals and estimates, but will usually use $\ell(J)$ when defining collections of intervals. For an interval $K \in \mathcal{D}$, we define

$$
\mathcal{G}[K] \equiv\{J \in \mathcal{G}: J \subset K\}
$$

to consist of all intervals $J$ in the other grid $\mathcal{G}$ that are contained in $K$. For an $A$-admissible collection $\mathcal{P}$ of pairs, define two atomic measures $\omega_{\mathcal{P}}$ and $\omega_{b \mathcal{P}}$ in the upper half space $\mathbb{R}_{+}^{2}$ by

$$
\omega_{\mathcal{P}} \equiv \sum_{J \in \Pi_{2} \mathcal{P}}\left\|\triangle_{J}^{\omega, \mathbf{b}^{*}} x\right\|_{L^{2}(\omega)}^{\boldsymbol{\omega}^{2}} \delta_{\left(c_{J}, \ell\left(J^{\boldsymbol{*}}\right)\right)} \text { and } \omega_{b \mathcal{P}} \equiv \sum_{J \in \Pi_{2} \mathcal{P}}\left\|\triangle_{J}^{\omega, \mathbf{b}^{*}} x\right\|_{L^{2}(\omega)}^{\boldsymbol{\omega}^{2}} \delta_{\left(c_{J^{b}}, \ell\left(J^{b}\right)\right)},
$$

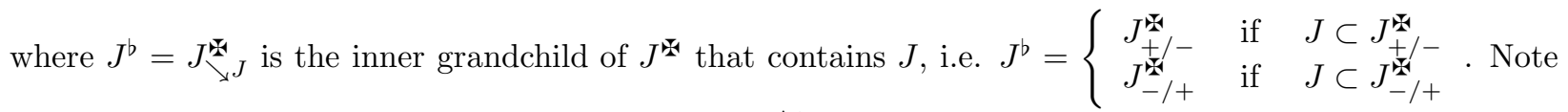
that each interval $J \in \Pi_{2} \mathcal{P}$ has its 'energy' $\left\|\triangle_{J}^{\omega, \mathbf{b}^{*}} x\right\|_{L^{2}(\omega)}^{\mathbf{\omega}^{2}}$ in the measure $\omega_{b} \mathcal{P}$ assigned to exactly one of the two points $\left(c_{J_{-/+}^{*}}, \frac{1}{4} \ell\left(J^{*}\right)\right)$ and $\left(c_{J_{+/-}^{*}}, \frac{1}{4} \ell\left(J^{*}\right)\right)$ in the upper half plane $\mathbb{R}_{+}^{2}$ since $J$ is either contained in $J_{-/+}$or in $J_{+/-}^{W_{1}}$ by Key Fact \#2 in (7.19). Note also that the atomic measure $\omega_{b \mathcal{P}}$ differs from the measure $\mu$ in (10.19) in Appendix B below - which is used there to control the functional energy condition - in that here we bundle together all the $J^{\prime} s$ having a common $J^{b}$. This is in order to rewrite the augmented size functional in terms of the measure $\omega_{b \mathcal{P}}$. We can get away with this here, as opposed to in Appendix B, due to the 'smaller and decoupled' nature of the augmented size functional to which we will relate $\omega_{b} \mathcal{P}$. 
Define the tent $\mathbf{T}(L)$ over an interval $L$ to be the convex hull of the interval $L \times\{0\}$ and the point $\left(c_{L}, \ell(L)\right) \in \mathbb{R}_{+}^{2}$. Then for $J \in \Pi_{2} \mathcal{P}$ we have $J \in \Pi_{2}^{K \text {,aug }} \mathcal{P}$ iff $\left\{J \subset K\right.$ and $\left.J^{*} \subset \pi_{\mathcal{D}}^{(2)} K\right\}$ iff $J^{b}=J_{\searrow} \subset K$ iff $\left(c_{J^{b}}, \ell\left(J^{b}\right)\right) \in \mathbf{T}(K)$. We can now rewrite the augmented size functional of $\mathcal{P}$ in Definition 72 as

$$
\mathcal{S}_{\text {aug size }}^{\alpha, A}(\mathcal{P})^{2} \equiv \sup _{K \in \Pi_{1}^{\text {below }} \mathcal{P} \cap \mathcal{C}_{A}^{\text {restrict }}} \frac{1}{|K|_{\sigma}}\left(\frac{\mathrm{P}^{\alpha}\left(K, \mathbf{1}_{A \backslash K} \sigma\right)}{|K|}\right)^{2} \omega_{b \mathcal{P}}(\mathbf{T}(K)) .
$$

It will be convenient to write

$$
\Psi^{\alpha}(K ; \mathcal{P})^{2} \equiv\left(\frac{\mathrm{P}^{\alpha}\left(K, \mathbf{1}_{A \backslash K} \sigma\right)}{|K|}\right)^{2} \omega_{b \mathcal{P}}(\mathbf{T}(K)),
$$

so that we have simply

$$
\mathcal{S}_{\text {aug size }}^{\alpha, A}(\mathcal{P})^{2}=\sup _{K \in \Pi_{1}^{\text {below }} \mathcal{P} \cap \mathcal{C}_{A}^{\text {restrict }}} \frac{\Psi^{\alpha}(K ; \mathcal{P})^{2}}{|K|_{\sigma}} .
$$

Remark 84. The functional $\omega_{b \mathcal{P}}(\mathbf{T}(K))$ is increasing in $K$, while the functional $\frac{\mathrm{P}^{\alpha}\left(K, \mathbf{1}_{A \backslash K} \sigma\right)}{|K|}$ is 'almost decreasing' in $K:$ if $K_{0} \subset K$ then

$$
\begin{aligned}
\frac{\mathrm{P}^{\alpha}\left(K, \mathbf{1}_{A \backslash K} \sigma\right)}{|K|} & =\int_{A \backslash K} \frac{d \sigma(y)}{\left(|K|+\left|y-c_{K}\right|\right)^{2-\alpha}} \\
& \lesssim \int_{A \backslash K} \frac{d \sigma(y)}{\left(\left|K_{0}\right|+\left|y-c_{K_{0}}\right|\right)^{2-\alpha}} \\
& \leq C_{\alpha} \int_{A \backslash K_{0}} \frac{d \sigma(y)}{\left(\left|K_{0}\right|+\left|y-c_{K_{0}}\right|\right)^{2-\alpha}}=C_{\alpha} \frac{\mathrm{P}^{\alpha}\left(K_{0}, \mathbf{1}_{A \backslash K_{0}} \sigma\right)}{\left|K_{0}\right|}
\end{aligned}
$$

since $\left|K_{0}\right|+\left|y-c_{K_{0}}\right| \leq|K|+\left|y-c_{K}\right|+\frac{1}{2} \operatorname{diam}(K)$ for $y \in A \backslash K$.

Recall that if $\mathcal{P}$ is an admissible collection for a dyadic interval $A$, the corresponding sublinear form in (17.9) and (7.10) is given by

$$
\begin{aligned}
|\mathrm{B}|_{\text {stop }, \triangle \omega}^{A, \mathcal{P}}(f, g) & \equiv \sum_{J \in \Pi_{2} \mathcal{P}} \frac{\mathrm{P}^{\alpha}\left(J,\left|\varphi_{J}^{\mathcal{P}}\right| \mathbf{1}_{A \backslash I_{\mathcal{P}}(J)} \sigma\right)}{|J|}\left\|\triangle_{J}^{\omega, \mathbf{b}^{*}} x\right\|_{L^{2}(\omega)}^{\boldsymbol{\omega}}\left\|\square_{J}^{\omega, \mathbf{b}^{*}} g\right\|_{L^{2}(\omega)}^{\star} ; \\
\text { where } \varphi_{J}^{\mathcal{P}} & \equiv \sum_{I \in \mathcal{C}_{A}^{\text {restrict }:(I, J) \in \mathcal{P}}} b_{A} E_{I}^{\sigma}\left(\widehat{\square}_{\pi I}^{\sigma, b, \mathbf{b}} f\right) \mathbf{1}_{A \backslash I} .
\end{aligned}
$$

In the notation for $|\mathrm{B}|_{\mathrm{stop}, \triangle \omega}^{A, \mathcal{P}}$, we are omitting dependence on the parameter $\alpha$, and to avoid clutter, we will often do so from now on when the dependence on $\alpha$ is inconsequential.

Recall further that the 'size testing collection' of intervals $\Pi_{1}^{\text {below } \mathcal{P}}$ for the initial size testing functional $\mathcal{S}_{\text {init size }}^{\alpha, A}(\mathcal{P})$ is the collection of all subintervals of intervals in $\Pi_{1} \mathcal{P}$, and moreover, by Key Fact \#1 in (7.17), that we can restrict the collection to $\Pi_{1}^{\text {below }} \mathcal{P} \cap \mathcal{C}_{A}^{\text {restrict. }}$. This latter set is used for the augmented size functional.

Assumption: We may assume that the corona $\mathcal{C}_{A}$ is finite, and that each $A$-admissible collection $\mathcal{P}$ is a finite collection, and hence so are $\Pi_{1} \mathcal{P}, \Pi_{1}^{\text {below }} \mathcal{P} \cap \mathcal{C}_{A}^{\text {restrict }}$ and $\Pi_{2} \mathcal{P}$, provided all of the bounds we obtain are independent of the cardinality of these latter collections.

Consider $0<\varepsilon<1$, where $\rho=1+\varepsilon$ will be chosen later in (7.43). Begin by defining the collection $\mathcal{L}_{0}$ to consist of the minimal dyadic intervals $K$ in $\Pi_{1}^{\text {below }} \mathcal{P} \cap \mathcal{C}_{A}^{\text {restrict }}$ such that

$$
\frac{\Psi^{\alpha}(K ; \mathcal{P})^{2}}{|K|_{\sigma}} \geq \varepsilon \mathcal{S}_{\text {aug size }}^{\alpha, A}(\mathcal{P})^{2} .
$$

where we recall that

$$
\Psi^{\alpha}(K ; \mathcal{P})^{2} \equiv\left(\frac{\mathrm{P}^{\alpha}\left(K, \mathbf{1}_{A \backslash K} \sigma\right)}{|K|}\right)^{2} \omega_{b \mathcal{P}}(\mathbf{T}(K)) .
$$


Note that such minimal intervals exist when $0<\varepsilon<1$ because $\mathcal{S}_{\text {aug size }}^{\alpha, A}(\mathcal{P})^{2}$ is the supremum over $K \in$ $\Pi_{1}^{\text {below }} \mathcal{P} \cap \mathcal{C}_{A}^{\text {restrict }}$ of $\frac{\Psi^{\alpha}(K ; \mathcal{P})^{2}}{|K|_{\sigma}}$. A key property of the minimality requirement is that

$$
\frac{\Psi^{\alpha}\left(K^{\prime} ; \mathcal{P}\right)^{2}}{\left|K^{\prime}\right|_{\sigma}}<\varepsilon \mathcal{S}_{\text {aug size }}^{\alpha, A}(\mathcal{P})^{2}
$$

whenever there is $K^{\prime} \in \Pi_{1}^{\text {below }} \mathcal{P} \cap \mathcal{C}_{A}^{\text {restrict }}$ with $K^{\prime} \varsubsetneqq K$ and $K \in \mathcal{L}_{0}$.

We now perform a stopping time argument 'from the bottom up' with respect to the atomic measure $\omega_{\mathcal{P}}$ in the upper half space. This construction of a stopping time 'from the bottom up', together with the subsequent applications of the Orthogonality Lemma and the Straddling Lemma, comprise the key innovations in Lacey's argument [Lac. However, in our situation the intervals $I$ belonging to $\Pi_{1}^{\text {below }} \mathcal{P}$ are no longer 'good' in any sense, and we must include an additional top/down stopping criterion in the next subsection to accommodate this lack of 'goodness'. The argument in [Lac] will apply to these special stopping intervals, called 'indented' intervals, and the remaining intervals form towers with a common endpoint, that are controlled using all three straddling lemmas.

We refer to $\mathcal{L}_{0}$ as the initial or level 0 generation of stopping intervals. Set

$$
\rho=1+\varepsilon \text {. }
$$

As in [SaShUr7], SaShUr9] and [SaShUr10, we follow Lacey Lac by recursively defining a finite sequence of generations $\left\{\mathcal{L}_{m}\right\}_{m \geq 0}$ by letting $\mathcal{L}_{m}$ consist of the minimal dyadic intervals $L$ in $\Pi_{1}^{\text {below }} \mathcal{P} \cap \mathcal{C}_{A}^{\text {restrict }}$ that contain an interval from some previous level $\mathcal{L}_{\ell}, \ell<m$, such that

$$
\omega_{b \mathcal{P}}(\mathbf{T}(L)) \geq \rho \omega_{b \mathcal{P}}\left(\begin{array}{l}
\bigcup_{L^{\prime} \in \bigcup_{\ell=0}^{m-1} \mathcal{L}_{\ell}: L^{\prime} \subset L} \\
\mathbf{T}\left(L^{\prime}\right)
\end{array}\right) .
$$

Since $\mathcal{P}$ is finite this recursion stops at some level $M$. We then let $\mathcal{L}_{M+1}$ consist of all the maximal intervals in $\Pi_{1}^{\text {below }} \mathcal{P} \cap \mathcal{C}_{A}^{\text {restrict }}$ that are not already in some $\mathcal{L}_{m}$ with $m \leq M$. Thus $\mathcal{L}_{M+1}$ will contain either none,

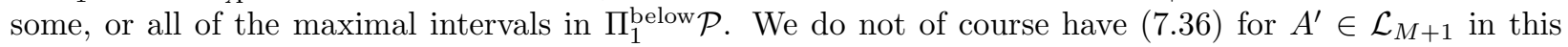
case, but we do have that (7.36) fails for subintervals $K$ of $A^{\prime} \in \mathcal{L}_{M+1}$ that are not contained in any other $L \in \mathcal{L}_{m}$ with $m \leq M$, and this is sufficient for the arguments below.

We now decompose the collection of pairs $(I, J)$ in $\mathcal{P}$ into collections $\mathcal{P}^{\text {bsmall }}$ and $\mathcal{P}^{\text {bbig }}$ according to the location of $I$ and $J^{b}$, but only after introducing below the indented corona $\mathcal{H}$. The collection $\mathcal{P}^{\text {bbig }}$ will then essentially consist of those pairs $(I, J) \in \mathcal{P}$ for which there are $L^{\prime}, L \in \mathcal{H}$ with $L^{\prime} \varsubsetneqq L$ and such that $J^{b} \in \mathcal{C}_{L^{\prime}}^{\mathcal{H}}$ and $I \in \mathcal{C}_{L}^{\mathcal{H}}$. The collection $\mathcal{P}^{\text {bsmall }}$ will consist of the remaining pairs $(I, J) \in \mathcal{P}$ for which there is $L \in \mathcal{H}$ such that $J^{b}, I \in \mathcal{C}_{L}^{\mathcal{H}}$, along with the pairs $(I, J) \in \mathcal{P}$ such that $I \subset I_{0}$ for some $I_{0} \in \mathcal{L}_{0}$. This will cover all pairs $(I, J)$ in $\mathcal{P} \subset \mathcal{P}_{A}$, since for such pairs, $I \in \mathcal{C}_{A}^{\text {restrict }}$ and $J \in \mathcal{C}_{A}^{\mathcal{G} \text { shift }}$, which in turn implies $I \in \mathcal{C}_{L}^{\mathcal{H}}$ and $J^{b} \in \mathcal{C}_{L^{\prime}}^{\mathcal{H}}$ for some $L, L^{\prime} \in \mathcal{H}$. But a considerable amount of further analysis is required to prove (7.10).

First recall that $\mathcal{L} \equiv \bigcup_{m=0}^{M+1} \mathcal{L}_{m}$ is the tree of stopping $\omega_{\mathcal{P}}$-energy intervals defined above. By the construction above, the maximal elements in $\mathcal{L}$ are the maximal intervals in $\Pi_{1}^{\text {below }} \mathcal{P} \cap \mathcal{C}_{A}^{\text {restrict }}$. For $L \in \mathcal{L}$, denote by $\mathcal{C}_{L}^{\mathcal{L}}$ the corona associated with $L$ in the tree $\mathcal{L}$,

$$
\mathcal{C}_{L}^{\mathcal{L}} \equiv\left\{K \in \mathcal{D}: K \subset L \text { and there is no } L^{\prime} \in \mathcal{L} \text { with } K \subset L^{\prime} \varsubsetneqq L\right\},
$$

and define the shifted $\mathcal{L}$-corona and the bshifted $\mathcal{L}$-corona by

$$
\begin{aligned}
\mathcal{C}_{L}^{\mathcal{L}, \text { shift }} & \equiv\left\{J \in \mathcal{G}: J^{\mathscr{W}} \in \mathcal{C}_{L}^{\mathcal{L}}\right\}, \\
\mathcal{C}_{L}^{\mathcal{L}, \text { bshift }} & \equiv\left\{J \in \mathcal{G}: J^{b} \in \mathcal{C}_{L}^{\mathcal{L}}\right\} .
\end{aligned}
$$

It is the second flat shifted corona $\mathcal{C}_{L}^{\mathcal{L}}$,b shift that will be used in our decompositions below, but we retain the more natural shifted coronas $\mathcal{C}_{L}^{\mathcal{L}}$,shift in order to make useful comparisons. Now the parameter $m$ in $\mathcal{L}_{m}$ 
refers to the level at which the stopping construction was performed, but for $L \in \mathcal{L}_{m}$, the corona children $L^{\prime}$ of $L$ are not all necessarily in $\mathcal{L}_{m-1}$, but may be in $\mathcal{L}_{m-t}$ for $t$ large.

At this point we introduce the notion of geometric depth $d$ in the tree $\mathcal{L}$ by defining

$$
\begin{aligned}
\mathcal{G}_{0} \equiv & \{L \in \mathcal{L}: L \text { is maximal }\} \\
\mathcal{G}_{1} \equiv & \left\{L \in \mathcal{L}: L \text { is maximal wrt } L \varsubsetneqq L_{0} \text { for some } L_{0} \in \mathcal{G}_{0}\right\}, \\
& \vdots \\
\mathcal{G}_{d+1} \equiv & \left\{L \in \mathcal{L}: L \text { is maximal wrt } L \varsubsetneqq L_{d} \text { for some } L_{d} \in \mathcal{G}_{d}\right\},
\end{aligned}
$$

We refer to $\mathcal{G}_{d}$ as the $d^{\text {th }}$ generation of intervals in the tree $\mathcal{L}$, and say that the intervals in $\mathcal{G}_{d}$ are at depth $d$ in the tree $\mathcal{L}$ (the generations $\mathcal{G}_{d}$ here are not related to the grid $\mathcal{G}$ ), and we write $d_{\text {geom }}(L)$ for the geometric depth of $L$. Thus the intervals in $\mathcal{G}_{d}$ are the stopping intervals in $\mathcal{L}$ that are $d$ levels in the geometric sense below the top level. While the geometric depth $d_{\text {geom }}$ is about to be superceded by the 'indented' depth $d_{\text {indent }}$ defined in the next subsection, we will return to the geometric depth in order to iterate Lacey's bottom/up stopping criterion when proving the second line in (7.42) in Proposition 85 below.

7.5. The indented corona construction. Now we address the lack of goodness in $\Pi_{1}^{\text {below }} \mathcal{P} \cap \mathcal{C}_{A}^{\text {restrict }}$. For this we introduce an additional top/down stopping time $\mathcal{H}$ over the collection $\mathcal{L}$. Given the initial generation

$$
\mathcal{H}_{0} \equiv \mathcal{L}_{M+1}=\{\text { maximal } L \in \mathcal{L}\}=\left\{\text { maximal } I \in \Pi_{1}^{\text {below }} \mathcal{P}\right\},
$$

define subsequent generations $\mathcal{H}_{k}$ as follows. For $k \geq 1$ and each $H \in \mathcal{H}_{k-1}$, let

$$
\mathcal{H}_{k}(H) \equiv\{\text { maximal } L \in \mathcal{L}: 3 L \subset H\}
$$

consist of the next $\mathcal{H}$-generation of $\mathcal{L}$-intervals below $H$, and set $\mathcal{H}_{k} \equiv \bigcup_{H \in \mathcal{H}_{k-1}} \mathcal{H}_{k}(H)$. Finally set $\mathcal{H} \equiv$ $\bigcup_{k=0}^{\infty} \mathcal{H}_{k}$. We refer to the stopping intervals $H \in \mathcal{H}$ as indented stopping intervals since $3 H \subset \pi_{\mathcal{H}} H$ for all $H \in \mathcal{H}$ at indented generation one or more, i.e. each successive such $H$ is 'indented' in its $\mathcal{H}$-parent. This property of indentation is precisely what is required in order to generate geometric decay in indented generations at the end of the proof. We refer to $k$ as the indented depth of the stopping interval $H \in \mathcal{H}_{k}$, written $k=d_{\text {indent }}(H)$, which is a refinement of the geometric depth $d_{\text {geom }}$ introduced above. We will often revert to writing the dummy variable for intervals in $\mathcal{H}$ as $L$ instead of $H$. For $L \in \mathcal{H}$ define the $\mathcal{H}$-corona $\mathcal{C}_{L}^{\mathcal{H}, \text { shift }}$ and the $\mathcal{H}$-shifted corona $\mathcal{C}_{L}^{\mathcal{H}, \text { shift }}$ and $\mathcal{H}$-bshifted corona $\mathcal{C}_{L}^{\mathcal{H}, \text { bshift }}$ by

$$
\begin{aligned}
\mathcal{C}_{L}^{\mathcal{H}} & \equiv\left\{I \in \mathcal{D}: I \subset L \text { and } I \not \subset L^{\prime} \text { for any } L^{\prime} \in \mathfrak{C}_{\mathcal{H}}(L)\right\}, \\
\mathcal{C}_{L}^{\mathcal{H}, \text { shift }} & \equiv\left\{J \in \mathcal{G}: J^{\mathcal{W}} \in \mathcal{C}_{L}^{\mathcal{H}}\right\}, \\
\mathcal{C}_{L}^{\mathcal{H}, \text { bshift }} & \equiv\left\{J \in \mathcal{G}: J^{\mathrm{b}} \in \mathcal{C}_{L}^{\mathcal{H}}\right\} .
\end{aligned}
$$

We will also need recourse to the coronas $\mathcal{C}_{L}^{\mathcal{H}}$ restricted to intervals in $\mathcal{L}$, i.e.

$$
\mathcal{C}_{L}^{\mathcal{H}}(\mathcal{L}) \equiv \mathcal{C}_{L}^{\mathcal{H}} \cap \mathcal{L}=\left\{T \in \mathcal{L}: T \subset L \text { and } T \not \subset L^{\prime} \text { for any } L^{\prime} \in \mathcal{H} \text { with } L^{\prime} \varsubsetneqq L\right\} .
$$

Then for $L \in \mathcal{H}$ and $t \geq 0$ define

$$
\mathcal{P}_{L, t}^{\mathcal{H}} \equiv\left\{(I, J) \in \mathcal{P}: I \in \mathcal{C}_{L}^{\mathcal{H}} \text { and } J \in \mathcal{C}_{L^{\prime}}^{\mathcal{H} \text {,shift }} \text { for some } L^{\prime} \in \mathcal{H}_{d_{\text {indent }}(L)+t} \text { with } L^{\prime} \subset L\right\} .
$$

In particular, $(I, J) \in \mathcal{P}_{L, t}^{\mathcal{H}}$ implies that $I$ is in the corona $\mathcal{C}_{L}^{\mathcal{H}}$, and that $J$ is in a shifted corona $\mathcal{C}_{L^{\prime}}^{\mathcal{H} \text {,shift }}$ that is $t$ levels of indented generation below $\mathcal{C}_{L}^{\mathcal{H}}$ (when $t=0$ we have $L^{\prime}=L$ ). We emphasize the distinction 'indented generation' as this refers to the indented depth rather than either the level of initial stopping construction of $\mathcal{L}$, or the geometric depth. The point of introducing the tree $\mathcal{H}$ of indented stopping intervals, is that the inclusion $3 L \subset \pi_{\mathcal{H}} L$ for all $L \in \mathcal{H}$ with $d_{\text {indent }}(L) \geq 1$ turns out to be an adequate substitute for the standard 'goodness' lost in the process of infusing the weak goodness of Hytönen and Martikainen in Subsection 3.1 above. 
FiguRE 2. Line segments (not to scale) are the bottom/up stopping intervals in Lacey's tree $\mathcal{L}$. Red segments are the indented intervals and green segments are the intervals in towers. The top indented interval is boxed in purple, the first generation of indented intervals are boxed in orange, and the second generation in blue. Vertical lines indicate common endpoints.

Now within the $\mathcal{H}$-corona $\mathcal{C}_{L}^{\mathcal{H}}(\mathcal{L})$, there are in general further intervals $T \in \mathcal{L}$ in addition to $L \in \mathcal{H}$ itself, but all of these further intervals are contained in the two endpoint $\mathcal{L}$-towers

$$
\begin{aligned}
\mathcal{T}_{\text {left }}(L) & \equiv\left\{L^{\prime} \in \mathcal{L}: L^{\prime} \varsubsetneqq L \text { and left end }\left(L^{\prime}\right)=\text { left end }(L)\right\} \\
\mathcal{T}_{\text {right }}(L) & \equiv\left\{L^{\prime} \in \mathcal{L}: L^{\prime} \varsubsetneqq L \text { and right end }\left(L^{\prime}\right)=\operatorname{right} \text { end }(L)\right\}
\end{aligned}
$$

where left end $(I)$ and right end $(I)$ denote the left and right hand endpoints of $I$ respectively. Thus $\mathcal{C}_{L}^{\mathcal{H} \text {,restrict }}(\mathcal{L}) \equiv$ $\mathcal{C}_{L}^{\mathcal{H}}(\mathcal{L}) \backslash\{L\}$ consists of two 'connected' $\mathcal{L}$-towers (possibly one or both empty), one in $\mathcal{T}_{\text {left }}(L)$ and the other in $\mathcal{T}_{\text {right }}(L)$. Set $\mathcal{T}(L) \equiv \mathcal{T}_{\text {left }}(L) \cup \dot{\cup} \mathcal{T}_{\text {right }}(L) \dot{\cup}\{L\}$. See Figure 2 ,

7.5.1. Decomposition of coronas. Here we describe the decomposition of admissible collections of pairs according to the regular shifted coronas $\mathcal{C}_{L}^{\mathcal{H} \text {,shift }}$ and $\mathcal{C}_{L}^{\mathcal{L} \text {,shift }}$. Strictly speaking, these decompositions will not be used in the sequel, but they do help to provide insight via comparison with the flat shifted decompositions introduced in the next subsubsection, which will be used to finish the proof. For $L \in \mathcal{H}$ and $t=0$ we further decompose $\mathcal{P}_{L, 0}^{\mathcal{H}}$ in (7.38) with $t=0$, i.e.

$$
\mathcal{P}_{L, 0}^{\mathcal{H}}=\left\{(I, J) \in \mathcal{P}: I \in \mathcal{C}_{L}^{\mathcal{H}} \text { and } J \in \mathcal{C}_{L}^{\mathcal{H}, \text { shift }}\right\}
$$

as

$$
\begin{aligned}
& \mathcal{P}_{L, 0}^{\mathcal{H}}=\mathcal{P}_{L, 0}^{\mathcal{H}-\text { small }} \dot{\cup} \mathcal{P}_{L, 0}^{\mathcal{H}-\text { big }} \\
& \mathcal{P}_{L, 0}^{\mathcal{H}-\text { small }} \equiv\left\{(I, J) \in \mathcal{P}_{L, 0}^{\mathcal{H}} \text { : there is no } L^{\prime} \in \mathcal{T}(L) \text { with } J^{\text {s }} \subset L^{\prime} \subset I\right\} \\
& =\left\{(I, J) \in \mathcal{P}_{L, 0}^{\mathcal{H}}: I \in \mathcal{C}_{L^{\prime}}^{\mathcal{L}} \backslash\left\{L^{\prime}\right\} \text { and } J \in \mathcal{C}_{L^{\prime}}^{\mathcal{L} \text {,shift }} \text { for some } L^{\prime} \in \mathcal{T}(L)\right\} \text {, } \\
& \mathcal{P}_{L, 0}^{\mathcal{H}-b i g} \equiv\left\{(I, J) \in \mathcal{P}_{L, 0}^{\mathcal{H}}: \text { there is } L^{\prime} \in \mathcal{T}(L) \text { with } J^{\mathscr{*}} \subset L^{\prime} \subset I\right\} \text {, }
\end{aligned}
$$


with one exception: if $L \in \mathcal{H}_{0}=\mathcal{L}_{M+1}$ we set $\mathcal{P}_{L, 0}^{\mathcal{H}-\text { small }} \equiv \mathcal{P}_{L, 0}^{\mathcal{H}}$ and $\mathcal{P}_{L, 0}^{\mathcal{H}-\text { big }} \equiv \emptyset$ since in this case $L$ fails to satisfy (7.36) as pointed out above. Finally, for $L \in \mathcal{H}$ we further decompose $\mathcal{P}_{L, 0}^{\mathcal{H}-\text { small }}$ as

$$
\begin{aligned}
\mathcal{P}_{L, 0}^{\mathcal{H}-\text { small }} & =\bigcup_{L^{\prime} \in \mathcal{T}(L)} \mathcal{P}_{L^{\prime}, 0}^{\mathcal{L}-\text { small }} ; \\
\mathcal{P}_{L^{\prime}, 0}^{\mathcal{L}-\text { small }} & \equiv\left\{(I, J) \in \mathcal{P}: I \in \mathcal{C}_{L^{\prime}}^{\mathcal{L}} \backslash\left\{L^{\prime}\right\} \text { and } J \in \mathcal{C}_{L^{\prime}}^{\mathcal{L} \text {,shift }}\right\} .
\end{aligned}
$$

Then we set

$$
\begin{aligned}
\mathcal{P}^{\text {big }} & \equiv\left\{\bigcup_{L \in \mathcal{H}} \mathcal{P}_{L, 0}^{\mathcal{H}-\text { big }}\right\} \bigcup\left\{\bigcup_{t \geq 1} \bigcup_{L \in \mathcal{H}} \mathcal{P}_{L, t}^{\mathcal{H}}\right\} ; \\
\mathcal{P}^{\text {small }} & \equiv \bigcup_{L \in \mathcal{L}} \mathcal{P}_{L, 0}^{\mathcal{L}-\text { small }} .
\end{aligned}
$$

Note that every pair $(I, J) \in \mathcal{P}$ is included in either $\mathcal{P}^{\text {small }}$ or $\mathcal{P}^{\text {big }}$ since every $I \in \Pi_{1}^{\text {below }} \mathcal{P}$ is contained in some $L \in \mathcal{H}_{0}=\mathcal{L}_{M+1}$.

7.5.2. Flat shifted coronas. More importantly, we now define the corresponding bshifted admissible collections of pairs $\mathcal{P}_{L, t}^{\text {bH }}$, etc., in which we replace $\mathcal{C}_{L}^{\mathcal{H} \text {,shift }}$ and $\mathcal{C}_{L}^{\mathcal{L} \text {,shift }}$ with

$$
\mathcal{C}_{L}^{\mathcal{H}, \text { bshift }} \equiv\left\{J \in \Pi_{2} \mathcal{P}: J^{\mathrm{b}} \in \mathcal{C}_{L}^{\mathcal{H}}\right\} \text { and } \mathcal{C}_{L}^{\mathcal{L}, \text { bhift }} \equiv\left\{J \in \Pi_{2} \mathcal{P}: J^{\mathrm{b}} \in \mathcal{C}_{L}^{\mathcal{L}}\right\}
$$

In these flat shifted $\mathcal{H}$ and $\mathcal{L}$ coronas, we have effectively shift the intervals $J$ two levels 'up' by requiring $J^{\mathrm{b}} \in \mathcal{C}_{L}^{\mathcal{L}}$ instead of $J^{\mathcal{L}} \in \mathcal{C}_{L}^{\mathcal{L}}$, etc., but because $\mathcal{P}$ is admissible, we always have $J^{\mathbb{A}} \in \mathcal{C}_{A}^{\mathcal{A} \text {,restrict }}$. We define

$$
\begin{aligned}
\mathcal{P}_{L, t}^{b \mathcal{H}} & \equiv\left\{(I, J) \in \mathcal{P}: I \in \mathcal{C}_{L}^{\mathcal{H}} \text { and } J \in \mathcal{C}_{L^{\prime}}^{\mathcal{H}, \text { shift }} \text { for some } L^{\prime} \in \mathcal{H}_{d_{\text {indent }}(L)+t} \text { with } L^{\prime} \subset L\right\}, \\
\mathcal{P}_{L, 0}^{\text {bH }} & =\left\{(I, J) \in \mathcal{P}: I \in \mathcal{C}_{L}^{\mathcal{H}} \text { and } J \in \mathcal{C}_{L}^{\mathcal{H}, \text { bshift }}\right\},
\end{aligned}
$$

and

$$
\begin{aligned}
& \mathcal{P}_{L, 0}^{b \mathcal{H}}=\mathcal{P}_{L, 0}^{b \mathcal{H}-\text { small }} \dot{\cup} \mathcal{P}_{L, 0}^{b \mathcal{H}-b i g} ; \\
& \mathcal{P}_{L, 0}^{b \mathcal{H}-\text { small }} \equiv\left\{(I, J) \in \mathcal{P}_{L, 0}^{b \mathcal{H}}: \text { there is no } L^{\prime} \in \mathcal{T}(L) \text { with } J^{\mathrm{b}} \subset L^{\prime} \subset I\right\} \\
& =\left\{(I, J) \in \mathcal{P}_{L, 0}^{\text {bH }}: I \in \mathcal{C}_{L^{\prime}}^{\mathcal{L}} \backslash\left\{L^{\prime}\right\} \text { and } J \in \mathcal{C}_{L^{\prime}}^{\mathcal{L}, \text { shift }} \text { for some } L^{\prime} \in \mathcal{T}(L)\right\} \text {, } \\
& \mathcal{P}_{L, 0}^{b \mathcal{H}-b i g} \equiv\left\{(I, J) \in \mathcal{P}_{L, 0}^{b \mathcal{H}} \text { : there is } L^{\prime} \in \mathcal{T}(L) \text { with } J^{b} \subset L^{\prime} \subset I\right\},
\end{aligned}
$$

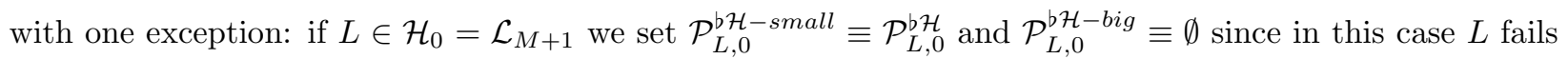
to satisfy (7.36) as pointed out above. Finally, for $L \in \mathcal{H}$ we further decompose $\mathcal{P}_{L, 0}^{\text {bH-small }}$ as

$$
\begin{aligned}
\mathcal{P}_{L, 0}^{\text {bH-small }} & =\bigcup_{L^{\prime} \in \mathcal{T}(L)} \mathcal{P}_{L^{\prime}, 0}^{\text {b } \mathcal{L} \text {-small }} ; \\
\mathcal{P}_{L^{\prime}, 0}^{b \mathcal{L}-\text { small }} & \equiv\left\{(I, J) \in \mathcal{P}: I \in \mathcal{C}_{L^{\prime}}^{\mathcal{L}} \backslash\left\{L^{\prime}\right\} \text { and } J \in \mathcal{C}_{L^{\prime}}^{\mathcal{L}, \text { shift }}\right\} .
\end{aligned}
$$

Then we set

$$
\begin{aligned}
\mathcal{P}^{\text {bbig }} & \equiv\left\{\bigcup_{L \in \mathcal{H}} \mathcal{P}_{L, 0}^{b \mathcal{H}-\text { big }}\right\} \bigcup\left\{\bigcup_{t \geq 1} \bigcup_{L \in \mathcal{H}} \mathcal{P}_{L, t}^{b \mathcal{H}}\right\} ; \\
\mathcal{P}^{\text {bsmall }} & \equiv \bigcup_{L \in \mathcal{L}} \mathcal{P}_{L, 0}^{b \mathcal{L}-\text { small }} .
\end{aligned}
$$

We observed above that every pair $(I, J) \in \mathcal{P}$ is included in either $\mathcal{P}^{\text {small }}$ or $\mathcal{P}^{\text {big }}$, and it follows that every pair $(I, J) \in \mathcal{P}$ is thus included in either $\mathcal{P}^{\text {bsmall }}$ or $\mathcal{P}^{\text {bbig }}$, simply because the pairs $(I, J)$ have been shifted up by two dyadic levels in the interval $J$. Thus the coronas $\mathcal{P}_{L, 0}^{\mathrm{b} \mathcal{L} \text {-small }}$ are now even smaller than the regular coronas $\mathcal{P}_{L, 0}^{\mathcal{L} \text {-small }}$, which permits the estimate (7.41) below to hold for the larger augmented 
size functional. On the other hand, the coronas $\mathcal{P}_{L, 0}^{b \mathcal{H}-\text { big }}$ and $\mathcal{P}_{L, t}^{b \mathcal{H}}$ are now bigger than before, requiring the stronger straddling lemmas above in order to obtain the estimates (7.42) below. More specifically, we will see that stopping forms with pairs in $\mathcal{P}^{\text {bbig }}$ will be estimated using the bStraddling and Substraddling Lemmas (Substraddling applies to part of $\mathcal{P}_{L, 0}^{b \mathcal{H}-b i g}$ and bStraddling applies to the remaining part of $\mathcal{P}_{L, 0}^{b \mathcal{H}-b i g}$ and to $\mathcal{P}_{L, t}^{b \mathcal{H}}$ ), and it is here that the removal of the corona-straddling collection $\mathcal{P}_{\text {cor }}^{A}$ is essential, while forms with pairs in $\mathcal{P}^{\text {bsmall }}$ will be absorbed.

7.6. Size estimates. Now we turn to proving the size estimates we need for these collections. Recall that the restricted norm $\widehat{\mathfrak{N}}_{\text {stop, } \triangle \omega}^{A, \mathcal{P}}$ is the best constant in the inequality

$$
|\mathrm{B}|_{\text {stop }, \triangle \omega}^{A, \mathcal{P}}(f, g) \leq \widehat{\mathfrak{N}}_{\text {stop }, \triangle \omega}^{A, \mathcal{P}}\left\|\mathrm{P}_{\Pi_{1} \mathcal{P}}^{\sigma, \mathbf{b}} f\right\|_{L^{2}(\sigma)}^{\star}\left\|\mathrm{P}_{\Pi_{2} \mathcal{P}}^{\omega, \mathbf{b}^{*}} g\right\|_{L^{2}(\omega)}^{\star},
$$

where $f \in L^{2}(\sigma)$ satisfies $E_{I}^{\sigma}|f| \leq \alpha_{\mathcal{A}}(A)$ for all $I \in \mathcal{C}_{A}$, and $g \in L^{2}(\omega)$.

Proposition 85. Suppose $\rho$ in (7.35) is greater than 1 , and $\mathcal{P}$ is a reduced admissible collection of pairs for a dyadic interval $A$. Let $\mathcal{P}=\mathcal{P}^{\text {big }} \dot{\cup}^{\text {bsmall }}$ be the decomposition satisfying (7.39) above, i.e.

$$
\mathcal{P}=\left\{\bigcup_{L \in \mathcal{H}} \mathcal{P}_{L, 0}^{b \mathcal{H}-b i g}\right\} \bigcup\left\{\bigcup_{t \geq 1} \bigcup_{L \in \mathcal{H}} \mathcal{P}_{L, t}^{b \mathcal{H}}\right\} \cup\left(\bigcup_{L \in \mathcal{L}} \mathcal{P}_{L, 0}^{b \mathcal{L}-\text { small }}\right) .
$$

Then all of these collections $\mathcal{P}_{L, 0}^{b \mathcal{L}-\text { small }}, \mathcal{P}_{L, 0}^{b \mathcal{H}-\text { big }}$ and $\mathcal{P}_{L, t}^{b \mathcal{H}}$ are reduced admissible, and we have the estimate

$$
\mathcal{S}_{\text {aug size }}^{\alpha, A}\left(\mathcal{P}_{L, 0}^{b \mathcal{L}-\text { small }}\right)^{2} \leq(\rho-1) \mathcal{S}_{\text {aug size }}^{\alpha, A}(\mathcal{P})^{2}, \quad L \in \mathcal{L}
$$

and the localized norm bounds,

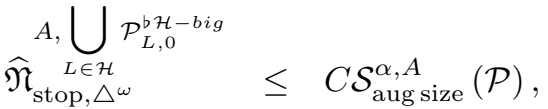

$$
\begin{aligned}
& \underset{\widehat{\mathfrak{N}}_{\text {stop }, \triangle \omega}^{A, \bigcup_{L \in \mathcal{H}} \mathcal{P}_{L, t}^{\text {fH }}}}{i} \leq C \rho^{-\frac{t}{2}} \mathcal{S}_{\text {aug size }}^{\alpha, A}(\mathcal{P}), \quad t \geq 1 .
\end{aligned}
$$

Using this proposition on size estimates, we can finish the proof of (7.10), and hence the proof of (17.1).

Corollary 86. The sublinear stopping form inequality (7.10) holds.

Proof. Recall that $\widehat{\mathfrak{N}}_{\text {stop }, \triangle \omega}^{A, \mathcal{P}}$ is the best constant in the inequality (7.13). Since $\left\{\mathcal{P}_{L, 0}^{b \mathcal{L}-\text { small }}\right\}_{L \in \mathcal{L}}$ is a mutually orthogonal family of $A$-admissible pairs, the Orthogonality Lemma 68 implies that

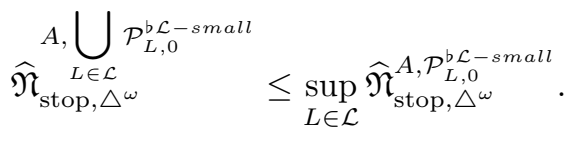

Using this, together with the decomposition of $\mathcal{P}$ and (7.42) above, we obtain

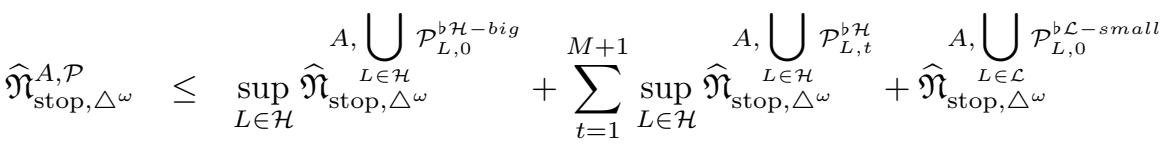

$$
\begin{aligned}
& \lesssim \mathcal{S}_{\text {aug size }}^{\alpha, A}(\mathcal{P})+\left(\sum_{t=1}^{M+1} \rho^{-\frac{t}{2}}\right) \mathcal{S}_{\text {aug size }}^{\alpha, A}(\mathcal{P})+\sup _{L \in \mathcal{L}} \widehat{\mathfrak{N}}_{\text {stop, } \triangle \omega}^{A, \mathcal{P}_{L}^{b \mathcal{L}-\text { small }}} .
\end{aligned}
$$

Since the admissible collection $\mathcal{P}^{A}$ in (7.4) that arises in the stopping form is finite, we can define $\mathfrak{L}$ to be the best constant in the inequality

$$
\widehat{\mathfrak{N}}_{\text {stop, } \triangle \omega}^{A, \mathcal{P}} \leq \mathfrak{L} \mathcal{S}_{\text {aug size }}^{\alpha, A}(\mathcal{P}) \text { for all } A \text {-admissible collections } \mathcal{P} \text {. }
$$

Now choose $\mathcal{P}$ so that

$$
\frac{\widehat{\mathfrak{N}}_{\text {stop }, \triangle \omega}^{A, \mathcal{P}}}{\mathcal{S}_{\text {aug size }}^{\alpha, A}(\mathcal{P})}>\frac{1}{2} \mathfrak{L}=\frac{1}{2} \sup _{\mathcal{Q} \text { is } A \text {-admissible }} \frac{\widehat{\mathfrak{N}}_{\text {stop }, \triangle \omega}^{A, \mathcal{Q}}}{\mathcal{S}_{\text {aug size }}^{\alpha, A}(\mathcal{Q})} .
$$


Then using $\sum_{t=1}^{M+1} \rho^{-\frac{t}{2}} \leq \frac{1}{\sqrt{\rho}-1}$ we have

$$
\begin{aligned}
\mathfrak{L} & <2 \frac{\widehat{\mathfrak{N}}_{\text {stop }, \Delta \omega}^{A, \mathcal{P}}}{\mathcal{S}_{\text {aug size }}^{\alpha, A}(\mathcal{P})} \leq \frac{C \frac{1}{\sqrt{\rho}-1} \mathcal{S}_{\text {aug size }}^{\alpha, A}(\mathcal{P})+C \sup _{L \in \mathcal{L}} \widehat{\mathfrak{N}}_{\text {stop }, \triangle \omega}^{A, \mathcal{P}_{L, 0}^{\text {b }}-\text { small }}}{\mathcal{S}_{\text {aug size }}^{\alpha, A}(\mathcal{P})} \\
& \leq C \frac{1}{\sqrt{\rho}-1}+C \sup _{L \in \mathcal{L}} \mathfrak{L} \frac{\mathcal{S}_{\text {aug size }}^{\alpha, A}\left(\mathcal{P}_{L, 0}^{b \mathcal{L}-\text { small }}\right)}{\mathcal{S}_{\text {aug size }}^{\alpha, A}(\mathcal{P})} \leq C \frac{1}{\sqrt{\rho}-1}+C \mathfrak{L} \sqrt{\rho-1},
\end{aligned}
$$

where we have used (7.41) in the last line. If we choose $\rho>1$ so that

$$
C \sqrt{\rho-1}<\frac{1}{2}
$$

then we obtain $\mathfrak{L} \leq 2 C \frac{1}{\sqrt{\rho}-1}$. Together with Lemma 73, this yields

$$
\widehat{\mathfrak{N}}_{\text {stop }, \triangle \omega}^{A, \mathcal{P}} \leq \mathfrak{L} \mathcal{S}_{\text {aug size }}^{\alpha, A}(\mathcal{P}) \leq 2 C \frac{1}{\sqrt{\rho}-1}\left(\mathcal{E}_{2}^{\alpha}+\sqrt{A_{2}^{\alpha}}+\sqrt{A_{2}^{\alpha, \text { punct }}}\right)
$$

as desired, and completes the proof of inequality (7.10).

Thus, in view of Conclusion 83 it remains only to prove Proposition 85 using the Orthogonality and Straddling and Substraddling Lemmas above, and we now turn to this task.

Proof of Proposition 85. We split the proof into three parts.

Proof of (7.41): To prove the inequality (7.41), suppose first that $L \notin \mathcal{L}_{M+1}$. In the case that $L \in \mathcal{L}_{0}$ is an initial generation interval, then from (7.34) and the fact that every $I \in \mathcal{P}_{L, 0}^{\text {b } \mathcal{L}-\text { small }}$ satisfies $I \varsubsetneqq L$, we obtain that

$$
\begin{aligned}
\mathcal{S}_{\text {aug size }}^{\alpha, A}\left(\mathcal{P}_{L, 0}^{\text {b } \mathcal{L}-\text { small }}\right)^{2}= & \sup _{K^{\prime} \in \Pi_{1}^{\text {below }} \mathcal{P}_{L, 0}^{\text {b } \mathcal{L}-\text { small }} \cap \mathcal{C}_{A}^{\text {restrict }}} \frac{\Psi^{\alpha}\left(K^{\prime} ; \mathcal{P}_{L, 0}^{b \mathcal{L}-\text { small }}\right)^{2}}{\left|K^{\prime}\right|_{\sigma}} \\
\leq & \sup _{K^{\prime} \in \Pi_{1}^{\text {below }} \mathcal{P} \cap \mathcal{C}_{A}^{\text {restrict }}: K^{\prime} \varsubsetneqq L} \frac{\Psi^{\alpha}\left(K^{\prime} ; \mathcal{P}_{L, 0}^{b \mathcal{L}-\text { small }}\right)^{2}}{\left|K^{\prime}\right|_{\sigma}} \leq \varepsilon \mathcal{S}_{\text {aug size }}^{\alpha, A}(\mathcal{P})^{2} .
\end{aligned}
$$

Now suppose that $L \notin \mathcal{L}_{0}$ in addition to $L \notin \mathcal{L}_{M+1}$. Pick a pair $(I, J) \in \mathcal{P}_{L, 0}^{\mathrm{b} \mathcal{L}-\text { small }}$. Then $I$ is in the restricted corona $\mathcal{C}_{L}^{\mathcal{L} \text {,restrict }}$ and $J$ is in the bshifted corona $\mathcal{C}_{L}^{\mathcal{L} \text {,bshift }}$. Since $\mathcal{P}_{L, 0}^{\text {b } \mathcal{L} \text {-small }}$ is a finite collection, the definition of $\mathcal{S}_{\text {aug size }}^{\alpha, A}\left(\mathcal{P}_{L, 0}^{\mathrm{b} \mathcal{L}-\text { small }}\right)$ shows that there is an interval $K \in \Pi_{1}^{\text {below }} \mathcal{P}_{L, 0}^{\text {b } \mathcal{L}-\text { small }} \cap \mathcal{C}_{A}^{\text {restrict }}$ so that

$$
\mathcal{S}_{\text {aug size }}^{\alpha, A}\left(\mathcal{P}_{L, 0}^{b \mathcal{L}-\text { small }}\right)^{2}=\frac{1}{|K|_{\sigma}}\left(\frac{\mathrm{P}^{\alpha}\left(K, \mathbf{1}_{A \backslash K} \sigma\right)}{|K|}\right)^{2} \omega_{b \mathcal{P}}(\mathbf{T}(K)) .
$$

Note that $K \varsubsetneqq L$ by definition of $\mathcal{P}_{L, 0}^{b \mathcal{L}-\text { small }}$. Now let $t$ be such that $L \in \mathcal{L}_{t}$, and define

$$
t^{\prime}=t^{\prime}(K) \equiv \max \left\{s: \text { there is } L^{\prime} \in \mathcal{L}_{s} \text { with } L^{\prime} \subset K\right\},
$$

and note that $t^{\prime}<t$. First, suppose that $t^{\prime}=0$ so that $K$ does not contain any $L^{\prime} \in \mathcal{L}$. Then it follows from the construction at level $\ell=0$ that

$$
\frac{1}{|K|_{\sigma}}\left(\frac{\mathrm{P}^{\alpha}\left(K, \mathbf{1}_{A \backslash K} \sigma\right)}{|K|}\right)^{2} \omega_{b \mathcal{P}}(\mathbf{T}(K))<\varepsilon \mathcal{S}_{\text {aug size }}^{\alpha, A}(\mathcal{P})^{2},
$$

and hence from $\rho=1+\varepsilon$ we obtain

$$
\mathcal{S}_{\text {aug size }}^{\alpha, A}\left(\mathcal{P}_{L, 0}^{\text {b } \mathcal{L}-\text { small }}\right)^{2}<\varepsilon \mathcal{S}_{\text {aug size }}^{\alpha, A}(\mathcal{P})^{2}=(\rho-1) \mathcal{S}_{\text {aug size }}^{\alpha, A}(\mathcal{P})^{2}
$$


Now suppose that $t^{\prime} \geq 1$. Then $K$ fails the stopping condition (7.36) with $m=t^{\prime}+1$, since otherwise it would contain an interval $L^{\prime \prime} \in \mathcal{L}_{t^{\prime}+1}$ contradicting our definition of $t^{\prime}$, and so

$$
\begin{aligned}
& \omega_{b \mathcal{P}}(\mathbf{T}(K))<\rho \omega_{b \mathcal{P}}(\mathbf{V}(K)) \text { where } \mathbf{V}(K) \equiv \quad \bigcup \mathbf{T}\left(L^{\prime}\right) . \\
& L^{\prime} \in \bigcup_{\ell=0}^{t^{\prime}} \mathcal{L}_{\ell}: L^{\prime} \subset K
\end{aligned}
$$

Now we use the crucial fact that the positive measure $\omega_{b \mathcal{P}}$ is additive and finite to obtain from this that

$$
\omega_{b \mathcal{P}}(\mathbf{T}(K) \backslash \mathbf{V}(K))=\omega_{b \mathcal{P}}(\mathbf{T}(K))-\omega_{b \mathcal{P}}(\mathbf{V}(K)) \leq(\rho-1) \omega_{b \mathcal{P}}(\mathbf{V}(K))
$$

Now recall that

$$
\mathcal{S}_{\text {aug size }}^{\alpha, A}(\mathcal{Q})^{2} \equiv \sup _{K \in \Pi_{1}^{\text {below }} \mathcal{Q} \cap C_{A}^{\text {restrict }}} \frac{1}{|K|_{\sigma}}\left(\frac{\mathrm{P}^{\alpha}\left(K, \mathbf{1}_{A \backslash K} \sigma\right)}{|K|}\right)^{2}\left\|\mathrm{Q}_{\Pi_{2}^{K, \text { aug }} \mathcal{Q}^{\omega, \mathbf{b}^{*}}}^{x}\right\|_{L^{2}(\omega)}^{\mathbf{\omega} 2}
$$

We claim it follows that for each $J \in \Pi_{2}^{K, \text { aug }} \mathcal{P}_{L, 0}^{b \mathcal{L}-\text { small }}$, the support $\left(c_{J^{b}}, \ell\left(J^{b}\right)\right)$ of the atom $\delta_{\left(c_{J^{b}}, \ell\left(J^{b}\right)\right)}$ is contained in the set $\mathbf{T}(K)$, but not in the set

$$
\mathbf{V}(K) \equiv \bigcup\left\{\mathbf{T}\left(L^{\prime}\right): L^{\prime} \in \bigcup_{\ell=0}^{t^{\prime}} \mathcal{L}_{\ell}: L^{\prime} \subset K\right\}
$$

Indeed, suppose in order to derive a contradiction, that $\left(c_{J^{b}}, \ell\left(J^{b}\right)\right) \in \mathbf{T}\left(L^{\prime}\right)$ for some $L^{\prime} \in \mathcal{L}_{\ell}$ with $0 \leq \ell \leq t^{\prime}$. Recall that $L \in \mathcal{L}_{t}$ with $t^{\prime}<t$ so that $L^{\prime} \varsubsetneqq L$. Thus $\left(c_{J^{\mathrm{b}}}, \ell\left(J^{b}\right)\right) \in \mathbf{T}\left(L^{\prime}\right)$ implies $J^{b} \subset L^{\prime}$, which contradicts the fact that

$$
J \in \Pi_{2}^{K} \mathcal{P}_{L, 0}^{b \mathcal{L}-\text { small }} \subset \Pi_{2} \mathcal{P}_{L, 0}^{b \mathcal{L}-\text { small }}=\left\{(I, J) \in \mathcal{P}: I \in \mathcal{C}_{L}^{\mathcal{L}} \backslash\{L\} \text { and } J \in \mathcal{C}_{L}^{\mathcal{L}, \text { bshift }}\right\}
$$

implies $J^{b} \in \mathcal{C}_{L}^{\mathcal{L}}$ - because $L^{\prime} \notin \mathcal{C}_{L}^{\mathcal{L}}$.

Thus from the definition of $\omega_{b \mathcal{P}}$ in (7.32), the 'energy' $\left\|Q_{\Pi_{2}^{K, \text { aug }} \mathcal{P}_{L, 0}^{\mathrm{b}-\text { small }}}^{\omega, \mathbf{b}^{*}}\right\|_{L^{2}(\omega)}^{\mathbf{\$} 2}$ is at most the $\omega_{b \mathcal{P}}$-measure of $\mathbf{T}(K) \backslash \mathbf{V}(K)$. Using

$$
\omega_{b \mathcal{P}}^{b \mathcal{L}-\text {-small }}(\mathbf{T}(K)) \leq \omega_{\mathrm{b} \mathcal{P}}(\mathbf{T}(K) \backslash \mathbf{V}(K))
$$

and (7.44), we then have

$$
\begin{aligned}
& \mathcal{S}_{\text {aug size }}^{\alpha, A}\left(\mathcal{P}_{L, 0}^{b \mathcal{L}-\text { small }}\right)^{2} \\
\leq & \sup _{K \in \Pi_{1}^{\text {below }} \mathcal{P}_{L, 0}^{\text {b } \mathcal{L}-\text { small }} \cap \mathcal{C}_{A}^{\text {restrict }}} \frac{1}{|K|_{\sigma}}\left(\frac{\mathrm{P}^{\alpha}\left(K, \mathbf{1}_{A \backslash K} \sigma\right)}{|K|}\right)^{2} \omega_{b \mathcal{P}}(\mathbf{T}(K) \backslash \mathbf{V}(K)) \\
\leq & (\rho-1) \sup _{K \in \Pi_{1}^{\text {below }} \mathcal{P}_{L, 0}^{b \mathcal{L}-\text { small }} \cap \mathcal{C}_{A}^{\text {restrict }}} \frac{1}{|K|_{\sigma}}\left(\frac{\mathrm{P}^{\alpha}\left(K, \mathbf{1}_{A \backslash K} \sigma\right)}{|K|}\right)^{2} \omega_{b \mathcal{P}}(\mathbf{V}(K)),
\end{aligned}
$$

and we can continue with

$$
\begin{aligned}
& \mathcal{S}_{\text {aug size }}^{\alpha, A}\left(\mathcal{P}_{L, 0}^{b \mathcal{L}-\text { small }}\right)^{2} \\
\leq & (\rho-1) \sup _{K \in \Pi_{1}^{\text {below }} \mathcal{P} \cap \mathcal{C}_{A}^{\text {restrict }}} \frac{1}{|K|_{\sigma}}\left(\frac{\mathrm{P}^{\alpha}\left(K, \mathbf{1}_{A \backslash K} \sigma\right)}{|K|}\right)^{2} \omega_{b \mathcal{P}}(\mathbf{T}(K)) \\
\leq & (\rho-1) \mathcal{S}_{\text {aug size }}^{\alpha, A}(\mathcal{P})^{2} .
\end{aligned}
$$

In the remaining case where $L \in \mathcal{L}_{M+1}$ we can include $L$ as a testing interval $K$ and the same reasoning applies. This completes the proof of (7.41). 
To prove the other inequality (7.42) in Proposition 85, we will use the Straddling and Substraddling Lemmas to bound the norm of certain 'straddled' stopping forms by the augmented size functional $\mathcal{S}_{\text {aug size }}^{\alpha, A}$, and the Orthogonality Lemma to bound sums of 'mutually orthogonal' stopping forms. Recall that

$$
\begin{aligned}
\mathcal{P}^{b b i g} & =\left\{\bigcup_{L \in \mathcal{H}} \mathcal{P}_{L, 0}^{b \mathcal{H}-b i g}\right\} \bigcup\left\{\bigcup_{t \geq 1} \bigcup_{L \in \mathcal{H}} \mathcal{P}_{L, t}^{b \mathcal{H}}\right\} \equiv \mathcal{Q}_{0}^{b \mathcal{H}-b i g} \bigcup \mathcal{Q}_{1}^{b \mathcal{H}-b i g} ; \\
\mathcal{Q}_{0}^{b \mathcal{H}-b i g} & \equiv \bigcup_{L \in \mathcal{L}} \mathcal{P}_{L, 0}^{b \mathcal{H}-b i g}, \quad \mathcal{Q}_{1}^{b \mathcal{H}-b i g} \equiv \bigcup_{t \geq 1} \mathcal{P}_{t}^{b \mathcal{H}-b i g}, \quad \mathcal{P}_{t}^{b \mathcal{H}-b i g} \equiv \bigcup_{L \in \mathcal{H}} \mathcal{P}_{L, t}^{b \mathcal{H}} .
\end{aligned}
$$

Proof of the second line in (7.42): We first turn to the collection

$$
\begin{aligned}
\mathcal{Q}_{1}^{b \mathcal{H}-b i g} & =\bigcup_{t \geq 1} \bigcup_{L \in \mathcal{H}} \mathcal{P}_{L, t}^{b \mathcal{H}}=\bigcup_{t \geq 1} \mathcal{P}_{t}^{b \mathcal{H}-b i g} ; \\
\mathcal{P}_{t}^{b \mathcal{H}-b i g} & \equiv \bigcup_{L \in \mathcal{L}} \mathcal{P}_{L, t}^{b \mathcal{H}}, \quad t \geq 1
\end{aligned}
$$

where

$$
\mathcal{P}_{L, t}^{b \mathcal{H}}=\left\{(I, J) \in \mathcal{P}: I \in \mathcal{C}_{L}^{\mathcal{H}} \text { and } J \in \mathcal{C}_{L^{\prime}}^{\mathcal{H}, \text { shift }} \text { for some } L^{\prime} \in \mathcal{H}_{d_{\text {indent }}(L)+t} \text { with } L^{\prime} \subset L\right\} .
$$

We now claim that the second line in (7.42) holds, i.e.

$$
\widehat{\mathfrak{N}}_{\text {stop }, \triangle \omega}^{A, \mathcal{P}_{t}^{\text {bH } \mathcal{H}-b i g}} \leq C \rho^{-\frac{t}{2}} \mathcal{S}_{\text {aug size }}^{\alpha, A}(\mathcal{P}), \quad t \geq 1,
$$

which recovers the key geometric gain obtained by Lacey in [Lac, except that here we are only gaining this decay relative to the indented subtree $\mathcal{H}$ of the tree $\mathcal{L}$.

The case $t=1$ can be handled with relative ease since decay is not relevant here. Indeed, $\mathcal{P}_{L, 1}^{\text {bH }}$ straddles the collection $\mathfrak{C}_{\mathcal{H}}(L)$ of $\mathcal{H}$-children of $L$, and so the localized bStraddling Lemma 80 applies to give

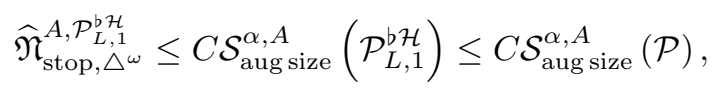

and then the Orthogonality Lemma 68 applies to give

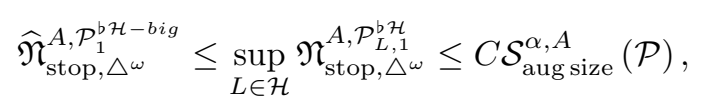

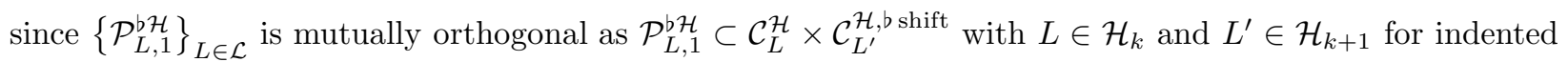
depth $k=k(L)$. The case $t=2$ is equally easy.

Now we consider the case $t \geq 2$, where it is essential to obtain geometric decay in $t$. We remind the reader that all of our admissible collections $\mathcal{P}_{L, t}^{b \mathcal{H}}$ are reduced by Conclusion 83, We again apply Lemma 80 to $\mathcal{P}_{L, t}^{b \mathcal{H}}$ with $\mathcal{S}=\mathfrak{C}_{\mathcal{H}}(L)$, so that for any $(I, J) \in \mathcal{P}_{L, t}^{\text {b }}$, there is $H^{\prime} \in \mathfrak{C}_{\mathcal{H}}(L)$ with $J^{\mathrm{b}} \subset H^{\prime} \varsubsetneqq I \in \mathcal{C}_{L}^{\mathcal{H}}$. But this time we must use the stronger localized bounds $\mathcal{S}_{\text {loc size }}^{\alpha, A}$ with an $S$-hole, that give

$$
\begin{aligned}
& \widehat{\mathfrak{N}}_{\text {stop }, \triangle \omega}^{A, \mathcal{P}_{L}^{b \mathcal{H}}, t} \leq C \sup _{H^{\prime} \in \mathfrak{C}_{\mathcal{H}}(L)} \mathcal{S}_{\text {loc size }}^{\alpha, A ; H^{\prime}}\left(\mathcal{P}_{L, t}^{b \mathcal{H}}\right), \quad t \geq 0
\end{aligned}
$$

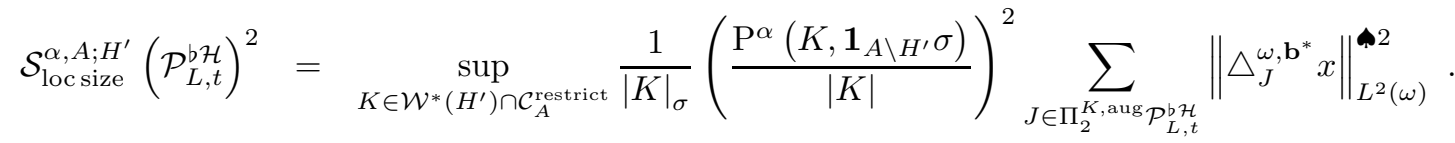

It remains to show that

$$
\begin{aligned}
& \sum_{J \in \Pi_{2}^{K, \operatorname{aug}} \mathcal{P}_{L, t}^{b \mathcal{H}}}\left\|\triangle_{J}^{\omega, \mathbf{b}^{*}} x\right\|_{L^{2}(\omega)}^{\mathbf{\omega} 2} \leq \rho^{-(t-2)} \omega_{\mathrm{b} \mathcal{P}}(\mathbf{T}(K)), \\
& \text { for } t \geq 2, K \in \mathcal{W}^{*}\left(H^{\prime}\right) \cap \mathcal{C}_{A}^{\text {restrict }}, H^{\prime} \in \mathfrak{C}_{\mathcal{H}}(L) \text {. }
\end{aligned}
$$


so that we then have

$$
\begin{aligned}
& \frac{1}{|K|_{\sigma}}\left(\frac{\mathrm{P}^{\alpha}\left(K, \mathbf{1}_{A \backslash H^{\prime}} \sigma\right)}{|K|}\right)^{2} \sum_{J \in \Pi_{2}^{K, \text { aug }} \mathcal{P}_{L, t}^{b \mathcal{H}}}\left\|\triangle_{J}^{\omega, \mathbf{b}^{*}} x\right\|_{L^{2}(\omega)}^{\mathbf{A}^{2}} \\
\leq & \rho^{-(t-2)} \frac{1}{|K|_{\sigma}}\left(\frac{\mathrm{P}^{\alpha}\left(K, \mathbf{1}_{A \backslash K} \sigma\right)}{|K|}\right)^{2} \omega_{b \mathcal{P}}(\mathbf{T}(K)) \leq \rho^{-(t-2)} \mathcal{S}_{\text {aug size }}^{\alpha, A}(\mathcal{P})^{2},
\end{aligned}
$$

by (17.33), and hence conclude the required bound for $\mathfrak{N}_{\text {stop, } \triangle}^{A, \mathcal{P}_{L}^{\mathrm{b} \mathcal{H}}}$, namely that

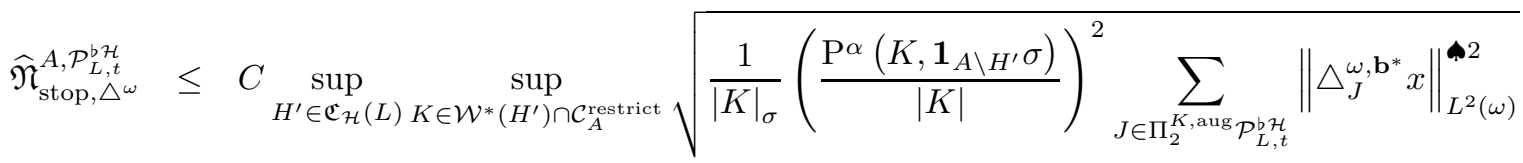

$$
\begin{aligned}
& \leq C \sqrt{\rho^{-(t-2)}} \mathcal{S}_{\text {aug size }}^{\alpha, A}(\mathcal{P})=C^{\prime} \rho^{-\frac{t}{2}} \mathcal{S}_{\text {aug size }}^{\alpha, A}(\mathcal{P}) \text {. }
\end{aligned}
$$

Remark on lack of usual goodness: To prove (7.47), it is essential that the intervals $H^{k+2} \in \mathcal{H}_{k+2}$ at the next indented level down from $H^{k+1} \in \mathfrak{C}_{\mathcal{H}}(L)$ are each contained in one of the Whitney intervals $K \in \mathcal{W}\left(H^{k+1}\right) \cap \mathcal{C}_{A}^{\text {restrict }}$ for some $H^{k+1} \in \mathfrak{C}_{\mathcal{H}}(L)$. And this is the reason we introduced the indented corona - namely so that $3 H^{k+2} \subset H^{k+1}$ for some $H^{k+1} \in \mathfrak{C}_{\mathcal{H}}(L)$, and hence $H^{k+2} \subset K$ for some $K \in \mathcal{W}\left(H^{k+1}\right)$. In the argument of Lacey in Lac], the corresponding intervals were good in the usual sense, and so the above triple property was automatic.

So we begin by fixing $K \in \mathcal{W}^{*}\left(H^{k+1}\right) \cap \mathcal{C}_{A}^{\text {restrict }}$ with $H^{k+1} \in \mathfrak{C}_{\mathcal{H}}(L)$, and noting from the above that each $J \in \Pi_{2}^{K, \operatorname{aug}_{\mathcal{P}}^{\text {b }}}{ }_{L, t}^{\mathcal{H}}$ satisfies

$$
J^{b} \subset H^{k+t} \subset H^{k+t-1} \subset \ldots \subset H^{k+2} \subset K
$$

for $H^{k+j} \in \mathcal{H}_{k+j}$ uniquely determined by $J^{b}$. Thus for $t \geq 2$ we have

$$
\begin{aligned}
& \sum_{J \in \Pi_{2}^{K, \text { aug }}}\left\|\triangle_{\mathcal{P}_{L, t}^{b \mathcal{H}}}^{\omega, \mathbf{b}^{*}} x\right\|_{L^{2}(\omega)}^{\mathbf{\mathbf { p } 2}}=\sum_{H^{k+t} \in \mathcal{H}_{k+t}: H^{k+t} \subset K} \sum_{J \in \Pi_{2}^{K, \text { aug }} \mathcal{P}_{L, t}^{\mathrm{b} \mathcal{H}}: J^{\mathrm{b}} \subset H^{k+t}}\left\|\triangle_{J}^{\omega, \mathbf{b}^{*}} x\right\|_{L^{2}(\omega)}^{\mathbf{\omega} 2} \\
& \leq \sum_{H^{k+t} \in \mathcal{H}_{k+t}: H^{k+t} \subset K} \omega_{b \mathcal{P}}\left(\mathbf{T}\left(H^{k+t}\right)\right) \text {. }
\end{aligned}
$$

In the case $t=2$ we are done since the final sum above is at most $\omega_{b \mathcal{P}}(\mathbf{T}(K))$.

Now suppose $t \geq 3$. In order to obtain geometric gain in $t$, we will apply the stopping criterion (7.36) in the following form,

$$
\sum_{L^{\prime} \in \mathfrak{C}_{\mathcal{L}}\left(L_{0}\right)} \omega_{b \mathcal{P}}\left(\mathbf{T}\left(L^{\prime}\right)\right)=\omega_{b \mathcal{P}}\left(\bigcup_{L^{\prime} \in \mathfrak{C}_{\mathcal{L}}\left(L_{0}\right)} \mathbf{T}\left(L^{\prime}\right)\right) \leq \frac{1}{\rho} \omega_{b \mathcal{P}}\left(\mathbf{T}\left(L_{0}\right)\right), \quad \text { for all } L_{0} \in \mathcal{L},
$$

where we have used the fact that the maximal intervals $L^{\prime}$ in the collection $\bigcup_{\ell=0}^{m-1}\left\{L^{\prime} \in \mathcal{L}_{\ell}: L^{\prime} \subset L_{0}\right\}$ for $L_{0} \in \mathcal{L}_{m}$ (that appears in (7.36) $)$ are precisely the $\mathcal{L}$-children of $L_{0}$ in the tree $\mathcal{L}$ (the intervals $L^{\prime}$ above are strictly contained in $L_{0}$ since $\rho>1$ in $(7.36)$ ), so that

$$
\bigcup_{L^{\prime} \in \Gamma} L^{\prime}=\bigcup_{L^{\prime} \in \mathfrak{C}_{\mathcal{L}}\left(L_{0}\right)} L^{\prime} \text { where } \Gamma \equiv \bigcup_{\ell=0}^{m-1}\left\{L^{\prime} \in \mathcal{L}_{\ell}: L^{\prime} \subset L_{0}\right\}
$$

In order to apply (7.49), we collect the pairwise disjoint intervals $H^{k+t} \in \mathcal{H}_{k+t}$ such that $H^{k+t} \subset H^{k+2} \subset$ $K$, into groups according to which interval $L^{k^{\prime}+t-2} \in \mathcal{G}_{k^{\prime}+t-2}$ they are contained in, where $k^{\prime}=d_{\text {geom }}\left(H^{k+2}\right)$ is the geometric depth of $H^{k+2}$ in the tree $\mathcal{L}$ introduced in (7.37). It follows that each interval $H^{k+t} \in \mathcal{H}_{k+t}$ 
is contained in a unique interval $L^{d_{\text {geom }}\left(H^{k+2}\right)+t-2} \in \mathcal{G}_{d_{\text {geom }}\left(H^{k+2}\right)+t-2}$. Thus we obtain from the previous inequality that

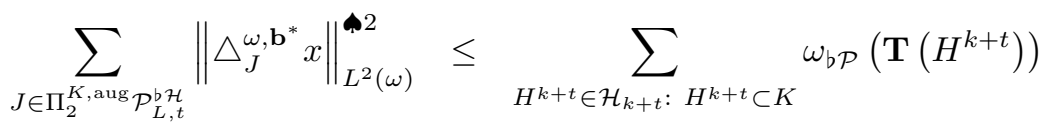

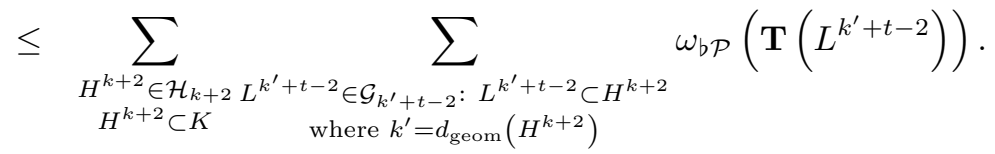

In the case $t=2$ we are done since the final sum above is dominated by

$$
\sum_{H^{k+2} \in \mathcal{H}_{k+2}: H^{k+2} \subset K} \omega_{\mathrm{b}} \mathcal{P}\left(\mathbf{T}\left(H^{k+2}\right)\right) \leq \omega_{\mathrm{b}} \mathcal{P}(\mathbf{T}(K)) .
$$

For $t \geq 3$, we have

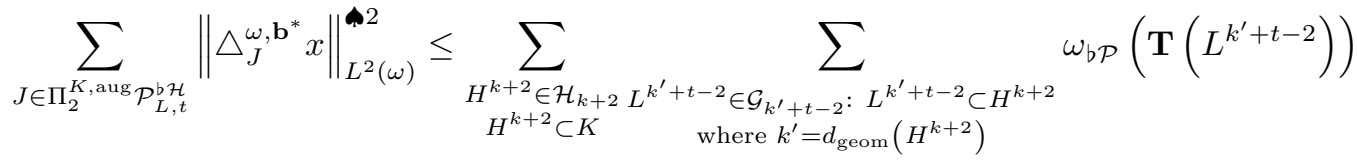

$$
\begin{aligned}
& =\sum_{\substack{H^{k+2} \in \mathcal{H}_{k+2} \\
H^{k+2} \subset K}} \sum_{\substack{L^{k^{\prime}+t-3} \in \mathcal{G}_{k^{\prime}+t-3}: L^{k^{\prime}+t-3} \subset H^{k+2} \\
\text { where } k^{\prime}=d_{\text {geom }}\left(H^{k+2}\right)}}\left\{\sum_{\begin{array}{c}
\sum_{k^{\prime}+t-2} \in \mathcal{G}_{k^{\prime}+t-2}: L^{k^{\prime}+t-2} \subset L^{k^{\prime}+t-3} \\
\text { where } k^{\prime}=d_{\text {geom }}\left(H^{k+2}\right)
\end{array}} \omega_{\mathrm{b} \mathcal{P}}\left(\mathbf{T}\left(L^{k^{\prime}+t-2}\right)\right)\right\} \\
& \leq \sum_{\substack{H^{k+2} \in \mathcal{H}_{k+2} \\
H^{k+2} \subset K}} \sum_{\substack{L^{k^{\prime}+t-3} \in \mathcal{G}_{k^{\prime}+t-3}: L^{k^{\prime}+t-3} \subset H^{k+2} \\
\text { where } k^{\prime}=d_{\text {geom }}\left(H^{k+2}\right)}}\left\{\frac{1}{\rho} \omega_{b \mathcal{P}}\left(\mathbf{T}\left(L^{k^{\prime}+t-3}\right)\right)\right\},
\end{aligned}
$$

where in the last line we have used (17.49) with $L_{0}=L^{k^{\prime}+t-3}$ on the sum in braces. We then continue (if necessary) with

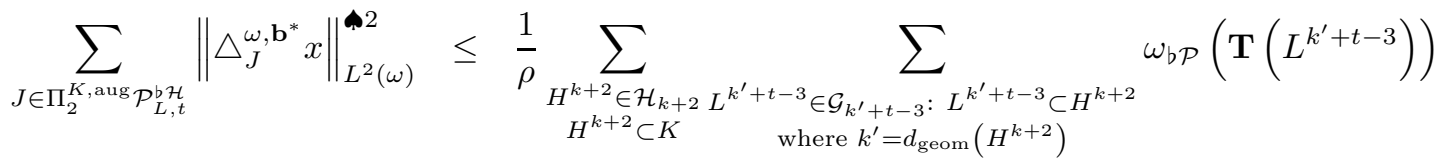

$$
\begin{aligned}
& \leq \frac{1}{\rho^{2}} \sum_{\substack{H^{k+2} \in \mathcal{H}_{k+2} \\
H^{k+2} \subset K}} \sum_{\substack{L^{k^{\prime}+t-4} \in \mathcal{G}_{k^{\prime}+t-4}: L^{k^{\prime}+t-4} \subset H^{k+2} \\
\text { where } k^{\prime}=d_{\text {geom }}\left(H^{k+2}\right)}} \omega_{b \mathcal{P}}\left(\mathbf{T}\left(L^{k^{\prime}+t-4}\right)\right) \\
& \leq \frac{1}{\rho^{t-2}} \sum_{\substack{H^{k+2} \in \mathcal{H}_{k+2} \\
H^{k+2} \subset K}} \sum_{\substack{L^{k^{\prime}} \in \mathcal{G}_{k^{\prime}}: L^{k^{\prime}} \subset H^{k+2} \\
\text { where } \\
k^{\prime}=d_{\text {geom }}\left(H^{k+2}\right)}} \omega_{\text {bP }}\left(\mathbf{T}\left(L^{k^{\prime}}\right)\right) .
\end{aligned}
$$

Since $L^{k^{\prime}} \subset H^{k+2}$ implies $L^{k^{\prime}}=H^{k+2}$, we now obtain

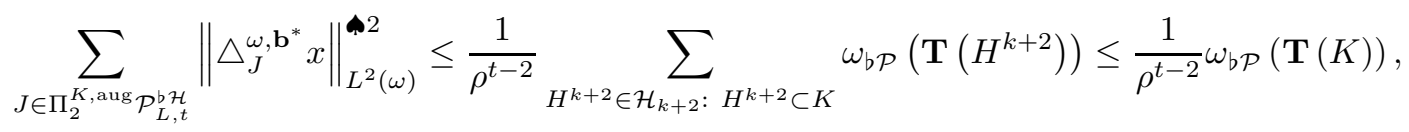

which completes the proof of (7.47), and hence that of (7.48). Finally, an application of the Orthogonality Lemma 68 proves (7.45). 
Proof of the first line in (7.42): At last we turn to proving the first line in (7.42). Recalling that $\mathcal{T}(L)=\mathcal{T}_{\text {left }}(L) \dot{\cup} \mathcal{T}_{\text {right }}(L) \dot{\cup}\{L\}$, we consider the collection

$$
\mathcal{Q}_{0}^{b \mathcal{H}-b i g}=\bigcup_{L \in \mathcal{H}} \mathcal{P}_{L, 0}^{b \mathcal{H}-b i g},
$$

where $\quad \mathcal{P}_{L, 0}^{b \mathcal{H}-b i g}=\left\{(I, J) \in \mathcal{P}_{L, 0}^{b \mathcal{H}}\right.$ : there is $L^{\prime} \in \mathcal{T}(L)$ with $\left.J^{b} \subset L^{\prime} \subset I\right\}, \quad L \in \mathcal{H}$,

$$
\text { and } \quad \mathcal{P}_{L, 0}^{b \mathcal{H}}=\left\{(I, J) \in \mathcal{P}: I \in \mathcal{C}_{L}^{\mathcal{H}} \text { and } J \in \mathcal{C}_{L}^{\mathcal{H}, \text { bhift }} \text { for some } L \in \mathcal{H}\right\}, \quad L \in \mathcal{H},
$$

and begin by claiming that

$$
\widehat{\mathfrak{N}}_{\text {stop }, \triangle \omega}^{A, \mathcal{P}_{L}^{b \mathcal{H}-b i g}} \leq C \mathcal{S}_{\text {aug size }}^{\alpha, A}\left(\mathcal{P}_{L, 0}^{b \mathcal{H}-b i g}\right) \leq C \mathcal{S}_{\text {aug size }}^{\alpha, A}(\mathcal{P}), \quad L \in \mathcal{H}
$$

To see this, we fix $L \in \mathcal{H}$ and order the 'left' tower of intervals $\mathcal{T}_{\text {left }}(L)=\left\{L^{k}\right\}_{k=1}^{\infty}$ that lie in the restricted corona $\mathcal{C}_{L}^{\mathcal{H} \text {,restricted }}$ by decreasing side length, i.e. $\ell\left(L^{k+1}\right) \leq \ell\left(L^{k}\right)$ for all $k \geq 1$, and set $L^{0}=L$ (of course the tower may be finite, but for convenience in notation, we won't reflect this in the notation). The 'right' tower of intervals $\mathcal{T}_{\text {right }}(L)$ is handled similarly and so not considered further here. Then $\mathcal{P}_{L, 0}^{\text {bH }}$-big can be decomposed as follows, remembering that $J^{\mathrm{b}} \subset I \subset L$ for $(I, J) \in \mathcal{P}_{L, 0}^{b \mathcal{H}-b i g} \subset \mathcal{P}_{L, 0}^{b \mathcal{H}}$ :

$$
\begin{aligned}
& \mathcal{P}_{L, 0}^{b \mathcal{H}-b i g}=\bigcup_{k=1}^{\infty}\left\{\mathcal{R}_{L_{\text {left }}^{b}}^{b \mathcal{L}} \dot{\mathcal{L}} \mathcal{R}_{L_{\text {right }}^{b}}^{b \mathcal{L}} \dot{\cup} \mathcal{R}_{L_{\text {disjoint }}^{b \mathcal{L}}}^{k}\right\} \\
& =\left(\bigcup_{k=1}^{\infty} \mathcal{R}_{L_{\text {left }}^{b \mathcal{L}}}^{k}\right) \dot{U}\left(\bigcup_{k=1}^{\infty} \mathcal{R}_{L_{\text {right }}^{b}}^{\mathcal{L}}\right) \dot{\cup}\left(\bigcup_{k=1}^{\infty} \mathcal{R}_{L_{\text {disjoint }}^{b}}^{b \mathcal{L}}\right) ; \\
& \mathcal{R}_{L_{\text {right }}^{b}}^{\text {L }} \equiv\left\{(I, J) \in \mathcal{P}_{L, 0}^{b \mathcal{H}-b i g}: I \in \mathcal{C}_{L^{k-1}}^{\mathcal{L}} \text { and } J^{b} \subset L_{\text {right }}^{k}\right\}, \\
& \mathcal{R}_{L_{\text {left }}^{k}}^{b \mathcal{L}} \equiv\left\{(I, J) \in \mathcal{P}_{L, 0}^{b \mathcal{H}-b i g}: I \in \mathcal{C}_{L^{k-1}}^{\mathcal{L}} \text { and } J^{b} \subset L_{\text {left }}^{k}\right\} \\
& \mathcal{R}_{L_{\text {disjoint }}^{b \mathcal{L}}} \equiv\left\{(I, J) \in \mathcal{P}_{L, 0}^{b \mathcal{H}-b i g}: I \in \mathcal{C}_{L^{k-1}}^{\mathcal{L}} \text { and } J^{b} \in \mathcal{C}_{L^{k-1}}^{\mathcal{L}} \text { and } J^{b} \cap L^{k}=\emptyset\right\} \\
& =\left\{(I, J) \in \mathcal{P}_{L, 0}^{b \mathcal{H}-b i g}: I=L^{k-1} \text { and } J^{b} \in \mathcal{C}_{L^{k-1}}^{\mathcal{L}} \text { and } J^{b} \cap L^{k}=\emptyset\right\} \text {, }
\end{aligned}
$$

and where in the last line we have used the fact that if $I, J^{b} \in \mathcal{C}_{L^{k-1}}^{\mathcal{L}}$ and there is $L^{\prime} \in \mathcal{T}(L)$ with $J^{b} \subset L^{\prime} \subset I$, then we must have $I=L^{k-1}$. All of the pairs $(I, J) \in \mathcal{P}_{L, 0}^{b \mathcal{H}-b i g}$ are included in either $\mathcal{R}_{L_{\text {right }}^{b}}^{b \mathcal{L}}, \mathcal{R}_{L_{\text {left }}^{b}}^{\mathcal{L}}$ or $\mathcal{R}_{L_{\text {disjoint }}^{b \mathcal{L}}}^{\mathcal{L}}$ for some $k$, since if $J^{b} \supset L^{k}$, then $J^{b}$ shares an endpoint with $L$, which contradicts the fact that $3 J^{b} \subset J^{*} \subset I \subset L$.

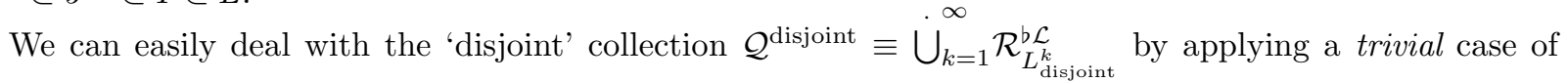
the bStraddling Lemma to $\mathcal{R}_{L_{\text {disjoint }}^{b} \mathcal{L}}^{\mathcal{L}}$ with a single straddling interval, followed by an application of the Orthogonality Lemma to $\mathcal{Q}^{\text {disjoint }}$. More precisely, every pair $(I, J) \in \mathcal{R}_{L_{\text {disjoint }}^{b}}^{\mathcal{L}}$ satisfies $J^{b} \subset L^{k-1}=I$, so that the reduced admissible collection $\mathcal{R}_{L_{\text {disjoint }}^{b} \mathcal{L}}^{b}$ bstraddles the trivial choice $\mathcal{S}=\left\{L^{k-1}\right\}$, the singleton consisting of just the interval $L^{k-1}$. Then the inequality

$$
\widehat{\mathfrak{N}}_{\text {stop }, \triangle \omega}^{A, \mathcal{R}_{L_{\text {disjoint }}^{b}}^{b \mathcal{L}}} \leq C \mathcal{S}_{\text {aug size }}^{\alpha, A}\left(\mathcal{R}_{L_{\text {disjoint }}^{k}}^{b \mathcal{L}}\right)
$$

follows from bStraddling Lemma 80, The collection $\left\{\mathcal{R}_{L_{\text {disjoint }}^{b \mathcal{L}}}\right\}_{k=1}^{\infty}$ is mutually orthogonal since

$$
\begin{aligned}
\mathcal{R}_{L_{\text {disjoint }}^{\mathrm{b}}}^{\mathcal{L}} & \subset \mathcal{C}_{L^{k-1}}^{\mathcal{L}} \times \mathcal{C}_{L^{k-1}}^{\mathcal{L}, \text { b shift }}, \\
\sum_{k=1}^{\infty} \mathbf{1}_{\mathcal{C}_{L^{k-1}}^{\mathcal{L}}} & \leq \mathbf{1} \text { and } \sum_{k=1}^{\infty} \mathbf{1}_{\mathcal{C}_{L^{k-1}}^{\mathcal{L}, \text { bs shift }}} \leq \mathbf{1}
\end{aligned}
$$


Since $\dot{\bigcup}_{k=1}^{\infty} \mathcal{R}_{L_{\text {disjoint }}^{b} \mathcal{L}}^{\text {is }}$ is reduced and admissible (each $J \in \Pi_{2}\left(\dot{\bigcup}_{k=1}^{\infty} \mathcal{R}_{L_{\text {disjoint }}^{b} \mathcal{L}}^{k}\right)$ is paired with a single $I$, namely the top of the $\mathcal{L}$-corona to which $J^{b}$ belongs), the Orthogonality Lemma 68 applies to obtain the estimate

$$
\widehat{\mathfrak{N}}_{\text {stop }, \triangle^{\omega}}^{A, \dot{\cup}_{k=1}^{\infty} \mathcal{R}_{L_{\text {disjoint }}^{b \mathcal{L}}}^{L}}=\widehat{\mathfrak{N}}_{\text {stop }, \triangle^{\omega}}^{A, \mathcal{Q}^{\text {disjoint }}} \leq \sup _{k \geq 1} \widehat{\mathfrak{N}}_{\text {stop }, \triangle \omega}^{A, \mathcal{R}_{L_{\text {disjoint }}^{b \mathcal{L}}}^{k}} \leq C \sup _{k \geq 1} \mathcal{S}_{\text {aug size }}^{\alpha, A}\left(\mathcal{R}_{L_{\text {disjoint }}^{b} \mathcal{L}}^{b}\right) \leq C \mathcal{S}_{\text {aug size }}^{\alpha, A}\left(\mathcal{P}_{L, 0}^{b \mathcal{H}-b i g}\right)
$$

Now we turn to estimating the norm of the 'right' collection $\mathcal{Q}^{\text {right }} \equiv \bigcup_{k=1}^{\infty} \mathcal{R}_{L_{\text {right }}^{b} \mathcal{L}}$. First we note that $L_{\text {right }}^{k} \in \mathcal{C}_{A}^{\mathcal{A} \text {,restrict }}$ if $(I, J) \in \mathcal{R}_{L_{\text {right }}^{b \mathcal{L}}}^{\mathcal{L}}$ since $\mathcal{R}_{L_{\text {right }}^{b \mathcal{L}}}^{\mathcal{L}}$ is reduced, i.e. doesn't contain any pairs $(I, J)$ with $J^{b} \subset A^{\prime}$ for some $A^{\prime} \in \mathfrak{C}_{\mathcal{A}}(A)$. Next we note that $\mathcal{Q}^{\text {right }}$ is admissible since if $J \in \Pi_{2} \mathcal{Q}^{\text {right }}$, then $J \in \Pi_{2} \mathcal{R}_{L_{\text {right }}^{k}}^{\text {b }}$ for a unique index $k$, and of course $\mathcal{R}_{L_{\text {right }}^{b}}^{b}$ is admissible, so that the intervals $I$ that are paired with $J$ are tree-connected. Thus we can apply the Straddling Lemma 80 to the reduced admissible collection $\mathcal{Q}^{\text {right }}$, with the 'straddling' set $\mathcal{S} \equiv\left\{L_{\text {right }}^{k}\right\}_{k=1}^{\infty} \cap \mathcal{C}_{A}^{\mathcal{A} \text {,restrict }}$, to obtain the estimate

$$
\widehat{\mathfrak{N}}_{\text {stop }, \triangle^{\omega}}^{A, \bigcup_{k=1}^{\infty} \mathcal{R}_{L_{\text {right }}^{b \mathcal{L}}}^{L}}=\widehat{\mathfrak{N}}_{\text {stop }, \triangle^{\omega}}^{A, \mathcal{Q}^{\text {right }}} \leq C \mathcal{S}_{\text {aug size }}^{\alpha, A}\left(\mathcal{Q}^{\text {right }}\right) \leq C \mathcal{S}_{\text {aug size }}^{\alpha, A}\left(\mathcal{P}_{L, 0}^{b \mathcal{H}-b i g}\right)
$$

As for the remaining 'left' form $|\mathrm{B}|_{\text {stop, } \triangle^{\omega}}^{A, \bigcup_{k=0}^{\infty} \mathcal{R}_{L_{\text {left }}^{b} \mathcal{L}}^{k}}(f, g)$, if the interval pair $(I, J) \in \mathcal{R}_{L_{\text {left }}^{b}}^{b \mathcal{L}}$, then either $J^{b} \subset L_{\text {left }}^{k} \varsubsetneqq J^{*}$ or $J^{*} \subset L_{\text {left }}^{k}$. But $J^{b} \subset L_{\text {left }}^{k} \varsubsetneqq J^{*}$ implies that either $J^{b}=L_{\text {left }}^{k} \varsubsetneqq J^{*} \subset I \subset L$, which is impossible since $J^{b}$ cannot share an endpoint with $L$, or that $J^{b}=L_{-/+}^{k}=\left(L_{\text {left }}^{k}\right)_{\text {right }}$ and $J^{\natural}=L^{k}$. So we conclude that if $(I, J) \in \mathcal{R}_{L_{\text {left }}^{b}}^{\text {L }}$, then

$$
\text { either } J^{\mathbf{W}} \subset L_{\text {left }}^{k} \text { or " } J^{*}=L^{k} \text { and } J \subset L_{\text {left }}^{k} \text {. }
$$

In either case in (7.53), there is a unique interval $K=K[J] \in \mathcal{W}(L)$ that contains $J$. It follows that there are now two remaining cases:

Case 1: $K[J] \in \mathcal{C}_{A}^{\text {restrict }}$,

Case 2: $K[J] \subset A^{\prime} \varsubsetneqq I$ for some $A^{\prime} \in \mathfrak{C}_{\mathcal{A}}(A)$.

However, by Key Fact \#2 in (7.19), and the fact that the great grandparent $\pi_{\mathcal{D}}^{(3)} A^{\prime}$ of $A^{\prime}$ contains $A^{\prime}$ inside its leftmost grandchild $\left(\pi_{\mathcal{D}}^{(3)} A^{\prime}\right)_{-/-}$, the pairs $(I, J)$ in Case 2 lie in the 'corona straddling' collection $P_{\text {cor }}^{A}$ that was removed from all $A$-admissible collections in (7.31) of Conclusion 83 above, and thus there are no pairs in Case 2 here. (We note in passing that a given $A^{\prime} \in \mathfrak{C}_{A}(A)$ can occur as one of the Whitney intervals $K$ in $\mathcal{W}(L)$ for at most one $L \in \mathcal{H}$, the indented corona.) Thus we conclude that $K[J] \in \mathcal{C}_{A}^{\text {restrict }}$.

We now claim that $3 K[J] \subset I$ for all pairs $(I, J) \in \bigcup_{k=1}^{\infty} \mathcal{R}_{L_{\text {left }}^{b}}^{\mathcal{L}}$. To see this, suppose that $(I, J) \in \mathcal{R}_{L_{\text {left }}^{k}}^{b \mathcal{L}}$ for some $k \geq 1$. Then by (7.53) we have both that $K[J] \subset L_{\text {left }}^{k}$ and $L^{k} \varsubsetneqq I$. But then $K[J] \subset L_{\text {left }}^{k}$ implies that $3 K[J] \subset L^{k} \subset I$ as claimed. See Figure 3 .

Now the 'left' collection $\mathcal{Q}^{\text {left }} \equiv \bigcup_{k=1}^{\infty} \mathcal{R}_{L_{\text {left }}^{b}}^{b \mathcal{L}}$ is admissible, since if $J \in \Pi_{2} \mathcal{Q}^{\text {left }}$ and $I_{j} \in \Pi_{1} \mathcal{Q}^{\text {left }}$ with $\left(I_{j}, J\right) \in \mathcal{Q}^{\text {left }}$ for $j=1,2$, then $I_{j} \in \mathcal{C}_{L^{k_{j}-1}}^{\mathcal{L}}$ for some $k_{j}$ and all of the intervals $I \in\left[I_{1}, I_{2}\right]$ lie in one of the coronas $\mathcal{C}_{L^{k-1}}^{\mathcal{L}}$ for $k$ between $k_{1}$ and $k_{2}$. Thus $(I, J) \in \mathcal{R}_{L_{\text {left }}^{b}}^{\mathcal{L}} \subset \mathcal{Q}^{\text {left }}$, and we have proved the required connectedness. From the containment $3 K[J] \subset I \subset L$ for all $(I, J) \in \bigcup_{k=1}^{\infty} \mathcal{R}_{L_{\text {left }}^{k}}^{\mathcal{L}}$, we now see that the reduced admissible collection $\mathcal{Q}^{\text {left }}$ substraddles the interval $L$. Hence the Substraddling Lemma 82 yields the bound

$$
\widehat{\mathfrak{N}}_{\text {stop }, \triangle^{\omega}}^{A, \bigcup_{k=1}^{\infty} \mathcal{R}_{L_{\text {left }}^{\text {b }} \mathcal{L}}^{k}}=\widehat{\mathfrak{N}}_{\text {stop }, \triangle \omega}^{A, \mathcal{Q}^{\text {left }}} \leq C \mathcal{S}_{\text {aug size }}^{\alpha, A}\left(\mathcal{Q}^{\text {left }}\right) \leq C \mathcal{S}_{\text {aug size }}^{\alpha, A}\left(\mathcal{P}_{L, 0}^{b \mathcal{H}-b i g}\right)
$$

Combining the bounds (7.51), (7.52) and (7.54), we obtain (7.50). 


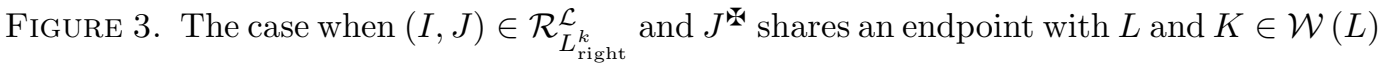
equals $J_{-/+}^{\text {出 }}$. In this case $3 K \subset I$.

Finally, we observe that the collections $\mathcal{P}_{L, 0}^{b \mathcal{H}-\text { big }}$ themselves are mutually orthogonal, namely

$$
\begin{aligned}
& \mathcal{P}_{L, 0}^{b \mathcal{H}-b i g} \subset \mathcal{C}_{L}^{\mathcal{H}} \times \mathcal{C}_{L}^{\mathcal{H}, b \text { shift }}, \quad L \in \mathcal{H}, \\
& \sum_{L \in \mathcal{H}} \mathbf{1}_{\mathcal{C}_{L}^{\mathcal{H}}} \leq \mathbf{1} \text { and } \sum_{L \in \mathcal{H}} \mathbf{1}_{\mathcal{C}_{L}^{\mathcal{H}, b \text { shift }}} \leq \mathbf{1} .
\end{aligned}
$$

Thus an application of the Orthogonality Lemma 68 shows that

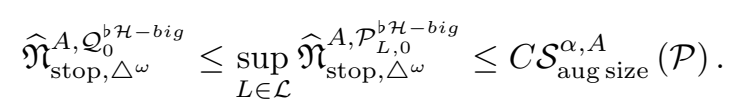

Altogether, the proof of Proposition 85] is now complete.

This finishes the proofs of the inequalities (7.10) and (7.1).

\section{Wrapup of THE PROOF}

At this point we have controlled, either directly or probabilistically, the norms of all of the forms in our decompositions - namely the disjoint, nearby, far below, paraproduct, neighbour, broken and stopping forms - in terms of the Muckenhoupt, energy and functional energy conditions, along with an arbitrarily small multiple of the operator norm. Thus it only remains to control the functional energy condition by the Muckenhoupt and energy conditions, since then, using $\int\left(T_{\sigma}^{\alpha} f\right) g d \omega=\Theta(f, g)+\Theta^{*}(f, g)$ with the further decompositions above, we will have shown that for any fixed tangent line truncation of the operator $T_{\sigma}^{\alpha}$, as defined in Definition 2, we have

$$
\begin{gathered}
\left|\int\left(T_{\sigma}^{\alpha} f\right) g d \omega\right|=\boldsymbol{E}_{\Omega}^{\mathcal{D}} \boldsymbol{E}_{\Omega}^{\mathcal{G}}\left|\int\left(T_{\sigma}^{\alpha} f\right) g d \omega\right| \leq \boldsymbol{E}_{\Omega}^{\mathcal{D}} \boldsymbol{E}_{\Omega}^{\mathcal{G}} \sum_{i=1}^{3}\left(\left|\Theta_{i}(f, g)\right|+\left|\Theta_{i}^{*}(f, g)\right|\right) \\
\leq\left(C_{\eta} \mathcal{N} \mathcal{T} \mathcal{V}_{\alpha}+\eta \mathfrak{N}_{T^{\alpha}}\right)\|f\|_{L^{2}(\sigma)}\|g\|_{L^{2}(\omega)}, \quad f \in L^{2}(\sigma) \text { and } g \in L^{2}(\omega)
\end{gathered}
$$

for an arbitarily small positive constant $\eta>0$, and a correspondingly large finite constant $C_{\eta}$. Note that the testing constants $\mathfrak{T}_{T^{\alpha}}$ and $\mathfrak{T}_{T^{\alpha, *}}$ in $\mathcal{N} \mathcal{T} \mathcal{V}_{\alpha}$ already include the supremum over all tangent line truncations of $T^{\alpha}$, while the operator norm $\mathfrak{N}_{T^{\alpha}}$ on the left refers to a fixed tangent line truncation of $T^{\alpha}$. This gives

$$
\mathfrak{N}_{T^{\alpha}}=\sup _{\|f\|_{L^{2}(\sigma)}=1} \sup _{\|g\|_{L^{2}(\omega)}=1}\left|\int\left(T_{\sigma}^{\alpha} f\right) g d \omega\right| \leq C_{\eta} \mathcal{N} \mathcal{T} \mathcal{V}_{\alpha}+\eta \mathfrak{N}_{T^{\alpha}},
$$


and since the truncated operators have finite operator norm $\mathfrak{N}_{T^{\alpha}}$, we can absorb the term $\eta \mathfrak{N}_{T^{\alpha}}$ into the left hand side for $\eta<1$ and obtain $\mathfrak{N}_{T^{\alpha}} \leq C_{\eta}^{\prime} \mathcal{N} \mathcal{T} \mathcal{V}_{\alpha}$ for each tangent line truncation of $T^{\alpha}$. Taking the supremum over all such truncations of $T^{\alpha}$ finishes the proof of Theorem 7 .

The task of controlling functional energy is taken up in Appendix B, after first establishing weak frame and weak Riesz inequalities for martingale and dual martingale differences in Appendix A (except for the lower weak Riesz inequality for the martingale difference $\triangle_{Q}^{\mu, \mathbf{b}}$ ).

\section{Appendix A: Martingale differences}

Most of the material in this appendix is known, see e.g. [NTV3 and HyMa. First, we recall the construction in SaShUr9] of a Haar basis in $\mathbb{R}$ that is adapted to a measure $\mu$ (c.f. NTV2] where this type of construction is made explicit). Given a dyadic interval $Q \in \mathcal{D}$, where $\mathcal{D}$ is a dyadic grid of intervals from $\mathcal{P}$, let $\triangle_{Q}^{\mu}$ denote orthogonal projection onto the one-dimensional subspace $L_{Q}^{2}(\mu)$ of $L^{2}(\mu)$ that consists of linear combinations of the indicators of the children $\mathfrak{C}(Q)$ of $Q$ that have $\mu$-mean zero over $Q$ :

$$
L_{Q}^{2}(\mu) \equiv\left\{f=\sum_{Q^{\prime} \in \mathfrak{C}(Q)} a_{Q^{\prime}} \mathbf{1}_{Q^{\prime}}: a_{Q^{\prime}} \in \mathbb{R}, \int_{Q} f d \mu=0\right\} .
$$

Then we have the important telescoping property for dyadic intervals $Q_{1} \subset Q_{2}$ :

$$
\mathbf{1}_{Q_{0}}(x)\left(\sum_{Q \in\left[Q_{1}, Q_{2}\right]} \triangle_{Q}^{\mu} f(x)\right)=\mathbf{1}_{Q_{0}}(x)\left(\mathbb{E}_{Q_{0}}^{\mu} f-\mathbb{E}_{Q_{2}}^{\mu} f\right), \quad Q_{0} \in \mathfrak{C}\left(Q_{1}\right), f \in L^{2}(\mu),
$$

Notation 87. Here $\mathbb{E}_{Q}^{\mu} f(x) \equiv \mathbf{1}_{Q}(x) \frac{1}{|Q|_{\mu}} \int_{Q} f d \mu$ denotes the projection of $f$ onto $\operatorname{Span}\left\{\mathbf{1}_{Q}\right\}$, the onedimensional subspace of multiples of the indicator of $Q$. We will also denote the average value itself by $E_{Q}^{\mu} f \equiv \frac{1}{|Q|_{\mu}} \int_{Q} f d \mu$

It is convenient at times to use a fixed normalized basis $\left\{h_{Q}^{\mu}\right\}$ of $L_{Q}^{2}(\mu)$. Then $\left\{h_{Q}^{\mu}\right\}_{Q \in \mathcal{D}}$ is an orthonormal basis for $L^{2}(\mu)$, with the understanding that we add the constant function $\mathbf{1}$ if $\mu$ is a finite measure. In particular we have

$$
\|f\|_{L^{2}(\mu)}^{2}=\sum_{Q \in \mathcal{D}}\left\|\triangle_{Q}^{\mu} f\right\|_{L^{2}(\mu)}^{2}=\sum_{Q \in \mathcal{D}}|\widehat{f}(Q)|^{2}, \quad \widehat{f}(Q) \equiv\left\langle f, h_{Q}^{\mu}\right\rangle_{\mu},
$$

where the measure is suppressed in the notation $\widehat{f}$. Indeed, this follows from (9.1) and Lebesgue's differentiation theorem for dyadic intervals. We also record the following useful estimate. If $I^{\prime}$ is either of the two $\mathcal{D}$-children of $I$, then

$$
\left|\mathbb{E}_{I^{\prime}}^{\mu} h_{I}^{\mu}\right| \leq \sqrt{\mathbb{E}_{I^{\prime}}^{\mu}\left(h_{I}^{\mu}\right)^{2}} \leq \frac{1}{\sqrt{\left|I^{\prime}\right|_{\mu}}} .
$$

In the next subsection, we introduce martingale and dual martingale differences for various $p$-weakly $\mu$ accretive families $\mathbf{b}=\left\{b_{Q}\right\}_{Q \in \mathcal{P}}$, and establish convergence properties for their expansions. Then in later subsections, we turn to frame inequalities, and weak Riesz inequalities.

9.1. Convergence for weakly and controlled accretive families. Supposes that $\mathbf{b}=\left\{b_{Q}\right\}_{Q \in \mathcal{P}}$ is a $p$ weakly $\mu$-accretive family on $\mathbb{R}$. Define the $\mathbf{b}$-expectation operator $\mathbb{E}_{Q}^{\mu, \mathbf{b}}$ and the dual b-expectation operator $\mathbb{F}_{Q}^{\mu, \mathbf{b}}$ by

$$
\begin{aligned}
\mathbb{E}_{Q}^{\mu, \mathbf{b}} f(x) & \equiv \mathbf{1}_{Q}(x) \frac{1}{\int_{Q} b_{Q} d \mu} \int_{Q} f b_{Q} d \mu, \quad Q \in \mathcal{P}, \\
\mathbb{F}_{Q}^{\mu, \mathbf{b}} f(x) & \equiv \mathbf{1}_{Q}(x) b_{Q}(x) \frac{1}{\int_{Q} b_{Q} d \mu} \int_{Q} f d \mu, \quad Q \in \mathcal{P} .
\end{aligned}
$$


Occasionally we will use the modification of $\mathbb{F}_{Q}^{\mu, \mathbf{b}}$ given by 'dividing out' the factor $b_{Q}$ :

$$
\widehat{\mathbb{F}}_{Q}^{\mu, \mathbf{b}} f(x) \equiv \mathbf{1}_{Q}(x) \frac{1}{\int_{Q} b_{Q} d \mu} \int_{Q} f d \mu, \quad Q \in \mathcal{P} .
$$

Then define the corresponding martingale and dual martingale differences by

$$
\begin{aligned}
\triangle_{Q}^{\mu, \mathbf{b}} f(x) & \equiv\left(\sum_{Q^{\prime} \in \mathfrak{C}(Q)} \mathbb{E}_{Q^{\prime}}^{\mu, \mathbf{b}} f(x)\right)-\mathbb{E}_{Q}^{\mu, \mathbf{b}} f(x)=\sum_{Q^{\prime} \in \mathfrak{C}(Q)} \mathbf{1}_{Q^{\prime}}(x)\left(\mathbb{E}_{Q^{\prime}}^{\mu, \mathbf{b}} f(x)-\mathbb{E}_{Q}^{\mu, \mathbf{b}} f(x)\right), \\
\square_{Q}^{\mu, \mathbf{b}} f(x) & \equiv\left(\sum_{Q^{\prime} \in \mathfrak{C}(Q)} \mathbb{F}_{Q^{\prime}}^{\mu, \mathbf{b}} f(x)\right)-\mathbb{F}_{Q}^{\mu, \mathbf{b}} f(x)=\sum_{Q^{\prime} \in \mathfrak{C}(Q)} \mathbf{1}_{Q^{\prime}}(x)\left(\mathbb{F}_{Q^{\prime}}^{\mu, \mathbf{b}} f(x)-\mathbb{F}_{Q}^{\mu, \mathbf{b}} f(x)\right) .
\end{aligned}
$$

Exception: In the special case that $\mathfrak{C}(Q)=\left\{Q_{1}, Q_{2}\right\}$ where $\left|Q_{1}\right|_{\mu}=0$ and $|Q|_{\mu}>0$, we set both $\triangle_{Q}^{\mu, \mathbf{b}} \equiv 0$ and $\square_{Q}^{\mu, \mathbf{b}} \equiv 0$, and redefine the parent differences $\triangle_{\pi Q}^{\mu, \mathbf{b}}$ and $\square_{\pi Q}^{\mu, \mathbf{b}}$ as follows. Let $\mathfrak{C}(\pi Q)=$ $\{Q, \widetilde{Q}\}$ and set

$$
\begin{aligned}
& \triangle_{\pi Q}^{\mu, \mathbf{b}} f(x) \equiv\left(\mathbb{E}_{Q}^{\mu, b_{Q_{2}}} f(x)+\mathbb{E}_{\widetilde{Q}}^{\mu, \mathbf{b}} f(x)\right)-\mathbb{E}_{\pi Q}^{\mu, \mathbf{b}} f(x), \\
& \square_{\pi Q}^{\mu, \mathbf{b}} f(x) \equiv\left(\mathbb{F}_{Q}^{\mu, b_{Q_{2}}} f(x)+\mathbb{F}_{\widetilde{Q}}^{\mu, \mathbf{b}} f(x)\right)-\mathbb{F}_{\pi Q}^{\mu, \mathbf{b}} f(x),
\end{aligned}
$$

where we have used the test function $b_{Q_{2}}$ in place of the expected $b_{Q}$ (because $\left|Q_{1}\right|_{\mu}=0$ ). With the analogous modification when only one grandchild at level $k$ below $Q$ is charged by $\mu$, the telescoping property holds for these differences, and the reader can easily verify all of the convergence statements and formulas below. For the sake of convenience only, we will ignore these exceptions in the sequel, and proceed under the assumption that all intervals are charged by $\mu$.

Note that in NTV3] and HyMa this notation is reversed - they use $\triangle_{Q}^{\mu, \mathbf{b}}$ for our $\square_{Q}^{\mu, \mathbf{b}}$. Finally, define the dual b-expectation operator $\mathbb{F}_{m}^{\mu, \mathbf{b}}$ on a function $f$ at level $m$ by

$$
\mathbb{F}_{m}^{\mu, \mathbf{b}} f(x) \equiv \sum_{Q \in \mathcal{D}_{m}} \mathbb{F}_{Q}^{\mu, \mathbf{b}} f(x)=\sum_{Q \in \mathcal{D}_{m}} \mathbf{1}_{Q}(x) b_{Q}(x) \frac{1}{\int_{Q} b_{Q} d \mu} \int_{Q} f d \mu,
$$

where $\mathcal{D}_{m} \equiv\left\{Q \in \mathcal{D}: \ell(Q)=2^{-m}\right\}$, and define the operators $\square_{m}^{\mu, \mathbf{b}}$ by

$$
\square_{m}^{\mu, \mathbf{b}} \equiv \mathbb{F}_{m}^{\mu, \mathbf{b}}-\mathbb{F}_{m-1}^{\mu, \mathbf{b}}
$$

Definition 88. Let $\mu$ be a locally finite positive Borel measure on $\mathbb{R}$, let $\mathbf{b}=\left\{b_{Q}\right\}_{Q \in \mathcal{P}}$ be a p-weakly $\mu$ accretive family on $\mathbb{R}$ with reverse Hölder control 2.15) on children, and let $\mathcal{A}$ be subset of a dyadic grid $\mathcal{D}$. We say that the subfamily $\mathbf{b}=\left\{b_{Q}\right\}_{Q \in \mathcal{D}}$ is a p-weakly $\mu$-controlled accretive family on $\mathcal{D}$ (note we omit dependence $\mathcal{A}$ on in this notation) if

$$
b_{Q}=\mathbf{1}_{Q} b_{A}, \quad Q \in \mathcal{C}_{A}, A \in \mathcal{A},
$$

and the set $\mathcal{A}$ satisfies a Carleson condition:

$$
\sum_{Q \in \mathcal{A}: Q \subset K}|Q|_{\mu} \leq C|K|_{\mu}, \quad \text { for all } K \in \mathcal{D},
$$

equivalently

$$
\sum_{Q \in \mathcal{A}: Q \subset \Omega}|Q|_{\mu} \leq C^{\prime}|\Omega|_{\mu}, \quad \text { for all open sets } \Omega \subset \mathbb{R} .
$$

Denote the coronas associated to $\mathcal{A}$ by $\left\{\mathcal{C}_{A}\right\}_{A \in \mathcal{A}}$, and now suppose that $\mathbf{b}=\left\{b_{Q}\right\}_{Q \in \mathcal{P}}$ is $p$-weakly $\mu$ controlled accretive, i.e.

$$
0<1 \leq\left|\frac{1}{|Q|_{\mu}} \int_{Q} b_{A} d \mu\right| \leq\left\|b_{A}\right\|_{L^{\infty}(\mu)} \leq C_{\mathbf{b}}<\infty, \quad Q \in \mathcal{C}_{A}, A \in \mathcal{A}
$$


where in addition we have reverse Hölder control (2.15) on children, and $\mathcal{A}$ satisfies a $\mu$-Carleson condition. Now decompose the children $Q^{\prime} \in \mathfrak{C}(Q)$ into the collection $\mathfrak{C}_{\text {broken }}(Q)$ of broken children $Q^{\prime} \in \mathcal{A}$ and the remaining collection $\mathfrak{C}_{\text {natural }}(Q)$ of natural children $Q^{\prime} \notin \mathcal{A}$, i.e.

$$
\mathfrak{C}_{\text {broken }}(Q) \equiv \mathfrak{C}(Q) \cap \mathcal{A} \text { and } \mathfrak{C}_{\text {natural }}(Q) \equiv \mathfrak{C}(Q) \backslash \mathcal{A}
$$

Let $\left\{\square_{I}^{\mu, \mathbf{b}}\right\}_{I \in \mathcal{D}}$ be associated to the coronas $\left\{\mathcal{C}_{A}\right\}_{A \in \mathcal{A}}$ and the $p$-weakly $\mu$-controlled accretive family $\mathbf{b}=$ $\left\{b_{A}\right\}_{A \in \mathcal{A}}$ as in Definition 88 above. We will refer to the collection of dual martingale differences $\left\{\square_{I}^{\mu, \mathbf{b}}\right\}_{I \in \mathcal{D}}$ as a 'broken corona decomposition' in light of the fact that the testing functions $b_{Q}$ 'break' when passing from one corona to another. For $A \in \mathcal{A}$, define the corona 'pseudoprojections' $\mathrm{P}_{\mathcal{C}_{A}}^{\mu, \mathbf{b}}$ by

$$
\mathrm{P}_{\mathcal{C}_{A}}^{\mu, \mathbf{b}} f=\sum_{I \in \mathcal{C}_{A}} \square_{I}^{\mu, \mathbf{b}} f
$$

We have the broken corona decomposition,

$$
f=\sum_{I \in \mathcal{D}} \square_{I}^{\mu, \mathbf{b}} f=\sum_{A \in \mathcal{A}} \mathrm{P}_{\mathcal{C}_{A}}^{\mu, \mathbf{b}} f
$$

whose convergence properties we investigate in the next subsubsection.

9.1.1. Convergence of controlled martingale differences. As shown by Hytönen and Martikainen HyMa, in the setting of a 2 -weakly $\mu$-controlled accretive family, we have strong convergence in $L^{2}(\mu)$ for the dual martingale differences - and also for the martingale differences under the stronger assumption of a $p$-weakly $\mu$-controlled accretive family for some $p>2$ (the proofs given there carry over to general measures). We only use the case $p=\infty$.

Lemma 89. (NTV3, HyMa, Lemma 3.5]) Suppose $\mathbf{b}$ is an $\infty$-weakly $\mu$-controlled accretive family on a grid $\mathcal{D}$. Then we have the dual martingale and martingale identities

$$
\begin{aligned}
f & =\sum_{I \in \mathcal{D}_{N}} \mathbb{F}_{I}^{\mu, \mathbf{b}} f+\sum_{I \in \mathcal{D}: \ell(I) \geq N+1} \square_{I}^{\mu, \mathbf{b}} f, \quad N \in \mathbb{Z}, \\
f & =\sum_{I \in \mathcal{D}_{N}} \mathbb{E}_{I}^{\mu, \mathbf{b}} f+\sum_{I \in \mathcal{D}: \ell(I) \geq N+1} \triangle_{I}^{\mu, \mathbf{b}} f, \quad N \in \mathbb{Z},
\end{aligned}
$$

in the sense of pointwise $\mu$-almost everywhere convergence, and also in the sense of strong convergence in $L^{2}(\mu)$.

9.2. Frame inequalities. Define the positive sublinear operators

$$
\begin{aligned}
\nabla_{Q}^{\mu} f & \equiv \sum_{Q^{\prime} \in \mathfrak{C}_{\text {broken }}(Q)}\left(\frac{1}{\left|Q^{\prime}\right|_{\mu}} \int_{Q^{\prime}}|f| d \mu\right) \mathbf{1}_{Q^{\prime}} \\
\hat{\nabla}_{Q}^{\mu} f & \equiv \sum_{Q^{\prime} \in \mathfrak{C}_{\text {broken }}(Q)}\left(\frac{1}{\left|Q^{\prime}\right|_{\mu}} \int_{Q^{\prime}}|f| d \mu+\frac{1}{|Q|_{\mu}} \int_{Q}|f| d \mu\right) \mathbf{1}_{Q^{\prime}}
\end{aligned}
$$

where we are suppressing here the dependence of both $\nabla_{Q}^{\mu}$ and its larger version $\widehat{\nabla}_{Q}^{\mu}$ on the breaking intervals. Note also that $\widehat{\nabla}_{Q}^{\mu}=0$ if $Q$ has no broken children. We also set $Q_{\text {broken }} \equiv \bigcup_{Q^{\prime} \in \mathfrak{C}_{\text {broken }}(Q)} Q^{\prime}$. We now show that the Carleson condition on broken children gives the inequality

$$
\sum_{Q \in \mathcal{D}}\left\|\hat{\nabla}_{Q}^{\mu} f\right\|_{L^{2}(\mu)}^{2} \lesssim\|f\|_{L^{2}(\mu)}^{2} .
$$


Indeed,

$$
\begin{aligned}
\sum_{Q \in \mathcal{D}}\left\|\hat{\nabla}_{Q}^{\mu} f\right\|_{L^{2}(\mu)}^{2} & =\sum_{Q \in \mathcal{D}} \int \sum_{Q^{\prime} \in \mathfrak{C}_{\text {broken }}(Q)} \mathbf{1}_{Q^{\prime}}\left(\frac{1}{\left|Q^{\prime}\right|_{\mu}} \int_{Q^{\prime}}|f| d \mu+\frac{1}{|Q|_{\mu}} \int_{Q}|f| d \mu\right)^{2} d \mu \\
& \lesssim \sum_{Q \in \mathcal{D}} \sum_{Q^{\prime} \in \mathfrak{C}_{\text {broken }(Q)}\left|Q^{\prime}\right|_{\mu}\left(\frac{1}{\left|Q^{\prime}\right|_{\mu}} \int_{Q^{\prime}}|f| d \mu+\frac{1}{|Q|_{\mu}} \int_{Q}|f| d \mu\right)^{2}} \\
& \lesssim \sum_{A \in \mathcal{A}}|A|_{\mu}\left(\frac{1}{|A|_{\mu}} \int_{A}|f| d \mu\right)^{2}+\sum_{A \in \mathcal{A}}|A|_{\mu}\left(\frac{1}{\left|\pi_{\mathcal{D}} A\right|_{\mu}} \int_{\pi_{\mathcal{D}} A}|f| d \mu\right)^{2}
\end{aligned}
$$

where $\mathcal{A}$ is a collection of stopping times that satisfy a Carleson condition as in Definition 88 . The first term in the last line above is at most $C\|f\|_{L^{2}(\mu)}^{2}$ by the Carleson embedding theorem. We claim that the second term is controlled by a variant of the Carleson embedding theorem,

$$
\sum_{A \in \mathcal{A}}|A|_{\mu}\left(\frac{1}{\left|\pi_{\mathcal{D}} A\right|_{\mu}} \int_{\pi_{\mathcal{D}} A}|f| d \mu\right)^{2} \lesssim \int|f|^{2} d \mu, \quad f \in L^{2}(\mu) .
$$

Indeed, with the measure $\nu(A) \equiv|A|_{\mu}$ on $\mathcal{A}$, the sublinear map $T$ defined by $T f(A) \equiv \frac{1}{\left|\pi_{\mathcal{D}} A\right|_{\mu}} \int_{\pi_{\mathcal{D}} A}|f| d \mu$ takes $L^{\infty}(\mu)$ to $L^{\infty}(\nu)$, and also $L^{1}(\mu)$ to $L^{1, \infty}(\nu)$, since if $\{M\}$ are the maximal dyadic intervals $\pi_{\mathcal{D}} A$ such that

$$
\frac{1}{\left|\pi_{\mathcal{D}} A\right|_{\mu}} \int_{\pi_{\mathcal{D}} A}|f| d \mu>\lambda>0
$$

then

$$
\begin{aligned}
|\{A \in \mathcal{A}: \operatorname{Tf}(A)>\lambda\}|_{\nu} & =\sum_{A: T f(A)>\lambda}|A|_{\mu} \leq \sum_{M} \sum_{A: A \subset M}|A|_{\mu} \lesssim \sum_{M}|M|_{\mu} \\
& <\sum_{M} \frac{1}{\lambda} \int_{M}|f| d \mu \leq \frac{1}{\lambda} \int|f| d \mu,
\end{aligned}
$$

since the maximal intervals $M$ are pairwise disjoint. Now interpolation shows that $T$ takes $L^{2}(\mu)$ to $L^{2}(\nu)$, which is (9.8). These inequalities provide the reason for referring to $\nabla_{Q}^{\mu}$ and $\widehat{\nabla}_{Q}^{\mu}$ as Carleson averaging operators.

From NTV3] we have that in the case $\mathbf{b}$ is an $\infty$-weakly $\mu$-controlled accretive family on a grid $\mathcal{D}$, and the underlying measure $\mu$ is upper doubling, then the following frame equivalences hold:

$$
\begin{aligned}
& \|f\|_{L^{2}(\mu)}^{2} \approx \sum_{Q \in \mathcal{D}}\left\{\left\|\square_{Q}^{\mu, \mathbf{b}} f\right\|_{L^{2}(\mu)}^{2}+\left\|\nabla_{Q}^{\mu} f\right\|_{L^{2}(\mu)}^{2}\right\} \\
& \approx \sum_{Q \in \mathcal{D}}\left\{\left\|\triangle_{Q}^{\mu, \mathbf{b}} f\right\|_{L^{2}(\mu)}^{2}+\left\|\nabla_{Q}^{\mu} f\right\|_{L^{2}(\mu)}^{2}\right\} \text {. }
\end{aligned}
$$

It appears however that the arguments in NTV3 hold for more general measures9, and in any case, we will extend most of these frame inequalities to certain of the weak Riesz inequalities below for arbitrary positive Borel measures $\mu$. For this it will be convenient to refer directly to the proofs of the frame inequalities, and since notation in [NTV3] is very different from that used in this paper, we will instead extend a similar argument of Hytönen and Martikainen [HyMa, Proposition 3.10] to general measures, adding a small additional argument for the martingale differences in term $I I I_{A}$ in the proof of Proposition 91 below. This uses the following unweighted square function estimate, which is essentially just the orthogonality of the standard Haar projections $\triangle_{I}^{\mu}$ adapted to the measure $\mu$. For this, we recall the general Haar projections $\mathrm{P}_{\mathcal{C}_{A}}^{\mu} h=\sum_{Q \in \mathcal{C}_{A}} \triangle_{Q}^{\mu} h$

\footnotetext{
${ }^{9}$ But in NTV3, a proof of their inequality (3.3), which is our lower frame inequality for $\triangle_{Q}^{\mu, \mathbf{b}}$, seems not to be explicitly given.
} 


\section{Lemma 90.}

$$
\sum_{A \in \mathcal{A}} \sum_{Q \in \mathcal{C}_{A}} \sum_{Q^{\prime} \in \mathfrak{C}_{\text {natural }}(Q)}\left|Q^{\prime}\right|_{\mu}\left|E_{Q^{\prime}}^{\mu} h-E_{Q}^{\mu} h\right|^{2} \lesssim\|h\|_{L^{2}(\mu)}^{2}, \quad h \in L^{2}(\mu) .
$$

Proof. Recall that the Haar projection $\triangle_{I}^{\mu}$ is given by

$$
\triangle_{I}^{\mu} h=\left(\sum_{I^{\prime} \in \mathfrak{C}(I)} \mathbb{E}_{I^{\prime}}^{\mu} h\right)-\mathbb{E}_{I}^{\mu} h=\sum_{I^{\prime} \in \mathfrak{C}(I)} \mathbf{1}_{I^{\prime}}\left(\mathbb{E}_{I^{\prime}}^{\mu} h-\mathbb{E}_{I}^{\mu} h\right),
$$

and so

$$
\sum_{Q^{\prime} \in \mathfrak{C}(Q)}\left|Q^{\prime}\right|_{\mu}\left|\mathbb{E}_{Q^{\prime}}^{\mu} h-\mathbb{E}_{Q}^{\mu} h\right|^{2}=\int\left|\sum_{Q^{\prime} \in \mathfrak{C}(Q)} \mathbf{1}_{Q^{\prime}}\left(\mathbb{E}_{Q^{\prime}}^{\mu} h-\mathbb{E}_{Q}^{\mu} h\right)\right|^{2} d \mu=\left\|\triangle_{Q}^{\mu} h\right\|_{L^{2}(\mu)}^{2}
$$

Thus we have

$$
\begin{aligned}
\sum_{A \in \mathcal{A}} \sum_{Q \in \mathcal{C}_{A}} \sum_{Q^{\prime} \in \mathfrak{C}_{\text {natural }}(Q)}\left|Q^{\prime}\right|_{\mu}\left|\mathbb{E}_{Q^{\prime}}^{\mu} h-\mathbb{E}_{Q}^{\mu} h\right|^{2} & \leq \sum_{A \in \mathcal{A}} \sum_{Q \in \mathcal{C}_{A}} \sum_{Q^{\prime} \in \mathfrak{C}(Q)}\left|Q^{\prime}\right|_{\mu}\left|\mathbb{E}_{Q^{\prime}}^{\mu} h-\mathbb{E}_{Q}^{\mu} h\right|^{2} \\
& =\sum_{A \in \mathcal{A}} \sum_{Q \in \mathcal{C}_{A}}\left\|\triangle_{Q}^{\mu} h\right\|_{L^{2}(\mu)}^{2}=\|h\|_{L^{2}(\mu)}^{2} .
\end{aligned}
$$

Proposition 91. (see HyMa, Remark 3.11] for the case of a doubling measure with $p>2$ ) Suppose that $\mathbf{b}$ is an $\infty$-weakly $\mu$-controlled accretive family on a grid $\mathcal{D}$ with corona tops $\mathcal{A} \subset \mathcal{D}$. Then we have the lower frame inequality

$$
\sum_{I \in \mathcal{D}}\left\|\triangle_{Q}^{\mu, \mathbf{b}} f\right\|_{L^{2}(\mu)}^{2} \lesssim\|f\|_{L^{2}(\mu)}^{2}
$$

Proof. Given $A \in \mathcal{A}$, we begin with

$$
\begin{aligned}
\sum_{Q \in \mathcal{C}_{A}}\left\|\triangle_{Q}^{\mu, \mathbf{b}} f\right\|_{L^{2}(\mu)}^{2}= & \sum_{Q \in \mathcal{C}_{A}} \sum_{Q^{\prime} \in \mathfrak{C}(Q)} \int_{Q^{\prime}}\left|\mathbb{E}_{Q^{\prime}}^{\mu, \mathbf{b}} f(x)-\mathbb{E}_{Q}^{\mu, \mathbf{b}} f(x)\right|^{2} d \mu(x) \\
= & \sum_{Q \in \mathcal{C}_{A}} \sum_{Q^{\prime} \in \mathfrak{C}_{\text {natural }}(Q)} \int_{Q^{\prime}}\left|\frac{\int_{Q^{\prime}} f b_{A} d \mu}{\int_{Q^{\prime}} b_{A} d \mu}-\frac{\int_{Q} f b_{A} d \mu}{\int_{Q} b_{A} d \mu}\right|^{2} d \mu(x) \\
& +\sum_{Q \in \mathcal{C}_{A}} \sum_{Q^{\prime} \in \mathfrak{C}_{\text {broken }}(Q)} \int_{Q^{\prime}}\left|\frac{\int_{Q^{\prime}} f b_{Q^{\prime}} d \mu}{\int_{Q^{\prime}} b_{Q^{\prime}} d \mu}-\frac{\int_{Q} f b_{A} d \mu}{\int_{Q} b_{A} d \mu}\right|^{2} d \mu(x) \\
= & \sum_{Q \in \mathcal{C}_{A}} \sum_{Q^{\prime} \in \mathfrak{C}_{\text {natural }}(Q)}\left|\frac{\int_{Q^{\prime}} f b_{A} d \mu}{\int_{Q^{\prime}} b_{A} d \mu}-\frac{\int_{Q} f b_{A} d \mu}{\int_{Q} b_{A} d \mu}\right|^{2}\left|Q^{\prime}\right|_{\mu} \\
& +\sum_{Q \in \mathcal{C}_{A}} \sum_{Q^{\prime} \in \mathfrak{C}_{\text {broken }}(Q)}\left|\frac{\int_{Q^{\prime}} f b_{Q^{\prime}} d \mu}{\int_{Q^{\prime}} b_{Q^{\prime}} d \mu}-\frac{\int_{Q} f b_{A} d \mu}{\int_{Q} b_{A} d \mu}\right|^{2}\left|Q^{\prime}\right|_{\mu} \\
\equiv & I_{A}+I I_{A} .
\end{aligned}
$$


To estimate term $I I_{A}$ we write

$$
\begin{aligned}
& \left|\frac{\int_{Q^{\prime}} f b_{Q^{\prime}} d \mu}{\int_{Q^{\prime}} b_{Q^{\prime}} d \mu}-\frac{\int_{Q} f b_{A} d \mu}{\int_{Q} b_{A} d \mu}\right| \\
= & \frac{\left|\left(\frac{1}{\left|Q^{\prime}\right|_{\mu}} \int_{Q^{\prime}} f b_{Q^{\prime}} d \mu\right)\left(\frac{1}{|Q|_{\mu}} \int_{Q} b_{A} d \mu\right)-\left(\frac{1}{|Q|_{\mu}} \int_{Q} f b_{A} d \mu\right)\left(\frac{1}{\left|Q^{\prime}\right|_{\mu}} \int_{Q^{\prime}} b_{Q^{\prime}} d \mu\right)\right|}{\left|\frac{1}{\left|Q^{\prime}\right|_{\mu}} \int_{Q^{\prime}} b_{Q^{\prime}} d \mu\right|\left|\frac{1}{|Q|_{\mu}} \int_{Q^{\prime}} b_{A} d \mu\right|} \\
\lesssim & \left|E_{Q^{\prime}}^{\mu}\left(f b_{Q^{\prime}}\right) E_{Q}^{\mu}\left(b_{A}\right)-E_{Q}^{\mu}\left(f b_{A}\right) E_{Q^{\prime}}^{\mu}\left(b_{Q^{\prime}}\right)\right| \\
= & \left|E_{Q^{\prime}}^{\mu}\left(f b_{Q^{\prime}}\right) E_{Q}^{\mu}\left(b_{A}\right)-E_{Q^{\prime}}^{\mu}\left(f b_{A}\right) E_{Q^{\prime}}^{\mu}\left(b_{Q^{\prime}}\right)-\left[E_{Q}^{\mu}\left(f b_{A}\right)-E_{Q^{\prime}}^{\mu}\left(f b_{A}\right)\right] E_{Q^{\prime}}^{\mu}\left(b_{Q^{\prime}}\right)\right| \\
\lesssim & \left|E_{Q^{\prime}}^{\mu}\left(f b_{Q^{\prime}}\right)\right|+\left|E_{Q^{\prime}}^{\mu}\left(f b_{A}\right)\right|+\left|E_{Q}^{\mu}\left(f b_{A}\right)-E_{Q^{\prime}}^{\mu}\left(f b_{A}\right)\right|,
\end{aligned}
$$

and then

$$
\begin{aligned}
I I_{A} & \lesssim \sum_{Q \in \mathcal{C}_{A}} \sum_{Q^{\prime} \in \mathfrak{C}_{\text {broken }}(Q)}\left(\left|E_{Q^{\prime}}^{\mu}\left(f b_{Q^{\prime}}\right)\right|^{2}+\left|E_{Q^{\prime}}^{\mu}\left(f b_{A}\right)\right|^{2}+\left|E_{Q}^{\mu}\left(f b_{A}\right)\right|^{2}\right)\left|Q^{\prime}\right|_{\mu} \\
& \lesssim \sum_{Q \in \mathcal{C}_{A}} \sum_{Q^{\prime} \in \mathfrak{C}_{\text {broken }}(Q)}\left(\left(E_{Q^{\prime}}^{\mu}|f|\right)^{2}+\left(E_{Q}^{\mu}|f|\right)^{2}\right)\left|Q^{\prime}\right|_{\mu} .
\end{aligned}
$$

Now we turn to term $I_{A}$ and use the analogue of (9.9) for natural children, along with the natural child bounds $\left|\int_{Q^{\prime}} b_{A} d \mu\right| \gtrsim\left|Q^{\prime}\right|_{\mu}$ and $\left|\int_{Q} b_{A} d \mu\right| \gtrsim|Q|_{\mu}$, to obtain

$$
\begin{aligned}
I_{A}= & \sum_{Q \in \mathcal{C}_{A}} \sum_{Q^{\prime} \in \mathfrak{C}_{\text {natural }}(Q)}\left|\frac{\int_{Q^{\prime}} f b_{A} d \mu}{\int_{Q^{\prime}} b_{A} d \mu}-\frac{\int_{Q} f b_{A} d \mu}{\int_{Q} b_{A} d \mu}\right|^{2}\left|Q^{\prime}\right|_{\mu} \\
= & \sum_{Q \in \mathcal{C}_{A}} \sum_{Q^{\prime} \in \mathfrak{C}_{\text {natural }}(Q)}\left|\frac{\left(\int_{Q^{\prime}} f b_{A} d \mu\right)\left(\int_{Q} b_{A} d \mu\right)-\left(\int_{Q^{\prime}} b_{A} d \mu\right)\left(\int_{Q} f b_{A} d \mu\right)}{\left(\int_{Q^{\prime}} b_{A} d \mu\right)\left(\int_{Q} b_{A} d \mu\right)}\right|^{2}\left|Q^{\prime}\right|_{\mu} \\
\lesssim & \sum_{Q \in \mathcal{C}_{A}} \sum_{Q^{\prime} \in \mathfrak{C}_{\text {natural }}(Q)}\left|E_{Q}^{\mu}\left(b_{A}\right)\right|^{2}\left|E_{Q^{\prime}}^{\mu}\left(f b_{A}\right)-E_{Q}^{\mu}\left(f b_{A}\right)\right|^{2}\left|Q^{\prime}\right|_{\mu} \\
& +\sum_{Q \in \mathcal{C}_{A}} \sum_{Q^{\prime} \in \mathfrak{C}_{\text {natural }}(Q)}\left|E_{Q}^{\mu}\left(b_{A}\right)-E_{Q^{\prime}}^{\mu} b_{A}\right|^{2}\left|E_{Q}^{\mu}\left(f b_{A}\right)\right|^{2}\left|Q^{\prime}\right|_{\mu} \\
\equiv & I I I_{A}+I V_{A} .
\end{aligned}
$$

Now we have

$$
I I I_{A} \lesssim \sum_{Q \in \mathcal{C}_{A}} \sum_{Q^{\prime} \in \mathfrak{C}_{\text {natural }}(Q)}\left|E_{Q^{\prime}}^{\mu}\left(f b_{A}\right)-E_{Q}^{\mu}\left(f b_{A}\right)\right|^{2}\left|Q^{\prime}\right|_{\mu},
$$

and for term $I V_{A}$, we introduce the quantities

$$
\gamma_{Q} \equiv \sum_{Q^{\prime} \in \mathfrak{C}_{\text {natural }}(Q)}\left|E_{Q}^{\mu} b_{A}-E_{Q^{\prime}}^{\mu} b_{A}\right|^{2}\left|Q^{\prime}\right|_{\mu}, \quad \text { for } Q \in \mathcal{C}_{A}, A \in \mathcal{A},
$$

so that

$$
\begin{aligned}
I V_{A} & =\sum_{Q \in \mathcal{C}_{A}} \sum_{Q^{\prime} \in \mathfrak{C}_{\text {natural }}(Q)}\left|E_{Q}^{\mu}\left(b_{A}\right)-E_{Q^{\prime}}^{\mu} b_{A}\right|^{2}\left|Q^{\prime}\right|_{\mu}\left|E_{Q}^{\mu}\left(f b_{A}\right)\right|^{2} \\
& =\sum_{Q \in \mathcal{C}_{A}} \gamma_{Q}\left|E_{Q}^{\mu}\left(f b_{A}\right)\right|^{2} \lesssim \sum_{Q \in \mathcal{C}_{A}} \gamma_{Q}\left(E_{Q}^{\mu}|f|\right)^{2}
\end{aligned}
$$

Now note that the coefficients $\left\{\gamma_{Q}\right\}_{Q \in \mathcal{D}}$ satisfy the Carleson condition

$$
\sum_{Q \subset B} \gamma_{Q} \lesssim|B|_{\sigma}
$$


Indeed, if $B \in \mathcal{C}_{A}$, then using $E_{Q}^{\mu} b_{A}=E_{Q}^{\mu}\left(\mathbf{1}_{B} b_{A}\right)$ for $Q \subset B$ and the unweighted square function estimate, and denoting by $\mathfrak{G}_{\mathcal{A}}^{t}(A)$ the $\mathcal{A}$-grandchildren at level $t$ below $A$, we have

$$
\begin{aligned}
\sum_{Q \subset B} \gamma_{Q}= & \sum_{A \in \mathcal{A}} \sum_{Q \in \mathcal{C}_{A}: Q \subset B} \sum_{Q^{\prime} \in \mathfrak{C}_{\text {natural }}(Q)}\left|E_{Q}^{\mu} b_{A}-E_{Q^{\prime}}^{\mu} b_{A}\right|^{2}\left|Q^{\prime}\right|_{\mu} \\
& +\sum_{t=1}^{\infty} \sum_{H \in \mathfrak{G}_{\mathcal{A}}^{t}(A):} \sum_{H \subset B} \sum_{Q \in \mathcal{C}_{H}}\left|E_{Q^{\prime} \in \mathfrak{C}_{\text {natural }}^{\mu}(Q)} b_{H}-E_{Q^{\prime}}^{\mu} b_{H}\right|^{2}\left|Q^{\prime}\right|_{\mu} \\
\lesssim & \int_{B}\left|b_{A}\right|^{2} d \mu+\sum_{t=1}^{\infty} \sum_{H \in \mathfrak{G}_{\mathcal{A}}^{t}(A): H \subset B} \int\left|b_{H}\right|^{2} d \mu \\
\lesssim & |B|_{\mu}+\sum_{t=1}^{\infty} \sum_{H \in \mathfrak{G}_{\mathcal{A}}^{t}(A): H \subset B}|H|_{\mu} \lesssim|B|_{\mu} .
\end{aligned}
$$

Altogether then, using the above inequalities for $I I_{A}, I I I_{A}$ and $I V_{A}$, and then using the Carleson embedding theorem on the sum of the terms $I I_{A}$, and the Carleson embedding theorem again on the sum of the terms $I V_{A}$ with the Carleson condition (9.10), we conclude that

$$
\begin{aligned}
\sum_{A \in \mathcal{A}} \sum_{Q \in \mathcal{C}_{A}}\left\|\triangle_{Q}^{\mu, \mathbf{b}} f\right\|_{L^{2}(\mu)}^{2} \lesssim & \sum_{A \in \mathcal{A}}\left(I I_{A}+I I I_{A}+I V_{A}\right) \\
\lesssim & \sum_{A \in \mathcal{A}}|A|_{\mu}\left\{\left(\frac{1}{|A|_{\mu}} \int_{A}|f| d \mu\right)^{2}+\left(\frac{1}{|\pi A|_{\mu}} \int_{\pi A}|f| d \mu\right)^{2}\right\} \\
& +\sum_{A \in \mathcal{A}} I I I_{A}+\sum_{Q \in \mathcal{C}_{A}} \gamma_{Q}\left(E_{Q}^{\mu}|f|\right)^{2} \lesssim \int|f|^{2} d \mu+\sum_{A \in \mathcal{A}} I I I_{A} .
\end{aligned}
$$

Thus it remains only to estimate the final square function expression, which requires an additional argument due to the fact that the functions $f b_{A}$ differ from one corona to the next. For this we define

$$
b \equiv \sum_{A \in \mathcal{A}} b_{A} \mathbf{1}_{A \backslash \cup_{A^{\prime} \in \mathfrak{C}_{\mathcal{A}}(A)}}
$$

and write

$$
\begin{aligned}
& \sum_{A \in \mathcal{A}} I I I_{A} \lesssim \sum_{A \in \mathcal{A}} \sum_{Q \in \mathcal{C}_{A}} \sum_{Q^{\prime} \in \mathfrak{C}_{\text {natural }}(Q)}\left|E_{Q^{\prime}}^{\mu}\left(f b_{A}\right)-E_{Q}^{\mu}\left(f b_{A}\right)\right|^{2}\left|Q^{\prime}\right|_{\mu} \\
\lesssim & \sum_{A \in \mathcal{A}} \sum_{Q \in \mathcal{C}_{A}} \sum_{Q^{\prime} \in \mathfrak{C}_{\text {natural }}(Q)}\left|E_{Q^{\prime}}^{\mu}\left(f b_{A}-f b\right)-E_{Q}^{\mu}\left(f b_{A}-f b\right)\right|^{2}\left|Q^{\prime}\right|_{\mu} \\
& +\sum_{A \in \mathcal{A}} \sum_{Q \in \mathcal{C}_{A}} \sum_{Q^{\prime} \in \mathfrak{C}_{\text {natural }}(Q)}\left|E_{Q^{\prime}}^{\mu}(f b)-E_{Q}^{\mu}(f b)\right|^{2}\left|Q^{\prime}\right|_{\mu} .
\end{aligned}
$$

Now the unweighted square function estimate applies to the second sum to give

$$
\sum_{A \in \mathcal{A}} \sum_{Q \in \mathcal{C}_{A}} \sum_{Q^{\prime} \in \mathfrak{C}_{\text {natural }}(Q)}\left|E_{Q^{\prime}}^{\mu}(f b)-E_{Q}^{\mu}(f b)\right|^{2}\left|Q^{\prime}\right|_{\mu} \lesssim \int|f b|^{2} d \mu \lesssim \int|f|^{2} d \mu .
$$

To handle the first sum we write both

$$
\begin{aligned}
& E_{Q^{\prime}}^{\mu}\left(f b_{A}-f b\right)=E_{Q^{\prime}}^{\mu}\left(\sum_{A^{\prime} \in \mathfrak{C}_{\mathcal{A}}(A)} \mathbf{1}_{A^{\prime}}\left(f b_{A}-f b\right)\right)=E_{Q^{\prime}}^{\mu}\left(\sum_{A^{\prime} \in \mathfrak{C}_{\mathcal{A}}(A)} \mathbb{E}_{A^{\prime}}^{\mu}\left(f b_{A}-f b\right)\right), \\
& E_{Q}^{\mu}\left(f b_{A}-f b\right)=E_{Q}^{\mu}\left(\sum_{A^{\prime} \in \mathfrak{C}_{\mathcal{A}}(A)} \mathbf{1}_{A^{\prime}}\left(f b_{A}-f b\right)\right)=E_{Q}^{\mu}\left(\sum_{A^{\prime} \in \mathfrak{C}_{\mathcal{A}}(A)} \mathbb{E}_{A^{\prime}}^{\mu}\left(f b_{A}-f b\right)\right),
\end{aligned}
$$


and use the unweighted square function estimate on each corona $\mathcal{C}_{A}$, applied to the function $f b_{A}-f b$, to obtain

$$
\begin{aligned}
& \sum_{Q \in \mathcal{C}_{A}} \sum_{Q^{\prime} \in \mathfrak{C}_{\text {natural }}(Q)}\left|E_{Q^{\prime}}^{\mu}\left(f b_{A}-f b\right)-E_{Q}^{\mu}\left(f b_{A}-f b\right)\right|^{2}\left|Q^{\prime}\right|_{\mu} \\
\lesssim & \int_{A}\left|\sum_{A^{\prime} \in \mathfrak{C}_{\mathcal{A}}(A)} \mathbb{E}_{A^{\prime}}^{\mu}\left(f b_{A}-f b\right)\right|^{2} d \mu=\sum_{A^{\prime} \in \mathfrak{C}_{\mathcal{A}}(A)}\left|E_{A^{\prime}}^{\mu}\left(f b_{A}-f b\right)\right|^{2}\left|A^{\prime}\right|_{\mu} \lesssim \sum_{A^{\prime} \in \mathfrak{C}_{\mathcal{A}}(A)}\left(E_{A^{\prime}}^{\mu}|f|\right)^{2}\left|A^{\prime}\right|_{\mu},
\end{aligned}
$$

We can now sum over $A \in \mathcal{A}$ to obtain

$$
\begin{aligned}
& \sum_{A \in \mathcal{A}} \sum_{Q \in \mathcal{C}_{A}} \sum_{Q^{\prime} \in \mathfrak{C}_{\text {natural }}(Q)}\left|E_{Q^{\prime}}^{\mu}\left(f b_{A}-f b\right)-E_{Q}^{\mu}\left(f b_{A}-f b\right)\right|^{2}\left|Q^{\prime}\right|_{\mu} \\
\lesssim & \sum_{A \in \mathcal{A}} \sum_{A^{\prime} \in \mathfrak{C}_{\mathcal{A}}(A)}\left(E_{A^{\prime}}^{\mu}|f|\right)^{2}\left|A^{\prime}\right|_{\mu} \lesssim \int|f|^{2} d \mu
\end{aligned}
$$

by the Carleson embedding theorem yet again.

Essentially the same proof gives the lower frame inequality for the dual martingale difference $\square_{Q}^{\mu, \mathbf{b}} f=$ $\left(\triangle_{Q}^{\mu, \mathbf{b}}\right)^{*} f:$

$$
\sum_{Q \in \mathcal{D}}\left\|\square_{Q}^{\mu, \mathbf{b}} f\right\|_{L^{2}(\mu)}^{2} \lesssim\|f\|_{L^{2}(\mu)}^{2}
$$

but matters are made slightly simpler by the fact that we do not need the function $b$ introduced in (9.11) above because in the definition of $\square_{Q}^{\mu, \mathbf{b}} f$ the function $b_{Q}$ doesn't multiply $f$, rather it sits outside the integral where it can be estimated crudely, leaving the unweighted square function applied to $f$ alone. We leave this for the reader, pointing out that the general idea of the proof can be found in the proof given below in Proposition 98 for the lower weak Riesz inequality for $\square_{Q}^{\mu, \mathbf{b}}$.

The corresponding upper weak frame inequalities

$$
\begin{aligned}
\|f\|_{L^{2}(\mu)}^{2} & \lesssim \sum_{Q \in \mathcal{D}}\left\|\triangle_{Q}^{\mu, \mathbf{b}} f\right\|_{L^{2}(\mu)}^{2}+\sum_{Q \in \mathcal{D}}\left\|\nabla_{Q}^{\mu} f\right\|_{L^{2}(\mu)}^{2}, \\
\|f\|_{L^{2}(\mu)}^{2} & \lesssim \sum_{Q \in \mathcal{D}}\left\|\square_{Q}^{\mu, \mathbf{b}} f\right\|_{L^{2}(\mu)}^{2}+\sum_{Q \in \mathcal{D}}\left\|\nabla_{Q}^{\mu} f\right\|_{L^{2}(\mu)}^{2},
\end{aligned}
$$

are proved by duality using the 'Calderon reproducing formulas'

$$
\triangle_{Q}^{\mu, \mathbf{b}}=\left(\triangle_{Q}^{\mu, \pi, \mathbf{b}}\right)^{2}+\triangle_{Q, \text { broken }}^{\mu, \mathbf{b}} \text { and } \square_{Q}^{\mu, \mathbf{b}}=\left(\square_{Q}^{\mu, \pi, \mathbf{b}}\right)^{2}+\square_{Q, \text { broken }}^{\mu, \mathbf{b}}
$$

introduced in the following subsection. We will actually prove stronger inequalities below, namely upper weak Riesz inequalities.

9.3. Unbroken projections, broken differences and a Calderón reproducing formula. Here we assume that $\int_{Q^{\prime}} b_{Q} d \mu \neq 0$, noting that in applications we will have the stronger inequality $\left|\int_{Q^{\prime}} b_{Q} d \omega\right| \gtrsim$ $\mathbf{1}_{Q^{\prime}}\left\|b_{Q}\right\|_{L^{\infty}(\omega)}>0$ due to the assumed reverse Hölder control on children (2.15). Define

$$
\mathbb{E}_{Q}^{\mu, \pi, \mathbf{b}} f \equiv \mathbf{1}_{Q} \frac{1}{\int_{Q} b_{\pi Q} d \mu} \int_{Q} b_{\pi Q} f d \mu \text { and } \mathbb{F}_{Q}^{\mu, \pi, \mathbf{b}} f \equiv \mathbf{1}_{Q} \frac{b_{\pi Q}}{\int_{Q} b_{\pi Q} d \mu} \int_{Q} f d \mu
$$

and

$$
\begin{aligned}
& \triangle_{Q}^{\mu, \pi, \mathbf{b} f}=\left[\sum_{Q^{\prime} \in \mathfrak{C}(Q)} \mathbb{E}_{Q^{\prime}}^{\mu, \pi, \mathbf{b}} f\right]-\mathbb{E}_{Q}^{\mu, \mathbf{b}} f=\sum_{Q^{\prime} \in \mathfrak{C}(Q)} \mathbb{E}_{Q^{\prime}}^{\mu, b_{Q}} f-\mathbb{E}_{Q}^{\mu, b_{Q}} f, \\
& \square_{Q}^{\mu, \pi, \mathbf{b} f}=\left[\sum_{Q^{\prime} \in \mathfrak{C}(Q)} \mathbb{F}_{Q^{\prime}}^{\mu, \pi, \mathbf{b}} f\right]-\mathbb{F}_{Q}^{\mu, \mathbf{b}} f=\sum_{Q^{\prime} \in \mathfrak{C}(Q)} \mathbb{F}_{Q^{\prime}}^{\mu, b_{Q}} f-\mathbb{F}_{Q}^{\mu, b_{Q}} f,
\end{aligned}
$$


where on the far right of (9.16) we are using the notation $\mathbb{E}_{Q^{\prime}}^{\mu, b}=\mathbf{1}_{Q^{\prime}} \frac{1}{\int_{Q^{\prime}} b d \mu} \int_{Q^{\prime}} b f d \mu$ when $b$ is simply a function, rather than a family of functions $\mathbf{b}$, in order to specify the testing function $b$ we use if it differs from the function $b_{Q^{\prime}}$ that is selected in the notation $\mathbb{E}_{Q^{\prime}}^{\mu, \mathbf{b}}$ when the family $\mathbf{b}$ appears in boldface as an exponent. Similarly for other pseudoprojections.

For convenience of notation, we set $\triangle_{Q}^{\mu, \pi, \mathbf{b}}=\triangle_{Q}^{\mu, b}$ and $\square_{Q}^{\mu, \pi, \mathbf{b}}=\square_{Q}^{\mu, b}$ with $b=b_{Q}$. Note that

$$
\begin{aligned}
\mathbb{E}_{Q}^{\mu, b}(1) & =\mathbf{1}_{Q} \frac{1}{\int_{Q} b d \mu} \int_{Q}(1) b d \mu=\mathbf{1}_{Q}, \\
\mathbb{F}_{Q}^{\mu, b}(b) & =\mathbf{1}_{Q} \frac{b}{\int_{Q} b d \mu} \int_{Q}(b) d \mu=\mathbf{1}_{Q} b, \\
\triangle_{Q}^{\mu, b}(1) & =\left[\sum_{Q^{\prime \prime} \in \mathfrak{C}(Q)} \mathbb{E}_{Q^{\prime \prime}}^{\mu, b}\right]-\mathbb{E}_{Q}^{\mu, b} 1=\left[\sum_{Q^{\prime \prime} \in \mathfrak{C}(Q)} \mathbf{1}_{Q^{\prime \prime}}\right]-\mathbf{1}_{Q}=0, \\
\square_{Q}^{\mu, b} b & =\left[\sum_{Q^{\prime \prime} \in \mathfrak{C}(Q)} \mathbb{F}_{Q^{\prime \prime \prime}}^{\mu, b}\right]-\mathbb{F}_{Q}^{\mu, b} b=\left[\sum_{Q^{\prime \prime} \in \mathfrak{C}(Q)} \mathbf{1}_{Q^{\prime \prime}}\right]-\mathbf{1}_{Q} b=0 .
\end{aligned}
$$

The next two lemmas are in [NTV2, see page 193].

Lemma 92. For dyadic cubes $R$ and $Q$ we have

$$
\mathbb{E}_{R}^{\mu, b} \triangle_{Q}^{\mu, b}=\left\{\begin{array}{clc}
0 & \text { if } & R \supset Q \text { or } R \cap Q=\emptyset \\
\mathbf{1}_{R} E_{Q_{R}}^{\mu, b} \triangle_{Q}^{\mu, b} f & \text { if } & R \varsubsetneqq Q
\end{array}\right.
$$

Proof. If $R \supset Q$, then since $\square_{Q}^{\mu, b} b=0$, we have

$$
\mathbb{E}_{R}^{\mu, b} \triangle_{Q}^{\mu, b} f=\mathbf{1}_{R} \frac{1}{\int_{R} b d \mu} \int_{R}\left(\triangle_{Q}^{\mu, b} f\right) b d \mu=\mathbf{1}_{R} \frac{\left\langle\triangle_{Q}^{\mu, b} f, b\right\rangle_{L^{2}(\mu)}}{\int_{R} b d \mu}=\mathbf{1}_{R} \frac{\left\langle f, \square_{Q}^{\mu, b} b\right\rangle_{L^{2}(\mu)}}{\int_{R} b d \mu}=0 .
$$

On the other hand, if $R \varsubsetneqq Q$, then $R \subset Q^{\prime}$ for some $Q^{\prime} \in \mathfrak{C}(Q)$, and since $\triangle_{Q}^{\mu, b} f$ equals the constant $A=E_{Q_{R}}^{\mu, b}\left(\triangle_{Q}^{\mu, b} f\right)$ on $Q^{\prime}$, and $E_{I}^{\mu, b} 1=1$ for all cubes $I$, we have

$$
\mathbb{E}_{R}^{\mu, b} \triangle_{Q}^{\mu, b} f=\mathbb{E}_{R}^{\mu, b} A=\mathbf{1}_{R} A=\mathbf{1}_{R} E_{Q_{R}}^{\mu, b}\left(\triangle_{Q}^{\mu, b} f\right)
$$

Lemma 93. For dyadic cubes $R$ and $Q$ we have

$$
\triangle_{R}^{\mu, b} \triangle_{Q}^{\mu, b}=\left\{\begin{array}{ccc}
\triangle_{Q}^{\mu, b} & \text { if } & R=Q \\
0 & \text { if } & R \neq Q
\end{array}\right.
$$

Proof. By the top line in Lemma 92, it suffices to consider the case $R \cap Q \neq \emptyset$. First we suppose that $R \varsubsetneqq Q$. Then $R \subset Q^{\prime}$ for some $Q^{\prime} \in \mathfrak{C}(Q)$, and since $\triangle_{Q}^{\mu, b} f=A$ is constant on $Q^{\prime}$, and $\mathbb{E}_{I}^{\mu, b} 1=\mathbf{1}_{I}$ for any cube $I$, we obtain

$$
\triangle_{R}^{\mu, b} \triangle_{Q}^{\mu, b} f=\left[\sum_{R^{\prime} \in \mathfrak{C}(R)} \mathbb{E}_{R^{\prime}}^{\mu, b}\left\{\triangle_{Q}^{\mu, b} f\right\}\right]-\mathbb{E}_{R}^{\mu, b}\left\{\triangle_{Q}^{\mu, b} f\right\}=\left[\sum_{R^{\prime} \in \mathfrak{C}(R)} \mathbf{1}_{R^{\prime}} A\right]-\mathbf{1}_{R} A=0 .
$$


Next we suppose that $R=Q$ and obtain

$$
\begin{aligned}
& \triangle_{Q}^{\mu, b} \triangle_{Q}^{\mu, b} f \\
= & {\left[\sum_{Q^{\prime} \in \mathfrak{C}(Q)} \mathbb{E}_{Q^{\prime}}^{\mu, b}\left\{\triangle_{Q}^{\mu, b} f\right\}-\mathbb{E}_{Q}^{\mu, b}\left\{\triangle_{Q}^{\mu, b} f\right\}\right.} \\
= & {\left[\sum_{Q^{\prime} \in \mathfrak{C}(Q)} \mathbb{E}_{Q^{\prime}}^{\mu, b}\left\{\left[\sum_{Q^{\prime \prime} \in \mathfrak{C}(Q)} \mathbb{E}_{Q^{\prime \prime}}^{\mu, b}\right]-\mathbb{E}_{Q}^{\mu, b} f\right\}\right]-\mathbb{E}_{Q}^{\mu, b}\left[\left\{\left[\sum_{Q^{\prime \prime} \in \mathfrak{C}(Q)} \mathbb{E}_{Q^{\prime \prime}}^{\mu, b}\right]-\mathbb{E}_{Q}^{\mu, b} f\right\}\right] } \\
= & \left\{\left[\sum_{Q^{\prime} \in \mathfrak{C}(Q)} \mathbb{E}_{Q^{\prime}}^{\mu, b} f\right]-\mathbb{E}_{Q}^{\mu, b} f\right\}-\mathbb{E}_{Q}^{\mu, b}\left[\left\{\left[\sum_{Q^{\prime \prime} \in \mathfrak{C}(Q)} \mathbb{E}_{Q^{\prime \prime}}^{\mu, b}\right]-\mathbb{E}_{Q}^{\mu, b} f\right\}\right] \\
= & \triangle_{Q}^{\mu, b} f-\mathbb{E}_{Q}^{\mu, b} \triangle_{Q}^{\mu, b} f=\triangle_{Q}^{\mu, b} f,
\end{aligned}
$$

where we have used Lemma 92 with $R=Q$ for the final equality.

Finally we suppose that $R \supsetneqq Q$. Then $R_{Q} \supset Q$, and so by the top line in Lemma 92 we have

$$
\triangle_{R}^{\mu, b} \triangle_{Q}^{\mu, b} f=\sum_{R^{\prime} \in \mathfrak{C}(R)} \mathbb{E}_{R^{\prime}}^{\mu, b} \triangle_{Q}^{\mu, b} f-\mathbb{E}_{R}^{\mu, b} \triangle_{Q}^{\mu, b} f=0-0=0 .
$$

Now since we are assuming that $\int_{Q^{\prime}} b_{Q} \neq 0$, we can define

$$
\begin{aligned}
\triangle_{Q, \text { broken }}^{\mu, \pi, \mathbf{b}} f & =\triangle_{Q}^{\mu, \mathbf{b}} f-\triangle_{Q}^{\mu, \pi, \mathbf{b}} f \\
& =\left(\sum_{Q^{\prime} \in \mathfrak{C}(Q)} \mathbb{E}_{Q^{\prime}}^{\mu, b_{Q^{\prime}}} f-\mathbb{E}_{Q}^{\mu, b_{Q}} f\right)-\left(\sum_{Q^{\prime} \in \mathfrak{C}(Q)} \mathbb{E}_{Q^{\prime}}^{\mu, b_{Q}} f-\mathbb{E}_{Q}^{\mu, b_{Q}} f\right) \\
& =\sum_{Q^{\prime} \in \mathfrak{C}_{\text {broken }}(Q)} \mathbb{E}_{Q^{\prime}}^{\mu, b_{Q^{\prime}}} f-\mathbb{E}_{Q^{\prime}}^{\mu, b_{Q}} f,
\end{aligned}
$$

with a similar definition for $\square_{Q, \text { broken }}^{\mu, \mathbf{b}} f$. Altogether, with $\square_{Q}^{\mu, \mathbf{b}}=\left(\triangle_{Q}^{\mu, \mathbf{b}}\right)^{*}, \square_{Q}^{\mu, \pi, \mathbf{b}}=\left(\triangle_{Q}^{\mu, \pi, \mathbf{b}}\right)^{*}$ and $\square_{Q, \text { broken }}^{\mu, \mathbf{b}}=\left(\triangle_{Q, \text { broken }}^{\mu, \mathbf{b}}\right)^{*}$, we have

$$
\triangle_{Q}^{\mu, \mathbf{b}}=\triangle_{Q}^{\mu, \pi, \mathbf{b}}+\triangle_{Q, \text { broken }}^{\mu, \pi, \mathbf{b}} \text { and } \square_{Q}^{\mu, \mathbf{b}}=\square_{Q}^{\mu, \pi, \mathbf{b}}+\square_{Q, \text { broken }}^{\mu, \pi, \mathbf{b}}
$$

where $\triangle_{Q}^{\mu, \pi, \mathbf{b}}$ and $\square_{Q}^{\mu, \pi, \mathbf{b}}$ are projections and

$$
\begin{aligned}
\triangle_{Q, \text { broken }}^{\mu, \pi, \mathbf{b}} f & =\sum_{Q^{\prime} \in \mathfrak{C}_{\text {broken }}(Q)}\left(\mathbb{E}_{Q^{\prime}}^{\mu, b_{Q^{\prime}}} f-\mathbb{E}_{Q^{\prime}}^{\mu, b_{Q}} f\right), \\
\left|\triangle_{Q, \text { broken }}^{\mu, \pi, \mathbf{b}} f\right| & \leq \sum_{Q^{\prime} \in \mathfrak{C}_{\text {broken }}(Q)}\left(\frac{\left\|\mathbf{1}_{Q^{\prime}} b_{Q}\right\|_{L^{\infty}(\mu)}}{\left|\int_{Q^{\prime}} b_{Q} d \mu\right|}+\left\|b_{Q^{\prime}}\right\|_{L^{\infty}(\mu)}\right) \frac{1}{\left|Q^{\prime}\right|_{\mu}} \int_{Q^{\prime}}|f| d \mu \leq C \sum_{Q^{\prime} \in \mathfrak{C}_{\text {broken }}(Q)} \frac{1}{\left|Q^{\prime}\right|_{\mu}} \int_{Q^{\prime}}|f| d \mu, \\
\square_{Q, \text { broken }}^{\mu, \pi, \mathbf{b}} f & =\sum_{Q^{\prime} \in \mathfrak{C}_{\text {broken }}(Q)}\left(\mathbb{F}_{Q^{\prime}}^{\left.\mu, b_{Q^{\prime}} f-\mathbb{F}_{Q^{\prime}}^{\mu, b_{Q}} f\right),}\right. \\
\left|\square_{Q, \text { broken }}^{\mu, \pi, \mathbf{b}} f\right| & \leq \sum_{Q^{\prime} \in \mathfrak{C}_{\text {broken }}(Q)}\left(\frac{\left\|\mathbf{1}_{Q^{\prime}} b_{Q}\right\|_{L^{\infty}(\mu)}}{\left|\int_{Q^{\prime}} b_{Q} d \mu\right|}+\left\|b_{Q^{\prime}}\right\|_{L^{\infty}(\mu)}\right) \frac{1}{\left|Q^{\prime}\right|_{\mu}} \int_{Q^{\prime}}|f| d \mu \leq C \sum_{Q^{\prime} \in \mathfrak{C}_{\text {broken }}(Q)} \frac{1}{\left|Q^{\prime}\right|_{\mu}} \int_{Q^{\prime}}|f| d \mu,
\end{aligned}
$$

where $C$ depends on both $C_{\mathbf{b}}$ and the constant in the reverse Hölder condition on children in (2.15).

Altogether then we have when $\int_{Q_{i}} b_{Q} d \sigma \neq 0$ for both children $Q_{i}$ of $Q$, the 'Calderón reproducing formula',

$$
\triangle_{Q}^{\mu, \mathbf{b}} f=\triangle_{Q}^{\mu, \pi, \mathbf{b}} \triangle_{Q}^{\mu, \pi, \mathbf{b}} f+\triangle_{Q, \text { broken }}^{\mu, \pi, \mathbf{b}} f \text { and } \square_{Q}^{\mu, \mathbf{b}} f=\square_{Q}^{\mu, \pi, \mathbf{b}} \square_{Q}^{\mu, \pi, \mathbf{b}} f+\square_{Q, \text { broken }}^{\mu, \pi, \mathbf{b}} f,
$$


and the pointwise estimates

$$
\left|\triangle_{Q}^{\mu, \mathbf{b}} f\right|,\left|\square_{Q}^{\mu, \mathbf{b}} f\right| \leq C_{\delta, \mathbf{b}} \sum_{Q^{\prime} \in \mathfrak{C}(Q)} \mathbf{1}_{Q^{\prime}}\left(\frac{1}{\left|Q^{\prime}\right|_{\mu}} \int_{Q^{\prime}}|f| d \mu+\frac{1}{|Q|_{\mu}} \int_{Q}|f| d \mu\right),
$$

and

$$
\left|\triangle_{Q, \text { broken }}^{\mu, \pi, \mathbf{b}} f\right| \lesssim\left|\widehat{\nabla}_{Q}^{\mu} f\right| \text { and }\left|\square_{Q, \text { broken }}^{\mu, \pi, \mathbf{b}} f\right| \lesssim\left|\widehat{\nabla}_{Q}^{\mu} f\right|,
$$

which follow from the reverse Hölder property in Lemma 18 of the children of $Q$,

$$
\left\|\mathbf{1}_{Q_{i}} b_{Q}\right\|_{L^{\infty}(\sigma)}<\frac{16 C_{b_{Q}}}{\delta}\left|\frac{1}{\left|Q_{i}\right|_{\sigma}} \int_{Q_{i}} b_{Q} d \sigma\right|, \quad Q_{i} \in \mathfrak{C}(Q) .
$$

Note again that the formulas in (9.18) always hold because our reverse Hölder assumption (2.15) in the triple corona construction implies in particular that $\left\|\mathbf{1}_{Q_{i}} b_{Q}\right\|_{L^{\infty}(\sigma)}>0$.

9.3.1. Another modified dual martingale difference. Define another modified dual martingale difference by

$$
\square_{I}^{\sigma, b, \mathbf{b}} f \equiv \square_{I}^{\sigma, \mathbf{b}} f-\sum_{I^{\prime} \in \mathfrak{C}_{\text {broken }}(I)} \mathbb{F}_{I^{\prime}}^{\sigma, \mathbf{b}} f=\left(\sum_{I^{\prime} \in \mathfrak{C}_{\text {natural }}(I)} \mathbb{F}_{I^{\prime}}^{\sigma, \mathbf{b}} f\right)-\mathbb{F}_{I}^{\sigma, \mathbf{b}} f
$$

where we have removed the averages over broken children from $\square_{I}^{\sigma, \mathbf{b}} f$, but left the average over $I$ intact. On any child $I^{\prime}$ of $I$, the function $\square_{I}^{\sigma, b, \mathbf{b}} f$ is thus a constant multiple of $b_{I}$, and so we have

$$
\begin{aligned}
\square_{I}^{\sigma, b, \mathbf{b}} f & =b_{I} \sum_{I^{\prime} \in \mathfrak{C}(I)} \mathbf{1}_{I^{\prime}} E_{I^{\prime}}^{\sigma}\left(\frac{1}{b_{I}} \square_{I}^{\sigma, b, \mathbf{b}} f\right)=b_{I} \sum_{I^{\prime} \in \mathfrak{C}(I)} \mathbf{1}_{I^{\prime}} E_{I^{\prime}}^{\sigma}\left(\widehat{\square}_{I}^{\sigma, b, \mathbf{b}} f\right) ; \\
\hat{\square}_{I}^{\sigma, b, \mathbf{b}} f & \equiv \sum_{I^{\prime} \in \mathfrak{C}(I)} \mathbf{1}_{I^{\prime}} E_{I^{\prime}}^{\sigma}\left(\frac{1}{b_{I}} \square_{I}^{\sigma, b, \mathbf{b}} f\right),
\end{aligned}
$$

where we have denoted the constants in question by the expressions $E_{I^{\prime}}^{\sigma}\left(\widehat{\square}_{I}^{\sigma, b, \mathbf{b}} f\right)$, and then defined $\widehat{\square}_{I}^{\sigma, b, \mathbf{b}} f$ to be the corresponding operator. We record the precise formula,

$$
\widehat{\square}_{I}^{\sigma, b, \mathbf{b}} f=\sum_{I^{\prime} \in \mathfrak{C}_{\text {natural }}(I)} \mathbf{1}_{I^{\prime}}\left[\frac{1}{\int_{I^{\prime}} b_{I} d \mu} \int_{I^{\prime}} f d \mu-\frac{1}{\int_{I} b_{I} d \mu} \int_{I} f d \mu\right]-\sum_{I^{\prime} \in \mathfrak{C}_{\text {broken }}(I)} \mathbf{1}_{I^{\prime}}\left[\frac{1}{\int_{I} b_{I} d \mu} \int_{I} f d \mu\right] .
$$

Thus for $I \in \mathcal{C}_{A}$ we have

$$
\square_{I}^{\sigma, b, \mathbf{b}} f=b_{A} \sum_{I^{\prime} \in \mathfrak{C}(I)} \mathbf{1}_{I^{\prime}} E_{I^{\prime}}^{\sigma}\left(\widehat{\square}_{I}^{\sigma, b, \mathbf{b}} f\right)=b_{A} \widehat{\square}_{I}^{\sigma, b, \mathbf{b}} f,
$$

where the averages $E_{I^{\prime}}^{\sigma}\left(\widehat{\square}_{I}^{\sigma, b, \mathbf{b}} f\right)$ satisfy the following telescoping property for all $K \in\left(\mathcal{C}_{A} \backslash\{A\}\right) \cup$ $\left(\bigcup_{A^{\prime} \in \mathfrak{C}_{\mathcal{A}}(A)} A^{\prime}\right)$ and $L \in \mathcal{C}_{A}$ with $K \subset L$ :

$$
\sum_{I: \pi K \subset I \subset L} E_{I_{K}}^{\sigma}\left(\widehat{\square}_{I}^{\sigma, b, \mathbf{b}} f\right)=\left\{\begin{array}{ccc}
-E_{L}^{\sigma} \widehat{\mathbb{F}}_{L}^{\sigma} f & \text { if } & K \in \mathfrak{C}_{\mathcal{A}}(A) \\
E_{K}^{\sigma} \widehat{\mathbb{F}}_{K}^{\sigma} f-E_{L}^{\sigma} \widehat{\mathbb{F}}_{L}^{\sigma} f & \text { if } & K \in \mathcal{C}_{A}
\end{array},\right.
$$

where $\widehat{\mathbb{F}}_{K}^{\sigma}$ is defined in (9.4) above. Indeed, recalling that $I_{K}$ denotes the child of $I$ that contains $K$, this is evident if we write

$$
\mathbf{1}_{K} b_{A} E_{I_{K}}^{\sigma}\left(\widehat{\square}_{I}^{\sigma, b, \mathbf{b}} f\right)=\mathbf{1}_{K} \square_{I}^{\sigma, b, \mathbf{b}} f=\mathbf{1}_{K}\left(\square_{I}^{\sigma, \mathbf{b}} f-\sum_{I^{\prime} \in \mathfrak{C}_{\text {broken }}(I)} \mathbf{1}_{I^{\prime}} \mathbb{F}_{I^{\prime}}^{\sigma, \mathbf{b}} f\right),
$$

and use the telescoping properties of $\left\{\square_{I}^{\sigma, \mathbf{b}}\right\}_{I \in \mathcal{D}}$, together with the fact that the set

$$
\left\{I^{\prime}: I^{\prime} \in \mathfrak{C}_{\text {broken }}(I) \text { for some } I \in \mathcal{C}_{A}\right\}=\mathfrak{C}_{\mathcal{A}}(A)
$$

is pairwise disjoint and lies beneath the corona $\mathcal{C}_{A}$. Of course we can similarly define $\triangle_{I}^{\sigma, b, \mathbf{b}}$. 
Finally, in analogy with the broken differences $\triangle_{Q, \text { broken }}^{\mu, \pi, \mathbf{b}}$ and $\square_{Q, \text { broken }}^{\mu, \pi}$ introduced above, we define

$$
\triangle_{I, \text { broken }}^{\mu, \mathrm{b}, \mathbf{b}} f \equiv \sum_{I^{\prime} \in \mathfrak{C}_{\text {broken }}(I)} \mathbb{E}_{I^{\prime}}^{\sigma, \mathbf{b}} f \text { and } \square_{I, \text { broken }}^{\mu, b, \mathbf{b}} f \equiv \sum_{I^{\prime} \in \mathfrak{C}_{\text {broken }}(I)} \mathbb{F}_{I^{\prime}}^{\sigma, \mathbf{b}} f,
$$

so that

$$
\triangle_{I}^{\mu, \mathbf{b}}=\triangle_{I}^{\mu, b, \mathbf{b}}+\triangle_{I, \text { broken }}^{\mu, b, \mathbf{b}} \text { and } \square_{I}^{\mu, \mathbf{b}}=\square_{I}^{\mu, b, \mathbf{b}}+\square_{I, \text { broken }}^{\mu, b, \mathbf{b}} .
$$

These modified differences and the identities (9.23) and (9.24) play a useful role in the analysis of the nearby and paraproduct forms.

9.4. Weak Riesz inequalities. We begin with a strengthening of the upper frame inequality for dual martingale pseudoprojections,

$$
\|f\|_{L^{2}(\mu)}^{2} \lesssim \sum_{Q \in \mathcal{D}}\left\|\square_{Q}^{\mu, \mathbf{b}} f\right\|_{L^{2}(\mu)}^{2}+\sum_{Q \in \mathcal{D}}\left\|\widehat{\nabla}_{Q}^{\mu} f\right\|_{L^{2}(\mu)}^{2}
$$

and then proceed to consider the corresponding lower inequalities and martingale versions as well. We refer to these strengthened inequalities as weak Riesz inequalities for the following reason. A family of pseudoprojections $\left\{\Psi_{Q}^{\mu, \mathbf{b}} f\right\}_{Q \in \mathcal{D}}$ is said to be a Riesz basis for $L^{2}(\mu)$ if for all subsets $\mathcal{B} \subset \mathcal{D}$ of the dyadic grid we have

$$
\sum_{Q \in \mathcal{B}}\left\|\Psi_{Q}^{\mu, \mathbf{b}} f\right\|_{L^{2}(\mu)}^{2} \lesssim\left\|\sum_{Q \in \mathcal{B}} \Psi_{Q}^{\mu, \mathbf{b}} f\right\|_{L^{2}(\mu)}^{2} \lesssim \sum_{Q \in \mathcal{B}}\left\|\Psi_{Q}^{\mu, \mathbf{b}} f\right\|_{L^{2}(\mu)}^{2}, \quad f \in L^{2}(\mu) .
$$

We refer to the left (respectively right) hand inequality above as the lower (respectively upper) Riesz inequality. The families $\left\{\square_{Q}^{\mu, \mathbf{b}} f\right\}_{Q \in \mathcal{D}}$ and $\left\{\triangle_{Q}^{\mu, \mathbf{b}} f\right\}_{Q \in \mathcal{D}}$ can fail to be a Riesz basis for $L^{2}(\mu)$, but in the case $p=\infty$, we show that each of these families is a Riesz basis in a certain weak sense, involving Carleson averaging operators, that is made precise below.

Definition 94. For any subset $\mathcal{B}$ of the grid $\mathcal{D}$, and any sequence of real numbers $\boldsymbol{\lambda}=\left\{\lambda_{I}\right\}_{I \in \mathcal{B}}$, define the linear operator

$$
\Psi_{\mathcal{B}, \boldsymbol{\lambda}}^{\mu, \mathbf{b}} f \equiv \sum_{I \in \mathcal{B}} \lambda_{I} \square_{I}^{\mu, \mathbf{b}} f
$$

which, with abuse of notation, we will refer to as a 'pseudoprojection'. In the event that all $\lambda_{I}=1$, we denote the operator by $\Psi_{\mathcal{B}}^{\mu, \mathbf{b}}$, and note that, despite the fact it is a sum of dual martingale averages, it is typically not a projection on $L^{2}(\mu)$.

Note that the failure of $\square_{I}^{\mu, \mathbf{b}}$ to be a projection in general is what motivated the introduction of the projections $\square_{I}^{\mu, \pi, \mathbf{b}}$ above. These projections have already played a significant role in the proof of the Monotonicity Lemma earlier, and will continue to play a role in other 'duality' situations below.

\subsubsection{An upper weak Riesz inequality.}

Proposition 95. Suppose that $\mathbf{b}$ is an $\infty$-weakly $\mu$-controlled accretive family on a grid $\mathcal{D}$. Then we have the following ' $\square_{I}^{\mu, \mathbf{b}}$-upper weak Riesz' inequality:

$$
\left\|\Psi_{\mathcal{B}, \boldsymbol{\lambda}}^{\mu, \mathbf{b}} f\right\|_{L^{2}(\mu)}^{2} \leq C\|\boldsymbol{\lambda}\|_{\infty}^{2}\left(\sum_{I \in \mathcal{B}}\left\|\square_{I}^{\mu, \mathbf{b}} f\right\|_{L^{2}(\mu)}^{2}+\sum_{I \in \mathcal{B}}\left\|\widehat{\nabla}_{I}^{\mu} f\right\|_{L^{2}(\mu)}^{2}\right)
$$

for all $f \in L^{2}(\mu)$ and all subsets $\mathcal{B}$ of the grid $\mathcal{D}$ and all sequences $\boldsymbol{\lambda}$,

where $\|\boldsymbol{\lambda}\|_{\infty} \equiv \sup _{I \in \mathcal{B}}\left|\lambda_{I}\right|$ and the positive constant $C$ is independent of the subset $\mathcal{B}$. In particular, the pseudoprojection $\Psi_{\mathcal{B}, \boldsymbol{\lambda}}^{\mu, \mathbf{b}}$ is a bounded linear operator on $L^{2}(\mu)$ if $\|\boldsymbol{\lambda}\|_{\infty}<\infty$ :

$$
\left\|\Psi_{\mathcal{B}, \boldsymbol{\lambda}}^{\mu, \mathbf{b}} f\right\|_{L^{2}(\mu)}^{2} \leq C\|\boldsymbol{\lambda}\|_{\infty}^{2}\|f\|_{L^{2}(\mu)}^{2}
$$


Proof. We may suppose that the subset $\mathcal{B}$ is finite provided the estimates we get are independent of the size of $\mathcal{B}$. Now let $g=\Psi_{\mathcal{B}, \boldsymbol{\lambda}}^{\mu, \mathbf{b}} f=\sum_{I \in \mathcal{B}} \lambda_{I} \square_{I}^{\mu, \mathbf{b}} f$. Then from (9.18) we have

$$
\begin{aligned}
\|g\|_{L^{2}(\mu)}^{2}= & \int\left(\sum_{I \in \mathcal{B}} \lambda_{I} \square_{I}^{\mu, \mathbf{b}} f\right) g d \mu=\int\left(\sum_{I \in \mathcal{B}} \lambda_{I}\left[\square_{I}^{\mu, \pi, \mathbf{b}} \square_{I}^{\mu, \pi, \mathbf{b}} f+\square_{I, \text { broken }}^{\mu, \pi, \mathbf{b}} f\right]\right) g d \mu \\
= & \sum_{I \in \mathcal{B}} \lambda_{I} \int\left(\square_{I}^{\mu, \pi, \mathbf{b}} f\right)\left(\square_{I}^{\mu, \pi, \mathbf{b}}\right)^{*} g d \mu+\sum_{I \in \mathcal{B}} \lambda_{I} \int\left(\square_{I, \text { broken }}^{\mu, \pi, \mathbf{b}} f\right) g d \mu \\
\lesssim & \|\boldsymbol{\lambda}\|_{\infty}\left(\sum_{I \in \mathcal{B}}\left\|\square_{I}^{\mu, \pi, \mathbf{b}} f\right\|_{L^{2}(\mu)}^{2}\right)^{\frac{1}{2}}\left(\sum_{I \in \mathcal{B}}\left\|\triangle_{I}^{\mu, \pi, \mathbf{b}} g\right\|_{L^{2}(\mu)}^{2}\right)^{\frac{1}{2}} \\
& +\|\boldsymbol{\lambda}\|_{\infty}\left(\sum_{I \in \mathcal{B}}\left\|\widehat{\nabla}_{I}^{\mu} f\right\|_{L^{2}(\mu)}^{2}\right)^{\frac{1}{2}}\left(\sum_{I \in \mathcal{B}}\left\|\widehat{\nabla}_{I}^{\mu} g\right\|_{L^{2}(\mu)}^{2}\right)^{\frac{1}{2}},
\end{aligned}
$$

where we have used (9.19) in the last line. Now using $\triangle_{I}^{\mu, \pi, \mathbf{b}}=\triangle_{I}^{\mu, \mathbf{b}}-\triangle_{I, \text { broken }}^{\mu, \pi, \mathbf{b}}$ and $\left|\triangle_{I, \text { broken }}^{\mu, \pi, \mathbf{b}} g\right| \leq C_{\mathbf{b}} \widehat{\nabla}_{I}^{\mu}|g|$, and $\square_{I}^{\mu, \pi, \mathbf{b}}=\square_{I}^{\mu, \mathbf{b}}-\square_{I, \text { broken }}^{\mu, \pi, \mathbf{b}}$, together with the lower frame inequalities in Proposition 91 (note that we do not use lower Riesz inequalities for $\triangle$ !)

$$
\begin{aligned}
& \sum_{I \in \mathcal{B}}\left\|\triangle_{I}^{\mu, \mathbf{b}} g\right\|_{L^{2}(\mu)}^{2} \leq \sum_{I \in \mathcal{D}}\left\|\triangle_{I}^{\mu, \mathbf{b} g}\right\|_{L^{2}(\mu)}^{2} \lesssim\|g\|_{L^{2}(\mu)}^{2}, \\
& \sum_{I \in \mathcal{B}}\left\|\widehat{\nabla}_{I}^{\mu}|g|\right\|_{L^{2}(\mu)}^{2} \leq \sum_{I \in \mathcal{D}}\left\|\widehat{\nabla}_{I}^{\mu}|g|\right\|_{L^{2}(\mu)}^{2} \lesssim\|g\|_{L^{2}(\mu)}^{2},
\end{aligned}
$$

we obtain

$$
\|g\|_{L^{2}(\mu)}^{2} \lesssim\|\boldsymbol{\lambda}\|_{\infty}\left(\sum_{I \in \mathcal{B}}\left\|\square_{I}^{\mu, \mathbf{b}} f\right\|_{L^{2}(\mu)}^{2}+\sum_{I \in \mathcal{B}}\left\|\widehat{\nabla}_{I}^{\mu} f\right\|_{L^{2}(\mu)}^{2}\right)^{\frac{1}{2}}\|g\|_{L^{2}(\mu)},
$$

which gives the desired upper weak Riesz inequality upon dividing through by the finite positive number $\|g\|_{L^{2}(\mu)}$, and then squaring the resulting inequality.

An analogous argument yields the next proposition. Recall that $\Psi_{\mathcal{B}, \boldsymbol{\lambda}}^{\mu, \mathbf{b}} f \equiv \sum_{I \in \mathcal{B}} \lambda_{I} \square_{I}^{\mu, \mathbf{b}} f$ and so

$$
\left(\Psi_{\mathcal{B}, \boldsymbol{\lambda}}^{\mu, \mathbf{b}}\right)^{*} f \equiv \sum_{I \in \mathcal{B}} \lambda_{I}\left(\square_{I}^{\mu, \mathbf{b}}\right)^{*} f=\sum_{I \in \mathcal{B}} \lambda_{I} \triangle_{I}^{\mu, \mathbf{b}} f
$$

Proposition 96. Suppose that $\mathbf{b}$ is an $\infty$-weakly $\mu$-controlled accretive family. Then we have the ' $\triangle_{I}^{\mu, \mathbf{b}}$ upper weak Riesz' inequality:

$$
\left\|\left(\Psi_{\mathcal{B}, \boldsymbol{\lambda}}^{\mu, \mathbf{b}}\right)^{*} f\right\|_{L^{2}(\mu)}^{2} \leq C\|\boldsymbol{\lambda}\|_{\infty}^{2}\left(\sum_{I \in \mathcal{B}}\left\|\triangle_{I}^{\mu, \mathbf{b}} f\right\|_{L^{2}(\mu)}^{2}+\sum_{I \in \mathcal{B}}\left\|\widehat{\nabla}_{I}^{\mu} f\right\|_{L^{2}(\mu)}^{2}\right)
$$

for all $f \in L^{2}(\mu)$ and all subsets $\mathcal{B}$ of the grid $\mathcal{D}$ and all sequences $\boldsymbol{\lambda}$,

and where the positive constant $C$ is independent of the subset $\mathcal{B}$ and the sequence $\boldsymbol{\lambda}$. In particular, the pseudoprojection $\left(\Psi_{\mathcal{B}, \boldsymbol{\lambda}}^{\mu, \mathbf{b}}\right)^{*}$ is a bounded linear operator on $L^{2}(\mu)$ :

$$
\left\|\left(\Psi_{\mathcal{B}, \boldsymbol{\lambda}}^{\mu, \mathbf{b}}\right)^{*} f\right\|_{L^{2}(\mu)}^{2} \leq C\|\boldsymbol{\lambda}\|_{\infty}^{2}\|f\|_{L^{2}(\mu)}^{2} .
$$

Proof. We may again suppose that the subset $\mathcal{B}$ is finite provided the estimates we get are independent of the size of $\mathcal{B}$. Now let $g=\sum_{I \in \mathcal{B}} \lambda_{I} \triangle_{I}^{\mu, \mathbf{b}} f$. Recall from (9.18) that $\triangle_{Q}^{\mu, \mathbf{b}}=\triangle_{Q}^{\mu, \pi, \mathbf{b}} \triangle_{Q}^{\mu, \pi, \mathbf{b}}+\triangle_{Q, \text { broken }}^{\mu, \mathbf{b}}$, and 
hence we have

$$
\begin{aligned}
\|g\|_{L^{2}(\mu)}^{2}= & \int\left(\sum_{I \in \mathcal{B}} \lambda_{I} \triangle_{I}^{\mu, \mathbf{b}} f\right) g d \mu=\int\left(\sum_{I \in \mathcal{B}} \lambda_{I}\left[\triangle_{I}^{\mu, \pi, \mathbf{b}} \triangle_{I}^{\mu, \pi, \mathbf{b}} f+\triangle_{I, \text { broken }}^{\mu, \pi, \mathbf{b}} f\right]\right) g d \mu \\
= & \sum_{I \in \mathcal{B}} \lambda_{I} \int\left(\triangle_{I}^{\mu, \pi, \mathbf{b}} f\right)\left(\triangle_{I}^{\mu, \pi, \mathbf{b}}\right)^{*} g d \mu+\sum_{I \in \mathcal{B}} \lambda_{I} \int\left(\triangle_{I, \text { broken }}^{\mu, \pi, \mathbf{b}} f\right) g d \mu \\
\lesssim & \|\boldsymbol{\lambda}\|_{\infty}\left(\sum_{I \in \mathcal{B}}\left\|\triangle_{I}^{\mu, \pi, \mathbf{b}} f\right\|_{L^{2}(\mu)}^{2}\right)^{\frac{1}{2}}\left(\sum_{I \in \mathcal{B}}\left\|\square_{I}^{\mu, \pi, \mathbf{b}} g\right\|_{L^{2}(\mu)}^{2}\right)^{\frac{1}{2}} \\
& +\|\boldsymbol{\lambda}\|_{\infty}\left(\sum_{I \in \mathcal{B}}\left\|\widehat{\nabla}_{I}^{\mu} f\right\|_{L^{2}(\mu)}^{2}\right)^{\frac{1}{2}}\left(\sum_{I \in \mathcal{B}}\left\|\nabla_{I}^{\mu} g\right\|_{L^{2}(\mu)}^{2}\right)^{\frac{1}{2}} .
\end{aligned}
$$

Now we continue as in the proof of Propostion 95, but using instead the lower frame inequality (9.13), to obtain

$$
\|g\|_{L^{2}(\mu)}^{2} \lesssim\|\boldsymbol{\lambda}\|_{\infty}\left(\sum_{I \in \mathcal{B}}\left\|\triangle_{I}^{\mu, \mathbf{b}} f\right\|_{L^{2}(\mu)}^{2}+\sum_{I \in \mathcal{B}}\left\|\widehat{\nabla}_{I}^{\mu} f\right\|_{L^{2}(\mu)}^{2}\right)\|g\|_{L^{2}(\mu)}
$$

which completes the proof of Propostion 96 upon dividing through by $\|g\|_{L^{2}(\mu)}$ and squaring.

Remark 97. The boundedness of the pseudoprojections $\Psi_{\mathcal{B}}^{\mu, \mathbf{b}}$ and $\left(\Psi_{\mathcal{B}}^{\mu, \mathbf{b}}\right)^{*}$ on $L^{2}(\mu)$ (where the absence of the sequence $\boldsymbol{\lambda}$ in the subscript implies all $\lambda_{I}=1$ ) given by 9.27) and (9.28), can fail for a 2-weakly $\mu$-controlled accretive family on a grid $\mathcal{D}$. Indeed, if (9.28) holds, then for a 2-weakly $\mu$-controlled accretive family,

$$
\left\|\sum_{I \in \mathcal{B}_{+}} \triangle_{I}^{\mu, \mathbf{b}} f-\sum_{I \in \mathcal{B}_{-}} \triangle_{I}^{\mu, \mathbf{b}} f\right\|_{L^{2}(\mu)}^{2} \leq 2\left\|\sum_{I \in \mathcal{B}_{+}} \triangle_{I}^{\mu, \mathbf{b}} f\right\|_{L^{2}(\mu)}^{2}+2\left\|\sum_{I \in \mathcal{B}_{-}} \triangle_{I}^{\mu, \mathbf{b}} f\right\|_{L^{2}(\mu)}^{2} \leq C\|f\|_{L^{2}(\mu)}^{2}
$$

holds for all decompositions of $\mathcal{B}$ into a disjoint union $\mathcal{B}=\mathcal{B}_{+} \cup \mathcal{B}_{-}$. Then

$$
\sum_{I \in \mathcal{B}}\left\|\triangle_{I}^{\mu, \mathbf{b}} f\right\|_{L^{2}(\mu)}^{2}=\mathbb{E}_{ \pm}\left\|\sum_{I \in \mathcal{B}} \pm \triangle_{I}^{\mu, \mathbf{b}} f\right\|_{L^{2}(\mu)}^{2} \leq \mathbb{E}_{ \pm} C\|f\|_{L^{2}(\mu)}^{2}=C\|f\|_{L^{2}(\mu)}^{2}
$$

which contradicts the example of Hytönen and Martikainen in [HyMa, Section 3.9] if $p=2$. Thus in order to prove a two weight local Tb theorem for $p=2$, one cannot appeal in general to the boundedness of pseudoprojections $\left(\Psi_{\mathcal{B}, \boldsymbol{\lambda}}^{\mu, \mathbf{b}}\right)^{*}=\sum_{I \in \mathcal{B}} \lambda_{I} \triangle_{I}^{\mu, \mathbf{b}}$ on $L^{2}(\mu)$ even when $\lambda_{I}=1$ for all $I \in \mathcal{B}$.

9.4.2. A lower weak Riesz inequality. The next proposition also assumes an $\infty$-weakly $\mu$-controlled accretive family on a grid $\mathcal{D}$. For a subset $\mathcal{B}$ of a dyadic grid $\mathcal{D}$, let

$$
\mathrm{P}_{\mathcal{B}}^{\mu} f=\sum_{Q \in \mathcal{B}} \triangle_{Q}^{\mu} f
$$

denote the orthogonal projection of $f$ onto the closed linear span of the collection $\left\{\triangle_{Q}^{\mu}\right\}_{Q \in \mathcal{B}}$ of Haar projections $\triangle_{Q}^{\mu}$ with $Q \in \mathcal{B}$. We obtain an appropriate form of a weak lower Riesz inequality for the dual martingale differences $\square_{Q}^{\mu, \mathbf{b}}$ since for these operators we no longer need the disruptive device of introducing the function $b$ in (9.11) above. 
Proposition 98. Suppose that $\mathbf{b}$ is an $\infty$-weakly $\mu$-controlled accretive family. Then we have a 'weak lower Riesz' inequality for dual martingale differences:

$$
\begin{aligned}
& \sum_{Q \in \mathcal{B}}\left\|\square_{Q}^{\mu, \mathbf{b}} f\right\|_{L^{2}(\mu)}^{2} \leq C\left(\left\|\mathrm{P}_{\mathcal{B}}^{\mu} f\right\|_{L^{2}(\mu)}^{2}+\sum_{Q \in \mathcal{B}}\left\|\hat{\nabla}_{Q}^{\mu} f\right\|_{L^{2}(\mu)}^{2}+\sum_{Q \in \mathcal{B}} \gamma_{Q}\left|E_{Q}^{\mu} f\right|^{2}\right) \\
& \text { for all } f \in L^{2}(\mu) \text { and all subsets } \mathcal{B} \text { of the grid } \mathcal{D} \text {, and where }\left\|\mathrm{P}_{\mathcal{B}}^{\mu} f\right\|_{L^{2}(\mu)}^{2}=\sum_{Q \in \mathcal{B}}\left\|\triangle_{Q}^{\mu} f\right\|_{L^{2}(\mu)}^{2} .
\end{aligned}
$$

and where the positive constant $C$ depends only on the accretivity constants, but is independent of the subset $\mathcal{B}$ and the testing family $\mathbf{b}$. Here the coefficients $\left\{\gamma_{Q}\right\}_{Q \in \mathcal{D}}$ form a Carleson sequence indexed by $\mathcal{D}$, i.e.

$$
\sum_{Q \in \mathcal{D}: Q \subset P} \gamma_{Q} \leq C|P|_{\mu}, \quad \text { for all } P \in \mathcal{D} .
$$

The third term on the right hand side above is additive in $\mathcal{B}$ and, by the Carleson embedding theorem, satisfies

$$
\sum_{Q \in \mathcal{D}} \gamma_{Q}\left|E_{Q}^{\mu} f\right|^{2} \lesssim\|f\|_{L^{2}(\mu)}^{2}
$$

Proof. The main modifications in the proof of Proposition 91 that are needed here, are that we use $\square_{Q}^{\mu, \mathbf{b}}$ in place of $\triangle_{Q}^{\mu, \mathbf{b}}$, which results in the testing functions $b_{Q}$ appearing outside the integrals rather than inside the integrals, and that we restrict the sums over $Q$ to $\mathcal{B}$, which results in the presence of the term $\sum_{Q \in \mathcal{B}} \gamma_{Q}\left|E_{Q}^{\mu} f\right|^{2}$ on the right hand side above. Since we are working with $\square_{Q}^{\mu, \mathbf{b}}$ we will not need the extra complications arising from the introduction of the function $b$ in (9.11). With these modifications in mind, we now describe the estimates we obtain for the terms analogous to $I I_{A}, I I I_{A}$ and $I V_{A}$ in the proof of Proposition 91, Given $A \in \mathcal{A}$, we begin with

$$
\begin{aligned}
\sum_{Q \in \mathcal{B} \cap \mathcal{C}_{A}}\left\|\square_{Q}^{\mu, \mathbf{b}} f\right\|_{L^{2}(\mu)}^{2}= & \sum_{Q \in \mathcal{B} \cap \mathcal{C}_{A}} \sum_{Q^{\prime} \in \mathfrak{C}(Q)} \int_{Q^{\prime}}\left|\mathbb{F}_{Q^{\prime}}^{\mu, \mathbf{b}} f(x)-\mathbb{F}_{Q}^{\mu, \mathbf{b}} f(x)\right|^{2} d \mu(x) \\
= & \sum_{Q \in \mathcal{B} \cap \mathcal{C}_{A}} \sum_{Q^{\prime} \in \mathfrak{C}_{\text {natural }}(Q)}\left|\frac{b_{A} \int_{Q^{\prime}} f d \mu}{\int_{Q^{\prime}} b_{A} d \mu}-\frac{b_{A} \int_{Q} f d \mu}{\int_{Q} b_{A} d \mu}\right|^{2}\left|Q^{\prime}\right|_{\mu} \\
& +\sum_{Q \in \mathcal{B} \cap \mathcal{C}_{A}} \sum_{Q^{\prime} \in \mathfrak{C}_{\text {broken }}(Q)}\left|\frac{b_{Q^{\prime}} \int_{Q^{\prime}} f d \mu}{\int_{Q^{\prime}} b_{Q^{\prime}} d \mu}-\frac{b_{A} \int_{Q} f d \mu}{\int_{Q} b_{A} d \mu}\right|^{2}\left|Q^{\prime}\right|_{\mu} \\
\equiv & I_{A}+I I_{A} .
\end{aligned}
$$

To estimate term $I I_{A}$ we write

$$
\begin{aligned}
& \left|\frac{b_{Q^{\prime}} \int_{Q^{\prime}} f d \mu}{\int_{Q^{\prime}} b_{Q^{\prime}} d \mu}-\frac{b_{A} \int_{Q} f d \mu}{\int_{Q} b_{A} d \mu}\right| \\
= & \frac{\left|\left(\frac{1}{\left|Q^{\prime}\right|_{\mu}} b_{Q^{\prime}} \int_{Q^{\prime}} f d \mu\right)\left(\frac{1}{|Q|_{\mu}} \int_{Q} b_{A} d \mu\right)-\left(\frac{1}{|Q|_{\mu}} b_{A} \int_{Q} f d \mu\right)\left(\frac{1}{\left|Q^{\prime}\right|_{\mu}} \int_{Q^{\prime}} b_{Q^{\prime}} d \mu\right)\right|}{\left|\frac{1}{\left|Q^{\prime}\right|_{\mu}} \int_{Q^{\prime}} b_{Q^{\prime}} d \mu\right|\left|\frac{1}{|Q|_{\mu}} \int_{Q} b_{A} d \mu\right|} \\
\lesssim & \left|b_{Q^{\prime}}\left(E_{Q^{\prime}}^{\mu} f\right)\left(E_{Q}^{\mu} b_{A}\right)-b_{A}\left(E_{Q}^{\mu} f\right)\left(E_{Q^{\prime}}^{\mu} b_{Q^{\prime}}\right)\right| \\
= & \left|b_{Q^{\prime}}\left(E_{Q^{\prime}}^{\mu} f\right)\left(E_{Q}^{\mu} b_{A}\right)-b_{A}\left(E_{Q^{\prime}}^{\mu} f\right)\left(E_{Q^{\prime}}^{\mu} b_{Q^{\prime}}\right)-\left[b_{A}\left(E_{Q}^{\mu} f\right)-b_{A}\left(E_{Q^{\prime}}^{\mu} f\right)\right]\left(E_{Q^{\prime}}^{\mu} b_{Q^{\prime}}\right)\right| \\
\lesssim & \left|b_{Q^{\prime}}\left(E_{Q^{\prime}}^{\mu} f\right)\right|+\left|b_{A}\left(E_{Q^{\prime}}^{\mu} f\right)\right|+\left|b_{A}\left(E_{Q}^{\mu} f\right)-b_{A}\left(E_{Q^{\prime}}^{\mu} f\right)\right|, \\
\lesssim & \left|E_{Q^{\prime}}^{\mu} f\right|+\left|E_{Q^{\prime}}^{\mu} f\right|+\left|\left(E_{Q}^{\mu} f\right)-\left(E_{Q^{\prime}}^{\mu} f\right)\right|,
\end{aligned}
$$


and then

$$
I I_{A} \lesssim \sum_{Q \in \mathcal{B} \cap \mathcal{C}_{A}} \sum_{Q^{\prime} \in \mathfrak{C}_{\text {broken }}(Q)}\left(\left|E_{Q^{\prime}}^{\mu} f\right|^{2}+\left|E_{Q}^{\mu} f\right|^{2}\right)\left|Q^{\prime}\right|_{\mu} \approx \sum_{Q \in \mathcal{B} \cap \mathcal{C}_{A}}\left\|\widehat{\nabla}_{Q}^{\mu} f\right\|_{L^{2}(\mu)}^{2} .
$$

Now we write

$$
\begin{aligned}
I_{A} & =\sum_{Q \in \mathcal{B} \cap \mathcal{C}_{A}} \sum_{Q^{\prime} \in \mathfrak{C}_{\text {natural }}(Q)}\left|\frac{b_{A} \int_{Q^{\prime}} f d \mu}{\int_{Q^{\prime}} b_{A} d \mu}-\frac{b_{A} \int_{Q} f d \mu}{\int_{Q} b_{A} d \mu}\right|^{2}\left|Q^{\prime}\right|_{\mu} \\
= & \sum_{Q \in \mathcal{B} \cap \mathcal{C}_{A}} \sum_{Q^{\prime} \in \mathfrak{C}_{\text {natural }}(Q)}\left|\frac{\left(b_{A} \int_{Q^{\prime}} f d \mu\right)\left(\int_{Q} b_{A} d \mu\right)-\left(\int_{Q^{\prime}} b_{A} d \mu\right)\left(b_{A} \int_{Q} f d \mu\right)}{\left(\int_{Q^{\prime}} b_{A} d \mu\right)\left(\int_{Q} b_{A} d \mu\right)}\right|^{2}\left|Q^{\prime}\right|_{\mu} \\
& \lesssim \sum_{Q \in \mathcal{B} \cap \mathcal{C}_{A}} \sum_{Q^{\prime} \in \mathfrak{C}_{\text {natural }}(Q)}\left|E_{Q}^{\mu} b_{A}\right|^{2}\left|E_{Q^{\prime}}^{\mu} f-E_{Q}^{\mu} f\right|^{2}\left|Q^{\prime}\right|_{\mu} \\
& +\sum_{Q \in \mathcal{B} \cap \mathcal{C}_{A} Q_{Q^{\prime} \in \mathfrak{C}_{\text {natural }}(Q)}} \sum_{E^{\mu} b_{A}-\left.E_{Q^{\prime}}^{\mu} b_{A}\right|^{2}\left|E_{Q}^{\mu} f\right|^{2}\left|Q^{\prime}\right|_{\mu}} \\
& \equiv I I I_{A}+I V_{A} .
\end{aligned}
$$

Then we use the following stronger form of an inequality used in the proof of the unweighted square function estimate in Lemma 90, namely

$$
\sum_{Q \in \mathcal{B} \cap \mathcal{C}_{A}} \sum_{Q^{\prime} \in \mathfrak{C}_{\text {natural }}(Q)}\left|Q^{\prime}\right|_{\mu}\left|E_{Q^{\prime}}^{\mu} h-E_{Q}^{\mu} h\right|^{2} \lesssim \sum_{Q \in \mathcal{B} \cap \mathcal{C}_{A}}\left\|\triangle_{Q}^{\mu} h\right\|_{L^{2}(\mu)}^{2} \lesssim\left\|\mathrm{P}_{\mathcal{B} \cap \mathcal{C}_{A}}^{\mu} h\right\|_{L^{2}(\mu)}^{2},
$$

to dominate term $I I I_{A}$ by

$$
I I I_{A} \lesssim\left\|b_{A} \mathrm{P}_{\mathcal{B} \cap \mathcal{C}_{A}}^{\mu} f\right\|_{L^{2}(\mu)}^{2} \lesssim\left\|\mathrm{P}_{\mathcal{B} \cap \mathcal{C}_{A}}^{\mu} f\right\|_{L^{2}(\mu)}^{2}
$$

and we write term $I V_{A}$ as

$$
\begin{aligned}
I V_{A} & =\sum_{Q \in \mathcal{B} \cap \mathcal{C}_{A}} \gamma_{Q}\left|E_{Q}^{\mu} f\right|^{2}, \\
\text { where } \gamma_{Q} & \equiv \sum_{Q^{\prime} \in \mathfrak{C}_{\text {natural }}(Q)}\left|E_{Q}^{\mu} b_{A}-E_{Q^{\prime}}^{\mu} b_{A}\right|^{2}\left|Q^{\prime}\right|_{\mu}, \quad \text { for } Q \in \mathcal{C}_{A}, A \in \mathcal{A} .
\end{aligned}
$$

Now we sum over $A \in \mathcal{A}$ to obtain

$$
\begin{aligned}
\sum_{Q \in \mathcal{B}}\left\|\square_{Q}^{\mu, \mathbf{b}} f\right\|_{L^{2}(\mu)}^{2} & =\sum_{A \in \mathcal{A}} \sum_{Q \in \mathcal{B} \cap \mathcal{C}_{A}}\left\|\square_{Q}^{\mu, \mathbf{b}} f\right\|_{L^{2}(\mu)}^{2} \lesssim \sum_{A \in \mathcal{A}}\left(I I_{A}+I I I_{A}+I V_{A}\right) \\
& \lesssim \sum_{Q \in \mathcal{B}}\left\|\widehat{\nabla}_{Q}^{\mu, \mathbf{b}} f\right\|_{L^{2}(\mu)}^{2}+\left\|\mathrm{P}_{\mathcal{B}}^{\mu} f\right\|_{L^{2}(\mu)}^{2}+\sum_{Q \in \mathcal{B}} \gamma_{Q}\left|E_{Q}^{\mu} f\right|^{2},
\end{aligned}
$$

where the sequence $\left\{\gamma_{Q}\right\}_{Q \in \mathcal{D}}$ satisfies the Carleson condition by (9.10) in the proof of Proposition 91

Remark 99. We are unable to obtain a corresponding lower weak Riesz inequality for the martingale differences $\triangle_{Q}^{\mu, \mathbf{b}}$ due to the need for introducing the function b in (9.11) as in the proof of Proposition 91, which does not interact well with $\mathcal{B}$ - see the argument surrounding 9.12) in the proof of Proposition 91. However, lower weak Riesz inequalities for the martingale differences $\triangle_{Q}^{\mu, \mathbf{b}}$ are not needed in this paper - in fact, only upper weak Riesz inequalities are needed for both $\square_{Q}^{\mu, \mathbf{b}}$ and $\triangle_{Q}^{\mu, \mathbf{b}}$.

\section{Appendix B: Control of functional energy}

Now we arrive at one of the main propositions used in the proof of our theorem. This result is proved independently of the main theorem, and only using the results on dual martingale differences established in the previous appendix. The organization of the proof is almost identical to that of the corresponding result in [SaShUr7, pages 128-151], together with the modifications in [SaShUr9, pages 348-360] to accommodate common point masses, but we repeat the organization here with modifications required for the use of two

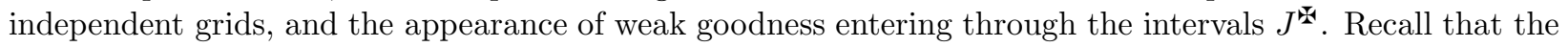


functional energy constant $\mathfrak{F}_{\alpha}=\mathfrak{F}_{\alpha}^{\mathbf{b}^{*}}(\mathcal{D}, \mathcal{G})$ in $\underline{6.6}, 0 \leq \alpha<n$, namely the best constant in the inequality (see (10.6) below for the definition of $\mathcal{W}(F)$ ),

$$
\sum_{F \in \mathcal{F}} \sum_{M \in \mathcal{W}(F)}\left(\frac{\mathrm{P}^{\alpha}(M, h \sigma)}{|M|}\right)^{2}\left\|\mathrm{Q}_{\mathcal{C}_{F}^{\mathcal{G}, \text { shift }} ; M}^{\omega, \mathbf{b}^{*}} x\right\|_{L^{2}(\omega)}^{\mathbf{a} 2} \leq \mathfrak{F}_{\alpha}\|h\|_{L^{2}(\sigma)},
$$

depends on the grids $\mathcal{D}$ and $\mathcal{G}$, the goodness parameter $\varepsilon>0$ used in the definition of $J^{\mathcal{*}}$ through the shifted corona $\mathcal{C}_{F}^{\mathcal{G} \text {,shift }}$, and on the family of martingale differences $\left\{\triangle_{J}^{\omega, \mathbf{b}^{*}}\right\}_{J \in \mathcal{G}}$ associated with $x \in L_{\text {loc }}^{2}(\omega)$, but not on the family of dual martingale differences $\left\{\square_{I}^{\sigma, \mathbf{b}}\right\}_{I \in \mathcal{D}}$, since the function $h \in L^{2}(\sigma)$ appearing in the definition of functional energy is not decomposed as a sum of pseudoprojections $\square_{I}^{\sigma, \mathbf{b}} h$. Finally, we emphasize that the pseudoprojection

$$
\mathrm{Q}_{\mathcal{C}_{F}^{\mathcal{G}, \text { shift } ; M}}^{\omega, \mathbf{b}^{*}} \equiv \sum_{J \in \mathcal{C}_{F}^{\mathcal{G}, \text { shift }}: J \subset M} \triangle_{J}^{\omega, \mathbf{b}^{*}}
$$

here uses the shifted restricted corona in

$$
\begin{aligned}
\mathcal{C}_{F}^{\mathcal{G}, \text { shift }} & =\left\{J \in \mathcal{G}: J^{\mathscr{W}} \in \mathcal{C}_{F}^{\mathcal{D}}\right\}, \\
\mathcal{C}_{F}^{\mathcal{G}, \text { shift }} ; K & \equiv\left\{J \in \mathcal{C}_{F}^{\mathcal{G}, \text { shift }}: J \subset K\right\},
\end{aligned}
$$

where $J^{\text {is }}$ is defined using the body of an interval as in Definition 42, and where we have defined here the 'restriction' $\mathcal{C}_{F}^{\mathcal{G} \text {,shift }} ; K$ to the interval $K$ of the corona $\mathcal{C}_{F}^{\mathcal{G} \text {,shift }}$ (c.f. $\Pi_{2}^{K} \mathcal{P}$ in Definition 69 , which uses the stronger requirement $\left.J^{\omega} \subset K\right)$. Moreover, recall from Notation 32 and the definition of $\nabla_{J}^{\omega}$ in $(\underline{9.6})$, that for any subset $\mathcal{H}$ of the grid $\mathcal{G}$,

$$
\left\|\mathrm{Q}_{\mathcal{H}}^{\omega, \mathbf{b}^{*}} x\right\|_{L^{2}(\omega)}^{\mathbf{\omega} 2} \equiv \sum_{J \in \mathcal{H}}\left\|\triangle_{J}^{\omega, \mathbf{b}^{*}} x\right\|_{L^{2}(\omega)}^{\mathbf{\$} 2}=\sum_{J \in \mathcal{H}}\left(\left\|\triangle_{J}^{\omega, \mathbf{b}^{*}} x\right\|_{L^{2}(\omega)}^{2}+\inf _{z \in \mathbb{R}}\left\|\widehat{\nabla}_{J}^{\omega}(x-z)\right\|_{L^{2}(\omega)}^{2}\right),
$$

so that we never need to consider the norm squared $\left\|\mathrm{Q}_{\mathcal{C}_{F}^{\mathcal{G}, \text { shift }} ; M}^{\omega}\right\|_{L^{2}(\omega)}^{2}$ of the pseudoprojection $\mathrm{Q}_{\mathcal{C}_{F}^{\mathcal{G}}}^{\omega, \mathbf{b}^{*} \text { shift } ; M}{ }^{x,}$ something for which we have no lower Riesz inequality. Note moreover that for $J \in \mathcal{G}$ and an arbitrary interval $K$, we have by the frame inequality in Proposition 91 .

$$
\begin{aligned}
\sum_{J \in \mathcal{G}: J \subset K}\left\|\triangle_{J}^{\omega, \mathbf{b}^{*}} x\right\|_{L^{2}(\omega)}^{2} & \lesssim\left\|x-m_{K}^{\omega}\right\|_{L^{2}\left(\mathbf{1}_{K} \omega\right)}^{2}, \\
\sum_{J \in \mathcal{G}: J \subset K} \inf _{z \in \mathbb{R}}\left\|\hat{\nabla}_{J}^{\omega}(x-z)\right\|_{L^{2}(\omega)}^{2} & \leq \sum_{J \in \mathcal{G}: J \subset K}\left\|\widehat{\nabla}_{J}^{\omega}\left\{(x-p) \mathbf{1}_{K}(x)\right\}\right\|_{L^{2}(\omega)}^{2} \lesssim\|(x-p)\|_{L^{2}\left(\mathbf{1}_{K} \omega\right)}^{2}, \quad p \in K,
\end{aligned}
$$

where the second line follows from (9.7).

Important note: If $J \in \mathcal{C}_{F}^{\mathcal{G} \text {,shift }}$, then in particular $J \Subset_{\boldsymbol{\rho}, \varepsilon} F$ with $\boldsymbol{\rho}=\left[\frac{3}{\varepsilon}\right]$ by Lemma 59, and so $J \cap M \neq \emptyset$ for a unique $M \in \mathcal{W}(F)$.

We will show that, uniformly in pairs of grids $\mathcal{D}$ and $\mathcal{G}$, the functional energy constants $\mathfrak{F}_{\alpha}(\mathcal{D}, \mathcal{G})$ in $(6.6)$ are controlled by $\mathcal{A}_{2}^{\alpha}, A_{2}^{\alpha, \text { punct }}$ and the large energy constant $\mathfrak{E}_{2}^{\alpha}$ - actually the proof shows that we have control by the Whitney plugged energy constant as defined in (10.15) below. More precisely this is our control of functional energy proposition.

Proposition 100. For all grids $\mathcal{D}$ and $\mathcal{G}$, and $\varepsilon>0$ sufficiently small, we have

$$
\begin{aligned}
\mathfrak{F}_{\alpha}^{\mathbf{b}^{*}}(\mathcal{D}, \mathcal{G}) & \lesssim \mathfrak{E}_{2}^{\alpha}+\sqrt{\mathcal{A}_{2}^{\alpha}}+\sqrt{\mathcal{A}_{2}^{\alpha, *}}+\sqrt{A_{2}^{\alpha, \text { punct }}} \\
\mathfrak{F}_{\alpha}^{\mathbf{b}, *}(\mathcal{G}, \mathcal{D}) & \lesssim \mathfrak{E}_{2}^{\alpha, *}+\sqrt{\mathcal{A}_{2}^{\alpha}}+\sqrt{\mathcal{A}_{2}^{\alpha, *}}+\sqrt{A_{2}^{\alpha, *, \text { punct }}}
\end{aligned}
$$

with implied constants independent of the grids $\mathcal{D}$ and $\mathcal{G}$.

In order to prove this proposition, we first turn to recalling these more refined notions of energy constants. 
10.1. Various energy conditions. In this subsection we recall various refinements of the strong energy conditions appearing in the main theorem above. Variants of this material already appear in earlier papers, but we repeat it here both for convenience and in order to introduce some arguments we will use repeatedly later on. These refinements represent the 'weakest' energy side conditions that suffice for use in our proof, but despite this, we will usually use the large energy constant $\mathfrak{E}_{2}^{\alpha}$ in estimates to avoid having to pay too much attention to which of the energy conditions we need to use - leaving the determination of the weakest conditions in such situations to the interested reader. We begin with the notion of 'deeply embedded'. Recall that the goodness parameter $\mathbf{r} \in \mathbb{N}$ is determined by $\varepsilon>0$ in (3.19), and that $0<\varepsilon<\frac{1}{2}<\frac{1}{2-\alpha}$.

For arbitrary intervals in $J, K \in \mathcal{P}$, we say that $J$ is $(\boldsymbol{\rho}, \varepsilon)$-deeply embedded in $K$, which we write as $J \Subset_{\rho, \varepsilon} K$, when $J \subset K$ and both

$$
\begin{aligned}
\ell(J) & \leq 2^{-\boldsymbol{\rho} \ell}(K), \\
d(J, \partial K) & \geq 2 \ell(J)^{\varepsilon} \ell(K)^{1-\varepsilon} .
\end{aligned}
$$

Note that we use the boundary of $K$ for the definition of $J \Subset_{\rho, \varepsilon} K$, rather than the skeleton or body of $K$, which would result in a more restrictive notion of $(\boldsymbol{\rho}, \varepsilon)$-deeply embedded. We will use this notion for the purpose of grouping $\varepsilon-$ good intervals into the following collections. Fix grids $\mathcal{D}$ and $\mathcal{G}$. For $K \in \mathcal{D}$, define the collections,

$$
\begin{aligned}
\mathcal{M}_{(\boldsymbol{\rho}, \varepsilon)-\operatorname{deep}, \mathcal{G}}(K) & \equiv\left\{J \in \mathcal{G}: J \text { is maximal w.r.t } J \Subset_{\boldsymbol{\rho}, \varepsilon} K\right\}, \\
\mathcal{M}_{(\boldsymbol{\rho}, \varepsilon)-\operatorname{deep}, \mathcal{D}}(K) & \equiv\left\{M \in \mathcal{D}: M \text { is maximal w.r.t } M \Subset_{\boldsymbol{\rho}, \varepsilon} K\right\}, \\
\mathcal{W}(K) & \equiv\{M \in \mathcal{D}: M \text { is maximal w.r.t } 3 M \subset K\}
\end{aligned}
$$

where the first two consist of maximal $(\rho, \varepsilon)$-deeply embedded dyadic $\mathcal{G}$-subintervals $J$, respectively $\mathcal{D}$ subintervals $M$, of a $\mathcal{D}$-interval $K$, and the third consists of the maximal $\mathcal{D}$-subintervals $M$ whose triples are contained in $K$.

Let $\gamma>1$. Then the following bounded overlap property holds where $\mathcal{M}_{(\boldsymbol{\rho}, \varepsilon) \text {-deep }}(K)$ can be taken to be either $\mathcal{M}_{(\boldsymbol{\rho}, \varepsilon)-\mathrm{deep}, \mathcal{G}}(K)$ or $\mathcal{M}_{(\boldsymbol{\rho}, \varepsilon)-\mathrm{deep}, \mathcal{D}}(K)$ or $\mathcal{W}(K)$ throughout.

Lemma 101. Let $0<\varepsilon \leq 1<\gamma \leq 1+4 \cdot 2^{\rho(1-\varepsilon)}$. Then

$$
\sum_{J \in \mathcal{M}_{(\boldsymbol{\rho}, \varepsilon)-\text { deep }}(K)} \mathbf{1}_{\gamma J} \leq \beta \mathbf{1}\left[\bigcup_{J \in \mathcal{M}_{(\boldsymbol{\rho}, \varepsilon)-\operatorname{deep}}(K)} \gamma J\right]
$$

holds for some positive constant $\beta$ depending only on $\gamma, \boldsymbol{\rho}$ and $\varepsilon$. In addition $\gamma J \subset K$ for all $J \in$ $\mathcal{M}_{(\rho, \varepsilon)-\operatorname{deep}}(K)$, and consequently

$$
\sum_{J \in \mathcal{M}_{(\rho, \varepsilon) \text {-deep }}(K)} \mathbf{1}_{\gamma J} \leq \beta \mathbf{1}_{K}
$$

A similar result holds for $\mathcal{W}(K)$.

Proof. We suppose $0<\varepsilon<1$ and leave the simpler case $\varepsilon=1$ for the reader. To prove (10.7), we first note that there are at most $2^{\rho+1}$ intervals $J$ contained in $K$ for which $\ell(J)>2^{-\rho} \ell(K)$. On the other hand, the maximal $(\boldsymbol{\rho}, \varepsilon)$-deeply embedded subintervals $J$ of $K$ also satisfy the comparability condition

$$
2 \ell(J)^{\varepsilon} \ell(K)^{1-\varepsilon} \leq d(J, \partial K) \leq d(\pi J, \partial K)-\ell(J) \leq 2(2 \ell(J))^{\varepsilon} \ell(K)^{1-\varepsilon}-\ell(J) \leq 4 \ell(J)^{\varepsilon} \ell(K)^{1-\varepsilon}-\ell(J) .
$$

Now with $0<\varepsilon<1$ and $\gamma>1$ fixed, let $y \in K$. Then if $y \in \gamma J$, we have

$$
\begin{aligned}
2 \ell(J)^{\varepsilon} \ell(K)^{1-\varepsilon} & \leq d(J, \partial K) \leq \gamma \ell(J)+d(\gamma J, \partial K) \\
& \leq \gamma \ell(J)+d(y, \partial K) .
\end{aligned}
$$

Now assume that $\frac{\ell(J)}{\ell(K)} \leq\left(\frac{1}{\gamma}\right)^{\frac{1}{1-\varepsilon}}$. Then we have $\gamma \ell(J) \leq \ell(J)^{\varepsilon} \ell(K)^{1-\varepsilon}$ and so

$$
\ell(J)^{\varepsilon} \ell(K)^{1-\varepsilon} \leq d(y, \partial K) .
$$

But we also have

$$
d(y, \partial K) \leq \ell(J)+d(J, \partial K) \leq \ell(J)+4 \ell(J)^{\varepsilon} \ell(K)^{1-\varepsilon}-\ell(J) \leq 4 \ell(J)^{\varepsilon} \ell(K)^{1-\varepsilon},
$$


and so altogether, under the assumption that $\frac{\ell(J)}{\ell(K)} \leq\left(\frac{1}{\gamma}\right)^{\frac{1}{1-\varepsilon}}$, we have

$$
\begin{aligned}
\frac{1}{4} d(y, \partial K) & \leq \ell(J)^{\varepsilon} \ell(K)^{1-\varepsilon} \leq d(y, \partial K), \\
\text { i.e. }\left(\frac{1}{4} \frac{d(y, \partial K)}{\ell(K)^{1-\varepsilon}}\right)^{\frac{1}{\varepsilon}} & \leq \ell(J) \leq\left(\frac{d(y, \partial K)}{\ell(K)^{1-\varepsilon}}\right)^{\frac{1}{\varepsilon}},
\end{aligned}
$$

which shows that the number of $J^{\prime} s$ satisfying $y \in \gamma J$ and $\frac{\ell(J)}{\ell(K)} \leq\left(\frac{1}{\gamma}\right)^{\frac{1}{1-\varepsilon}}$ is at most $C^{\prime} \frac{1}{\varepsilon}$. On the other hand, the number of $J^{\prime} s$ contained in $K$ satisfying $y \in \gamma J$ and $\frac{\ell(J)}{\ell(K)}>\left(\frac{1}{\gamma}\right)^{\frac{1}{1-\varepsilon}}$ is at most $C^{\prime} \frac{1}{1-\varepsilon}\left(1+\log _{2} \gamma\right)$. This proves (10.7) with

$$
\beta=2^{\rho+1}+C^{\prime} \frac{1}{\varepsilon}+C^{\prime} \frac{1}{1-\varepsilon}\left(1+\log _{2} \gamma\right) .
$$

In order to prove (10.8) it suffices, by (10.7), to prove $\gamma J \subset K$ for all $J \in \mathcal{M}_{(\boldsymbol{\rho}, \varepsilon) \text {-deep }}(K)$. But $J \in \mathcal{M}_{(\rho, \varepsilon)-\text { deep }}(K)$ implies

$$
2 \ell(J)^{\varepsilon} \ell(K)^{1-\varepsilon} \leq d(J, \partial K)=d\left(c_{J}, \partial K\right)+\frac{1}{2} \ell(J) .
$$

We wish to show $\gamma J \subset K$, which is implied by

$$
\gamma \frac{1}{2} \ell(J) \leq d\left(c_{J}, K^{c}\right)=d(J, \partial K)+\frac{1}{2} \ell(J) .
$$

But we have

$$
d(J, \partial K)+\frac{1}{2} \ell(J) \geq 2 \ell(J)^{\varepsilon} \ell(K)^{1-\varepsilon}+\frac{1}{2} \ell(J),
$$

and so it suffices to show that

$$
2 \ell(J)^{\varepsilon} \ell(K)^{1-\varepsilon}+\frac{1}{2} \ell(J) \geq \gamma \frac{1}{2} \ell(J)
$$

which is equivalent to

$$
\gamma-1 \leq 4 \ell(J)^{\varepsilon-1} \ell(K)^{1-\varepsilon} .
$$

But the smallest that $\ell(J)^{\varepsilon-1} \ell(K)^{1-\varepsilon}$ can get for $J \in \mathcal{M}_{(\boldsymbol{\rho}, \varepsilon)-\operatorname{deep}}(K)$ is $2^{\rho(1-\varepsilon)} \geq 1$, and so $\gamma \leq 1+4 \cdot 2^{\rho(1-\varepsilon)}$ implies $\gamma-1 \leq 4 \ell(J)^{\varepsilon-1} \ell(K)^{1-\varepsilon}$, which completes the proof.

The reader can easily verify the same argument works for the Whitney collection $\mathcal{W}(K)$.

Now we recall the notion of alternate dyadic intervals from [SaShUr7, which we rename augmented dyadic intervals here.

Definition 102. Given a dyadic grid $\mathcal{D}$, the augmented dyadic grid $\mathcal{A D}$ consists of those intervals I whose dyadic children $I^{\prime}$ belong to the grid $\mathcal{D}$.

Of course an augmented grid is not actually a grid because the nesting property fails, but this terminology should cause no confusion. These augmented grids will be needed in order to use the 'prepare to puncture' argument (introduced in SaShUr9]) at several places below.

Now we proceed to recall certain of the definitions of various energy conditions from SaShUr5] and SaShUr7. While these definitions are not explicitly used in the proof of functional energy, some of the arguments we give to control them will be appealed to later, and so we take the time to develop these definitions in detail.

10.1.1. Whitney energy conditions. The following definition of Whitney energy condition uses the Whitney decomposition $\mathcal{M}_{(\boldsymbol{\rho}, 1)-\text { deep }, \mathcal{D}}\left(I_{r}\right)$ into $\mathcal{D}$-dyadic intervals in which $\varepsilon=1$, as well as the 'large' pseudoprojections

$$
\mathrm{Q}_{K}^{\omega, \mathbf{b}^{*}} \equiv \sum_{J \in \mathcal{G}: J \subset K} \triangle_{J}^{\omega, \mathbf{b}^{*}}
$$


Definition 103. Suppose $\sigma$ and $\omega$ are locally finite positive Borel measures on $\mathbb{R}$ and fix $\gamma>1$. Then the Whitney energy condition constant $\mathcal{E}_{2}^{\alpha, \text { Whitney }}$ is given by

$$
\left(\mathcal{E}_{2}^{\alpha, \text { Whitney }}\right)^{2} \equiv \sup _{\mathcal{D}, \mathcal{G}} \sup _{I=\cup \cup} \frac{1}{\mid I I_{r}} \sum_{\sigma=1}^{\infty} \sum_{M \in \mathcal{W}\left(I_{r}\right)}\left(\frac{\mathrm{P}^{\alpha}\left(M, \mathbf{1}_{I \backslash \gamma M} \sigma\right)}{|M|}\right)^{2}\left\|Q_{M}^{\omega, \mathbf{b}^{*}} x\right\|_{L^{2}(\omega)}^{\boldsymbol{\omega}^{2}},
$$

where $\sup _{\mathcal{D}, \mathcal{G}} \sup _{I=\dot{U} I_{r}}$ is taken over

(1) all dyadic grids $\mathcal{D}$ and $\mathcal{G}$,

(2) all $\mathcal{D}$-dyadic intervals $I$,

(3) and all partitions $\left\{I_{r}\right\}_{r=1}^{N}$ or $\infty$ of the interval I into $\mathcal{D}$-dyadic subintervals $I_{r}$.

If the parameter $\gamma>1$ above is chosen sufficiently close to 1 , then the collection of intervals $\{\gamma M\}_{M \in \mathcal{W}\left(I_{r}\right)}$

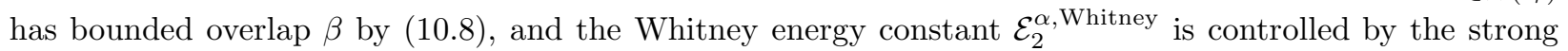
energy constant $\mathcal{E}_{2}^{\alpha}$ in (1.12),

$$
\mathcal{E}_{2}^{\alpha, \text { Whitney }} \lesssim \mathcal{E}_{2}^{\alpha} \text {. }
$$

Indeed, to see this, fix a decomposition of an interval

$$
I=\bigcup_{1 \leq r<\infty} \bigcup_{M \in \mathcal{W}\left(I_{r}\right)} M
$$

as in Definition 103, Then consider the subdecomposition

$$
I \supset \bigcup_{1 \leq r<\infty} \bigcup_{M \in \mathcal{W}\left(I_{r}\right)} M
$$

of the interval $I$ given by the collection of intervals,

We then have

$$
\mathcal{I} \equiv \bigcup_{1 \leq r<\infty} \mathcal{W}\left(I_{r}\right)
$$

$$
\left(\mathcal{E}_{2}^{\alpha}\right)^{2} \geq \frac{1}{|I|_{\sigma}} \sum_{r=1}^{\infty} \sum_{M \in \mathcal{W}\left(I_{r}\right)}\left(\frac{\mathrm{P}^{\alpha}\left(M, \mathbf{1}_{I} \sigma\right)}{|M|}\right)^{2}\left\|x-m_{M}^{\omega}\right\|_{L^{2}\left(\mathbf{1}_{M} \omega\right)}^{2} .
$$

Now $\mathrm{P}^{\alpha}\left(M, \mathbf{1}_{I} \sigma\right) \geq \mathrm{P}^{\alpha}\left(M, \mathbf{1}_{I \backslash \gamma M} \sigma\right)$ and from (10.4),

$$
\left\|x-m_{M}^{\omega}\right\|_{L^{2}\left(\mathbf{1}_{M} \omega\right)}^{2} \gtrsim\left\|Q_{M}^{\omega, \mathbf{b}^{*}} x\right\|_{L^{2}(\omega)}^{\mathbf{\omega} 2},
$$

and combining these two inequalities, we obtain that

$$
\left(\mathcal{E}_{2}^{\alpha}\right)^{2} \geq c \frac{1}{|I|_{\sigma}} \sum_{r=1}^{\infty} \sum_{M \in \mathcal{W}\left(I_{r}\right)}\left(\frac{\mathrm{P}^{\alpha}\left(M, \mathbf{1}_{I \backslash \gamma M} \sigma\right)}{|M|}\right)^{2}\left\|Q_{M}^{\omega, \mathbf{b}^{*}} x\right\|_{L^{2}(\omega)}^{\mathbf{\omega} 2} .
$$

Thus we conclude that

$$
\frac{1}{|I|_{\sigma}} \sum_{r=1}^{\infty} \sum_{M \in \mathcal{W}\left(I_{r}\right)}\left(\frac{\mathrm{P}^{\alpha}\left(M, \mathbf{1}_{I \backslash \gamma M} \sigma\right)}{|M|}\right)^{2}\left\|\mathrm{Q}_{M}^{\omega, \mathbf{b}^{*}} x\right\|_{L^{2}(\omega)}^{\boldsymbol{\aleph}^{2}} \leq \frac{C}{c} \beta\left(\mathcal{E}_{2}^{\alpha}\right)^{2},
$$

and taking the supremum over all decompositions (10.11) as in Definition 103, we obtain (10.10).

There is a similar definition for the dual (backward) Whitney energy conditions that simply interchanges $\sigma$ and $\omega$ everywhere. These definitions of the Whitney energy conditions depend on the choice of $\gamma>1$.

Commentary on proofs: We now introduce a number of results concerning partial plugging of the hole for Whitney energy conditions.

Note that we can 'partially' plug the $\gamma$-hole in the Poisson integral $\mathrm{P}^{\alpha}\left(J, \mathbf{1}_{I \backslash \gamma J} \sigma\right)$ for $\mathcal{E}_{2}^{\alpha, \text { Whitney }}$ using the offset $A_{2}^{\alpha}$ condition and the bounded overlap property (10.8). Indeed, define

$$
\left(\mathcal{E}_{2}^{\alpha, \text { Whitney partial }}\right)^{2} \equiv \sup _{\mathcal{D}, \mathcal{G}} \sup _{I=\dot{\cup} I_{r}} \frac{1}{|I|_{\sigma}} \sum_{r=1}^{\infty} \sum_{M \in \mathcal{W}\left(I_{r}\right)}\left(\frac{\mathrm{P}^{\alpha}\left(M, \mathbf{1}_{I \backslash M} \sigma\right)}{|M|}\right)^{2}\left\|\mathrm{Q}_{M}^{\omega, \mathbf{b}^{*}} x\right\|_{L^{2}(\omega)}^{\boldsymbol{\oplus}^{2}}
$$


Recall from (10.8) that

$$
\gamma M \subset I_{r} \text { for all } M \in \mathcal{W}\left(I_{r}\right) \text { provided } \gamma \leq 5 .
$$

At this point we need the following analogues of the 'energy $A_{2}^{\alpha}$ conditions' from [SaShUr9], which we denote by $A_{2}^{\alpha, \text { energy }}$ and $A_{2}^{\alpha, *, \text { energy }}$, and define by

$$
\begin{gathered}
A_{2}^{\alpha, \text { energy }}(\sigma, \omega) \equiv \sup _{Q \in \mathcal{P}} \frac{\left\|Q_{Q}^{\omega, \mathbf{b}^{*}} \frac{x}{\ell(Q)}\right\|_{L^{2}(\omega)}^{\mathbf{p}^{2}}}{|Q|^{1-\alpha}} \frac{|Q|_{\sigma}}{|Q|^{1-\alpha}}, \\
A_{2}^{\alpha, *, \text { energy }}(\sigma, \omega) \equiv \sup _{Q \in \mathcal{P}} \frac{|Q|_{\omega}}{|Q|^{1-\alpha}} \frac{\left\|Q_{Q}^{\sigma, \mathbf{b}} \frac{x}{\ell(Q)}\right\|_{L^{2}(\sigma)}^{\mathbf{\omega} 2}}{|Q|^{1-\alpha}} .
\end{gathered}
$$

Then if $\gamma \leq 5$, we have

$$
\begin{aligned}
& \left(\mathcal{E}_{2}^{\alpha, \text { Whitney partial }}\right)^{2} \\
\lesssim & \sup _{\mathcal{D}, \mathcal{G}} \sup _{I=\cup \dot{ } I_{r}} \frac{1}{|I|_{\sigma}} \sum_{r=1}^{\infty} \sum_{M \in \mathcal{W}\left(I_{r}\right)}\left(\frac{\mathrm{P}^{\alpha}\left(M, \mathbf{1}_{I \backslash \gamma M} \sigma\right)}{|M|}\right)^{2}\left\|\mathrm{Q}_{M}^{\omega, \mathbf{b}^{*}} x\right\|_{L^{2}(\omega)}^{\mathbf{\omega} 2} \\
& +\sup _{\mathcal{D}, \mathcal{G}} \sup _{I=\cup \dot{ } I_{r}} \frac{1}{|I|_{\sigma}} \sum_{r=1}^{\infty} \sum_{M \in \mathcal{W}\left(I_{r}\right)}\left(\frac{\mathrm{P}^{\alpha}\left(M, \mathbf{1}_{\gamma M \backslash M} \sigma\right)}{|M|}\right)^{2}\left\|\mathrm{Q}_{M}^{\omega, \mathbf{b}^{*}} x\right\|_{L^{2}(\omega)}^{\mathbf{\omega} 2} \\
\lesssim & \left(\mathcal{E}_{2}^{\alpha, \text { Whitney }}\right)^{2}+\sup _{\mathcal{D}, \mathcal{G}} \sup _{I=\dot{\cup} I_{r}} \frac{1}{|I|_{\sigma}} \sum_{r=1}^{\infty} \sum_{M \in \mathcal{W}\left(I_{r}\right)} A_{2}^{\alpha, \text { energy }}|\gamma M|_{\sigma} \lesssim\left(\mathcal{E}_{2}^{\alpha, \text { deep }}\right)^{2}+\beta A_{2}^{\alpha, \text { energy }},
\end{aligned}
$$

by (10.8).

10.1.2. Plugged energy conditions. We continue to recall some results from [SaShUr9] and [SaShUr10] that we will use repeatedly here. For example, we will use the punctured Muckenhoupt conditions $A_{2}^{\alpha, \text { punct }}$ and $A_{2}^{\alpha, *, p u n c t}$ introduced earlier in (1.8) to control the plugged energy conditions, where the hole in the argument of the Poisson term $\mathrm{P}^{\alpha}\left(M, \mathbf{1}_{I \backslash M} \sigma\right)$ in the partially plugged energy condition above, is replaced with the 'plugged' term $\mathrm{P}^{\alpha}\left(M, \mathbf{1}_{I} \sigma\right)$, for example

$$
\left(\mathcal{E}_{2}^{\alpha, \text { Whitney plug }}\right)^{2} \equiv \sup _{\mathcal{D}, \mathcal{G}} \sup _{I=\cup \dot{\cup} I_{r}} \frac{1}{|I|_{\sigma}} \sum_{r=1}^{\infty} \sum_{M \in \mathcal{W}\left(I_{r}\right)}\left(\frac{\mathrm{P}^{\alpha}\left(M, \mathbf{1}_{I} \sigma\right)}{|M|}\right)^{2}\left\|\mathrm{Q}_{M}^{\omega, \mathbf{b}^{*}} x\right\|_{L^{2}(\omega)}^{\mathbf{\omega} 2} .
$$

By an argument similar to that in (10.14), we obtain

$$
\mathcal{E}_{2}^{\alpha, \text { Whitney plug }} \lesssim \mathcal{E}_{2}^{\alpha, \text { Whitney partial }}+A_{2}^{\alpha, \text { energy }}
$$

We first show that the punctured Muckenhoupt conditions $A_{2}^{\alpha, \text { punct }}$ and $A_{2}^{\alpha, *, p u n c t}$ control respectively the 'energy $A_{2}^{\alpha}$ conditions' in (10.13). We will make reference to the proof of the next lemma (for the $T 1$ theorem this is from [SaShUr9, Lemma 3.2 on page 328.]) several times in the sequel. We repeat the proof from SaShUr9, Lemma 3.2 on page 328.] but with modifications to accommodate the differences that arise here in the setting of a local $T b$ theorem. Recall that $\mathfrak{P}_{(\sigma, \omega)}$ is defined in (1.9) above.

Lemma 104. For any positive locally finite Borel measures $\sigma, \omega$ we have

$$
\begin{aligned}
A_{2}^{\alpha, \text { energy }}(\sigma, \omega) & \lesssim A_{2}^{\alpha, \text { punct }}(\sigma, \omega), \\
A_{2}^{\alpha, *, \text { energy }}(\sigma, \omega) & \lesssim A_{2}^{\alpha, *, \text { punct }}(\sigma, \omega) .
\end{aligned}
$$

Proof. Fix an interval $Q \in \mathcal{D}$. Recall the definition of $\omega\left(Q, \mathfrak{P}_{(\sigma, \omega)}\right)$ in (1.8). If $\omega\left(Q, \mathfrak{P}_{(\sigma, \omega)}\right) \geq \frac{1}{2}|Q|_{\omega}$, then we trivially have

$$
\begin{aligned}
\frac{\left\|Q_{Q}^{\omega, \mathbf{b}^{*}} \frac{x}{\ell(Q)}\right\|_{L^{2}(\omega)}^{\boldsymbol{\omega}^{2}}}{|Q|^{1-\alpha}} \frac{|Q|_{\sigma}}{|Q|^{1-\alpha}} & \lesssim \frac{|Q|_{\omega}}{|Q|^{1-\alpha}} \frac{|Q|_{\sigma}}{|Q|^{1-\alpha}} \\
& \leq 2 \frac{\omega(Q, \mathfrak{P}(\sigma, \omega)}{|Q|^{1-\alpha}} \frac{|Q|_{\sigma}}{|Q|^{1-\alpha}} \leq 2 A_{2}^{\alpha, \text { punct }}(\sigma, \omega) .
\end{aligned}
$$


On the other hand, if $\omega\left(Q, \mathfrak{P}_{(\sigma, \omega)}\right)<\frac{1}{2}|Q|_{\omega}$ then there is a point $p \in Q \cap \mathfrak{P}_{(\sigma, \omega)}$ such that

$$
\omega(\{p\})>\frac{1}{2}|Q|_{\omega},
$$

and consequently, $p$ is the largest $\omega$-point mass in $Q$. Thus if we define $\widetilde{\omega}=\omega-\omega(\{p\}) \delta_{p}$, then we have

$$
\omega\left(Q, \mathfrak{P}_{(\sigma, \omega)}\right)=|Q|_{\widetilde{\omega}} .
$$

Now we observe from the construction of martingale differences that

$$
\triangle_{J}^{\widetilde{\omega}, \mathbf{b}^{*}}=\triangle_{J}^{\omega, \mathbf{b}^{*}}, \quad \text { for all } J \in \mathcal{D} \text { with } p \notin J .
$$

So for each $s \geq 0$ there is a unique interval $J_{s} \in \mathcal{D}$ with $\ell\left(J_{s}\right)=2^{-s} \ell(Q)$ that contains the point $p$. Now observe that, just as for the Haar projection, the one-dimensional projection $\triangle_{J_{s}}^{\omega, \mathbf{b}^{*}}$ is given by $\triangle_{J_{s}}^{\omega, \mathbf{b}^{*}} f=$ $\left\langle h_{J_{s}}^{\omega, \mathbf{b}^{*}}, f\right\rangle_{\omega} h_{J_{s}}^{\omega, \mathbf{b}^{*}}$ for a unique up to \pm unit vector $h_{J_{s}}^{\omega, \mathbf{b}^{*}}$. For this interval we then have

$$
\begin{aligned}
\left\|\triangle_{J_{s}}^{\omega, \mathbf{b}^{*}} x\right\|_{L^{2}(\omega)}^{2} & =\left|\left\langle h_{J_{s}}^{\omega, \mathbf{b}^{*}}, x\right\rangle_{\omega}\right|^{2}=\left|\left\langle h_{J_{s}}^{\omega, \mathbf{b}^{*}}, x-p\right\rangle_{\omega}\right|^{2} \\
& =\left|\int_{J_{s}} h_{J_{s}}^{\omega, \mathbf{b}^{*}}(x)(x-p) d \omega(x)\right|^{2}=\left|\int_{J_{s}} h_{J_{s}}^{\omega, \mathbf{b}^{*}}(x)(x-p) d \widetilde{\omega}(x)\right|^{2} \\
& \leq\left\|h_{J_{s}}^{\omega, \mathbf{b}^{*}}\right\|_{L^{2}(\widetilde{\omega})}^{2}\left\|\mathbf{1}_{J_{s}}(x-p)\right\|_{L^{2}(\widetilde{\omega})}^{2} \leq\left\|h_{J_{s}}^{\omega, \mathbf{b}^{*}}\right\|_{L^{2}(\omega)}^{2}\left\|\mathbf{1}_{J_{s}}(x-p)\right\|_{L^{2}(\widetilde{\omega})}^{2} \\
& \leq \ell\left(J_{s}\right)^{2}\left|J_{s}\right|_{\widetilde{\omega}} \leq 2^{-2 s} \ell(Q)^{2}|Q|_{\widetilde{\omega}},
\end{aligned}
$$

as well as

$$
\inf _{z \in \mathbb{R}}\left\|\widehat{\nabla}_{J_{s}}^{\omega}(x-z)\right\|_{L^{2}(\omega)}^{2} \lesssim\|(x-p)\|_{L^{2}\left(\mathbf{1}_{J_{s}} \omega\right)}^{2}=\|(x-p)\|_{L^{2}\left(\mathbf{1}_{s} \widetilde{\omega}\right)}^{2} \leq \ell\left(J_{s}\right)^{2}\left|J_{s}\right|_{\widetilde{\omega}} \leq 2^{-2 s} \ell(Q)^{2}|Q|_{\widetilde{\omega}},
$$

from (10.4). Thus we can estimate

$$
\begin{aligned}
\left\|Q_{Q}^{\omega, \mathbf{b}^{*}} \frac{x}{\ell(Q)}\right\|_{L^{2}(\omega)}^{\mathbf{\omega} 2} & \leq \frac{1}{\ell(Q)^{2}}\left(\sum_{J \in \mathcal{D}: J \subset Q}\left\|\triangle_{J}^{\omega, \mathbf{b}^{*}} x\right\|_{L^{2}(\omega)}^{2}+\inf _{z \in \mathbb{R}}\left\|\widehat{\nabla}_{J_{s}}^{\omega}(x-z)\right\|_{L^{2}(\omega)}^{2}\right) \\
& =\frac{1}{\ell(Q)^{2}}\left(\sum_{J \in \mathcal{D}: p \notin J \subset Q}\left\|\triangle_{J}^{\widetilde{\omega}, \mathbf{b}^{*}} x\right\|_{L^{2}(\widetilde{\omega})}^{2}+\sum_{s=0}^{\infty}\left\|\triangle_{J_{s}}^{\omega, \mathbf{b}^{*}} x\right\|_{L^{2}(\omega)}^{2}+\inf _{z \in \mathbb{R}}\left\|\widehat{\nabla}_{J_{s}}^{\omega}(x-z)\right\|_{L^{2}(\omega)}^{2}\right) \\
& \lesssim \frac{1}{\ell(Q)^{2}}\left(\left\|Q_{Q}^{\widetilde{\omega}, \mathbf{b}^{*}} x\right\|_{L^{2}(\widetilde{\omega})}^{\mathbf{\omega} 2}+\sum_{s=0}^{\infty} 2^{-2 s} \ell(Q)^{2}|Q|_{\widetilde{\omega}}\right) \\
& \lesssim \frac{1}{\ell(Q)^{2}}\left(\ell(Q)^{2}|Q|_{\widetilde{\omega}}+\sum_{s=0}^{\infty} 2^{-2 s} \ell(Q)^{2}|Q|_{\widetilde{\omega}}\right) \\
& \leq 3|Q|_{\widetilde{\omega}}=3 \omega\left(Q, \mathfrak{P}_{(\sigma, \omega)}\right),
\end{aligned}
$$

and so

$$
\frac{\left\|Q_{Q}^{\omega, \mathbf{b}^{*}} \frac{x}{\ell(Q)}\right\|_{L^{2}(\omega)}^{\boldsymbol{\omega}^{2}}}{|Q|^{1-\alpha}} \frac{|Q|_{\sigma}}{|Q|^{1-\alpha}} \lesssim \frac{3 \omega\left(Q, \mathfrak{P}_{(\sigma, \omega)}\right)}{|Q|^{1-\alpha}} \frac{|Q|_{\sigma}}{|Q|^{1-\alpha}} \leq 3 A_{2}^{\alpha, \text { punct }}(\sigma, \omega) .
$$

Now take the supremum over $Q \in \mathcal{D}$ to obtain $A_{2}^{\alpha, \text { energy }}(\sigma, \omega) \lesssim A_{2}^{\alpha, \text { punct }}(\sigma, \omega)$. The dual inequality follows upon interchanging the measures $\sigma$ and $\omega$.

We isolate a simple but key fact that will be used repeatedly in what follows:

$$
\sum_{Q \in \mathcal{D}: Q \subset P} \ell(Q)^{2}|Q|_{\mu} \lesssim \ell(P)^{2}|P|_{\mu}, \quad \text { for } P \in \mathcal{D} \text { and } \mu \text { a positive measure. }
$$

Indeed, to see (10.18), simply pigeonhole the length of $Q$ relative to that of $P$ and sum. The next corollary follows immediately from Lemma 104 (10.14) and (10.16). 
Corollary 105. Provided $1<\gamma \leq 5$,

$$
\mathcal{E}_{2}^{\alpha, \text { Whitney plug }} \lesssim \mathcal{E}_{2}^{\alpha, \text { Whitney partial }}+A_{2}^{\alpha, \text { punct }} \lesssim \mathcal{E}_{2}^{\alpha, \text { Whitney }}+A_{2}^{\alpha, \text { punct }},
$$

and similarly for the dual plugged energy condition.

10.1.3. Plugged $\mathcal{A}_{2}^{\alpha, \text { energy plug }}$ conditions. Using Lemma 104 we can control the 'plugged' energy $\mathcal{A}_{2}^{\alpha}$ conditions:

$$
\begin{aligned}
\mathcal{A}_{2}^{\alpha, \text { energy plug }}(\sigma, \omega) & \equiv \sup _{Q \in \mathcal{P}} \frac{\left\|Q_{Q}^{\omega, \mathbf{b}^{*}} \frac{x}{\ell(Q)}\right\|_{L^{2}(\omega)}^{\mathbf{\omega} 2}}{|Q|^{1-\alpha}} \mathcal{P}^{\alpha}(Q, \sigma), \\
\mathcal{A}_{2}^{\alpha, *, \text { energy plug }}(\sigma, \omega) & \equiv \sup _{Q \in \mathcal{P}} \mathcal{P}^{\alpha}(Q, \omega) \frac{\left\|\mathrm{Q}_{Q}^{\sigma, \mathbf{b}} \frac{x}{\ell(Q)}\right\|_{L^{2}(\sigma)}^{\mathbf{\omega} 2}}{|Q|^{1-\alpha}} .
\end{aligned}
$$

Lemma 106. We have

$$
\begin{aligned}
\mathcal{A}_{2}^{\alpha, \text { energy plug }}(\sigma, \omega) & \lesssim \mathcal{A}_{2}^{\alpha}(\sigma, \omega)+A_{2}^{\alpha, \text { energy }}(\sigma, \omega), \\
\mathcal{A}_{2}^{\alpha, *, \text { energy plug }}(\sigma, \omega) & \lesssim \mathcal{A}_{2}^{\alpha, *}(\sigma, \omega)+A_{2}^{\alpha, *, \text { energy }}(\sigma, \omega) .
\end{aligned}
$$

Proof. We have

$$
\begin{aligned}
\frac{\left\|Q_{Q}^{\omega, \mathbf{b}^{*}} \frac{x}{\ell(Q)}\right\|_{L^{2}(\omega)}^{\mathbf{\omega} 2}}{|Q|^{1-\alpha}} \mathcal{P}^{\alpha}(Q, \sigma) & =\frac{\left\|Q_{Q}^{\omega, \mathbf{b}^{*}} \frac{x}{\ell(Q)}\right\|_{L^{2}(\omega)}^{\mathbf{\omega}^{2}}}{|Q|^{1-\alpha}} \mathcal{P}^{\alpha}\left(Q, \mathbf{1}_{Q^{c} \sigma} \sigma\right)+\frac{\left\|Q_{Q}^{\omega, \mathbf{b}^{*}} \frac{x}{\ell(Q)}\right\|_{L^{2}(\omega)}^{\mathbf{\omega} 2}}{|Q|^{1-\alpha}} \mathcal{P}^{\alpha}\left(Q, \mathbf{1}_{Q} \sigma\right) \\
& \lesssim \frac{|Q|_{\omega}}{|Q|^{1-\alpha}} \mathcal{P}^{\alpha}\left(Q, \mathbf{1}_{Q^{c}} \sigma\right)+\frac{\left\|Q_{Q}^{\omega, \mathbf{b}^{*}} \frac{x}{\ell(Q)}\right\|_{L^{2}(\omega)}^{\mathbf{\omega} 2}}{|Q|_{\sigma}} \\
& \left.\lesssim\right|^{1-\alpha} \\
& \lesssim \mathcal{A}_{2}^{\alpha}(\sigma, \omega)+A_{2}^{\alpha, \text { energy }}(\sigma, \omega) .
\end{aligned}
$$

10.2. The Poisson formulation. Recall from Definitions 42 and 52 that

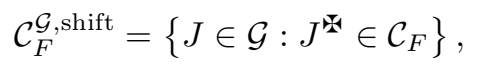

where $F \in \mathcal{F}$ is a stopping interval in the dyadic grid $\mathcal{D}$. For convenience we repeat here the main result of this section, Proposition 100 .

Proposition 107. For all grids $\mathcal{D}$ and $\mathcal{G}$, and $\varepsilon>0$ sufficiently small, we have

$$
\begin{aligned}
\mathfrak{F}_{\alpha}^{\mathbf{b}^{*}}(\mathcal{D}, \mathcal{G}) & \lesssim \mathfrak{E}_{2}^{\alpha}+\sqrt{\mathcal{A}_{2}^{\alpha}}+\sqrt{\mathcal{A}_{2}^{\alpha, *}}+\sqrt{A_{2}^{\alpha, \text { punct }}}, \\
\mathfrak{F}_{\alpha}^{\mathbf{b}, *}(\mathcal{G}, \mathcal{D}) & \lesssim \mathfrak{E}_{2}^{\alpha, *}+\sqrt{\mathcal{A}_{2}^{\alpha}}+\sqrt{\mathcal{A}_{2}^{\alpha, *}}+\sqrt{A_{2}^{\alpha, *, \text { punct }}},
\end{aligned}
$$

with implied constants independent of the grids $\mathcal{D}$ and $\mathcal{G}$.

To prove Proposition 107, we fix grids $\mathcal{D}$ and $\mathcal{G}$ and a subgrid $\mathcal{F}$ of $\mathcal{D}$ as in (6.6), and set

$$
\mu \equiv \sum_{F \in \mathcal{F}} \sum_{M \in \mathcal{W}(F)}\left\|Q_{F, M}^{\omega, \mathbf{b}^{*}} x\right\|_{L^{2}(\omega)}^{\mathbf{A}^{2}} \cdot \delta_{\left(c_{M}, \ell(M)\right)} \text { and } d \bar{\mu}(x, t) \equiv \frac{1}{t^{2}} d \mu(x, t)
$$

where $\mathcal{W}(F)$ consists of the maximal $\mathcal{D}$-subintervals of $F$ whose triples are contained in $F$, and where $\delta_{\left(c_{M}, \ell(M)\right)}$ denotes the Dirac unit mass at the point $\left(c_{M}, \ell(M)\right)$ in the upper half-space $\mathbb{R}_{+}^{2}$. Here $M \in \mathcal{D}$ is a dyadic interval with center $c_{M}$ and side length $\ell(M)$, and for any interval $K \in \mathcal{P}$, the shorthand notation

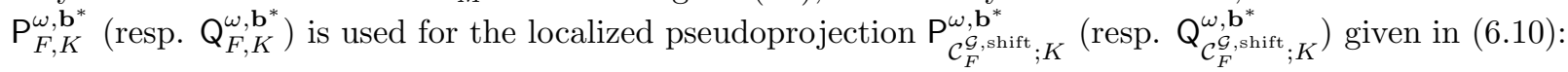

$$
\mathrm{P}_{F, K}^{\omega, \mathbf{b}^{*}} \equiv \mathrm{P}_{\mathcal{C}_{F}^{\mathcal{G}, \text { shift }} ; K}^{\omega, \mathbf{b}^{*}}=\sum_{J \subset K: J \in \mathcal{C}_{F}^{\mathcal{G}, \text { shift }}} \square_{J}^{\omega, \mathbf{b}^{*}}\left(\operatorname{resp.} \mathrm{Q}_{F, K}^{\omega, \mathbf{b}^{*}} \equiv \mathrm{Q}_{\mathcal{C}_{F}^{\mathcal{G}, \text { shift }} ; K}^{\omega, \mathbf{s}^{*}}=\sum_{J \subset K: J \in \mathcal{C}_{F}^{\mathcal{G}, \text { shift }}} \triangle_{J}^{\omega, \mathbf{b}^{*}}\right)
$$


We emphasize that all the subintervals $J$ that arise in the projection $\mathbf{Q}_{F, M}^{\omega, \mathbf{b}^{*}}$ are good inside the intervals $F$ and beyond since $J^{w} \subset F$. Here $J^{*}$ is defined in Definition 42 using the body of an interval. Thus every $J \in Q_{F}^{\omega, \mathbf{b}^{*}}$ is contained in a unique $M \in \mathcal{W}(F)$, so that $Q_{F}^{\omega, \mathbf{b}^{*}}=\bigcup_{M \in \mathcal{W}(F)} Q_{F, M}^{\omega, \mathbf{b}^{*}}$. We can replace $x$ by $x-c$ inside the projection for any choice of $c$ we wish; the projection is unchanged. More generally, $\delta_{q}$ denotes a Dirac unit mass at a point $q$ in the upper half-space $\mathbb{R}_{+}^{2}$.

We will prove the two-weight inequality

$$
\left\|\mathbb{P}^{\alpha}(f \sigma)\right\|_{L^{2}\left(\mathbb{R}_{+}^{2}, \bar{\mu}\right)} \lesssim\left(\mathfrak{E}_{2}^{\alpha}+\sqrt{\mathcal{A}_{2}^{\alpha}}+\sqrt{\mathcal{A}_{2}^{\alpha, *}}+\sqrt{A_{2}^{\alpha, \text { punct }}}\right)\|f\|_{L^{2}(\sigma)},
$$

for all nonnegative $f$ in $L^{2}(\sigma)$, noting that $\mathcal{F}$ and $f$ are not related here. Above, $\mathbb{P}^{\alpha}(\cdot)$ denotes the $\alpha$ fractional Poisson extension to the upper half-space $\mathbb{R}_{+}^{2}$,

so that in particular

$$
\mathbb{P}^{\alpha} \rho(x, t) \equiv \int_{\mathbb{R}} \frac{t}{\left(t^{2}+|x-y|^{2}\right)^{\frac{2-\alpha}{2}}} d \rho(y),
$$

$$
\left\|\mathbb{P}^{\alpha}(f \sigma)\right\|_{L^{2}\left(\mathbb{R}_{+}^{2}, \bar{\mu}\right)}^{2}=\sum_{F \in \mathcal{F}} \sum_{M \in \mathcal{W}(F)} \mathbb{P}^{\alpha}(f \sigma)(c(M), \ell(M))^{2}\left\|Q_{F, M}^{\omega, \mathbf{b}^{*}} \frac{x}{|M|}\right\|_{L^{2}(\omega)}^{\mathbf{\omega} 2},
$$

and so (10.21) proves the first line in Proposition 100 upon inspecting (6.6). Note also that we can equivalently write $\left\|\mathbb{P}^{\alpha}(f \sigma)\right\|_{L^{2}\left(\mathbb{R}_{+}^{2}, \bar{\mu}\right)}=\left\|\widetilde{\mathbb{P}}^{\alpha}(f \sigma)\right\|_{L^{2}\left(\mathbb{R}_{+}^{2}, \mu\right)}$ where $\widetilde{\mathbb{P}}^{\alpha} \nu(x, t) \equiv \frac{1}{t} \mathbb{P}^{\alpha} \nu(x, t)$ is the renormalized Poisson operator. Here we have simply shifted the factor $\frac{1}{t^{2}}$ in $\bar{\mu}$ to $\left|\widetilde{\mathbb{P}}^{\alpha}(f \sigma)\right|^{2}$ instead, and we will do this shifting often throughout the proof when it is convenient to do so.

One version of the characterization of the two-weight inequality for fractional and Poisson integrals in Saw was stated in terms of a fixed dyadic grid $\mathcal{D}$ of intervals in $\mathbb{R}$ with sides parallel to the coordinate axes. Using this theorem for the two-weight Poisson inequality, but adapted to the $\alpha$-fractional Poisson integral $\mathbb{P} a 10$, we see that inequality (10.21) requires checking these two inequalities for dyadic intervals $I \in \mathcal{D}$ and boxes $\widehat{I}=I \times[0, \ell(I))$ in the upper half-space $\mathbb{R}_{+}^{2}$ :

$$
\begin{gathered}
\int_{\mathbb{R}_{+}^{2}} \mathbb{P}^{\alpha}\left(\mathbf{1}_{I} \sigma\right)(x, t)^{2} d \bar{\mu}(x, t) \equiv\left\|\mathbb{P}^{\alpha}\left(\mathbf{1}_{I} \sigma\right)\right\|_{L^{2}(\bar{\mu})}^{2} \lesssim\left(\left(\mathfrak{E}_{2}^{\alpha}\right)^{2}+\mathcal{A}_{2}^{\alpha}+\mathcal{A}_{2}^{\alpha, *}+A_{2}^{\alpha, \text { punct }}\right) \sigma(I), \\
\int_{\mathbb{R}^{\prime}}\left[\mathbb{Q}^{\alpha}\left(t \mathbf{1}_{\widehat{I}} \bar{\mu}\right)\right]^{2} d \sigma(x) \lesssim\left(\left(\mathfrak{E}_{2}^{\alpha}\right)^{2}+\mathcal{A}_{2}^{\alpha}+A_{2}^{\alpha, \text { punct }}\right) \int_{\widehat{I}} t^{2} d \bar{\mu}(x, t),
\end{gathered}
$$

for all dyadic intervals $I \in \mathcal{D}$, and where the dual Poisson operator $\mathbb{Q}^{\alpha}$ is given by

$$
\mathbb{Q}^{\alpha}\left(t \mathbf{1}_{\widehat{I}} \bar{\mu}\right)(x)=\int_{\widehat{I}} \frac{t^{2}}{\left(t^{2}+|x-y|^{2}\right)^{\frac{2-\alpha}{2}}} d \bar{\mu}(y, t) .
$$

It is important to note that we can choose for $\mathcal{D}$ any fixed dyadic grid, the compensating point being that the integrations on the left sides of (10.22) and (10.23) are taken over the entire spaces $\mathbb{R}_{+}^{2}$ and $\mathbb{R}$ respectively 11 .

10.3. Poisson testing. We now turn to proving the Poisson testing conditions (10.22) and (10.23). Similar testing conditions have been considered in [SaShUr5], SaShUr7], SaShUr9] and [SaShUr10], and the proofs there essentially carry over to the situation here, but careful attention must now be paid to the changed definition of functional energy and the weaker notion of goodness. We continue to circumvent the difficulty of permitting common point masses here by using the energy Muckenhoupt constants $A_{2}^{\alpha, \text { energy }}$ and $A_{2}^{\alpha, *, \text { energy }}$, which require control by the punctured Muckenhoupt constants $A_{2}^{\alpha, \text { punct }}$ and $A_{2}^{\alpha, *, \text { punct }}$. The following elementary Poisson inequalities (see e.g. [Vol]) will be used extensively.

\footnotetext{
${ }^{10}$ The proof for $0 \leq \alpha<1$ is essentially identical to that for $\alpha=0$ given in $\underline{\mathrm{Saw}}$.

${ }^{11}$ There is a gap in the proof of the Poisson inequality at the top of page 542 in Saw. However, this gap can be fixed as in SaWh] or LaSaUr1].
} 
Lemma 108. Suppose that $J, K, I$ are intervals in $\mathbb{R}$, and that $\mu$ is a positive measure supported in $\mathbb{R} \backslash I$. If $J \subset K \subset \beta K \subset I$ for some $\beta>1$, then

$$
\frac{\mathrm{P}^{\alpha}(J, \mu)}{|J|} \approx \frac{\mathrm{P}^{\alpha}(K, \mu)}{|K|}
$$

while if $J \subset \beta K$, then

$$
\frac{\mathrm{P}^{\alpha}(K, \mu)}{|K|} \lesssim \frac{\mathrm{P}^{\alpha}(J, \mu)}{|J|}
$$

Proof. We have

$$
\frac{\mathrm{P}^{\alpha}(J, \mu)}{|J|}=\frac{1}{|J|} \int \frac{|J|}{\left(|J|+\left|x-c_{J}\right|\right)^{2-\alpha}} d \mu(x),
$$

where $J \subset K \subset \beta K \subset I$ implies that

$$
|J|+\left|x-c_{J}\right| \approx|K|+\left|x-c_{K}\right|, \quad x \in \mathbb{R} \backslash I,
$$

and where $J \subset \beta K$ implies that

$$
|J|+\left|x-c_{J}\right| \lesssim|J|+\left|c_{K}-c_{J}\right|+\left|x-c_{K}\right| \lesssim|K|+\left|x-c_{K}\right|, \quad x \in \mathbb{R} .
$$

Recall that in the case of the $T 1$ theorem in [SaShUr7, where we assumed traditional goodness in a single family of grids $\mathcal{D}$, we had a strong bounded overlap property associated with the projections $\mathrm{P}_{F, J}^{\omega, \mathbf{b}^{*}}$ defined there; namely, that for each interval $I_{0} \in \mathcal{D}$, there were a bounded number of intervals $F \in \mathcal{F}$ with the property that $F \supsetneqq I_{0} \supset J$ for some $J \in \mathcal{M}_{(\boldsymbol{\rho}, \varepsilon) \text {-deep }}(F)$ with $\mathrm{P}_{F, J}^{\omega, \mathbf{b}^{*}} \neq 0$ (see the first part of Lemma 10.4 in [SaShUr7]). However, we no longer have this strong bounded overlap property when ordinary goodness is replaced with the weak goodness of Hytönen and Martikainen. Indeed, there may now be an unbounded number of intervals $F \in \mathcal{F}$ with $F \supsetneqq I_{0} \supset J$ and $\mathrm{P}_{F, J}^{\omega, \mathbf{b}^{*}} \neq 0$, simply because there can be $J^{\prime} \in \mathcal{G}$ with both

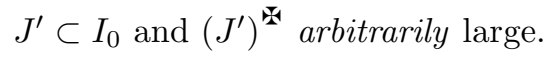

What will save us in obtaining the following lemma is that the Whitney intervals $M$ in $\mathcal{W}(F)$ that happen to lie in some $I \in \mathcal{D}$ with $I \subset F$ have one of just two different forms: if $I$ shares an endpoint with $F$ then the intervals $M$ near that endpoint are the same as those in $\mathcal{W}(I)$ - note that $F$ has been replaced with $I$ here - while otherwise there are a bounded number of Whitney intervals $M$ in $I$, and each such $M$ has side length comparable to $\ell(I)$.

The next lemma will be used in bounding both of the local Poisson testing conditions. Recall from Definition 102 that $\mathcal{A D}$ consists of all augmented $\mathcal{D}$-dyadic intervals where $K$ is an augmented dyadic interval if it is a union of $2 \mathcal{D}$-dyadic intervals $K^{\prime}$ with $\ell\left(K^{\prime}\right)=\frac{1}{2} \ell(K)$.

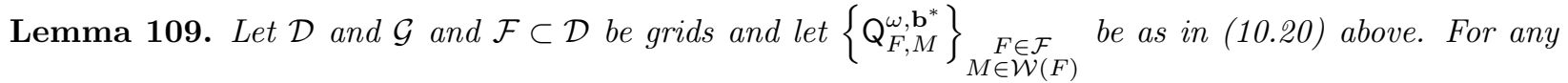
augmented interval $I \in \mathcal{A D}$ define

$$
B(I) \equiv \sum_{F \in \mathcal{F}: F \supsetneqq I^{\prime}} \sum_{\text {for some } I^{\prime} \in \mathfrak{C}(I)} \sum_{M \in \mathcal{W}(F): M \subset I}\left(\frac{\mathrm{P}^{\alpha}\left(M, \mathbf{1}_{I} \sigma\right)}{|M|}\right)^{2}\left\|\mathrm{Q}_{F, M}^{\omega, \mathbf{b}^{*}} x\right\|_{L^{2}(\omega)}^{\boldsymbol{\omega}^{2}} .
$$

Then

$$
B(I) \lesssim\left(\left(\mathfrak{E}_{2}^{\alpha}\right)^{2}+A_{2}^{\alpha, \text { energy }}\right)|I|_{\sigma} .
$$

Proof. We first prove the bound (10.25) for $B(I)$ ignoring for the moment the possible case when $M=I$ in the sum defining $B(I)$. So suppose that $I \in \mathcal{A D}$ is an augmented $\mathcal{D}$-dyadic interval. Define

$$
\Lambda^{*}(I) \equiv\left\{M \varsubsetneqq I: M \in \mathcal{W}(F) \text { for some } F \supsetneqq I^{\prime}, I^{\prime} \in \mathfrak{C}(I) \text { with } Q_{F, M}^{\omega, \mathbf{b}^{*}} x \neq 0\right\},
$$

and pigeonhole this collection as $\Lambda^{*}(I)=\bigcup_{I^{\prime} \in \mathfrak{C}(I)} \Lambda\left(I^{\prime}\right)$, where for each $I^{\prime} \in \mathfrak{C}(I)$ we define

$$
\Lambda\left(I^{\prime}\right) \equiv\left\{M \subset I^{\prime}: M \in \mathcal{W}(F) \text { for some } F \supsetneqq I^{\prime} \text { with } Q_{F, M}^{\omega, \mathbf{b}^{*}} x \neq 0\right\} \text {. }
$$


Consider first the case when $3 I^{\prime} \subset F$, so that $d\left(I^{\prime}, \partial F\right) \geq \ell\left(I^{\prime}\right)$. Then if $M \in \mathcal{W}(F)$ for some $F \supsetneqq I^{\prime}$ we have $\ell(M)=d(M, \partial F)$, and if in addition $M \subset I^{\prime}$, then $M=I^{\prime}$. Consider the sum over all $F \supsetneqq I^{\prime}=M$ :

$$
\begin{aligned}
B_{M}(I) & \equiv \sum_{F \in \mathcal{F}: F \supsetneqq M \text { for some } M \in \mathfrak{C}(I) \cap \mathcal{W}(F)}\left(\frac{\mathrm{P}^{\alpha}\left(M, \mathbf{1}_{I} \sigma\right)}{|M|}\right)^{2}\left\|\mathrm{Q}_{F, M}^{\omega, \mathbf{b}^{*}} x\right\|_{L^{2}(\omega)}^{\mathbf{\omega}^{2}} \\
& \leq\left(\frac{\mathrm{P}^{\alpha}\left(M, \mathbf{1}_{I} \sigma\right)}{|M|}\right)^{2}\left\|\mathrm{Q}_{M}^{\omega, \mathbf{b}^{*}} x\right\|_{L^{2}(\omega)}^{\boldsymbol{\aleph}^{2}} \lesssim\left(\frac{\mathrm{P}^{\alpha}\left(I, \mathbf{1}_{I} \sigma\right)}{|I|}\right)^{2}\left\|\mathrm{Q}_{I}^{\omega, \mathbf{b}^{*}} x\right\|_{L^{2}(\omega)}^{\mathbf{\omega}^{2}} \lesssim A_{2}^{\alpha, \text { energy }}|I|_{\sigma},
\end{aligned}
$$

where we have used the definitions (10.20) and (10.9). Thus we have obtained the bound

$$
\sum_{F \in \mathcal{F}: F \supsetneqq M \text { for some } M \in \mathfrak{C}(I) \cap \mathcal{W}(F)}\left(\frac{\mathrm{P}^{\alpha}\left(M, \mathbf{1}_{I} \sigma\right)}{|M|}\right)^{2}\left\|\mathrm{Q}_{F, M}^{\omega, \mathbf{b}^{*}} x\right\|_{L^{2}(\omega)}^{\mathbf{\omega}^{2}} \lesssim A_{2}^{\alpha, \text { energy }}|I|_{\sigma} .
$$

Now we turn to the case $3 I^{\prime} \not \subset F$, i.e. when $\partial I^{\prime} \cap \partial F$ consists of exactly one boundary point. In this case, if both $M \subset I^{\prime}$ and $M \in \mathcal{W}(F)$ for some $F \supsetneqq I^{\prime}$, then we must have either $M \in \mathcal{W}\left(I^{\prime}\right)$ or $M \in \mathfrak{C}\left(I^{\prime}\right)$, since both $M$ and $I^{\prime}$ are then close to the same boundary point in $\partial F$. Note that it is here that we use the Whitney decompositions to full advantage. So again we can estimate

$$
\begin{aligned}
& \sum_{F \in \mathcal{F}: F \supsetneqq I^{\prime} \text { for some }} \sum_{\substack{I^{\prime} \in \mathfrak{C}(I) \\
3 I^{\prime} \not \subset F}}\left(\frac{\mathrm{P}^{\alpha}\left(M, \mathbf{1}_{I} \sigma\right)}{|M|}\right)^{2}\left\|\mathrm{Q}_{F, M}^{\omega, \mathbf{b}^{*}} x\right\|_{L^{2}(\omega)}^{\mathbf{\omega}^{2}} \\
& \leq \sum_{M \in\left\{\mathcal{W}\left(I^{\prime}\right) \cup \mathfrak{C}\left(I^{\prime}\right)\right\} \cap \mathcal{W}(F)}\left(\frac{\mathrm{P}^{\alpha}\left(M, \mathbf{1}_{I} \sigma\right)}{|M|}\right)^{2}\left\|\mathrm{Q}_{M}^{\omega, \mathbf{b}^{*}} x\right\|_{L^{2}(\omega)}^{\boldsymbol{\oplus}^{2}} \lesssim\left(\mathfrak{E}_{2}^{\alpha}\right)^{2}|I|_{\sigma} .
\end{aligned}
$$

Finally, we consider the case $M=I$. In this case $I \in \mathcal{D}$ and so $F \supsetneqq I^{\prime}$ implies $F \supset I$ and we can estimate

$$
\sum_{F \in \mathcal{F}: F \supset I}\left(\frac{\mathrm{P}^{\alpha}\left(I, \mathbf{1}_{I} \sigma\right)}{|I|}\right)^{2}\left\|\mathrm{Q}_{F, I}^{\omega, \mathbf{b}^{*}} x\right\|_{L^{2}(\omega)}^{\mathbf{\omega} 2} \leq\left(\frac{\mathrm{P}^{\alpha}\left(I, \mathbf{1}_{I} \sigma\right)}{|I|}\right)^{2}\left\|\mathrm{Q}_{I}^{\omega, \mathbf{b}^{*}} x\right\|_{L^{2}(\omega)}^{\mathbf{\omega} 2} \lesssim A_{2}^{\alpha, \text { energy }}|I|_{\sigma} .
$$

This completes the proof of Lemma 109 .

10.4. The forward Poisson testing inequality. Fix $I \in \mathcal{D}$. We split the integration on the left side of (10.22) into a local and global piece:

$$
\int_{\mathbb{R}_{+}^{2}} \mathbb{P}^{\alpha}\left(\mathbf{1}_{I} \sigma\right)^{2} d \bar{\mu}=\int_{\widehat{I}} \mathbb{P}^{\alpha}\left(\mathbf{1}_{I} \sigma\right)^{2} d \bar{\mu}+\int_{\mathbb{R}_{+}^{2} \backslash \widehat{I}} \mathbb{P}^{\alpha}\left(\mathbf{1}_{I} \sigma\right)^{2} d \bar{\mu} \equiv \mathbf{L o c a l}(I)+\operatorname{Global}(I),
$$

where more explicitly,

$$
\begin{array}{r}
\operatorname{Local}(I) \equiv \int_{\widehat{I}}\left[\mathbb{P}^{\alpha}\left(\mathbf{1}_{I} \sigma\right)(x, t)\right]^{2} d \bar{\mu}(x, t) ; \quad \bar{\mu} \equiv \frac{1}{t^{2}} \mu, \\
\text { i.e. } \bar{\mu} \equiv \sum_{F \in \mathcal{F}} \sum_{M \in \mathcal{W}(F)}\left\|Q_{F, M}^{\omega, \mathbf{b}^{*}} \frac{x}{\ell(M)}\right\|_{L^{2}(\omega)}^{\mathbf{\omega} 2} \cdot \delta_{\left(c_{M}, \ell(M)\right)},
\end{array}
$$

where we recall $\mathbf{Q}_{F, M}^{\omega, \mathbf{b}^{*}}$ is defined in (10.20) above. Here is a brief schematic diagram of the decompositions, with bounds in $\square$, used in this subsection:

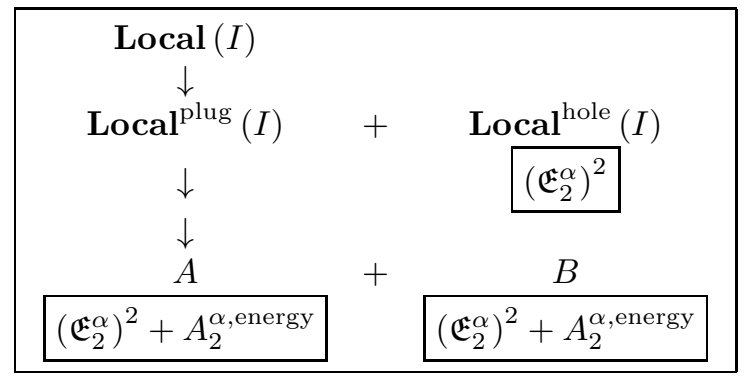


and

$$
\begin{gathered}
\text { Global }(I) \\
\downarrow \\
\stackrel{\downarrow}{A} \\
A_{2}^{\alpha}
\end{gathered}+\frac{B}{\left(\mathfrak{E}_{2}^{\alpha}\right)^{2}+A_{2}^{\alpha}+A_{2}^{\alpha, \text { energy }}+\mathcal{A}^{\alpha, *}+\frac{D}{\mathcal{A}_{2}^{\alpha, *}+A_{2}^{\alpha, \text { punct }}}}
$$

As in our earlier papers [SaShUr2]-SaShUr10] that used a single family of random grids, we have the useful equivalence that

$$
(c(M), \ell(M)) \in \widehat{I} \text { if and only if } M \subset I,
$$

since $M$ and $I$ live in the common grid $\mathcal{D}$. We thus have

$$
\begin{aligned}
& \operatorname{Local}(I)=\int_{\widehat{I}} \mathbb{P}^{\alpha}\left(\mathbf{1}_{I} \sigma\right)(x, t)^{2} d \bar{\mu}(x, t) \\
= & \sum_{F \in \mathcal{F}} \sum_{M \in \mathcal{W}(F): M \subset I} \mathbb{P}^{\alpha}\left(\mathbf{1}_{I} \sigma\right)\left(c_{M}, \ell(M)\right)^{2}\left\|\mathbf{Q}_{F, M}^{\omega, \mathbf{b}^{*}} \frac{x}{|M|}\right\|_{L^{2}(\omega)}^{\boldsymbol{\omega}^{2}} \\
\approx & \sum_{F \in \mathcal{F}} \sum_{M \in \mathcal{W}(F): M \subset I} \mathrm{P}^{\alpha}\left(M, \mathbf{1}_{I} \sigma\right)^{2}\left\|\mathrm{Q}_{F, M}^{\omega, \mathbf{b}^{*}} \frac{x}{|M|}\right\|_{L^{2}(\omega)}^{\mathbf{\omega}^{2}} \\
\approx & \text { Local }^{\text {plug }}(I)+\text { Local }^{\text {hole }}(I),
\end{aligned}
$$

where

$$
\begin{aligned}
\text { Local }^{\text {plug }}(I) & \equiv \sum_{F \in \mathcal{F}} \sum_{M \in \mathcal{W}(F)): M \subset I}\left(\frac{\mathrm{P}^{\alpha}\left(M, \mathbf{1}_{F \cap I} \sigma\right)}{|M|}\right)^{2}\left\|\mathrm{Q}_{F, M}^{\omega, \mathbf{b}^{*}} x\right\|_{L^{2}(\omega)}^{2}, \\
\text { Local }^{\text {hole }}(I) & \equiv \sum_{F \in \mathcal{F}} \sum_{M \in \mathcal{W}(F): M \subset I}\left(\frac{\mathrm{P}^{\alpha}\left(M, \mathbf{1}_{I \backslash F} \sigma\right)}{|M|}\right)^{2}\left\|\mathrm{Q}_{F, M}^{\omega, \mathbf{b}^{*}} x\right\|_{L^{2}(\omega)}^{\boldsymbol{\omega}^{2}}
\end{aligned}
$$

The 'plugged' local sum Local ${ }^{\text {plug }}(I)$ can be further decomposed into

$$
\begin{aligned}
& \text { Local }^{\text {plug }}(I)=\left\{\sum_{F \in \mathcal{F}: F \subset I}+\sum_{F \in \mathcal{F}: F \supsetneqq I}\right\} \sum_{M \in \mathcal{W}(F): M \subset I}\left(\frac{\mathrm{P}^{\alpha}\left(M, \mathbf{1}_{F \cap I} \sigma\right)}{|M|}\right)^{2}\left\|\mathrm{Q}_{F, M}^{\omega, \mathbf{b}^{*}} x\right\|_{L^{2}(\omega)}^{\boldsymbol{\omega}^{2}} \\
& =A+B .
\end{aligned}
$$

Then an application of the Whitney plugged energy condition gives

$$
\begin{aligned}
A & =\sum_{F \in \mathcal{F}: F \subset I} \sum_{M \in \mathcal{W}(F)}\left(\frac{\mathrm{P}^{\alpha}\left(M, \mathbf{1}_{F \cap I} \sigma\right)}{|M|}\right)^{2}\left\|Q_{F, M}^{\omega, \mathbf{b}^{*}} x\right\|_{L^{2}(\omega)}^{\boldsymbol{中} 2} \\
& \leq \sum_{F \in \mathcal{F}: F \subset I}\left(\mathfrak{E}_{2}^{\alpha}+\sqrt{A_{2}^{\alpha, \text { energy }}}\right)^{2}|F|_{\sigma} \lesssim\left(\mathfrak{E}_{2}^{\alpha}+\sqrt{A_{2}^{\alpha, \text { energy }}}\right)^{2}|I|_{\sigma},
\end{aligned}
$$

since $\left\|Q_{F, M}^{\omega, \mathbf{b}^{*} x}\right\|_{L^{2}(\omega)}^{\mathbf{\omega} 2} \leq\left\|Q_{M}^{\omega, \mathbf{b}^{*}} x\right\|_{L^{2}(\omega)}^{\mathbf{\omega} 2}$. We also used here that the stopping intervals $\mathcal{F}$ satisfy a $\sigma$-Carleson measure estimate,

$$
\sum_{F \in \mathcal{F}: F \subset F_{0}}|F|_{\sigma} \lesssim\left|F_{0}\right|_{\sigma}
$$

Lemma 109 applies to the remaining term $B$ to obtain the bound

$$
B \lesssim\left(\left(\mathfrak{E}_{2}^{\alpha}\right)^{2}+A_{2}^{\alpha, \text { energy }}\right)|I|_{\sigma} .
$$

Next we show the inequality with 'holes', where the support of $\sigma$ is restricted to the complement of the interval $F$. 
Lemma 110. We have

$$
\text { Local }^{\text {hole }}(I) \lesssim\left(\mathfrak{E}_{2}^{\alpha}\right)^{2}|I|_{\sigma}
$$

Proof. Fix $I \in \mathcal{D}$ and define

$$
\mathcal{F}_{I} \equiv\{F \in \mathcal{F}: F \subset I\} \cup\{I\},
$$

and denote by $\pi F$, for this proof only, the parent of $F$ in the tree $\mathcal{F}_{I}$. Also denote by $d\left(F, F^{\prime}\right) \equiv d_{\mathcal{F}_{I}}\left(F, F^{\prime}\right)$ the distance from $F$ to $F^{\prime}$ in the tree $\mathcal{F}_{I}$, and denote by $d(F) \equiv d_{\mathcal{F}_{I}}(F, I)$ the distance of $F$ from the root $I$. Since $I \backslash F$ appears in the argument of the Poisson integral, those $F \in \mathcal{F} \backslash \mathcal{F}_{I}$ do not contribute to the sum and so we estimate

$$
S \equiv \text { Local }^{\text {hole }}(I)=\sum_{F \in \mathcal{F}_{I}} \sum_{M \in \mathcal{W}(F): M \subset I}\left(\frac{\mathrm{P}^{\alpha}\left(M, \mathbf{1}_{I \backslash F} \sigma\right)}{|M|}\right)^{2}\left\|\mathrm{Q}_{F, M}^{\omega, \mathbf{b}^{*}} x\right\|_{L^{2}(\omega)}^{\boldsymbol{\omega}^{2}}
$$

by using $\sum_{F^{\prime} \in \mathcal{F}: F \subset F^{\prime} \varsubsetneqq I} \frac{1}{d\left(F^{\prime}\right)^{2}} \leq C$ to obtain 12

$$
\begin{aligned}
& S=\sum_{F \in \mathcal{F}_{I}} \sum_{M \in \mathcal{W}(F): M \subset I}\left(\sum_{F^{\prime} \in \mathcal{F}: F \subset F^{\prime} \varsubsetneqq I} \frac{d\left(F^{\prime}\right)}{d\left(F^{\prime}\right)} \frac{\mathrm{P}^{\alpha}\left(M, \mathbf{1}_{\pi F^{\prime} \backslash F^{\prime}} \sigma\right)}{|M|}\right)^{2}\left\|Q_{F, M}^{\omega, \mathbf{b}^{*}} x\right\|_{L^{2}(\omega)}^{\mathbf{\omega} 2} \\
& \leq \sum_{F \in \mathcal{F}_{I}} \sum_{M \in \mathcal{W}(F): M \subset I}\left(\sum_{F^{\prime} \in \mathcal{F}: F \subset F^{\prime} \varsubsetneqq I} \frac{1}{d\left(F^{\prime}\right)^{2}}\right) \\
& \times\left(\sum_{F^{\prime} \in \mathcal{F}: F \subset F^{\prime} \varsubsetneqq I} d\left(F^{\prime}\right)^{2}\left(\frac{\mathrm{P}^{\alpha}\left(M, \mathbf{1}_{\pi F^{\prime} \backslash F^{\prime}} \sigma\right)}{|M|}\right)^{2}\right)\left\|Q_{F, M}^{\omega, \mathbf{b}^{*}} x\right\|_{L^{2}(\omega)}^{\mathbf{\omega} 2} \\
& \leq C \sum_{F^{\prime} \in \mathcal{F}_{I}} d\left(F^{\prime}\right)^{2} \sum_{F \in \mathcal{F}: F \subset F^{\prime}} \sum_{M \in \mathcal{W}(F): M \subset I}\left(\frac{\mathrm{P}^{\alpha}\left(M, \mathbf{1}_{\left.\pi F^{\prime} \backslash F^{\prime} \sigma\right)}\right.}{|M|}\right)^{2}\left\|\mathrm{Q}_{F, M}^{\omega, \mathbf{b}^{*}} x\right\|_{L^{2}(\omega)}^{\boldsymbol{\omega}^{2}} \\
& =C \sum_{F^{\prime} \in \mathcal{F}_{I}} d\left(F^{\prime}\right)^{2} \sum_{K \in \mathcal{W}\left(F^{\prime}\right)} \sum_{F \in \mathcal{F}: F \subset F^{\prime}} \sum_{M \in \mathcal{W}(F): M \subset I}\left(\frac{\mathrm{P}^{\alpha}\left(M, \mathbf{1}_{\pi F^{\prime} \backslash F^{\prime}} \sigma\right)}{|M|}\right)^{2}\left\|\mathrm{Q}_{F, M \cap K^{*}}^{\omega, \mathbf{b}^{*}} x\right\|_{L^{2}(\omega)}^{\boldsymbol{中} 2} \\
& \lesssim \sum_{F^{\prime} \in \mathcal{F}_{I}} d\left(F^{\prime}\right)^{2} \sum_{K \in \mathcal{W}\left(F^{\prime}\right)}\left(\frac{\mathrm{P}^{\alpha}\left(K, \mathbf{1}_{\pi F^{\prime} \backslash F^{\prime}} \sigma\right)}{|K|}\right)^{2} \sum_{F \in \mathcal{F}: F \subset F^{\prime}} \sum_{M \in \mathcal{W}(F): M \subset I}\left\|Q_{F, M \cap K}^{\omega, \mathbf{b}^{*}} x\right\|_{L^{2}(\omega)}^{\mathbf{\omega}^{2}},
\end{aligned}
$$

where in the fifth line we have used that each $J^{\prime}$ appearing in $\mathbf{Q}_{F, M}^{\omega, \mathbf{b}^{*}}$ occurs in one of the $\mathbf{Q}_{F, M \cap K}^{\omega, \mathbf{b}^{*}}$ since each $M$ is contained in a unique $K$. We have also used there the Poisson inequalities in Lemma 108 ,

We now use the lower frame inequality from Appendix A applied to the function $\mathbf{1}_{K}\left(x-m_{K}^{\omega}\right)$ to obtain

$$
\sum_{F \in \mathcal{F}: F \subset F^{\prime}} \sum_{M \in \mathcal{W}(F): M \subset I}\left\|Q_{F, M \cap K}^{\omega, \mathbf{b}^{*}} x\right\|_{L^{2}(\omega)}^{\mathbf{\omega} 2} \lesssim\left\|\mathbf{1}_{K}\left(x-m_{K}^{\omega}\right)\right\|_{L^{2}(\omega)}^{\mathbf{\omega}^{2}} .
$$

Since the collection $\mathcal{F}_{I}$ satisfies a Carleson condition, namely $\sum_{F \in \mathcal{F}_{I}}\left|F \cap I^{\prime}\right|_{\sigma} \leq C\left|I^{\prime}\right|_{\sigma}$ for all intervals $I^{\prime}$, we have geometric decay in generations:

$$
\sum_{F \in \mathcal{F}_{I}: d(F)=k}|F|_{\sigma} \lesssim 2^{-\delta k}|I|_{\sigma}, \quad k \geq 0
$$

Indeed, with $m>2 C$ we have for each $F^{\prime} \in \mathcal{F}_{I}$,

$$
\sum_{F \in \mathcal{F}_{I}: F \subset F^{\prime} \text { and } d\left(F, F^{\prime}\right)=m}\left|F \cap F^{\prime}\right|_{\sigma}<\frac{1}{2}\left|F^{\prime}\right|_{\sigma},
$$

\footnotetext{
${ }^{12}$ In SaShUr7 and SaShUr6] the first line of this display incorrectly avoided the use of the Cauchy-Schwarz inequality. In the earlier versions [SaShUr5] and version \#2 of [SaShUr6], the argument was correctly given by duality. The fix used here is taken from pages $94-95$ of version \#4 of [SaShUr5].
} 
since otherwise

$$
\sum_{F \in \mathcal{F}_{I}: F \subset F^{\prime} \text { and } d\left(F, F^{\prime}\right) \leq m}\left|F \cap F^{\prime}\right|_{\sigma} \geq m \frac{1}{2}\left|F^{\prime}\right|_{\sigma},
$$

a contradiction. Now iterate (10.30) to obtain (10.29).

Thus we can write

$$
\begin{aligned}
S & \lesssim \sum_{F^{\prime} \in \mathcal{F}_{I}} d\left(F^{\prime}\right)^{2} \sum_{K \in \mathcal{W}\left(F^{\prime}\right)}\left(\frac{\mathrm{P}^{\alpha}\left(K, \mathbf{1}_{\pi F^{\prime} \backslash F^{\prime}} \sigma\right)}{|K|}\right)^{2}\left\|\mathbf{1}_{K}\left(x-m_{K}^{\omega}\right)\right\|_{L^{2}(\omega)}^{\mathbf{\phi}^{2}} \\
& =\sum_{k=1}^{\infty} k^{2} \sum_{F^{\prime} \in \mathcal{F}_{I}: d\left(F^{\prime}\right)=k} \sum_{K \in \mathcal{W}\left(F^{\prime}\right)}\left(\frac{\mathrm{P}^{\alpha}\left(K, \mathbf{1}_{\left.\pi F^{\prime} \backslash F^{\prime} \sigma\right)}\right.}{|K|}\right)^{2}\left\|\mathbf{1}_{K}\left(x-m_{K}^{\omega}\right)\right\|_{L^{2}(\omega)}^{\boldsymbol{\phi}^{2}} \equiv \sum_{k=1}^{\infty} A_{k},
\end{aligned}
$$

where $A_{k}$ is defined at the end of the above display. Hence using the strong energy condition,

$$
\begin{aligned}
A_{k} & =k^{2} \sum_{F^{\prime} \in \mathcal{F}_{I}: d\left(F^{\prime}\right)=k} \sum_{K \in \mathcal{W}\left(F^{\prime}\right)}\left(\frac{\mathrm{P}^{\alpha}\left(K, \mathbf{1}_{\left.\pi F^{\prime} \backslash F^{\prime} \sigma\right)}\right.}{|K|}\right)^{2}\left\|\mathbf{1}_{K}\left(x-m_{K}^{\omega}\right)\right\|_{L^{2}(\omega)}^{\mathbf{\phi}^{2}} \\
& \lesssim k^{2}\left(\mathfrak{E}_{2}^{\alpha}\right)^{2} \sum_{F^{\prime \prime} \in \mathcal{F}_{I}: d\left(F^{\prime \prime}\right)=k-1}\left|F^{\prime \prime}\right|_{\sigma} \lesssim\left(\mathfrak{E}_{2}^{\alpha}\right)^{2} k^{2} 2^{-\delta k}|I|_{\sigma},
\end{aligned}
$$

where we have applied the strong energy condition for each $F^{\prime \prime} \in \mathcal{F}_{I}$ with $d\left(F^{\prime \prime}\right)=k-1$ to obtain

$$
\sum_{F^{\prime} \in \mathcal{F}_{I}: \pi F^{\prime}=F^{\prime \prime}} \sum_{K \in \mathcal{W}\left(F^{\prime}\right)}\left(\frac{\mathrm{P}^{\alpha}\left(K, \mathbf{1}_{F^{\prime \prime} \backslash F^{\prime}} \sigma\right)}{|K|}\right)^{2}\left\|\mathbf{1}_{K}\left(x-m_{K}^{\omega}\right)\right\|_{L^{2}(\omega)}^{\boldsymbol{\phi}^{2}} \leq\left(\mathfrak{E}_{2}^{\alpha}\right)^{2}\left|F^{\prime \prime}\right|_{\sigma} .
$$

Finally then we obtain

$$
S \lesssim \sum_{k=1}^{\infty}\left(\mathfrak{E}_{2}^{\alpha}\right)^{2} k^{2} 2^{-\delta k}|I|_{\sigma} \lesssim\left(\mathfrak{E}_{2}^{\alpha}\right)^{2}|I|_{\sigma}
$$

which is (10.28).

Altogether we have now proved the estimate $\operatorname{Local}(I) \lesssim\left(\left(\mathfrak{E}_{2}^{\alpha}\right)^{2}+A_{2}^{\alpha, \text { energy }}\right)|I|_{\sigma}$ when $I \in \mathcal{D}$, i.e. for every dyadic interval $I \in \mathcal{D}$,

$$
\begin{aligned}
\operatorname{Local}(I) & \approx \sum_{F \in \mathcal{F}} \sum_{M \in \mathcal{W}(F): M \subset I}\left(\frac{\mathrm{P}^{\alpha}\left(M, \mathbf{1}_{I} \sigma\right)}{|M|}\right)^{2}\left\|\mathrm{Q}_{F, M}^{\omega, \mathbf{b}^{*}} x\right\|_{L^{2}(\omega)}^{\mathbf{\omega}^{2}} \\
& \lesssim\left(\left(\mathfrak{E}_{2}^{\alpha}\right)^{2}+A_{2}^{\alpha, \text { energy }}\right)|I|_{\sigma}, \quad I \in \mathcal{D} .
\end{aligned}
$$

10.4.1. The augmented local estimate. For future use in the 'prepare to puncture' arguments below, we prove a strengthening of the local estimate Local $(I)$ to augmented intervals $L \in \mathcal{A D}$.

Lemma 111. With notation as above and $L \in \mathcal{A D}$ an augmented interval, we have

$$
\begin{aligned}
\operatorname{Local}(L) & \equiv \sum_{F \in \mathcal{F}} \sum_{M \in \mathcal{W}(F): M \subset L}\left(\frac{\mathrm{P}^{\alpha}\left(M, \mathbf{1}_{L} \sigma\right)}{|M|}\right)^{2}\left\|Q_{F, M}^{\omega, \mathbf{b}^{*}} x\right\|_{L^{2}(\omega)}^{\mathbf{\omega}^{2}} \\
& \lesssim\left(\left(\mathfrak{E}_{2}^{\alpha}\right)^{2}+A_{2}^{\alpha, \text { energy }}\right)|L|_{\sigma}, \quad L \in \mathcal{A D} .
\end{aligned}
$$

Proof. We prove (10.33) by repeating the above proof of (10.32) and noting the points requiring change. First we decompose

$$
\text { Local }(L) \lesssim \text { Local }^{\text {plug }}(L)+\text { Local }^{\text {hole }}(L)+\text { Local }^{\text {offset }}(L)
$$


where Local $^{\text {plug }}(L)$ and Local ${ }^{\text {hole }}(L)$ are analogous to Local ${ }^{\text {plug }}(I)$ and Local ${ }^{\text {hole }}(I)$ above, and where Local $^{\text {offset }}(L)$ is an additional term arising because $L \backslash F$ need not be empty when $L \cap F \neq \emptyset$ and $F$ is not contained in $L$ :

$$
\begin{aligned}
\text { Local }^{\text {plug }}(L) & \equiv \sum_{F \in \mathcal{F}} \sum_{M \in \mathcal{W}(F): M \subset L}\left(\frac{\mathrm{P}^{\alpha}\left(M, \mathbf{1}_{L \cap F} \sigma\right)}{|M|}\right)^{2}\left\|Q_{F, M}^{\omega, \mathbf{b}^{*}} x\right\|_{L^{2}(\omega)}^{\mathbf{\omega} 2} \\
\text { Local }^{\text {hole }}(L) & \equiv \sum_{F \in \mathcal{F}: F \subset L} \sum_{M \in \mathcal{W}(F): M \subset L}\left(\frac{\mathrm{P}^{\alpha}\left(M, \mathbf{1}_{L \backslash F} \sigma\right)}{|M|}\right)^{2}\left\|\mathrm{Q}_{F, M}^{\omega, \mathbf{b}^{*} x}\right\|_{L^{2}(\omega)}^{\boldsymbol{\omega}^{2}}, \\
\text { Local }^{\text {offset }}(L) & \equiv \sum_{F \in \mathcal{F}: F \not \subset L} \sum_{M \in \mathcal{W}(F): M \subset L}\left(\frac{\mathrm{P}^{\alpha}\left(M, \mathbf{1}_{L \backslash F} \sigma\right)}{|M|}\right)^{2}\left\|\mathrm{Q}_{F, M}^{\omega, \mathbf{b}^{*} x}\right\|_{L^{2}(\omega)}^{\boldsymbol{\omega}^{2}}
\end{aligned}
$$

We have

$$
\begin{aligned}
& \text { Local } \left.^{\text {plug }}(L)=\left\{\sum_{F \in \mathcal{F}: F \subset \text { some } L^{\prime} \in \mathfrak{C}(L)}+\sum_{F \in \mathcal{F}: F \supsetneqq}\right\} \sum_{\text {some }} \sum_{L^{\prime} \in \mathfrak{C}_{\mathcal{D}}(L)}\right\} \\
& \times\left(\frac{\mathrm{P}^{\alpha}\left(M, \mathbf{1}_{F \cap L} \sigma\right)}{|M|}\right)^{2}\left\|\mathrm{Q}_{F, M}^{\omega, \mathbf{b}^{*}} x\right\|_{L^{2}(\omega)}^{\infty 2} \\
& =A+B .
\end{aligned}
$$

Term $A$ satisfies

$$
A \lesssim\left(\mathfrak{E}_{2}^{\alpha}+\sqrt{A_{2}^{\alpha, \text { energy }}}\right)^{2}|L|_{\sigma}
$$

just as above using $\left\|\mathrm{Q}_{F, M}^{\omega} x\right\|_{L^{2}(\omega)}^{2} \leq\left\|\mathrm{Q}_{M}^{\omega} x\right\|_{L^{2}(\omega)}^{2}$, and the fact that the stopping intervals $\mathcal{F}$ satisfy a $\sigma$-Carleson measure estimate,

$$
\sum_{F \in \mathcal{F}: F \subset L}|F|_{\sigma} \lesssim|L|_{\sigma}
$$

Term $B$ is handled directly by Lemma 109 with the augmented interval $I=L$ to obtain

$$
B \lesssim\left(\left(\mathfrak{E}_{2}^{\alpha}\right)^{2}+A_{2}^{\alpha, \text { energy }}\right)|L|_{\sigma}
$$

To handle Local ${ }^{\text {hole }}(L)$, we define

$$
\mathcal{F}_{L} \equiv\{F \in \mathcal{F}: F \subset L\} \cup\{L\},
$$

and follow along the proof there with only trivial changes. The analogue of (10.31) is now

$$
\sum_{F^{\prime} \in \mathcal{F}_{L}: \pi F^{\prime}=F^{\prime \prime}} \sum_{K \in \mathcal{W}\left(F^{\prime}\right)}\left(\frac{\mathrm{P}^{\alpha}\left(K, \mathbf{1}_{F^{\prime \prime} \backslash F^{\prime}} \sigma\right)}{|K|}\right)^{2}\left\|\mathbf{1}_{K}\left(x-m_{K}^{\omega}\right)\right\|_{L^{2}(\omega)}^{\boldsymbol{\phi}^{2}} \leq\left(\mathfrak{E}_{2}^{\alpha}\right)^{2}\left|F^{\prime \prime}\right|_{\sigma},
$$

the only change being that $\mathcal{F}_{L}$ now appears in place of $\mathcal{F}_{I}$, so that the energy condition still applies. We conclude that

$$
\text { Local }^{\text {hole }}(L) \lesssim\left(\mathfrak{E}_{2}^{\alpha}\right)^{2}|L|_{\sigma} .
$$

Finally, the additional term Local ${ }^{\text {offset }}(L)$ is handled directly by Lemma 109, and this completes the proof of the estimate (10.33) in Lemma 111.

10.4.2. The global estimate. Now we turn to proving the following estimate for the global part of the first testing condition (10.22):

$$
\operatorname{Global}(I)=\int_{\mathbb{R}_{+}^{2} \backslash \widehat{I}} \mathbb{P}^{\alpha}\left(\mathbf{1}_{I} \sigma\right)^{2} d \bar{\mu} \lesssim\left(\left(\mathfrak{E}_{2}^{\alpha}\right)^{2}+\mathcal{A}_{2}^{\alpha, *}+A_{2}^{\alpha, \text { punct }}\right)|I|_{\sigma} .
$$


We begin by decomposing the integral above into four pieces. We have from (10.27):

$$
\begin{aligned}
& \int_{\mathbb{R}_{+}^{2} \backslash \widehat{I}} \mathbb{P}^{\alpha}\left(\mathbf{1}_{I} \sigma\right)^{2} d \bar{\mu}=\sum_{M:\left(c_{M}, \ell(M)\right) \in \mathbb{R}_{+}^{2} \backslash \widehat{I}} \mathbb{P}^{\alpha}\left(\mathbf{1}_{I} \sigma\right)\left(c_{M}, \ell(M)\right)^{2} \sum_{\substack{F \in \mathcal{F}: \\
M \in \mathcal{W}(F)}}\left\|\mathbf{Q}_{F, M}^{\omega, \mathbf{b}^{*}} \frac{x}{|M|}\right\|_{L^{2}(\omega)}^{\boldsymbol{\omega}^{2}} \\
& =\left\{\sum_{\substack{M \cap 3 I=\emptyset \\
\ell(M) \leq \ell(I)}}+\sum_{M \subset 3 I \backslash I}+\sum_{\substack{M \cap I=\emptyset \\
\ell(M)>\ell(I)}}+\sum_{M \supsetneqq I}\right\} \mathbb{P}^{\alpha}\left(\mathbf{1}_{I} \sigma\right)\left(c_{M}, \ell(M)\right)^{2} \sum_{\substack{F \in \mathcal{F}: \\
M \in \mathcal{W}(F)}}\left\|Q_{F, M}^{\omega, \mathbf{b}^{*}} \frac{x}{|M|}\right\|_{L^{2}(\omega)} \\
& =A+B+C+D \text {. }
\end{aligned}
$$

We further decompose term $A$ according to the length of $M$ and its distance from $I$, and then use the pairwise disjointedness of the projections $Q_{F, M}^{\omega, \mathbf{b}^{*}}$ in $F$ (see the definition in (10.20) ) to obtain:

$$
\begin{aligned}
A & \lesssim \sum_{m=0}^{\infty} \sum_{k=1}^{\infty} \sum_{\substack{M \subset 3^{k+1} I 3^{k} I \\
\ell(M)=2^{-m} \ell(I)}}\left(\frac{2^{-m}|I|}{d(M, I)^{2-\alpha}}|I|_{\sigma}\right)^{2}|M|_{\omega} \\
& \lesssim \sum_{m=0}^{\infty} 2^{-2 m} \sum_{k=1}^{\infty} \frac{|I|^{2}|I|_{\sigma}\left|3^{k+1} I \backslash 3^{k} I\right|_{\omega}}{\left|3^{k} I\right|^{2(2-\alpha)}}|I|_{\sigma} \\
& \lesssim \sum_{m=0}^{\infty} 2^{-2 m} \sum_{k=1}^{\infty} 3^{-2 k}\left\{\frac{\left|3^{k+1} I \backslash 3^{k} I\right|_{\omega}\left|3^{k} I\right|_{\sigma}}{\left|3^{k} I\right|^{2(1-\alpha)}}\right\}|I|_{\sigma} \lesssim A_{2}^{\alpha}|I|_{\sigma}
\end{aligned}
$$

where the offset Muckenhoupt constant $A_{2}^{\alpha}$ applies because $3^{k+1} I$ has only three times the side length of $3^{k} I$.

For term $B$ we first dispose of the nearby sum $B_{\text {nearby }}$ that consists of the sum over those $M$ which satisfy in addition $2^{-\boldsymbol{\rho}} \ell(I) \leq \ell(M) \leq \ell(I)$. But it is a straightforward task to bound $B_{\text {nearby }}$ by $C A_{2}^{\alpha, \text { energy }}|I|_{\sigma}$ as there are at most $2^{\rho+1}$ such intervals $M$. To bound $B_{\text {away }} \equiv B-B_{\text {nearby, }}$, we further decompose the sum over $F \in \mathcal{F}$ according to whether or not $F \subset 3 I \backslash I$ :

$$
\begin{aligned}
B_{\text {away }} \approx & \sum_{\substack{M \subset 3 I \backslash I \text { and } \ell(M)<2^{-\rho \ell} \ell(I)}}\left(\frac{\mathrm{P}^{\alpha}\left(M, \mathbf{1}_{I} \sigma\right)}{|M|}\right)^{2} \sum_{\substack{F \in \mathcal{F}: F \subset 3 I \backslash I \\
M \in \mathcal{W}(F)}}\left\|\mathrm{Q}_{F, M}^{\omega, \mathbf{b}^{*}} x\right\|_{L^{2}(\omega)}^{\mathbf{\omega}^{2}} \\
& +\sum_{M \subset 3 I \backslash I \text { and } \ell(M)<2^{-\rho} \ell(I)}\left(\frac{\mathrm{P}^{\alpha}\left(M, \mathbf{1}_{I} \sigma\right)}{|M|}\right)^{2} \sum_{\substack{F \in \mathcal{F}: F \not \subset B \backslash I \\
M \in \mathcal{W}(F)}}\left\|Q_{F, M}^{\omega, \mathbf{b}^{*}} x\right\|_{L^{2}(\omega)}^{\mathbf{\omega}^{2}} \\
\equiv & B_{\text {away }}^{1}+B_{\text {away }}^{2} .
\end{aligned}
$$

To estimate $B_{\text {away }}^{1}$, let

$$
\mathcal{J}^{*} \equiv \bigcup_{\substack{F \in \mathcal{F} \\ F \subset 3 I \backslash I}} \bigcup_{\substack{M \in \mathcal{W}(F) \\ M \subset 3 I \backslash I \text { and } \ell(M)<2^{-\rho} \ell(I)}}\left\{J \in \mathcal{C}_{F}^{\mathcal{G}, \text { shift }}: J \subset M\right\}
$$

consist of all intervals $J \in \mathcal{G}$ for which the projection $\triangle_{J}^{\omega, \mathbf{b}^{*}}$ occurs in one of the projections $\mathrm{Q}_{F, M}^{\omega, \mathbf{b}^{*}}$ in term $B_{\text {away }}^{1}$. In order to use $\mathcal{J}^{*}$ in the estimate for $B_{\text {away }}^{1}$ we need the following inequality. For any interval $M \in \mathcal{W}(F)$ we have

$$
\begin{aligned}
\left(\frac{\mathrm{P}^{\alpha}\left(M, \mathbf{1}_{I} \sigma\right)}{|M|}\right)^{2}\left\|\mathrm{Q}_{F ; M}^{\omega, \mathbf{b}^{*} x}\right\|_{L^{2}(\omega)}^{\boldsymbol{\omega} 2} & =\left(\frac{\mathrm{P}^{\alpha}\left(M, \mathbf{1}_{I} \sigma\right)}{|M|}\right)^{2} \sum_{J \in \mathcal{C}_{F}^{\mathcal{G}, \text { shift }:}}\left\|\triangle_{J \subset M}^{\omega, \mathbf{b}^{*}} x\right\|_{L^{2}(\omega)}^{\mathbf{\omega} 2} \\
& \lesssim \sum_{J \in \mathcal{C}_{F}^{\mathcal{G}, \text { shift }}: J \subset M}\left(\frac{\mathrm{P}^{\alpha}\left(J, \mathbf{1}_{I} \sigma\right)}{|J|}\right)^{2}\left\|\triangle_{J}^{\omega, \mathbf{b}^{*}} x\right\|_{L^{2}(\omega)}^{\mathbf{\omega} 2}
\end{aligned}
$$


since

$$
\begin{aligned}
\frac{\mathrm{P}^{\alpha}\left(M, \mathbf{1}_{I} \sigma\right)}{|M|} & =\int_{I} \frac{1}{\left(\ell(M)+\left|x-c_{M}\right|\right)^{2-\alpha}} d \sigma(x) \\
& \lesssim \int_{I} \frac{1}{\left(\ell(J)+\left|x-c_{J}\right|\right)^{2-\alpha}} d \sigma(x)=\frac{\mathrm{P}^{\alpha}\left(J, \mathbf{1}_{I} \sigma\right)}{|J|}
\end{aligned}
$$

for $J \subset M$ because

$$
\ell(J)+\left|x-c_{J}\right| \lesssim \ell(M)+\left|x-c_{M}\right|, \quad J \subset M \text { and } x \in \mathbb{R} .
$$

We now use (10.35) to replace the sum over $M \in \mathcal{W}(F)$ in $B_{\text {away }}^{1}$, with a sum over $J \in \mathcal{J}^{*}$ :

$$
\begin{aligned}
& B_{\text {away }}^{1}=\sum_{M \subset 3 I \backslash I \text { and } \ell(M)<2^{-} \rho_{\ell(I)}}\left(\frac{\mathrm{P}^{\alpha}\left(M, \mathbf{1}_{I} \sigma\right)}{|M|}\right)^{2} \sum_{\substack{F \in \mathcal{F}: F \subset 3 I \backslash I \\
M \in \mathcal{W}(F)}}\left\|Q_{F, M}^{\omega, \mathbf{b}^{*}} x\right\|_{L^{2}(\omega)}^{\boldsymbol{\omega}^{2}} \\
& \lesssim \sum_{M \subset 3 I \backslash I \text { and } \ell(M)<2^{-\rho} \ell(I)} \sum_{\substack{F \in \mathcal{F}: F \subset \mathcal{F} \subset \mathcal{M} \backslash \backslash \backslash I \\
M \in \mathcal{W}(F)}} \sum_{J \in \mathcal{C}_{F}^{\mathcal{G}, \text { shift }}:}\left(\frac{\mathrm{P}^{\alpha}\left(J, \mathbf{1}_{I} \sigma\right)}{|J|}\right)^{2}\left\|\triangle_{J}^{\omega, \mathbf{b}^{*}} x\right\|_{L^{2}(\omega)}^{\mathbf{\omega} 2} \\
& \lesssim \sum_{J \in \mathcal{J}^{*}}\left(\frac{\mathrm{P}^{\alpha}\left(J, \mathbf{1}_{I} \sigma\right)}{|J|}\right)^{2}\left\|\triangle_{J}^{\omega, \mathbf{b}^{*}} x\right\|_{L^{2}(\omega)}^{\mathbf{\omega}^{2}}
\end{aligned}
$$

where the final line follows since for each $J \in \mathcal{J}^{*}$ there is a unique pair $(F, M)$ satisfying the conditions in the second line.

We will now exploit the smallness of $\varepsilon>0$ in the weak goodness condition by decomposing the sum over $J \in \mathcal{J}^{*}$ according to the length of $J$, and then using the fractional Poisson inequality (6.22) in Lemma 63 on the neighbour $I^{\prime}$ of $I$ containing $J$. Indeed, for $J \subset I^{\prime} \subset \mathbb{R}$ and $I \subset \mathbb{R} \backslash I^{\prime}$, we have

$$
\mathrm{P}^{\alpha}\left(J, \mathbf{1}_{I} \sigma\right)^{2} \lesssim\left(\frac{\ell(J)}{\ell(I)}\right)^{2-2(2-\alpha) \varepsilon} \mathrm{P}^{\alpha}\left(I, \mathbf{1}_{I} \sigma\right)^{2}, \quad J \in \mathcal{J}^{*}
$$

where we have used that $\ell\left(I^{\prime}\right)=\ell(I)$ and $\mathrm{P}^{\alpha}\left(I^{\prime}, \mathbf{1}_{I} \sigma\right) \approx \mathrm{P}^{\alpha}\left(I, \mathbf{1}_{I} \sigma\right)$, and that the intervals $J \in \mathcal{J}^{*}$ are good in $I^{\prime}$ and beyond, and have side length at most $2^{-\boldsymbol{\rho}} \ell(I)$, all because $J^{\text {w }} \subset F \subset 3 I \backslash I$ and we have already dealt with the term $B_{\text {nearby. }}$ Moreover, we may also assume here that the exponent $2-2(2-\alpha) \varepsilon$ is positive, i.e. $\varepsilon<\frac{1}{2-\alpha}$, which is of course implied by $0<\varepsilon<\frac{1}{2}$. We then obtain from (10.36), the inequality $\left\|\triangle_{J}^{\omega, \mathbf{b}^{*}} x\right\|_{L^{2}(\omega)}^{\mathbf{\omega}^{2}} \lesssim|J|^{2}|J|_{\omega}$, the pairwise disjointedness of the $M \in \mathcal{W}(F)$, the uniqueness of $F$ with $J \in \mathcal{C}_{F}^{\mathcal{G} \text {,shift }}$, and since $F \subset 3 I \backslash I$ in the sum over $J \in \mathcal{J}^{*}$, that

$$
\begin{aligned}
B_{\text {away }}^{1} & \lesssim \sum_{J \in \mathcal{J}^{*}}\left(\frac{\mathrm{P}^{\alpha}\left(J, \mathbf{1}_{I} \sigma\right)}{|J|}\right)^{2}\left\|\triangle_{J}^{\omega, \mathbf{b}^{*}} x\right\|_{L^{2}(\omega)}^{\mathbf{\omega} 2} \lesssim \sum_{m=\rho}^{\infty} \sum_{\substack{J \in \mathcal{J}^{*} \\
\ell(J)=2^{-m} \ell(I)}}\left(2^{-m}\right)^{2-2(2-\alpha) \varepsilon} \mathrm{P}^{\alpha}\left(I, \mathbf{1}_{I} \sigma\right)^{2}|J|_{\omega} \\
& \lesssim \sum_{m=\boldsymbol{\rho}}^{\infty}\left(2^{-m}\right)^{2-2(2-\alpha) \varepsilon}\left(\frac{|I|_{\sigma}}{|I|^{1-\alpha}}\right)^{2} \sum_{\substack{J \subset 3 I \backslash I \\
\ell(J)=2^{-m} \ell(I)}}|J|_{\omega} \lesssim \sum_{m=\rho}^{\infty}\left(2^{-m}\right)^{2-2(2-\alpha) \varepsilon} \frac{|I|_{\sigma}|3 I \backslash I|_{\omega}}{|3 I|^{2(1-\alpha)}}|I|_{\sigma} \lesssim A_{2}^{\alpha}|I|_{\sigma},
\end{aligned}
$$

since $2-2(2-\alpha) \varepsilon>0$.

To complete the bound for term $B=B_{\text {nearby }}+B_{\text {away }}^{1}+B_{\text {away }}^{2}$, it remains to estimate term $B_{\text {away }}^{2}$ in which we sum over $F \not \subset 3 I \backslash I$. In this case $F \nsupseteq I^{\prime}$ for one of the two neighbours $I^{\prime}$ of $I$, and so we can apply Lemma 109, with $I$ there replaced by the augmented intervals $I^{\prime} \cup I$, to obtain the estimate

$$
B_{\text {away }}^{2} \lesssim\left(\left(\mathfrak{E}_{2}^{\alpha}\right)^{2}+A_{2}^{\alpha, \text { energy }}\right)|I|_{\sigma} .
$$


Next we turn to term $D$. The intervals $M$ occurring here are included in the set of ancestors $A_{k} \equiv \pi_{\mathcal{D}}^{(k)} I$ of $I, 1 \leq k<\infty$.

$$
\begin{aligned}
& D=\sum_{k=1}^{\infty} \mathbb{P}^{\alpha}\left(\mathbf{1}_{I} \sigma\right)\left(c\left(A_{k}\right),\left|A_{k}\right|\right)^{2} \sum_{\substack{F \in \mathcal{F}: \\
A_{k} \in \mathcal{W}(F)}}\left\|Q_{F, A_{k}}^{\omega, \mathbf{b}^{*}} \frac{x}{\left|A_{k}\right|}\right\|_{L^{2}(\omega)}^{\infty 2} \\
& =\sum_{k=1}^{\infty} \mathbb{P}^{\alpha}\left(\mathbf{1}_{I} \sigma\right)\left(c\left(A_{k}\right),\left|A_{k}\right|\right)^{2} \sum_{\substack{F \in \mathcal{F}: \\
A_{k} \in \mathcal{W}(F)}} \sum_{J^{\prime} \in \mathcal{C}_{F}^{\mathcal{G}, \text { shift }:}: J^{\prime} \subset A_{k} \backslash I}\left\|\triangle_{J^{\prime}}^{\omega, \mathbf{b}^{*}} \frac{x}{\left|A_{k}\right|}\right\|_{L^{2}(\omega)}^{\boldsymbol{\omega}^{2}} \\
& +\sum_{k=1}^{\infty} \mathbb{P}^{\alpha}\left(\mathbf{1}_{I} \sigma\right)\left(c\left(A_{k}\right),\left|A_{k}\right|\right)^{2} \sum_{\substack{F \in \mathcal{F}: \\
A_{k} \in \mathcal{W}(F)}} \sum_{\substack{J^{\prime} \in \mathcal{C}_{F}^{\mathcal{G}, \text { shift }}: \\
J^{\prime} \cap I \neq \emptyset \text { and } \ell\left(J^{\prime}\right) \leq \ell(I)}}\left\|\triangle_{J^{\prime}}^{\omega, \mathbf{b}^{*}} \frac{x}{\left|A_{k}\right|}\right\|_{L^{2}(\omega)}^{\boldsymbol{\omega}^{2}} \\
& +\sum_{k=1}^{\infty} \mathbb{P}^{\alpha}\left(\mathbf{1}_{I} \sigma\right)\left(c\left(A_{k}\right),\left|A_{k}\right|\right)^{2} \sum_{\substack{F \in \mathcal{F}: \\
A_{k} \in \mathcal{W}(F)}} \sum_{\substack{J^{\prime} \in \mathcal{C}_{F}^{\mathcal{G}, \text { shift }}: J^{\prime} \subset A_{k} \\
J^{\prime} \cap I \neq \emptyset \text { and } \ell\left(J^{\prime}\right)>\ell(I)}}\left\|\triangle_{J^{\prime}}^{\omega, \mathbf{b}^{*}} \frac{x}{\left|A_{k}\right|}\right\|_{L^{2}(\omega)}^{\mathbf{\omega}^{2}} \\
& \equiv D_{\text {disjoint }}+D_{\text {descendent }}+D_{\text {ancestor }} .
\end{aligned}
$$

We thus have from the pairwise disjointedness of the projections $Q_{F, A_{k}}^{\omega, \mathbf{b}^{*}}$ in $F$ once again,

$$
\begin{aligned}
D_{\text {disjoint }} & =\sum_{k=1}^{\infty} \mathbb{P}^{\alpha}\left(\mathbf{1}_{I} \sigma\right)\left(c\left(A_{k}\right),\left|A_{k}\right|\right)^{2} \sum_{\substack{F \in \mathcal{F}: \\
A_{k} \in \mathcal{W}(F)}} \sum_{J^{\prime} \in \mathcal{C}_{F}^{\mathcal{G}, \text { shift }: J^{\prime} \subset A_{k} \backslash I}}\left\|\triangle_{J^{\prime}}^{\omega, \mathbf{b}^{*}} \frac{x}{\left|A_{k}\right|}\right\|_{L^{2}(\omega)} \\
& \lesssim \sum_{k=1}^{\infty}\left(\frac{|I|_{\sigma}\left|A_{k}\right|}{\left|A_{k}\right|^{2-\alpha}}\right)^{2}\left|A_{k} \backslash I\right|_{\omega}=\left\{\frac{|I|_{\sigma}}{|I|^{1-\alpha}} \sum_{k=1}^{\infty} \frac{|I|^{1-\alpha}}{\left|A_{k}\right|^{2(1-\alpha)}}\left|A_{k} \backslash I\right|_{\omega}\right\}|I|_{\sigma} \\
& \lesssim\left\{\frac{|I|_{\sigma}}{|I|^{1-\alpha}} \mathcal{P}^{\alpha}\left(I, \mathbf{1}_{I^{c}} \omega\right)\right\}|I|_{\sigma} \lesssim \mathcal{A}_{2}^{\alpha, *}|I|_{\sigma},
\end{aligned}
$$

since

$$
\begin{aligned}
\sum_{k=1}^{\infty} \frac{|I|^{1-\alpha}}{\left|A_{k}\right|^{2(1-\alpha)}}\left|A_{k} \backslash I\right|_{\omega} & =\int \sum_{k=1}^{\infty} \frac{|I|^{1-\alpha}}{\left|A_{k}\right|^{2(1-\alpha)}} \mathbf{1}_{A_{k} \backslash I}(x) d \omega(x) \\
& =\int \sum_{k=1}^{\infty} \frac{1}{2^{2(1-\alpha) k}} \frac{|I|^{1-\alpha}}{|I|^{2(1-\alpha)}} \mathbf{1}_{A_{k} \backslash I}(x) d \omega(x) \\
& \lesssim \int_{I^{c}}\left(\frac{|I|}{[|I|+d(x, I)]^{2}}\right)^{1-\alpha} d \omega(x)=\mathcal{P}^{\alpha}\left(I, \mathbf{1}_{I c} \omega\right)
\end{aligned}
$$

upon summing a geometric series with $2(1-\alpha)>0$.

The next term $D_{\text {descendent }}$ satisfies

$$
\begin{aligned}
D_{\text {descendent }} & \lesssim \sum_{k=1}^{\infty}\left(\frac{|I|_{\sigma}\left|A_{k}\right|}{\left|A_{k}\right|^{2-\alpha}}\right)^{2}\left\|Q_{3 I}^{\omega, \mathbf{b}^{*}} \frac{x}{2^{k}|I|}\right\|_{L^{2}(\omega)}^{\infty}{ }^{2} \\
& =\sum_{k=1}^{\infty} 2^{-2 k(2-\alpha)}\left(\frac{|I|_{\sigma}}{|I|^{1-\alpha}}\right)^{2}\left\|Q_{3 I}^{\omega, \mathbf{b}^{*}} \frac{x}{|I|}\right\|_{L^{2}(\omega)}^{\infty 2} \\
& \lesssim\left\{\frac{|I|_{\sigma}\left\|Q_{3 I}^{\omega, \mathbf{b}^{*}} \frac{x}{|I|}\right\|_{L^{2}(\omega)}^{\boldsymbol{\omega}^{2}}}{|I|^{2(1-\alpha)}}\right\}|I|_{\sigma} \lesssim A_{2}^{\alpha, \text { energy }}|I|_{\sigma} .
\end{aligned}
$$


Lastly, for $D_{\text {ancestor }}$ we note that there are at most two intervals $K_{1}$ and $K_{2}$ in $\mathcal{G}$ having side length $\ell(I)$

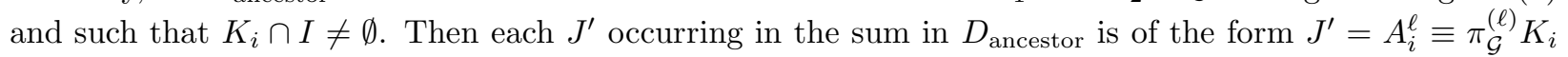
with $J^{\prime} \subset A_{k}$ for some $1 \leq \ell \leq k$ and $i \in\{1,2\}$. Now we write

$$
\begin{aligned}
& D_{\text {ancestor }}=\sum_{k=1}^{\infty} \mathbb{P}^{\alpha}\left(\mathbf{1}_{I} \sigma\right)\left(c\left(A_{k}\right),\left|A_{k}\right|\right)^{2} \sum_{\substack{F \in \mathcal{F} ; \\
A_{k} \in \mathcal{W}(F)}} \sum_{\substack{J^{\prime} \in \mathcal{C}_{F}^{\mathcal{G}, \text { shift }}: \\
J^{\prime} \cap I \neq \emptyset \text { and } \ell\left(J^{\prime}\right)>\ell(I)}}\left\|\triangle_{J^{\prime}}^{\omega, \mathbf{b}^{*}} \frac{x}{\left|A_{k}\right|}\right\|_{L^{2}(\omega)}^{\boldsymbol{\omega}^{2}} \\
& \lesssim \sum_{k=1}^{\infty}\left(\frac{|I|_{\sigma}\left|A_{k}\right|}{\left|A_{k}\right|^{2-\alpha}}\right)^{2} \sum_{i=1}^{2} \sum_{\ell=1}^{k}\left\|\triangle_{A_{i}^{\ell}}^{\omega, \mathbf{b}^{*}} \frac{x}{\left|A_{k}\right|}\right\|_{L^{2}(\omega)}^{\boldsymbol{\omega}^{2}} \\
& \leq 2 \sum_{k=1}^{\infty}\left(\frac{|I|_{\sigma}\left|A_{k}\right|}{\left|A_{k}\right|^{2-\alpha}}\right)^{2}\left\|Q_{A_{k}}^{\omega, \mathbf{b}^{*}} \frac{x}{\left|A_{k}\right|}\right\|_{L^{2}(\omega)}^{\omega^{2}} \text {. }
\end{aligned}
$$

At this point we need a 'prepare to puncture' argument, as we will want to derive geometric decay from $\left\|Q_{J^{\prime}}^{\omega, \mathbf{b}^{*}} x\right\|_{L^{2}(\omega)}^{\mathbf{\omega}^{2}}$ by dominating it by the 'nonenergy' term $\left|J^{\prime}\right|^{2}\left|J^{\prime}\right|_{\omega}$, as well as using the Muckenhoupt energy constant. For this we define $\widetilde{\omega}=\omega-\omega(\{p\}) \delta_{p}$ where $p$ is an atomic point in $I$ for which

$$
\omega(\{p\})=\sup _{q \in \mathfrak{P}_{(\sigma, \omega)}: q \in I} \omega(\{q\}) .
$$

(If $\omega$ has no atomic point in common with $\sigma$ in $I$ set $\widetilde{\omega}=\omega$.) Then we have $|I|_{\widetilde{\omega}}=\omega\left(I, \mathfrak{P}_{(\sigma, \omega)}\right)$ and

$$
\frac{|I|_{\widetilde{\omega}}}{|I|^{1-\alpha}} \frac{|I|_{\sigma}}{|I|^{1-\alpha}}=\frac{\omega\left(I, \mathfrak{P}_{(\sigma, \omega)}\right)}{|I|^{1-\alpha}} \frac{|I|_{\sigma}}{|I|^{1-\alpha}} \leq A_{2}^{\alpha, \text { punct }}
$$

A key observation, already noted in the proof of Lemma 104 above, is that

$$
\left\|\triangle_{K}^{\omega, \mathbf{b}^{*}} x\right\|_{L^{2}(\omega)}^{2}=\left\{\begin{array}{ccc}
\left\|\triangle_{K}^{\omega, \mathbf{b}^{*}}(x-p)\right\|_{L^{2}(\omega)}^{2} & \text { if } & p \in K \\
\left\|\triangle_{K}^{\omega, \mathbf{b}^{*}} x\right\|_{L^{2}(\widetilde{\omega})}^{2} & \text { if } \quad p \notin K
\end{array} \leq \ell(K)^{2}|K|_{\widetilde{\omega}}, \quad \text { for all } K \in \mathcal{D}\right.
$$

and so, as in the proof of (10.17) in Lemma 104,

$$
\left\|Q_{A_{k}}^{\omega, \mathbf{b}^{*}} \frac{x}{\left|A_{k}\right|}\right\|_{L^{2}(\omega)}^{\mathbf{\omega}^{2}} \lesssim\left|A_{k}\right|_{\widetilde{\omega}} .
$$

Then we continue with

$$
\begin{aligned}
& \sum_{k=1}^{\infty}\left(\frac{|I|_{\sigma}\left|A_{k}\right|}{\left|A_{k}\right|^{2-\alpha}}\right)^{2}\left\|Q_{A_{k}}^{\omega, \mathbf{b}^{*}} \frac{x}{\left|A_{k}\right|}\right\|_{L^{2}(\omega)}^{\mathbf{\omega} 2} \\
\lesssim & \sum_{k=1}^{\infty}\left(\frac{|I|_{\sigma}\left|A_{k}\right|}{\left|A_{k}\right|^{2-\alpha}}\right)^{2}\left|A_{k}\right|_{\widetilde{\omega}} \\
= & \sum_{k=1}^{\infty}\left(\frac{|I|_{\sigma}}{\left|A_{k}\right|^{1-\alpha}}\right)^{2}\left|A_{k} \backslash I\right|_{\omega}+\sum_{k=1}^{\infty}\left(\frac{|I|_{\sigma}}{2^{k(1-\alpha)}|I|^{1-\alpha}}\right)^{2}|I|_{\widetilde{\omega}} \\
\lesssim & \left(\mathcal{A}_{2}^{\alpha, *}+A_{2}^{\alpha, \text { punct }}\right)|I|_{\sigma},
\end{aligned}
$$

where the inequality $\sum_{k=1}^{\infty}\left(\frac{|I|_{\sigma}}{\left|A_{k}\right|^{1-\alpha}}\right)^{2}\left|A_{k} \backslash I\right|_{\omega} \lesssim \mathcal{A}_{2}^{\alpha, *}|I|_{\sigma}$ is already proved above in the display estimating $D_{\text {disjoint }}$. 
Finally, for term $C$ we will have to group the intervals $M$ into blocks $B_{i}$. We first split the sum according to whether or not $I$ intersects the triple of $M$ :

$$
\begin{aligned}
C & \approx\left\{\sum_{\substack{M: I \cap 3 M=\emptyset \\
\ell(M)>\ell(I)}}+\sum_{\substack{M: I \subset 3 M \backslash M \\
\ell(M)>\ell(I)}}\right\}\left(\frac{|M|}{(|M|+d(M, I))^{2-\alpha}}|I|_{\sigma}\right)^{2} \sum_{\substack{F \in \mathcal{F}: \\
M \in \mathcal{W}(F)}}\left\|Q_{F, M}^{\omega \mathbf{b}^{*}} \frac{x}{|M|}\right\|_{L^{2}(\omega)} \\
& =C_{1}+C_{2} .
\end{aligned}
$$

We first consider $C_{1}$. Let $\mathcal{M}$ consist of the maximal dyadic intervals in the collection $\{Q: 3 Q \cap I=\emptyset\}$, and then let $\left\{B_{i}\right\}_{i=1}^{\infty}$ be an enumeration of those $Q \in \mathcal{M}$ whose side length is at least $\ell(I)$. Note in particular that $3 B_{i} \cap I=\emptyset$. Now we further decompose the sum in $C_{1}$ by grouping the intervals $M$ into the 'Whitney' intervals $B_{i}$, and then using the pairwise disjointedness of the martingale supports of the pseudoprojections $\mathrm{Q}_{F, M}^{\omega, \mathbf{b}^{*}}$ in $F:$

$$
\begin{aligned}
& C_{1} \leq \sum_{i=1}^{\infty} \sum_{M: M \subset B_{i}}\left(\frac{1}{(|M|+d(M, I))^{2-\alpha}}|I|_{\sigma}\right)^{2} \sum_{\substack{F \in \mathcal{F}: \\
M \in \mathcal{W}(F)}}\left\|Q_{F, M}^{\omega, \mathbf{b}^{*}} x\right\|_{L^{2}(\omega)}^{\mathbf{\omega}^{2}} \\
& \lesssim \sum_{i=1}^{\infty}\left(\frac{1}{\left(\left|B_{i}\right|+d\left(B_{i}, I\right)\right)^{2-\alpha}}|I|_{\sigma}\right)^{2} \sum_{M: M \subset B_{i}} \sum_{\substack{F \in \mathcal{F}: \\
M \in \mathcal{W}(F)}}\left\|\mathbf{Q}_{F, M}^{\omega, \mathbf{b}^{*}} x\right\|_{L^{2}(\omega)}^{\mathbf{\omega}^{2}} \\
& \lesssim \sum_{i=1}^{\infty}\left(\frac{1}{\left(\left|B_{i}\right|+d\left(B_{i}, I\right)\right)^{2-\alpha}}|I|_{\sigma}\right)^{2} \sum_{M: M \subset B_{i}}|M|^{2}|M|_{\omega} \\
& \lesssim \sum_{i=1}^{\infty}\left(\frac{1}{\left(\left|B_{i}\right|+d\left(B_{i}, I\right)\right)^{2-\alpha}}|I|_{\sigma}\right)^{2}\left|B_{i}\right|^{2}\left|B_{i}\right|_{\omega} \\
& \lesssim\left\{\sum_{i=1}^{\infty} \frac{\left|B_{i}\right|_{\omega}|I|_{\sigma}}{\left|B_{i}\right|^{2(1-\alpha)}}\right\}|I|_{\sigma},
\end{aligned}
$$

Now since $\left|B_{i}\right| \approx d(x, I)$ for $x \in B_{i}$,

$$
\begin{aligned}
\sum_{i=1}^{\infty} \frac{\left|B_{i}\right|_{\omega}|I|_{\sigma}}{\left|B_{i}\right|^{2(1-\alpha)}} & =\frac{|I|_{\sigma}}{|I|^{1-\alpha}} \sum_{i=1}^{\infty} \frac{|I|^{1-\alpha}}{\left|B_{i}\right|^{2(1-\alpha)}}\left|B_{i}\right|_{\omega} \\
& \approx \frac{|I|_{\sigma}}{|I|^{1-\alpha}} \sum_{i=1}^{\infty} \int_{B_{i}} \frac{|I|^{1-\alpha}}{d(x, I)^{2(1-\alpha)}} d \omega(x) \\
& \approx \frac{|I|_{\sigma}}{|I|^{1-\alpha}} \sum_{i=1}^{\infty} \int_{B_{i}}\left(\frac{|I|}{[|I|+d(x, I)]^{2}}\right)^{1-\alpha} d \omega(x) \\
& \leq \frac{|I|_{\sigma}}{|I|^{1-\alpha}} \mathcal{P}^{\alpha}\left(I, \mathbf{1}_{I^{c}} \omega\right) \leq \mathcal{A}_{2}^{\alpha, *},
\end{aligned}
$$

we obtain

$$
C_{1} \lesssim \mathcal{A}_{2}^{\alpha, *}|I|_{\sigma}
$$

Next we turn to estimating term $C_{2}$ where the triple of $M$ contains $I$ but $M$ itself does not. Note that there are at most two such intervals $M$ of a given side length. So with this in mind, we sum over the intervals 
$M$ according to their lengths to obtain

$$
\begin{aligned}
C_{2} & =\sum_{\substack { m=1 \\
\begin{subarray}{c}{M: I \subset 3 M \backslash M \\
\ell(M)=2^{m} \ell(I){ m = 1 \\
\begin{subarray} { c } { M : I \subset 3 M \backslash M \\
\ell ( M ) = 2 ^ { m } \ell ( I ) } }\end{subarray}}^{\infty}\left(\frac{|M|}{(|M|+\operatorname{dist}(M, I))^{2-\alpha}}|I|_{\sigma}\right)^{2} \sum_{\substack{F \in \mathcal{F}: \\
M \in \mathcal{W}(F)}}\left\|Q_{F, M}^{\omega, \mathbf{b}^{*}} \frac{x}{|M|}\right\|_{L^{2}(\omega)}^{\mathbf{N}^{2}} \\
& \lesssim \sum_{m=1}^{\infty}\left(\frac{|I|_{\sigma}}{\left|2^{m} I\right|^{1-\alpha}}\right)^{2}\left|\left(5 \cdot 2^{m} I\right) \backslash I\right|_{\omega}=\left\{\frac{|I|_{\sigma}}{|I|^{1-\alpha}} \sum_{m=1}^{\infty} \frac{|I|^{1-\alpha}\left|\left(5 \cdot 2^{m} I\right) \backslash I\right|_{\omega}}{\left|2^{m} I\right|^{2(1-\alpha)}}\right\}|I|_{\sigma} \\
& \lesssim\left\{\frac{|I|_{\sigma}}{|I|^{1-\alpha}} \mathcal{P}^{\alpha}\left(I, \mathbf{1}_{I^{c}} \omega\right)\right\}|I|_{\sigma} \leq \mathcal{A}_{2}^{\alpha, *}|I|_{\sigma},
\end{aligned}
$$

since in analogy with the corresponding estimate above,

$$
\sum_{m=1}^{\infty} \frac{|I|^{1-\alpha}\left|\left(5 \cdot 2^{m} I\right) \backslash I\right|_{\omega}}{\left|2^{m} I\right|^{2(1-\alpha)}}=\int \sum_{m=1}^{\infty} \frac{|I|^{1-\alpha}}{\left|2^{m} I\right|^{2(1-\alpha)}} \mathbf{1}_{\left(5 \cdot 2^{m} I\right) \backslash I}(x) d \omega(x) \lesssim \mathcal{P}^{\alpha}\left(I, \mathbf{1}_{I^{c}} \omega\right) .
$$

10.5. The backward Poisson testing inequality. The argument here follows the broad outline of the analogous argument in [SaShUr7], but using modifications from [SaShUr9] that involve 'prepare to puncture arguments', using decompositions $\mathcal{W}(F)$ in place of $(\boldsymbol{\rho}, \varepsilon)$-decompositions, and using pseudoprojections $\mathrm{Q}_{F, M}^{\omega, \mathbf{b}^{*}} x$ (see (10.20) for the definition). The final change here is that there is no splitting into local and global parts as in [SaShUr7] - instead, we follow the treatment in [SaShUr6] in this regard.

Fix $I \in \mathcal{D}$. It suffices to prove

$$
\operatorname{Back}(\widehat{I}) \equiv \int_{\mathbb{R}}\left[\mathbb{Q}^{\alpha}\left(t \mathbf{1}_{\widehat{I}} \bar{\mu}\right)(y)\right]^{2} d \sigma(y) \lesssim\left\{\mathcal{A}_{2}^{\alpha}+\left(\mathfrak{E}_{2}^{\alpha}+\sqrt{A_{2}^{\alpha, \text { energy }}}\right) \sqrt{A_{2}^{\alpha, \text { punct }}}\right\} \int_{\widehat{I}} t^{2} d \bar{\mu}(x, t)
$$

Note that for a 'Poisson integral with holes' and a measure $\mu$ built with Haar projections, Hytönen obtained in Hyt2 the simpler bound $A_{2}^{\alpha}$ for a term analogous to, but significantly smaller than, (10.38). Here is a brief schematic diagram of the decompositions, with bounds in $\square$, used in this subsection:

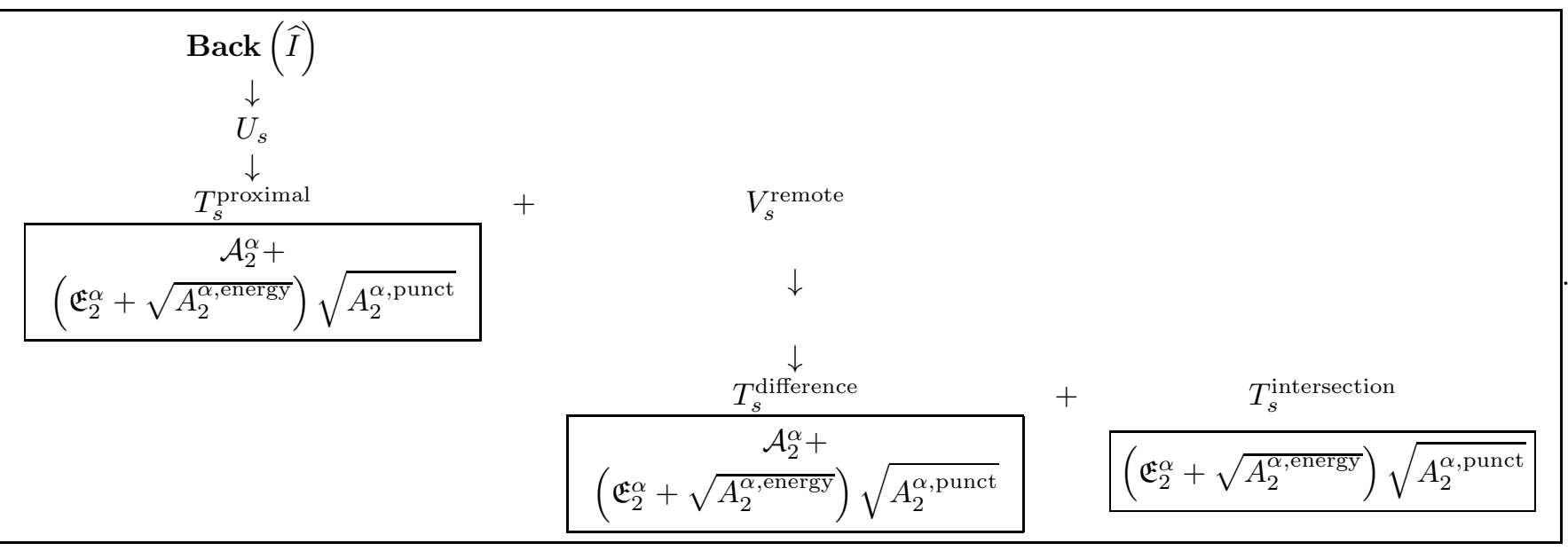

Using (10.27) we see that the integral on the right hand side of (10.38) is

$$
\int_{\widehat{I}} t^{2} d \bar{\mu}=\sum_{F \in \mathcal{F}} \sum_{M \in \mathcal{W}(F): M \subset I}\left\|Q_{F, M}^{\omega, \mathbf{b}^{*}} x\right\|_{L^{2}(\omega)}^{\mathbf{A}^{2}}
$$

where $\mathrm{Q}_{F, M}^{\omega, \mathbf{b}^{*}}$ was defined in (10.20). 
We now compute using (10.27) again that

$$
\begin{aligned}
\mathbb{Q}^{\alpha}\left(t \mathbf{1}_{\widehat{I}} \bar{\mu}\right)(y) & =\int_{\widehat{I}} \frac{t^{2}}{\left(t^{2}+|x-y|^{2}\right)^{\frac{2-\alpha}{2}}} d \bar{\mu}(x, t) \\
& \approx \sum_{F \in \mathcal{F}} \sum_{M \in \mathcal{W}(F): M \subset I} \frac{\left\|\mathbf{Q}_{F, M}^{\omega, \mathbf{b}^{*}} x\right\|_{L^{2}(\omega)}^{\mathbf{A}^{2}}}{\left(|M|+\left|y-c_{M}\right|\right)^{2-\alpha}},
\end{aligned}
$$

and then expand the square and integrate to obtain that the term Back $(\widehat{I})$ is

$$
\sum_{\substack{F \in \mathcal{F} \\ M \in \mathcal{W}(F) \\ M \subset I}} \sum_{\substack{F^{\prime} \in \mathcal{F}: \\ M^{\prime} \in \mathcal{W}\left(F^{\prime}\right) \\ M^{\prime} \subset I}} \int_{\mathbb{R}} \frac{\left\|Q_{F, M}^{\omega, \mathbf{b}^{*}} x\right\|_{L^{2}(\omega)}}{\left(|M|+\left|y-c_{M}\right|\right)^{2-\alpha}} \frac{\left\|Q_{F^{\prime}, M^{\prime}}^{\omega, \mathbf{b}^{*}} x\right\|_{L^{2}(\omega)}^{\mathbf{\omega}^{2}}}{\left(\left|M^{\prime}\right|+\left|y-c_{M^{\prime}}\right|\right)^{2-\alpha}} d \sigma(y) .
$$

By symmetry we may assume that $\ell\left(M^{\prime}\right) \leq \ell(M)$. We fix a nonnegative integer $s$, and consider those intervals $M$ and $M^{\prime}$ with $\ell\left(M^{\prime}\right)=2^{-s} \ell(M)$. For fixed $s$ we will control the expression

$$
\begin{aligned}
U_{s} \equiv & \sum_{F, F^{\prime} \in \mathcal{F}} \sum_{\substack{M \in \mathcal{W}(F), M^{\prime} \in \mathcal{W}\left(F^{\prime}\right) \\
M, M^{\prime} \subset I, \ell\left(M^{\prime}\right)=2^{-s} \ell(M)}} \\
& \times \int_{\mathbb{R}} \frac{\left\|\mathbf{Q}_{F, M}^{\omega, \mathbf{b}^{*}} x\right\|_{L^{2}(\omega)}^{\mathbf{\omega} 2}}{\left(|M|+\left|y-c_{M}\right|\right)^{2-\alpha}} \frac{\left\|Q_{F^{\prime}, M^{\prime}}^{\omega, \mathbf{b}^{*}} x\right\|_{L^{2}(\omega)}^{\mathbf{\omega} 2}}{\left(\left|M^{\prime}\right|+\left|y-c_{M^{\prime}}\right|\right)^{2-\alpha}} d \sigma(y),
\end{aligned}
$$

by proving that

$$
U_{s} \lesssim 2^{-\delta s}\left\{\mathcal{A}_{2}^{\alpha}+\left(\mathfrak{E}_{2}^{\alpha}+\sqrt{A_{2}^{\alpha, \text { energy }}}\right) \sqrt{A_{2}^{\alpha, \text { punct }}}\right\} \int_{\widehat{I}} t^{2} d \bar{\mu}, \quad \text { where } \delta=\frac{1}{2}
$$

With this accomplished, we can sum in $s \geq 0$ to control the term $\operatorname{Back}(\hat{I})$. We now decompose $U_{s}=$ $T_{s}^{\text {proximal }}+T_{s}^{\text {difference }}+T_{s}^{\text {intersection }}$ into three pieces.

Our first decomposition is to write

$$
U_{s}=T_{s}^{\text {proximal }}+V_{s}^{\text {remote }},
$$

where in the 'proximal' term $T_{s}^{\text {proximal }}$ we restrict the summation over pairs of intervals $M, M^{\prime}$ to those satisfying $d\left(c_{M}, c_{M^{\prime}}\right)<2^{s \delta} \ell(M)$; while in the 'remote' term $V_{s}^{\text {remote }}$ we restrict the summation over pairs of intervals $M, M^{\prime}$ to those satisfying the opposite inequality $d\left(c_{M}, c_{M^{\prime}}\right) \geq 2^{s \delta} \ell(M)$. Then we further decompose

$$
V_{s}^{\text {remote }}=T_{s}^{\text {difference }}+T_{s}^{\text {intersection }},
$$

where in the 'difference' term $T_{s}^{\text {difference }}$ we restrict integration in $y$ to the difference $\mathbb{R} \backslash B\left(M, M^{\prime}\right)$ of $\mathbb{R}$ and

$$
B\left(M, M^{\prime}\right) \equiv B\left(c_{M}, \frac{1}{2} d\left(c_{M}, c_{M^{\prime}}\right)\right),
$$

the ball centered at $c_{M}$ with radius $\frac{1}{2} d\left(c_{M}, c_{M^{\prime}}\right)$; while in the 'intersection' term $T_{s}^{\text {intersection }}$ we restrict integration in $y$ to the intersection $\mathbb{R} \cap B\left(M, M^{\prime}\right)$ of $\mathbb{R}$ with the ball $B\left(M, M^{\prime}\right)$; i.e.

$$
\begin{aligned}
T_{s}^{\text {intersection }} \equiv & \sum_{F, F^{\prime} \in \mathcal{F}} \sum_{\begin{array}{c}
M \in \mathcal{W}(F), M^{\prime} \in \mathcal{W}\left(F^{\prime}\right) \\
M, M^{\prime} \subset I, \ell\left(M^{\prime}\right)=2^{-s} \ell(M) \\
d\left(c_{M}, c_{M^{\prime}}\right) \geq 2^{s(1+\delta)} \ell\left(M^{\prime}\right)
\end{array}} \\
& \times \int_{B\left(M, M^{\prime}\right)} \frac{\left\|Q_{F, M}^{\omega, \mathbf{b}^{*}} x\right\|_{L^{2}(\omega)}^{\mathbf{\omega} 2}}{\left(|M|+\left|y-c_{M}\right|\right)^{2-\alpha}} \frac{\left\|Q_{F^{\prime}, M^{\prime}}^{\omega, \mathbf{b}^{*}} x\right\|_{L^{2}(\omega)}^{\mathbf{\omega} 2}}{\left(\left|M^{\prime}\right|+\left|y-c_{M^{\prime}}\right|\right)^{2-\alpha}} d \sigma(y) .
\end{aligned}
$$


Here is a schematic reminder of the these decompositions with the distinguishing points of the definitions boxed:

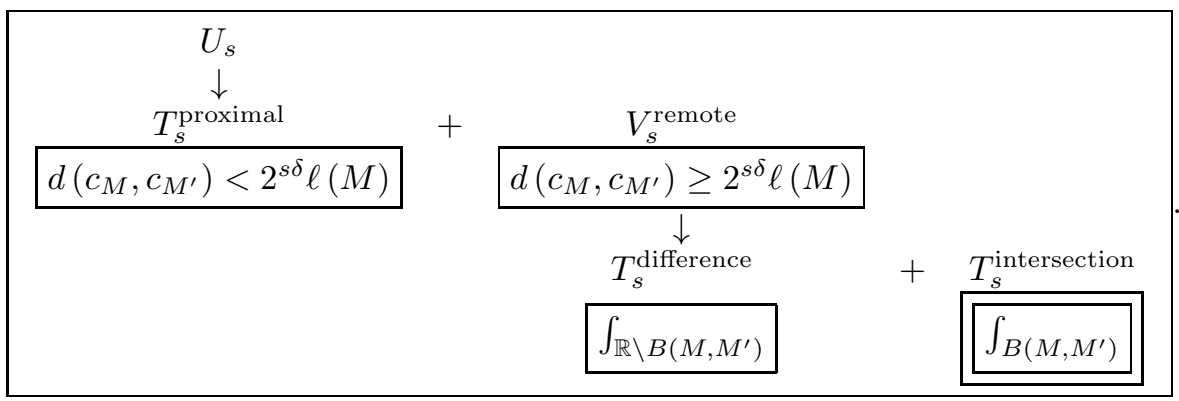

We will exploit the restriction of integration to $B\left(M, M^{\prime}\right)$, together with the condition

$$
d\left(c_{M}, c_{M^{\prime}}\right) \geq 2^{s(1+\delta)} \ell\left(M^{\prime}\right)=2^{s \delta} \ell(M),
$$

which will then give an estimate for the term $T_{s}^{\text {intersection }}$ using an argument dual to that used for the other terms $T_{s}^{\text {proximal }}$ and $T_{s}^{\text {difference }}$, to which we now turn.

10.5.1. The proximal and difference terms. We have

$$
\begin{aligned}
& T_{s}^{\text {proximal }} \equiv \sum_{F, F^{\prime} \in \mathcal{F}} \sum_{M \in \mathcal{W}(F), M^{\prime} \in \mathcal{W}\left(F^{\prime}\right)} \\
& M, M^{\prime} \subset I, \ell\left(M^{\prime}\right)=2^{-s} \ell(M) \text { and } d\left(c_{M}, c_{M^{\prime}}\right)<2^{s \delta} \ell(M) \\
& \times \int_{\mathbb{R}} \frac{\left\|\mathbf{Q}_{F, M}^{\omega, \mathbf{b}^{*}} x\right\|_{L^{2}(\omega)}^{\mathbf{\omega} 2}}{\left(|M|+\left|y-c_{M}\right|\right)^{2-\alpha}} \frac{\left\|\mathbf{Q}_{F^{\prime}, M^{\prime}}^{\omega, \mathbf{b}^{*}} x\right\|_{L^{2}(\omega)}^{\mathbf{\omega} 2}}{\left(\left|M^{\prime}\right|+\left|y-c_{M^{\prime}}\right|\right)^{2-\alpha}} d \sigma(y) \\
& \leq \mathcal{M}_{s}^{\text {proximal }} \sum_{F \in \mathcal{F}} \sum_{\substack{M \in \mathcal{W}(F) \\
M \subset I}}\left\|Q_{F, M}^{\omega, \mathbf{b}^{*}} z\right\|_{\omega}^{\mathbf{\omega} 2}=\mathcal{M}_{s}^{\text {proximal }} \int_{\widehat{I}} t^{2} d \bar{\mu},
\end{aligned}
$$

where

$$
\begin{aligned}
& \mathcal{M}_{s}^{\text {proximal }} \equiv \sup _{F \in \mathcal{F}} \sup _{\substack{M \in \mathcal{W}(F) \\
M \subset I}} \mathcal{A}_{s}^{\text {proximal }}(M) ; \\
& \mathcal{A}_{s}^{\text {proximal }}(M) \equiv \sum_{F^{\prime} \in \mathcal{F}} \sum_{\substack{M^{\prime} \in \mathcal{W}\left(F^{\prime}\right) \\
M^{\prime} \subset I, \ell\left(M^{\prime}\right)=2^{-s} \ell(M) \text { and } d\left(c_{M}, c_{M^{\prime}}\right)<2^{s \delta} \ell(M)}} \int_{\mathbb{R}} S_{\left(M^{\prime}, M\right)}^{F^{\prime}}(y) d \sigma(y) ; \\
& S_{\left(M^{\prime}, M\right)}^{F^{\prime}}(x) \equiv \frac{1}{\left(|M|+\left|y-c_{M}\right|\right)^{2-\alpha}} \frac{\left\|Q_{F^{\prime}, M^{\prime}}^{\omega} x\right\|_{L^{2}(\omega)}^{\mathbf{\omega} 2}}{\left(\left|M^{\prime}\right|+\left|y-c_{M^{\prime}}\right|\right)^{2-\alpha}}
\end{aligned}
$$

and similarly

$$
\begin{aligned}
T_{s}^{\text {difference }} & \equiv \sum_{F, F^{\prime} \in \mathcal{F}} \sum_{\substack{M \in \mathcal{W}(F), M^{\prime} \in \mathcal{W}\left(F^{\prime}\right) \\
M, M^{\prime} \subset I, \ell\left(M^{\prime}\right)=2^{-s} \ell(M) \text { and } d\left(c_{M}, c_{M^{\prime}}\right) \geq 2^{s \delta} \ell(M)}} \\
& \times \int_{\mathbb{R} \backslash B\left(M, M^{\prime}\right)} \frac{\left\|Q_{F, M}^{\omega, \mathbf{b}^{*}} x\right\|_{L^{2}(\omega)}^{\mathbf{\omega} 2}}{\left(|M|+\left|y-c_{M}\right|\right)^{2-\alpha}} \frac{\left\|Q_{F^{\prime}, M^{\prime}}^{\omega, \mathbf{b}^{*}} x\right\|_{L^{2}(\omega)}}{\left(\left|M^{\prime}\right|+\left|y-c_{M^{\prime}}\right|\right)^{2-\alpha}} d \sigma(y) \\
& \leq \mathcal{M}_{s}^{\text {difference }} \sum_{F \in \mathcal{F}} \sum_{\substack{M \in \mathcal{W}(F) \\
M \subset I}}\left\|Q_{F, M}^{\omega, \mathbf{b}^{*}} z\right\|_{\omega}^{\mathbf{\omega}^{2}}=\mathcal{M}_{s}^{\text {difference }} \int_{\widehat{I}} t^{2} d \bar{\mu} ;
\end{aligned}
$$


where

$$
\begin{aligned}
& \mathcal{M}_{s}^{\text {difference }} \equiv \sup _{F \in \mathcal{F}} \sup _{\substack{M \in \mathcal{W}(F) \\
M \subset I}} \mathcal{A}_{s}^{\text {difference }}(M) \\
& \mathcal{A}_{s}^{\text {difference }}(M) \equiv \sum_{F^{\prime} \in \mathcal{F}} \sum_{\substack{M^{\prime} \in \mathcal{W}\left(F^{\prime}\right) \\
M^{\prime} \subset I, \ell\left(M^{\prime}\right)=2^{-s} \ell(M) \text { and } d\left(c_{M}, c_{M^{\prime}}\right) \geq 2^{s \delta} \ell(M)}} \int_{\mathbb{R} \backslash B\left(M, M^{\prime}\right)} S_{\left(M^{\prime}, M\right)}^{F^{\prime}}(y) d \sigma(y) \text {. }
\end{aligned}
$$

The restriction of integration in $\mathcal{A}_{s}^{\text {difference }}$ to $\mathbb{R} \backslash B\left(M, M^{\prime}\right)$ will be used to establish (10.49) below.

Notation 112. Since the intervals $F, M, F^{\prime}, M^{\prime}$ that arise in all of the sums here satisfy

$$
M \in \mathcal{W}(F), M^{\prime} \in \mathcal{W}\left(F^{\prime}\right) \text { and } \ell\left(M^{\prime}\right)=2^{-s} \ell(M) \text { and } M, M^{\prime} \subset I,
$$

we will often employ the notation $\sum^{*}$ to remind the reader that, as applicable, these four conditions are in force even when they are not explictly mentioned.

Now fix $M$ as in $\mathcal{M}_{s}^{\text {proximal }}$ respectively $\mathcal{M}_{s}^{\text {difference }}$, and decompose the sum over $M^{\prime}$ in $\mathcal{A}_{s}^{\text {proximal }}(M)$ respectively $\mathcal{A}_{s}^{\text {difference }}(M)$ by

$$
\begin{aligned}
& \mathcal{A}_{s}^{\text {proximal }}(M)=\sum_{F^{\prime} \in \mathcal{F}} \sum_{\substack{M^{\prime} \in \mathcal{W}\left(F^{\prime}\right) \\
M^{\prime} \subset I, \ell\left(M^{\prime}\right)=2^{-s} \ell(M) \text { and } d\left(c_{M}, c_{M^{\prime}}\right)<2^{s \delta} \ell(M)}} \int_{\mathbb{R}} S_{\left(M^{\prime}, M\right)}^{F^{\prime}}(y) d \sigma(y) \\
= & \sum_{F^{\prime} \in \mathcal{F}} \sum_{\substack{c_{M^{\prime}} \in 2 M \\
d\left(c_{M}, c_{M^{\prime}}\right)<2^{s \delta} \ell(M)}}^{*} \int_{\mathbb{R}} S_{\left(M^{\prime}, M\right)}^{F^{\prime}}(y) d \sigma(y)+\sum_{F^{\prime} \in \mathcal{F}} \sum_{\ell=1}^{\infty} \sum_{\substack{c_{M^{\prime}} \in 2^{\ell+1} M \backslash 2^{\ell} M \\
d\left(c_{M}, c_{M^{\prime}}\right)<2^{s \delta} \ell(M)}} \int_{\mathbb{R}} S_{\left(M^{\prime}, M\right)}^{\left.F^{\prime}, M\right) d \sigma(y)} \\
\equiv & \sum_{\ell=0}^{\infty} \mathcal{A}_{s}^{\text {proximal }, \ell}(M),
\end{aligned}
$$

respectively

$$
\begin{aligned}
& \mathcal{A}_{s}^{\text {difference }}(M)=\sum_{F^{\prime} \in \mathcal{F}} \sum_{\substack{M^{\prime} \in \mathcal{W}\left(F^{\prime}\right) \\
M^{\prime} \subset I, \ell\left(M^{\prime}\right)=2^{-s} \ell(M) \text { and } d\left(c_{M}, c_{M^{\prime}}\right) \geq 2^{s \delta} \ell(M)}} \int_{\mathbb{R} \backslash B\left(M, M^{\prime}\right)} S_{\left(M^{\prime}, M\right)}^{F^{\prime}}(y) d \sigma(y) \\
= & \sum_{F^{\prime} \in \mathcal{F}} \sum_{\substack{c_{M^{\prime}} \in 2 M \\
d\left(c_{M}, c_{M^{\prime}}\right) \geq 2^{s \delta} \ell(M)}}^{*} \int_{\mathbb{R} \backslash B\left(M, M^{\prime}\right)}^{F_{\left(M^{\prime}, M\right)}^{F^{\prime}}}(y) d \sigma(y) \\
& +\sum_{\ell=1}^{\infty} \sum_{F^{\prime} \in \mathcal{F}} \sum_{\substack{c_{M^{\prime}} \in 2^{\ell+1} M \backslash 2^{\ell} M \\
d\left(c_{M}, c_{M^{\prime}}\right) \geq 2^{s \delta} \ell(M)}} \int_{\mathbb{R} \backslash B\left(M, M^{\prime}\right)} S_{\left(M^{\prime}, M\right)}^{F^{\prime}}(y) d \sigma(y) \\
\equiv & \sum_{\ell=0}^{\infty} \mathcal{A}_{s}^{\text {difference }, \ell}(M) .
\end{aligned}
$$

Let $m=2$ so that

$$
2^{-m} \leq \frac{1}{3} .
$$


Now decompose the integrals over $\mathbb{R}$ in $\mathcal{A}_{s}^{\text {proximal }, \ell}(M)$ by

$$
\begin{aligned}
\mathcal{A}_{s}^{\text {proximal }, 0}(M)= & \sum_{F^{\prime} \in \mathcal{F}} \sum_{\substack{c_{M^{\prime}} \in 2 M \\
d\left(c_{M}, c_{M^{\prime}}\right)<2^{s \delta} \ell(M)}}^{*} \int_{\mathbb{R} \backslash 4 M} S_{\left(M^{\prime}, M\right)}^{F^{\prime}}(y) d \sigma(y) \\
& +\sum_{F^{\prime} \in \mathcal{F}} \sum_{\substack{c_{M^{\prime}} \in 2 M \\
d\left(c_{M}, c_{M^{\prime}}\right)<2^{s \delta} \ell(M)}}^{*} \int_{4 M} S_{\left(M^{\prime}, M\right)}^{F^{\prime}}(y) d \sigma(y) \\
\equiv & \mathcal{A}_{s, \text { par }}^{\text {proxal }, 0}(M)+\mathcal{A}_{s, \text { near }}^{\text {proximal }, 0}(M),
\end{aligned}
$$

and

$$
\begin{aligned}
& \mathcal{A}_{s}^{\text {proximal }, \ell}(M)=\sum_{F^{\prime} \in \mathcal{F}} \sum_{\substack{c_{M^{\prime}} \in 2^{\ell+1} M \backslash 2^{\ell} M \\
d\left(c_{M}, c_{M^{\prime}}\right)<2^{s^{s}} \ell(M)}}^{*} \int_{\mathbb{R} \backslash 2^{\ell+2} M} S_{\left(M^{\prime}, M\right)}^{F^{\prime}}(y) d \sigma(y) \\
& +\sum_{F^{\prime} \in \mathcal{F}} \sum_{\substack{c_{M^{\prime}} \in 2^{\ell+1} M \backslash 2^{\ell} M \\
d\left(c_{M}, c_{M^{\prime}}\right)<2^{s \delta} \ell(M)}}^{*} \int_{2^{\ell+2} M \backslash 2^{\ell-m} M} S_{\left(M^{\prime}, M\right)}^{F^{\prime}}(y) d \sigma(y) \\
& +\sum_{F^{\prime} \in \mathcal{F}} \sum_{\substack{c_{M^{\prime}} \in 2^{\ell+1} M \backslash 2^{\ell} M \\
d\left(c_{M}, c_{M^{\prime}}\right)<2^{s \delta} \ell(M)}}^{*} \int_{2^{\ell-m} M} S_{\left(M^{\prime}, M\right)}^{F^{\prime}}(y) d \sigma(y) \\
& \equiv \mathcal{A}_{s, \text { far }}^{\text {proximal }, \ell}(M)+\mathcal{A}_{s, \text { near }}^{\text {proximal }, \ell}(M)+\mathcal{A}_{s, \text { close }}^{\text {proximal }, \ell}(M), \quad \ell \geq 1 .
\end{aligned}
$$

Similarly we decompose the integrals over the difference

$$
B^{*} \equiv \mathbb{R} \backslash B\left(M, M^{\prime}\right)
$$

in $\mathcal{A}_{s}^{\text {difference, } \ell}(M)$ by

$$
\begin{aligned}
\mathcal{A}_{s}^{\text {difference, } 0}(M)= & \sum_{F^{\prime} \in \mathcal{F}} \sum_{\substack{c_{M^{\prime}} \in 2 M \\
d\left(c_{M}, c_{M^{\prime}}\right) \geq 2^{s \delta} \ell(M)}}^{*} \int_{B^{*} \backslash 4 M} S_{\left(M^{\prime}, M\right)}^{F^{\prime}}(y) d \sigma(y) \\
& +\sum_{F^{\prime} \in \mathcal{F}} \sum_{\substack{c_{M^{\prime}} \in 2 M \\
d\left(c_{M}, c_{M^{\prime}}\right) \geq 2^{s \delta} \ell(M)}}^{*} \int_{B^{*} \cap 4 M} S_{\left(M^{\prime}, M\right)}^{F^{\prime}}(y) d \sigma(y) \\
\equiv & \mathcal{A}_{s, \text { fireres }, 0}^{\text {diffence }}(M)+\mathcal{A}_{s, \text { near }}^{\text {difference } 0}(M)
\end{aligned}
$$

and

$$
\begin{aligned}
& \mathcal{A}_{s}^{\text {difference }, \ell}(M)=\sum_{F^{\prime} \in \mathcal{F}} \sum_{\substack{c_{M^{\prime}} \in 2^{\ell+1} M \backslash 2^{\ell} M \\
d\left(c_{M}, c_{M^{\prime}}\right) \geq 2^{s \delta} \ell(M)}}^{*} \int_{B^{*} \backslash 2^{\ell+2} M} S_{\left(M^{\prime}, M\right)}^{F^{\prime}}(y) d \sigma(y) \\
& +\sum_{F^{\prime} \in \mathcal{F}} \sum_{\begin{array}{c}
c_{M^{\prime}} \in 2^{\ell+1} M \backslash 2^{\ell} M \\
d\left(c_{M}, c_{M^{\prime}}\right) \geq 2^{s \delta} \ell(M)
\end{array}}^{*} \int_{B^{*} \cap\left(2^{\ell+2} M \backslash 2^{\ell-m} M\right)} S_{\left(M^{\prime}, M\right)}^{F^{\prime}}(y) d \sigma(y) \\
& +\sum_{F^{\prime} \in \mathcal{F}} \sum_{\substack{c_{M^{\prime}} \in 2^{\ell+1} M \backslash 2^{\ell} M \\
d\left(c_{M}, c_{M^{\prime}}\right) \geq 2^{s \delta} \ell(M)}}^{*} \int_{B^{*} \cap 2^{\ell-m} M} S_{\left(M^{\prime}, M\right)}^{F^{\prime}}(y) d \sigma(y) \\
& \equiv \mathcal{A}_{s, \text { far }}^{\text {difference }, \ell}(M)+\mathcal{A}_{s, \text { near }}^{\text {difference } \ell}(M)+\mathcal{A}_{s, \text { close }}^{\text {differe }, \ell}(M), \quad \ell \geq 1 \text {. }
\end{aligned}
$$


We now note the important point that the close terms $\mathcal{A}_{s, \text { close }}^{\text {proximal } \ell}(M)$ and $\mathcal{A}_{s, \text { close }}^{\text {differenc } \ell}(M)$ both vanish for $\ell>\delta s$ because of the decomposition (10.43):

$$
\mathcal{A}_{s, \text { close }}^{\text {proximal }, \ell}(M)=\mathcal{A}_{s, \text { close }}^{\text {difference } \ell}(M)=0, \quad \ell \geq 1+\delta s .
$$

Indeed, if $c_{M^{\prime}} \in 2^{\ell+1} M \backslash 2^{\ell} M$, then we have

$$
\frac{1}{2} 2^{\ell} \ell(M) \leq d\left(c_{M}, c_{M^{\prime}}\right) .
$$

Now the summands in $\mathcal{A}_{s, \text { close }}^{\text {proximal } \ell}(M)$ satisfy $d\left(c_{M}, c_{M^{\prime}}\right)<2^{\delta s} \ell(M)$, which by (10.50) is impossible if $\ell \geq 1+\delta s$ - indeed, if $\ell \geq 1+\delta s$, we get the contradiction

$$
2^{\delta s} \ell(M)=\frac{1}{2} 2^{1+\delta s} \ell(M) \leq \frac{1}{2} 2^{\ell} \ell(M) \leq d\left(c_{M}, c_{M^{\prime}}\right)<2^{\delta s} \ell(M) .
$$

It now follows that $\mathcal{A}_{s, \text { close }}^{\text {proximal } \ell}(M)=0$. Thus we are left to consider the term $\mathcal{A}_{s, \text { close }}^{\text {differen } \ell}(M)$, where the integration is taken over the set $\mathbb{R} \backslash B\left(M, M^{\prime}\right)$. But we are also restricted in $\mathcal{A}_{s, \text { close }}^{\text {difference } \ell}(M)$ to integrating over the interval $2^{\ell-m} M$, which is contained in $B\left(M, M^{\prime}\right)$ by (10.50). Indeed, the smallest ball centered at $c_{M}$ that contains $2^{\ell-m} M$ has radius $\frac{1}{2} 2^{\ell-m} \ell(M)$, which by (10.48) and (10.50) is at most $\frac{1}{4} 2^{\ell} \ell(M) \leq$ $\frac{1}{2} d\left(c_{M}, c_{M^{\prime}}\right)$, the radius of $B\left(M, M^{\prime}\right)$. Thus the range of integration in the term $\mathcal{A}_{s, \text { close }}^{\text {differe } \ell}(M)$ is the empty set, and so $\mathcal{A}_{s, \text { close }}^{\text {differe } \ell}(M)=0$ as well as $\mathcal{A}_{s, \text { close }}^{\text {proximal } \ell}(M)=0$. This proves (10.49).

From now on we treat $T_{s}^{\text {proximal }}$ and $T_{s}^{\text {difference }}$ in the same way since the terms $\mathcal{A}_{s, \text { close }}^{\text {proximal } \ell}(M)$ and $\mathcal{A}_{s, \text { close }}^{\text {differe } \ell}(M)$ both vanish for $\ell \geq 1+\delta s$. Thus we will suppress the superscripts proximal and difference in the far, near and close decomposition of $\mathcal{A}_{s}^{\text {proximal, } \ell}(M)$ and $\mathcal{A}_{s}^{\text {difference, } \ell}(M)$, and we will also suppress the conditions $d\left(c_{M}, c_{M^{\prime}}\right)<2^{s \delta} \ell(M)$ and $d\left(c_{M}, c_{M^{\prime}}\right) \geq 2^{s \delta} \ell(M)$ in the proximal and difference terms since they no longer play a role. Using the pairwise disjointedness of the shifted coronas $\mathcal{C}_{F}^{\mathcal{G} \text {,shift }}$, we have

$$
\sum_{F^{\prime} \in \mathcal{F}}\left\|Q_{F^{\prime}, A}^{\omega, \mathbf{b}^{*}} x\right\|_{L^{2}(\omega)}^{\mathbf{\omega} 2} \lesssim|A|^{2}|A|_{\omega}, \quad \text { for any interval } A .
$$

Note that if $c_{M^{\prime}} \in 2 M$, then $M^{\prime} \subset 3 M$. Then with

$$
\mathcal{W}_{M}^{s} \equiv \bigcup_{F^{\prime} \in \mathcal{F}}\left\{M^{\prime} \in \mathcal{W}\left(F^{\prime}\right): M^{\prime} \subset 3 M \text { and } \ell\left(M^{\prime}\right)=2^{-s} \ell(M)\right\}
$$

we have

$$
\begin{aligned}
\mathcal{A}_{s, f a r}^{0}(M) & \leq \sum_{F^{\prime} \in \mathcal{F}} \sum_{c_{M^{\prime}} \in 2 M}^{*} \int_{\mathbb{R} \backslash 4 M} S_{\left(M^{\prime}, M\right)}^{F^{\prime}}(y) d \sigma(y) \\
& \lesssim \sum_{A \in \mathcal{W}_{M}^{s}} \sum_{F^{\prime} \in \mathcal{F}:} \int_{A \in \mathcal{W}\left(F^{\prime}\right)} \frac{\left\|Q_{F^{\prime}, M^{\prime}}^{\omega, \mathbf{b}^{*}} x\right\|_{L^{2}(\omega)}^{\mathbf{\omega}^{2}}}{\left(|M|+\left|y-c_{M}\right|\right)^{2(2-\alpha)}} d \sigma(y) \\
& \lesssim \sum_{A \in \mathcal{W}_{M}^{s}} \int_{\mathbb{R} \backslash 4 M} \frac{|A|^{2}|A|_{\omega}}{\left(|M|+\left|y-c_{M}\right|\right)^{2(2-\alpha)}} d \sigma(y) \\
& =\left(\sum_{A \in \mathcal{W}_{M}^{s}}|A|^{2}|A|_{\omega}\right) \int_{\mathbb{R} \backslash 4 M} \frac{1}{\left(|M|+\left|y-c_{M}\right|\right)^{2(2-\alpha)}} d \sigma(y) .
\end{aligned}
$$

Now we use the standard pigeonholing of side length of $A$ to conclude that

$$
\begin{aligned}
(10.52) \sum_{A \in \mathcal{W}_{M}^{s}}|A|^{2}|A|_{\omega} & =\sum_{k=s}^{\infty} \sum_{A \in \mathcal{W}_{M}^{s}: \ell(A)=2^{-k} \ell(M)}|A|^{2}|A|_{\omega} \leq \sum_{k=s}^{\infty} 2^{-2 k}|M|^{2} \sum_{A \in \mathcal{W}_{M}^{s}: \ell(A)=2^{-k} \ell(M)}|A|_{\omega} \\
& \leq \sum_{k=s}^{\infty} 2^{-2 k}|M|^{2}|3 M|_{\omega} \lesssim 2^{-2 s}|M|^{2}|3 M|_{\omega},
\end{aligned}
$$


so that combining the previous two displays we have

$$
\begin{aligned}
\mathcal{A}_{s, f a r}^{0}(M) & \lesssim 2^{-2 s}|M|^{2}|3 M|_{\omega} \int_{\mathbb{R} \backslash 4 M} \frac{1}{\left(|M|+\left|y-c_{M}\right|\right)^{2(2-\alpha)}} d \sigma(y) \\
& \leq 2^{-2 s}|4 M|_{\omega} \int_{\mathbb{R} \backslash 4 M} \frac{1}{\left(|M|+\left|y-c_{M}\right|\right)^{2(1-\alpha)}} d \sigma(y) \\
& \approx 2^{-2 s} \frac{|4 M|_{\omega}}{|4 M|^{1-\alpha}} \int_{\mathbb{R} \backslash 4 M}\left(\frac{|M|}{\left(|M|+\left|y-c_{M}\right|\right)^{2}}\right)^{1-\alpha} d \sigma(y) \\
& \lesssim 2^{-2 s} \frac{|4 M|_{\omega}}{|4 M|^{1-\alpha}} \mathcal{P}^{\alpha}\left(4 M, \mathbf{1}_{\mathbb{R} \backslash 4 M} \sigma\right) \lesssim 2^{-2 s} \mathcal{A}_{2}^{\alpha} .
\end{aligned}
$$

To estimate the near term $\mathcal{A}_{s, \text { near }}^{0}(M)$, we initially keep the energy $\left\|\mathrm{Q}_{F^{\prime}, M^{\prime}}^{\omega, \mathbf{b}^{*}}\right\|_{L^{2}(\omega)}^{2}$ and write

$$
\begin{aligned}
\mathcal{A}_{s, \text { near }}^{0}(M) & \leq \sum_{F^{\prime} \in \mathcal{F}} \sum_{c_{M^{\prime}} \in 2 M}^{*} \int_{4 M} S_{\left(M^{\prime}, M\right)}^{F^{\prime}}(y) d \sigma(y) \\
& \approx \sum_{F^{\prime} \in \mathcal{F}} \sum_{c_{M^{\prime}} \in 2 M}^{*} \int_{4 M} \frac{1}{|M|^{2-\alpha}} \frac{\left\|\mathrm{Q}_{F^{\prime}, M^{\prime}}^{\omega, \mathbf{b}^{*}} x\right\|_{L^{2}(\omega)}^{\mathbf{N} 2}}{\left(\left|M^{\prime}\right|+\left|y-c_{M^{\prime}}\right|\right)^{2-\alpha}} d \sigma(y) \\
& =\sum_{F^{\prime} \in \mathcal{F}} \frac{1}{|M|^{2-\alpha}} \sum_{c_{M^{\prime} \in 2 M}}^{*}\left\|\mathrm{Q}_{F^{\prime}, M^{\prime}}^{\omega, \mathbf{b}^{*}} x\right\|_{L^{2}(\omega)}^{\mathbf{\omega} 2} \int_{4 M} \frac{1}{\left(\left|M^{\prime}\right|+\left|y-c_{M^{\prime}}\right|\right)^{2-\alpha}} d \sigma(y) \\
& =\sum_{F^{\prime} \in \mathcal{F}} \frac{1}{|M|^{2-\alpha}} \sum_{c_{M^{\prime}} \in 2 M}^{*}\left\|\mathrm{Q}_{F^{\prime}, M^{\prime}}^{\omega, \mathbf{b}^{*}} x\right\|_{L^{2}(\omega)}^{\mathbf{\omega} 2} \frac{\mathrm{P}^{\alpha}\left(M^{\prime}, \mathbf{1}_{4 M} \sigma\right)}{\left|M^{\prime}\right|}
\end{aligned}
$$

In order to estimate the final sum above, we must invoke the 'prepare to puncture' argument above, as we will want to derive geometric decay from $\left\|Q_{M^{\prime}}^{\omega, \mathbf{b}^{*}} x\right\|_{L^{2}(\omega)}^{\mathbf{A}^{2}}$ by dominating it by the 'nonenergy' term $\left|M^{\prime}\right|^{2}\left|M^{\prime}\right|_{\omega}$ ' as well as using the Muckenhoupt energy constant. Choose an augmented interval $\widetilde{M} \in \mathcal{A D}$ satisfying $\bigcup_{c_{M^{\prime}} \in 2 M} M^{\prime} \subset 4 M \subset \widetilde{M}$ and $\ell(\widetilde{M}) \leq C \ell(M)$. Define $\widetilde{\omega}=\omega-\omega(\{p\}) \delta_{p}$ where $p$ is an atomic point in $\widetilde{M}$ for which

$$
\omega(\{p\})=\sup _{q \in \mathfrak{P}_{(\sigma, \omega)}: q \in \widetilde{M}} \omega(\{q\}) .
$$

(If $\omega$ has no atomic point in common with $\sigma$ in $\widetilde{M}$, set $\widetilde{\omega}=\omega$.) Then we have $|\widetilde{M}|_{\widetilde{\omega}}=\omega\left(\widetilde{M}, \mathfrak{P}_{(\sigma, \omega)}\right)$ and

$$
\frac{|\widetilde{M}|_{\widetilde{\omega}}}{|\widetilde{M}|^{1-\alpha}} \frac{|\widetilde{M}|_{\sigma}}{|\widetilde{M}|^{1-\alpha}}=\frac{\omega\left(\widetilde{M}, \mathfrak{P}_{(\sigma, \omega)}\right)}{|\widetilde{M}|^{1-\alpha}} \frac{|\widetilde{M}|_{\sigma}}{|\widetilde{M}|^{1-\alpha}} \leq A_{2}^{\alpha, \text { punct }}
$$

From (10.37) and (10.18) we also have

$$
\sum_{F^{\prime} \in \mathcal{F}}\left\|Q_{F^{\prime}, A}^{\omega, \mathbf{b}^{*}} x\right\|_{L^{2}(\omega)}^{\mathbf{\aleph}^{2}} \lesssim \ell(A)^{2}|A|_{\widetilde{\omega}}, \quad \text { for any interval } A
$$

Now by Cauchy-Schwarz and the augmented local estimate (10.33) in Lemma 111 with $M=\widetilde{M}$ applied to the second line below, and with $\mathcal{W}_{M}^{s}$ as in (10.51), and noting (10.52), the last sum in (10.53) is dominated 
by

$$
\begin{aligned}
& \frac{1}{|M|^{2-\alpha}}\left(\sum_{F^{\prime} \in \mathcal{F}} \sum_{c\left(M^{\prime}\right) \in 2 M}^{*}\left\|Q_{F^{\prime}, M^{\prime}}^{\omega, \mathbf{b}^{*}} x\right\|_{L^{2}(\omega)}^{\mathbf{\omega} 2}\right)^{\frac{1}{2}} \\
& \times\left(\sum_{F^{\prime} \in \mathcal{F}} \sum_{c_{M^{\prime}} \in 2 M}^{*}\left\|Q_{F^{\prime}, M^{\prime}}^{\omega, \mathbf{b}^{*}} x\right\|_{L^{2}(\omega)}^{\mathbf{\omega}^{2}}\left(\frac{\mathrm{P}^{\alpha}\left(M^{\prime}, \mathbf{1}_{4 M} \sigma\right)}{\left|M^{\prime}\right|}\right)^{2}\right)^{\frac{1}{2}} \\
& \lesssim \frac{1}{|M|^{2-\alpha}}\left(\sum_{A \in \mathcal{W}_{M}^{s}}|A|^{2}|A|_{\widetilde{\omega}}\right)^{\frac{1}{2}} \sqrt{\left(\mathfrak{E}_{2}^{\alpha}\right)^{2}+A_{2}^{\alpha, \text { energy }}} \sqrt{|\widetilde{M}|_{\sigma}} \\
& \lesssim \frac{2^{-s}|M|}{|M|^{2-\alpha}} \sqrt{|4 M|_{\widetilde{\omega}}} \sqrt{\left(\mathfrak{E}_{2}^{\alpha}\right)^{2}+A_{2}^{\alpha, \text { energy }}} \sqrt{|\widetilde{M}|_{\sigma}}
\end{aligned}
$$

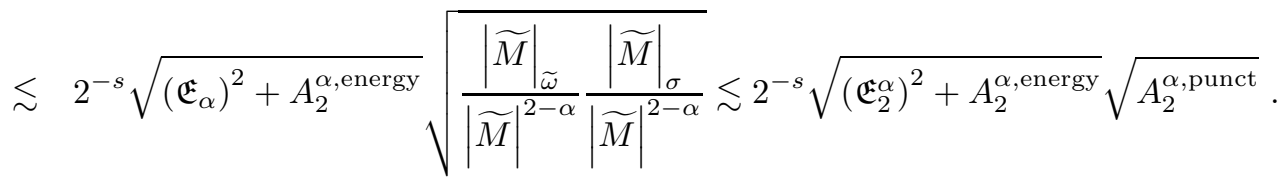

Similarly, for $\ell \geq 1$, we can estimate the far term $\mathcal{A}_{s, f a r}^{\ell}(M)$ by the argument used for $\mathcal{A}_{s, f a r}^{0}(M)$ but applied to $2^{\ell} M$ in place of $M$. For this need the following variant of $\mathcal{W}_{M}^{s}$ in (10.51) given by

$$
\mathcal{W}_{M}^{s, \ell} \equiv \bigcup_{F^{\prime} \in \mathcal{F}}\left\{M^{\prime} \in \mathcal{W}\left(F^{\prime}\right): M^{\prime} \subset 3\left(2^{\ell} M\right) \text { and } \ell\left(M^{\prime}\right)=2^{-s-\ell} \ell\left(2^{\ell} M\right)\right\}
$$

Then we have

$$
\begin{aligned}
\mathcal{A}_{s, f a r}^{\ell}(M) & \leq \sum_{F^{\prime} \in \mathcal{F}} \sum_{c_{M^{\prime}} \in\left(2^{\ell+1} M\right) \backslash\left(2^{\ell} M\right)}^{*} \int_{\mathbb{R} \backslash 2^{\ell+2} M} S_{\left(M^{\prime}, M\right)}^{F^{\prime}}(y) d \sigma(y) \\
& \lesssim \sum_{A \in \mathcal{W}_{M}^{s, \ell} F^{\prime} \in \mathcal{F}: A \in \mathcal{W}\left(F^{\prime}\right)} \int_{\mathbb{R} \backslash 4\left(2^{\ell} M\right)} \frac{\left\|Q_{F^{\prime}, A}^{\omega, \mathbf{b}^{*}} x\right\|_{L^{2}(\omega)}^{\mathbf{\omega} 2}}{\left(|M|+\left|y-c_{M}\right|\right)^{2(2-\alpha)}} d \sigma(y) \\
& \lesssim \sum_{A \in \mathcal{W}_{M}^{s, \ell}} \int_{\mathbb{R} \backslash 4\left(2^{\ell} M\right)} \frac{|A|^{2}|A|_{\omega}}{\left(|M|+\left|y-c_{M}\right|\right)^{2(2-\alpha)}} d \sigma(y) \\
& =\left(\sum_{A \in \mathcal{W}_{M}^{s, \ell}}|A|^{2}|A|_{\omega}\right) \int_{\mathbb{R} \backslash 4\left(2^{\ell} M\right)} \frac{1}{\left(|M|+\left|y-c_{M}\right|\right)^{2(2-\alpha)}} d \sigma(y),
\end{aligned}
$$

where, just as for the sum over $A \in \mathcal{W}_{M}^{s, 0}$, we have

$$
\begin{aligned}
& \sum_{A \in \mathcal{W}_{M}^{s, \ell}}|A|^{2}|A|_{\omega} \\
= & \sum_{k=s}^{\infty} \sum_{A \in \mathcal{W}_{M}^{s, \ell}: \ell(A)=2^{-k-\ell} \ell\left(2^{\ell} M\right)}|A|^{2}|A|_{\omega} \leq \sum_{k=s}^{\infty} 2^{-2 k-2 \ell}\left|2^{\ell} M\right|^{2} \sum_{A \in \mathcal{W}_{M}^{s, \ell}: \ell(A)=2^{-k-\ell} \ell\left(2^{\ell} M\right)}|A|_{\omega} \\
\leq & \sum_{k=s}^{\infty} 2^{-2 k-2 \ell}\left|2^{\ell} M\right|^{2}\left|3\left(2^{\ell} M\right)\right|_{\omega} \lesssim 2^{-2 s-2 \ell}\left|2^{\ell} M\right|^{2}\left|3\left(2^{\ell} M\right)\right|_{\omega} .
\end{aligned}
$$


Now using $\frac{\left|2^{\ell} M\right|^{2}}{\left(|M|+\left|y-c_{M}\right|\right)^{2(2-\alpha)}} \leq \frac{1}{\left(\left|2^{\ell} M\right|+\left|y-c_{2^{\ell} M}\right|\right)^{2(1-\alpha)}}$ for $y \notin 2^{\ell+2} M$, we can continue with

$$
\begin{aligned}
& \mathcal{A}_{s, f a r}^{\ell}(M) \lesssim 2^{-2 s} 2^{-2 \ell}\left|2^{\ell+2} M\right|_{\omega} \int_{\mathbb{R} \backslash 2^{\ell+2} M} \frac{1}{\left(\left|2^{\ell} M\right|+\left|y-c_{2^{\ell} M}\right|\right)^{2(1-\alpha)}} d \sigma(y) \\
\approx & 2^{-2 s} 2^{-2 \ell} \frac{\left|2^{\ell+2} M\right|_{\omega}}{\left|2^{\ell} M\right|^{1-\alpha}} \int_{\mathbb{R} \backslash 2^{\ell+2} M}\left(\frac{\left|2^{\ell} M\right|}{\left(\left|2^{\ell} M\right|+\left|y-c_{2^{\ell} M}\right|\right)^{2}}\right)^{1-\alpha} d \sigma(y) \\
\lesssim & 2^{-2 s} 2^{-2 \ell}\left\{\frac{\left|2^{\ell+2} M\right|_{\omega}}{\left|2^{\ell} M\right|^{1-\alpha}} \mathcal{P}^{\alpha}\left(2^{\ell+2} M, 1_{\mathbb{R} \backslash 2^{\ell+2} M} \sigma\right)\right\} \lesssim 2^{-2 s} 2^{-2 \ell} \mathcal{A}_{2}^{\alpha} .
\end{aligned}
$$

To estimate the near term $\mathcal{A}_{s, n e a r}^{\ell}(M)$ we must again invoke the 'prepare to puncture' argument. Choose an augmented interval $\widetilde{M} \in \mathcal{A D}$ such that $\bigcup_{c_{M^{\prime}} \in 2^{\ell+1} M \backslash 2^{\ell} M} M^{\prime} \subset 2^{\ell+2} M \subset \widetilde{M}$ and $\ell(\widetilde{M}) \leq C 2^{\ell} \ell(M)$. Define $\widetilde{\omega}=\omega-\omega(\{p\}) \delta_{p}$ where $p$ is an atomic point in $\widetilde{M}$ for which

$$
\omega(\{p\})=\sup _{q \in \mathfrak{P}_{(\sigma, \omega)}: q \in \widetilde{M}} \omega(\{q\}) .
$$

(If $\omega$ has no atomic point in common with $\sigma$ in $\widetilde{M}$ set $\widetilde{\omega}=\omega$.) Then we have $|\widetilde{M}|_{\widetilde{\omega}}=\omega\left(\widetilde{M}, \mathfrak{P}_{(\sigma, \omega)}\right)$, and just as in the argument above following (10.53), we have from (10.37) and (10.18) that both

$$
\frac{|\widetilde{M}|_{\widetilde{\omega}}}{|\widetilde{M}|^{1-\alpha}} \frac{|\widetilde{M}|_{\sigma}}{|\widetilde{M}|^{1-\alpha}} \leq A_{2}^{\alpha, \text { punct }} \text { and } \sum_{F^{\prime} \in \mathcal{F}}\left\|Q_{F^{\prime}, M^{\prime}}^{\omega, \mathbf{b}^{*}} x\right\|_{L^{2}(\omega)}^{\mathbf{\omega}^{2}} \lesssim \ell\left(M^{\prime}\right)^{2}\left|M^{\prime}\right|_{\widetilde{\omega}}
$$

Thus using that $m=2$ in the definition of $A_{s, \text { near }}^{\ell}(M)$, we see that

$$
\begin{aligned}
\mathcal{A}_{s, \text { near }}^{\ell}(M) & \leq \sum_{F^{\prime} \in \mathcal{F}} \sum_{c_{M^{\prime}} \in 2^{\ell+1} M \backslash 2^{\ell} M}^{*} \int_{2^{\ell+2} M \backslash 2^{\ell-m} M} S_{\left(M^{\prime}, M\right)}^{F^{\prime}}(y) d \sigma(y) \\
& \approx \sum_{F^{\prime} \in \mathcal{F}} \sum_{c_{M^{\prime}} \in 2^{\ell+1} M \backslash 2^{\ell} M}^{*} \int_{2^{\ell+2} M \backslash 2^{\ell-m} M} \frac{1}{\left|2^{\ell} M\right|^{2-\alpha}} \frac{\left\|Q_{F^{\prime}, M^{\prime}}^{\omega, \mathbf{b}^{*}} x\right\|_{L^{2}(\omega)}^{\mathbf{\omega}^{2}}}{\left(\left|M^{\prime}\right|+\left|y-c_{M^{\prime}}\right|\right)^{2-\alpha}} d \sigma(y) \\
& \lesssim \frac{1}{\left|2^{\ell} M\right|^{2-\alpha}} \sum_{F^{\prime} \in \mathcal{F}} \sum_{c_{M^{\prime}} \in 2^{\ell+1} M \backslash 2^{\ell} M}^{*}\left\|Q_{F^{\prime}, M^{\prime}}^{\omega, \mathbf{b}^{*}} x\right\|_{L^{2}(\omega)}^{\boldsymbol{\omega}^{2}} \\
& \quad \times \int_{2^{\ell+2} M} \frac{1}{\left(\left|M^{\prime}\right|+\left|y-c_{M^{\prime}}\right|\right)^{2-\alpha}} d \sigma(y),
\end{aligned}
$$

is dominated by

$$
\begin{aligned}
& \frac{1}{\left|2^{\ell} M\right|^{2-\alpha}} \sum_{F^{\prime} \in \mathcal{F}} \sum_{c_{M^{\prime}} \in 2^{\ell+1} M \backslash 2^{\ell} M}^{*}\left\|Q_{F^{\prime}, M^{\prime}}^{\omega, \mathbf{b}^{*}}\right\|_{L^{2}(\omega)}^{\mathbf{\omega}^{2}} \frac{\mathrm{P}^{\alpha}\left(M^{\prime}, \mathbf{1}_{2^{\ell+2} M} \sigma\right)}{\left|M^{\prime}\right|} \\
& \leq \frac{1}{\left|2^{\ell} M\right|^{2-\alpha}}\left(\sum_{F^{\prime} \in \mathcal{F}} \sum_{c_{M^{\prime}} \in 2^{\ell+1} M \backslash 2^{\ell} M}^{*}\left\|Q_{F^{\prime}, M^{\prime}}^{\omega, \mathbf{b}^{*}} x\right\|_{L^{2}(\omega)}^{\mathbf{\omega} 2}\right)^{\frac{1}{2}} \\
& \times\left(\sum_{F^{\prime} \in \mathcal{F}^{\prime}} \sum_{c_{M^{\prime}} \in 2^{\ell+1}}^{*}\left\|\mathrm{Q}_{F^{\prime}, 2^{\ell}}^{\omega, \mathbf{b}^{*}} \times\right\|_{L^{2}(\omega)}^{\mathbf{\omega}^{2}}\left(\frac{\mathrm{P}^{\alpha}\left(M^{\prime}, \mathbf{1}_{2^{\ell+2} M} \sigma\right)}{\left|M^{\prime}\right|}\right)^{2}\right)^{\frac{1}{2}} .
\end{aligned}
$$


This can now be estimated as for the term $\mathcal{A}_{s, \text { near }}^{0}(M)$, along with the augmented local estimate (10.33) in Lemma 111 with $M=\widetilde{M}$ applied to the final line above, to get

$$
\begin{aligned}
\mathcal{A}_{s, \text { near }}^{\ell}(M) & \lesssim 2^{-s} 2^{-\ell} \frac{\left|2^{\ell} M\right|}{\left|2^{\ell} M\right|^{2-\alpha}} \sqrt{|\widetilde{M}|_{\widetilde{\omega}}} \sqrt{\left(\mathfrak{E}_{2}^{\alpha}\right)^{2}+A_{2}^{\alpha, \text { energy }}} \sqrt{|\widetilde{M}|_{\sigma}} \\
& \lesssim 2^{-s} 2^{-\ell} \sqrt{\left(\mathfrak{E}_{2}^{\alpha}\right)^{2}+A_{2}^{\alpha, \text { energy }}} \sqrt{\frac{|\widetilde{M}|_{\widetilde{\omega}}}{|\widetilde{M}|^{1-\alpha}} \mid \frac{|\widetilde{M}|_{\sigma}}{|\widetilde{M}|^{1-\alpha}}} \\
& \lesssim 2^{-s} 2^{-\ell} \sqrt{\left(\mathfrak{E}_{2}^{\alpha}\right)^{2}+A_{2}^{\alpha, \text { energy }}} \sqrt{A_{2}^{\alpha, \text { punct }}} .
\end{aligned}
$$

Each of the estimates for $\mathcal{A}_{s, f a r}^{\ell}(M)$ and $\mathcal{A}_{s, \text { near }}^{\ell}(M)$ is summable in both $s$ and $\ell$.

Now we turn to the terms $\mathcal{A}_{s, \text { close }}^{\ell}(M)$, and recall from (10.49) that $\mathcal{A}_{s, \text { close }}^{\ell}(M)=0$ if $\ell \geq 1+\delta s$. So we now suppose that $\ell \leq \delta s$. We have, with $m=2$ as in (10.48),

$$
\begin{gathered}
\mathcal{A}_{s, \text { close }}^{\ell}(M) \leq \sum_{F^{\prime} \in \mathcal{F}} \sum_{c_{M^{\prime}} \in 2^{\ell+1} \backslash \backslash 2^{\ell} M}^{*} \int_{2^{\ell-m} M} S_{\left(M^{\prime}, M\right)}^{F^{\prime}}(y) d \sigma(y) \\
\approx \sum_{F^{\prime} \in \mathcal{F} c_{M^{\prime}} \in 2^{\ell+1} M \backslash 2^{\ell} M} \sum_{2^{\ell-m} M}^{*} \frac{1}{\left(|M|+\left|y-c_{M}\right|\right)^{2-\alpha}} \frac{\left\|Q_{F^{\prime}, M^{\prime}}^{\omega, \mathbf{b}^{*}} x\right\|_{L^{2}(\omega)}^{\mathbf{\omega} 2}}{\left|2^{\ell} M\right|^{2-\alpha}} d \sigma(y) \\
=\left(\sum_{F^{\prime} \in \mathcal{F}} \sum_{c_{M^{\prime}} \in 2^{\ell+1} M \backslash 2^{\ell} M}^{*}\left\|Q_{F^{\prime}, M^{\prime}}^{\omega, \mathbf{b}^{*}} x\right\|_{L^{2}(\omega)}^{\boldsymbol{\omega}^{2}}\right) \frac{1}{\left|2^{\ell} M\right|^{2-\alpha}} \\
\times \int_{2^{\ell-m} M} \frac{1}{\left(|M|+\left|y-c_{M}\right|\right)^{2-\alpha}} d \sigma(y) .
\end{gathered}
$$

The argument used to prove (10.56) gives the analogous inequality with a hole $2^{\ell-1} M$,

$$
\sum_{F^{\prime} \in \mathcal{F}} \sum_{c_{M^{\prime}} \in 2^{\ell+1} M \backslash 2^{\ell} M}^{*}\left\|Q_{F^{\prime}, M^{\prime}}^{\omega, \mathbf{b}^{*}} x\right\|_{L^{2}(\omega)}^{\boldsymbol{\omega}^{2}} \lesssim 2^{-2 s}\left|2^{\ell} M\right|^{2}\left|2^{\ell+2} M \backslash 2^{\ell-1} M\right|_{\omega} .
$$

Thus we get

$$
\begin{aligned}
& \mathcal{A}_{s, \text { close }}^{\ell}(M) \\
\lesssim & 2^{-2 s}\left|2^{\ell} M\right|^{2}\left|2^{\ell+2} M \backslash 2^{\ell-1} M\right|_{\omega} \frac{1}{\left|2^{\ell} M\right|^{2-\alpha}} \int_{2^{\ell-m} M} \frac{1}{\left(|M|+\left|y-c_{M}\right|\right)^{2-\alpha}} d \sigma(y) \\
\lesssim & 2^{-2 s}\left|2^{\ell} M\right|^{2} \frac{\left|2^{\ell+2} M \backslash 2^{\ell-1} M\right|_{\omega}}{\left|2^{\ell} M\right|^{2-\alpha}} \frac{\left|2^{\ell-m} M\right|_{\sigma}}{|M|^{2-\alpha}} \\
\lesssim & 2^{-2 s} 2^{(2-\alpha) \ell} \frac{\left|2^{\ell+2} M \backslash 2^{\ell-1} M\right|_{\omega}}{\left|2^{\ell+2} M\right|^{1-\alpha}} \frac{\left|2^{\ell-m} M\right|_{\sigma}}{\left|2^{\ell-m} M\right|^{1-\alpha}} \lesssim 2^{-2 s} 2^{(2-\alpha) \ell} A_{2}^{\alpha},
\end{aligned}
$$

provided that $m=2>1$. Note that we can use the offset Muckenhoupt constant $A_{2}^{\alpha}$ here since $2^{\ell+2} M \backslash$ $2^{\ell-1} M$ and $2^{\ell-m} M$ are disjoint. If $\ell \leq s$, then we have the relatively crude estimate $\mathcal{A}_{s, \text { close }}(M) \lesssim$ $2^{-s} A_{2}^{\alpha}$ without decay in $\ell$. But we are assuming $\ell \leq \delta s$ here, and so we obtain a suitable estimate for $\mathcal{A}_{s, \text { close }}^{\ell}(M)$ provided we choose $0<\delta \leq \frac{1}{2-\alpha}$. Indeed, we then have

$$
\sum_{l=1}^{\delta s} 2^{-2 s} 2^{(2-\alpha) \ell} A_{2}^{\alpha}=2^{-2 s}\left(\sum_{l=1}^{\delta s} 2^{(2-\alpha) \ell}\right) A_{2}^{\alpha} \lesssim 2^{-2 s} 2^{(2-\alpha) \delta s} A_{2}^{\alpha} \leq 2^{-s} A_{2}^{\alpha},
$$

provided $\delta \leq \frac{1}{2-\alpha}$, and in particular we may take $\delta=\frac{1}{2}$. Altogether, the above estimates prove

$$
T_{s}^{\text {proximal }}+T_{s}^{\text {difference }} \lesssim 2^{-s}\left(\mathcal{A}_{2}^{\alpha}+\sqrt{\left(\mathfrak{E}_{2}^{\alpha}\right)^{2}+A_{2}^{\alpha, \text { energy }}} \sqrt{A_{2}^{\alpha, \text { punct }}}\right),
$$


which is summable in $s$.

10.5.2. The intersection term. Now we return to the term,

$$
\begin{aligned}
& T_{s}^{\text {intersection }} \equiv \sum_{F, F^{\prime} \in \mathcal{F}} \sum_{M \in \mathcal{W}(F), M^{\prime} \in \mathcal{W}\left(F^{\prime}\right)} \\
& M, M^{\prime} \subset I, \ell\left(M^{\prime}\right)=2^{-s} \ell(M) \\
& d\left(c_{M}, c_{M^{\prime}}\right) \geq 2^{s(1+\delta)} \ell\left(M^{\prime}\right) \\
& \times \int_{B\left(M, M^{\prime}\right)} \frac{\left\|\mathbf{Q}_{F, M}^{\omega, \mathbf{b}^{*}} x\right\|_{L^{2}(\omega)}^{\mathbf{\omega} 2}}{\left(|M|+\left|y-c_{M}\right|\right)^{2-\alpha}} \frac{\left\|\mathrm{Q}_{F^{\prime}, M^{\prime}}^{\omega, \mathbf{b}^{*}} x\right\|_{L^{2}(\omega)}^{\mathbf{\$} 2}}{\left(\left|M^{\prime}\right|+\left|y-c_{M^{\prime}}\right|\right)^{2-\alpha}} d \sigma(y) .
\end{aligned}
$$

It will suffice to show that $T_{s}^{\text {intersection }}$ satisfies the estimate,

$$
\begin{aligned}
& T_{s}^{\text {intersection }} \lesssim 2^{-s \delta} \sqrt{\left(\mathfrak{E}_{2}^{\alpha}\right)^{2}+A_{2}^{\alpha, \text { energy }}} \sqrt{A_{2}^{\alpha, \text { punct }}} \sum_{\substack{F^{\prime} \in \mathcal{F}^{\prime} \\
M^{\prime} \in \mathcal{M}_{(\boldsymbol{\rho}, \varepsilon) \text { deep }}\left(F^{\prime}\right) \\
M^{\prime} \subset I}}\left\|\mathrm{Q}_{F^{\prime}, M^{\prime}}^{\omega, \mathbf{b}^{*}} x\right\|_{L^{2}(\omega)}^{\mathbf{A}_{2} 2} \\
& =2^{-s \delta} \sqrt{\left(\mathfrak{E}_{2}^{\alpha}\right)^{2}+A_{2}^{\alpha, \text { energy }}} \sqrt{A_{2}^{\alpha, \text { punct }}} \int_{\widehat{I}} t^{2} \bar{\mu} .
\end{aligned}
$$

Recalling $B\left(M, M^{\prime}\right)=B\left(c_{M}, \frac{1}{2} d\left(c_{M}, c_{M^{\prime}}\right)\right)$, we can write (suppressing some notation for clarity),

$$
\begin{aligned}
& T_{s}^{\text {intersection }} \\
& =\sum_{F, F^{\prime}} \sum_{M, M^{\prime}} \int_{B\left(M, M^{\prime}\right)} \frac{\left\|\mathrm{Q}_{F, M^{\prime}}^{\omega, \mathbf{b}^{*}} x\right\|_{L^{2}(\omega)}^{\mathbf{\omega} 2}}{\left(|M|+\left|y-c_{M}\right|\right)^{2-\alpha}} \frac{\left\|\mathbf{Q}_{F^{\prime}, M^{\prime}}^{\omega, \mathbf{b}^{*}} x\right\|_{L^{2}(\omega)}^{\mathbf{A}^{2}}}{\left(\left|M^{\prime}\right|+\left|y-c_{M^{\prime}}\right|\right)^{2-\alpha}} d \sigma(y) \\
& \approx \sum_{F, F^{\prime}} \sum_{M, M^{\prime}}\left\|Q_{F, M}^{\omega, \mathbf{b}^{*}} x\right\|_{L^{2}(\omega)}^{\mathbf{\phi} 2}\left\|Q_{F^{\prime}, M^{\prime}}^{\omega, \mathbf{b}^{*}} x\right\|_{L^{2}(\omega)}^{\mathbf{\omega} 2} \frac{1}{\left|c_{M}-c_{M^{\prime}}\right|^{2-\alpha}} \int_{B\left(M, M^{\prime}\right)} \frac{d \sigma(y)}{\left(|M|+\left|y-c_{M}\right|\right)^{2-\alpha}} \\
& \leq \sum_{F^{\prime}} \sum_{M^{\prime}}\left\|\mathrm{Q}_{F^{\prime}, M^{\prime}}^{\omega, \mathbf{b}^{*}} x\right\|_{L^{2}(\omega)}^{\mathbf{\infty} 2} \sum_{F} \sum_{M} \frac{1}{\left|c_{M}-c_{M^{\prime}}\right|^{2-\alpha}}\left\|\mathbf{Q}_{F, M}^{\omega, \mathbf{b}^{*}} x\right\|_{L^{2}(\omega)}^{\mathbf{\infty} 2} \int_{B\left(M, M^{\prime}\right)} \frac{d \sigma(y)}{\left(|M|+\left|y-c_{M}\right|\right)^{2-\alpha}} \\
& \equiv \sum_{F^{\prime}} \sum_{M^{\prime}}\left\|\mathrm{Q}_{F^{\prime}, M^{\prime}}^{\omega, \mathbf{b}^{*}} x\right\|_{L^{2}(\omega)}^{\mathbf{\$} 2} S_{s}\left(M^{\prime}\right)
\end{aligned}
$$

and since $\int_{B\left(M, M^{\prime}\right)} \frac{d \sigma(y)}{\left(|M|+\left|y-c_{M}\right|\right)^{2-\alpha}} \approx \frac{\mathrm{P}^{\alpha}\left(M, \mathbf{1}_{B\left(M, M^{\prime}\right)} \sigma\right)}{|M|}$, it remains to show that for each fixed $M^{\prime}$,

$$
\begin{aligned}
S_{s}\left(M^{\prime}\right) & \approx \sum_{F} \sum_{M: d\left(c_{M}, c_{M^{\prime}}\right) \geq 2^{s(1+\delta)} \ell\left(M^{\prime}\right)}^{*} \frac{\left\|\mathrm{Q}_{F, M}^{\omega, \mathbf{b}^{*}} x\right\|_{L^{2}(\omega)}^{\mathbf{A}^{2}}}{\left|c_{M}-c_{M^{\prime}}\right|^{2-\alpha}} \frac{\mathrm{P}^{\alpha}\left(M, \mathbf{1}_{B\left(M, M^{\prime}\right)} \sigma\right)}{|M|} \\
& \lesssim 2^{-\delta s} \sqrt{\left(\mathfrak{E}_{2}^{\alpha}\right)^{2}+A_{2}^{\alpha, \text { energy }}} \sqrt{A_{2}^{\alpha}} .
\end{aligned}
$$

We write

$$
\begin{aligned}
(10.57) S_{s}\left(M^{\prime}\right) & \approx \sum_{k \geq s(1+\delta)} \frac{1}{\left(2^{k}\left|M^{\prime}\right|\right)^{2-\alpha}} \sum_{F} \sum_{M: d\left(c_{M}, c_{M^{\prime}}\right) \approx 2^{k} \ell\left(M^{\prime}\right)}^{*}\left\|\mathrm{Q}_{F, M^{*}}^{\omega, \mathbf{b}^{*}} x\right\|_{L^{2}(\omega)}^{\mathbf{\omega} 2} \frac{\mathrm{P}^{\alpha}\left(M, \mathbf{1}_{B\left(M, M^{\prime}\right)} \sigma\right)}{|M|} \\
& =\sum_{k \geq s(1+\delta)} \frac{1}{\left(2^{k}\left|M^{\prime}\right|\right)^{2-\alpha}} S_{s}^{k}\left(M^{\prime}\right) ; \\
S_{s}^{k}\left(M^{\prime}\right) & \equiv \sum_{F} \sum_{M: d\left(c_{M}, c_{M^{\prime}}\right) \approx 2^{k} \ell\left(M^{\prime}\right)}^{*}\left\|\mathrm{Q}_{F, M}^{\omega, \mathbf{b}^{*}} x\right\|_{L^{2}(\omega)}^{\mathbf{\omega} 2} \frac{\mathrm{P}^{\alpha}\left(M, \mathbf{1}_{B\left(M, M^{\prime}\right)} \sigma\right)}{|M|},
\end{aligned}
$$


where by $d\left(c_{M}, c_{M^{\prime}}\right) \approx 2^{k} \ell\left(M^{\prime}\right)$ we mean $2^{k} \ell\left(M^{\prime}\right) \leq d\left(c_{M}, c_{M^{\prime}}\right) \leq 2^{k+1} \ell\left(M^{\prime}\right)$. Moreover, if $d\left(c_{M}, c_{M^{\prime}}\right) \approx$ $2^{k} \ell\left(M^{\prime}\right)$, then from the fact that the radius of $B\left(M, M^{\prime}\right)$ is $\frac{1}{2} d\left(c_{M}, c_{M^{\prime}}\right)$, we obtain

$$
B\left(M, M^{\prime}\right) \subset C_{0} 2^{k} M^{\prime},
$$

where $C_{0}$ is a positive constant $\left(C_{0}=6\right.$ works).

For fixed $k \geq s(1+\delta)$, we invoke yet again the 'prepare to puncture' argument. Choose an augmented interval $\widetilde{M^{\prime}} \in \mathcal{A D}$ such that $C_{0} 2^{k} M \subset \widetilde{M^{\prime}}$ and $\ell\left(\widetilde{M^{\prime}}\right) \leq C 2^{k} \ell\left(M^{\prime}\right)$. Define $\widetilde{\omega}=\omega-\omega(\{p\}) \delta_{p}$ where $p$ is an atomic point in $\widetilde{M^{\prime}}$ for which

$$
\omega(\{p\})=\sup _{q \in \mathfrak{P}_{(\sigma, \omega)}: q \in \widetilde{M}^{\prime}} \omega(\{q\}) .
$$

(If $\omega$ has no atomic point in common with $\sigma$ in $\widetilde{M}^{\prime}$, set $\widetilde{\omega}=\omega$.) Then we have $\left|\widetilde{M}^{\prime}\right|_{\widetilde{\omega}}=\omega\left(\widetilde{M}^{\prime}, \mathfrak{P}_{(\sigma, \omega)}\right)$ and so from (10.37) and (10.18),

$$
\frac{\left|\widetilde{M}^{\prime}\right|_{\widetilde{\omega}}}{\left|\widetilde{M^{\prime}}\right|^{1-\alpha}} \frac{\left|\widetilde{M}^{\prime}\right|_{\sigma}}{\left|\widetilde{M^{\prime}}\right|^{1-\alpha}} \leq A_{2}^{\alpha, \text { punct }} \text { and } \sum_{F \in \mathcal{F}}\left\|Q_{F, A}^{\omega, \mathbf{b}^{*}} x\right\|_{L^{2}(\omega)}^{\mathbf{\omega}^{2}} \lesssim \ell(A)^{2}|A|_{\widetilde{\omega}} \text { for any interval } A \text {. }
$$

Now we are ready to apply Cauchy-Schwarz and the augmented local estimate (10.33) in Lemma 111 with $M=\widetilde{M^{\prime}}$ to the second line below, and to apply the argument in (10.56) to the first line below, to get the following estimate for $S_{s}^{k}\left(M^{\prime}\right)$ defined in (10.57) above:

$$
\begin{aligned}
& S_{s}^{k}\left(M^{\prime}\right) \leq\left(\sum_{F \quad M: d\left(c_{M}, c_{M^{\prime}}\right) \approx 2^{k} \ell\left(M^{\prime}\right)}\left\|Q_{F, M}^{\omega, \mathbf{b}^{*}} x\right\|_{L^{2}(\omega)}^{\mathbf{\omega} 2}\right)^{\frac{1}{2}} \\
& \times\left(\sum_{F} \sum_{M: d\left(c_{M}, c_{M^{\prime}}\right) \approx 2^{k} \ell\left(M^{\prime}\right)}\left\|Q_{F, M}^{\omega, \mathbf{b}^{*}} x\right\|_{L^{2}(\omega)}^{\boldsymbol{\omega}^{2}}\left(\frac{\mathrm{P}^{\alpha}\left(M, \mathbf{1}_{B\left(M, M^{\prime}\right)} \sigma\right)}{|M|}\right)^{2}\right)^{\frac{1}{2}} \\
& \lesssim\left(2^{2 s}\left|\widetilde{M}^{\prime}\right|^{2}\left|\widetilde{M}^{\prime}\right|_{\widetilde{\omega}}\right)^{\frac{1}{2}}\left(\left[\left(\mathfrak{E}_{2}^{\alpha}\right)^{2}+A_{2}^{\alpha, \text { energy }}\right]\left|\widetilde{M}^{\prime}\right|_{\sigma}\right)^{\frac{1}{2}} \\
& \lesssim \sqrt{\left(\mathfrak{E}_{2}^{\alpha}\right)^{2}+A_{2}^{\alpha, \text { energy }}} 2^{s}\left|\widetilde{M^{\prime}}\right| \sqrt{\left|\widetilde{M^{\prime}}\right|_{\widetilde{\omega}}} \sqrt{\left|\widetilde{M^{\prime}}\right|_{\sigma}} \\
& \lesssim \sqrt{\left(\mathfrak{E}_{2}^{\alpha}\right)^{2}+A_{2}^{\alpha, \text { energy }}} \sqrt{A_{2}^{\alpha, \text { punct }}} 2^{s}\left|\widetilde{M^{\prime}}\right|\left|\widetilde{M}^{\prime}\right|^{1-\alpha} \\
& \approx \sqrt{\left(\mathfrak{E}_{2}^{\alpha}\right)^{2}+A_{2}^{\alpha, \text { energy }}} \sqrt{A_{2}^{\alpha, \text { punct }}} 2^{s} 2^{k(1-\alpha)}\left|M^{\prime}\right|^{2-\alpha}, \quad \text { since } \ell\left(\widetilde{M^{\prime}}\right) \approx 2^{k} \ell\left(M^{\prime}\right) \text {. }
\end{aligned}
$$

Altogether then we have

$$
\begin{aligned}
S_{s}\left(M^{\prime}\right) & =\sum_{k \geq(1+\delta) s} \frac{1}{\left(2^{k}\left|M^{\prime}\right|\right)^{2-\alpha}} S_{s}^{k}\left(M^{\prime}\right) \\
& \lesssim \sqrt{\left(\mathfrak{E}_{2}^{\alpha}\right)^{2}+A_{2}^{\alpha, \text { energy }}} \sqrt{A_{2}^{\alpha, \text { punct }}} \sum_{k \geq(1+\delta) s} \frac{1}{\left(2^{k}\left|M^{\prime}\right|\right)^{2-\alpha}} 2^{s} 2^{k(1-\alpha)}\left|M^{\prime}\right|^{2-\alpha} \\
& =\sqrt{\left(\mathfrak{E}_{2}^{\alpha}\right)^{2}+A_{2}^{\alpha, \text { energy }}} \sqrt{A_{2}^{\alpha, \text { punct }}} \sum_{k \geq(1+\delta) s} 2^{s-k} \lesssim 2^{-\delta s} \sqrt{\left(\mathfrak{E}_{2}^{\alpha}\right)^{2}+A_{2}^{\alpha, \text { energy }}} \sqrt{A_{2}^{\alpha, \text { punct }}},
\end{aligned}
$$

which is summable in $s$. This completes the proof of (10.42), and hence of the estimate for Back $(\widehat{I})$ in (10.38).

The proof of Proposition 100 is now complete. 


\section{Appendix C: Errata for the Revista paper}

The current paper adapts the arguments of our 2016 Revista paper SaShUr7 whenever possible (which in turn adapted arguments from many earlier papers of various authors). To aid the reader in consulting [SaShUr7, we include here a list of up-to-date errata for the Revista paper [SaShUr7.

\#1: Lemma 3.1 on page 90 and its proof should be replaced with the following taken from [SaShUr6 arXiv: $1505.07816 \mathrm{v} 3]$.

Lemma 3.1 Given $\mathbf{r} \geq 3, \tau \geq 1$ and $\frac{1}{\mathbf{r}}<\varepsilon<1-\frac{1}{\mathbf{r}}$, we have

$$
\mathcal{D}_{(\mathbf{r}-1, \delta)-\text { good }} \subset \mathcal{D}_{(\mathbf{r}, \varepsilon) \text {-good }}^{\tau},
$$

provided

$$
0<\delta \leq \frac{\mathbf{r} \varepsilon-1}{\mathbf{r}+\boldsymbol{\tau}}
$$

Proof. Suppose that $I \in \mathcal{D}_{(\mathbf{r}-1, \delta) \text {-good }}$ where $\delta$ is as above. If $J$ is a child of $I$, then $J \in \mathcal{D}_{(\mathbf{r}, \delta) \text {-good }}$, and since $\delta<\varepsilon$, we also have $\mathcal{D}_{(\mathbf{r}, \delta) \text {-good }} \subset \mathcal{D}_{(\mathbf{r}, \varepsilon) \text {-good }}$. It remains to show that $\pi_{\mathcal{D}}^{(m)} I \in \mathcal{D}_{(\mathbf{r}, \varepsilon) \text {-good }}$ for $0 \leq m \leq \boldsymbol{\tau}$. For this it suffices to show that if $K \in \mathcal{D}$ satisfies $\pi_{\mathcal{D}}^{(m)} I \subset K$ and $\ell\left(\pi_{\mathcal{D}}^{(m)} I\right) \leq 2^{-\mathbf{r}} \ell(K)$, then

$$
\frac{1}{2}\left(\frac{\ell\left(\pi_{\mathcal{D}}^{(m)} I\right)}{\ell(K)}\right)^{\varepsilon} \ell(K) \leq \operatorname{dist}\left(\pi_{\mathcal{D}}^{(m)} I, K^{c}\right) .
$$

Now $\ell(I)=2^{-m} \ell\left(\pi_{\mathcal{D}}^{(m)} I\right) \leq 2^{-(m+\mathbf{r})} \ell(K) \leq 2^{-(\mathbf{r}-1)} \ell(K)$ and $I \in \mathcal{D}_{(\mathbf{r}-1, \delta)-\text { good }}$ imply that

$$
\frac{1}{2}\left(\frac{\ell(I)}{\ell(K)}\right)^{\delta} \ell(K) \leq \operatorname{dist}\left(I, K^{c}\right)
$$

and since the triangle inequality gives

$$
\operatorname{dist}\left(I, K^{c}\right) \leq \operatorname{dist}\left(\pi_{\mathcal{D}}^{(m)} I, K^{c}\right)+2^{m} \ell(I)
$$

we see that it suffices to show

$$
\frac{1}{2}\left(\frac{\ell\left(\pi_{\mathcal{D}}^{(m)} I\right)}{\ell(K)}\right)^{\varepsilon} \ell(K)+2^{m} \ell(I) \leq \frac{1}{2}\left(\frac{\ell(I)}{\ell(K)}\right)^{\delta} \ell(K), \quad 0 \leq m \leq \boldsymbol{\tau} .
$$

This is equivalent to successively,

$$
\begin{aligned}
\frac{1}{2}\left(\frac{2^{m} \ell(I)}{\ell(K)}\right)^{\varepsilon} \ell(K)+2^{m} \ell(I) & \leq \frac{1}{2}\left(\frac{\ell(I)}{\ell(K)}\right)^{\delta} \ell(K) ; \\
\left(\frac{2^{m} \ell(I)}{\ell(K)}\right)^{\varepsilon}+2^{m+1} \frac{\ell(I)}{\ell(K)} & \leq\left(\frac{\ell(I)}{\ell(K)}\right)^{\delta} ; \\
2^{m \varepsilon}\left(\frac{\ell(I)}{\ell(K)}\right)^{\varepsilon-\delta}+2^{m+1}\left(\frac{\ell(I)}{\ell(K)}\right)^{1-\delta} & \leq 1, \quad 0 \leq m \leq \tau .
\end{aligned}
$$

Since $0<\delta<\varepsilon<1$ by our restriction on $\varepsilon$ and our choice of $\delta$, and since $\frac{\ell(I)}{\ell(K)} \leq 2^{-(m+\mathbf{r})}$, it thus suffices to show that

$$
\begin{gathered}
2^{m \varepsilon}\left(2^{-(m+\mathbf{r})}\right)^{\varepsilon-\delta}+2^{m+1}\left(2^{-(m+\mathbf{r})}\right)^{1-\delta} \leq 1, \\
\text { i.e. } 2^{m \varepsilon-(m+\mathbf{r})(\varepsilon-\delta)}+2^{m+1-(m+\mathbf{r})(1-\delta)} \leq 1,
\end{gathered}
$$

for $0 \leq m \leq \boldsymbol{\tau}$. In particular then it suffices to show both

$$
\begin{aligned}
m \varepsilon-(m+\mathbf{r})(\varepsilon-\delta) & \leq-1, \\
m+1-(m+\mathbf{r})(1-\delta) & \leq-1,
\end{aligned}
$$


equivalently both

$$
\begin{aligned}
& (\mathbf{r}+m) \delta \leq \mathbf{r} \varepsilon-1, \\
& (\mathbf{r}+m) \delta \leq \mathbf{r}-2,
\end{aligned}
$$

for $0 \leq m \leq \boldsymbol{\tau}$. Finally then it suffices to show both

$$
\delta \leq \frac{\mathbf{r} \varepsilon-1}{\mathbf{r}+\boldsymbol{\tau}} \text { and } \delta \leq \frac{\mathbf{r}-2}{\mathbf{r}+\boldsymbol{\tau}} .
$$

Because of the restriction that $\frac{1}{\mathbf{r}}<\varepsilon<1-\frac{1}{\mathbf{r}}$, we see that $0<\mathbf{r} \varepsilon-1<\mathbf{r}-2$, and it is now clear that the above display holds for our choice of $\delta$.

\#2: In the integral in line 3 of page 93 , the factor $s_{I}(y)$ should be raised to the power $n-\alpha$.

\#3: The definition of $\varphi_{F}$ in line -2 of page 124 should read

$$
\varphi_{F} \equiv \sum_{k, \theta: \theta\left(I_{k}\right) \in \mathcal{F}} \mathbf{1}_{\theta\left(I_{k}\right)}\left(\mathbb{E}_{\theta\left(I_{k}\right)}^{\sigma} f-\mathbb{E}_{I_{K}}^{\sigma} f\right)
$$

and the display in line 3 of page 125 should read

$$
\left|\sum_{F \in \mathcal{F}}\left\langle T_{\sigma}^{\alpha} \varphi_{F}, g_{F}\right\rangle\right| \lesssim \sqrt{A_{2}^{\alpha}}\|f\|_{L^{2}(\sigma)}\|g\|_{L^{2}(\omega)},
$$

and finally in line -3 of page 125 , the constant $\mathcal{N} \mathcal{T} \mathcal{V}_{\alpha}$ should be replaced by $\mathfrak{T}_{T^{\alpha}}$ in both of its appearances.

\#4: The display beginning with the term $S$ in line 4 of page 134 should be replaced with

$$
\begin{aligned}
& S=\sum_{F \in \mathcal{F}_{I}} \sum_{J \in \mathcal{M}_{\mathbf{r}-\text { deep }}(F)}\left(\sum_{F^{\prime} \in \mathcal{F}: F \subset F^{\prime} \varsubsetneqq I} \frac{d\left(F^{\prime}\right)}{d\left(F^{\prime}\right)} \frac{\mathrm{P}^{\alpha}\left(J, \mathbf{1}_{\pi_{\mathcal{F}_{I}} F^{\prime} \backslash F^{\prime}} \sigma\right)}{|J|^{1 / n}}\right)^{2}\left\|\mathrm{P}_{F, J}^{\omega} \mathbf{x}\right\|_{L^{2}(\omega)}^{2} \\
& \leq \sum_{F^{\prime} \in \mathcal{F}_{I}} d\left(F^{\prime}\right)^{2} \sum_{F \in \mathcal{F}: F \subset F^{\prime}} \sum_{J \in \mathcal{M}_{\mathbf{r}-\text { deep }}(F)}\left(\sum_{F^{\prime} \in \mathcal{F}: F \subset F^{\prime} \varsubsetneqq I} \frac{1}{d\left(F^{\prime}\right)^{2}}\right)\left(\frac{\mathrm{P}^{\alpha}\left(J, \mathbf{1}_{\pi_{\mathcal{F}_{I}} F^{\prime} \backslash F^{\prime}} \sigma\right)}{|J|^{1 / n}}\right)^{2}\left\|\mathrm{P}_{F, J}^{\omega} \mathbf{x}\right\|_{L^{2}(\omega)}^{2} \\
& \leq C \sum_{F^{\prime} \in \mathcal{F}_{I}} d\left(F^{\prime}\right)^{2} \sum_{K \in \mathcal{M}_{\mathbf{r}-\text { deep }}\left(F^{\prime}\right)} \sum_{F \in \mathcal{F}: F \subset F^{\prime}} \sum_{J \in \mathcal{M}_{\mathbf{r}-\text { deep }}(F): J \subset K}\left(\frac{\mathrm{P}^{\alpha}\left(J, \mathbf{1}_{\pi_{\mathcal{F}_{I}} F^{\prime} \backslash F^{\prime}} \sigma\right)}{|J|^{1 / n}}\right)^{2}\left\|\mathrm{P}_{F, J}^{\omega} \mathbf{x}\right\|_{L^{2}(\omega)}^{2} \\
& \lesssim \sum_{F^{\prime} \in \mathcal{F}_{I}} d\left(F^{\prime}\right)^{2} \sum_{K \in \mathcal{M}_{\mathbf{r}-\text { deep }}\left(F^{\prime}\right)}\left(\frac{\mathrm{P}^{\alpha}\left(K, \mathbf{1}_{\pi_{\mathcal{F}_{I}} F^{\prime} \backslash F^{\prime}} \sigma\right)}{|K|^{1 / n}}\right)^{2} \sum_{F \in \mathcal{F}: F \subset F^{\prime}} \sum_{J \in \mathcal{M}_{\mathbf{r}-\text { deep }}(F): J \subset K}\left\|\mathrm{P}_{F, J}^{\omega} \mathbf{x}\right\|_{L^{2}(\omega)}^{2},
\end{aligned}
$$

and then the display at the bottom of page 134 should be replaced with

$$
\begin{aligned}
S & \lesssim \sum_{F^{\prime} \in \mathcal{F}_{I}} d\left(F^{\prime}\right)^{2} \sum_{K \in \mathcal{M}_{\mathbf{r}-\text { deep }}\left(F^{\prime}\right)}\left(\frac{\mathrm{P}^{\alpha}\left(K, \mathbf{1}_{\pi_{\mathcal{F}_{I}} F^{\prime} \backslash F^{\prime}} \sigma\right)}{|K|^{1 / n}}\right)^{2}\left\|\widehat{\mathrm{P}}_{F^{\prime}, K^{\prime}}^{\omega} \mathbf{x}\right\|_{L^{2}(\omega)}^{2} \\
& =\sum_{k=0}^{\infty} k^{2} \sum_{F^{\prime} \in \mathcal{F}_{I}: d\left(F^{\prime}\right)=k} \sum_{K \in \mathcal{M}_{\mathbf{r}-\text { deep }}\left(F^{\prime}\right)}\left(\frac{\mathrm{P}^{\alpha}\left(K, \mathbf{1}_{\left.\pi_{\mathcal{F}_{I}} F^{\prime} \backslash F^{\prime} \sigma\right)} \sigma\right.}{|K|^{1 / n}}\right)^{2}\left\|\widehat{\mathrm{P}}_{F^{\prime}, K^{\prime}}^{\omega, \mathbf{b}^{*}} \mathbf{x}\right\|_{L^{2}(\omega)}^{2} \equiv \sum_{k=0}^{\infty} A_{k} .
\end{aligned}
$$

\#5: Beginning two lines above the display at the bottom of page 144, and ending after the display at the top of page 145 , replace with the following:

$$
\sum_{F^{\prime} \in \mathcal{F}}\left\|Q_{F^{\prime}, A}^{\omega} x\right\|_{L^{2}(\omega)}^{2} \lesssim \tau|A|^{\frac{2}{n}}|A|_{\omega}, \quad \text { for any cube } A .
$$


Note that if $c_{J^{\prime}} \in 2 J$ and $\ell\left(J^{\prime}\right)<\ell(J)$, then $J^{\prime} \subset \frac{5}{2} J$. Then with

$$
\mathcal{W}_{J}^{s} \equiv \bigcup_{F^{\prime} \in \mathcal{F}}\left\{J^{\prime} \in \mathcal{M}_{\mathbf{r}-\text { deep }}\left(F^{\prime}\right): J^{\prime} \subset \frac{5}{2} J \text { and } \ell\left(J^{\prime}\right)=2^{-s} \ell(J)\right\},
$$

we have

$$
\begin{aligned}
\mathcal{A}_{s, f a r}^{0}(J) & \leq \sum_{F^{\prime} \in \mathcal{F}} \sum_{J_{J^{\prime}} \in 2 J}^{*} \int_{I \backslash(3 J)} S_{\left(J^{\prime}, J\right)}^{F^{\prime}}(y) d \sigma(y) \\
& \lesssim \sum_{A \in \mathcal{W}_{J}^{s} F^{\prime} \in \mathcal{F}: \sum_{A \in \mathcal{M}_{\mathbf{r}-\text { deep }}\left(F^{\prime}\right)}} \int_{I \backslash(3 J)} \frac{\left\|Q_{F^{\prime}, A^{\prime}}^{\omega} x\right\|_{L^{2}(\omega)}^{\mathbf{\omega}^{2}}}{\left(|J|^{\frac{2}{n}}+\left|y-c_{J}\right|\right)^{2(2-\alpha)}} d \sigma(y) \\
& \lesssim \sum_{A \in \mathcal{W}_{J}^{s}} \int_{I \backslash(3 J)} \frac{|A|^{\frac{2}{n}}|A|_{\omega}}{\left(|J|^{\frac{2}{n}}+\left|y-c_{M}\right|\right)^{2(2-\alpha)}} d \sigma(y) \\
& =\left(\sum_{A \in \mathcal{W}_{J}^{s}}|A|^{\frac{2}{n}}|A|_{\omega}\right) \int_{I \backslash(3 J)} \frac{1}{\left(|J|^{\frac{2}{n}}+\left|y-c_{J}\right|\right)^{2(2-\alpha)}} d \sigma(y)
\end{aligned}
$$

Now we use the standard pigeonholing of side length of $A$ to conclude that

$$
\begin{aligned}
\sum_{A \in \mathcal{W}_{J}^{s}}|A|^{\frac{2}{n}}|A|_{\omega} & =\sum_{k=s}^{\infty} \sum_{A \in \mathcal{W}_{J}^{s}: \ell(A)=2^{-k} \ell(J)}|A|^{\frac{2}{n}}|A|_{\omega} \leq \sum_{k=s}^{\infty} 2^{-2 k}|J|^{\frac{2}{n}} \sum_{A \in \mathcal{W}_{J}^{s}: \ell(A)=2^{-k} \ell(J)}|A|_{\omega} \\
& \leq \sum_{k=s}^{\infty} 2^{-2 k}|J|^{2}\left|\frac{5}{2} J\right|_{\omega} \lesssim 2^{-2 s}|J|^{2}\left|\frac{5}{2} J\right|_{\omega},
\end{aligned}
$$

so that combining the previous two displays we have

$$
\begin{aligned}
\mathcal{A}_{s, f a r}^{0}(J) & \lesssim 2^{-2 s}|J|^{2}\left|\frac{5}{2} J\right|_{\omega} \int_{I \backslash(3 J)} \frac{1}{\left(|J|^{\frac{2}{n}}+\left|y-c_{J}\right|\right)^{2(2-\alpha)}} d \sigma(y) \\
& \leq 2^{-2 s}\left|\frac{5}{2} J\right|_{\omega} \int_{I \backslash(3 J)} \frac{1}{\left(|J|^{\frac{2}{n}}+\left|y-c_{J}\right|\right)^{2(1-\alpha)}} d \sigma(y) \\
& \approx 2^{-2 s} \frac{\left|\frac{5}{2} J\right|_{\omega}}{\left|\frac{5}{2} J\right|^{1-\alpha}} \int_{I \backslash(3 J)}\left(\frac{|J|}{\left(|J|^{\frac{2}{n}}+\left|y-c_{J}\right|\right)^{2}}\right)^{1-\alpha} d \sigma(y) \\
& \lesssim 2^{-2 s} \frac{\left|\frac{5}{2} J\right|_{\omega}}{\left|\frac{5}{2} J\right|^{1-\alpha}} \mathcal{P}^{\alpha}\left(\frac{5}{2} J, \mathbf{1}_{I \backslash(3 J)} \sigma\right) \lesssim 2^{-2 s} \mathcal{A}_{2}^{\alpha} .
\end{aligned}
$$

\#6: Lines 3 through 11 on page 146 should be replaced with this:

Similarly, for $\ell \geq 1$, we can estimate the far term $\mathcal{A}_{s, \text { far }}^{\ell}(J)$ by the argument used for $\mathcal{A}_{s, \text { far }}^{0}(J)$ but applied to $2^{\ell} J$ in place of $J$. For this need the following variant of $\mathcal{W}_{J}^{s}$ given by

$$
\mathcal{W}_{J}^{s, \ell} \equiv \bigcup_{F^{\prime} \in \mathcal{F}}\left\{J^{\prime} \in \mathcal{W}\left(F^{\prime}\right): J^{\prime} \subset 3\left(2^{\ell} J\right) \text { and } \ell\left(J^{\prime}\right)=2^{-s-\ell} \ell\left(2^{\ell} J\right)\right\}
$$

Then we have 


$$
\begin{aligned}
\mathcal{A}_{s, f a r}^{\ell}(J) & \leq \sum_{F^{\prime} \in \mathcal{F}} \sum_{c_{J^{\prime}} \in\left(2^{\ell+1} J\right) \backslash\left(2^{\ell} J\right)}^{*} \int_{I \backslash 2^{\ell+2} J} S_{\left(J^{\prime}, J\right)}^{F^{\prime}}(y) d \sigma(y) \\
& \lesssim \sum_{A \in \mathcal{W}_{J}^{s, \ell} F^{\prime} \in \mathcal{F}: A \in \mathcal{M}_{\mathbf{r}-\text { deep }}\left(F^{\prime}\right)} \int_{I \backslash 4\left(2^{\ell} J\right)} \frac{\left\|Q_{F^{\prime}, A^{\prime}}^{\omega, \mathbf{b}^{*}} x\right\|_{L^{2}(\omega)}^{\mathbf{\omega}^{2}}}{\left(|J|^{\frac{2}{n}}+\left|y-c_{J}\right|\right)^{2(2-\alpha)}} d \sigma(y) \\
& \lesssim \sum_{A \in \mathcal{W}_{J}^{s, \ell}} \int_{I \backslash 4\left(2^{\ell} J\right)} \frac{|A|^{\frac{2}{n}}|A|_{\omega}}{\left(|J|^{\frac{2}{n}}+\left|y-c_{J}\right|\right)^{2(2-\alpha)}} d \sigma(y) \\
& =\left(\sum_{A \in \mathcal{W}_{J}^{s, \ell}}|A|^{\frac{2}{n}}|A|_{\omega}\right) \int_{I \backslash 4\left(2^{\ell} J\right)} \frac{1}{\left(|J|^{\frac{2}{n}}+\left|y-c_{J}\right|\right)^{2(2-\alpha)}} d \sigma(y),
\end{aligned}
$$

where, just as for the sum over $A \in \mathcal{W}_{J}^{s, 0}$,

$$
\begin{aligned}
\sum_{A \in \mathcal{W}_{J}^{s, \ell}}|A|^{\frac{2}{n}}|A|_{\omega} & =\sum_{k=s}^{\infty} \sum_{A \in \mathcal{W}_{J}^{s, \ell}: \ell(A)=2^{-k-\ell} \ell\left(2^{\ell} J\right)}|A|^{\frac{2}{n}}|A|_{\omega} \leq \sum_{k=s}^{\infty} 2^{-2 k-2 \ell}|J|^{\frac{2}{n}} \sum_{A \in \mathcal{W}_{M}^{s, \ell}: \ell(A)=2^{-k-\ell} \ell\left(2^{\ell} J\right)}|A|_{\omega} \\
& \leq \sum_{k=s}^{\infty} 2^{-2 k-2 \ell}\left|2^{\ell} J\right|^{\frac{2}{n}}\left|3\left(2^{\ell} J\right)\right|_{\omega} \lesssim 2^{-2 s-2 \ell}\left|2^{\ell} J\right|^{\frac{2}{n}}\left|3\left(2^{\ell} J\right)\right|_{\omega} .
\end{aligned}
$$

Now we can continue with

$$
\begin{aligned}
& \mathcal{A}_{s, f a r}^{\ell}(J) \lesssim 2^{-2 s} 2^{-2 \ell}\left|3\left(2^{\ell} J\right)\right|_{\omega} \int_{I \backslash 4\left(2^{\ell} J\right)} \frac{1}{\left(\left|2^{\ell} J\right|^{\frac{2}{n}}+\left|y-c_{2^{\ell} J}\right|\right)^{2(1-\alpha)}} d \sigma(y) \\
\approx & 2^{-2 s} 2^{-2 \ell} \frac{\left|3\left(2^{\ell} J\right)\right|_{\omega}}{\left|3\left(2^{\ell} J\right)\right|^{1-\alpha}} \int_{I \backslash 4\left(2^{\ell} J\right)}\left(\frac{\left|2^{\ell} J\right|^{\frac{2}{n}}}{\left(\left|2^{\ell} J\right|^{\frac{2}{n}}+\left|y-c_{2^{\ell} J}\right|\right)^{2}}\right)^{1-\alpha} d \sigma(y) \\
\lesssim & 2^{-2 s} 2^{-2 \ell}\left\{\frac{\left|3\left(2^{\ell} J\right)\right|_{\omega}}{\left|3\left(2^{\ell} J\right)\right|^{1-\alpha}} \mathcal{P}^{\alpha}\left(3\left(2^{\ell} J\right), \mathbf{1}_{I \backslash 4\left(2^{\ell} J\right)} \sigma\right)\right\} \lesssim 2^{-2 s} 2^{-2 \ell} \mathcal{A}_{2}^{\alpha} .
\end{aligned}
$$

\#7: The three lines above Section 11 should be replaced with these three lines in quotes: "13: and then expanding the square and calculating as in the proof of the local part given earlier to obtain the bound $\mathcal{A}_{2}^{\alpha}+\left(\mathcal{E}_{\alpha}^{\text {plug }}+\sqrt{A_{2}^{\alpha, \text { energy }}}\right) \sqrt{A_{2}^{\alpha, \text { punct }}}$. The details are similar and they are left to the reader."

\#8: In line -3 of page 164 the final factor on the right hand side should instead be $\omega_{\mathcal{P}}^{\text {small }}\left(\mathbf{T}^{\boldsymbol{\tau}-\text { deep }}(K)\right)$.

\#9: There is a gap in the proof of the Orthogonality Lemma at the top of page 170, where the restricted norm of the sublinear form used there doesn't match the definition on page 161. The required change in definition of the restricted norm, then forces an additional argument - due to Michael Lacey in Lac] - that uses another Calderón-Zygmund decomposition in the proof of the Straddling Lemma on page 166. Here are the changes required (see also Section 7 above, which adapts Lacey's additional argument to the $T b$ setting, in which the dual martingale differences are no longer orthogonal projections).

First, the display on page 161 right before Proposition 11.8 should be replaced with

$$
|\mathrm{B}|_{\text {stop }, 1, \triangle^{\omega}}^{A, \mathcal{P}}(f, g) \leq \mathfrak{N}_{\text {stop }, 1, \triangle^{\omega}}^{A, \mathcal{P}}\|f\|_{L^{2}(\sigma)}\|g\|_{L^{2}(\omega)},
$$

in which the term $\alpha_{\mathcal{A}}(A) \sqrt{|A|_{\sigma}}$ no longer appears on the right hand side.

\footnotetext{
${ }^{13}$ A detailed argument is given in Subsection 9.3 of [SaShUr6 v3], and in Subsection 10.5] of Appendix B above for the one dimensional case adapted to $T b$.
} 
Second, the sentence on page 169 right before Subsubsection 11.4.2 should be replaced with the following material:

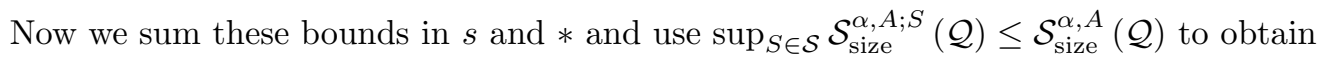

$$
|\mathrm{B}|_{\text {stop }, 1, \triangle^{\omega}}^{A, \mathcal{Q}}(f, g) \leq \mathcal{S}_{\text {size }}^{\alpha, A}(\mathcal{Q})\left\{\alpha_{\mathcal{A}}(A) \sqrt{|A|_{\sigma}}+\|f\|_{L^{2}(\sigma)}\right\}\|g\|_{L^{2}(\omega)} .
$$

However, this inequality has the unwanted term $\alpha_{\mathcal{A}}(A) \sqrt{|A|_{\sigma}}$ included on the right hand side, and we must apply an argument of M. Lacey [Lac, see the proof of Lemma 3.19] to eliminate this term. We begin with

$$
\begin{aligned}
|\mathrm{B}|_{\text {stop }, 1, \triangle^{\omega}}^{A, \mathcal{L}}(f, g) & =|\mathrm{B}|_{\text {stop }, 1, \triangle^{\omega}}^{A, \mathcal{L}}(h, g) \\
\text { where } h & \equiv \mathrm{P}_{\pi\left(\Pi_{1} \mathcal{Q}\right)}^{\sigma} f=\sum_{I \in \Pi_{1} \mathcal{Q}} \triangle_{\pi I}^{\sigma} f
\end{aligned}
$$

which follows from the formula $\varphi_{J}^{\mathcal{Q}} \equiv \sum_{I \in \mathcal{C}_{A}^{\prime}:(I, J) \in \mathcal{Q}} \mathbb{E}_{I}^{\sigma}\left(\triangle_{\pi I}^{\sigma} f\right) \mathbf{1}_{A \backslash I}$ since $\triangle_{\pi I}^{\sigma} f=\triangle_{\pi I}^{\sigma} h$ for $I \in \Pi_{1} \mathcal{Q}$, which holds in turn because the Haar projections are orthogonal. Now define Calderón-Zygmund stopping times $\mathcal{H}$ for the function $h=\mathrm{P}_{\pi\left(\Pi_{1} \mathcal{Q}\right)}^{\sigma} f \in L^{2}(\sigma)$, so that the quasiorthogonal inequality

$$
\sum_{H \in \mathcal{H}} \alpha_{\mathcal{H}}(H)^{2}|H|_{\sigma} \lesssim\|h\|_{L^{2}(\sigma)}^{2}=\left\|\mathrm{P}_{\pi\left(\Pi_{1} \mathcal{Q}\right)}^{\sigma} f\right\|_{L^{2}(\sigma)}^{2}
$$

holds with $\alpha_{\mathcal{H}}(H)=\mathbb{E}_{H}^{\sigma}|h|$. We also define in the usual way coronas $\mathcal{C}_{H}^{\mathcal{H}}$ and $\mathcal{C}_{H}^{\mathcal{H}, \boldsymbol{\tau}-\text { shift }}$.

Now we return to the previous inequalities we obtained for $|\mathrm{B}|_{\text {stop }, 1,{ }^{\omega}}^{A, \mathcal{Q}_{s}}(f, g)$ and $|\mathrm{B}|_{\text {stop }, 1, \triangle \omega}^{A, \mathcal{Q}_{*}}(f, g)$, and replace the collection $\mathcal{Q}$ with the $A$-admissible collection $\mathcal{Q}_{H} \equiv\left\{(I, J) \in \mathcal{Q}: J \in \mathcal{C}_{H}^{\mathcal{H}, \boldsymbol{\tau} \text {-shift }}\right\}$, so that the arguments given there can be adapted to yield

$$
\begin{aligned}
|\mathrm{B}|_{\text {stop }, 1, \triangle^{\omega}}^{A,\left(\mathcal{Q}_{H}\right)_{s}}(f, g) & \leq \sup _{S \in \mathcal{S}} \mathcal{S}_{\text {size }}^{\alpha, A ; S}\left(\mathcal{Q}_{H}\right) \alpha_{\mathcal{H}}(H) \sqrt{|H|_{\sigma}}\left\|\mathrm{P}_{\mathcal{C}_{H}^{\mathcal{H}, \tau-\text { shift }}}^{\omega} g\right\|_{L^{2}(\omega)}, \quad \boldsymbol{\tau} \leq s \leq \boldsymbol{\rho}-1, \\
|\mathrm{~B}|_{\text {stop }, 1, \triangle^{\prime} \omega}^{A,\left(\mathcal{Q}_{H}\right)_{*}}(f, g) & \leq \sup _{S \in \mathcal{S}} \mathcal{S}_{\text {size }}^{\alpha, A ; S}\left(\mathcal{Q}_{H}\right) \alpha_{\mathcal{H}}(H) \sqrt{|H|_{\sigma}}\left\|\mathrm{P}_{\mathcal{C}_{H}^{\mathcal{H}}, \boldsymbol{\tau}, \text { shift }}^{\omega,} g\right\|_{L^{2}(\omega)} .
\end{aligned}
$$

We then sum these improved bounds in $s$ and $*$ to obtain

$$
\begin{aligned}
|\mathrm{B}|_{\text {stop }, 1, \triangle \omega}^{A, \mathcal{Q}}(f, g) & \leq \sup _{S \in \mathcal{S}} \mathcal{S}_{\text {size }}^{\alpha, A ; S}(\mathcal{Q}) \sum_{H \in \mathcal{H}} \alpha_{\mathcal{H}}(H) \sqrt{|H|_{\sigma}}\left\|\mathrm{P}_{\mathcal{C}_{H}^{\mathcal{H}, \tau-\text { shift }}}^{\omega} g\right\|_{L^{2}(\omega)} \\
& \leq \sup _{S \in \mathcal{S}} \mathcal{S}_{\text {size }}^{\alpha, A ; S}(\mathcal{Q}) \sqrt{\sum_{H \in \mathcal{H}} \alpha_{\mathcal{H}}(H)^{2}|H|_{\sigma}} \sqrt{\sum_{H \in \mathcal{H}}\left\|\mathrm{P}_{\mathcal{C}_{H}^{\mathcal{H}, \tau-\text { shift }}}^{\omega} g\right\|_{L^{2}(\omega)}^{2}} \\
& \leq \mathcal{S}_{\text {size }}^{\alpha, A}(\mathcal{Q})\|f\|_{L^{2}(\sigma)}\|g\|_{L^{2}(\omega)},
\end{aligned}
$$

where we have used the quasiorthogonal inequality and $\left\|\mathrm{P}_{\pi\left(\Pi_{1} \mathcal{Q}\right)}^{\sigma} f\right\|_{L^{2}(\sigma)}^{2} \leq\|f\|_{L^{2}(\sigma)}^{2}$ in the last line.

\section{Appendix D: Glossary}

12.0.1. Section 1.

(1) $C_{C Z} ;(1.1)$

(2) $\mathfrak{N}_{T_{\sigma}^{\alpha}} ;(1.2)$

(3) $T_{\sigma, \delta, R}^{\alpha} f(x) ;(1.3)$

(4) $p$-weakly $\mu$-accretive family $\mathbf{b}=\left\{b_{Q}\right\}_{Q \in \mathcal{P}}$ of functions on $\mathbb{R}$; (1.4)

(5) $\mathfrak{T}_{T^{\alpha}}^{\mathbf{b}}, \mathfrak{T}_{T^{\alpha, *}}^{\mathbf{b}^{*}} ;(1.5)$

(6) $\mathrm{P}^{\alpha}(Q, \mu), \mathcal{P}^{\alpha}(Q, \mu) ;(1.6)$

(7) $\mathcal{A}_{2}^{\alpha}, \mathcal{A}_{2}^{\alpha, *} ;$ (1.7)

(8) $\mathfrak{P}_{(\sigma, \omega)}$; 1.9)

(9) $A_{2}^{\alpha, \text { punct }}, A_{2}^{\alpha, *, \text { punct }} ;(1.10)$

(10) $\mathfrak{A}_{2}^{\alpha} ; 1.11$

(11) $\mathcal{E}_{2}^{\alpha}, \mathcal{E}_{2}^{\alpha, *}, \mathfrak{E}_{2}^{\alpha}, \mathcal{N} \mathcal{T} \mathcal{V}_{\alpha} ;(1.12),(1.13),(1.14),(1.15)$ 


\subsubsection{Section 2.}

(1) gradient elliptic kernel $K^{\alpha}(x, y)$; (2.1)

(2) PLBP,pointwise lower bound property; (2.2)

(3) reverse Hölder control of children (2.15)

(4) Calderón-Zygmund stopping intervals; Definition 20

(5) b-accretive/weak testing stopping intervals; Definition 21

(6) $\sigma$-energy stopping intervals; Definition 23

(7) $\mathbf{X}_{\alpha}\left(\mathcal{C}_{S}\right)$ energy stopping times; (2.23)

(8) $\mathcal{D}_{\beta}:$ (2.28)

12.0.3. Section 3.

(1) skel $K, \mathbb{S}_{x}^{\mathrm{dy}}$, body $K$ skeleton, spray, body of an interval; (37)

(2) $\varepsilon$ - good with respect to an interval; (3.8)

(3) $\mathcal{G}_{(k, \varepsilon) \text {-good }}^{\mathcal{D}}$; Definition 39

(4) $k$-bad in a grid; (41)

(5) $R^{\text {w }}, \kappa(R): 42$

(6) $\Theta_{2}^{\text {bad }}(f, g)$; (3.15)

(7) $\mathcal{G}_{k-\text { bad }}^{\mathcal{D}}=\mathcal{G}_{(k, \varepsilon) \text {-bad }}^{\mathcal{D}}$; Definition 44

(8) $\Theta_{2}^{\text {good }}(f, g)$; (3.20)

(9) $3 \mathfrak{T}_{T^{\alpha}}^{\mathbf{b}} ;(3.23)$

(10) $\mathfrak{F T}_{T^{\alpha}}^{\mathbf{b}} ;(3.24)$

(11) $\mathcal{E}_{2}^{\alpha, \text { triple }} ;$ Definition 45

12.0.4. Section 4.

(1) $\Theta_{1}^{\text {long }}(f, g), \Theta_{1}^{\text {short }}(f, g)$; (4.1)

\subsubsection{Section 5.}

(1) $\partial_{\eta} L ;$ (5.7)

(2) $\mathcal{K}\left(I^{\prime}, J^{\prime}\right)$; (5.8)

(3) $\{E, F\}$; (5.11)

(4) $\mathrm{P}_{\delta}^{\alpha} \mathrm{Q}^{\omega}(J, v) ;$ (5.15)

(5) $\left\{K_{\text {out }}, K_{\text {in }}\right\}^{\text {orig }} ;$ (5.28)

12.0.6. Section 6.

(1) $\mathcal{C}_{B}^{\mathcal{G}}$,shift $;$ Definition 52

(2) $\left\langle T_{\sigma}^{\alpha}\left(\mathrm{P}_{\mathcal{C}_{A}^{\mathcal{D}}}^{\sigma, \mathbf{b}} f\right), \mathrm{P}_{\mathcal{C}_{B}^{\mathcal{G}, \text { shift }}}^{\omega, \mathbf{b}^{*}} g\right\rangle_{\omega}^{\epsilon_{\mathbf{r}, \varepsilon}} ;(6.2)$

(3) $\mathrm{B}_{\leftarrow_{\mathbf{r}, \varepsilon}}^{A}(f, g) ;(6.4)$

(4) Whitney subintervals $\mathcal{W}(F)$; (53)

(5) $\mathfrak{F}_{\alpha}=\mathfrak{F}_{\alpha}^{\mathbf{b}^{*}}(\mathcal{D}, \mathcal{G}) ;(54)$

(6) $\mathrm{B}_{\text {stop }}^{A}(f, g)$; (6.7), (6.17), (7.3)

(7) $\mathcal{C}_{F}^{\mathcal{G} \text {,shift }}$; Definition 56

(8) $\mathrm{Q}_{\mathcal{H}}^{\omega, \mathbf{b}^{*}} ; 6.10$

(9) $\mathrm{B}_{\text {broken }}^{A}(f, g)$; (6.18)

(10) $\mathrm{B}_{\text {neighbour }}^{A}(f, g)$; (6.20)

(11) $\mathrm{B}_{\text {stop }}^{A, \mathcal{P}}(f, g)$; 65)

12.0.7. Section 7.

(1) $\varphi_{J}^{\mathcal{P}} ; 7.6$

(2) $\widehat{\mathfrak{N}}_{\text {stop }, \triangle \omega}^{A, \mathcal{P}}$;67)

(3) $\Pi_{2}^{K} \mathcal{P}$; 69)

(4) $\Pi_{1}^{\text {below }} \mathcal{P}$; (70)

(5) $\mathcal{S}_{\text {init size }}^{\alpha, A}(\mathcal{P})^{2}$; (71) 
(6) $\Pi_{2}^{K \text {,aug }} \mathcal{P}$; Definition 72

(7) $\mathcal{S}_{\text {aug size }}^{\alpha, A}(\mathcal{P})$; Definition 72 (7.33)

(8) $\mathcal{P}_{\text {cor }}^{A} ; 7.20$

(9) $J^{\mathrm{b}}=J^{\mathrm{W}_{J}} ;$ (766)

(10) $\mathcal{Q}$ bstraddles $\mathcal{S}$; Definition 77

(11) $\mathcal{W}^{*}(S)$; (78)

(12) $\mathcal{S}_{\text {loc size }}^{\alpha, A ; S}(\mathcal{Q})^{2} ; 7.26$

(13) $\mathcal{Q}$ substraddles $L$; 81)

(14) $\omega_{\mathcal{P}}$ and $\omega_{b \mathcal{P}} ;$ (7.32)

(15) $\mathcal{G}_{d} ;$ (7.37)

(16) $\mathcal{P}_{L, t}^{\mathcal{H}} ;(7.38)$

(17) $\mathcal{P}^{\text {big }}, \mathcal{P}^{\text {small }} ;$ (7.39)

(18) $\mathcal{P}^{\text {bbig }}, \mathcal{P}^{\text {bsmall }} ;(7.40)$

12.0.8. Section 9.

(1) $\mathbb{E}_{Q}^{\mu, \mathbf{b}} f(x) ;(9.3)$

(2) $\widehat{\mathbb{F}}_{Q}^{\mu, \mathbf{b}} f(x) ;$ (9.4)

(3) $\triangle_{Q}^{\mu, \mathbf{b}} f(x), \square_{Q}^{\mu, \mathbf{b}} f(x) ;(9.5)$

(4) $\nabla_{Q}^{\mu}, \widehat{\nabla}_{Q}^{\mu} ; 9.6$

(5) $\mathbb{E}_{Q}^{\mu, \pi, \mathbf{b}} f, \mathbb{F}_{Q}^{\mu, \pi, \mathbf{b}} f ;(9.15)$

(6) $\triangle_{Q}^{\mu, \pi, \mathbf{b}} f, \square_{Q}^{\mu, \pi, \mathbf{b}} f ;$ (9.16)

(7) $\square_{I}^{\sigma, b, \mathbf{b}} f ;$ (9.21)

(8) $\widehat{\square}_{I}^{\sigma, b, \mathbf{b}} f ;(9.22)$

(9) $\triangle_{I, \text { broken }}^{\mu, b, \mathbf{b}} f, \square_{I, \text { broken }}^{\mu, b, \mathbf{b}} f ;$ (9.25)

(10) $\Psi_{\mathcal{B}, \boldsymbol{\lambda}}^{\mu, \mathbf{b}} f:$ Definition 94

(11) $\mathrm{P}_{\mathcal{B}}^{\mu} f ;(9.29)$

12.0.9. Section 10.

(1) $Q_{\mathcal{C}_{F}^{\mathcal{G} \text { shift }} ; M}^{\omega, \mathbf{w}^{*}} ;(10.2)$

(2) $\mathcal{C}_{F}^{\mathcal{G} \text {,shift }}, \mathcal{C}_{F}^{\mathcal{G} \text {,shift }} ; K ;(10.3$ )

(3) $J \Subset_{\rho, \varepsilon} K ;$ (10.5)

(4) $\mathcal{M}_{(\boldsymbol{\rho}, \varepsilon)-\operatorname{deep}, \mathcal{G}}(K), \mathcal{M}_{(\boldsymbol{\rho}, \varepsilon)-\operatorname{deep}, \mathcal{D}}(K), \mathcal{W}(K)$; 10.6)

(5) augmented dyadic grid $\mathcal{A D}$; Definition 102

(6) $Q_{K}^{\omega, b^{*}} ;(10.9)$

(7) $\mathcal{E}_{2}^{\alpha, \text { Whitney partial } ;(10.12)}$

(8) $A_{2}^{\alpha, \text { energy }}, A_{2}^{\alpha, * \text {,energy } ; ~(10.13) ~}$

(9) $\mathcal{E}_{2}^{\alpha, \text { Whitney plug ; 10.15) }}$

(10) $\mu$; 10.19)

(11) $\mathrm{P}_{F, K}^{\omega, \mathbf{b}^{*}} \equiv \mathrm{P}_{\mathcal{C}_{F}^{\mathcal{G}}, \omega \text {,shift }}^{\omega, \mathbf{b}_{K}^{*}} ;$ (10.20)

(12) Local $(I), \bar{\mu}$; 10.26)

(13) $\mathcal{J}^{*} ;$ (10.34)

(14) $\operatorname{Back}(\widehat{I})$; (10.38)

(15) $U_{s}$; (10.41)

(16) $B\left(M, M^{\prime}\right) ;(10.44)$

(17) $T_{s}^{\text {intersection; }}(10.45)$

(18) $T_{s}^{\text {proximal }} ;(10.46)$

(19) $T_{s}^{\text {difference; (10.47) }}$

(20) $\sum^{*}$; Notation 112

(21) $\mathcal{W}_{M}^{s} ;$ (10.51) 
(22) $\mathcal{W}_{M}^{s, \ell} ;(10.55)$

\section{REFERENCES}

[AAAHK] M. A. Alfonseca, P. Auscher, A. Axelsson, S. Hofmann, And S. Kim, Analyticity of layer potentials and $L^{2}$ solvability of boundary value problems for divergence form elliptic equations with complex $L^{\infty}$ coefficients, arXiv:0705.0836v1.

[AuHoMctc] Auscher, P., Hofmann, S., Lacey, M., McIntosh, A., and Tchamitchian, P., The Solution of the Kato Square Root Problem for Second Order Elliptic Operators on $\mathbb{R}^{n}$, Ann. of Math. 156 (2002), 633-654.

[AuHoMuTaTh] P. Auscher, S. Hofmann, C. Muscalu, T. TaO, and C. Thiele, Carleson measures, trees, extrapolation, and $T(b)$ theorems, Publ. Mat. 46 (2002), no. 2, 257-325.

[Chr] Christ, M., A T (b) theorem with remarks on analytic capacity and the Cauchy integral, Colloq. Math. 60/61 (1990), 601-628.

[CoJoSe] R. R. Coifman, P. W. Jones And S. Semmes, Two elementary proofs of the L $L^{2}$ boundedness of Cauchy integrals on Lipschitz curves, Journal of the A.M.S. 2 (1989), p. 553-564.

[Dav1] DAvID, G., Unrectifiable 1-sets have vanishing analytic capacity, Rev. Mat. Iberoamericana 14 (2) (1998), 369-479.

[Dav2] David, G., Analytic capacity, Calderón-Zygmund operators, and rectifiability, Publ. Mat. 43 (1) (1999), 3-25.

[DaJo] David, GuY, Journé, Jean-Lin, A boundedness criterion for generalized Calderón-Zygmund operators, Ann. of Math. (2) 120 (1984), 371-397, MR763911 (85k:42041).

[DaJoSe] David,G.,Journé,J.-L.,AndSemmes,S.,Opérateurs de Calderón-Zygmund, fonctions para-accrétives et interpolation. Rev. Mat. Iberoamericana 1 (1985), 1-56.

[Hof $]$ Hofmann, S., A proof of the local Tb theorem for standard Calderón-Zygmund operators, arXiv:0705.0840 1.

[HoLaMc] Hofmann, S., Lacey, M., and McIntosh, A., The solution of the Kato problem for divergence form elliptic operators with Gaussian heat kernel bounds. Ann. of Math. 156 (2002), 623-631.

[HoMc] Hofmann, S., And McIntosh, A., The solution of the Kato problem in two dimensions, In Proceedings of the Conference on Harmonic Analysis and PDE (El Escorial, 2000), Publ. Mat. Extra Vol. (2002), 143-160.

[HuMuWh] R. Hunt, B. Muckenhoupt And R. L. Wheeden, Weighted norm inequalities for the conjugate function and the Hilbert transform, Trans. Amer. Math. Soc. 176 (1973), 227-251.

[Hyt] Hytönen, Tuomas, On Petermichl's dyadic shift and the Hilbert transform, C. R. Math. Acad. Sci. Paris 346 (2008), MR2464252.

[Hyt2] Hytönen, TuOmas, The two weight inequality for the Hilbert transform with general measures, arXiv:1312.0843v2.

[HyMa] Hytönen, TuOmas and H. Martikainen, On general local Tb theorems, arXiv:1011.0642v1.

[Lac] Lacey, Michael T., Two weight inequality for the Hilbert transform: A real variable characterization, II, Duke Math. J. Volume 163, Number 15 (2014), 2821-2840.

[LaMa] M. T. LACEY AND H. MARTIKAINEN, Local Tb theorem with $L^{2}$ testing conditions and general measures: Calderón-Zygmund operators, arXiv:1310.08531v1.

[LaSaUr1] Lacey, Michael T., Sawyer, Eric T., Uriarte-Tuero, Ignacio, A characterization of two weight norm inequalities for maximal singular integrals with one doubling measure, Analysis \& PDE, Vol. 5 (2012), No. 1, $1-60$.

[LaSaUr2] Lacey, Michael T., Sawyer, Eric T., Uriarte-Tuero, Ignacio, A Two Weight Inequality for the Hilbert transform assuming an energy hypothesis, Journal of Functional Analysis, Volume 263 (2012), Issue 2, 305363.

[LaSaShUr2] Lacey, Michael T., Sawyer, Eric T., Shen, Chun-Yen, Uriarte-Tuero, Ignacio, Two Weight Inequality for the Hilbert Transform: A Real Variable Characterization, arXiv:1201.4319 (2012).

[LaSaShur3] Lacey, Michael T., Sawyer, Eric T., Shen, Chun-Yen, Uriarte-Tuero, Ignacio, Two weight inequality for the Hilbert transform: A real variable characterization I, Duke Math. J, Volume 163, Number 15 (2014), $2795-2820$.

[LaWi] Lacey, Michael T., Wick, Brett D., Two weight inequalities for Riesz transforms: uniformly full dimension weights, arXiv:1312.6163v3.

[MaMeVe] Mattila, P., Melnikov, M., and Verdera, J., The Cauchy integral, analytic capacity, and uniform rectifiability, Ann. of Math. (2) 144 (1) (1996), 127-136.

[NTV1] Nazarov, F., Treil, S., And Volberg, A., Weak type estimates and Cotlar inequalities for CalderónZygmund operators on nonhomogeneous spaces, 1998 (9) IMRN (1997).

[NTV2] Nazarov, F., Treil, S. And Volberg, A., The Tb-theorem on non-homogeneous spaces, Acta Math. 190 (2003), no. 2, MR 1998349 (2005d:30053).

[NTV3] Nazarov, F., Treil, S., and Volberg, A., Accretive system Tb-theorems on nonhomogeneous spaces, Duke Math. J. 113 (2) (2002), 259-312.

[NTV4] F. Nazarov, S. Treil and A. Volberg, Two weight estimate for the Hilbert transform and corona decomposition for non-doubling measures, preprint (2004) arxiv:1003.1596

[Saw] E. SAwYer, A characterization of two weight norm inequalities for fractional and Poisson integrals, Trans. A.M.S. 308 (1988), 533-545, MR $\{930072$ (89d:26009)\}.

[SaShUr2] Sawyer, Eric T., Shen, Chun-Yen, Uriarte-Tuero, Ignacio, A two weight theorem for $\alpha$-fractional singular integrals with an energy side condition, arXiv:1302.5093 $\mathrm{v}$. 
[SaShUr3] Sawyer, Eric T., Shen, Chun-Yen, Uriarte-Tuero, Ignacio, A geometric condition, necessity of energy, and two weight boundedness of fractional Riesz transforms, arXiv: $1310.4484 \mathrm{v} 1$.

[SaShUr4] Sawyer, Eric T., Shen, Chun-Yen, Uriarte-Tuero, Ignacio, A note on failure of energy reversal for classical fractional singular integrals, IMRN, Volume 2015, Issue 19, 9888-9920.

[SaShUr5] Sawyer, Eric T., Shen, Chun-Yen, Uriarte-Tuero, Ignacio, A two weight theorem for $\alpha$-fractional singular integrals with an energy side condition and quasicube testing, arXiv:1302.5093v10.

[SaShUr6] Sawyer, Eric T., Shen, Chun-Yen, Uriarte-Tuero, Ignacio, A two weight theorem for $\alpha$ fractional singular integrals with an energy side condition, quasicube testing and common point masses, arXiv: $1505.07816 \mathrm{v} 2$, v3

[SaShUr7] Sawyer, Eric T., Shen, Chun-Yen, Uriarte-Tuero, Ignacio, A two weight theorem for $\alpha$-fractional singular integrals with an energy side condition, Revista Mat. Iberoam. 32 (2016), no. 1, 79-174.

[SaShUr8] Sawyer, Eric T., Shen, Chun-Yen, Uriarte-Tuero, Ignacio, The two weight T1 theorem for fractional Riesz transforms when one measure is supported on a curve, arXiv:1505.07822v4.

[SaShUr9] Sawyer, Eric T., Shen, Chun-Yen, Uriarte-Tuero, Ignacio, A two weight fractional singular integral theorem with side conditions, energy and k-energy dispersed, Harmonic Analysis, Partial Differential Equations, Complex Analysis, Banach Spaces, and Operator Theory (Volume 2) (Celebrating Cora Sadosky's life), Springer 2017 (see also arXiv: 1603.04332v2).

[SaShUr10] Sawyer, Eric T., Shen, Chun-Yen, Uriarte-Tuero, Ignacio, A good- $\lambda$ lemma, two weight T1 theorems without weak boundedness, and a two weight accretive global Tb theorem, Harmonic Analysis, Partial Differential Equations and Applications (In Honor of Richard L. Wheeden), Birkhäuser 2017 (see also arXiv: $1609.08125 \mathrm{v} 2)$.

[SaShUr11] Sawyer, Eric T., Shen, Chun-Yen, Uriarte-Tuero, Ignacio, A counterexample in the theory of CalderónZygmund operators, arXiv:16079.06071v3v1.

[SaWh] E. SAWYER AND R. L. WHEEDEN, Weighted inequalities for fractional integrals on Euclidean and homogeneous spaces, Amer. J. Math. 114 (1992), 813-874.

[Ste] E. M. Stein, Harmonic Analysis: real-variable methods, orthogonality, and oscillatory integrals, Princeton University Press, Princeton, N. J., 1993.

[Tol] Tolsa, X., Painlevés problem and the semiadditivity of analytic capacity, Acta Math. 190 (1) (2003), 105149.

[Vol] A. Volberg, Calderón-Zygmund capacities and operators on nonhomogeneous spaces, CBMS Regional Conference Series in Mathematics (2003), MR\{2019058 (2005c:42015)\}.

Department of Mathematics \& Statistics, McMaster University, 1280 Main Street West, Hamilton, Ontario, CANADA L8S $4 \mathrm{~K} 1$

E-mail address: sawyer@mcmaster.ca

Department of Mathematics,, National Taiwan University, 10617, Taipei

E-mail address: cyshen@math.ntu.edu.tw

Department of Mathematics, Michigan State University, East Lansing Mi

E-mail address: ignacio@math.msu.edu 